Workplace

Safety and Health

\title{
WORK-RELATED LUNG DISEASE SURVEILLANCE REPORT 2002
}
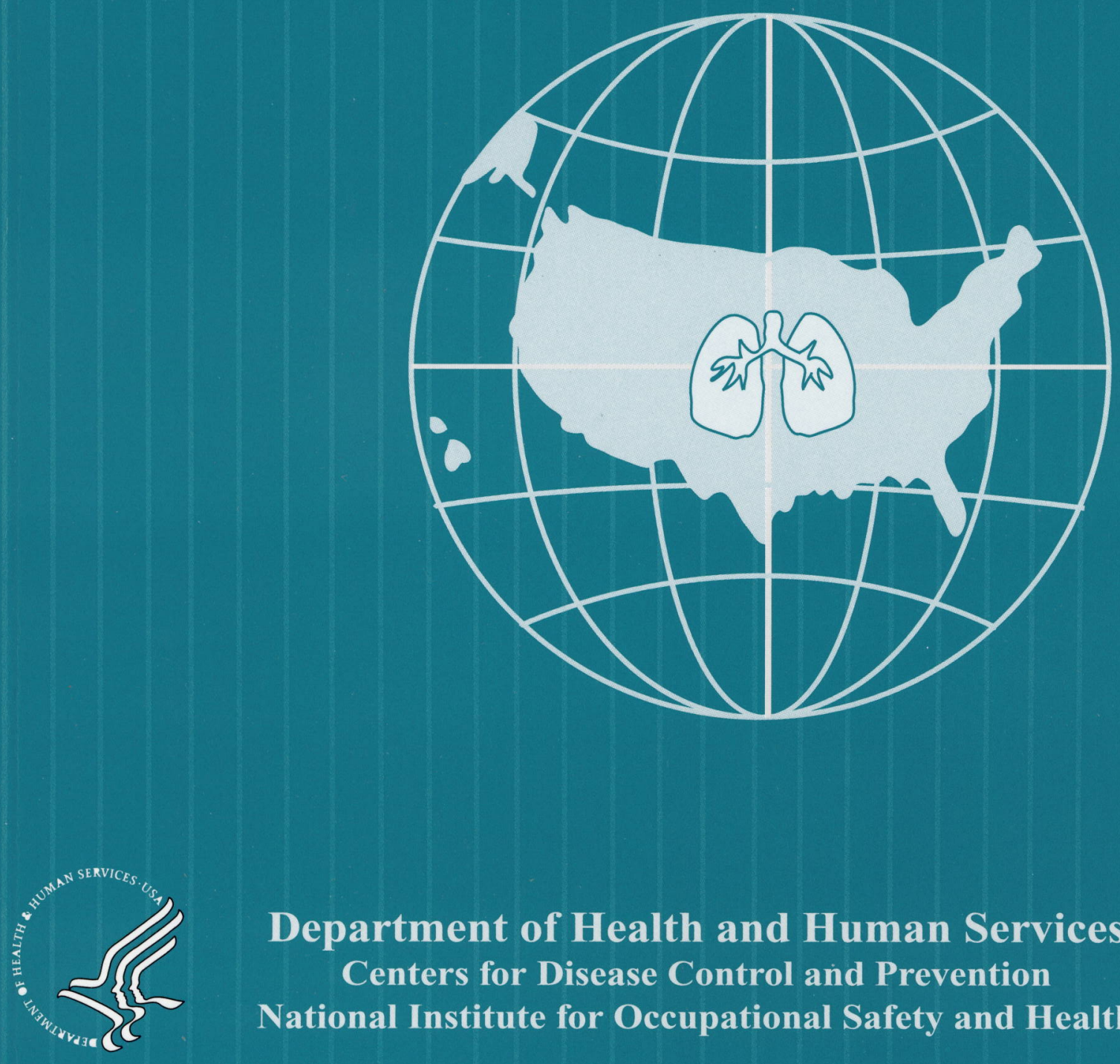

Department of Health and Human Services Centers for Disease Control and Prevention National Institute for Occupational Safety and Health Nosin 


\title{
Work-Related Lung Disease Surveillance Report 2002
}

\author{
Division of Respiratory Disease Studies \\ National Institute for Occupational Safety and Health
}

U.S. Department of Health and Human Services

Public Health Service

Centers for Disease Control and Prevention

May 2003 


\section{DISCLAIMER}

Mention of the name of any company or product does not constitute endorsement by the National Institute for Occupational Safety and Heath.

All web addresses referenced in this report were accessible as of the date this manuscript was prepared for publication.

This document is in the public domain and may be freely copied or reprinted.

Copies of this and other NIOSH documents are available from:

Publications Dissemination, EID

National Institute for Occupational Safety and Health

4676 Columbia Parkway

Cincinnati, OH 45226-1998

Fax (513) 533-8573

DHHS (NIOSH) Number 2003-111

For further information about occupational safety and health, call 1-800-35-NIOSH 
This Work-Related Lung Disease (WoRLD) Surveillance Report is the sixth in a series of occupational respiratory disease surveillance reports (see page $i v$ ) produced by the National Institute for Occupational Safety and Health (NIOSH). It presents summary tables and figures of occupational respiratory disease surveillance data focusing on various occupationally-relevant respiratory diseases, including pneumoconioses, occupational asthma and other airways diseases, and several other respiratory conditions. For many of these diseases, selected data on related exposures are also presented.

The 2002 WoRLD Surveillance Report has three major sections: (1) a section that provides data highlights and data usage limitations; (2) a section comprised of 15 subsections, each concerning a major disease category and (where available) related occupational exposures, and one subsection concerning smoking status; (3) a section of appendices that provide descriptions of data sources, methods, and other supplementary information.

Similar to the 1999 WoRLD Surveillance Report, this report includes data on hypersensitivity pneumonitis, asthma, chronic obstructive pulmonary disease, respiratory conditions due to chemical fumes and vapors, and other work-related respiratory conditions, in addition to the pneumoconioses. This report updates pneumoconiosis mortality data published in the 1999 WoRLD Surveillance Report by the addition of currently available data for 1997 through 1999. Pneumoconiosis conditions highlighted include asbestosis, coal workers' pneumoconiosis, silicosis, byssinosis, and pneumoconioses coded as either "unspecified" or "other," and all pneumoconioses aggregated. The current report presents data on conditions not included in earlier reports (e.g., malignant mesothelioma, lung cancer, and other interstitial pulmonary disease), plus data on smoking status by industry and occupation.

For many of the conditions reported on, the 2002 WoRLD Surveillance Report presents national and state summary statistics such as counts, crude and age-adjusted mortality rates, and years of potential life lost to age 65 and to life expectancy. Proportionate mortality ratios by industry and occupation are based on the most recent decade of data from a subset of states (see state list, Appendix E) for which usual industry and occupation have been coded for decedents. Also presented are U.S. state- and county-level maps showing the geographic distribution of mortality and, for the pneumoconioses, tables and figures summarizing selected occupational exposure data for asbestos, coal mine dust, silica dust, cotton dust, etc. (see agent categories, Appendix F).
Data contained in the 2002 WoRLD Surveillance Report originate from various publications, reports, data files, and tabulations provided by the Association of Occupational and Environmental Clinics (AOEC), the Bureau of Labor Statistics (BLS), the Department of Labor (DOL), the Mine Safety and Health Administration (MSHA), the National Center for Health Statistics (NCHS), the Occupational Safety and Health Administration (OSHA), NIOSH, and the Social Security Administration (SSA). Details on the major data sources and on the methods used to compute specific statistics can be found in Appendices A and B, respectively.

Interpreted with appropriate caution, the information contained in this report can help to establish priorities for research and prevention. It is also useful for tracking progress toward the elimination of important preventable occupational respiratory diseases, including those targeted in U.S. Public Health Service Healthy People objectives for the nation.

Comments and suggestions from users of earlier editions of the WoRLD Surveillance Report have influenced the content and format of this 2002 edition. To increase the utility of future editions, comments on the current report, descriptions of how the information is or could be used, and suggestions of other data for inclusion in future reports are invited.

See page $i i$ of this report for information on how to order copies of previous Work-Related Lung Disease Surveillance Reports, described on the next page.

\section{Send comments, suggestions, and other correspondence to:}

Work-Related Lung Disease Surveillance Report Public Health Surveillance Team

Surveillance Branch

Division of Respiratory Disease Studies

NIOSH

1095 Willowdale Road

Morgantown, WV 26505-2888

FAX: 304-285-6111

E-Mail: WoRLD@CDC.GOV 


\section{WoRLD Surveillance Report (1991)} www.cdc.gov/niosh/91-113.html

The 1991 report is the first in the series of WoRLD Surveillance Reports. Data presented in the report, most of which relates to the 1968-1987 time period, originated from the National Institute for Occupational Safety and Health (NIOSH), the National Center for Health Statistics (NCHS), the Bureau of Labor Statistics (BLS), the Mine Safety and Health Administration (MSHA), the Occupational Safety and Health Administration (OSHA), the Department of Labor (DOL), the Health Care Financing Administration (HCFA), and the Social Security Administration (SSA). The 1991 report is organized into two major sections, one of figures and the other of tables. Within each section, data are presented in the following subheadings: asbestosis, coal workers' pneumoconiosis, silicosis, exposure to cotton dust, pneumonopathy due to inhalation of other dust (i.e., byssinosis), hypersensitivity pneumonitis, toxic agents, dust diseases of the lung, and compensation.

\section{WoRLD Surveillance Report Supplement, 1992 www.cdc.gov/niosh/91-113s.html}

The 1992 supplement presents updated data for many of the figures and tables presented in the 1991 report, including mortality data through 1988. In addition, the 1992 supplement includes data not previously presented: (1) sex, race, geographic distribution, usual industry, and usual occupation, supplementing mortality data presented in the 1991 report; (2) number of discharges with silicosis, asbestosis, or coal workers' pneumoconiosis from the National Hospital Discharge Survey; and (3) reports of occupational asthma and silicosis from the Sentinel Event Notification Systems for Occupational Risks (SENSOR) Program.

\section{WoRLD Surveillance Report, 1994}

www.cdc.gov/niosh/94-120.html

Data presented in the 1994 report originate generally from programs and activities described in the 1991 and 1992 reports. The 1994 report is divided into 11 major sections, most containing both figures and data tables. Ten sections summarize mortality and morbidity data and other information, such as occupational exposures, for types of pneumoconiosis, malignant neoplasms of the pleura, hypersensitivity pneumonitis, occupational asthma, and other lung conditions. The final section provides data from the Association of Occupational and Environmental Clinics (AOEC) Disease Surveillance Database. The 1994 report contains major additions, including previously unreported data, such as those from the National Health Interview Survey (NHIS) and the AOEC, and additional statistical measures, such as proportionate mortality ratios, both crude and age-adjusted rates at national and state levels, and years of potential life lost to age 65 and to life expectancy.

\section{WoRLD Surveillance Report, 1996}

\section{www.cdc.gov/niosh/w7wrld96.html}

The 1996 report focuses entirely on pneumoconiosis mortality and related exposures, providing updated mortality data from 1968 through 1992. It has three sections: (1) a section that describes data highlights and data limitations; (2) a section that updates and expands national data provided in the 1994 report; and (3) a section that provides detailed profiles of relevant data for each state in the U.S. Surveillance data include counts, crude and age-adjusted rates, years of potential life lost, and proportionate mortality ratios by industry and occupation. The 1996 report presents detailed tables of pneumoconiosis mortality data for each state and for the District of Columbia, as well as for counties within each state. It also presents county-level maps showing the geographic distribution of mortality for each pneumoconiosis and showing results of federal occupational exposure inspection sampling for agents that cause pneumoconiosis.

\section{WoRLD Surveillance Report, 1999 www.cdc.gov/niosh/W99front.html}

The 1999 report is similar in content and organization to the 1994 WoRLD Surveillance Report. It is structured into three sections with 13 sub-sections which summarize mortality and morbidity data and other information, such as occupational exposures, for each type of pneumoconiosis and all pneumoconioses, malignant neoplasms of the pleura, hypersensitivity pneumonitis, occupational asthma, and other lung conditions. Major additions were sub-sections for pulmonary tuberculosis and chronic obstructive pulmonary disease. Mortality data published in the 1994 and 1996 World Surveillance Reports are updated through 1996. The 1994 report contains major additions, including previously unreported data, such as that from the National Health and Nutrition Examination Survey (NHANES). Reports of occupational asthma and silicosis from the Sentinel Event Notification Systems for Occupational Risks (SENSOR) Program are updated through 1995; updated summaries from the Association of Occupational and Environmental Clinics (AOEC) are provided for 1991-1996. 
This report was prepared primarily by staff of the Public Health Surveillance Team, Surveillance Branch, Division of Respiratory Disease Studies (DRDS), NIOSH. Major contributors include: Rochelle B. Althouse, Ki Moon Bang, Robert M. Castellan, Brent C. Doney, Margaret Filios, Mark F. Greskevitch, Ryan A. Heaslip, Kenneth D. Linch, Paul J. Middendorf, Cathy J. Rotunda, Girija Syamlal, and John M. Wood. Mei Lin Wang and Edward Lee Petsonk of the Workforce Screening and Surveillance Team, Surveillance Branch, and Janet M. Hale of the Communications and Information Activity, DRDS, provided helpful assistance. Michael D. Attfield, Chief, Surveillance Branch, and Gregory R. Wagner, Director, DRDS, provided guidance.

Special appreciation is expressed for the cooperation of staff at the Occupational Safety and Health Administration (OSHA) and at the Mine Safety and Health Administration (MSHA) for providing exposure data files, to the Association of Occupational and Environmental Clinics (AOEC) for providing data from their occupational disease database, and to state-level SENSOR Program staff, who provided data on silicosis and work-related asthma.

Draft portions of this report were provided for review and comment to individuals associated with public health agencies and other governmental organizations, as well as to others within NIOSH. Their comments have been considered in the final version of this report. 


\begin{tabular}{|c|c|c|c|}
\hline ACGIH ${ }^{\circ}$ & American Conference of Governmental & MRE & Mining Research Establishment \\
\hline & Industrial Hygienists & MSHA & Mine Safety and Health Administration \\
\hline AOEC & $\begin{array}{l}\text { Association of Occupational and } \\
\text { Environmental Clinics }\end{array}$ & NCHS & National Center for Health Statistics \\
\hline BLS & Bureau of Labor Statistics & n.e.c. & not elsewhere classified \\
\hline $\mathrm{BoC}$ & Bureau of the Census & NHANES & $\begin{array}{l}\text { National Health and Nutrition Examination } \\
\text { Survey }\end{array}$ \\
\hline $\mathrm{CDC}$ & Centers for Disease Control and Prevention & NHDS & National Hospital Discharge Survey \\
\hline CFR & Code of Federal Regulations & NIOSH & National Institute for Occupational Safety \\
\hline CI & confidence interval & & and Health \\
\hline CIC & Census Industry Code & n.o.s. & not otherwise specified \\
\hline $\mathrm{COC}$ & Census Occupation Code & OA & occupational asthma \\
\hline COPD & chronic obstructive pulmonary disease & OSHA & Occupational Safety and Health \\
\hline CWP & coal workers' pneumoconiosis & & Administration \\
\hline CWXSP & Coal Workers' X-ray Surveillance Program & PEFR & peak expiratory flow rate \\
\hline CXR & chest $x$-ray & PEL & permissible exposure limit \\
\hline DFR & Doctor's First Report & PHS & Public Health Service \\
\hline DHHS & Department of Health and Human Services & PMF & progressive massive fibrosis \\
\hline DOL & Department of Labor & PMR & proportionate mortality ratio \\
\hline DRDS & Division of Respiratory Disease Studies & PPD & purified protein derivative \\
\hline $\mathrm{f} / \mathrm{cc}$ & fibers per cubic centimeter & REL & NIOSH recommended exposure limit \\
\hline $\mathrm{FEV}_{1}$ & forced expiratory volume in one second & RADS & reactive airways dysfunction syndrome \\
\hline GM & geometric mean & SIC & Standard Industrial Classification \\
\hline HCFA & Health Care Financing Administration & SSA & Social Security Administration \\
\hline ICD & International Classification of Diseases & SENSOR & $\begin{array}{l}\text { Sentinel Event Notification Systems for } \\
\text { Occupational Risks }\end{array}$ \\
\hline ILO & International Labour Office & SOP & standard operating procedure \\
\hline IMIS & Integrated Management Information System & SUDAAN $®$ & Survey Data Analysis (software) \\
\hline LCL & lower confidence limit & TLV® & Threshold Limit Value \\
\hline MQC & minimum quantifiable concentration & TWA & time-weighted average \\
\hline$\mu \mathrm{g} / \mathrm{m}^{3}$ & micrograms per cubic meter & UCL & upper confidence limit \\
\hline $\mathrm{mg} / \mathrm{m}^{3}$ & milligrams per cubic meter & WOHL & Wisconsin Occupational Health Laboratory \\
\hline MMWR & Morbidity and Mortality Weekly Report & WoRLD & Work-Related Lung Disease \\
\hline MNMD & metal/nonmetal mine data & WRA & work-related asthma \\
\hline mppcf & millions of particles per cubic foot & YPLL & years of potential life lost \\
\hline
\end{tabular}




\section{Table of Contents}

Preface iii

Summary of Previous WoRLD Surveillance Reports ....................................................................... iv

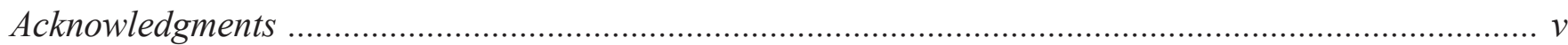

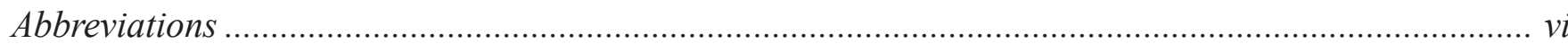

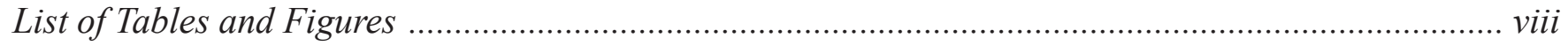

Highlights and Limitations

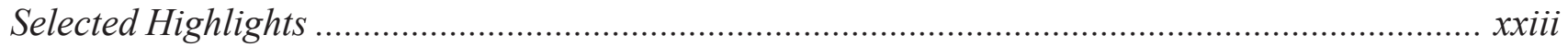

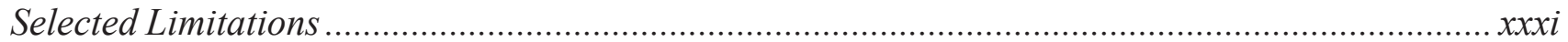

\section{Diseases and Related Exposures}

Section 1. Asbestosis and Related Exposures ...................................................................... 1

Section 2. Coal Workers' Pneumoconiosis and Related Exposures ............................................ 25

Section 3. Silicosis and Related Exposures .......................................................................... 51

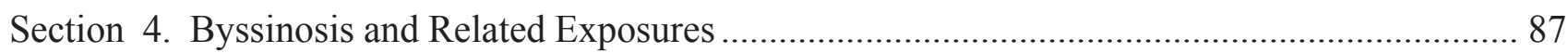

Section 5. Unspecified and Other Pneumoconiosis ........................................................ 107

Section 6. All Pneumoconioses and Related Exposures ....................................................... 125

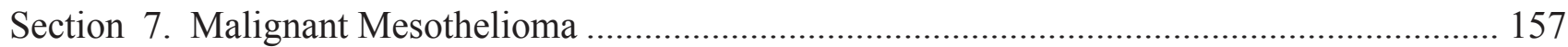

Section 8. Hypersensitivity Pneumonitis ............................................................................... 169

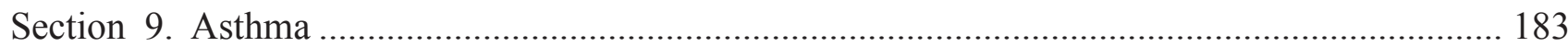

Section 10. Chronic Obstructive Pulmonary Disease ............................................................ 203

Section 11. Respiratory Conditions Due to Toxic Agents ..................................................... 213

Section 12. Respiratory Tuberculosis ................................................................................. 219

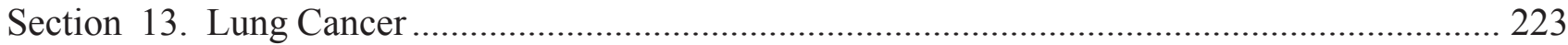

Section 14. Other Interstitial Pulmonary Diseases ............................................................ 227

Section 15. Various Work-Related Respiratory Conditions .................................................... 231

Section 16. Smoking Prevalence by Industry and Occupation .............................................. 239

\section{Appendices}

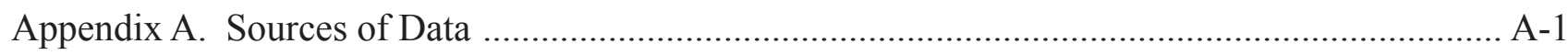

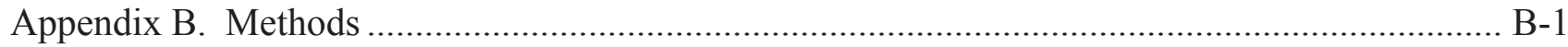

Appendix C. International Classification of Diseases (ICD) Codes ........................................ C-1

Appendix D. Changes in Bureau of Census Industry and Occupation Codes and Titles ............... D-1

Appendix E. States (and Years) for which Industry and Occupation Codes from Death Certificates Met NCHS Quality Criteria, 1985-1999 ............................................................... E-1

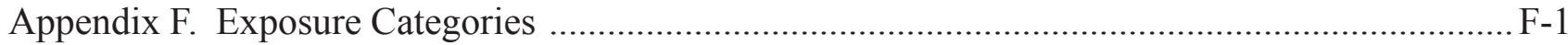

Appendix G. Surveillance Guidelines for State Health Departments ....................................... G-1

Appendix H. Split, Merged, or Renamed Counties and County Equivalents .............................. H-1 


\section{Asbestosis: Mortality}

Figure 1-1. Asbestosis: Number of deaths, crude and age-adjusted mortality rates, U.S. residents age 15 and over, 1968-1999

Figure 1-2. Asbestosis: Age-adjusted mortality rates by state, U.S. residents age 15 and over, 1990-1999

Table 1-1. Asbestosis: Number of deaths by sex, race, and age, and median age at death, U.S. residents age 15 and over, 1990-1999

Table 1-2. Asbestosis: Mortality rates (per million population) by race and sex, U.S. residents age 15 and over, 1990-1999

Table 1-3. Asbestosis: Years of potential life lost to age 65 and to life expectancy by race and sex, U.S. residents age 15 and over, 1990-1999

Table 1-4. Asbestosis: Number of deaths by state, U.S. residents age 15 and over, 1990-1999 ........ 7

Table 1-5. Asbestosis: Number of deaths, mortality rates (per million population), and years of potential life lost (YPLL) by state, U.S. residents age 15 and over, 1990-1999 ....................... 8

Table 1-6. Asbestosis: Most frequently recorded industries on death certificate, U.S. residents age 15 and over, selected states and years, 1990-1999.

Table 1-7. Asbestosis: Most frequently recorded occupations on death certificate, U.S. residents age 15 and over, selected states and years, 1990-1999

Table 1-8. Asbestosis: Proportionate mortality ratio (PMR) adjusted for age, sex, and race by usual industry, U.S. residents age 15 and over, selected states and years, 1990-1999

Table 1-9. Asbestosis: Proportionate mortality ratio (PMR) adjusted for age, sex, and race by usual occupation, U.S. residents age 15 and over, selected states and years, 1990-1999

Figure 1-3. Asbestosis: Age-adjusted mortality rates by county, U.S. residents age 15 and over, 1970-1999

Figure 1-4. Asbestosis: Age-adjusted mortality rates by county, U.S. residents age 15 and over, 1970-1984 and 1985-1999

Table 1-10. Asbestosis: Counties with highest age-adjusted mortality rates (per million population), U.S. residents age 15 and over, 1985-1999

\section{Asbestosis: Morbidity}

Table 1-11. Asbestosis: Estimated number of discharges from short-stay nonfederal hospitals, $1970-2000$

\section{Asbestosis: Asbestos Exposure}

Figure 1-5. Asbestos: Geometric mean exposures by major industry division, MSHA and OSHA samples, 1979-1999

Table 1-12. Asbestos: Geometric mean exposures and percent exceeding designated occupational exposure limits by major industry division, MSHA and OSHA samples, 1979-1999

Table 1-13. Asbestos: Number of samples, geometric mean exposures, and percent exceeding designated occupational exposure limits by industries with elevated asbestosis mortality, MSHA and OSHA samples, 1990-1999 
Table 1-14. Asbestos: Geometric mean exposures and percent exceeding designated occupational exposure limits by MSHA metal/nonmetal district and state, MSHA samples, 1979-1999 .... 19

Table 1-15. Asbestos: Geometric mean exposures and percent exceeding designated occupational exposure limits by OSHA region and state, OSHA samples, 1979-1999 ............................... 21

Figure 1-6. Asbestos: Geometric mean exposures by state, OSHA samples, 1979-1999 ............... 24

\section{Coal Workers' Pneumoconiosis: Mortality}

Figure 2-1. Coal workers' pneumoconiosis: Number of deaths, crude and age-adjusted mortality rates, U.S. residents age 15 and over, 1968-1999

Figure 2-2. Coal workers' pneumoconiosis: Age-adjusted mortality rates by state, U.S. residents age 15 and over, 1990-1999

Table 2-1. Coal workers' pneumoconiosis: Number of deaths by sex, race, and age, and median age at death, U.S. residents age 15 and over, 1990-1999

Table 2-2. Coal workers' pneumoconiosis: Mortality rates (per million population) by race and sex, U.S. residents age 15 and over, 1990-1999

Table 2-3. Coal workers' pneumoconiosis: Years of potential life lost to age 65 and to life expectancy by race and sex, U.S. residents age 15 and over, 1990-1999

Table 2-4. Coal workers' pneumoconiosis: Number of deaths by state, U.S. residents age 15 and over, 1990-1999

Table 2-5. Coal workers' pneumoconiosis: Number of deaths, mortality rates (per million population), and years of potential life lost (YPLL) by state, U.S. residents age 15 and over, 1990-1999

Table 2-6. Coal workers' pneumoconiosis: Most frequently recorded industries on death certificate, U.S. residents age 15 and over, selected states and years, 1990-1999

Table 2-7. Coal workers' pneumoconiosis: Most frequently recorded occupations on death certificate, U.S. residents age 15 and over, selected states and years, 1990-1999

Table 2-8. Coal workers' pneumoconiosis: Proportionate mortality ratio (PMR) adjusted for age, sex, and race by usual industry, U.S. residents age 15 and over, selected states and years, 1990-1999

Table 2-9. Coal workers' pneumoconiosis: Proportionate mortality ratio (PMR) adjusted for age, sex, and race by usual occupation, U.S. residents age 15 and over, selected states and years, 1990-1999

Figure 2-3. Coal workers' pneumoconiosis: Age-adjusted mortality rates by county, U.S. residents age 15 and over, 1970-1999

Figure 2-4. Coal workers' pneumoconiosis: Age-adjusted mortality rates by county, U.S. residents age 15 and over, 1970-1984 and 1985-1999

Table 2-10. Coal workers' pneumoconiosis: Counties with highest age-adjusted mortality rates (per million population), U.S. residents age 15 and over, 1985-1999 38 


\section{Coal Workers' Pneumoconiosis: Morbidity}

Table 2-11. Coal workers' pneumoconiosis: Estimated number of discharges from short-stay nonfederal hospitals, 1970-2000

Figure 2-5. CWXSP: Percentage of examined miners with coal workers' pneumoconiosis (category $1 / 0+$ ) by tenure in mining, 1970-1999

Figure 2-6. CWXSP: Estimated number of actively employed underground coal miners and number examined, 1970-1999

Table 2-12. CWXSP: Number and percentage of examined miners with coal workers' pneumoconiosis (category 1/0+), by round and tenure, 1970-1999

\section{Coal Workers' Pneumoconiosis: Black Lung Benefits}

Table 2-13. Federal Black Lung Program: Number of beneficiaries and total payments by the Social Security Administration and Department of Labor, 1980-1999

\section{Coal Workers' Pneumoconiosis: Coal Mine Dust Exposures}

Figure 2-7. Respirable coal mine dust: Geometric mean exposures by type of mine, MSHA inspector and mine operator samples, 1979-1999

Table 2-14. Respirable coal mine dust: Geometric mean exposures and percent exceeding designated occupational exposure limits by type of facility, MSHA inspector and mine operator samples, 1979-1999

Table 2-15. Respirable coal mine dust: Number of samples, geometric mean exposures, and percent exceeding designated occupational exposure limits by industries with elevated coal workers' pneumoconiosis mortality, MSHA inspector and mine operator samples, 1990-1999

Table 2-16. Respirable coal mine dust: Geometric mean exposures and percent exceeding designated occupational exposure limits by MSHA coal mine district and state, MSHA inspector and mine operator samples, 1979-1999

Figure 2-8. Respirable coal mine dust: Geometric mean exposures by state, MSHA inspector and mine operator samples, 1979-1999

\section{Silicosis: Mortality}

Figure 3-1. Silicosis: Number of deaths, crude and age-adjusted mortality rates, U.S. residents age 15 and over, 1968-1999

Figure 3-2. Silicosis: Age-adjusted mortality rates by state, U.S. residents age 15 and over, 1990-1999

Table 3-1. Silicosis: Number of deaths by sex, race, age, and median age at death, U.S. residents age 15 and over, 1990-1999

Table 3-2. Silicosis: Mortality rates (per million population) by race and sex, U.S. residents age 15 and over, 1990-1999

Table 3-3. Silicosis: Years of potential life lost to age 65 and to life expectancy by race and sex, U.S. residents age 15 and over, 1990-1999 56

Table 3-4. Silicosis: Number of deaths by state, U.S. residents age 15 and over, 1990-1999 
Table 3-5. Silicosis: Number of deaths, mortality rates (per million population), and years of potential life lost (YPLL) by state, U.S. residents age 15 and over, 1990-1999 _...................... 58

Table 3-6. Silicosis: Most frequently recorded industries on death certificate, U.S. residents age 15 and over, selected states and years, 1990-1999 ..................................................... 59

Table 3-7. Silicosis: Most frequently recorded occupations on death certificate, U.S. residents age 15 and over, selected states and years, 1990-1999

Table 3-8. Silicosis: Proportionate mortality ratio (PMR) adjusted for age, sex, and race by usual industry, U.S. residents age 15 and over, selected states and years, 1990-1999

Table 3-9. Silicosis: Proportionate mortality ratio (PMR) adjusted for age, sex, and race by usual occupation, U.S. residents age 15 and over, selected states and years, 1990-1999

Figure 3-3. Silicosis: Age-adjusted mortality rates by county, U.S. residents age 15 and over, 1970-1999

Figure 3-4. Silicosis: Age-adjusted mortality rates by county, U.S. residents age 15 and over, 1970-1984 and 1985-1999

Table 3-10. Silicosis: Counties with highest age-adjusted mortality rates (per million population), U.S. residents age 15 and over, 1985-1999

\section{Silicosis: Morbidity}

Table 3-11. Silicosis: Estimated number of discharges from short-stay nonfederal hospitals, $1970-2000$

\section{Silicosis: SENSOR}

Table 3-12. Silicosis: Number of cases by ascertainment source and state, 1989-1998 66

Table 3-13. Silicosis: Number of cases by duration of occupational exposure to silica and by state, 1989-1998

Figure 3-5. Silicosis: Decade of first exposure for 986 confirmed cases, 1989-1998 68

Table 3-14. Silicosis: Primary industries associated with silica exposure of silicosis cases by state, 1989-1998

Table 3-15. Silicosis: Primary occupations associated with silica exposure of silicosis cases by state, $1989-1998$

\section{Silicosis: Respirable Quartz Exposures}

Figure 3-6a. Respirable quartz: Geometric mean exposures in coal mining, MSHA inspector and mine operator samples, 1982-1999

Figure 3-6b. Respirable quartz: Geometric mean exposures by major industry division, MSHA and OSHA samples, 1979-1999

Table 3-16a. Respirable quartz: Geometric mean exposures and percent exceeding designated occupational exposure limits in coal mining, MSHA inspector and mine operator samples, 1982-1999

Table 3-16b. Respirable quartz: Geometric mean exposures and percent exceeding designated occupational exposure limits by major industry division, MSHA and OSHA samples, 1979-1999 
Table 3-17. Respirable quartz: Number of samples, geometric mean exposures, and percent exceeding designated occupational exposure limits by industries with elevated silicosis mortality, MSHA inspector and mine operator and OSHA samples, 1990-1999 75

Table 3-18. Respirable quartz: Geometric mean exposures and percent exceeding designated occupational exposure limits by MSHA coal mine district and state, MSHA inspector and mine operator samples, 1982-1999

Table 3-19. Respirable quartz: Geometric mean exposures and percent exceeding designated occupational exposure limits by MSHA metal/nonmetal mine district and state, MSHA samples, 1979-1999

Table 3-20. Respirable quartz: Geometric mean exposures and percent exceeding designated occupational exposure limits by OSHA region and state, OSHA samples, 1979-1999 80

Figure 3-7. Respirable quartz: Geometric mean coal mining exposures by state, MSHA inspector and mine operator samples, 1982-1999

Figure 3-8. Respirable quartz: Geometric mean metal/nonmetal mining exposures by state, MSHA samples, 1979-1999 84

Figure 3-9. Respirable quartz: Geometric mean exposures by state, OSHA samples, 1979-1999 ... 85

\section{Silicosis: Respirable Cristobalite Exposures}

Table 3-21. Respirable cristobalite: Geometric mean exposures and percent exceeding designated occupational exposure limits, MSHA and OSHA samples, 1979-1999 86

\section{Byssinosis: Mortality}

Figure 4-1. Byssinosis: Number of deaths, crude and age-adjusted mortality rates, U.S. residents age 15 and over, 1979-1999

Figure 4-2. Byssinosis: Age-adjusted mortality rates by state, U.S. residents age 15 and over, 1990-1999

Table 4-1. Byssinosis: Number of deaths by sex, race, and age, and median age at death, U.S. residents age 15 and over, 1990-1999

Table 4-2. Byssinosis: Mortality rates (per million population) by race and sex, U.S. residents age 15 and over, 1990-1999

Table 4-3. Byssinosis: Years of potential life lost to age 65 and to life expectancy by race and sex, U.S. residents age 15 and over, 1990-1999 ............................................................. 92

Table 4-4. Byssinosis: Number of deaths by state, U.S. residents age 15 and over, 1990-1999 ...... 93

Table 4-5. Byssinosis: Number of deaths, mortality rates (per million population), and years of potential life lost (YPLL) by state, U.S. residents age 15 and over, 1990-1999.

Table 4-6. Byssinosis: Most frequently recorded industries on death certificate, U.S. residents age 15 and over, selected states and years, 1990-1999.....

Table 4-7. Byssinosis: Most frequently recorded occupations on death certificate, U.S. residents age 15 and over, selected states and years, 1990-1999

Table 4-8. Byssinosis: Proportionate mortality ratio (PMR) adjusted for age, sex, and race by usual industry, U.S. residents age 15 and over, selected states and years, 1990-1999 
Table 4-9. Byssinosis: Proportionate mortality ratio (PMR) adjusted for age, sex, and race by usual occupation, U.S. residents age 15 and over, selected states and years, 1990-1999

Figure 4-3. Byssinosis: Age-adjusted mortality rates by county, U.S. residents age 15 and over,

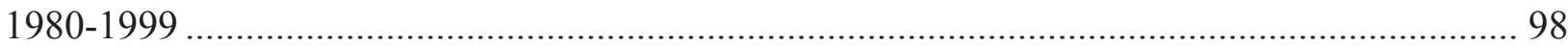

Table 4-10. Byssinosis: Counties with highest age-adjusted mortality rates (per million population), U.S. residents age 15 and over, 1985-1999

\section{Byssinosis: Cotton Dust Exposure}

Figure 4-4. Cotton dust: Geometric mean exposures by cotton process, OSHA samples, 1980-1999

Table 4-11. Cotton dust: Geometric mean exposures and percent exceeding designated occupational exposure limits by cotton process, OSHA samples, 1980-1999

Table 4-12. Cotton Dust: Number of samples, geometric mean exposures, and percent exceeding designated occupational exposure limits by industries with elevated byssinosis mortality, OSHA samples, 1990-1999

Table 4-13. Cotton dust: Geometric mean exposures and percent exceeding designated occupational exposure limits by OSHA region, state and process, OSHA samples, 1980-1999

Figure 4-5. Cotton dust: Percent of exposures exceeding the NIOSH recommended exposure limit by state, OSHA samples, 1980-1999 106

\section{Unspecified and Other Pneumoconiosis: Mortality}

Figure 5-1. Unspecified and other pneumoconioses: Number of deaths, crude and age-adjusted mortality rates, U.S. residents age 15 and over, 1968-1999

Figure 5-2. Unspecified and other pneumoconioses: Age-adjusted mortality rates by state, U.S. residents age 15 and over, 1990-1999

Table 5-1. Unspecified and other pneumoconioses: Number of deaths by sex, race, and age, and median age at death, U.S. residents age 15 and over, 1990-1999

Table 5-2. Unspecified and other pneumoconioses: Mortality rates (per million population) by race and sex, U.S. residents age 15 and over, 1990-1999.

Table 5-3. Unspecified and other pneumoconioses: Years of potential life lost to age 65 and to life expectancy by race and sex, U.S. residents age 15 and over, 1990-1999

Table 5-4. Unspecified and other pneumoconioses: Number of deaths by state, U.S. residents age 15 and over, 1990-1999

Table 5-5. Unspecified and other pneumoconioses: Number of deaths, mortality rates (per million population), and years of potential life lost (YPLL) by state, U.S. residents age 15 and over, 1990-1999

Table 5-6. Unspecified and other pneumoconioses: Most frequently recorded industries on death certificate, U.S. residents age 15 and over, selected states and years, 1990-1999

Table 5-7. Unspecified and other pneumoconioses: Most frequently recorded occupations on death certificate, U.S. residents age 15 and over, selected states and years, 1990-1999 
Table 5-8. Unspecified and other pneumoconioses: Proportionate mortality ratio (PMR) adjusted for age, sex, and race by usual industry, U.S. residents age 15 and over, selected states and years, 1990-1999

Table 5-9. Unspecified and other pneumoconioses: Proportionate mortality ratio (PMR) adjusted for age, sex, and race by usual occupation, U.S. residents age 15 and over, selected states and years, 1990-1999

Figure 5-3. Unspecified and other pneumoconiosis: Age-adjusted mortality rates by county, U.S. residents age 15 and over, 1970-1999

Figure 5-4. Unspecified and other pneumoconioses: Age-adjusted mortality rates by county, U.S. residents age 15 and over, 1970-1984 and 1985-1999

Table 5-10. Unspecified and other pneumoconioses: Counties with highest age-adjusted mortality rates (per million population), U.S. residents age 15 and over, 1985-1999

\section{Unspecified and Other Pneumoconiosis: Selected Agents Exposures}

Table 5-11. Selected pneumoconiotic agents: Geometric mean exposures and percent exceeding designated occupational exposure limits, MSHA and OSHA samples, 1979-1999

\section{All Pneumoconiosis: Mortality}

Figure 6-1. All pneumoconioses: Number of deaths, crude and age-adjusted mortality rates, U.S. residents age 15 and over, 1968-1999

Figure 6-2. All pneumoconioses: Age-adjusted mortality rates by state, U.S. residents age 15 and over, 1990-1999

Table 6-1. All pneumoconioses: Number of deaths by sex, race, and age, and median age at death, U.S. residents age 15 and over, 1990-1999

Table 6-2. All pneumoconioses: Mortality rates (per million population) by race and sex, U.S. residents age 15 and over, 1990-1999

Table 6-3. All pneumoconioses: Years of potential life lost to age 65 and to life expectancy by race and sex, U.S. residents age 15 and over, 1990-1999

Table 6-4. All pneumoconioses: Number of deaths by state, U.S. residents age 15 and over, 1990-1999

Table 6-5. All pneumoconioses: Number of deaths, mortality rates (per million population), and years of potential life lost (YPLL) by state, U.S. residents age 15 and over, 1990-1999

Table 6-6. All pneumoconioses: Percent of deaths by condition and state, U.S. residents age 15 and over, 1990-1999

Table 6-7. All pneumoconioses: Most frequently recorded industries on death certificate, U.S. residents age 15 and over, selected states and years, 1990-1999

Table 6-8. All pneumoconioses: Most frequently recorded occupations on death certificate, U.S. residents age 15 and over, selected states and years, 1990-1999

Table 6-9. All pneumoconioses: Proportionate mortality ratio (PMR) adjusted for age, sex, and race by usual industry, U.S. residents age 15 and over, selected states and years, 1990-1999 ... 135 
Table 6-10. All pneumoconioses: Proportionate mortality ratio (PMR) adjusted for age, sex, and race by usual occupation, U.S. residents age 15 and over, selected states and years, 1990-1999

Figure 6-3. All pneumoconioses: Age-adjusted mortality rates by county, U.S. residents age 15 and over, 1970-1999

Figure 6-4. All pneumoconioses: Age-adjusted mortality rates by county, U.S. residents age 15 and over, 1970-1984 and 1985-1999

Table 6-11. All pneumoconioses: Counties with highest age-adjusted mortality rates (per million population), U.S. residents age 15 and over, 1985-1999

\section{All Pneumoconiosis: Morbidity}

Table 6-12. Occupational dust diseases of the lungs: Estimated number of cases reported by employers, by industry division, U.S. private sector, 1973-2000

Table 6-13. Occupational dust diseases of the lungs: Estimated rate (based on cases reported by employers, per 10,000 full-time workers) by industry division, U.S. private sector, 1973-2000

Table 6-14. Occupational dust diseases of the lungs: Industries with highest estimated incidence rates (based on cases reported by employers, per 10,000 full-time workers) by industry division, U.S. private sector, 1996-2000

\section{All Pneumoconiosis: Pneumoconiotic Agents Exposures}

Figure 6-5. Pneumoconiotic agents: Percent of exposures exceeding the NIOSH recommended exposure limits by major industry division, MSHA inspector and mine operator and OSHA samples, 1979-1999

Table 6-15a. Pneumoconiotic agents: Percent of exposures exceeding designated occupational exposure limits by type of mine, MSHA inspector and mine operator samples, 1979-1999 ..... 145

Table 6-15b. Pneumoconiotic agents: Percent of exposures exceeding designated occupational exposure limits by major industry division, OSHA samples, 1979-1999

Table 6-16. Pneumoconiotic agents: Number of samples and percent of exposures exceeding designated occupational exposure limits by industries with elevated pneumoconiosis mortality, MSHA inspector and mine operator and OSHA samples, 1990-1999

Table 6-17. Pneumoconiotic agents: Percent of exposures exceeding designated occupational exposure limits by MSHA coal mine district and states, MSHA inspector and mine operator samples, 1979-1999

Table 6-18. Pneumoconiotic agents: Percent of exposures exceeding designated occupational exposure limits by MSHA metal/nonmetal district and state, MSHA samples, 1979-1999

Table 6-19. Pneumoconiotic agents: Percent of exposures exceeding designated occupational exposure limits by OSHA region and state, OSHA samples, 1979-1999

Figure 6-6. Pneumoconiotic agents: Percent of exposures exceeding the NIOSH recommended exposure limits by state, MSHA metal/nonmetal mine samples, 1979-1999

Figure 6-7. Pneumoconiotic agents: Percent of exposures exceeding the NIOSH recommended exposure limits by state, OSHA samples, 1979-1999 


\section{Malignant Mesothelioma: Mortality}

Figure 7-1. Malignant mesothelioma: Number of deaths by anatomical site, U.S. residents age 15 and over, 1999

Figure 7-2. Malignant mesothelioma: Age-adjusted mortality rates by state, U.S. residents age 15 and over, 1999

Table 7-1. Malignant mesothelioma: Number of deaths by sex, race, and age, and median age at death, U.S. residents age 15 and over, 1999

Table 7-2. Malignant mesothelioma: Mortality rates (per million population) by race and sex, U.S. residents age 15 and over, 1999

Table 7-3. Malignant mesothelioma: Years of potential life lost to age 65 and to life expectancy by race and sex, U.S. residents age 15 and over, 1999

Table 7-4. Malignant mesothelioma: Number of deaths, mortality rates (per million population), and years of potential life lost (YPLL) by state, U.S. residents age 15 and over, 1999

Table 7-5. Malignant mesothelioma: Most frequently recorded industries on death certificate, U.S. residents age 15 and over, selected states, 1999

Table 7-6. Malignant mesothelioma: Most frequently recorded occupations on death certificate, U.S. residents age 15 and over, selected states, 1999

Table 7-7. Malignant mesothelioma: Proportionate mortality ratio (PMR) adjusted for age, sex, and race by usual industry, U.S. residents age 15 and over, selected states, 1999 ................... 165

Table 7-8. Malignant mesothelioma: Proportionate mortality ratio (PMR) adjusted for age, sex, and race by usual occupation, U.S. residents age 15 and over, selected states, 1999 ............... 166

Figure 7-3. Malignant mesothelioma: Age-adjusted mortality rates by county, U.S. residents age 15 and over, 1999

Table 7-9. Malignant mesothelioma: Counties with highest age-adjusted mortality rates (per million population), U.S. residents age 15 and over, 1999

\section{Hypersensitivity Pneumonitis: Mortality}

Figure 8-1. Hypersensitivity pneumonitis: Number of deaths, crude and age-adjusted mortality rates, U.S. residents age 15 and over, 1979-1999

Figure 8-2. Hypersensitivity pneumonitis: Age-adjusted mortality rates by state, U.S. residents age 15 and over, 1990-1999

Table 8-1. Hypersensitivity pneumonitis: Number of deaths by sex, race, and age, and median age at death, U.S. residents age 15 and over, 1990-1999

Table 8-2. Hypersensitivity pneumonitis: Mortality rates (per million population) by race and sex, U.S. residents age 15 and over, 1990-1999

Table 8-3. Hypersensitivity pneumonitis: Years of potential life lost to age 65 and to life expectancy by race and sex, U.S. residents age 15 and over, 1990-1999

Table 8-4. Hypersensitivity pneumonitis: Number of deaths by state, U.S. residents age 15 and over, 1990-1999

Table 8-5. Hypersensitivity pneumonitis: Number of deaths, mortality rates (per million population), and years of potential life lost (YPLL) by state, U.S. residents age 15 and over, 1990-1999 
Table 8-6. Hypersensitivity pneumonitis: Most frequently recorded industries on death certificate, U.S. residents age 15 and over, selected states and years, 1990-1999

Table 8-7. Hypersensitivity pneumonitis: Most frequently recorded occupations on death certificate, U.S. residents age 15 and over, selected states and years, 1990-1999

Table 8-8. Hypersensitivity pneumonitis: Proportionate mortality ratio (PMR) adjusted for age, sex, and race by usual industry, U.S. residents age 15 and over, selected states and years, 1990-1999

Table 8-9. Hypersensitivity pneumonitis: Proportionate mortality ratio (PMR) adjusted for age, sex, and race by usual occupation, U.S. residents age 15 and over, selected states and years, 1990-1999

Figure 8-3. Hypersensitivity pneumonitis: Age-adjusted mortality rates by county, U.S. residents age 15 and over, 1980-1999

Table 8-10. Hypersensitivity pneumonitis: Counties with highest age-adjusted mortality rates (per million population), U.S. residents age 15 and over, 1985-1999

\section{Asthma: Mortality}

Table 9-1. Asthma: Proportionate mortality ratio (PMR) adjusted for age, sex, and race by usual industry, U.S. residents age 15 and over, selected states and years, 1990-1999

Table 9-2. Asthma: Proportionate mortality ratio (PMR) adjusted for age, sex, and race by usual occupation, U.S. residents age 15 and over, selected states and years, 1990-1999

\section{Work-Related Asthma: SENSOR}

Table 9-3. Work-related asthma: Number of cases by classification and state, 1993-1999

Table 9-4. Work-related asthma: Number of cases by ascertainment source and state, 1993-1999

Table 9-5. Work-related asthma: Most frequently reported putative agents associated with work-related asthma cases by state, 1993-1999

Figure 9-1. Work-related asthma: Most frequently reported agent categories associated with cases of work-related asthma, 1993-1999

Table 9-6. Work-related asthma: Primary industries associated with work-related asthma cases by state, 1993-1999

Table 9-7. Work-related asthma: Primary occupations associated with work-related asthma cases by state, 1993-1999

\section{Asthma: Morbidity}

Table 9-8. Asthma: Estimated prevalence by current industry and smoking status,

U.S. residents age 18 and over, 2000

Table 9-9. Asthma: Estimated prevalence by current industry and smoking status,

U.S. male residents age 18 and over, 2000

Table 9-10. Asthma: Estimated prevalence by current industry and smoking status,

U.S. female residents age 18 and over, 2000 
Table 9-11. Asthma: Estimated prevalence by current occupation and smoking status,

U.S. residents age 18 and over, 2000

Table 9-12. Asthma: Estimated prevalence by current occupation and smoking status,

U.S. male residents age 18 and over, 2000

Table 9-13. Asthma: Estimated prevalence by current occupation and smoking status,

U.S. female residents age 18 and over, 2000

\section{Chronic Obstructive Pulmonary Disease: Mortality}

Table 10-1. Chronic obstructive pulmonary disease: Proportionate mortality ratio (PMR) adjusted for age, sex, and race by usual industry, U.S. residents age 15 and over, selected states, 1999

Table 10-2. Chronic obstructive pulmonary disease: Proportionate mortality ratio (PMR) adjusted for age, sex, and race by usual occupation, U.S. residents age 15 and over, selected states, 1999

\section{Chronic Obstructive Pulmonary Disease: Morbidity}

Table 10-3. Chronic obstructive pulmonary disease: Estimated prevalence by current industry and smoking status, U.S. residents age 18 and over, 2000

Table 10-4. Chronic obstructive pulmonary disease: Estimated prevalence by current industry and smoking status, U.S. male residents age 18 and over, 2000 .

Table 10-5. Chronic obstructive pulmonary disease: Estimated prevalence by current industry and smoking status, U.S. female residents age 18 and over, 2000

Table 10-6. Chronic obstructive pulmonary disease: Estimated prevalence by current occupation and smoking status, U.S. residents age 18 and over, 2000

Table 10-7. Chronic obstructive pulmonary disease: Estimated prevalence by current occupation and smoking status, U.S. male residents age 18 and over, 2000.

Table 10-8. Chronic obstructive pulmonary disease: Estimated prevalence by current occupation and smoking status, U.S. female residents age 18 and over, 2000

\section{Occupational Respiratory Conditions Due to Toxic Agents: Morbidity}

Table 11-1. Occupational respiratory conditions due to toxic agents: Estimated number of cases reported by employers, by industry division, U.S. private sector, 1973-2000

Table 11-2. Occupational respiratory conditions due to toxic agents: Estimated rate (based on cases reported by employers, per 10,000 full-time workers) by industry division, U.S. private sector, 1973-2000

Table 11-3. Occupational respiratory conditions due to toxic agents: Industries with the highest estimated incidence rates (based on cases reported by employers, per 10,000 full-time workers), U.S. private sector, 1996-2000 


\section{Respiratory Tuberculosis: Mortality}

Table 12-1. Respiratory tuberculosis: Proportionate mortality ratio (PMR) adjusted for age, sex, and race by usual industry, U.S. residents age 15 and over, selected states and years, 1990-1999

Table 12-2. Respiratory tuberculosis: Proportionate mortality ratio (PMR) adjusted for age, sex, and race by usual occupation, U.S. residents age 15 and over, selected states and years, 1990-1999

\section{Lung Cancer: Mortality}

Table 13-1. Lung cancer: Proportionate mortality ratio (PMR) adjusted for age, sex, and race by usual industry, U.S. residents age 15 and over, selected states, 1999

Table 13-2. Lung cancer: Proportionate mortality ratio (PMR) adjusted for age, sex, and race by usual occupation, U.S. residents age 15 and over, selected states, 1999 226

\section{Other Interstitial Pulmonary Disease: Mortality}

Table 14-1. Other interstitial pulmonary diseases: Proportionate mortality ratio (PMR) adjusted for age, sex, and race by usual industry, U.S. residents age 15 and over, selected states, 1999

Table 14-2. Other interstitial pulmonary diseases: Proportionate mortality ratio (PMR) adjusted for age, sex, and race by usual occupation, U.S. residents age 15 and over, selected states, 1999

\section{Various Work-Related Respiratory Conditions: Morbidity}

Table 15-1. Work-related respiratory illnesses (with days away from work): Estimated number, 1996-2000

Table 15-2. Work-related respiratory illnesses (with days away from work): Median days away from work, 1996-2000....

Table 15-3a. Annual average employment: Estimated number (in thousands) by major industry division, 1996-2000

Table 15-3b. Work-related respiratory illnesses (with days away from work): Estimated number by industry division, 1996-2000 ....

Table 15-3c. Work-related respiratory illnesses (with days away from work): Estimated rate (per 100,000 workers) by industry division, 1996-2000

Table 15-4. Work-related respiratory conditions: Number of diagnoses related to asbestos or to other occupational exposures, based on physician's judgement, selected occupational and environmental medical clinics, 1991-2000

Table 15-5. Most frequent hazards associated with respiratory diagnoses, patients without asbestosrelated diagnoses, selected occupational and environmental medical clinics, 1991-2000 


\section{Smoking Status}

Table 16-1. Smoking status: Estimated prevalence by current industry, U.S. residents age 18 and over, 2000

Table 16-2. Smoking status: Estimated prevalence by current industry, U.S. male residents age 18 and over, 2000

Table 16-3. Smoking status: Estimated prevalence by current industry, U.S. female residents age 18 and over, 2000

Table 16-4. Smoking status: Estimated prevalence by current occupation, U.S. residents age 18 and over, 2000

Table 16-5. Smoking status: Estimated prevalence by current occupation, U.S. male residents age 18 and over, 2000

Table 16-6. Smoking status: Estimated prevalence by current occupation, U.S. female residents age 18 and over, 2000 


\section{Highlights and}

Limitations 



\section{Selected Highlights}

The following paragraphs highlight selected findings based on data from the United States presented in this and previous WoRLD Surveillance Reports.

\section{Asbestosis and Related Exposures}

- Asbestosis deaths among U.S. residents age 15 and over have increased from fewer than 100 in 1968 to more than 1,250 annually in 1999 , the most recent year for which data are available, with no apparent leveling off of this trend. (Figure 1-1)

- Over the 10-year period from 1990 to 1999 , there were more than 10,000 asbestosis deaths and annual asbestosis death counts increased by onethird. (Table 1-1)

- During the 10-year period from 1990 to 1999 , asbestosis deaths represented about one-third of all pneumoconiosis deaths. (Table 6-6)

- For 1998 and for 1999, asbestosis deaths outnumbered coal workers' pneumoconiosis (CWP) deaths, displacing CWP as the most frequent type of pneumoconiosis death. (Tables 1-1, 2-1, 6-1)

- Asbestosis was designated as the underlying cause of death in one-third of all asbestosis deaths from 1990 to 1999. (Table 1-1)

- Residents of California, Pennsylvania, New Jersey, Texas, Florida, Washington, and Virginia together accounted for nearly half of all asbestosis deaths in the 1990 to 1999 period. (Table 1-4)

- For the period from 1985 to 1999 , four counties (one in Virginia, one in Texas, one in Mississippi, and one in New Jersey) had age-adjusted asbestosis mortality rates that exceeded the national rate by more than 20-fold. (Table 1-10)

- Based on a large subset of the national data for which decedents' usual occupation and industry information was available, the construction industry accounted for one-fourth of decedents with asbestosis from 1990 through 1999. Apart from construction, asbestosis deaths were reported in a wide range of industries, with no particular industry predominating. Similarly, no one occupation emerged as being particularly common, though the most frequently listed occupational group was plumbers, pipefitters, and steamfitters. (Tables 1$6,1-7)$

- From 1990 to 1999 , decedents whose death certificate indicated that they worked in the miscellaneous nonmetallic mineral and stone products industry or the ship and boat building and repairing industry had proportionate asbestosis mortality more than 15 times higher than that of all industries combined. (Table 1-8)

- From 1990 to 1999 , decedents whose death certificate indicated that they were insulation workers or boilermakers had proportionate asbestosis mortality 20 times higher than that in all occupations combined. (Table 1-9)

- Hospital discharges associated with asbestosis have been rising rapidly between 1995 and 2000 . (Table 1-11)

- Data from the Occupational Safety and Health Administration (OSHA) and the Mine Safety and Health Administration (MSHA) indicate a trend towards lower exposure levels from 1979 to 1999 , concomitant with mandated reductions in the OSHA permissible exposure limit (PEL). (Figure 1-5, Table 1-12)

- For the period 1990 to 1999 , less than $5 \%$ of the MSHA and OSHA asbestos exposures exceeded the recommended exposure limit (REL). The miscellaneous nonmetallic mineral and stone products industry, which had the highest proportionate mortality ratio (PMR) for asbestosis, also had the highest geometric mean exposure and the highest percent of exposures 
exceeding the PEL and REL (26\% and $41 \%$, respectively). (Table 1-13)

\section{Coal Workers' Pneumoconiosis (CWP) and Related Exposures}

- CWP deaths among U.S. residents age 15 and over continue a long-term decline, from well over 2,500 deaths annually in the early 1980 s to just over 1,000 in the late 1990s. (Figure 2-1)

- Similarly, among active underground coal miners examined in a federally-administered CWP screening program, the prevalence of radiographically evident CWP continues to decline - from over $10 \%$ in the early 1970 s to less than $2 \%$ in the late 1990 s. (Table 2-12, Figure 2-5)

- CWP deaths accounted for nearly half of pneumoconiosis deaths during the 10 -year period from 1990 to 1999 , clearly outnumbering deaths associated with other types of pneumoconiosis. (Table 6-6)

- CWP was designated as the underlying cause of death in over one-third of all CWP deaths from 1990 to 1999. (Table 2-1)

- For the decade from 1990 to 1999 , more than three-fourths of all CWP decedents were residents of Pennsylvania, West Virginia, Virginia, and Kentucky. Pennsylvania alone accounted for about half of all CWP deaths in this period. (Table 2-4)

- For the period from 1985 to 1999 , four counties (one in Virginia, one in Pennsylvania, and two in West Virginia) had age-adjusted CWP mortality rates that exceeded the national rate by more than 100-fold. (Table 2-10)

- A large majority of CWP deaths are associated with employment in the coal mining industry, for which proportionate CWP mortality was more than 50 times higher than that of all occupations combined. (Tables 2-6, 2-8)

xxiv
- Federal "Black Lung" Program payments totaled more the $\$ 1.5$ billion for nearly 190,000 beneficiaries in 1999. These figures reflect a continuing slow decline from over $\$ 1.8$ billion paid out for over 500,000 beneficiaries in 1980 . The number of beneficiaries is now about one-third of what it was in 1980. (Table 2-13)

- Data from MSHA indicate that from the early 1980s to 1999 the underground coal mining industry experienced little change in level of exposure to respirable coal mine dust. Surface mine exposure levels have also remained fairly steady although there is some evidence of a decline in exposure levels since the early 1990s. (Figure 2-7, Table 2-14)

- During the period 1995 to 1999 , one-fourth of coal mine dust exposures recorded by MSHA exceeded the REL. (Tables 2-15, 2-16)

\section{Silicosis and Related Exposures}

- Silicosis deaths among U.S. residents age 15 and over represented nearly $8 \%$ of all pneumoconiosis deaths in the U.S. during the 10year period from 1990 to 1999. (Table 6-6)

- Over the past several decades, silicosis mortality has declined, from well over 1,000 deaths annually in the late 1960s to fewer than 200 per year in the late 1990s. (Figure 3-1)

- Silicosis was designated as the underlying cause of death in half of all silicosis deaths from 1990 to 1999. (Table 3-1)

- Compared to asbestosis, CWP, and byssinosis, silicosis mortality appears to be somewhat less concentrated by geographic region or by industry. However, Pennsylvania, alone, accounts for nearly $18 \%$ of silicosis deaths for the 1990-1999 period, ranking first among all states in number of silicosis deaths and fourth in age-adjusted silicosis mortality rate behind West Virginia, Vermont, and Colorado. (Tables 3-4, 3-5) 
- For the period from 1985 to 1999 , six counties (two in North Carolina and one each in Montana, Idaho, Colorado, and Georgia) had age-adjusted silicosis mortality rates that exceeded the national rate by more than 25 -fold. (Table 3-10)

- Based on a large subset of the national data for which decedents' usual occupation and industry information was available, the construction and mining industries accounted for at least one-third of decedents with silicosis from 1990 through 1999. (Table 3-6)

- Throughout the 1990-1999 period, silicosis mortality rates were higher among black males than among white males. (Table 3-2)

- Based on data from the SENSOR silicosis programs in Michigan, New Jersey, and Ohio, more than $8 \%$ of confirmed silicosis cases for which duration of exposure was ascertained had less than 10 years of occupational exposure to silica dust. (Table 3-13)

- Data from MSHA indicate that from 1979 to 1999 respirable quartz exposure levels have remained relatively constant in the coal mining industry. Levels in the metal mining and nonmetallic mining and quarrying industries appear to have declined from 1979 to 1987 , increased substantially in 1988 when MSHA implemented a different quartz analytical standard, declined from 1989 to 1995 and increased thereafter. Data from OSHA indicate that from 1979 to 1999 respirable quartz exposure levels have declined in the non-mining industries during the period 1989 to 1992 when the OSHA PEL was changed from a formula for respirable dust containing quartz to a respirable quartz concentration of $0.1 \mathrm{mg} / \mathrm{m}^{3}$. (Figures 3-6a, 3-6b, Tables 3-16a, 3-16b, 3-20)

- For the overall period 1993 to 1999 , the percentages of exposures greater than the PEL were about $29 \%$ in coal mining, about $6 \%$ in metal mining and nonmetallic mining and quarrying industries, and $31 \%$ in other industries. (Tables 3-18 to 3-20)

- For the overall period 1990 to 1999 , miscellaneous nonmetallic mineral and stone products, iron and steel foundries, and structural clay products were the industries with elevated PMRs for silicosis in which at least $29 \%$ of their exposures exceeded the PEL and about half exceeded the REL. The coal mining industry had an elevated PMR for silicosis and about $30 \%$ of its exposures exceeded the MSHA PEL. (Tables 3-8, 3-17)

- For the period 1993 to 1999, Indiana, Virginia, Tennessee, West Virginia, Oklahoma, Kentucky, Arizona, and Alabama had geometric mean respirable quartz exposure levels in the coal mining industry which exceeded $0.05 \mathrm{mg} / \mathrm{m}^{3}$ MRE and at least 10 samples analyzed by MSHA. (Table 3-18, Figure 3-7)

- For the period 1993 to 1999,16 states had geometric mean respirable quartz exposure levels in non-mining industries which exceeded the REL of $0.05 \mathrm{mg} / \mathrm{m}^{3}$ and at least 10 samples analyzed by OSHA. (Table 3-20, Figure 3-9)

\section{Byssinosis and Related Exposures}

- In comparison with other pneumoconioses, byssinosis deaths among U.S. residents age 15 and over (as enumerated from death certificate data) remain very few - fewer than 20 annually since 1990, and fewer than 10 annually in 1998 and 1999. (Table 4-1)

- Nearly one-third of byssinosis decedents in the 1990 to 1999 period were female. (Table 4-1)

- Byssinosis was designated as the underlying cause of death in about half of all byssinosis deaths from 1990 to 1999. (Table 4-1)

- Over one-half of byssinosis decedents in the period from 1990 to 1999 were residents of North Carolina, South Carolina, and Georgia. (Table 4-4) 
- For the period from 1985 to 1999 , three counties (all in North Carolina) had age-adjusted byssinosis mortality rates that exceeded the national rate by more than 50-fold. (Table 4-10)

- Only one industry - yarn, thread, and fabric mills - was associated with a significantly high byssinosis mortality for the 1990 to 1999 period. (Table 4-8)

- Although cotton dust exposure data are sparse, nearly one-third of the exposures measured by OSHA exceeded the REL for the period 1990 to 1999. (Table 4-12)

\section{Unspecified/Other Pneumoconioses}

- The pattern of deaths from unspecified/other pneumoconioses, which account for $10 \%$ of all pneumoconiosis deaths during the 1990-1999 period, tends to resemble coal workers' pneumoconiosis (and, less so, silicosis) mortality with respect to geographic distribution, a similar peak in 1972, and similar occupations and industries associated with high PMRs. This indicates that most unspecified pneumoconiosis deaths are likely to be CWP deaths. (Tables 5-1, $5-4,5-8,5-9,6-6)$

- For each year from 1979 to 1999 , at least five percent of OSHA iron oxide fume exposures exceeded the REL. (Table 5-11)

\section{All Pneumoconioses and Related Exposures}

- During the 10-year period from 1990 to 1999 , there were more than 31,000 pneumoconiosis deaths nationwide, accounting for more than 300,000 years of potential life lost. (Tables 6-1, 6-3)

- Overall pneumoconiosis mortality in the U.S. has been gradually declining over the past two-andone-half decades, from a peak of more than 5,000 deaths in 1972 to 2,745 pneumoconiosis deaths in 1999. (Figure 6-1, Table 6-1)
- Pneumoconiosis was designated as the underlying cause of death in over one-third of all pneumoconiosis deaths from 1990 to 1999. (Table 6-1)

- The pattern of all pneumoconiosis mortality is largely influenced by coal workers' pneumoconiosis (CWP), given that certified CWP deaths represent nearly half of all pneumoconiosis deaths from 1990 to 1999 . However, asbestosis deaths have been increasing and exceeded CWP deaths in 1998 and 1999. (Tables 1-1, 2-1, 6-6)

- Based on a major survey of private industry employers, annual estimates for the number of new cases of pneumoconiosis over the late 1990s have ranged from 1,700 to 3,500 among employees. There is no clear trend in these estimates since 1980. The highest estimated rates have been consistently associated with mining, particularly with coal mining. (Tables 6-12 to 6-14)

- For the overall period 1990 to 1999 , the coal mining industry had the highest PMR (33) for pneumoconiosis and over one-fourth of its exposures exceeded the REL. (Table 6-16)

\section{Malignant Mesothelioma}

- There were nearly 2,500 malignant mesothelioma deaths among U.S. residents age 15 and over in 1999. (Table 7-1)

- Mesothelioma was designated as the underlying cause of death in nearly $95 \%$ of all malignant mesothelioma deaths in 1999. (Table 7-1)

- Nearly $20 \%$ of mesothelioma decedents were female. (Table 7-1)

- For 1999, more than one-third of mesothelioma decedents were residents of just five states (California, Florida, Pennsylvania, New York and Ohio). (Table 7-4) 
- For 1999, seven counties (two in Virginia, and one each in Ohio, Maine, New Jersey, Mississippi, and Michigan) had age-adjusted malignant mesothelioma mortality rates that exceeded the national rate by more than 5-fold. (Table 7-9)

- Based on a large subset of the national data for which decedents' usual occupation and industry information was available, the construction industry accounted for nearly $15 \%$ of decedents with malignant mesothelioma in 1999. (Table 7-5)

- In addition to the construction industry, other industries associated with significantly increased mesothelioma mortality in 1999 include: ship and boat building and repairing; industrial and miscellaneous chemicals; petroleum refining; and electric light and power. (Table 7-7)

- Occupations associated with significantly elevated mesothelioma mortality in 1999 include: plumbers, pipefitters, and steamfitters; mechanical engineers; electricians; and elementary school teachers. (Table 7-8)

\section{Hypersensitivity Pneumonitis (HP)}

- The annual number of hypersensitivity pneumonitis (HP) deaths has been generally increasing, from less than 20 per year in 1979 to 57 in 1999. (Figure 8-1, Table 8-1)

- HP was designated as the underlying cause of death in two-thirds of all HP deaths from 1990 to 1999. (Table 8-1)

- The highest HP mortality rates for the 19901999 period are in the upper Midwest, northern Plains, Mountain, and New England states. (Table 8-5)

- For the 1985-1999 period, two counties in Wisconsin had age-adjusted HP mortality rates that exceeded the national rate by more than 5 -fold. (Table 8-10)
- For the 1990-1999 period, Agricultural production industries (both livestock and crops) and farmers, except horticulture were associated with significantly elevated PMRs for HP. (Tables $8-8,8-9$ )

\section{Asthma}

- For the 1990-1999 period, agriculture production, livestock and farm machinery and equipment were associated with the highest proportionate mortality ratios for asthma. Among the other top five industries with significantly elevated PMRs for asthma were: child day care services; drug stores; and health services, not elsewhere classified. Among the top ten industries associated with significantly elevated PMRs for asthma are two others related to the health care industry: hospitals and offices and clinics of physicians. (Table 9-1)

- For the 1990-1999 period, half of the 23 occupational groups associated with significantly elevated PMRs for asthma were related to health care and education. (Table 9-2)

- Public health surveillance programs in four states (California, Massachusetts, Michigan, and New Jersey) have identified over 2,500 cases of work-related asthma over a recent seven-year period (1993-1999). About 80\% represented asthma caused by occupational exposure, while $20 \%$ represented preexisting asthma aggravated by occupational exposure. (Table 9-3)

- Of all the work-related asthma cases from California, Massachusetts, Michigan, and New Jersey associated with various categories of reported putative agents for 1993-1999, 20\% were associated with miscellaneous chemicals, $12 \%$ with cleaning materials, $11 \%$ with mineral and inorganic dust, $10 \%$ with indoor air pollutants, and $4 \%$ with welding exposures, among others. (Figure 9-1) 
- Based on a recent national survey of the U.S. population in which respondents' current industry was ascertained, elementary and secondary schools and colleges was the current industry sector associated with an estimated asthma prevalence among nonsmokers that significantly exceeded the estimated $8 \%$ prevalence of asthma among all U.S. adult nonsmokers. (Table 9-8)

- Based on the survey noted above, teachers, librarians and counselors was the current occupation associated with estimated asthma prevalence among nonsmokers that significantly exceeded the estimated $8 \%$ prevalence of asthma among all U.S. adult nonsmokers. (Table 9-11)

\section{Chronic Obstructive Pulmonary Disease (COPD)}

- Coal mining led the list of industries with significantly elevated PMRs for COPD in 1999. Two other mining sectors were in the top five industries for COPD mortality, as were trucking service and automotive repair and related services. (Table 10-1)

- The top five occupations for COPD mortality in 1999 included: washing, cleaning, and pickling machine operators; helpers, mechanics and repairers; textile cutting machine operators; mining machine operators; and construction trades, not elsewhere classified. (Table 10-2)

\section{Respiratory Conditions due to Toxic Agents}

- Based on a major survey of private employers, the average annual estimated number of new cases of respiratory conditions due to toxic agents has decreased to approximately 15,000 for 1999 and 2000, down from annual estimates of about 25,000 in the early and mid-1990s. (Table 11-1)

- The major industry groups associated with the highest annual estimated rates of work-related respiratory conditions due to toxic agents in 2000 are manufacturing (3.0 per 10,000 full-time xxviii workers), services (1.9 per 10,000 full-time workers), and transportation and public utilities (1.5 per 10,000 full-time workers). The transportation equipment industry, with annual estimated rates of about 10 per 10,000 , has consistently ranked in the top three industry sectors during the 1996 to 2000 period. (Tables 11-2, 11-3)

\section{Respiratory Tuberculosis}

- Among the industry sectors associated with significantly elevated tuberculosis mortality in the 1990-1999 period were: health-care industries (offices and clinics of health practitioners; hospitals; and miscellaneous personal services); agricultural production, crops; and industries with significantly elevated silicosis mortality (nonmetallic mining and quarrying, except fuel; metal mining; other primary metal industries; coal mining; and construction). (Tables 12-1, 3-8)

- Among occupations associated with significantly elevated tuberculosis mortality in the 1990-1999 period were agricultural occupations (farm workers and farmers, except horticulture), sailors and deckhands, garbage collectors, and occupations associated with significantly elevated silicosis mortality (crushing and grinding machine operators; mining machine operators; construction laborers; and laborers, except construction). (Tables 12-2, 3-9)

\section{Lung Cancer}

- This edition of the Work-Related Lung Disease Surveillance Report is the first of the series to include a section on lung cancer - specifically PMRs by industry and occupation. A variety of industries and occupations associated with significantly elevated lung cancer mortality are listed in this section. (Tables 13-1, 13-2)

\section{Other Interstitial Pulmonary Diseases}

- This edition of the Work-Related Lung Disease Surveillance Report is the first of the series to include a section on other interstitial pulmonary 
diseases - specifically PMRs by industry and occupation. A variety of industries and occupations associated with significantly elevated other interstitial pulmonary diseases mortality are listed in this section. (Tables 14-1, 14-2)

\section{Various Work-Related Respiratory Conditions}

- Data from the Bureau of Labor Statistics Annual Survey and the Association of Occupational and Environmental Clinics Database, both of which include information on a wide range of work-related respiratory diseases, serve to remind readers that there is much more to work-related lung disease and other occupational respiratory diseases than they might otherwise realize. Data are presented on work-related upper airway disorders (e.g., allergic rhinitis, nasal septum perforation), malignant diseases (e.g., nasal and laryngeal, as well as pulmonary and pleural), infectious diseases (e.g., influenza, pneumonia, and Legionnaires' disease), and other respiratory diseases (e.g., pneumonitis, interstitial fibrosis, etc.). (Tables 15-1 to 15-5)

\section{Smoking Prevalence by Occupation and Industry}

- This edition of the Work-Related Lung Disease Surveillance Report is the first of the series to include a section on smoking prevalence by industry and occupation. Smoking by itself is an important cause of lung disease and smoking can also compound the adverse effects of occupational exposures. Based on recent data from the National Health Interview Survey, estimated smoking prevalences range widely from $12 \%$ among elementary and secondary schools and colleges workers to over $40 \%$ among repair services workers. Similar wide-ranging smoking prevalences are seen among occupational groups. (Tables 16-1 to 16-6) 



\section{Selected Limitations}

In addition to the following cautions, readers should see Appendix A for other limitations relating to specific sources of data presented in this report.

\section{General}

- In this report, every reasonable attempt has been made with the available resources to present comprehensive data on health outcomes and exposures of relevance to work-related lung diseases. The data are drawn from the major existing databases. However, other data may exist which would improve the completeness and reliability of the findings presented in this report. Readers who are aware of other data that should be considered for inclusion in future editions are encouraged to make their suggestions known (see Preface for contact information).

- Statistics in many tables and figures in this report are based on small numbers. Readers are cautioned that these can be unstable. Hence, inferences should be drawn with care, and should take the numerical basis into account.

- A decedent's or survey respondent's usual or current industry and occupation are not always indicative of the industry and occupation associated with the exposure responsible for that individual's work-related disease. Readers are therefore cautioned not to make definitive causative inferences about industries and occupations based solely on the various mortality and morbidity tables presented in this report.

\section{Disease Data}

- Work-related respiratory diseases are typically, though not always, chronic and may also have long latencies. As reflected in median ages at death presented in this report for the pneumoconioses, many affected individuals live to or even beyond average life expectancy. The fact that many affected individuals do not die as a direct result of their workrelated respiratory disease led to a decision to consider all causes of death, underlying and contributing, in the development of the summary tables and figures of mortality data presented in this report. In the absence of national incidence and prevalence morbidity data specific to occupational diseases, the intent is to provide a better assessment of disease occurrence and distribution than would be possible if consideration were restricted to underlying causes of death.

- Certifying physicians typically do not list all of a decedent's diseases on the death certificate. Therefore, even though contributing causes of death are considered, the mortality data presented in this report probably underestimate the total occurrence of pneumoconioses and other diseases.

- As with any analysis based on death certificate data, there is undoubtedly some misclassification of cause of death. A treating physician may not correctly diagnose a particular disease during a patient's life or, as mentioned above, a certifying physician may fail to list a correctly diagnosed disease on the death certificate, particularly if another disease was directly responsible for the decedent's death. In addition, the diagnoses listed on the death certificate are sometimes miscoded.

- Data that depend, either directly or indirectly, on physician reporting or recording of occupational disease diagnoses can be influenced significantly by the physician's ability or willingness to suspect and evaluate a relationship between work and health. These, in turn, are influenced by evolving medical/ scientific information, and by the legal, political, and social environment. Some factors may lead to increased diagnosis and recording/reporting (e.g., the Coal Mine Health and Safety Act of 1969 increasing recognition and recording of coal workers' pneumoconiosis), while other factors may reduce occupational disease recognition or reporting by physicians (e.g., long latency between a work exposure and disease development, or concern about involvement in litigation). 
- Byssinosis and asthma lack the characteristic fibrosis and associated radiographic appearance commonly observed in mineral dust pneumoconioses. In addition, advanced stages of asthma and byssinosis may be difficult to distinguish from other chronic obstructive pulmonary diseases, including those due solely to cigarette smoking. For both these reasons, under-diagnosis may be more likely for byssinosis and work-related asthma than for the radiographically apparent pneumoconioses.

- Categorization of lung diseases for which mortality data are presented in this report is limited by the ICD coding system used for the NCHS multiple cause of death data. Also, ICD-8, ICD-9, and ICD-10 disease rubrics differ somewhat for all types of pneumoconioses (see Appendix C). However, the effect of ICD changes is not substantial for most of the diseases under consideration (e.g., there is no indication of any changes in the yearly trend in national silicosis mortality related to changes in the rubrics for the ICD code related to silicosis).

- Prior to ICD-10, there was no discrete ICD code for malignant mesothelioma, a disease strongly associated with exposure to asbestos. ICD-10 coding of national death data in the United States began with 1999 deaths; thus, only one year of malignant mesothelioma data is presented in this report. Past reports in this Work-Related Lung Disease Surveillance Report series have presented data on mortality associated with "malignant neoplasm of the pleura," but that former ICD category lacked specificity and sensitivity for malignant mesothelioma.

- A general assumption of work-relatedness for pneumoconiosis deaths is reasonable for surveillance purposes. However, a very small proportion of pneumoconiosis decedents may have developed their disease as a result of non-occupational (e.g., avocational) exposure to pneumoconiotic agents.

xxxii
- Although respiratory diseases other than the pneumoconioses can be caused by occupational exposure to respiratory hazards, it is generally unreasonable to assume an automatic occupational etiology because of the strong influence of nonoccupational factors. As a result, readers will note that the types of mortality tables presented in this report differ depending on the specific disease. More comprehensive tables are presented for those diseases that are highly specific for occupational etiology, while a more limited approach is used for diseases that are less likely to be caused solely by occupational exposure.

- Individuals affected by chronic diseases with long latency have much more time to change residences prior to death than individuals affected by acute diseases with short latency. Thus, state of residence at death does not necessarily represent the location of a decedent's occupational exposure, even for a death that results directly from occupational respiratory disease.

- Readers are reminded that only about half the states provide data on usual industry and occupation of decedents which meet the National Center for Health Statistics' quality criteria for the national death data files used to develop many of the tables presented in this report (see Appendix E).

- Apparent differences in mortality rates may reflect, in part or in whole, geographical as well as temporal changes in employment patterns affecting the number of workers at risk to various respiratory hazards. Denominators used to calculate mortality rates presented in this report are based on general population estimates for the location (e.g., national, state, or county) and for the years in which the deaths occurred. The resulting rates have clear public health significance. However, as suggested by some very high proportionate mortality ratios presented in this report for specific industrial and occupational groups, national and state-specific rates typically represent a dilution of very high mortality among 
exposed groups of workers by very low mortality within the general population that is not significantly exposed.

- To comply with current CDC policy, populationbased mortality rates for this edition of the WorkRelated Lung Disease Surveillance Report have been adjusted to the U.S. Year 2000 Standard Population. This is a change from prior editions in which rates were adjusted to the 1940 standard population. Readers are cautioned that rates are not directly comparable with those shown in earlier editions.

- Proportionate mortality ratios (PMRs) reported in this edition of the Work-Related Lung Disease Surveillance Report are not directly comparable to those reported in earlier editions because PMRs in the current edition have been adjusted for age (in five-year categories), sex, and race, whereas PMRs in earlier editions were adjusted only for age (in 20 -year categories). Readers are also reminded that, because of the lack of smoking information in the national death files, PMRs presented in this report have not been adjusted for smoking.

- Over the period covered by data presented in this report, median ages at death have generally increased for all pneumoconioses. The reader is cautioned to realize that this increase is the result of many factors, only one of which may be a general reduction of disease severity (e.g., due to enhanced diagnostic sensitivity and fewer severe cases). Another possible factor is a reduced number of younger workers at risk due to changing employment patterns. Reduced mortality from other causes of death is undoubtedly another important factor.

- Data from the Coal Workers X-Ray Surveillance Program (CWXSP) have a number of limitations. The program is restricted to currently employed miners and participation rates are generally low. Disease prevalence estimates may be biased due to selective participation, and missing or inaccurate work history information may affect tenure calculations. Also, radiographic detection of pneumoconiosis is imperfect. Pathologic disease in some individuals may not be detected radiographically and, although rare among working populations, various non-occupational conditions may result in radiographic abnormalities consistent with pneumoconiosis.

- The main usefulness of the Bureau of Labor Statistics (BLS) Annual Survey of Injuries and Illnesses is to assess occupational injuries, because work-attribution of traumatic injuries is typically quite clear to the employers. In contrast, workrelated diseases are generally under-recognized and under-reported by employers.

\section{Exposure Data}

- The reported OSHA and MSHA exposure data should be considered provisional and subject to revision. The samples were collected for regulatory compliance purposes, rather than for the surveillance of worker exposures, and therefore may not represent exposures typically experienced by workers. Nonetheless, these data provide the best available national exposure information for industries in the U.S.

- MSHA and OSHA data for similar agents are presented in this report in a parallel format. The reader is cautioned that MSHA and OSHA are separate agencies with separate regulatory jurisdictions over different industries. The number of compliance samples collected by an agency depends upon many factors, including the size and nature of an industry, congressional actions, and regulatory policies.

- To identify pneumoconiotic agents included in the MSHA and OSHA data systems, the following documents were reviewed: Documentation of $T L V S{ }^{\circledR}$ and BEIS ${ }^{\circledR}, 6^{\text {th }}$ edition (ACGIH7); Occupational Respiratory Diseases (NIOSH Pub. 
No. 86-102); the Pocket Guide to Chemical Hazards (NIOSH Pub No. 97-140); and various NIOSH Criteria Documents. The resulting list of pneumoconiotic agents (see Table F-1 of Appendix F) represents those agents associated with the most prevalent types of pneumoconiosis, but is not intended to be a complete listing of all agents that may cause pneumoconiosis.

- Many of the reported geometric mean exposures include samples that could not be quantified with the sampling and analytical methods used. Rather than assume the values of these samples were zero, estimates of the sample results were used to calculate the geometric mean. The methods for estimating the sample result are described in the exposure section, and readers should keep in mind this uncertainty underlying the geometric mean concentrations presented in this report.

- Although OSHA adopted permissible exposure limits (PELs) of $0.1 \mathrm{mg} / \mathrm{m}^{3}$ for quartz and $0.05 \mathrm{mg} / \mathrm{m}^{3}$ for cristobalite that were enforced from March 1, 1989 through March 22, 1993, neither OSHA nor MSHA currently has a PEL specific to any form of crystalline silica. Instead, the relevant PELs are for respirable dust containing crystalline silica. These PELs take the form of formulas in which the PEL for respirable dust is reduced as the crystalline silica content of the dust increases. The PEL formulas vary with the agency and the industry, but, with all of them, the effective allowable exposure to quartz is less than or equal to $0.1 \mathrm{mg} / \mathrm{m}^{3}$ and the effective allowable exposure to cristobalite is less than or equal to $0.05 \mathrm{mg} / \mathrm{m}^{3}$, regardless of silica content. Thus, the percentage of OSHA samples exceeding the PEL is greater in the years when the formula PEL is applied (in this report, all years except 1989 through 1993) than it would be if a $0.1 \mathrm{mg} / \mathrm{m}^{3}$ quartz or $0.05 \mathrm{mg} / \mathrm{m}^{3}$ cristobalite PEL had been applied for these years. Readers should keep the preceding explanation in mind when considering data presented in this report showing apparent temporal discontinuities in the annual percentage of OSHA silica samples exceeding the PEL.
- The percentage of respirable coal mine dust samples exceeding the PEL was calculated using the MSHA PEL of $2 \mathrm{mg} / \mathrm{m}^{3}$ MRE for respirable coal mine dust containing no more than $5 \%$ quartz. Because the quartz content could not be reliably identified for most of the respirable coal mine dust samples, no attempt was made to use the MSHA formula for reducing the PEL when the quartz content exceeded 5\%. Thus, as presented in this report, the percentage of respirable coal mine dust samples exceeding the PEL is a lower limit, and the actual percentage exceeding the PEL is very likely higher than reported.

- In addition to samples in which quartz was identified, the respirable quartz data reported in Section 3 include MSHA samples identified as:

- nuisance dust, respirable fraction, less than $1 \%$ quartz;

- unlisted particulate, respirable fraction, less than $1 \%$ quartz; and

- respirable dust (not analyzed or below detection limit) from metal/nonmetal mines because, although the samples did not indicate quartz exposure, they were collected, in part, to assess exposure to quartz. This provides a more accurate estimate of the geometric mean exposures and the percentage of exposures that exceed a PEL or recommended exposure limit (REL).

- Available exposure data for agents associated with each type of pneumoconiosis are presented in this report following the presentation of mortality data for that same condition. The reader is reminded that the time period over which the exposure data were collected does not necessarily correspond to the time period during which most of the decedents represented in the mortality data acquired their disease. For most pneumoconiosis deaths, there is a latency period of at least several years between first occupational exposure and onset of disease. Subsequent death typically occurs many years after disease onset. 


\section{Section 1}

Asbestosis

and

Related Exposures 

Figure 1-1. Asbestosis: Number of deaths, crude and age-adjusted mortality rates, U.S. residents age 15 and over, 1968-1999

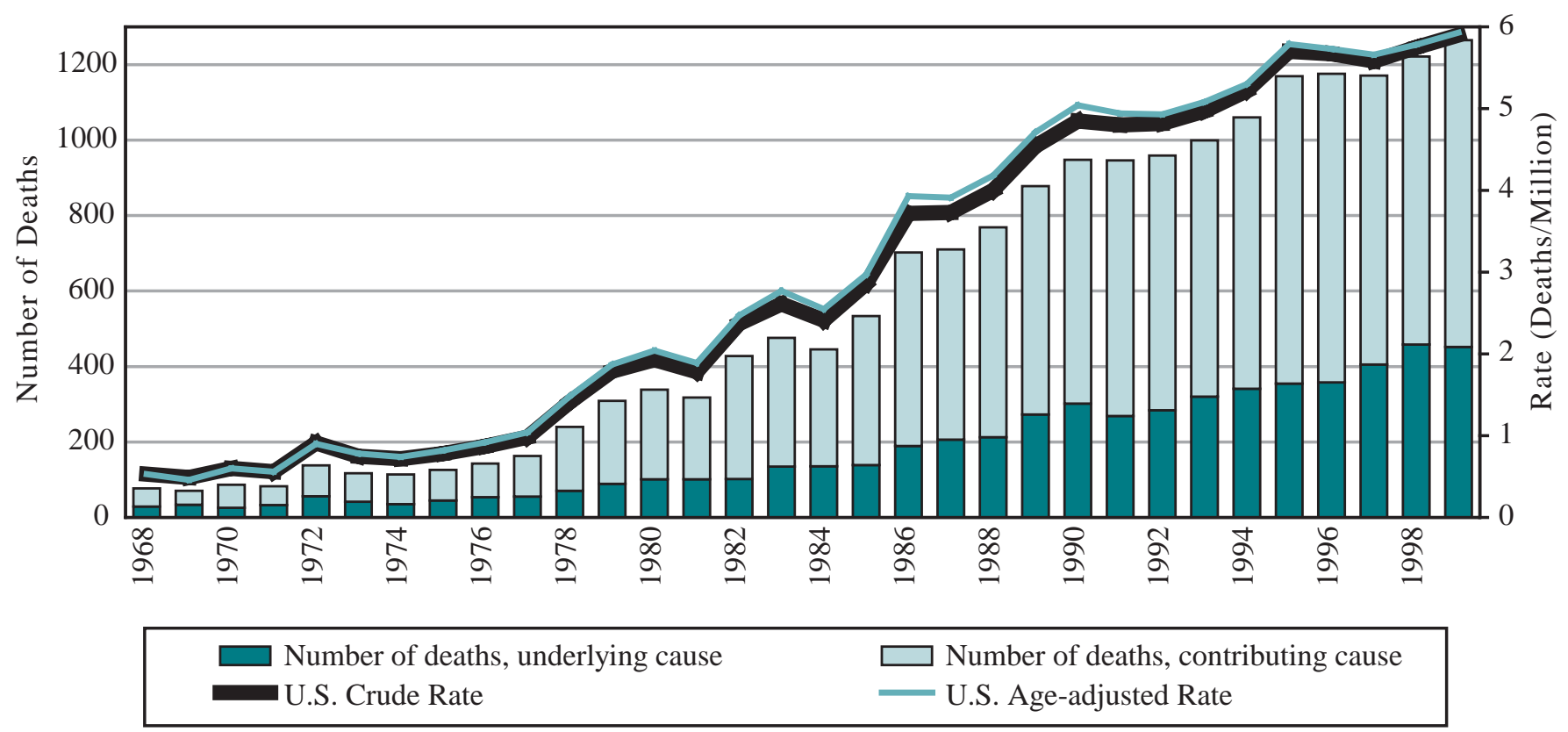

NOTE: See appendices for source description, methods, and ICD codes.

SOURCE: National Center for Health Statistics multiple cause of death data. Population estimates from U.S. Bureau of the Census.

Figure 1-2. Asbestosis: Age-adjusted mortality rates by state, U.S. residents age 15 and over, 1990-1999

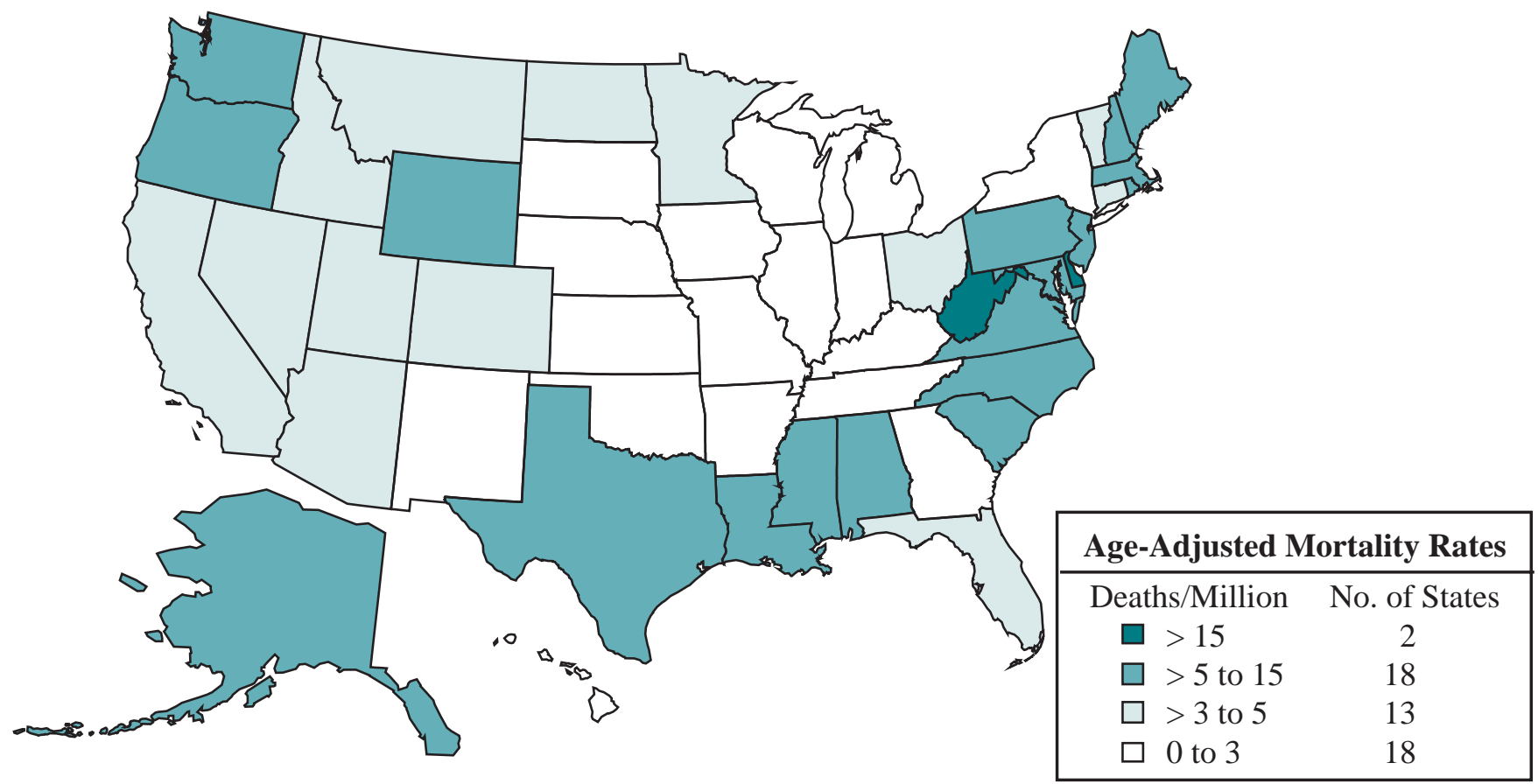

NOTE: See appendices for source description, methods, and ICD codes.

SOURCE: National Center for Health Statistics multiple cause of death data. Population estimates from U.S. Bureau of the Census. 
Table 1-1. Asbestosis: Number of deaths by sex, race, and age, and median age at death, U.S. residents age 15 and over, 1990-1999

\begin{tabular}{|c|c|c|c|c|c|c|c|c|c|c|c|c|c|c|c|c|}
\hline \multirow[b]{2}{*}{ Year } & \multirow{2}{*}{$\begin{array}{l}\text { No. of } \\
\text { Deaths }\end{array}$} & \multirow{2}{*}{$\begin{array}{c}\text { Under- } \\
\text { lying } \\
\text { Cause } \\
(\%) \\
\end{array}$} & \multicolumn{2}{|c|}{ Sex } & \multicolumn{3}{|c|}{ Race } & \multicolumn{8}{|c|}{ Age Group (yrs) } & \multirow{2}{*}{$\begin{array}{c}\text { Median } \\
\text { Age } \\
\text { (yrs) }\end{array}$} \\
\hline & & & Male & Female & White & Black & Other & $15-24$ & 25-34 & $35-44$ & 45-54 & $55-64$ & $65-74$ & 75-84 & $85+$ & \\
\hline 1990 & 948 & 31.9 & 901 & 47 & 864 & 78 & 6 & - & - & 4 & 18 & 142 & 359 & 336 & 89 & 74.0 \\
\hline 1991 & 946 & 28.4 & 908 & 38 & 877 & 63 & 6 & 1 & - & 1 & 25 & 114 & 370 & 358 & 77 & 74.0 \\
\hline 1992 & 959 & 29.6 & 923 & 36 & 898 & 57 & 4 & - & - & 3 & 13 & 124 & 371 & 355 & 93 & 74.0 \\
\hline 1993 & 999 & 32.0 & 969 & 30 & 934 & 58 & 7 & - & - & 1 & 20 & 110 & 365 & 396 & 107 & 75.0 \\
\hline 1994 & 1,060 & 32.2 & 1,026 & 34 & 993 & 62 & 5 & - & - & 2 & 21 & 94 & 410 & 422 & 111 & 75.0 \\
\hline 1995 & 1,169 & 30.4 & 1,138 & 31 & 1,095 & 69 & 5 & - & - & 3 & 24 & 118 & 411 & 477 & 136 & 75.0 \\
\hline 1996 & 1,176 & 30.4 & 1,123 & 53 & 1,088 & 84 & 4 & - & - & 3 & 16 & 104 & 428 & 480 & 145 & 75.0 \\
\hline 1997 & 1,171 & 34.6 & 1,128 & 43 & 1,106 & 60 & 5 & - & - & - & 15 & 95 & 363 & 516 & 182 & 77.0 \\
\hline 1998 & 1,221 & 37.5 & 1,177 & 44 & 1,153 & 62 & 6 & - & 1 & 1 & 11 & 97 & 394 & 524 & 193 & 76.0 \\
\hline 1999 & 1,265 & 35.7 & 1,225 & 40 & 1,190 & 64 & 11 & - & - & - & 15 & 89 & 390 & 563 & 208 & 77.0 \\
\hline TOTAL & 10,914 & 32.5 & 10,518 & 396 & 10,198 & 657 & 59 & 1 & 1 & 18 & 178 & 1,087 & 3,861 & 4,427 & 1,341 & 75.0 \\
\hline
\end{tabular}

- indicates no deaths listed.

NOTE: See appendices for source description, methods, and ICD codes.

SOURCE: National Center for Health Statistics multiple cause of death data. 
Table 1-2. Asbestosis: Mortality rates (per million population) by race and sex, U.S. residents age 15 and over, 1990-1999

\begin{tabular}{|c|c|c|c|c|c|c|c|}
\hline \multirow[b]{2}{*}{ Year } & \multirow[b]{2}{*}{ Overall } & \multicolumn{2}{|c|}{ White } & \multicolumn{2}{|c|}{ Black } & \multicolumn{2}{|c|}{ Other } \\
\hline & & Male & Female & Male & Female & Male & Female \\
\hline & \multicolumn{7}{|c|}{ Crude Mortality Rate } \\
\hline 1990 & 4.85 & 10.23 & 0.51 & 7.40 & 0.17 & 1.44 & 0.27 \\
\hline 1991 & 4.80 & 10.42 & 0.42 & 5.86 & 0.16 & 1.66 & - \\
\hline 1992 & 4.82 & 10.63 & 0.37 & 5.00 & 0.32 & 1.06 & - \\
\hline 1993 & 4.97 & 11.03 & 0.32 & 5.19 & 0.16 & 1.82 & - \\
\hline 1994 & 5.22 & 11.62 & 0.35 & 5.38 & 0.23 & 1.26 & - \\
\hline 1995 & 5.70 & 12.74 & 0.35 & 6.19 & - & 1.23 & - \\
\hline 1996 & 5.67 & 12.26 & 0.57 & 7.22 & 0.15 & 0.93 & - \\
\hline 1997 & 5.58 & 12.49 & 0.44 & 4.93 & 0.22 & 1.13 & - \\
\hline 1998 & 5.75 & 12.94 & 0.45 & 5.09 & 0.15 & 1.09 & 0.28 \\
\hline 1999 & 5.90 & 13.28 & 0.42 & 5.26 & 0.07 & 2.33 & - \\
\hline \multirow[t]{2}{*}{ 1990-1999 } & 5.32 & 11.76 & 0.42 & 5.70 & 0.16 & 1.40 & 0.04 \\
\hline & \multicolumn{7}{|c|}{ Age-Adjusted Mortality Rate } \\
\hline 1990 & 5.04 & 12.34 & 0.43 & 12.59 & 0.18 & 3.43 & 0.40 \\
\hline 1991 & 4.94 & 12.31 & 0.35 & 10.25 & 0.19 & 3.23 & - \\
\hline 1992 & 4.93 & 12.54 & 0.30 & 8.51 & 0.38 & 1.94 & - \\
\hline 1993 & 5.08 & 13.05 & 0.27 & 9.37 & 0.22 & 3.53 & - \\
\hline 1994 & 5.30 & 13.57 & 0.29 & 8.96 & 0.31 & 2.58 & - \\
\hline 1995 & 5.79 & 14.78 & 0.28 & 11.74 & - & 2.66 & - \\
\hline 1996 & 5.73 & 14.21 & 0.46 & 12.30 & 0.18 & 1.89 & - \\
\hline 1997 & 5.66 & 14.69 & 0.35 & 8.59 & 0.29 & 2.35 & - \\
\hline 1998 & 5.78 & 14.89 & 0.36 & 9.08 & 0.19 & 1.87 & 0.36 \\
\hline 1999 & 5.93 & 15.17 & 0.34 & 9.97 & 0.09 & 3.83 & - \\
\hline 1990-1999 & 5.41 & 13.76 & 0.34 & 10.10 & 0.20 & 2.78 & 0.08 \\
\hline
\end{tabular}

- indicates no deaths listed.

NOTE: See appendices for source description, methods, and ICD codes.

SOURCE: National Center for Health Statistics multiple cause of death data. Population estimates from U.S. Bureau of the Census. 
Table 1-3. Asbestosis: Years of potential life lost to age 65 and to life expectancy by race and sex, U.S. residents age 15 and over, 1990-1999

\begin{tabular}{|c|c|c|c|c|c|c|c|}
\hline \multirow[b]{2}{*}{ Year } & \multicolumn{2}{|c|}{ White } & \multicolumn{2}{|c|}{ Black } & \multicolumn{2}{|c|}{ Other } & \multirow[b]{2}{*}{ Total } \\
\hline & Male & Female & Male & Female & Male & Female & \\
\hline & \multicolumn{7}{|c|}{ Years of Potential Life Lost to Age 65} \\
\hline 1990 & 930 & 25 & 90 & 25 & 10 & - & 1,080 \\
\hline 1991 & 845 & 30 & 130 & - & 10 & - & 1,015 \\
\hline 1992 & 780 & 15 & 50 & 30 & 15 & - & 890 \\
\hline 1993 & 820 & 20 & 35 & - & - & - & 875 \\
\hline 1994 & 710 & 15 & 110 & - & - & - & 835 \\
\hline 1995 & 930 & 10 & 55 & - & 30 & - & 1,025 \\
\hline 1996 & 715 & 25 & 70 & - & 25 & - & 835 \\
\hline 1997 & 660 & - & 40 & - & - & - & 700 \\
\hline 1998 & 605 & 45 & 50 & - & 10 & - & 710 \\
\hline 1999 & 585 & 10 & 50 & - & 25 & - & 670 \\
\hline \multirow[t]{2}{*}{ Total } & 7,580 & 195 & 680 & 55 & 125 & - & 8,635 \\
\hline & \multicolumn{7}{|c|}{ Years of Potential Life Lost to Life Expectancy } \\
\hline 1990 & 9,038 & 558 & 771 & 51 & 70 & 14 & 10,502 \\
\hline 1991 & 9,294 & 466 & 664 & 28 & 87 & - & 10,539 \\
\hline 1992 & 9,419 & 392 & 546 & 81 & 66 & - & 10,504 \\
\hline 1993 & 9,541 & 357 & 529 & 17 & 87 & - & 10,531 \\
\hline 1994 & 10,074 & 353 & 657 & 29 & 59 & - & 11,172 \\
\hline 1995 & 11,245 & 351 & 637 & - & 87 & - & 12,320 \\
\hline 1996 & 10,741 & 571 & 840 & 28 & 67 & - & 12,247 \\
\hline 1997 & 10,652 & 408 & 582 & 30 & 54 & - & 11,726 \\
\hline 1998 & 11,189 & 457 & 593 & 23 & 75 & 9 & 12,346 \\
\hline 1999 & 11,436 & 407 & 592 & 14 & 170 & - & 12,619 \\
\hline Total & 102,629 & 4,320 & 6,411 & 301 & 822 & 23 & 114,506 \\
\hline
\end{tabular}

- indicates no deaths listed.

NOTE: See appendices for source description, methods, and ICD codes.

SOURCE: National Center for Health Statistics multiple cause of death data. 
Table 1-4. Asbestosis: Number of deaths by state, U.S. residents age 15 and over, 1990-1999

\begin{tabular}{|c|c|c|c|c|c|c|c|c|c|c|c|}
\hline State & 1990 & 1991 & 1992 & 1993 & 1994 & 1995 & 1996 & 1997 & 1998 & 1999 & Total \\
\hline Alabama & 21 & 23 & 18 & 23 & 29 & 36 & 43 & 41 & 40 & 44 & 318 \\
\hline Alaska & 1 & 1 & 1 & 2 & 2 & - & 2 & 2 & 5 & 2 & 18 \\
\hline Arizona & 6 & 6 & 8 & 12 & 9 & 13 & 13 & 15 & 12 & 21 & 115 \\
\hline Arkansas & 4 & 6 & 5 & 9 & 5 & 9 & 6 & 6 & 5 & 7 & 62 \\
\hline California & 102 & 94 & 95 & 93 & 101 & 113 & 100 & 104 & 91 & 107 & 1,000 \\
\hline Colorado & 4 & 5 & 4 & 7 & 12 & 6 & 13 & 9 & 6 & 7 & 73 \\
\hline Connecticut & 11 & 14 & 17 & 8 & 7 & 13 & 18 & 13 & 12 & 15 & 128 \\
\hline Delaware & 6 & 14 & 8 & 8 & 12 & 10 & 10 & 7 & 13 & 21 & 109 \\
\hline District of Columbia & - & 1 & - & 1 & 2 & - & 1 & - & - & - & 5 \\
\hline Florida & 43 & 54 & 52 & 39 & 60 & 67 & 84 & 61 & 65 & 95 & 620 \\
\hline Georgia & 13 & 10 & 18 & 9 & 12 & 11 & 9 & 12 & 15 & 16 & 125 \\
\hline Hawaii & 3 & 4 & 4 & 1 & 2 & 5 & 1 & 1 & 1 & 1 & 23 \\
\hline Idaho & 4 & 6 & 3 & 2 & 3 & 4 & 4 & 4 & 3 & 7 & 40 \\
\hline Illinois & 18 & 20 & 21 & 24 & 22 & 21 & 17 & 22 & 28 & 25 & 218 \\
\hline Indiana & 8 & 4 & 4 & 4 & 6 & 6 & 7 & 7 & 11 & 7 & 64 \\
\hline Iowa & 4 & 3 & 7 & 3 & 8 & 8 & 4 & 3 & 9 & 5 & 54 \\
\hline Kansas & 2 & 3 & 7 & 9 & 5 & 10 & 6 & 6 & 4 & 4 & 56 \\
\hline Kentucky & 1 & 5 & 5 & 11 & 5 & 3 & 9 & 12 & 10 & 4 & 65 \\
\hline Louisiana & 20 & 20 & 14 & 20 & 15 & 18 & 20 & 21 & 27 & 19 & 194 \\
\hline Maine & 17 & 8 & 8 & 13 & 12 & 8 & 6 & 8 & 16 & 15 & 111 \\
\hline Maryland & 36 & 27 & 33 & 35 & 44 & 53 & 50 & 43 & 45 & 46 & 412 \\
\hline Massachusetts & 36 & 27 & 48 & 25 & 45 & 40 & 39 & 43 & 40 & 37 & 380 \\
\hline Michigan & 17 & 15 & 16 & 16 & 17 & 27 & 21 & 24 & 16 & 30 & 199 \\
\hline Minnesota & 8 & 6 & 17 & 19 & 17 & 18 & 7 & 12 & 18 & 20 & 142 \\
\hline Mississippi & 16 & 25 & 25 & 20 & 25 & 34 & 33 & 31 & 25 & 27 & 261 \\
\hline Missouri & 9 & 11 & 14 & 18 & 13 & 11 & 11 & 11 & 13 & 13 & 124 \\
\hline Montana & 6 & 2 & 4 & 4 & 4 & - & 4 & 2 & 6 & 2 & 34 \\
\hline Nebraska & 3 & 3 & 2 & 6 & 4 & 4 & 2 & 5 & 1 & 6 & 36 \\
\hline Nevada & 2 & 3 & 1 & 3 & 6 & 5 & 5 & 3 & 7 & 7 & 42 \\
\hline New Hampshire & 2 & 1 & 4 & 8 & 6 & 6 & 4 & 7 & 6 & 6 & 50 \\
\hline New Jersey & 115 & 93 & 80 & 80 & 81 & 93 & 109 & 78 & 93 & 93 & 915 \\
\hline New Mexico & 2 & 3 & 1 & 3 & 6 & 8 & 2 & 1 & 1 & 4 & 31 \\
\hline New York & 44 & 37 & 30 & 26 & 34 & 43 & 42 & 46 & 47 & 54 & 403 \\
\hline North Carolina & 25 & 21 & 25 & 12 & 32 & 29 & 33 & 37 & 50 & 34 & 298 \\
\hline North Dakota & - & 3 & - & 2 & 3 & - & 3 & 3 & 2 & 4 & 20 \\
\hline Ohio & 27 & 24 & 32 & 29 & 31 & 35 & 43 & 43 & 31 & 45 & 340 \\
\hline Oklahoma & 6 & 6 & 5 & 1 & 5 & 5 & 5 & 8 & 9 & 6 & 56 \\
\hline Oregon & 12 & 22 & 22 & 29 & 26 & 18 & 30 & 21 & 33 & 31 & 244 \\
\hline Pennsylvania & 67 & 83 & 100 & 114 & 90 & 114 & 106 & 112 & 99 & 77 & 962 \\
\hline Rhode Island & 7 & 1 & 5 & 4 & 6 & 5 & 2 & 4 & 9 & 4 & 47 \\
\hline South Carolina & 11 & 8 & 13 & 21 & 13 & 17 & 18 & 24 & 23 & 19 & 167 \\
\hline South Dakota & - & - & - & - & - & - & 3 & - & - & 1 & 4 \\
\hline Tennessee & 6 & 8 & 4 & 8 & 13 & 12 & 14 & 18 & 16 & 12 & 111 \\
\hline Texas & 91 & 95 & 54 & 72 & 80 & 93 & 87 & 88 & 98 & 80 & 838 \\
\hline Utah & 3 & - & 4 & 5 & 2 & 4 & 5 & 7 & - & 6 & 36 \\
\hline Vermont & 1 & - & 3 & 3 & - & 2 & 2 & 1 & 2 & 2 & 16 \\
\hline Virginia & 47 & 36 & 43 & 47 & 38 & 44 & 37 & 41 & 55 & 60 & 448 \\
\hline Washington & 34 & 50 & 40 & 59 & 60 & 44 & 48 & 56 & 60 & 75 & 526 \\
\hline West Virginia & 17 & 20 & 28 & 18 & 20 & 32 & 32 & 34 & 28 & 27 & 256 \\
\hline Wisconsin & 9 & 11 & 6 & 10 & 9 & 10 & 3 & 13 & 13 & 15 & 99 \\
\hline Wyoming & 1 & 4 & 1 & 4 & 1 & 2 & 3 & 1 & 2 & - & 19 \\
\hline TOTAL & 948 & 946 & 959 & 999 & 1,060 & 1,169 & 1,176 & 1,171 & 1,221 & 1,265 & 10,914 \\
\hline
\end{tabular}

- indicates no deaths listed.

NOTE: See appendices for source description, methods, and ICD codes. SOURCE: National Center for Health Statistics multiple cause of death data. 
Table 1-5. Asbestosis: Number of deaths, mortality rates (per million population), and years of potential life lost (YPLL) by state, U.S. residents age 15 and over, 1990-1999

\begin{tabular}{|c|c|c|c|c|c|c|c|c|c|c|}
\hline \multirow[b]{2}{*}{ State } & \multirow{2}{*}{\begin{tabular}{|l|} 
No. of \\
Deaths
\end{tabular}} & \multirow[b]{2}{*}{ Rank } & \multicolumn{2}{|c|}{ Crude Mortality } & \multicolumn{2}{|c|}{ Age-Adjusted Mortality } & \multicolumn{4}{|c|}{ YPLL to Life Expectancy } \\
\hline & & & Rate & Rank & Rate & Rank & Total & Rank & YPLL/death & Rank \\
\hline Alabama & 318 & 12 & 9.59 & 10 & 9.29 & 11 & 4,047 & 12 & 12.7 & 8 \\
\hline Alaska & 18 & 48 & 4.12 & 24 & 10.62 & 8 & 242 & 47 & 13.4 & 2 \\
\hline Arizona & 115 & 25 & 3.54 & 29 & 3.48 & 31 & 1,358 & 26 & 11.8 & 28 \\
\hline Arkansas & 62 & 33 & 3.21 & 32 & 2.82 & 35 & 819 & 31 & 13.2 & 3 \\
\hline California & 1,000 & 1 & 4.10 & 25 & 4.90 & 21 & 11,489 & 1 & 11.5 & 38 \\
\hline Colorado & 73 & 30 & 2.52 & 40 & 3.10 & 33 & 905 & 30 & 12.4 & 14 \\
\hline Connecticut & 128 & 22 & 4.86 & 22 & 4.67 & 23 & 1,548 & 23 & 12.1 & 19 \\
\hline Delaware & 109 & 28 & 19.29 & 1 & 20.63 & 1 & 1,311 & 27 & 12.0 & 23 \\
\hline District of Columbia & 5 & 50 & 1.08 & 50 & 1.12 & 50 & 57 & 50 & 11.3 & 40 \\
\hline Florida & 620 & 5 & 5.40 & 18 & 4.05 & 26 & 7,087 & 5 & 11.4 & 39 \\
\hline Georgia & 125 & 23 & 2.25 & 46 & 2.75 & 38 & 1,641 & 22 & 13.1 & 4 \\
\hline Hawaii & 23 & 45 & 2.49 & 42 & 2.89 & 34 & 285 & 45 & 12.4 & 14 \\
\hline Idaho & 40 & 40 & 4.65 & 23 & 4.59 & 24 & 453 & 40 & 11.3 & 40 \\
\hline Illinois & 218 & 17 & 2.37 & 45 & 2.37 & 44 & 2,579 & 17 & 11.8 & 28 \\
\hline Indiana & 64 & 32 & 1.42 & 49 & 1.41 & 49 & 778 & 33 & 12.2 & 18 \\
\hline Iowa & 54 & 36 & 2.44 & 44 & 2.02 & 48 & 654 & 35 & 12.1 & 19 \\
\hline Kansas & 56 & 34 & 2.84 & 35 & 2.55 & 42 & 649 & 36 & 11.6 & 35 \\
\hline Kentucky & 65 & 31 & 2.15 & 48 & 2.13 & 46 & 782 & 32 & 12.0 & 23 \\
\hline Louisiana & 194 & 19 & 5.96 & 13 & 6.55 & 16 & 2,466 & 19 & 12.7 & 8 \\
\hline Maine & 111 & 26 & 11.38 & 6 & 10.75 & 7 & 1,216 & 28 & 11.0 & 44 \\
\hline Maryland & 412 & 8 & 10.35 & 7 & 12.40 & 6 & 5,219 & 8 & 12.7 & 8 \\
\hline Massachusetts & 380 & 10 & 7.76 & 12 & 7.47 & 13 & 4,165 & 11 & 11.0 & 44 \\
\hline Michigan & 199 & 18 & 2.69 & 37 & 2.79 & 36 & 2,575 & 18 & 12.9 & 7 \\
\hline Minnesota & 142 & 21 & 4.01 & 27 & 4.01 & 27 & 1,774 & 21 & 12.5 & 13 \\
\hline Mississippi & 261 & 14 & 12.81 & 4 & 12.71 & 5 & 3,398 & 14 & 13.0 & 6 \\
\hline Missouri & 124 & 24 & 2.99 & 33 & 2.69 & 40 & 1,505 & 24 & 12.1 & 19 \\
\hline Montana & 34 & 43 & 5.11 & 21 & 4.87 & 22 & 407 & 41 & 12.0 & 23 \\
\hline Nebraska & 36 & 41 & 2.87 & 34 & 2.46 & 43 & 367 & 43 & 10.2 & 51 \\
\hline Nevada & 42 & 39 & 3.49 & 31 & 4.26 & 25 & 528 & 39 & 12.6 & 12 \\
\hline New Hampshire & 50 & 37 & 5.56 & 17 & 6.17 & 18 & 580 & 37 & 11.6 & 35 \\
\hline New Jersey & 915 & 3 & 14.39 & 3 & 14.31 & 3 & 10,230 & 3 & 11.2 & 43 \\
\hline New Mexico & 31 & 44 & 2.46 & 43 & 2.73 & 39 & 365 & 44 & 11.8 & 28 \\
\hline New York & 403 & 9 & 2.79 & 36 & 2.79 & 36 & 4,752 & 9 & 11.8 & 28 \\
\hline North Carolina & 298 & 13 & 5.21 & 20 & 5.46 & 19 & 3,702 & 13 & 12.4 & 14 \\
\hline North Dakota & 20 & 46 & 4.08 & 26 & 3.63 & 30 & 271 & 46 & 13.5 & 1 \\
\hline Ohio & 340 & 11 & 3.91 & 28 & 3.82 & 28 & 4,446 & 10 & 13.1 & 4 \\
\hline Oklahoma & 56 & 34 & 2.21 & 47 & 2.05 & 47 & 668 & 34 & 11.9 & 27 \\
\hline Oregon & 244 & 16 & 9.94 & 9 & 9.32 & 10 & 2,604 & 16 & 10.7 & 47 \\
\hline Pennsylvania & 962 & 2 & 9.97 & 8 & 8.53 & 12 & 11,316 & 2 & 11.8 & 28 \\
\hline Rhode Island & 47 & 38 & 5.89 & 15 & 5.21 & 20 & 546 & 38 & 11.6 & 35 \\
\hline South Carolina & 167 & 20 & 5.85 & 16 & 6.62 & 15 & 1,967 & 20 & 11.8 & 28 \\
\hline South Dakota & 4 & 51 & 0.73 & 51 & 0.60 & 51 & 45 & 51 & 11.3 & 40 \\
\hline Tennessee & 111 & 26 & 2.68 & 39 & 2.69 & 40 & 1,404 & 25 & 12.7 & 8 \\
\hline Texas & 838 & 4 & 5.92 & 14 & 7.11 & 14 & 10,131 & 4 & 12.1 & 19 \\
\hline Utah & 36 & 41 & 2.69 & 37 & 3.42 & 32 & 380 & 42 & 10.6 & 48 \\
\hline Vermont & 16 & 49 & 3.50 & 30 & 3.73 & 29 & 174 & 49 & 10.9 & 46 \\
\hline Virginia & 448 & 7 & 8.56 & 11 & 10.50 & 9 & 5,259 & 7 & 11.7 & 34 \\
\hline Washington & 526 & 6 & 12.50 & 5 & 13.49 & 4 & 5,490 & 6 & 10.4 & 49 \\
\hline West Virginia & 256 & 15 & 17.60 & 2 & 15.29 & 2 & 3,074 & 15 & 12.0 & 23 \\
\hline Wisconsin & 99 & 29 & 2.50 & 41 & 2.35 & 45 & 1,215 & 29 & 12.3 & 17 \\
\hline Wyoming & 19 & 47 & 5.31 & 19 & 6.27 & 17 & 198 & 48 & 10.4 & 49 \\
\hline
\end{tabular}

- indicates no deaths listed.

NOTE: See appendices for source description, methods, and ICD codes.

SOURCE: National Center for Health Statistics multiple cause of death data. Population estimates from U.S. Bureau of the Census. 
Table 1-6. Asbestosis: Most frequently recorded industries on death certificate, U.S. residents age 15 and over, selected states and years, 1990-1999

\begin{tabular}{clrc}
\hline CIC & Industry & Number of Deaths & Percent \\
\hline 060 & Construction & 702 & 24.6 \\
360 & Ship and boat building and repairing & 171 & 6.0 \\
192 & Industrial and miscellaneous chemicals & 124 & 4.3 \\
400 & Railroads & 89 & 3.1 \\
262 & Miscellaneous nonmetallic and stone products & 75 & 2.6 \\
901 & General government, n.e.c. & 71 & 2.5 \\
270 & Blast furnaces, steelworks, rolling and finishing mills & 67 & 2.3 \\
392 & Not specified manufacturing industries & 61 & 2.1 \\
460 & Electric light and power & 55 & 1.9 \\
842 & Elementary and secondary schools & 53 & 1.9 \\
& All other industries & 1,286 & 45.0 \\
& Industry not reported & 105 & 3.7 \\
& TOTAL & $\mathbf{2 , 8 5 9}$ & $\mathbf{1 0 0 . 0}$ \\
\hline
\end{tabular}

CIC - Census Industry Code

n.e.c. - not elsewhere classified

NOTE: Percentages may not total to $100 \%$ due to rounding. See appendices for source description, methods, and ICD codes, industry and occupation codes, and list of selected states and years.

SOURCE: National Center for Health Statistics multiple cause of death data.

Table 1-7. Asbestosis: Most frequently recorded occupations on death certificate, U.S. residents age 15 and over, selected states and years, 1990-1999

\begin{tabular}{clrc}
\hline COC & Occupation & Number of Deaths & Percent \\
\hline 585 & Plumbers, pipefitters, and steamfitters & 238 & 8.3 \\
019 & Managers and administrators, n.e.c. & 129 & 4.5 \\
575 & Electricians & 125 & 4.4 \\
567 & Carpenters & 120 & 4.2 \\
593 & Insulation workers & 108 & 3.8 \\
889 & Laborers, except construction & 95 & 3.3 \\
633 & Supervisors, production occupations & 85 & 3.0 \\
783 & Welders and cutters & 78 & 2.7 \\
453 & Janitors and cleaners & 74 & 2.6 \\
804 & Truck drivers & 66 & 2.3 \\
& All other occupations & 1,639 & 57.3 \\
& Occupation not reported & 102 & 3.6 \\
& TOTAL & $\mathbf{2 , 8 5 9}$ & $\mathbf{1 0 0 . 0}$ \\
\hline
\end{tabular}

COC - Census Occupation Code n.e.c. - not elsewhere classified

NOTE: Percentages may not total to $100 \%$ due to rounding. See appendices for source description, methods, and ICD codes, industry and occupation codes, and list of selected states and years.

SOURCE: National Center for Health Statistics multiple cause of death data. 
Table 1-8. Asbestosis: Proportionate mortality ratio (PMR) adjusted for age, sex, and race by usual industry, U.S. residents age 15 and over, selected states and years, 1990-1999

\begin{tabular}{llrrrr}
\hline & & & \multicolumn{3}{c}{ 95\% Confidence Interval } \\
\cline { 5 - 6 } CIC & Industry & Number & Deaths & PMR & UCL \\
\hline 262 & Miscellaneous nonmetallic mineral and stone products & 75 & 16.39 & 13.00 & 20.70 \\
360 & Ship and boat building and repairing & 171 & 15.70 & 13.48 & 18.29 \\
502 & Lumber and construction materials & 20 & 6.95 & 4.24 & 10.75 \\
192 & Industrial and miscellaneous chemicals & 124 & 4.78 & 4.00 & 5.73 \\
211 & Other rubber products, and plastics footwear and belting & 40 & 4.31 & 3.08 & 5.87 \\
462 & Electric and gas, and other combinations & 14 & 3.05 & 1.66 & 5.11 \\
180 & Plastics, synthetics, and resins & 12 & 2.80 & 1.44 & 4.89 \\
200 & Petroleum refining & 31 & 2.74 & 1.86 & 3.89 \\
272 & Primary aluminum industries & 16 & 2.65 & 1.52 & 4.31 \\
460 & Electric light and power & 55 & 2.65 & 2.02 & 3.48 \\
250 & Glass and glass products & 30 & 2.58 & 1.74 & 3.68 \\
881 & Membership organizations & 13 & 2.47 & 1.31 & 4.22 \\
060 & Construction & 702 & 2.38 & 2.21 & 2.57 \\
282 & Fabricated structural metal products & 28 & 2.29 & 1.52 & 3.30 \\
420 & Water transportation & 24 & 2.28 & 1.46 & 3.39 \\
210 & Tires and inner tubes & 15 & 2.23 & 1.25 & 3.69 \\
400 & Railroads & 89 & 1.64 & 1.33 & 2.03 \\
270 & Blast furnaces, steelworks, rolling and finishing mills & 67 & 1.30 & 1.02 & 1.67 \\
\hline
\end{tabular}

CIC - Census Industry Code $\quad$ n.e.c. - not elsewhere classified $\quad$ LCL - lower confidence limit $\quad$ UCL - upper confidence limit NOTE: See appendices for source description, methods, and ICD codes, industry and occupation codes, and list of selected states and years. SOURCE: National Center for Health Statistics multiple cause of death data. 
Table 1-9. Asbestosis: Proportionate mortality ratio (PMR) adjusted for age, sex, and race by usual occupation, U.S. residents age 15 and over, selected states and years, 1990-1999

\begin{tabular}{|c|c|c|c|c|c|}
\hline \multirow[b]{2}{*}{$\mathrm{COC}$} & \multirow[b]{2}{*}{ Occupation } & \multirow{2}{*}{$\begin{array}{c}\text { Number } \\
\text { of Deaths }\end{array}$} & \multirow[b]{2}{*}{ PMR } & \multicolumn{2}{|c|}{ 95\% Confidence Interval } \\
\hline & & & & LCL & $\mathbf{U C L}$ \\
\hline 593 & Insulation workers & 108 & 84.30 & 69.54 & 102.28 \\
\hline 643 & Boilermakers & 59 & 20.26 & 15.59 & 26.38 \\
\hline 585 & Plumbers, pipefitters, and steamfitters & 238 & 9.38 & 8.25 & 10.68 \\
\hline 058 & Marine and naval architects & 7 & 8.25 & 3.31 & 17.01 \\
\hline 646 & Lay-out workers & 7 & 8.21 & 3.30 & 16.92 \\
\hline 584 & Plasterers & 9 & 6.43 & 2.95 & 12.20 \\
\hline 676 & Patternmakers, lay-out workers, and cutters & 5 & 6.34 & 2.05 & 14.82 \\
\hline 653 & Sheet metal workers & 53 & 6.14 & 4.66 & 8.12 \\
\hline 557 & Supervisors: plumbers, pipefitters, and steamfitters & 7 & 5.34 & 2.14 & 11.01 \\
\hline 224 & Chemical technicians & 8 & 4.89 & 2.11 & 9.62 \\
\hline 757 & Separating, filtering, and clarifying machine operators & 21 & 4.77 & 2.94 & 7.29 \\
\hline 829 & Sailors and deckhands & 12 & 4.22 & 2.18 & 7.37 \\
\hline 534 & Heating, air conditioning, and refrigeration mechanics & 16 & 4.13 & 2.36 & 6.71 \\
\hline 544 & Millwrights & 34 & 4.10 & 2.85 & 5.73 \\
\hline 575 & Electricians & 125 & 4.10 & 3.43 & 4.91 \\
\hline 555 & Supervisors, electricians, power transmission installers & 9 & 3.62 & 1.66 & 6.87 \\
\hline 783 & Welders and cutters & 78 & 3.08 & 2.46 & 3.87 \\
\hline 547 & Specified mechanics and repairers, n.e.c. & 21 & 2.55 & 1.58 & 3.90 \\
\hline 518 & Industrial machinery repairers & 34 & 2.22 & 1.54 & 3.10 \\
\hline 563 & Brickmasons and stonemasons & 26 & 2.14 & 1.40 & 3.14 \\
\hline 856 & Industrial truck and tractor equipment operators & 17 & 2.09 & 1.22 & 3.35 \\
\hline 738 & Winding and twisting machine operators & 11 & 2.09 & 1.04 & 3.73 \\
\hline 849 & Crane and tower operators & 15 & 2.05 & 1.14 & 3.38 \\
\hline 696 & Stationary engineers & 26 & 1.88 & 1.23 & 2.76 \\
\hline 503 & Supervisors, mechanics and repairers & 17 & 1.85 & 1.08 & 2.96 \\
\hline 567 & Carpenters & 120 & 1.83 & 1.52 & 2.20 \\
\hline 507 & Bus, truck, and stationary engine mechanics & 16 & 1.80 & 1.03 & 2.92 \\
\hline 549 & Not specified mechanics and repairers & 22 & 1.75 & 1.09 & 2.65 \\
\hline 777 & Miscellaneous machine operators, n.e.c. & 38 & 1.62 & 1.15 & 2.22 \\
\hline 779 & Machine operators, not specified & 49 & 1.53 & 1.13 & 2.02 \\
\hline 633 & Supervisors, production occupations & 85 & 1.34 & 1.08 & 1.67 \\
\hline 869 & Construction laborers & 58 & 1.34 & 1.03 & 1.74 \\
\hline
\end{tabular}

COC - Census Occupation Code n.e.c. - not elsewhere classified $\quad$ LCL - lower confidence limit UCL - upper confidence limit NOTE: See appendices for source description, methods, and ICD codes, industry and occupation codes, and list of selected states and years. SOURCE: National Center for Health Statistics multiple cause of death data. 
$\nwarrow$ Figure 1-3. Asbestosis: Age-adjusted mortality rates by county, U.S. residents age 15 and over, 1970-1999

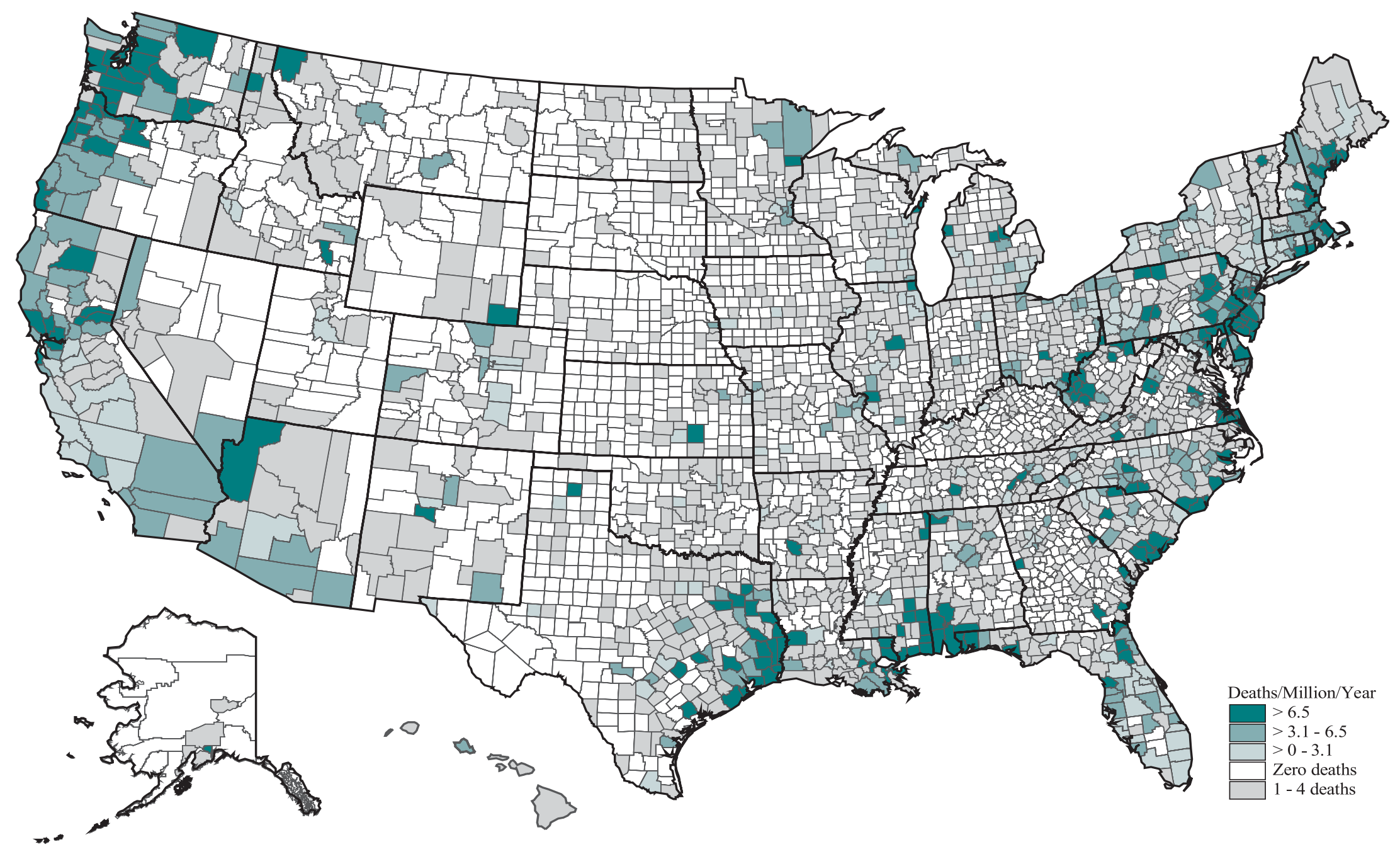

NOTE: Age-adjusted rates are not calculated for those counties with 1-4 deaths. See appendices for source description, methods, and ICD codes. SOURCE: National Center for Health Statistics multiple cause of death data. Population estimates from U.S. Bureau of the Census. 
Figure 1-4. Asbestosis: Age-adjusted mortality rates by county, U.S. residents age 15 and over, 1970-1984 and 1985-1999
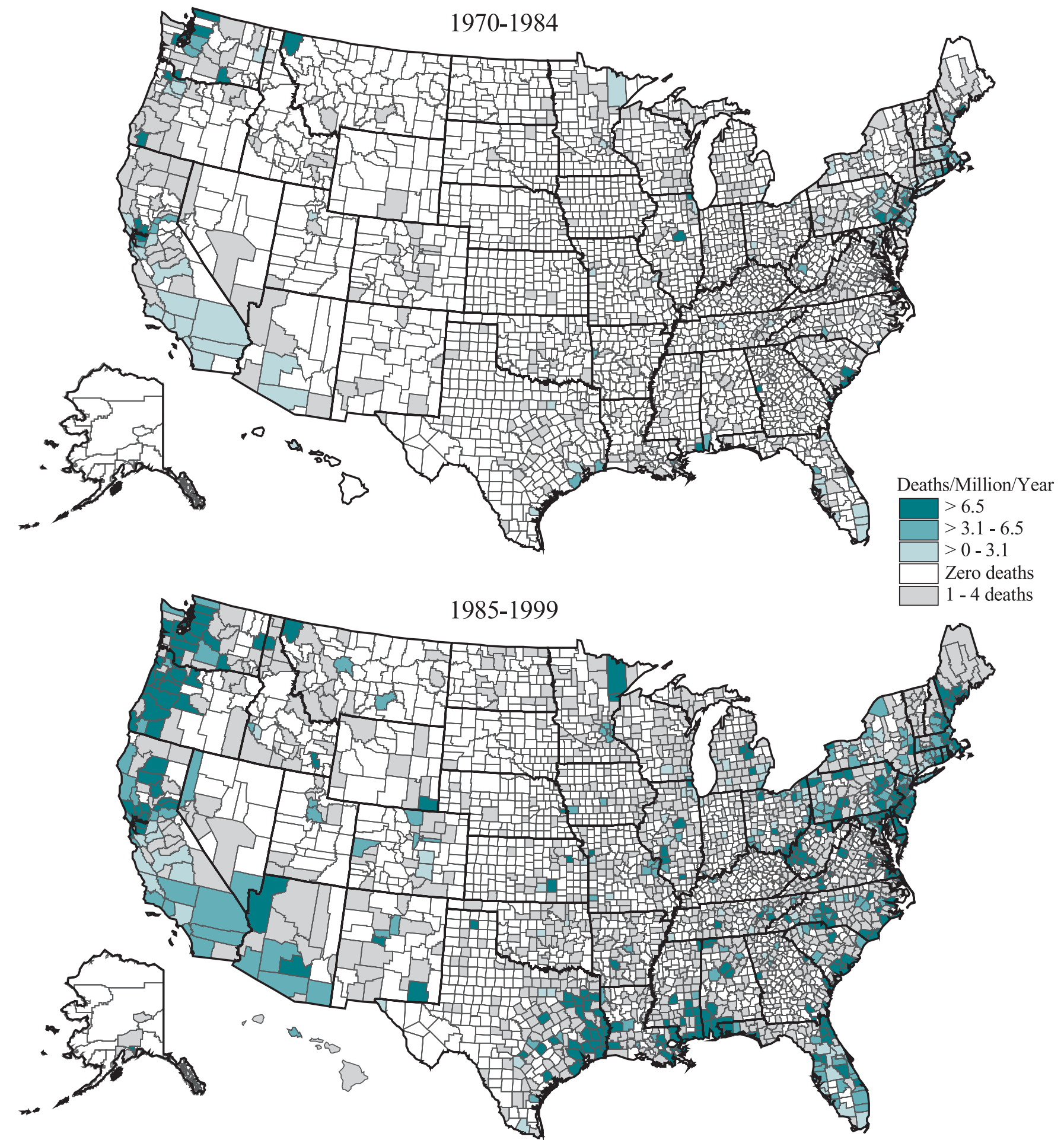

NOTE: Age-adjusted rates are not calculated for those counties with 1-4 deaths. See appendices for source description, methods, and ICD codes. SOURCE: National Center for Health Statistics multiple cause of death data. Population estimates from U.S. Bureau of the Census. 
Table 1-10. Asbestosis: Counties with highest age-adjusted mortality rates (per million population), U.S. residents age 15 and over, 1985-1999

\begin{tabular}{|c|c|c|c|c|c|}
\hline County & State & Age-Adjusted Rate & Crude Rate & Number of Deaths & \% Female \\
\hline Poquoson City & Virginia & 140.6 & 100.8 & 13 & 0.0 \\
\hline Orange County & Texas & 127.3 & 108.3 & 102 & 3.9 \\
\hline Jackson County & Mississippi & 111.2 & 81.0 & 110 & 3.6 \\
\hline Somerset County & New Jersey & 105.7 & 86.5 & 263 & 17.1 \\
\hline George County & Mississippi & 98.0 & 88.1 & 17 & 0.0 \\
\hline Lincoln County & Montana & 96.2 & 96.2 & 19 & 5.3 \\
\hline Sagadahoc County & Maine & 82.2 & 71.2 & 28 & 7.1 \\
\hline Kitsap County & Washington & 68.1 & 57.2 & 137 & 2.2 \\
\hline Newport News City & Virginia & 62.8 & 42.3 & 86 & 4.7 \\
\hline Greene County & Mississippi & 61.6 & 57.9 & 7 & 0.0 \\
\hline Hampton City & Virginia & 57.6 & 39.8 & 64 & 1.6 \\
\hline Isle of Wight County & Virginia & 56.0 & 49.2 & 15 & 0.0 \\
\hline Camden County & New Jersey & 52.6 & 49.6 & 291 & 3.1 \\
\hline Jefferson County & Texas & 51.4 & 56.9 & 159 & 2.5 \\
\hline York County & Virginia & 49.9 & 26.3 & 14 & 0.0 \\
\hline Gloucester County & New Jersey & 48.6 & 40.0 & 109 & 1.8 \\
\hline Mason County & West Virginia & 47.8 & 54.6 & 16 & 0.0 \\
\hline Jones County & Mississippi & 44.7 & 50.2 & 36 & 2.8 \\
\hline Portsmouth City & Virginia & 43.7 & 47.5 & 57 & 0.0 \\
\hline Currituck County & North Carolina & 42.9 & 46.6 & 8 & 0.0 \\
\hline Washington County & Alabama & 41.9 & 42.5 & 8 & 0.0 \\
\hline Putnam County & West Virginia & 41.7 & 36.0 & 19 & 0.0 \\
\hline Suffolk City & Virginia & 39.8 & 39.2 & 24 & 4.2 \\
\hline Jasper County & Texas & 37.7 & 44.5 & 16 & 0.0 \\
\hline Chesapeake City & Virginia & 37.7 & 25.1 & 47 & 4.3 \\
\hline Hardin County & Texas & 37.0 & 36.7 & 18 & 0.0 \\
\hline Mobile County & Alabama & 36.9 & 35.3 & 156 & 1.9 \\
\hline Newton County & Texas & 36.4 & 39.7 & 6 & 0.0 \\
\hline Burleson County & Texas & 35.6 & 43.5 & 7 & 0.0 \\
\hline Charleston County & South Carolina & 34.3 & 25.1 & 89 & 10.1 \\
\hline Kanawha County & West Virginia & 34.2 & 39.9 & 100 & 4.0 \\
\hline Berkeley County & South Carolina & 33.8 & 15.0 & 22 & 22.7 \\
\hline Sabine County & Texas & 29.6 & 58.6 & 7 & 0.0 \\
\hline Solano County & California & 27.3 & 18.1 & 73 & 1.4 \\
\hline Delaware County & Pennsylvania & 27.3 & 32.5 & 214 & 1.9 \\
\hline Tyler County & Texas & 27.0 & 37.7 & 8 & 0.0 \\
\hline Lincoln County & West Virginia & 26.0 & 28.0 & 7 & 0.0 \\
\hline Sussex County & Delaware & 25.6 & 31.0 & 44 & 4.5 \\
\hline Galveston County & Texas & 25.2 & 19.9 & 52 & 0.0 \\
\hline Jasper County & Mississippi & 25.1 & 31.1 & 6 & 16.7 \\
\hline Napa County & California & 25.0 & 30.0 & 41 & 0.0 \\
\hline Salem County & New Jersey & 24.9 & 27.4 & 21 & 4.8 \\
\hline Clarke County & Alabama & 24.0 & 25.5 & 8 & 0.0 \\
\hline Wayne County & Mississippi & 23.8 & 23.2 & 5 & 0.0 \\
\hline Benton County & Washington & 23.7 & 18.7 & 25 & 4.0 \\
\hline Boone County & West Virginia & 22.8 & 19.3 & 6 & 0.0 \\
\hline Gloucester County & Virginia & 22.2 & 22.2 & 8 & 0.0 \\
\hline Lincoln County & Maine & 22.0 & 27.6 & 10 & 10.0 \\
\hline Effingham County & Georgia & 21.8 & 16.5 & 5 & 0.0 \\
\hline Allegany County & Maryland & 21.6 & 29.5 & 27 & 7.4 \\
\hline Overall United States & & 5.0 & 4.9 & 14,507 & 3.6 \\
\hline
\end{tabular}

NOTE: Only counties with at least 5 deaths from the disease of interest are included. See appendices for source description, methods, and ICD codes. SOURCE: National Center for Health Statistics multiple cause of death data. Population estimates from U.S. Bureau of the Census. 
Table 1-11. Asbestosis: Estimated number of discharges from short-stay nonfederal hospitals, 1970-2000

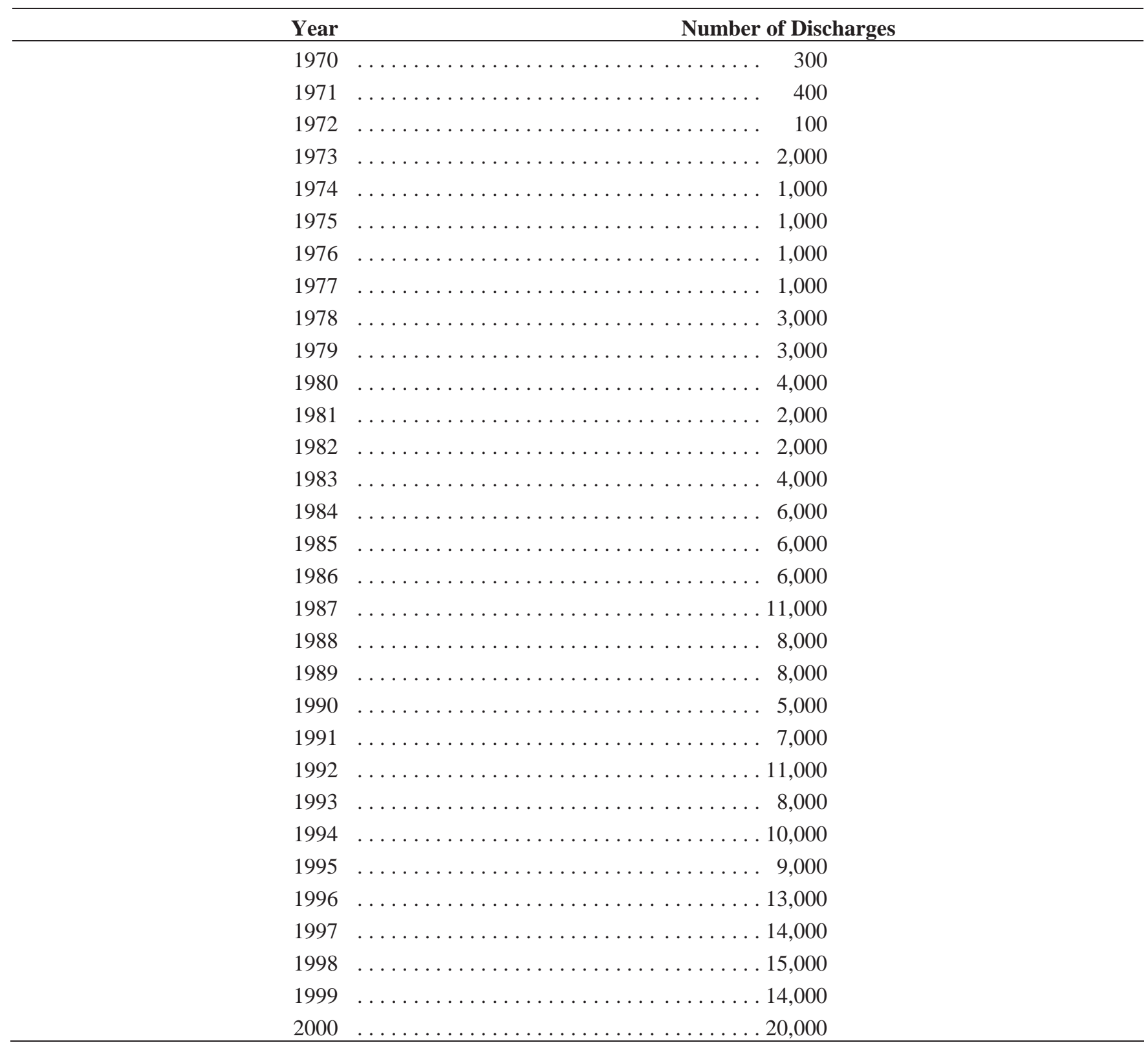

NOTE: Number of discharges has been rounded. NCHS recommends that, in statistical comparisons, estimates of less than 5,000 not be used and that estimates of 5,000 to 10,000 be used with caution. See appendices for source description and methods. SOURCE: National Center for Health Statistics National Hospital Discharge Survey. 
Figure 1-5. Asbestos: Geometric mean exposures by major industry division, MSHA and OSHA samples, 1979-1999

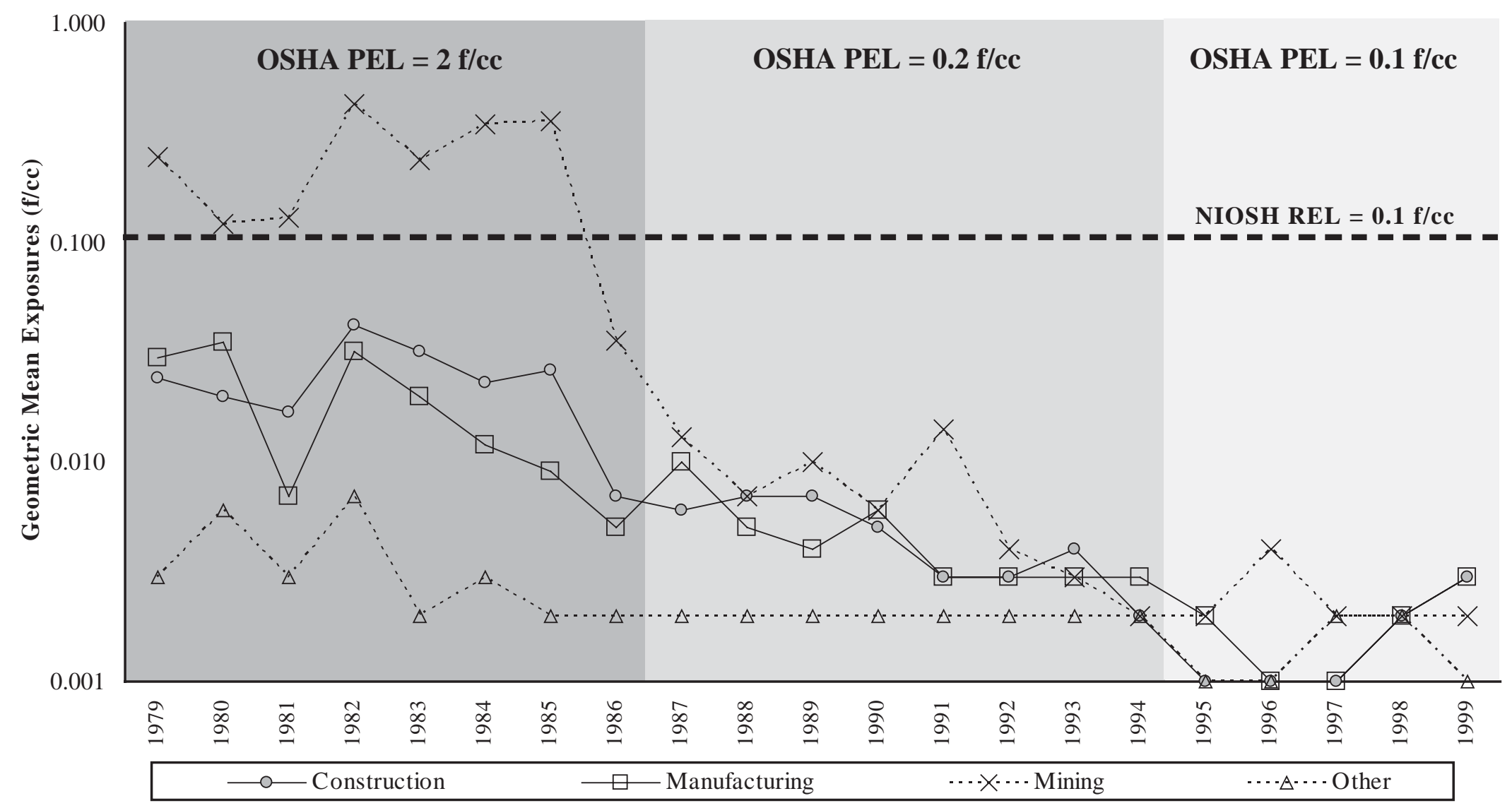

PEL - permissible exposure limit $\quad$ REL - recommended exposure limit $\quad \mathrm{f} / \mathrm{cc}$ - fibers per cubic centimeter

NOTE: The MSHA PEL is $2 \mathrm{f} / \mathrm{cc}$. See appendices for source description, methods, and agents.

SOURCE: Mine Safety and Health Administration (MSHA) metal/nonmetal mine data. Occupational Safety and Health Administation (OSHA) Integrated Management Information System. 
Table 1-12. Asbestos: Geometric mean exposures and percent exceeding designated occupational exposure limits by major industry division, MSHA and OSHA samples, 1979-1999

\begin{tabular}{|c|c|c|c|c|c|c|c|c|c|c|c|c|c|c|c|c|c|c|c|c|c|c|}
\hline \multirow{2}{*}{\multicolumn{2}{|c|}{ Industry Division }} & \multicolumn{8}{|c|}{ OSHA PEL $=2 \mathrm{f} / \mathrm{cc}$} & \multicolumn{8}{|c|}{ OSHA PEL = 0.2 f/cc } & \multicolumn{5}{|c|}{ OSHA PEL = 0.1 f/cc } \\
\hline & & 1979 & 1980 & 1981 & 1982 & 1983 & 1984 & 1985 & 1986 & 1987 & 1988 & 1989 & 1990 & 1991 & 1992 & 1993 & 1994 & 1995 & 1996 & 1997 & 1998 & 1999 \\
\hline \multirow{4}{*}{$\begin{array}{l}\text { Construction } \\
\text { SIC 15-17 }\end{array}$} & GM (f/cc) & 0.024 & 0.020 & 0.017 & 0.042 & 0.032 & 0.023 & 0.026 & 0.007 & 0.006 & 0.007 & 0.007 & 0.005 & 0.003 & 0.003 & 0.004 & 0.002 & 0.001 & 0.001 & 0.001 & 0.002 & 0.003 \\
\hline & No. of samples & 97 & 172 & 112 & 168 & 383 & 629 & 474 & 279 & 254 & 254 & 266 & 175 & 214 & 200 & 144 & 82 & 45 & 75 & 52 & 35 & 31 \\
\hline & $\%>$ PEL & 3.1 & 3.5 & 1.8 & 7.7 & 4.2 & 5.1 & 5.3 & 3.2 & 4.7 & 9.1 & 11.3 & 9.7 & 2.8 & 4.5 & 1.4 & 3.7 & 2.2 & 0.0 & 0.0 & 0.0 & 12.9 \\
\hline & $\%>$ REL & 36.1 & 30.2 & 22.3 & 32.1 & 37.9 & 32.4 & 30.4 & 12.9 & 9.1 & 12.2 & 15.0 & 13.7 & 4.7 & 7.5 & 4.9 & 6.1 & 2.2 & 0.0 & 0.0 & 0.0 & 12.9 \\
\hline \multirow{4}{*}{$\begin{array}{l}\text { Manufacturing } \\
\text { SIC 20-39 }\end{array}$} & GM (f/cc) & 0.030 & 0.035 & 0.007 & 0.032 & 0.020 & 0.012 & 0.009 & 0.005 & 0.010 & 0.005 & 0.004 & 0.006 & 0.003 & 0.003 & 0.003 & 0.003 & 0.002 & 0.001 & 0.001 & 0.002 & 0.003 \\
\hline & No. of samples & 335 & 313 & 153 & 181 & 372 & 438 & 428 & 278 & 447 & 347 & 292 & 203 & 277 & 142 & 131 & 76 & 108 & 149 & 48 & 33 & 18 \\
\hline & $\%>$ PEL & 3.0 & 7.7 & 0.7 & 1.1 & 4.6 & 2.3 & 2.3 & 2.5 & 16.6 & 6.3 & 6.2 & 11.8 & 7.9 & 0.0 & 5.3 & 0.0 & 0.9 & 1.3 & 0.0 & 0.0 & 5.6 \\
\hline & $\%>$ REL & 37.3 & 41.2 & 14.4 & 35.4 & 27.2 & 26.3 & 21.0 & 10.1 & 21.5 & 13.3 & 10.6 & 15.8 & 10.5 & 1.4 & 9.9 & 3.9 & 0.9 & 1.3 & 0.0 & 0.0 & 5.6 \\
\hline \multirow{4}{*}{$\begin{array}{l}\text { Mining } \\
\text { SIC 10-12, } 14\end{array}$} & GM (f/cc) & 0.244 & 0.121 & 0.129 & 0.430 & 0.239 & 0.346 & 0.359 & 0.036 & 0.013 & 0.007 & 0.010 & 0.006 & 0.014 & 0.004 & 0.003 & 0.002 & 0.002 & 0.004 & 0.002 & 0.002 & 0.002 \\
\hline & No. of samples & 204 & 301 & 276 & 64 & 73 & 23 & 43 & 37 & 16 & 53 & 29 & 46 & 34 & 28 & 14 & 157 & 77 & 28 & 7 & 3 & 2 \\
\hline & $\%>$ PEL & 10.8 & 5.6 & 5.4 & 0.0 & 0.0 & 0.0 & 0.0 & 0.0 & 0.0 & 0.0 & 0.0 & 4.3 & 2.9 & 7.1 & 0.0 & 0.0 & 0.0 & 0.0 & 0.0 & 0.0 & 0.0 \\
\hline & $\%>$ REL & 70.6 & 60.8 & 60.9 & 90.6 & 78.1 & 91.3 & 86.0 & 48.6 & 12.5 & 18.9 & 20.7 & 19.6 & 32.4 & 7.1 & 7.1 & 0.6 & 0.0 & 10.7 & 0.0 & 0.0 & 0.0 \\
\hline \multirow{4}{*}{$\begin{array}{l}\text { Other } \\
\text { SIC 1-9, 13, } \\
\quad 40-99\end{array}$} & GM (f/cc) & 0.003 & 0.006 & 0.003 & 0.007 & 0.002 & 0.003 & 0.002 & 0.002 & 0.002 & 0.002 & 0.002 & 0.002 & 0.002 & 0.002 & 0.002 & 0.002 & 0.001 & 0.001 & 0.002 & 0.002 & 0.001 \\
\hline & No. of samples & 94 & 124 & 68 & 51 & 469 & 834 & 732 & 505 & 814 & 509 & 625 & 472 & 462 & 285 & 231 & 214 & 144 & 166 & 136 & 93 & 71 \\
\hline & $\%>$ PEL & 0.0 & 0.0 & 0.0 & 0.0 & 0.0 & 0.0 & 0.1 & 0.6 & 1.0 & 0.6 & 0.5 & 0.2 & 0.0 & 0.0 & 0.0 & 0.0 & 0.7 & 0.6 & 0.0 & 0.0 & 0.0 \\
\hline & $\%>$ REL & 7.4 & 13.7 & 2.9 & 9.8 & 1.5 & 2.6 & 2.3 & 1.0 & 1.4 & 1.8 & 1.0 & 0.6 & 0.4 & 1.1 & 1.3 & 0.5 & 0.7 & 0.6 & 0.0 & 0.0 & 0.0 \\
\hline \multicolumn{23}{|c|}{$\begin{array}{l}\text { SIC - Standard Industrial Classification } \quad \text { PEL - permissible exposure limit } \quad \text { REL - recommended exposure limit } \quad \text { GM - geometric mean } \\
\text { NOTE: The MSHA PEL is } 2 \mathrm{f} / \mathrm{cc} \text {. The NIOSH REL is } 0.1 \mathrm{f} / \mathrm{cc} \text {. See appendices for source description, methods, and agents. } \\
\text { SOURCE: Mine Safety and Health Administration (MSHA) metal/nonmetal mine data. Occupational Safety and Health Administation (OSHA) Integrated Management Information System. }\end{array}$} \\
\hline
\end{tabular}


Table 1-13. Asbestos: Number of samples, geometric mean exposures, and percent exceeding designated occupational exposure limits by industries with elevated asbestosis mortality, MSHA and OSHA samples, 1990-1999

\begin{tabular}{|c|c|c|c|c|c|c|c|}
\hline \multicolumn{4}{|c|}{$\begin{array}{c}\text { Asbestosis Mortality, } \\
\text { Selected States and Years, 1990-1999 }\end{array}$} & \multirow[b]{2}{*}{\begin{tabular}{|c|} 
Number of \\
Samples
\end{tabular}} & \multirow[b]{2}{*}{$\begin{array}{c}\text { GM } \\
(\mathbf{f} / \mathbf{c c})\end{array}$} & \multirow[b]{2}{*}{$\begin{array}{l}\%> \\
\text { PEL }\end{array}$} & \multirow[b]{2}{*}{$\begin{array}{l}\%> \\
\text { REL }\end{array}$} \\
\hline CIC & $\begin{array}{l}\text { Industries with elevated PMRs and most } \\
\text { frequently recorded on death certificates }\end{array}$ & $\begin{array}{l}\text { Number of } \\
\text { Deaths }\end{array}$ & PMR & & & & \\
\hline 262 & $\begin{array}{l}\text { Miscellaneous nonmetallic mineral and stone } \\
\text { products }\end{array}$ & 75 & 16.39 & 115 & 0.031 & 26.1 & 40.9 \\
\hline 360 & Ship and boat building and repairing & 171 & 15.70 & 21 & 0.002 & 0.0 & 9.5 \\
\hline 192 & Industrial and miscellaneous chemicals & 124 & 4.78 & 19 & 0.003 & 0.0 & 5.3 \\
\hline 211 & $\begin{array}{l}\text { Other rubber products, and plastics footwear } \\
\text { and belting }\end{array}$ & 40 & 4.31 & 28 & 0.002 & 0.0 & 0.0 \\
\hline 200 & Petroleum refining & 31 & 2.74 & 11 & 0.001 & 0.0 & 0.0 \\
\hline 460 & Electric light and power & 55 & 2.65 & 41 & 0.002 & 0.0 & 0.0 \\
\hline 250 & Glass and glass products & 30 & 2.58 & 8 & 0.004 & 0.0 & 0.0 \\
\hline 060 & Construction & 702 & 2.38 & 1,051 & 0.003 & 4.0 & 6.3 \\
\hline 400 & Railroads & 89 & 1.64 & 9 & 0.001 & 0.0 & 0.0 \\
\hline 270 & $\begin{array}{l}\text { Blast furnaces, steelworks, rolling and } \\
\text { finishing mills }\end{array}$ & 67 & 1.30 & 26 & 0.002 & 0.0 & 0.0 \\
\hline & All other industries & 1,370 & & 3,561 & 0.002 & 1.0 & 2.1 \\
\hline & TOTAL & & & 4,890 & 0.002 & 2.2 & 3.9 \\
\hline
\end{tabular}

CIC - Census Industry Code PEL - permissible exposure limit

NOTE: The MSHA PEL is $2 \mathrm{f} / \mathrm{cc}$. The OSHA PEL is $2 \mathrm{f} / \mathrm{cc}$ before July $21,1986,0.2 \mathrm{f} / \mathrm{cc}$ from July 21,1986 to October 10, 1994, and $0.1 \mathrm{f} / \mathrm{cc}$ after October 10, 1994. The NIOSH REL is $0.1 \mathrm{f} / \mathrm{cc}$. See appendices for source description, methods, ICD codes, industry codes, agents, and list of selected states (and years) for which usual industry has been reported.

SOURCE: Mine Safety and Health Administration (MSHA) metal/nonmetal mine data. Occupational Safety and Health Administration (OSHA): Integrated Management Information System. National Center for Health Statistics multiple cause of death data. 
Table 1-14 (page 1 of 2). Asbestos: Geometric mean exposures and percent exceeding designated occupational exposure limits by MSHA metal/nonmetal district and state, MSHA samples, 1979-1999

\begin{tabular}{|c|c|c|c|c|c|c|c|c|c|c|c|c|c|c|}
\hline \multirow[b]{2}{*}{ MSHA Metal/Nonmetal District } & \multicolumn{2}{|c|}{ All years } & \multicolumn{4}{|c|}{$1979-1986$} & \multicolumn{4}{|c|}{$1987-1994$} & \multicolumn{4}{|c|}{1995 - 1999} \\
\hline & $\begin{array}{c}\text { No. of } \\
\text { Samples }\end{array}$ & $\begin{array}{c}\text { GM } \\
\text { (f/cc) }\end{array}$ & $\begin{array}{c}\text { No. of } \\
\text { Samples }\end{array}$ & $\begin{array}{c}\text { GM } \\
(\mathbf{f} / \mathbf{c c})\end{array}$ & $\begin{array}{l}\%> \\
\text { PEL }\end{array}$ & $\begin{array}{l}\%> \\
\text { REL }\end{array}$ & \begin{tabular}{|c|}
$\begin{array}{c}\text { No. of } \\
\text { Samples }\end{array}$ \\
\end{tabular} & $\begin{array}{c}\text { GM } \\
\text { (f/cc) }\end{array}$ & $\begin{array}{l}\%> \\
\text { PEL }\end{array}$ & $\begin{array}{l}\%> \\
\text { REL }\end{array}$ & \begin{tabular}{|c|}
$\begin{array}{c}\text { No. of } \\
\text { Samples }\end{array}$ \\
\end{tabular} & $\begin{array}{c}\text { GM } \\
\text { (f/cc) }\end{array}$ & $\begin{array}{l}\%> \\
\text { PEL }\end{array}$ & $\begin{array}{r}\%> \\
\text { REL }\end{array}$ \\
\hline Northeast & 318 & 0.159 & 240 & 0.430 & 6.3 & 85.0 & 70 & 0.009 & 7.1 & 21.4 & 8 & 0.002 & 0.0 & 0.0 \\
\hline Connecticut & 0 & - & 0 & - & - & - & 0 & - & - & - & 0 & - & - & - \\
\hline Delaware & 0 & - & 0 & - & - & - & 0 & - & - & - & 0 & - & - & - \\
\hline District of Columbia & 0 & - & 0 & - & - & - & 0 & - & - & - & 0 & - & - & - \\
\hline Maine & 0 & - & 0 & - & - & - & 0 & - & - & - & 0 & - & - & - \\
\hline Maryland & 45 & 0.021 & 26 & 0.119 & 0.0 & 53.8 & 18 & 0.002 & 0.0 & 0.0 & 1 & 0.002 & 0.0 & 0.0 \\
\hline Massachusetts & 1 & 0.002 & 0 & - & - & - & 1 & 0.002 & 0.0 & 0.0 & 0 & - & - & - \\
\hline New Hampshire & 0 & - & 0 & - & - & - & 0 & - & - & - & 0 & - & - & - \\
\hline New Jersey & 14 & 0.191 & 14 & 0.191 & 0.0 & 78.6 & 0 & - & - & - & 0 & - & - & - \\
\hline New York & 40 & 0.187 & 34 & 0.439 & 0.0 & 91.2 & 6 & 0.002 & 0.0 & 0.0 & 0 & - & - & - \\
\hline Pennsylvania & 19 & 0.001 & 0 & - & - & - & 12 & 0.002 & 0.0 & 0.0 & 7 & 0.002 & 0.0 & 0.0 \\
\hline Rhode Island & 0 & - & 0 & - & - & - & 0 & - & - & - & 0 & - & - & - \\
\hline Vermont & 176 & 0.560 & 154 & 0.599 & 9.7 & 89.6 & 22 & 0.350 & 22.7 & 68.2 & 0 & - & - & - \\
\hline Virginia & 20 & 0.031 & 12 & 0.239 & 0.0 & 83.3 & 8 & 0.002 & 0.0 & 0.0 & 0 & - & - & - \\
\hline West Virginia & 3 & 0.002 & 0 & - & - & - & 3 & 0.002 & 0.0 & 0.0 & 0 & - & - & - \\
\hline Southeast & 91 & 0.036 & 71 & 0.086 & 5.6 & 42.3 & 14 & 0.002 & 0.0 & 0.0 & 6 & 0.002 & 0.0 & 0.0 \\
\hline Alabama & 1 & 0.020 & 0 & - & - & - & 1 & 0.020 & 0.0 & 0.0 & 0 & - & - & - \\
\hline Florida & 6 & 0.013 & 6 & 0.013 & 0.0 & 33.3 & 0 & - & - & - & 0 & - & - & - \\
\hline Georgia & 8 & 0.124 & 4 & 10.3 & 100.0 & 100.0 & 2 & 0.002 & 0.0 & 0.0 & 2 & 0.002 & 0.0 & 0.0 \\
\hline Kentucky & 0 & - & 0 & - & - & - & 0 & - & - & - & 0 & - & - & - \\
\hline Mississippi & 0 & - & 0 & - & - & - & 0 & - & - & - & 0 & - & - & - \\
\hline North Carolina & 0 & - & 0 & - & - & - & 0 & - & - & - & 0 & - & - & - \\
\hline Puerto Rico & 0 & - & 0 & - & - & - & 0 & - & - & - & 0 & - & - & - \\
\hline South Carolina & 76 & 0.035 & 61 & 0.076 & 0.0 & 39.3 & 11 & 0.002 & 0.0 & 0.0 & 4 & 0.002 & 0.0 & 0.0 \\
\hline Tennessee & 0 & - & 0 & - & - & - & 0 & - & - & - & 0 & - & - & - \\
\hline Virgin Islands & 0 & - & 0 & - & - & - & 0 & - & - & - & 0 & - & - & - \\
\hline North Central & 354 & 0.007 & 119 & 0.075 & 0.8 & 45.4 & 172 & 0.002 & 0.0 & 1.7 & 63 & 0.002 & 0.0 & 0.0 \\
\hline Illinois & 11 & 0.038 & 9 & 0.079 & 0.0 & 55.6 & 2 & 0.002 & 0.0 & 0.0 & 0 & - & - & - \\
\hline Indiana & 1 & 0.010 & 1 & 0.010 & 0.0 & 0.0 & 0 & - & - & - & 0 & - & - & - \\
\hline Iowa & 0 & - & 0 & - & - & - & 0 & - & - & - & 0 & - & - & - \\
\hline Michigan & 176 & 0.002 & 2 & 0.002 & 0.0 & 0.0 & 134 & 0.002 & 0.0 & 0.0 & 40 & 0.001 & 0.0 & 0.0 \\
\hline Minnesota & 161 & 0.027 & 106 & 0.083 & 0.9 & 46.2 & 34 & 0.005 & 0.0 & 8.8 & 21 & 0.001 & 0.0 & 0.0 \\
\hline Ohio & 1 & 0.010 & 1 & 0.010 & 0.0 & 0.0 & 0 & - & - & - & 0 & - & - & - \\
\hline Wisconsin & 4 & 0.002 & 0 & - & - & - & 2 & 0.002 & 0.0 & 0.0 & 2 & 0.004 & 0.0 & 0.0 \\
\hline
\end{tabular}


Table 1-14 (page 2 of 2). Asbestos: Geometric mean exposures and percent exceeding designated occupational exposure limits by MSHA metal/nonmetal district and state, MSHA samples, 1979-1999

\begin{tabular}{|c|c|c|c|c|c|c|c|c|c|c|c|c|c|c|}
\hline \multirow[b]{2}{*}{ MSHA Metal/Nonmetal District } & \multicolumn{2}{|c|}{ All years } & \multicolumn{4}{|c|}{$1979-1986$} & \multicolumn{4}{|c|}{$1987-1994$} & \multicolumn{4}{|c|}{1995 - 1999} \\
\hline & $\begin{array}{c}\text { No. of } \\
\text { Samples }\end{array}$ & $\begin{array}{c}\text { GM } \\
\text { (f/cc) }\end{array}$ & $\begin{array}{c}\text { No. of } \\
\text { Samples }\end{array}$ & $\begin{array}{c}\text { GM } \\
\text { (f/cc) }\end{array}$ & $\begin{array}{l}\%> \\
\text { PEL }\end{array}$ & $\begin{array}{l}\%> \\
\text { REL }\end{array}$ & \begin{tabular}{|c|}
$\begin{array}{c}\text { No. of } \\
\text { Samples }\end{array}$ \\
\end{tabular} & $\begin{array}{c}\text { GM } \\
\text { (f/cc) }\end{array}$ & $\begin{array}{l}\%> \\
\text { PEL }\end{array}$ & $\begin{array}{l}\%> \\
\text { REL }\end{array}$ & \begin{tabular}{|c|}
$\begin{array}{c}\text { No. of } \\
\text { Samples }\end{array}$ \\
\end{tabular} & $\begin{array}{c}\text { GM } \\
\text { (f/cc) }\end{array}$ & $\begin{array}{l}\%> \\
\text { PEL }\end{array}$ & $\begin{array}{r}\%> \\
\text { REL }\end{array}$ \\
\hline South Central & 304 & 0.017 & 231 & 0.035 & 1.3 & 39.0 & 47 & 0.002 & 0.0 & 2.1 & 26 & 0.001 & 0.0 & 0.0 \\
\hline Arkansas & 0 & - & 0 & - & - & - & 0 & - & - & - & 0 & - & - & - \\
\hline Louisiana & 45 & 0.002 & 0 & - & - & - & 45 & 0.002 & 0.0 & 2.2 & 0 & - & - & - \\
\hline Missouri & 0 & - & 0 & - & - & - & 0 & - & - & - & 0 & - & - & - \\
\hline New Mexico & 112 & 0.042 & 112 & 0.042 & 1.8 & 42.9 & 0 & - & - & - & 0 & - & - & - \\
\hline Oklahoma & 27 & 0.033 & 27 & 0.033 & 0.0 & 40.7 & 0 & - & - & - & 0 & - & - & - \\
\hline Texas & 120 & 0.014 & 92 & 0.028 & 1.1 & 33.7 & 2 & 0.002 & 0.0 & 0.0 & 26 & 0.001 & 0.0 & 0.0 \\
\hline Rocky Mountain & 254 & 0.211 & 225 & 0.324 & 4.9 & 87.1 & 29 & 0.008 & 0.0 & 20.7 & $\mathbf{0}$ & 0.000 & 0.0 & 0.0 \\
\hline Arizona & 89 & 0.525 & 89 & 0.525 & 11.2 & 92.1 & 0 & - & - & - & 0 & - & - & - \\
\hline Colorado & 35 & 0.055 & 31 & 0.088 & 0.0 & 64.5 & 4 & 0.002 & 0.0 & 0.0 & 0 & - & - & - \\
\hline Kansas & 0 & - & 0 & - & - & - & 0 & - & - & - & 0 & - & - & - \\
\hline Montana & 74 & 0.278 & 62 & 0.376 & 1.6 & 91.9 & 12 & 0.058 & 0.0 & 50.0 & 0 & - & - & - \\
\hline Nebraska & 0 & - & 0 & - & - & - & 0 & - & - & - & 0 & - & - & - \\
\hline Nevada & 6 & 0.002 & 0 & - & - & - & 6 & 0.002 & 0.0 & 0.0 & 0 & - & - & - \\
\hline North Dakota & 0 & - & 0 & - & - & - & 0 & - & - & - & 0 & - & - & - \\
\hline South Dakota & 47 & 0.157 & 41 & 0.287 & 0.0 & 90.2 & 6 & 0.003 & 0.0 & 0.0 & 0 & - & - & - \\
\hline Utah & 3 & 0.005 & 2 & 0.010 & 0.0 & 0.0 & 1 & 0.002 & 0.0 & 0.0 & 0 & - & - & - \\
\hline Wyoming & 0 & - & 0 & - & - & - & 0 & - & - & - & 0 & - & - & - \\
\hline Western & 194 & 0.158 & 135 & 0.389 & 14.8 & 83.0 & 45 & 0.025 & 0.0 & 37.8 & 14 & 0.010 & 0.0 & 21.4 \\
\hline Alaska & 0 & - & 0 & - & - & - & 0 & - & - & - & 0 & - & - & - \\
\hline California & 185 & 0.195 & 128 & 0.515 & 15.6 & 87.5 & 43 & 0.029 & 0.0 & 39.5 & 14 & 0.010 & 0.0 & 21.4 \\
\hline Hawaii & 1 & 0.002 & 0 & - & - & - & 1 & 0.002 & 0.0 & 0.0 & 0 & - & - & - \\
\hline Idaho & 1 & 0.002 & 0 & - & - & - & 1 & 0.002 & 0.0 & 0.0 & 0 & - & - & - \\
\hline Oregon & 0 & - & 0 & - & - & - & 0 & - & - & - & 0 & - & - & - \\
\hline Washington & 7 & 0.002 & 7 & 0.002 & 0.0 & 0.0 & 0 & - & - & - & 0 & - & - & - \\
\hline TOTAL & 1,515 & 0.046 & 1,021 & 0.165 & 5.3 & 67.2 & 377 & 0.004 & 1.3 & 11.1 & 117 & 0.002 & 0.0 & 2.6 \\
\hline
\end{tabular}

- indicates incalculable field

PEL - permissible exposure limit REL - recommended exposure limit

GM - geometric mean

$\mathrm{f} / \mathrm{cc}$ - fibers per cubic centimeter

NOTE: The MSHA PEL is $2 \mathrm{f} / \mathrm{cc}$. The NIOSH REL is $0.1 \mathrm{f} / \mathrm{cc}$. See appendices for source description, methods, and agents.

SOURCE: Mine Safety and Health Administration (MSHA) metal/nonmetal mine data. 
Table 1-15 (page 1 of 3). Asbestos: Geometric mean exposures and percent exceeding designated occupational exposure limits by OSHA region and state, OSHA samples, 1979-1999

\begin{tabular}{|c|c|c|c|c|c|c|c|c|c|c|c|c|c|}
\hline \multirow[b]{2}{*}{ OSHA Region } & \multicolumn{2}{|c|}{ All years } & \multicolumn{4}{|c|}{$\begin{array}{c}1979-1986 \\
\text { OSHA } P E L=2 \text { f/cc }\end{array}$} & \multicolumn{4}{|c|}{$\begin{array}{c}1987-1994 \\
\text { OSHA PEL }=0.2 \text { f/cc }\end{array}$} & \multicolumn{3}{|c|}{$\begin{array}{c}1995-1999 \\
\text { OSHA PEL=0.1 f/cc } \\
\text { NIOSH REL }=0.1 \mathrm{f} / \mathrm{cc}\end{array}$} \\
\hline & \begin{tabular}{|c|}
$\begin{array}{c}\text { Number } \\
\text { of Samples }\end{array}$ \\
\end{tabular} & $\begin{array}{c}\text { GM } \\
\text { (f/cc) }\end{array}$ & \begin{tabular}{|c|c} 
Number \\
of Samples
\end{tabular} & $\begin{array}{c}\text { GM } \\
\text { (f/cc) }\end{array}$ & $\begin{array}{l}\%> \\
\text { PEL }\end{array}$ & $\begin{array}{l}\%> \\
\text { REL }\end{array}$ & \begin{tabular}{|c|c} 
Number \\
of Samples
\end{tabular} & $\begin{array}{c}\text { GM } \\
(\mathbf{f} / \mathbf{c c})\end{array}$ & $\begin{array}{l}\%> \\
\text { PEL }\end{array}$ & $\begin{array}{l}\%> \\
\text { REL }\end{array}$ & $\begin{array}{c}\text { Number } \\
\text { of Samples }\end{array}$ & $\begin{array}{c}\text { GM } \\
(\mathbf{f} / \mathbf{c c})\end{array}$ & $\begin{array}{l}\%> \\
\text { PEL }\end{array}$ \\
\hline Region 1 & 1,430 & 0.006 & 836 & 0.014 & 5.0 & 27.0 & 500 & 0.002 & 4.2 & 5.6 & 94 & 0.001 & 0.0 \\
\hline Connecticut & 619 & 0.003 & 319 & 0.005 & 1.3 & 16.0 & 254 & 0.001 & 0.0 & 0.4 & 46 & 0.001 & 0.0 \\
\hline Maine & 103 & 0.008 & 83 & 0.010 & 2.4 & 16.9 & 20 & 0.004 & 20.0 & 20.0 & 0 & - & - \\
\hline Massachusetts & 481 & 0.014 & 285 & 0.032 & 10.2 & 38.6 & 172 & 0.005 & 7.0 & 10.5 & 24 & 0.001 & 0.0 \\
\hline New Hampshire & 156 & 0.007 & 89 & 0.021 & 1.1 & 37.1 & 44 & 0.002 & 2.3 & 2.3 & 23 & 0.001 & 0.0 \\
\hline Rhode Island & 70 & 0.033 & 60 & 0.032 & 10.0 & 30.0 & 9 & 0.062 & 44.4 & 44.4 & 1 & 0.002 & 0.0 \\
\hline Vermont & 1 & 0.002 & 0 & - & - & - & 1 & 0.002 & 0.0 & 0.0 & 0 & - & - \\
\hline Region 2 & 2,891 & 0.004 & 1,279 & 0.006 & 1.5 & 14.9 & 1,359 & 0.003 & 4.3 & 6.7 & 253 & 0.002 & 2.4 \\
\hline New Jersey & 700 & 0.008 & 384 & 0.010 & 2.9 & 20.8 & 288 & 0.006 & 14.9 & 20.8 & 28 & 0.001 & 0.0 \\
\hline New York & 2,061 & 0.003 & 861 & 0.005 & 0.9 & 12.5 & 991 & 0.003 & 1.2 & 2.6 & 209 & 0.002 & 2.9 \\
\hline Puerto Rico & 109 & 0.002 & 29 & 0.003 & 0.0 & 6.9 & 64 & 0.002 & 1.6 & 4.7 & 16 & 0.001 & 0.0 \\
\hline Virgin Islands & 21 & 0.005 & 5 & 0.002 & 0.0 & 0.0 & 16 & 0.007 & 12.5 & 12.5 & 0 & - & - \\
\hline Region 3 & 1,773 & 0.010 & 1,043 & 0.013 & 3.4 & 25.8 & 659 & 0.007 & 14.0 & 18.4 & 71 & 0.001 & 0.0 \\
\hline Delaware & 22 & 0.022 & 20 & 0.029 & 0.0 & 40.0 & 2 & 0.002 & 0.0 & 0.0 & 0 & - & - \\
\hline District of Columbia & 131 & 0.003 & 114 & 0.003 & 0.9 & 10.5 & 17 & 0.001 & 0.0 & 0.0 & 0 & - & - \\
\hline Maryland & 129 & 0.003 & 71 & 0.003 & 0.0 & 4.2 & 31 & 0.008 & 16.1 & 19.4 & 27 & 0.001 & 0.0 \\
\hline Pennsylvania & 1,014 & 0.008 & 587 & 0.013 & 3.4 & 25.7 & 394 & 0.004 & 5.6 & 7.9 & 33 & 0.001 & 0.0 \\
\hline Virginia & 362 & 0.041 & 163 & 0.060 & 7.4 & 45.4 & 198 & 0.031 & 29.8 & 39.4 & 1 & 0.0003 & 0.0 \\
\hline West Virginia & 115 & 0.010 & 88 & 0.012 & 2.3 & 23.9 & 17 & 0.015 & 35.3 & 35.3 & 10 & 0.001 & 0.0 \\
\hline Region 4 & 2,128 & 0.004 & 714 & 0.014 & 4.1 & 24.6 & 1,085 & 0.003 & 2.0 & 4.2 & 331 & 0.002 & 0.9 \\
\hline Alabama & 187 & 0.009 & 104 & 0.020 & 6.7 & 28.8 & 74 & 0.003 & 0.0 & 0.0 & 9 & 0.008 & 11.1 \\
\hline Florida & 269 & 0.003 & 93 & 0.008 & 3.2 & 14.0 & 161 & 0.002 & 1.9 & 3.7 & 15 & 0.001 & 0.0 \\
\hline Georgia & 336 & 0.009 & 217 & 0.015 & 7.4 & 25.3 & 112 & 0.003 & 3.6 & 8.9 & 7 & 0.001 & 0.0 \\
\hline Kentucky & 351 & 0.003 & 104 & 0.004 & 0.0 & 5.8 & 194 & 0.003 & 0.5 & 2.6 & 55 & 0.002 & 0.0 \\
\hline Mississippi & 138 & 0.005 & 33 & 0.099 & 3.0 & 63.6 & 87 & 0.002 & 0.0 & 0.0 & 18 & 0.001 & 0.0 \\
\hline North Carolina & 472 & 0.005 & 87 & 0.055 & 2.3 & 47.1 & 230 & 0.005 & 4.3 & 7.4 & 155 & 0.001 & 0.0 \\
\hline South Carolina & 188 & 0.002 & 45 & 0.008 & 0.0 & 20.0 & 114 & 0.001 & 2.6 & 2.6 & 29 & 0.001 & 0.0 \\
\hline Tennessee & 187 & 0.004 & 31 & 0.003 & 0.0 & 3.2 & 113 & 0.004 & 0.9 & 4.4 & 43 & 0.003 & 4.7 \\
\hline
\end{tabular}

See footnotes at end of table. 
Table 1-15 (page 2 of 3). Asbestos: Geometric mean exposures and percent exceeding designated occupational exposure limits by OSHA region and state, OSHA samples, 1979-1999

\begin{tabular}{|c|c|c|c|c|c|c|c|c|c|c|c|c|c|}
\hline \multirow[b]{2}{*}{ OSHA Region } & \multicolumn{2}{|c|}{ All years } & \multicolumn{4}{|c|}{$\begin{array}{l}1979-1986 \\
\text { PEL }=2 \text { f/cc }\end{array}$} & \multicolumn{4}{|c|}{$\begin{array}{c}1987-1994 \\
\text { PEL }=0.2 \mathrm{f} / \mathrm{cc}\end{array}$} & \multicolumn{3}{|c|}{$\begin{array}{c}1995-1999 \\
\text { OSHA PEL }=0.1 \mathrm{f} / \mathrm{cc} \\
\text { NIOSH REL }=0.1 \mathrm{f} / \mathrm{cc}\end{array}$} \\
\hline & \begin{tabular}{|c|}
$\begin{array}{c}\text { Number } \\
\text { of Samples }\end{array}$ \\
\end{tabular} & $\begin{array}{c}\text { GM } \\
\text { (f/cc) }\end{array}$ & \begin{tabular}{|c|}
$\begin{array}{c}\text { Number } \\
\text { of Samples }\end{array}$ \\
\end{tabular} & $\begin{array}{c}\text { GM } \\
\text { (f/cc) }\end{array}$ & $\begin{array}{l}\%> \\
\text { PEL }\end{array}$ & $\begin{array}{l}\%> \\
\text { REL }\end{array}$ & \begin{tabular}{|c|}
$\begin{array}{c}\text { Number } \\
\text { of Samples }\end{array}$ \\
\end{tabular} & $\begin{array}{c}\text { GM } \\
\text { (f/cc) }\end{array}$ & $\begin{array}{l}\%>> \\
\text { PEL }\end{array}$ & $\begin{array}{l}\%> \\
\text { REL }\end{array}$ & \begin{tabular}{|c|}
$\begin{array}{c}\text { Number } \\
\text { of Samples }\end{array}$ \\
\end{tabular} & $\begin{array}{c}\text { GM } \\
\text { (f/cc) }\end{array}$ & $\begin{array}{l}\%> \\
\text { PEL }\end{array}$ \\
\hline Region 5 & 3,255 & 0.004 & 1,301 & 0.010 & 1.9 & 19.4 & 1,722 & 0.003 & 2.0 & 3.4 & 232 & 0.001 & 0.4 \\
\hline Illinois & 835 & 0.003 & 352 & 0.007 & 0.0 & 12.8 & 434 & 0.002 & 0.0 & 0.7 & 49 & 0.001 & 0.0 \\
\hline Indiana & 440 & 0.006 & 213 & 0.033 & 4.2 & 41.8 & 152 & 0.001 & 0.0 & 0.7 & 75 & 0.001 & 0.0 \\
\hline Michigan & 453 & 0.002 & 36 & 0.001 & 0.0 & 0.0 & 376 & 0.002 & 1.9 & 2.7 & 41 & 0.003 & 2.4 \\
\hline Minnesota & 61 & 0.003 & 14 & 0.010 & 14.3 & 21.4 & 47 & 0.002 & 0.0 & 2.1 & 0 & - & - \\
\hline Ohio & 863 & 0.005 & 381 & 0.009 & 0.8 & 18.4 & 435 & 0.004 & 5.5 & 7.8 & 47 & 0.001 & 0.0 \\
\hline Wisconsin & 603 & 0.006 & 305 & 0.012 & 3.6 & 15.1 & 278 & 0.003 & 1.4 & 3.2 & 20 & 0.001 & 0.0 \\
\hline Region 6 & 1,315 & 0.005 & 695 & 0.007 & 1.7 & 15.5 & 557 & 0.004 & 5.6 & 9.9 & 63 & 0.001 & 0.0 \\
\hline Arkansas & 253 & 0.005 & 100 & 0.005 & 4.0 & 13.0 & 143 & 0.006 & 7.0 & 14.0 & 10 & 0.001 & 0.0 \\
\hline Louisiana & 121 & 0.005 & 69 & 0.006 & 1.4 & 8.7 & 52 & 0.003 & 1.9 & 1.9 & 0 & - & - \\
\hline New Mexico & 30 & 0.002 & 2 & 0.002 & 0.0 & 0.0 & 27 & 0.002 & 0.0 & 0.0 & 1 & 0.001 & 0.0 \\
\hline Oklahoma & 166 & 0.006 & 108 & 0.008 & 4.6 & 15.7 & 49 & 0.003 & 4.1 & 6.1 & 9 & 0.004 & 0.0 \\
\hline Texas & 745 & 0.005 & 416 & 0.008 & 0.5 & 17.3 & 286 & 0.004 & 6.3 & 10.8 & 43 & 0.001 & 0.0 \\
\hline Region 7 & 1,304 & 0.004 & 910 & 0.006 & 1.9 & 10.9 & 373 & 0.002 & 1.1 & 1.1 & 21 & 0.001 & 0.0 \\
\hline Iowa & 339 & 0.004 & 230 & 0.006 & 2.6 & 14.8 & 106 & 0.002 & 0.0 & 0.0 & 3 & 0.001 & 0.0 \\
\hline Kansas & 247 & 0.010 & 177 & 0.018 & 2.8 & 19.2 & 64 & 0.003 & 3.1 & 3.1 & 6 & 0.001 & 0.0 \\
\hline Missouri & 548 & 0.003 & 416 & 0.004 & 1.4 & 6.0 & 126 & 0.002 & 1.6 & 1.6 & 6 & 0.001 & 0.0 \\
\hline Nebraska & 170 & 0.003 & 87 & 0.004 & 0.0 & 6.9 & 77 & 0.002 & 0.0 & 0.0 & 6 & 0.001 & 0.0 \\
\hline Region 8 & 628 & 0.005 & 445 & 0.007 & 0.9 & 15.1 & 158 & 0.003 & 2.5 & 3.2 & 25 & 0.001 & 0.0 \\
\hline Colorado & 320 & 0.005 & 226 & 0.005 & 0.4 & 14.2 & 82 & 0.004 & 4.9 & 6.1 & 12 & 0.001 & 0.0 \\
\hline Montana & 164 & 0.009 & 134 & 0.012 & 2.2 & 21.6 & 27 & 0.003 & 0.0 & 0.0 & 3 & 0.001 & 0.0 \\
\hline North Dakota & 16 & 0.003 & 9 & 0.006 & 0.0 & 11.1 & 0 & - & - & - & 7 & 0.001 & 0.0 \\
\hline South Dakota & 66 & 0.002 & 32 & 0.003 & 0.0 & 6.3 & 33 & 0.001 & 0.0 & 0.0 & 1 & 0.001 & 0.0 \\
\hline Utah & 35 & 0.012 & 22 & 0.023 & 0.0 & 13.6 & 12 & 0.005 & 0.0 & 0.0 & 1 & 0.001 & 0.0 \\
\hline Wyoming & 27 & 0.003 & 22 & 0.003 & 0.0 & 0.0 & 4 & 0.010 & 0.0 & 0.0 & 1 & 0.001 & 0.0 \\
\hline
\end{tabular}

See footnotes at end of table. 
Table 1-15 (page 3 of 3). Asbestos: Geometric mean exposures and percent exceeding designated occupational exposure limits by OSHA region and state, OSHA samples, 1979-1999

\begin{tabular}{|c|c|c|c|c|c|c|c|c|c|c|c|c|c|}
\hline \multirow[b]{2}{*}{ OSHA Region } & \multicolumn{2}{|c|}{ All years } & \multicolumn{4}{|c|}{$\begin{array}{l}1979-1986 \\
\text { PEL }=2 \mathrm{f} / \mathrm{cc}\end{array}$} & \multicolumn{4}{|c|}{$\begin{array}{c}1987-1994 \\
\text { PEL }=0.2 \text { f/cc }\end{array}$} & \multicolumn{3}{|c|}{$\begin{array}{c}1995-1999 \\
\text { OSHA PEL=0.1 f/cc } \\
\text { NIOSH REL }=0.1 \mathrm{f} / \mathrm{cc}\end{array}$} \\
\hline & \begin{tabular}{|c|c|} 
Number \\
of Samples
\end{tabular} & $\begin{array}{c}\text { GM } \\
\text { (f/cc) }\end{array}$ & \begin{tabular}{|c|c} 
Number \\
of Samples
\end{tabular} & $\begin{array}{c}\text { GM } \\
\text { (f/cc) }\end{array}$ & $\begin{array}{c}\%> \\
\text { PEL }\end{array}$ & $\begin{array}{l}\%> \\
\text { REL }\end{array}$ & \begin{tabular}{|c} 
Number \\
of Samples
\end{tabular} & $\begin{array}{c}\text { GM } \\
\text { (f/cc) }\end{array}$ & $\begin{array}{l}\%> \\
\text { PEL }\end{array}$ & $\begin{array}{l}\%> \\
\text { REL }\end{array}$ & \begin{tabular}{|c} 
Number \\
of Samples
\end{tabular} & $\begin{array}{c}\text { GM } \\
\text { (f/cc) }\end{array}$ & $\begin{array}{l}\%> \\
\text { PEL }\end{array}$ \\
\hline Region 9 & 715 & 0.002 & 177 & 0.003 & 0.6 & 9.0 & 471 & 0.002 & 3.2 & 6.2 & 67 & 0.004 & 0.0 \\
\hline American Samoa & 0 & - & 0 & - & - & - & 0 & - & - & - & 0 & - & - \\
\hline Arizona & 103 & 0.004 & 45 & 0.002 & 0.0 & 8.9 & 56 & 0.006 & 1.8 & 3.6 & 2 & 0.043 & 0.0 \\
\hline California & 450 & 0.003 & 71 & 0.004 & 1.4 & 12.7 & 320 & 0.002 & 3.4 & 7.2 & 59 & 0.004 & 0.0 \\
\hline Guam & 3 & 0.000 & 3 & 0.0003 & 0.0 & 0.0 & 0 & - & - & - & 0 & - & - \\
\hline Hawaii & 50 & 0.001 & 29 & 0.001 & 0.0 & 3.4 & 19 & 0.001 & 0.0 & 0.0 & 2 & 0.001 & 0.0 \\
\hline Nevada & 109 & 0.002 & 29 & 0.004 & 0.0 & 6.9 & 76 & 0.001 & 3.9 & 5.3 & 4 & 0.003 & 0.0 \\
\hline Region 10 & 568 & 0.005 & 289 & 0.007 & 2.4 & 16.3 & 232 & 0.003 & 0.9 & 3.4 & 47 & 0.001 & 2.1 \\
\hline Alaska & 220 & 0.003 & 149 & 0.003 & 1.3 & 2.7 & 50 & 0.004 & 2.0 & 2.0 & 21 & 0.002 & 0.0 \\
\hline Idaho & 95 & 0.003 & 27 & 0.013 & 0.0 & 22.2 & 66 & 0.002 & 0.0 & 0.0 & 2 & 0.001 & 0.0 \\
\hline Oregon & 154 & 0.011 & 107 & 0.013 & 2.8 & 29.9 & 41 & 0.010 & 0.0 & 9.8 & 6 & 0.003 & 16.7 \\
\hline Washington & 99 & 0.003 & 6 & 0.385 & 33.3 & 83.3 & 75 & 0.003 & 1.3 & 4.0 & 18 & 0.001 & 0.0 \\
\hline TOTAL & 16,007 & 0.005 & 7,689 & 0.009 & 2.5 & 18.9 & 7,116 & 0.003 & 4.0 & 6.3 & 1,204 & 0.001 & 0.9 \\
\hline
\end{tabular}

- indicates incalculable field

PEL - permissible exposure linit

REL - recommended exposure limit

GM - geometric mean

$\mathrm{f} / \mathrm{cc}$ - fibers per cubic centimeter

NOTE: The NIOSH REL is $0.1 \mathrm{f} / \mathrm{cc}$. See appendices for source description, methods, and agents.

SOURCE: Occupational Safety and Health Administation (OSHA) Integrated Management Information System. 
Figure 1-6. Asbestos: Geometric mean exposures by state, OSHA samples, 19791999

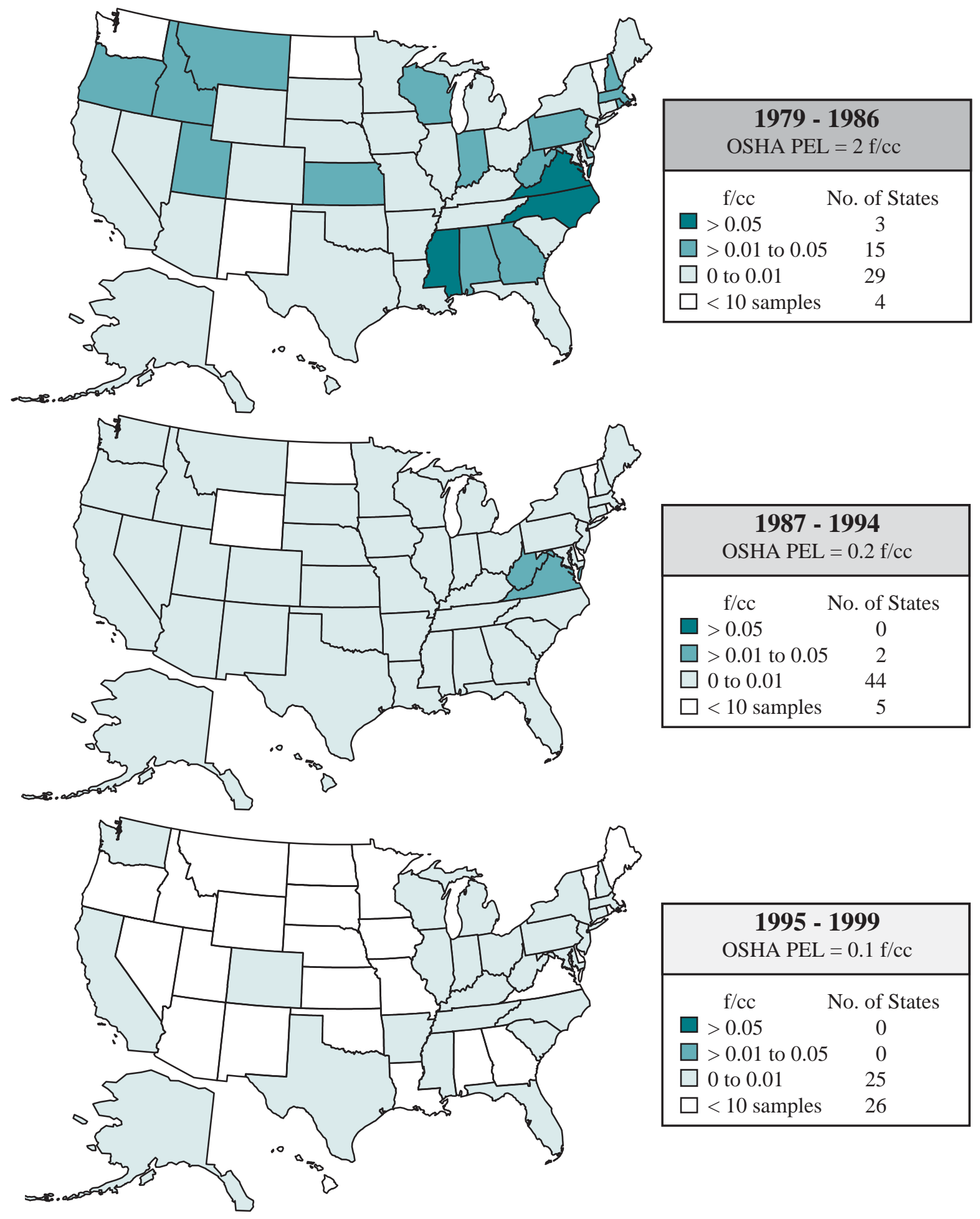

PEL - permissible exposure limit $\quad$ REL - recommended exposure limit $\quad \mathrm{f} / \mathrm{cc}$ - fibers per cubic centimeter NOTE: The NIOSH REL is $0.1 \mathrm{f} / \mathrm{cc}$. See appendices for source description, methods, and agents.

SOURCE: Occupational Safety and Health Administration (OSHA) Integrated Management Information System. 


\section{Section 2}

Coal Workers'

Pneumoconiosis

and

Related Exposures 

Figure 2-1. Coal workers' pneumoconiosis: Number of deaths, crude and ageadjusted mortality rates, U.S. residents age 15 and over, 1968-1999

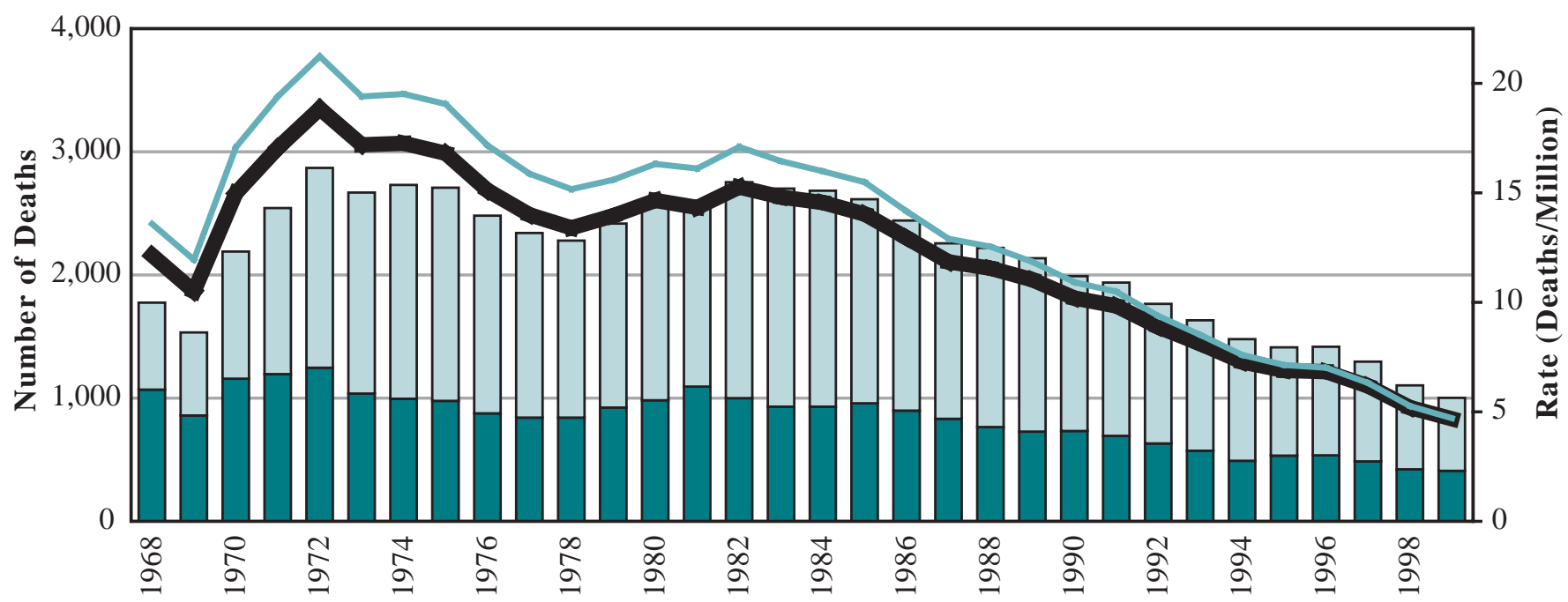

Figure 2-2. Coal workers' pneumoconiosis: Age-adjusted mortality rates by state, U.S. residents age 15 and over, 1990-1999

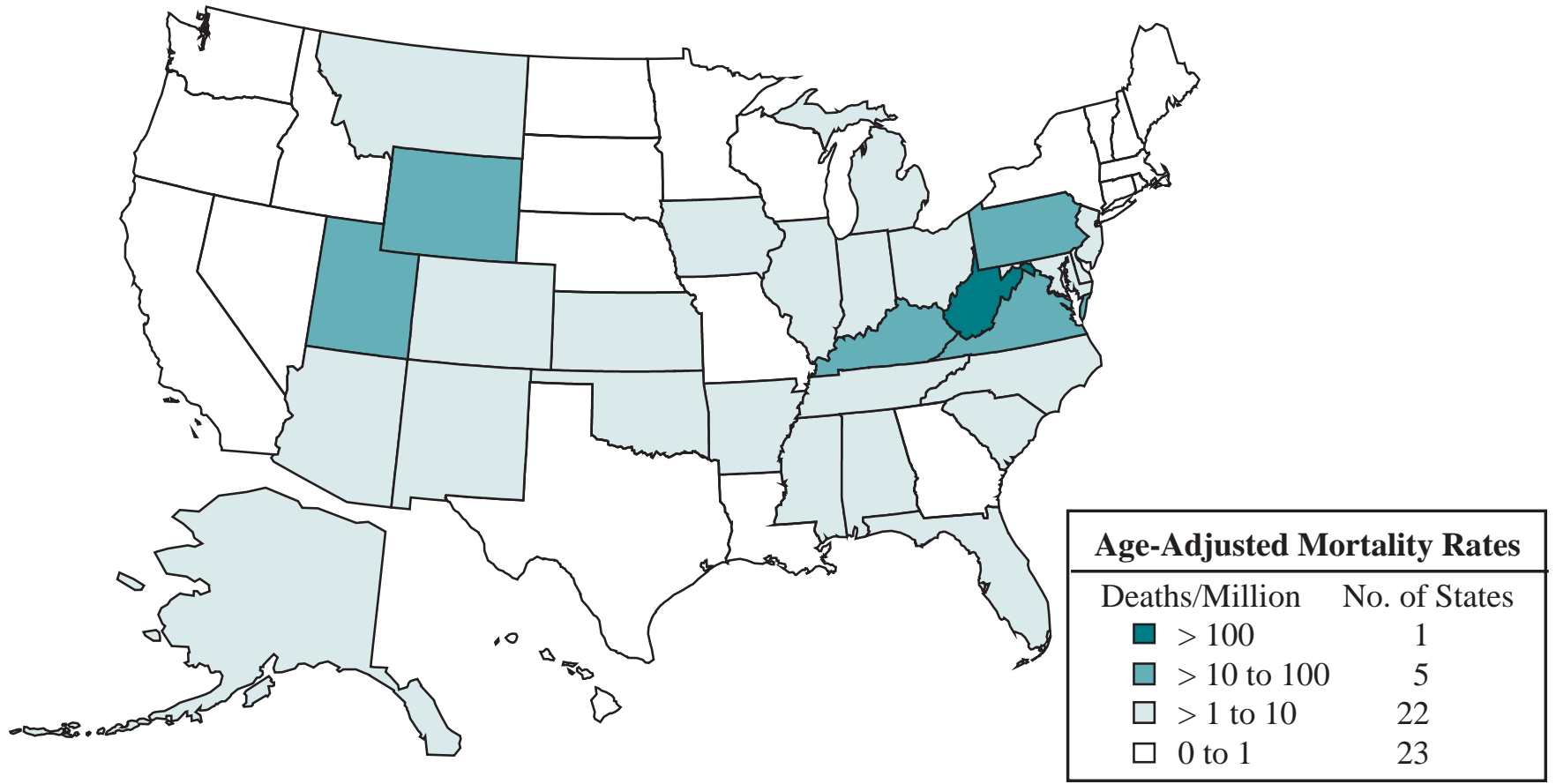

NOTE: See appendices for source description, methods, and ICD codes.

SOURCE: National Center for Health Statistics multiple cause of death data. Population estimates from U.S. Bureau of the Census. 
Table 2-1. Coal workers' pneumoconiosis: Number of deaths by sex, race, and age, and median age at death, U.S. residents age 15 and over, 1990-1999

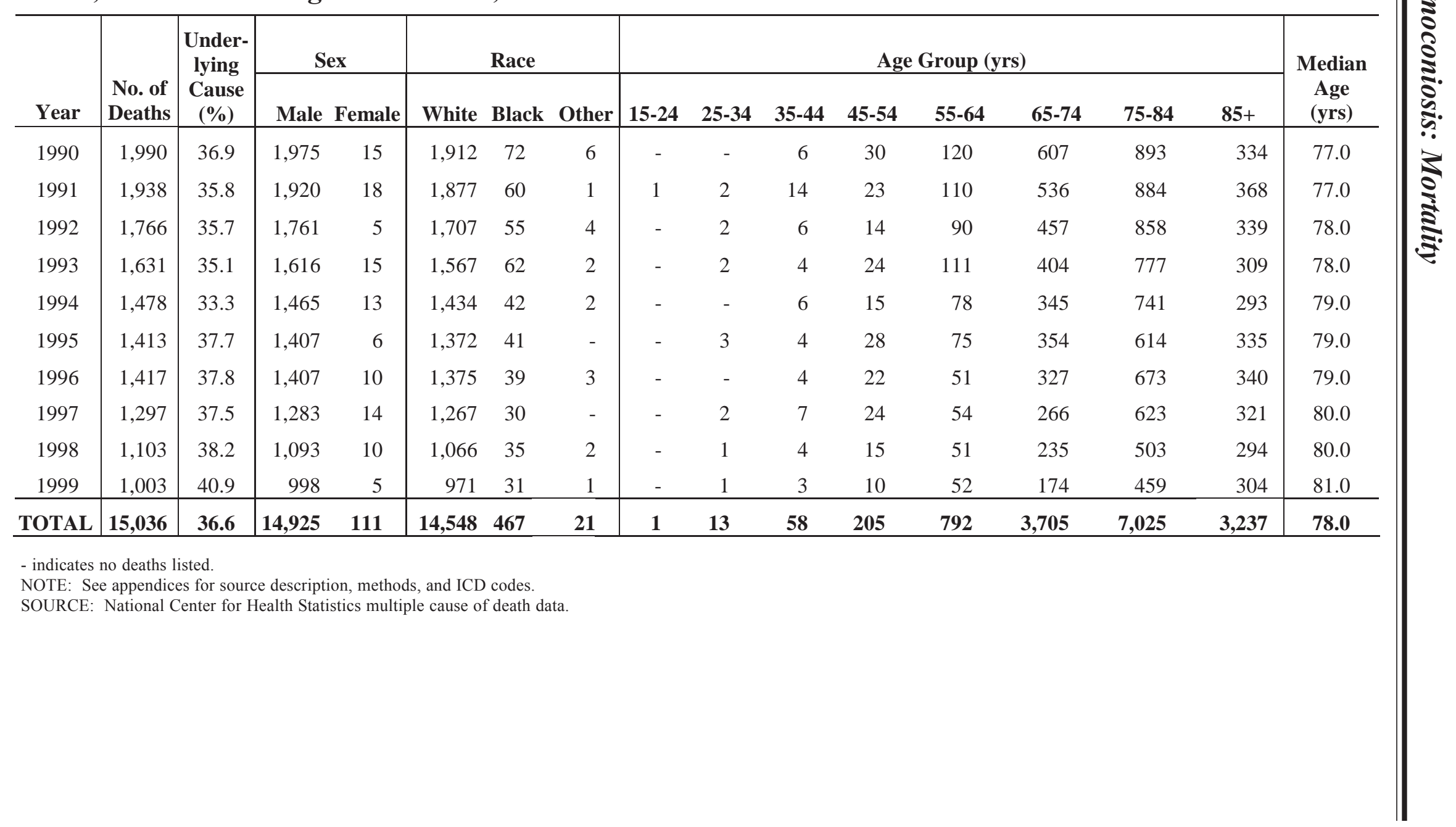


Table 2-2. Coal workers' pneumoconiosis: Mortality rates (per million population) by race and sex, U.S. residents age 15 and over, 1990-1999

\begin{tabular}{|c|c|c|c|c|c|c|c|}
\hline \multirow[b]{2}{*}{ Year } & \multirow[b]{2}{*}{ Overall } & \multicolumn{2}{|c|}{ White } & \multicolumn{2}{|c|}{ Black } & \multicolumn{2}{|c|}{ Other } \\
\hline & & Male & Female & Male & Female & Male & Female \\
\hline & \multicolumn{7}{|c|}{ Crude Mortality Rate } \\
\hline 1990 & 10.19 & 23.70 & 0.14 & 6.82 & 0.17 & 1.44 & 0.27 \\
\hline 1991 & 9.84 & 23.03 & 0.21 & 5.76 & - & 0.28 & - \\
\hline 1992 & 8.87 & 20.89 & 0.06 & 5.19 & - & 1.06 & - \\
\hline 1993 & 8.11 & 18.90 & 0.17 & 5.75 & - & 0.52 & - \\
\hline 1994 & 7.28 & 17.16 & 0.15 & 3.83 & - & 0.50 & - \\
\hline 1995 & 6.89 & 16.35 & 0.07 & 3.68 & - & - & - \\
\hline 1996 & 6.83 & 16.15 & 0.10 & 3.43 & - & 0.46 & 0.21 \\
\hline 1997 & 6.18 & 14.71 & 0.12 & 2.33 & 0.22 & - & - \\
\hline 1998 & 5.20 & 12.31 & 0.09 & 2.89 & 0.07 & 0.22 & 0.20 \\
\hline 1999 & 4.68 & 11.14 & 0.05 & 2.59 & - & 0.21 & - \\
\hline \multirow[t]{2}{*}{ 1990-1999 } & 7.33 & 17.29 & 0.11 & 4.13 & 0.05 & 0.44 & 0.07 \\
\hline & \multicolumn{7}{|c|}{ Age-Adjusted Mortality Rate } \\
\hline 1990 & 10.91 & 31.16 & 0.12 & 14.22 & 0.21 & 3.68 & 0.66 \\
\hline 1991 & 10.48 & 30.29 & 0.19 & 12.48 & - & 0.39 & - \\
\hline 1992 & 9.38 & 27.29 & 0.05 & 10.84 & - & 2.69 & - \\
\hline 1993 & 8.51 & 24.18 & 0.14 & 11.78 & - & 0.67 & - \\
\hline 1994 & 7.60 & 21.82 & 0.12 & 8.58 & - & 1.08 & - \\
\hline 1995 & 7.14 & 20.72 & 0.05 & 7.87 & - & - & - \\
\hline 1996 & 7.02 & 20.30 & 0.09 & 7.25 & - & 1.30 & 0.42 \\
\hline 1997 & 6.32 & 18.82 & 0.11 & 4.96 & 0.31 & - & - \\
\hline 1998 & 5.26 & 14.98 & 0.07 & 5.96 & 0.09 & 0.49 & 0.40 \\
\hline 1999 & 4.71 & 13.57 & 0.05 & 5.41 & - & 0.46 & - \\
\hline 1990-1999 & 7.59 & 21.75 & 0.10 & 8.72 & 0.06 & 1.04 & 0.16 \\
\hline
\end{tabular}

- indicates no deaths listed.

NOTE: See appendices for source description, methods, and ICD codes.

SOURCE: National Center for Health Statistics multiple cause of death data. Population estimates from U.S. Bureau of the Census. 
Table 2-3. Coal workers' pneumoconiosis: Years of potential life lost to age 65 and to life expectancy by race and sex, U.S. residents age 15 and over, 1990-1999

\begin{tabular}{|c|c|c|c|c|c|c|c|}
\hline \multirow[b]{2}{*}{ Year } & \multicolumn{2}{|c|}{ White } & \multicolumn{2}{|c|}{ Black } & \multicolumn{2}{|c|}{ Other } & \multirow[b]{2}{*}{ Total } \\
\hline & Male & Female & Male & Female & Male & Female & \\
\hline & \multicolumn{7}{|c|}{ Years of Potential Life Lost to Age 65} \\
\hline 1990 & 1,145 & - & 25 & 5 & 25 & - & 1,200 \\
\hline 1991 & 1,205 & 115 & 35 & - & 5 & - & 1,360 \\
\hline 1992 & 810 & 20 & 50 & - & - & - & 880 \\
\hline 1993 & 990 & 5 & 65 & - & 25 & - & 1,085 \\
\hline 1994 & 745 & 5 & 15 & - & - & - & 765 \\
\hline 1995 & 960 & 5 & 35 & - & - & - & 1,000 \\
\hline 1996 & 650 & 30 & 5 & - & - & - & 685 \\
\hline 1997 & 815 & 55 & 5 & - & - & - & 875 \\
\hline 1998 & 580 & - & 35 & - & - & - & 615 \\
\hline 1999 & 410 & 50 & 60 & - & - & - & 520 \\
\hline \multirow[t]{2}{*}{ TOTAL } & 8,310 & 285 & 330 & 5 & 55 & - & 8,985 \\
\hline & \multicolumn{7}{|c|}{ Years of Potential Life Lost to Life Expectancy } \\
\hline 1990 & 17,883 & 126 & 548 & 29 & 75 & 8 & 18,669 \\
\hline 1991 & 17,780 & 336 & 472 & - & 21 & - & 18,609 \\
\hline 1992 & 15,428 & 89 & 463 & - & 43 & - & 16,023 \\
\hline 1993 & 14,298 & 157 & 540 & - & 52 & - & 15,047 \\
\hline 1994 & 12,828 & 137 & 306 & - & 22 & - & 13,293 \\
\hline 1995 & 12,663 & 66 & 331 & - & - & - & 13,060 \\
\hline 1996 & 12,135 & 124 & 296 & - & 15 & 8 & 12,578 \\
\hline 1997 & 11,389 & 201 & 208 & 25 & - & - & 11,823 \\
\hline 1998 & 9,563 & 94 & 296 & 14 & 9 & 6 & 9,982 \\
\hline 1999 & 8,403 & 126 & 285 & - & 9 & - & 8,823 \\
\hline TOTAL & 132,370 & 1,456 & 3,745 & 68 & 246 & 22 & 137,907 \\
\hline
\end{tabular}

- indicates no deaths listed.

NOTE: See appendices for source description, methods, and ICD codes.

SOURCE: National Center for Health Statistics multiple cause of death data. 
Table 2-4. Coal workers' pneumoconiosis: Number of deaths by state, U.S. residents age 15 and over, 1990-1999

\begin{tabular}{|c|c|c|c|c|c|c|c|c|c|c|c|}
\hline State & 1990 & 1991 & 1992 & 1993 & 1994 & 1995 & 1996 & 1997 & 1998 & 1999 & Total \\
\hline Alabama & 29 & 28 & 23 & 19 & 21 & 15 & 14 & 15 & 13 & 8 & 185 \\
\hline Alaska & - & - & 1 & - & 1 & - & - & - & - & 1 & 3 \\
\hline Arizona & 5 & 4 & 2 & 4 & 6 & 6 & 7 & 2 & 1 & 4 & 41 \\
\hline Arkansas & 9 & 7 & 6 & 7 & 3 & 3 & 5 & 4 & 3 & 7 & 54 \\
\hline California & 28 & 41 & 22 & 27 & 28 & 13 & 12 & 4 & 17 & 8 & 200 \\
\hline Colorado & 15 & 15 & 19 & 18 & 6 & 10 & 7 & 11 & 8 & 9 & 118 \\
\hline Connecticut & 2 & 1 & 3 & 1 & 7 & 1 & - & - & 1 & 1 & 17 \\
\hline Delaware & 4 & 4 & 1 & 2 & 3 & - & - & 2 & - & - & 16 \\
\hline District of Columbia & - & - & - & - & - & 1 & - & - & - & - & 1 \\
\hline Florida & 25 & 33 & 23 & 25 & 15 & 21 & 28 & 23 & 14 & 16 & 223 \\
\hline Georgia & 4 & 3 & 2 & 7 & 3 & 5 & 3 & 3 & 1 & 2 & 33 \\
\hline Hawaii & - & - & - & - & - & - & - & - & - & - & - \\
\hline Idaho & - & - & 1 & 1 & - & - & 1 & 2 & 2 & 1 & 8 \\
\hline Illinois & 49 & 43 & 46 & 30 & 24 & 36 & 41 & 39 & 26 & 24 & 358 \\
\hline Indiana & 19 & 33 & 19 & 15 & 16 & 19 & 16 & 22 & 22 & 12 & 193 \\
\hline Iowa & 6 & 6 & 6 & 7 & 1 & 4 & 7 & 4 & 3 & 4 & 48 \\
\hline Kansas & 2 & 2 & 2 & 4 & 3 & 6 & 4 & 1 & 2 & 1 & 27 \\
\hline Kentucky & 115 & 112 & 116 & 114 & 81 & 84 & 129 & 99 & 90 & 90 & 1,030 \\
\hline Louisiana & 1 & 3 & - & 2 & 2 & 1 & 2 & 2 & 1 & 1 & 15 \\
\hline Maine & - & - & - & 1 & - & - & - & - & 11 & - & 12 \\
\hline Maryland & 12 & 5 & 13 & 6 & 10 & 7 & 8 & 7 & - & 7 & 75 \\
\hline Massachusetts & 2 & 2 & 2 & - & - & - & - & - & 1 & - & 7 \\
\hline Michigan & 21 & 15 & 10 & 11 & 11 & 6 & 10 & 9 & 7 & 6 & 106 \\
\hline Minnesota & - & - & - & - & - & - & - & - & - & - & - \\
\hline Mississippi & 2 & 3 & 1 & 1 & 2 & 6 & 1 & 7 & 6 & 6 & 35 \\
\hline Missouri & 4 & 6 & 11 & 7 & 5 & 3 & 1 & 2 & 2 & 3 & 44 \\
\hline Montana & - & - & - & - & 1 & 3 & 2 & 2 & - & - & 8 \\
\hline Nebraska & - & - & - & - & - & - & - & 1 & 1 & - & 2 \\
\hline Nevada & 1 & 1 & 1 & 1 & 1 & - & 1 & - & - & 1 & 7 \\
\hline New Hampshire & - & - & - & 1 & - & - & - & 1 & - & - & 2 \\
\hline New Jersey & 11 & 8 & 14 & 7 & 3 & 8 & 8 & 4 & 5 & 4 & 72 \\
\hline New Mexico & 3 & 3 & 4 & 4 & 4 & 5 & 7 & 5 & 5 & 4 & 44 \\
\hline New York & 9 & 4 & 2 & 9 & 11 & 1 & 4 & 4 & 2 & 6 & 52 \\
\hline North Carolina & 12 & 13 & 10 & 7 & 8 & 15 & 9 & 4 & 6 & 9 & 93 \\
\hline North Dakota & 1 & - & - & - & 1 & - & - & 1 & - & - & 3 \\
\hline Ohio & 79 & 83 & 74 & 64 & 57 & 71 & 64 & 41 & 52 & 31 & 616 \\
\hline Oklahoma & 5 & 4 & 1 & 4 & 3 & 4 & 1 & 5 & 2 & 2 & 31 \\
\hline Oregon & 1 & 1 & 1 & 2 & - & 1 & 1 & 1 & 1 & 1 & 10 \\
\hline Pennsylvania & 1,030 & 961 & 836 & 730 & 681 & 592 & 622 & 550 & 464 & 409 & 6,875 \\
\hline Rhode Island & - & - & - & 1 & - & - & 1 & - & - & - & 2 \\
\hline South Carolina & 1 & - & 5 & 3 & 5 & 4 & 3 & 1 & 5 & 2 & 29 \\
\hline South Dakota & - & - & - & - & - & - & - & 1 & - & - & 1 \\
\hline Tennessee & 37 & 23 & 30 & 25 & 28 & 30 & 25 & 19 & 23 & 26 & 266 \\
\hline Texas & 5 & 7 & 9 & 4 & 6 & 2 & 8 & 6 & 2 & 7 & 56 \\
\hline Utah & 18 & 12 & 13 & 12 & 16 & 13 & 6 & 8 & 9 & 5 & 112 \\
\hline Vermont & - & 1 & - & - & - & 1 & - & 129 & - & - & 131 \\
\hline Virginia & 131 & 134 & 147 & 151 & 100 & 118 & 124 & - & 82 & 86 & 1,073 \\
\hline Washington & 6 & 1 & 1 & 6 & 4 & 3 & 5 & 6 & 4 & - & 36 \\
\hline West Virginia & 279 & 308 & 283 & 287 & 289 & 291 & 228 & 245 & 207 & 196 & 2,613 \\
\hline Wisconsin & 3 & 1 & - & 1 & 1 & 1 & - & 3 & 1 & 1 & 12 \\
\hline Wyoming & 4 & 7 & 6 & 3 & 11 & 3 & 2 & 2 & 1 & 2 & 41 \\
\hline TOTAL & 1,990 & 1,938 & 1,766 & 1,631 & 1,478 & 1,413 & 1,417 & 1,297 & 1,103 & 1,003 & 15,036 \\
\hline
\end{tabular}

- indicates no deaths listed.

NOTE: See appendices for source description, methods, and ICD codes.

SOURCE: National Center for Health Statistics multiple cause of death data. 
Table 2-5. Coal workers' pneumoconiosis: Number of deaths, mortality rates (per million population), and years of potential life lost (YPLL) by state, U.S. residents age 15 and over, 1990-1999

\begin{tabular}{|c|c|c|c|c|c|c|c|c|c|c|}
\hline \multirow[b]{2}{*}{ State } & \multirow{2}{*}{$\begin{array}{l}\text { No. of } \\
\text { Deaths }\end{array}$} & \multirow[b]{2}{*}{ Rank } & \multicolumn{2}{|c|}{ Crude Mortality } & \multicolumn{2}{|c|}{ Age-Adjusted Mortality } & \multicolumn{4}{|c|}{ YPLL to Life Expectancy } \\
\hline & & & Rate & Rank & Rate & Rank & Total & Rank & YPLL/death & Rank \\
\hline Alabama & 185 & 11 & 5.58 & 9 & 5.75 & 9 & 1,756 & 11 & 9.5 & 31 \\
\hline Alaska & 3 & 41 & 0.69 & 32 & 2.59 & 16 & 23 & 42 & 7.7 & 44 \\
\hline Arizona & 41 & 24 & 1.26 & 23 & 1.35 & 23 & 363 & 26 & 8.9 & 34 \\
\hline Arkansas & 54 & 19 & 2.79 & 15 & 2.39 & 17 & 487 & 21 & 9.0 & 32 \\
\hline California & 200 & 9 & 0.82 & 31 & 0.97 & 30 & 3,063 & 7 & 15.3 & 5 \\
\hline Colorado & 118 & 12 & 4.07 & 11 & 5.34 & 10 & 1,009 & 14 & 8.5 & 38 \\
\hline Connecticut & 17 & 32 & 0.65 & 33 & 0.63 & 35 & 142 & 33 & 8.3 & 41 \\
\hline Delaware & 16 & 33 & 2.83 & 14 & 3.25 & 14 & 142 & 33 & 8.9 & 34 \\
\hline District of Columbia & 1 & 47 & 0.22 & 44 & 0.24 & 44 & 8 & 48 & 8.3 & 41 \\
\hline Florida & 223 & 8 & 1.94 & 18 & 1.50 & 22 & 2,312 & 9 & 10.4 & 18 \\
\hline Georgia & 33 & 28 & 0.59 & 35 & 0.76 & 34 & 357 & 27 & 10.8 & 16 \\
\hline Hawaii & - & - & - & - & - & - & - & - & - & - \\
\hline Idaho & 8 & 37 & 0.93 & 29 & 1.00 & 29 & 99 & 37 & 12.3 & 9 \\
\hline Illinois & 358 & 6 & 3.89 & 12 & 4.01 & 13 & 3,711 & 6 & 10.4 & 18 \\
\hline Indiana & 193 & 10 & 4.28 & 10 & 4.33 & 11 & 1,931 & 10 & 10.0 & 24 \\
\hline Iowa & 48 & 21 & 2.17 & 16 & 1.68 & 20 & 405 & 24 & 8.4 & 39 \\
\hline Kansas & 27 & 31 & 1.37 & 22 & 1.29 & 24 & 418 & 23 & 15.5 & 4 \\
\hline Kentucky & 1,030 & 4 & 34.13 & 3 & 34.39 & 3 & 11,919 & 4 & 11.6 & 12 \\
\hline Louisiana & 15 & 34 & 0.46 & 37 & 0.53 & 36 & 263 & 32 & 17.6 & 3 \\
\hline Maine & 1 & 47 & 0.10 & 49 & 0.10 & 49 & 8 & 48 & 8.3 & 41 \\
\hline Maryland & 86 & 16 & 2.16 & 17 & 2.79 & 15 & 842 & 17 & 9.8 & 25 \\
\hline Massachusetts & 7 & 39 & 0.14 & 48 & 0.14 & 47 & 95 & 38 & 13.5 & 6 \\
\hline Michigan & 106 & 14 & 1.43 & 21 & 1.56 & 21 & 1,131 & 12 & 10.7 & 17 \\
\hline Minnesota & - & - & - & - & - & - & - & - & - & - \\
\hline Mississippi & 35 & 27 & 1.72 & 19 & 1.80 & 19 & 914 & 16 & 26.1 & 2 \\
\hline Missouri & 44 & 22 & 1.06 & 27 & 0.95 & 31 & 459 & 22 & 10.4 & 18 \\
\hline Montana & 8 & 37 & 1.20 & 25 & 1.17 & 26 & 58 & 40 & 7.3 & 47 \\
\hline Nebraska & 2 & 43 & 0.16 & 47 & 0.13 & 48 & 15 & 45 & 7.4 & 46 \\
\hline Nevada & 7 & 39 & 0.58 & 36 & 0.82 & 33 & 68 & 39 & 9.7 & 27 \\
\hline New Hampshire & 2 & 43 & 0.22 & 44 & 0.25 & 43 & 17 & 44 & 8.4 & 39 \\
\hline New Jersey & 72 & 17 & 1.13 & 26 & 1.19 & 25 & 702 & 18 & 9.8 & 25 \\
\hline New Mexico & 44 & 22 & 3.49 & 13 & 4.23 & 12 & 384 & 25 & 8.7 & 36 \\
\hline New York & 52 & 20 & 0.36 & 41 & 0.37 & 40 & 537 & 20 & 10.3 & 21 \\
\hline North Carolina & 93 & 15 & 1.62 & 20 & 1.83 & 18 & 951 & 15 & 10.2 & 22 \\
\hline North Dakota & 3 & 41 & 0.61 & 34 & 0.50 & 37 & 23 & 42 & 7.6 & 45 \\
\hline Ohio & 616 & 5 & 7.08 & 7 & 7.13 & 7 & 5,968 & 5 & 9.7 & 27 \\
\hline Oklahoma & 31 & 29 & 1.22 & 24 & 1.16 & 27 & 355 & 28 & 11.5 & 13 \\
\hline Oregon & 10 & 36 & 0.41 & 39 & 0.37 & 40 & 135 & 36 & 13.5 & 6 \\
\hline Pennsylvania & 6,875 & 1 & 71.22 & 2 & 62.73 & 2 & 66,262 & 1 & 9.6 & 29 \\
\hline Rhode Island & 2 & 43 & 0.25 & 43 & 0.23 & 45 & 14 & 47 & 7.2 & 49 \\
\hline South Carolina & 29 & 30 & 1.02 & 28 & 1.14 & 28 & 341 & 30 & 11.8 & 10 \\
\hline South Dakota & 1 & 47 & 0.18 & 46 & 0.19 & 46 & 39 & 41 & 38.7 & 1 \\
\hline Tennessee & 266 & 7 & 6.41 & 8 & 6.65 & 8 & 2,677 & 8 & 10.1 & 23 \\
\hline Texas & 56 & 18 & 0.40 & 40 & 0.49 & 38 & 693 & 19 & 12.4 & 8 \\
\hline Utah & 112 & 13 & 8.37 & 6 & 10.74 & 6 & 1,073 & 13 & 9.6 & 29 \\
\hline Vermont & 2 & 43 & 0.44 & 38 & 0.46 & 39 & 15 & 45 & 7.3 & 47 \\
\hline Virginia & 1,202 & 3 & 22.96 & 4 & 28.26 & 4 & 14,223 & 3 & 11.8 & 10 \\
\hline Washington & 36 & 26 & 0.86 & 30 & 0.94 & 32 & 323 & 31 & 9.0 & 32 \\
\hline West Virginia & 2,613 & 2 & 179.64 & 1 & 157.33 & 1 & 29,547 & 2 & 11.3 & 14 \\
\hline Wisconsin & 12 & 35 & 0.30 & 42 & 0.29 & 42 & 136 & 35 & 11.3 & 14 \\
\hline Wyoming & 41 & 24 & 11.45 & 5 & 13.91 & 5 & 351 & 29 & 8.6 & 37 \\
\hline
\end{tabular}

- indicates no deaths listed.

NOTE: See appendices for source description, methods, and ICD codes.

SOURCE: National Center for Health Statistics multiple cause of death data. Population estimates from U.S. Bureau of the Census. 
Table 2-6. Coal workers' pneumoconiosis: Most frequently recorded industries on death certificate, U.S. residents age 15 and over, selected states and years, 1990-1999

\begin{tabular}{llrc}
\hline CIC & Industry & Number of Deaths & Percent \\
\hline 041 & Coal mining & 3,765 & 77.0 \\
060 & Construction & 188 & 3.8 \\
270 & Blast furnaces, steelworks, rolling and finishing mills & 56 & 1.1 \\
392 & Not specified manufacturing industries & 48 & 1.0 \\
400 & Railroads & 39 & 0.8 \\
010 & Agricultural production, crops & 35 & 0.7 \\
351 & Motor vehicles and motor vehicle equipment & 30 & 0.6 \\
410 & Trucking service & 29 & 0.6 \\
040 & Metal mining & 25 & 0.5 \\
961 & Non-paid worker or non-worker or own home/at home & 24 & 0.5 \\
& All other industries & 461 & 9.4 \\
& Industry not reported & 193 & 3.9 \\
& TOTAL & $\mathbf{4 , 8 9 3}$ & $\mathbf{1 0 0 . 0}$ \\
\hline
\end{tabular}

CIC - Census Industry Code

n.e.c. - not elsewhere classified

NOTE: Percentages may not total to $100 \%$ due to rounding. See appendices for source description, methods, and ICD codes, industry and occupation codes, and list of selected states and years.

SOURCE: National Center for Health Statistics multiple cause of death data.

Table 2-7. Coal workers' pneumoconiosis: Most frequently recorded occupations on death certificate, U.S. residents age 15 and over, selected states and years, 1990-1999

\begin{tabular}{clrc}
\hline COC & Occupation & Number of Deaths & Percent \\
\hline 616 & Mining machine operators & 3,440 & 70.3 \\
889 & Laborers, except construction & 147 & 3.0 \\
575 & Electricians & 64 & 1.3 \\
804 & Truck drivers & 62 & 1.3 \\
019 & Managers and administrators, n.e.c. & 59 & 1.2 \\
613 & Supervisors, extractive occupations & 49 & 1.0 \\
567 & Carpenters & 48 & 1.0 \\
453 & Janitors and cleaners & 47 & 1.0 \\
869 & Construction laborers & 45 & 0.9 \\
473 & Farmers, except horticulture & 39 & 0.8 \\
844 & Operating engineers & 39 & 0.8 \\
& All other occupations & 655 & 13.4 \\
& Occupation not reported & 199 & 4.1 \\
& TOTAL & $\mathbf{4 , 8 9 3}$ & $\mathbf{1 0 0 . 0}$ \\
\hline
\end{tabular}


Table 2-8. Coal workers' pneumoconiosis: Proportionate mortality ratio (PMR) adjusted for age, sex, and race by usual industry, U.S. residents age 15 and over, selected states and years, 1990-1999

\begin{tabular}{llrrrr}
\hline & & \multicolumn{2}{c}{$\begin{array}{c}\text { Number } \\
\text { CIC }\end{array}$} & Industry & \multicolumn{2}{c}{ 95\% Confidence Interval } \\
\cline { 5 - 6 } of Deaths & PMR & LCL & UCL \\
\hline 041 & Coal mining & 3,765 & 53.18 & 51.50 & 54.91 \\
040 & Metal mining & 25 & 1.98 & 1.28 & 2.92 \\
\hline
\end{tabular}

CIC - Census Industry Code n.e.c. - not elsewhere classified LCL - lower confidence limit UCL - upper confidence limit
NOTE: See appendices for source description, methods, and ICD codes, industry and occupation codes, and list of selected states and years.

SOURCE: National Center for Health Statistics multiple cause of death data. 
Table 2-9. Coal workers' pneumoconiosis: Proportionate mortality ratio (PMR) adjusted for age, sex, and race by usual occupation, U.S. residents age 15 and over, selected states and years, 1990-1999

\begin{tabular}{|c|c|c|c|c|c|}
\hline \multirow[b]{2}{*}{$\mathrm{COC}$} & \multirow[b]{2}{*}{ Occupation } & \multirow{2}{*}{$\begin{array}{c}\text { Number } \\
\text { of Deaths }\end{array}$} & \multirow[b]{2}{*}{ PMR } & \multicolumn{2}{|c|}{ 95\% Confidence Interval } \\
\hline & & & & LCL & UCL \\
\hline 616 & Mining machine operators & 3,440 & 51.67 & 49.97 & 53.44 \\
\hline 613 & Supervisors, extractive occupations & 49 & 14.36 & 10.64 & 18.99 \\
\hline 046 & Mining engineers & 8 & 6.03 & 2.60 & 11.86 \\
\hline 617 & Mining occupations, n.e.c. & 14 & 4.45 & 2.43 & 7.46 \\
\hline 859 & Miscellaneous material moving equipment operators & 12 & 2.27 & 1.17 & 3.96 \\
\hline 824 & Locomotive operating occupations & 23 & 2.03 & 1.29 & 3.05 \\
\hline
\end{tabular}

COC - Census Occupation Code n.e.c. - not elsewhere classified LCL - lower confidence limit UCL - upper confidence limit NOTE: See appendices for source description, methods, and ICD codes, industry and occupation codes, and list of selected states and years. SOURCE: National Center for Health Statistics multiple cause of death data. 
ఒ Figure 2-3. Coal workers' pneumoconiosis: Age-adjusted mortality rates by county, U.S. residents age 15 and over, 1970-1999

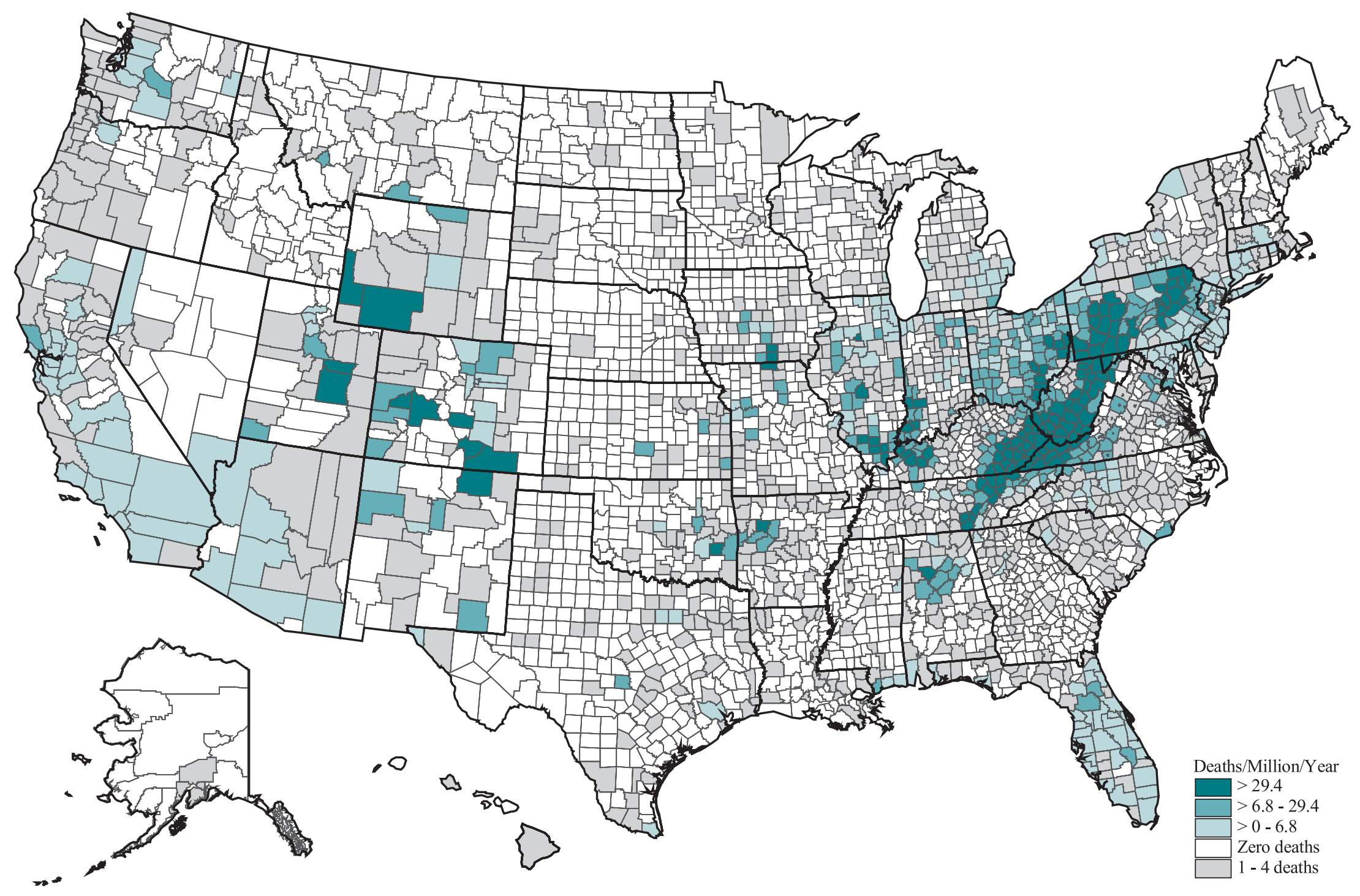

NOTE: Age-adjusted rates are not calculated for those counties with 1-4 deaths. See appendices for source description, methods, and ICD codes. SOURCE: National Center for Health Statistics multiple cause of death data. Population estimates from U.S. Bureau of the Census. 
Figure 2-4. Coal workers' pneumoconiosis: Age-adjusted mortality rates by county, U.S. residents age 15 and over, 1970-1984 and 1985-1999

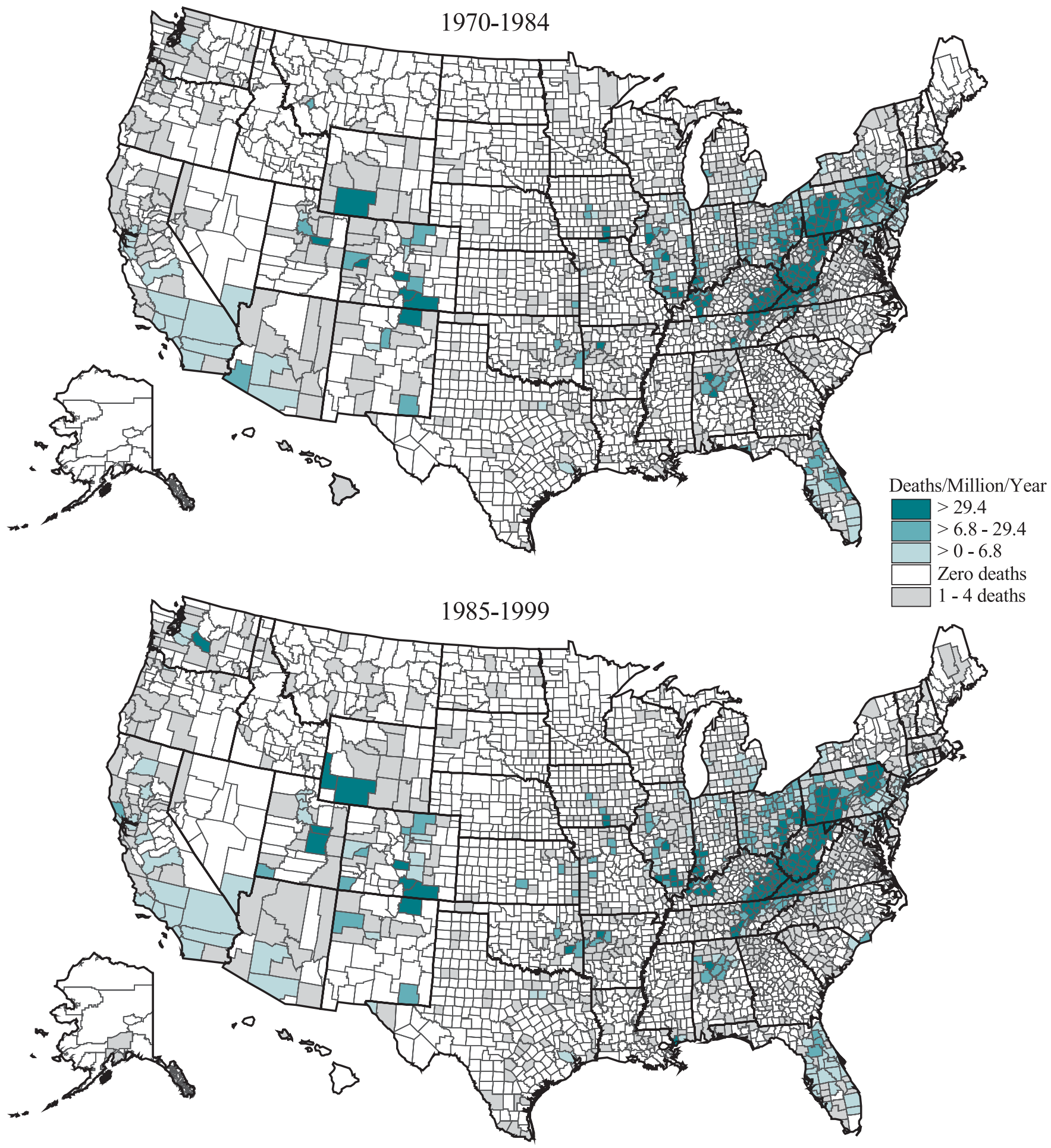

NOTE: Age-adjusted rates are not calculated for those counties with 1-4 deaths. See appendices for source description, methods, and ICD codes. SOURCE: National Center for Health Statistics multiple cause of death data. Population estimates from U.S. Bureau of the Census. 
Table 2-10. Coal workers' pneumoconiosis: Counties with highest age-adjusted mortality rates (per million population), U.S. residents age 15 and over, 1985-1999

\begin{tabular}{|c|c|c|c|c|c|}
\hline County & State & Age-Adjusted Rate & Crude Rate & Number of Deaths & \% Female \\
\hline Buchanan County & Virginia & $1,659.8$ & $1,170.4$ & 430 & 0.2 \\
\hline Schuylkill County & Pennsylvania & $1,042.4$ & $1,454.2$ & 2,744 & 0.1 \\
\hline McDowell County & West Virginia & $1,019.1$ & $1,141.7$ & 447 & 0.0 \\
\hline Raleigh County & West Virginia & 999.2 & $1,134.7$ & 1,035 & 0.0 \\
\hline Wyoming County & West Virginia & 922.0 & 769.3 & 258 & 0.0 \\
\hline Floyd County & Kentucky & 890.2 & 799.2 & 401 & 0.0 \\
\hline Tazewell County & Virginia & 741.0 & 763.6 & 426 & 0.2 \\
\hline Wise County & Virginia & 739.4 & 746.4 & 349 & 0.3 \\
\hline Dickenson County & Virginia & 716.8 & 703.8 & 146 & 0.7 \\
\hline Norton City & Virginia & 660.8 & 655.7 & 33 & 0.0 \\
\hline Luzerne County & Pennsylvania & 652.4 & 916.8 & 3,718 & 0.3 \\
\hline Boone County & West Virginia & 581.3 & 557.2 & 173 & 0.0 \\
\hline Fayette County & West Virginia & 536.8 & 677.8 & 388 & 0.0 \\
\hline Letcher County & Kentucky & 524.8 & 492.4 & 152 & 0.7 \\
\hline Logan County & West Virginia & 514.9 & 495.4 & 247 & 0.4 \\
\hline Harlan County & Kentucky & 477.8 & 471.6 & 196 & 0.0 \\
\hline Russell County & Virginia & 468.3 & 442.0 & 152 & 0.0 \\
\hline Northumberland County & Pennsylvania & 458.2 & 640.6 & 744 & 0.1 \\
\hline Carbon County & Utah & 408.8 & 449.4 & 97 & 0.0 \\
\hline Cambria County & Pennsylvania & 388.3 & 512.4 & 1,008 & 0.1 \\
\hline Knott County & Kentucky & 370.5 & 299.6 & 62 & 0.0 \\
\hline Somerset County & Pennsylvania & 360.0 & 444.3 & 415 & 0.0 \\
\hline Lee County & Virginia & 352.6 & 413.3 & 119 & 0.8 \\
\hline Mingo County & West Virginia & 350.5 & 290.5 & 109 & 0.0 \\
\hline Webster County & West Virginia & 341.7 & 409.2 & 50 & 0.0 \\
\hline Carbon County & Pennsylvania & 332.6 & 437.4 & 308 & 0.3 \\
\hline Bell County & Kentucky & 323.9 & 320.0 & 115 & 0.0 \\
\hline Lackawanna County & Pennsylvania & 313.8 & 439.7 & 1,173 & 0.3 \\
\hline Fayette County & Pennsylvania & 307.1 & 391.7 & 686 & 0.0 \\
\hline Nicholas County & West Virginia & 300.2 & 322.6 & 101 & 1.0 \\
\hline Mercer County & West Virginia & 288.3 & 353.4 & 277 & 0.4 \\
\hline Johnson County & Kentucky & 287.1 & 240.9 & 66 & 0.0 \\
\hline Emery County & Utah & 284.9 & 226.5 & 22 & 0.0 \\
\hline Pike County & Kentucky & 253.1 & 209.2 & 177 & 0.6 \\
\hline Greene County & Pennsylvania & 244.2 & 306.8 & 144 & 0.0 \\
\hline Franklin County & Illinois & 241.0 & 347.9 & 168 & 0.0 \\
\hline Muhlenberg County & Kentucky & 213.3 & 241.4 & 89 & 0.0 \\
\hline Knox County & Kentucky & 203.6 & 206.8 & 72 & 1.4 \\
\hline Leslie County & Kentucky & 201.0 & 162.6 & 25 & 0.0 \\
\hline Campbell County & Tennessee & 198.1 & 213.1 & 90 & 0.0 \\
\hline Indiana County & Pennsylvania & 194.2 & 196.6 & 214 & 0.0 \\
\hline Martin County & Kentucky & 193.8 & 149.2 & 21 & 0.0 \\
\hline Perry County & Kentucky & 187.1 & 157.9 & 56 & 0.0 \\
\hline Greenbrier County & West Virginia & 184.9 & 237.4 & 101 & 0.0 \\
\hline Clay County & West Virginia & 180.9 & 194.9 & 22 & 0.0 \\
\hline Preston County & West Virginia & 176.3 & 196.2 & 67 & 0.0 \\
\hline Marion County & West Virginia & 155.9 & 207.6 & 146 & 0.0 \\
\hline Whitley County & Kentucky & 155.4 & 156.8 & 62 & 0.0 \\
\hline Grundy County & Tennessee & 142.8 & 154.1 & 24 & 0.0 \\
\hline Sweetwater County & Wyoming & 137.2 & 79.0 & 34 & 0.0 \\
\hline Overall United States & & 9.4 & 8.9 & 26,706 & 0.7 \\
\hline
\end{tabular}

NOTE: Only counties with at least 5 deaths from the disease of interest are included. See appendices for source description, methods, and ICD codes. SOURCE: National Center for Health Statistics multiple cause of death data. Population estimates from U.S. Bureau of the Census. 


\section{Table 2-11. Coal workers' pneumoconiosis: Estimated number of discharges from} short-stay nonfederal hospitals, 1970-2000

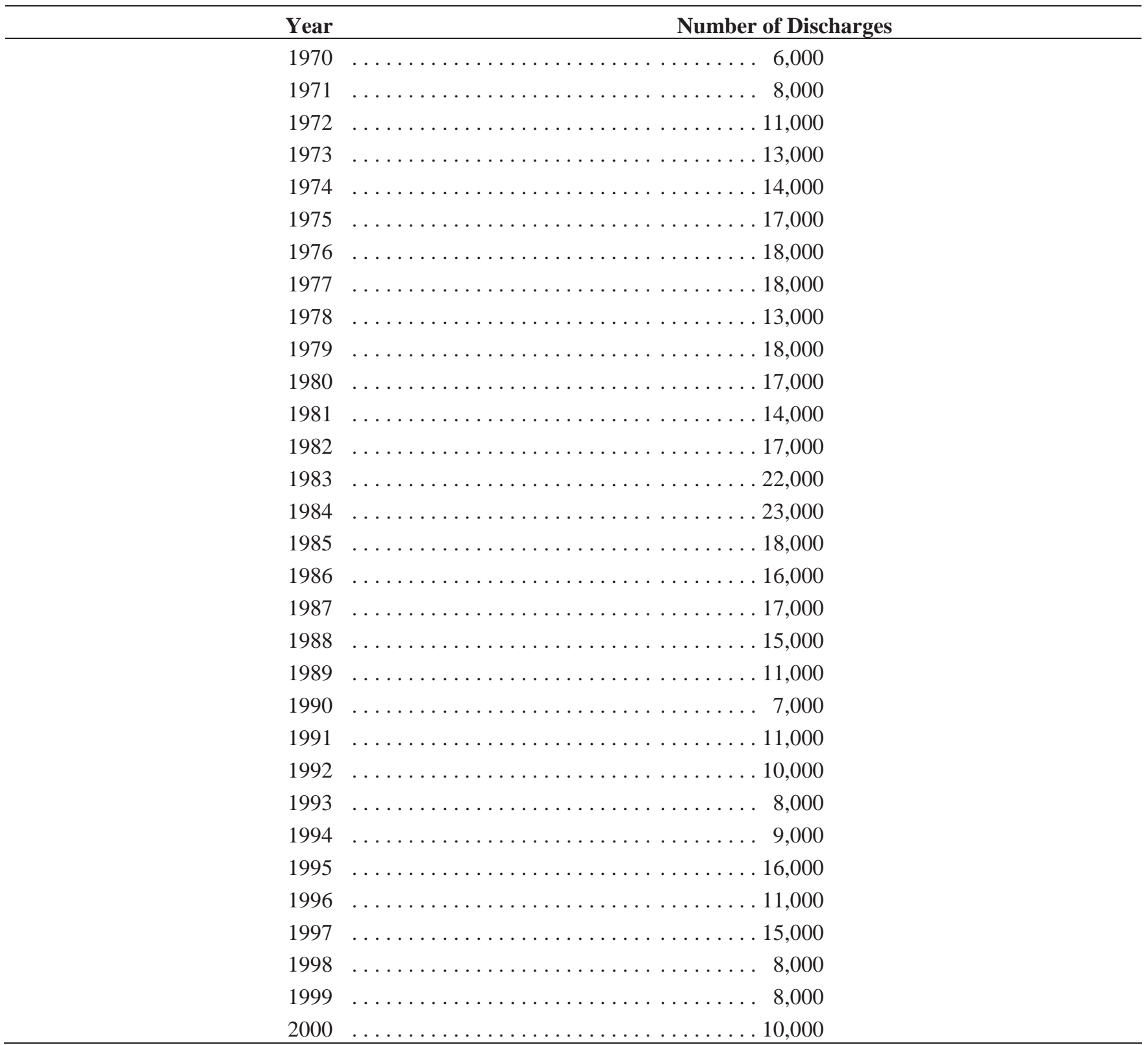

NOTE: Number of discharges has been rounded. NCHS recommends that, in statistical comparisons, estimates of less than 5,000 not be used and that estimates of 5,000 to 10,000 be used with caution. See appendices for source description and methods.

SOURCE: National Center for Health Statistics National Hospital Discharge Survey. 
Figure 2-5. CWXSP: Percentage of examined miners with coal workers' pneumoconiosis (category 1/0+) by tenure in mining, 1970-1999

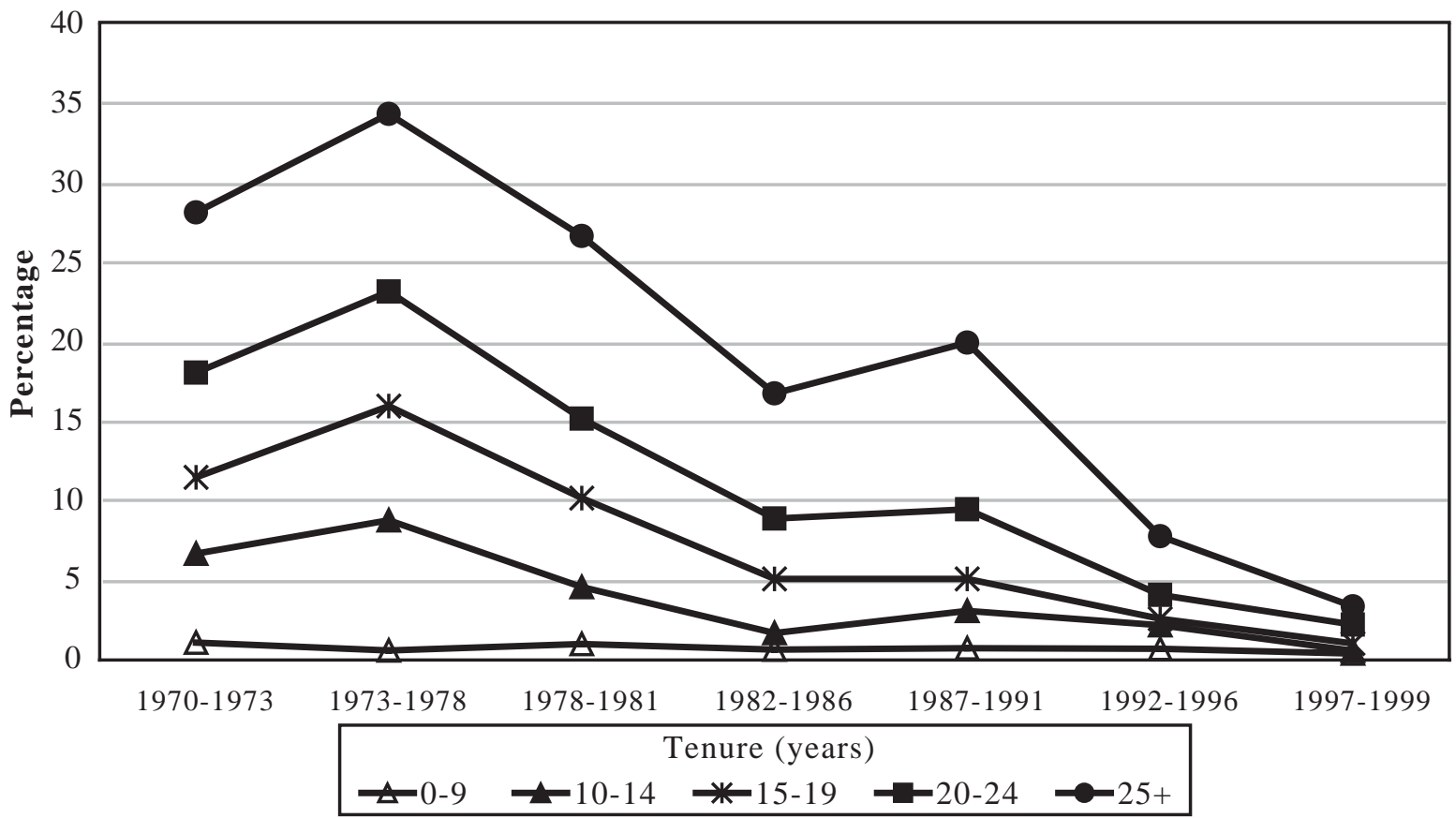

NOTE: 1997-1999 represents a partial round. See appendices for source description. SOURCE: NIOSH Coal Workers' X-ray Surveillance Program.

Figure 2-6. CWXSP: Estimated number of actively employed underground coal miners and number examined, 1970-1999

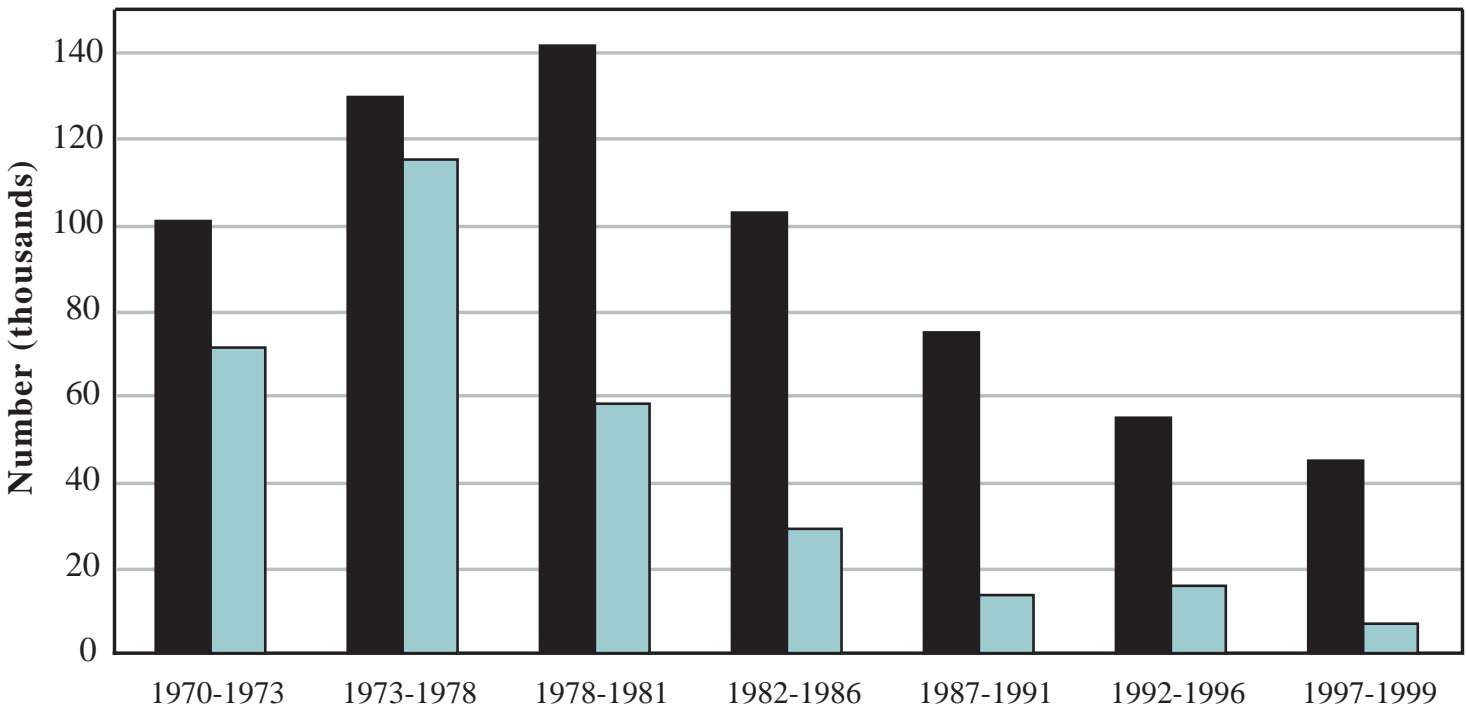

number employed $\quad$ Number x-rayed

NOTE: 1997-1999 represents a partial round. See appendices for source description.

SOURCE: NIOSH Coal Workers' X-ray Surveillance Program. Mine Safety and Health Administration (MSHA) coal mine employment data 
Table 2-12. CWXSP: Number and percentage of examined miners with coal workers' pneumoconiosis (category 1/0+), by round and tenure, 1970-1999

\begin{tabular}{|c|c|c|c|c|c|c|c|c|c|c|c|c|c|c|c|c|c|c|c|c|c|}
\hline \multirow{3}{*}{$\begin{array}{c}\text { Tenure } \\
\text { Years } \\
\text { in } \\
\text { Mining }\end{array}$} & \multicolumn{3}{|c|}{$\begin{array}{c}\text { Round 1 } \\
1970-1973\end{array}$} & \multicolumn{3}{|c|}{$\begin{array}{c}\text { Round 2 } \\
1973-1978\end{array}$} & \multicolumn{3}{|c|}{$\begin{array}{c}\text { Round 3 } \\
\text { 1978-1981 }\end{array}$} & \multicolumn{3}{|c|}{$\begin{array}{c}\text { Round } 4 \\
1982-1986\end{array}$} & \multicolumn{3}{|c|}{$\begin{array}{c}\text { Round 5 } \\
1987-1991 \\
\end{array}$} & \multicolumn{3}{|c|}{$\begin{array}{c}\text { Round } 6 \\
1992-1996 \\
\end{array}$} & \multicolumn{3}{|c|}{$\begin{array}{c}\text { Round } 7 \\
1997-1999\end{array}$} \\
\hline & \multirow{2}{*}{\begin{tabular}{|c|} 
Miners \\
examined \\
No. \\
\end{tabular}} & \multicolumn{2}{|c|}{ Cat. 1/0+ } & \multirow{2}{*}{\begin{tabular}{|c|} 
Miners \\
examined \\
No. \\
\end{tabular}} & \multicolumn{2}{|c|}{ Cat. 1/0+ } & \multirow{2}{*}{\begin{tabular}{|c|} 
Miners \\
examined \\
No.
\end{tabular}} & \multicolumn{2}{|c|}{ Cat. 1/0+ } & \multirow{2}{*}{$\begin{array}{c}\text { Miners } \\
\text { examined } \\
\text { No. }\end{array}$} & \multicolumn{2}{|c|}{ Cat. 1/0+ } & \multirow{2}{*}{\begin{tabular}{|c|} 
Miners \\
examined \\
No. \\
\end{tabular}} & \multicolumn{2}{|c|}{ Cat. 1/0+ } & \multirow{2}{*}{$\begin{array}{c}\text { Miners } \\
\text { examined } \\
\text { No. }\end{array}$} & \multicolumn{2}{|c|}{ Cat. 1/0+ } & \multirow{2}{*}{\begin{tabular}{|c|} 
Miners \\
examined \\
No.
\end{tabular}} & \multicolumn{2}{|c|}{ Cat. $1 / 0+$} \\
\hline & & No. & $\%$ & & No. & $\%$ & & No. & $\%$ & & No. & $\%$ & & No. & $\%$ & & No. & $\%$ & & No. & $\%$ \\
\hline 0 & 15,844 & 100 & 0.6 & 50,341 & 31 & 0.1 & 14,528 & 94 & 0.6 & 3,577 & 18 & 0.5 & 2,007 & 10 & 0.5 & 1,812 & 13 & 0.7 & 969 & 6 & 0.6 \\
\hline 1 & 5,287 & 49 & 0.9 & 9,579 & 13 & 0.1 & 3,719 & 18 & 0.5 & 742 & 1 & 0.1 & 356 & 0 & 0.0 & 238 & 2 & 0.8 & 163 & 0 & 0.0 \\
\hline $2-4$ & 8,274 & 73 & 0.9 & 18,432 & 137 & 0.7 & 12,059 & 103 & 0.8 & 3,786 & 25 & 0.7 & 1,057 & 6 & 0.6 & 791 & 2 & 0.3 & 388 & 2 & 0.5 \\
\hline $5-9$ & 6,706 & 182 & 2.7 & 13,528 & 386 & 2.8 & 14,157 & 215 & 1.5 & 7,434 & 57 & 0.8 & 2,763 & 30 & 1.1 & 1,235 & 12 & 1.0 & 418 & 0 & 0.0 \\
\hline $10-14$ & 4,451 & 298 & 6.7 & 5,282 & 466 & 8.8 & 5,318 & 246 & 4.6 & 5,435 & 93 & 1.7 & 4,120 & 123 & 3.0 & 2,522 & 56 & 2.2 & 511 & 3 & 0.6 \\
\hline $15-19$ & 4,743 & 546 & 11.5 & 3,380 & 542 & 16.0 & 2,168 & 221 & 10.2 & 1,824 & 93 & 5.1 & 2,279 & 114 & 5.0 & 4,646 & 119 & 2.6 & 1,148 & 12 & 1.0 \\
\hline 20-24 & 7,279 & 1,316 & 18.1 & 3,214 & 745 & 23.2 & 1,505 & 228 & 15.2 & 711 & 63 & 8.9 & 769 & 71 & 9.2 & 3,220 & 132 & 4.1 & 1,983 & 44 & 2.2 \\
\hline $25-29$ & 6,260 & 1,368 & 21.8 & 4,437 & 1,279 & 28.8 & 1,294 & 257 & 19.9 & 491 & 64 & 13.0 & 257 & 52 & 20.2 & 938 & 51 & 5.4 & 1,057 & 28 & 2.7 \\
\hline 30+ & 12,602 & 3,947 & 31.3 & 7,193 & 2,722 & 37.8 & 3,546 & 1,034 & 29.2 & 1,154 & 213 & 18.5 & 312 & 61 & 19.6 & 365 & 51 & 14.0 & 277 & 17 & 6.1 \\
\hline TOTAL & 71,446 & $\mathbf{7 , 8 9 7}$ & 11.0 & 115,386 & 6,321 & 5.5 & $\mathbf{5 8 , 2 9 4}$ & 2,416 & 4.1 & 25,154 & 627 & 2.5 & 13,920 & 467 & 3.4 & 15,767 & 438 & 2.8 & 6,914 & 112 & 1.6 \\
\hline
\end{tabular}

NOTE: Tabulations are based on one chest $\mathrm{x}$-ray per round for each participating miner

Round 1: Jan. 1970 - July 1973

Round 2: Aug. 1973 - July 1978

Round 3: Aug. 1978 - Dec. 1981

Round 4: Jan. 1982 - Dec. 1986

Round 5: Jan. 1987 - Dec. 1991

Round 6: Jan. 1992 - Dec. 1996

Round 7: Jan. 1997 - Dec. 1999

NOTE: 1997-1999 represents a partial round. See appendices for source description.

SOURCE: NIOSH Coal Workers' X-ray Surveillance Program. 
Table 2-13. Federal Black Lung Program: Number of beneficiaries and total payments by the Social Security Administration and Department of Labor, 1980-1999

\begin{tabular}{|c|c|c|c|c|c|c|}
\hline \multirow[b]{2}{*}{ Year } & \multicolumn{2}{|c|}{ Social Security Administration (SSA) } & \multicolumn{2}{|c|}{ Department of Labor (DOL) } & \multicolumn{2}{|c|}{ SSA and DOL } \\
\hline & Beneficiaries & $\begin{array}{l}\text { Amount } \\
\text { (dollars) }\end{array}$ & Beneficiaries & $\begin{array}{l}\text { Amount } \\
\text { (dollars) }\end{array}$ & $\begin{array}{c}\text { Total } \\
\text { Beneficiaries } \\
\end{array}$ & $\begin{array}{c}\text { Total Amount } \\
\text { (dollars) }\end{array}$ \\
\hline 1980 & 399,477 & $1,032,000,000$ & 139,073 & $813,205,000$ & 538,550 & $1,845,205,000$ \\
\hline 1981 & 376,505 & $1,081,300,000$ & 163,401 & $805,627,000$ & 539,906 & $1,886,927,000$ \\
\hline 1982 & 354,569 & $1,076,000,000$ & 173,972 & $784,085,000$ & 528,541 & $1,860,085,000$ \\
\hline 1983 & 333,358 & $1,055,800,000$ & 166,043 & $858,854,000$ & 499,401 & $1,914,654,000$ \\
\hline 1984 & 313,822 & $1,038,000,000$ & 163,166 & $873,932,000$ & 476,988 & $1,911,932,000$ \\
\hline 1985 & 294,846 & $1,025,000,000$ & 160,441 & $905,517,000$ & 455,287 & $1,930,517,000$ \\
\hline 1986 & 275,783 & $971,000,000$ & 156,892 & $629,075,000$ & 432,675 & $1,600,075,000$ \\
\hline 1987 & 258,988 & $940,000,000$ & 153,769 & $655,290,000$ & 412,757 & $1,595,290,000$ \\
\hline 1988 & 241,626 & $904,000,000$ & 150,123 & $656,689,000$ & 391,749 & $1,560,689,000$ \\
\hline 1989 & 225,764 & $882,000,000$ & 145,289 & $650,123,000$ & 371,053 & $1,532,123,000$ \\
\hline 1990 & 210,678 & $863,400,000$ & 139,854 & $626,521,000$ & 350,532 & $1,489,921,000$ \\
\hline 1991 & 196,419 & $844,400,000$ & 134,205 & $942,428,000$ & 330,624 & $1,786,828,000$ \\
\hline 1992 & 182,396 & $822,500,000$ & 128,761 & $973,636,000$ & 311,157 & $1,796,136,000$ \\
\hline 1993 & 168,365 & $794,300,000$ & 123,213 & $984,666,000$ & 291,578 & $1,778,966,000$ \\
\hline 1994 & 155,122 & $751,900,000$ & 117,569 & $994,655,000$ & 272,691 & $1,746,555,000$ \\
\hline 1995 & 143,011 & $696,700,000$ & 111,769 & $995,722,000$ & 254,780 & $1,692,422,000$ \\
\hline 1996 & 131,143 & $654,600,000$ & 105,923 & $992,128,000$ & 237,066 & $1,646,728,000$ \\
\hline 1997 & 119,233 & $614,888,000$ & 100,352 & $1,004,672,000$ & 219,585 & $1,619,560,000$ \\
\hline 1998 & 109,271 & $576,389,000$ & 94,488 & $999,822,000$ & 203,759 & $1,576,211,000$ \\
\hline 1999 & 98,977 & $541,200,000$ & 88,716 & $1,005,246,000$ & 187,693 & $1,546,446,000$ \\
\hline
\end{tabular}

NOTE: The Social Security Administration (SSA) was assigned initial responsibility for administering the Black Lung benefits program. The Department of Labor (DOL) assumed responsibility for processing and paying claims on July 1, 1973. Most claims filed prior to July 1 , 1973 remain within the jurisdiction of SSA, which also continues to be responsible for processing and paying claims filed by the survivors of these miners. The dollar amounts from the Department of Labor are for fiscal years. See appendices for source description.

SOURCE: Social Security Bulletin Annual Statistical Supplement (annual reports) and Black Lung Benefits Act Annual Report to Congress (annual reports). 
Figure 2-7. Respirable coal mine dust: Geometric mean exposures by type of mine, MSHA inspector and mine operator samples, 1979-1999

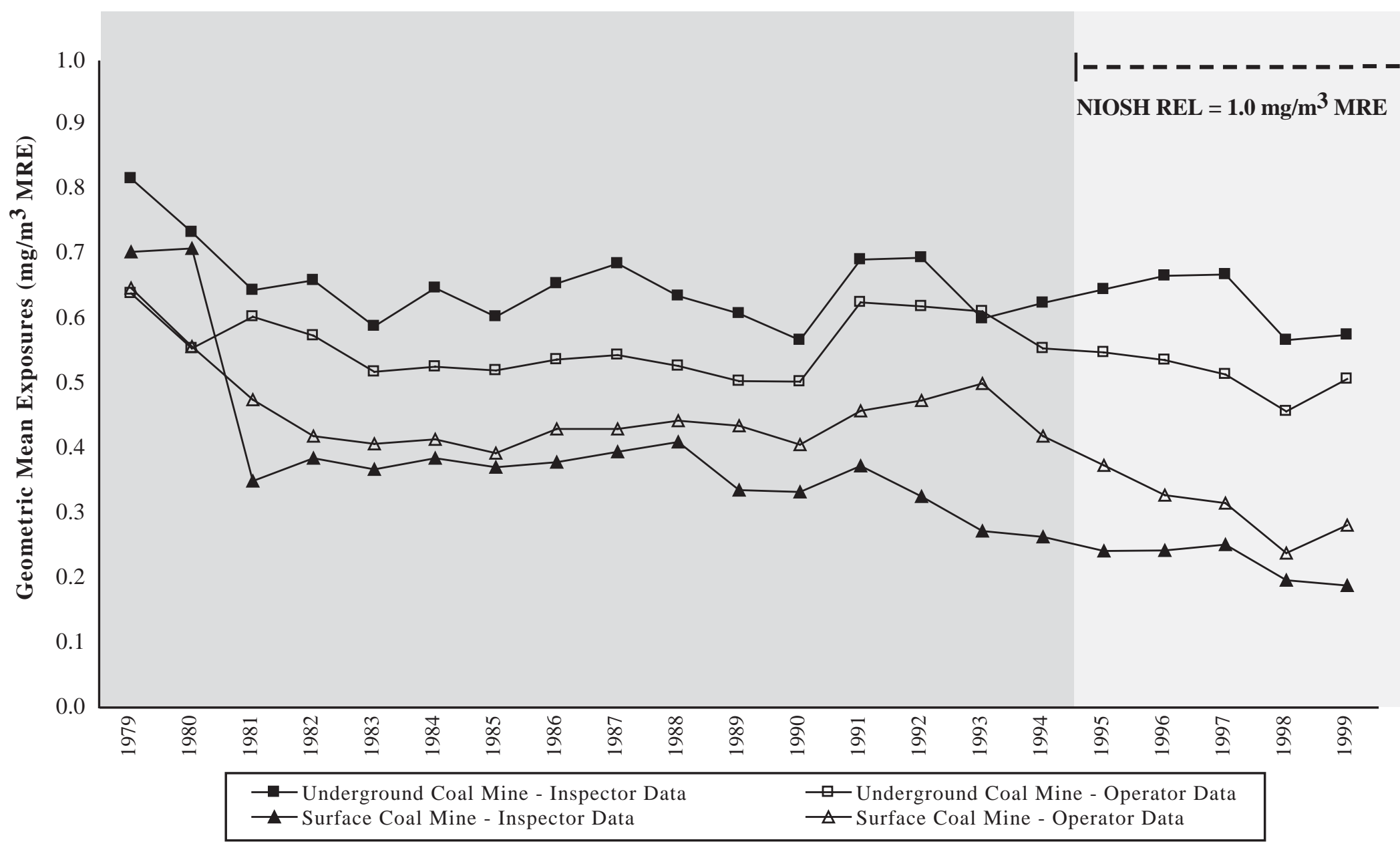

PEL - permissible exposure limit $\quad$ REL - recommended exposure limit $\quad \mathrm{mg} / \mathrm{m}^{3}$ - milligrams per cubic meter $\quad$ MRE - Mining Research Establishment

NOTE: In coal mining, for respirable dust containing less than $5 \%$ quartz, the MSHA PEL is $2 \mathrm{mg} / \mathrm{m}^{3} \mathrm{MRE}$; for respirable dust containing greater than $5 \%$ quartz, the MSHA PEL is [( 10 $\left.\mathrm{mg} / \mathrm{m}^{3} \mathrm{MRE}\right) /\left(\%\right.$ quartz)]. The NIOSH REL of $1 \mathrm{mg} / \mathrm{m}^{3}$ MRE for respirable coal mine dust was adopted in September of 1995. Geometric means are reported in MRE equivalent. See appendices for source description, methods, and agents.

SOURCE: Mine Safety and Health Administration (MSHA) coal mine inspector and mine operator dust data. 
Table 2-14. Respirable coal mine dust: Geometric mean exposures and percent exceeding designated occupational exposure limits by type of facility, MSHA inspector and mine operator samples, 1979-1999

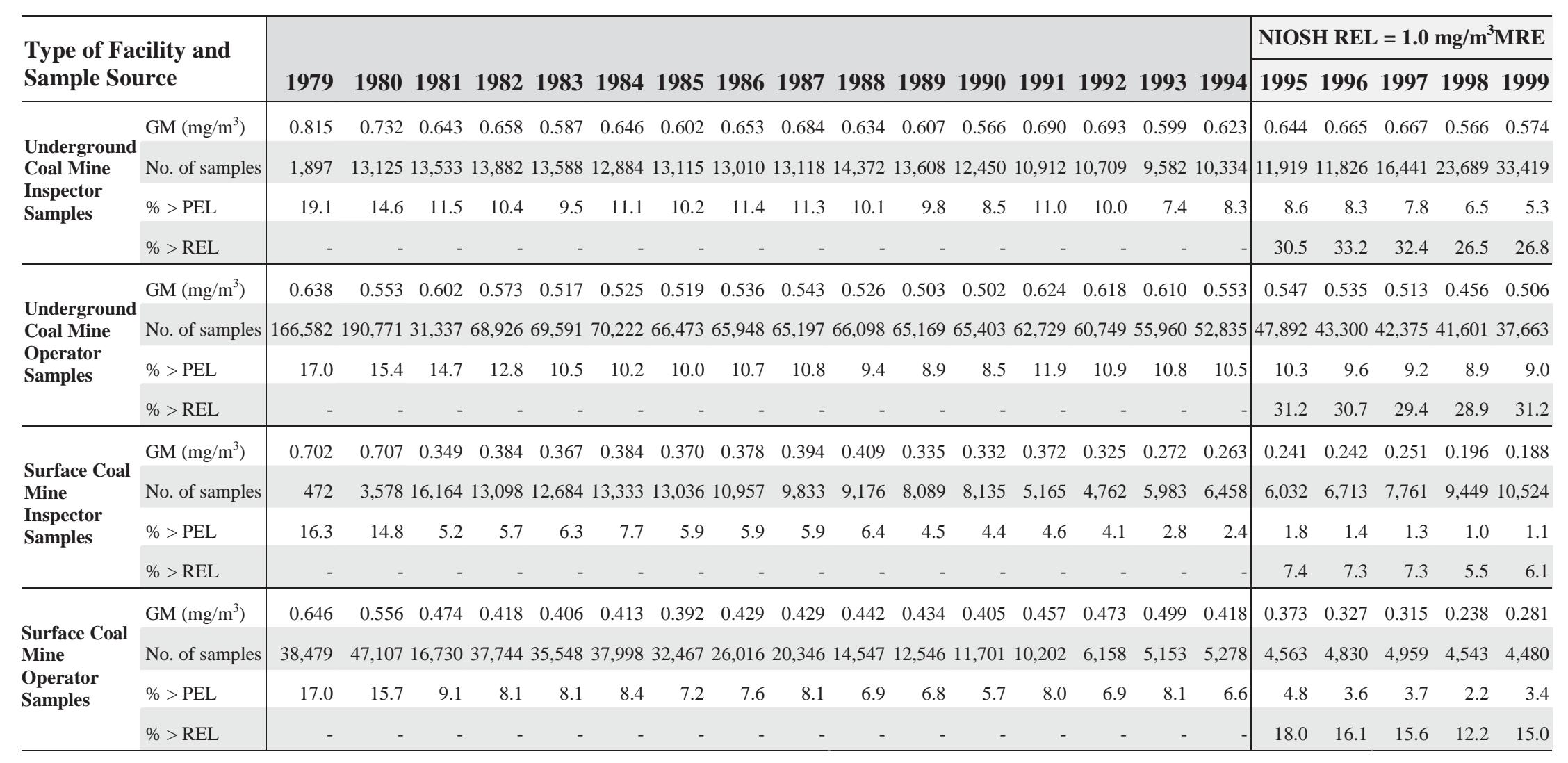

- indicates incalculable field

PEL - permissible exposure limit

MRE - Mining Research Establishment

NOTE: In coal mining, for respirable dust containing less than $5 \%$ quartz, the MSHA PEL is $2 \mathrm{mg} / \mathrm{m}^{3} \mathrm{MRE}$; for respirable dust containing greater than $5 \%$ quartz, the MSHA PEL is [(10 $\left.\mathrm{mg} / \mathrm{m}^{3} \mathrm{MRE}\right) /\left(\%\right.$ quartz)]. The NIOSH REL of $1 \mathrm{mg} / \mathrm{m}^{3}$ MRE for respirable coal mine dust was adopted in September of 1995. Geometric means are reported in MRE equivalent. All samples are compared to the MSHA PEL of $2 \mathrm{mg} / \mathrm{m}^{3}$ MRE for respirable coal mine dust containing less than $5 \%$ quartz, regardless of actual quartz content. See appendices for source description, methods, and agents.

SOURCE: Mine Safety and Health Administration (MSHA) coal mine inspector and mine operator dust data. 


\section{Table 2-15. Respirable coal mine dust: Number of samples, geometric mean} exposures, and percent exceeding designated occupational exposure limits by industries with elevated coal workers' pneumoconiosis mortality, MSHA inspector and mine operator samples, 1990-1999

\begin{tabular}{|c|c|c|c|c|c|c|c|}
\hline \multicolumn{4}{|c|}{$\begin{array}{l}\text { Coal Workers' Pneumoconiosis Mortality, } \\
\text { Selected States and Years, 1990-1999 }\end{array}$} & \multirow[b]{2}{*}{$\begin{array}{l}\text { Number of } \\
\text { Samples }\end{array}$} & \multirow[b]{2}{*}{$\begin{array}{c}\text { GM } \\
\left(\mathbf{m g} / \mathbf{m}^{3}\right)\end{array}$} & \multirow[b]{2}{*}{$\begin{array}{l}\%> \\
\text { PEL }\end{array}$} & \multirow[b]{2}{*}{$\begin{array}{c}\%> \\
\text { REL } \\
1995-1999\end{array}$} \\
\hline CIC & $\begin{array}{l}\text { Industries with elevated PMRs and most } \\
\text { frequently recorded on death certificates }\end{array}$ & $\begin{array}{l}\text { Number of } \\
\text { Deaths }\end{array}$ & PMR & & & & \\
\hline 041 & Coal mining & 3,765 & 53.18 & 794,637 & 0.509 & 8.6 & 26.2 \\
\hline & All other industries & 935 & & 0 & - & - & - \\
\hline & TOTAL & & & 794,637 & 0.509 & 8.6 & 26.2 \\
\hline
\end{tabular}

- indicates incalculable field

CIC - Census Industry Code

GM - geometric mean

PEL - permissible exposure limit

REL - recommended exposure limit

PMR - proportionate mortality ratio MRE - Mining Research Establishment $5 \%$ quartz, the MSHA PEL is $\left[\left(10 \mathrm{mg} / \mathrm{m}^{3} \mathrm{MRE}\right) /(\%\right.$ quartz $\left.)\right]$. The NIOSH REL of $1 \mathrm{mg} / \mathrm{m}^{3} \mathrm{MRE}$ for respirable coal mine dust was adopted in September of 1995. Geometric means are reported in MRE equivalent. All samples are compared to the MSHA PEL of $2 \mathrm{mg} / \mathrm{m}^{3} \mathrm{MRE}$ for respirable coal mine dust containing less than $5 \%$ quartz, regardless of actual quartz content. See appendices for source description, methods, ICD codes, industry codes, agents, and list of selected states and years for which usual industry has been reported.

SOURCE: Mine Safety and Health Administration (MSHA) coal mine inspector and mine operator dust data. National Center for Health Statistics multiple cause of death data. 
th Table 2-16 (page 1 of 3). Respirable coal mine dust: Geometric mean exposures and percent exceeding designated occupational exposure limits by MSHA coal mine district and state, MSHA inspector and mine operator samples, 1979-1999

\begin{tabular}{|c|c|c|c|c|c|c|c|c|c|c|c|c|}
\hline \multirow[b]{2}{*}{ MSHA Coal Mine District } & \multicolumn{2}{|c|}{ All years } & \multicolumn{3}{|c|}{$1979-1989$} & \multicolumn{3}{|c|}{$1990-1994$} & \multicolumn{4}{|c|}{$\begin{array}{c}1995-1999 \\
\text { NIOSH REL }=1.0 \mathrm{mg} / \mathrm{m}^{3} \text { MRE }\end{array}$} \\
\hline & $\begin{array}{c}\text { No. of } \\
\text { Samples }\end{array}$ & $\begin{array}{c}\mathbf{G M} \\
\left(\mathbf{m g} / \mathbf{m}^{3}\right)\end{array}$ & $\begin{array}{c}\text { No. of } \\
\text { Samples }\end{array}$ & $\underset{\left(\mathbf{m g} / \mathbf{m}^{3}\right)}{\mathbf{G M}}$ & $\begin{array}{l}\%> \\
\text { PEL }\end{array}$ & $\begin{array}{c}\text { No. of } \\
\text { Samples }\end{array}$ & $\begin{array}{c}\mathbf{G M} \\
\left(\mathrm{mg} / \mathbf{m}^{3}\right)\end{array}$ & $\begin{array}{l}\%> \\
\text { PEL }\end{array}$ & $\begin{array}{c}\text { No. of } \\
\text { Samples }\end{array}$ & $\underset{\left(\mathbf{m g} / \mathbf{m}^{3}\right)}{\mathbf{G M}}$ & $\begin{array}{l}\%> \\
\text { PEL }\end{array}$ & $\begin{array}{l}\%> \\
\text { REL }\end{array}$ \\
\hline $\begin{array}{l}\text { District } 1 \text { (Anthracite coal mining regions in } \\
\text { Pennsylvania) }\end{array}$ & 40,660 & 0.203 & 22,717 & 0.219 & 2.7 & 9,277 & 0.184 & 2.4 & 8,666 & 0.186 & 2.6 & 8.9 \\
\hline $\begin{array}{l}\text { District } 2 \text { (Bituminous coal mining regions in } \\
\text { Pennsylvania) }\end{array}$ & 289,781 & 0.512 & 208,709 & 0.515 & 9.8 & 40,164 & 0.564 & 9.5 & 40,908 & 0.451 & 5.8 & 23.9 \\
\hline District 3 & 258,453 & 0.558 & 184,655 & 0.590 & 12.7 & 38,816 & 0.531 & 7.5 & 34,982 & 0.436 & 5.2 & 23.3 \\
\hline Maryland & 9,818 & 0.621 & 5,668 & 0.674 & 13.1 & 1,501 & 0.614 & 6.9 & 2,649 & 0.523 & 6.1 & 32.3 \\
\hline Ohio & 85,691 & 0.606 & 66,440 & 0.672 & 16.3 & 9,792 & 0.479 & 6.0 & 9,459 & 0.372 & 4.7 & 22.5 \\
\hline Northern West Virginia & 162,944 & 0.531 & 112,547 & 0.543 & 10.5 & 27,523 & 0.547 & 8.1 & 22,874 & 0.457 & 5.4 & 22.5 \\
\hline District 4 (Southern West Virginia) & 451,111 & 0.551 & 306,083 & 0.572 & 14.6 & 76,284 & 0.531 & 10.6 & 68,744 & 0.485 & 9.0 & 27.4 \\
\hline District 5 (Virginia) & 287,317 & 0.467 & 189,909 & 0.465 & 9.3 & 55,143 & 0.512 & 8.4 & 42,265 & 0.421 & 5.9 & 20.7 \\
\hline District 6 (Eastern Kentucky) & 266,705 & 0.415 & 154,093 & 0.392 & 7.0 & 56,560 & 0.467 & 8.3 & 56,052 & 0.433 & 6.6 & 22.4 \\
\hline District 7 & 262,223 & 0.449 & 143,987 & 0.444 & 7.2 & 63,635 & 0.483 & 7.0 & 54,601 & 0.423 & 6.2 & 21.2 \\
\hline Central Kentucky & 216,315 & 0.457 & 112,157 & 0.453 & 7.5 & 54,830 & 0.492 & 7.2 & 49,328 & 0.430 & 6.3 & 21.4 \\
\hline North Carolina & 9 & 0.147 & 6 & 0.159 & 0.0 & 3 & 0.126 & 0.0 & 0 & - & - & - \\
\hline South Carolina & 0 & - & 0 & - & - & 0 & - & - & 0 & - & - & - \\
\hline Tennessee & 45,870 & 0.411 & 31,795 & 0.413 & 6.5 & 8,802 & 0.432 & 6.0 & 5,273 & 0.366 & 6.1 & 19.2 \\
\hline Northern Georgia & 29 & 0.328 & 29 & 0.328 & 0.0 & 0 & - & - & 0 & - & - & - \\
\hline District 8 & 146,515 & 0.743 & 99,969 & 0.681 & 14.7 & 26,146 & 0.899 & 14.3 & 20,400 & 0.891 & 15.5 & 50.5 \\
\hline Illinois & 131,785 & 0.794 & 90,886 & 0.731 & 15.6 & 23,477 & 0.974 & 15.0 & 17,422 & 0.934 & 15.4 & 52.0 \\
\hline Indiana & 13,033 & 0.424 & 7,575 & 0.342 & 5.2 & 2,514 & 0.459 & 8.6 & 2,944 & 0.687 & 16.6 & 42.8 \\
\hline Iowa & 886 & 0.384 & 813 & 0.388 & 5.3 & 73 & 0.344 & 0.0 & 0 & - & - & - \\
\hline Michigan & 0 & - & 0 & - & - & 0 & - & - & 0 & - & - & - \\
\hline Minnesota & 0 & - & 0 & - & - & 0 & - & - & 0 & - & - & - \\
\hline Northern Missouri & 811 & 0.249 & 695 & 0.260 & 1.7 & 82 & 0.240 & 2.4 & 34 & 0.107 & 0.0 & 0.0 \\
\hline Wisconsin & 0 & & 0 & - & - & 0 & - & - & 0 & - & - & - \\
\hline
\end{tabular}

See footnotes at end of table. 
Table 2-16 (page 2 of 3). Respirable coal mine dust: Geometric mean exposures and percent exceeding designated occupational exposure limits by MSHA coal mine district and state, MSHA inspector and mine operator samples, 1979-1999

\begin{tabular}{|c|c|c|c|c|c|c|c|c|c|c|c|c|}
\hline \multirow[b]{2}{*}{ MSHA Coal Mine District } & \multicolumn{2}{|c|}{ All years } & \multicolumn{3}{|c|}{$1979-1989$} & \multicolumn{3}{|c|}{1990 - 1994} & \multicolumn{4}{|c|}{$\begin{array}{c}1995-1999 \\
\text { NIOSH REL }=1.0 \mathrm{mg} / \mathrm{m}^{3} \text { MRE }\end{array}$} \\
\hline & $\begin{array}{c}\text { No. of } \\
\text { Samples }\end{array}$ & $\begin{array}{c}\mathbf{G M} \\
\left(\mathrm{mg} / \mathrm{m}^{3}\right)\end{array}$ & \begin{tabular}{|c|} 
No. of \\
Samples
\end{tabular} & $\begin{array}{c}\mathbf{G M} \\
\left(\mathrm{mg} / \mathbf{m}^{3}\right)\end{array}$ & $\begin{array}{l}\%> \\
\text { PEL }\end{array}$ & $\begin{array}{c}\text { No. of } \\
\text { Samples }\end{array}$ & $\begin{array}{c}\mathbf{G M} \\
\left(\mathrm{mg} / \mathbf{m}^{3}\right)\end{array}$ & $\begin{array}{l}\%> \\
\text { PEL }\end{array}$ & \begin{tabular}{|c|} 
No. of \\
Samples
\end{tabular} & $\begin{array}{c}\mathbf{G M} \\
\left(\mathrm{mg} / \mathbf{m}^{3}\right)\end{array}$ & $\begin{array}{l}\%> \\
\text { PEL }\end{array}$ & $\begin{array}{l}\%> \\
\text { REL }\end{array}$ \\
\hline District 9 & 120,599 & 0.683 & 78,749 & 0.696 & 19.8 & 22,044 & 0.759 & 15.0 & 19,806 & 0.565 & 9.3 & 33.8 \\
\hline Alaska & 246 & 0.338 & 181 & 0.408 & 6.6 & 48 & 0.237 & 2.1 & 17 & 0.123 & 0.0 & 0.0 \\
\hline Arizona & 913 & 0.252 & 484 & 0.310 & 5.0 & 201 & 0.308 & 4.5 & 228 & 0.136 & 1.3 & 1.9 \\
\hline Arkansas & 311 & 0.172 & 238 & 0.205 & 2.5 & 37 & 0.149 & 2.7 & 36 & 0.064 & 0.0 & 0.0 \\
\hline California & 12 & 0.218 & 0 & - & - & 1 & 0.400 & 0.0 & 11 & 0.206 & 0.0 & 9.1 \\
\hline Colorado & 38,040 & 0.844 & 25,744 & 0.845 & 24.1 & 6,616 & 0.915 & 16.4 & 5,680 & 0.765 & 10.4 & 40.7 \\
\hline Hawaii & 0 & - & 0 & - & - & 0 & - & - & 0 & - & - & - \\
\hline Idaho & 0 & - & 0 & - & - & 0 & - & - & 0 & - & - & - \\
\hline Kansas & 510 & 0.216 & 383 & 0.235 & 2.6 & 49 & 0.190 & 0.0 & 78 & 0.152 & 1.3 & 1.4 \\
\hline Louisiana & 105 & 0.186 & 14 & 0.163 & 0.0 & 39 & 0.228 & 0.0 & 52 & 0.165 & 0.0 & 0.0 \\
\hline Southern Missouri & 647 & 0.236 & 453 & 0.255 & 2.0 & 56 & 0.160 & 0.0 & 138 & 0.216 & 1.4 & 7.0 \\
\hline Montana & 1,720 & 0.296 & 1,096 & 0.368 & 7.2 & 213 & 0.250 & 2.8 & 411 & 0.181 & 2.7 & 5.6 \\
\hline Nebraska & 0 & - & 0 & - & - & 0 & - & - & 0 & - & - & - \\
\hline Nevada & 0 & - & 0 & - & - & 0 & - & - & 0 & - & - & - \\
\hline New Mexico & 5,222 & 0.615 & 3,535 & 0.767 & 23.7 & 876 & 0.753 & 20.2 & 811 & 0.188 & 3.5 & 6.2 \\
\hline North Dakota & 1,345 & 0.187 & 1,029 & 0.221 & 1.9 & 163 & 0.157 & 0.6 & 153 & 0.073 & 0.0 & 0.0 \\
\hline Oklahoma & 4,857 & 0.331 & 2,972 & 0.345 & 6.9 & 985 & 0.325 & 6.7 & 900 & 0.297 & 3.4 & 9.8 \\
\hline Oregon & 0 & - & 0 & - & - & 0 & - & - & 0 & - & - & \\
\hline Texas & 4,005 & 0.179 & 2,740 & 0.194 & 1.4 & 664 & 0.198 & 3.9 & 601 & 0.109 & 1.0 & 3.5 \\
\hline Utah & 52,952 & 0.852 & 34,017 & 0.834 & 21.8 & 10,277 & 0.928 & 16.4 & 8,658 & 0.834 & 12.1 & 43.6 \\
\hline Washington & 346 & 0.178 & 124 & 0.166 & 0.0 & 74 & 0.257 & 2.7 & 148 & 0.158 & 0.7 & 4.3 \\
\hline Wyoming & 9,368 & 0.463 & 5,739 & 0.479 & 12.5 & 1,745 & 0.569 & 14.1 & 1,884 & 0.345 & 7.0 & 21.9 \\
\hline
\end{tabular}

See footnotes at end of table. 
Table 2-16 (page 3 of 3). Respirable coal mine dust: Geometric mean exposures and percent exceeding designated occupational exposure limits by MSHA coal mine district and state, MSHA inspector and mine operator samples, 1979-1999

\begin{tabular}{|c|c|c|c|c|c|c|c|c|c|c|c|c|}
\hline \multirow[b]{2}{*}{ MSHA Coal Mine District } & \multicolumn{2}{|c|}{ All years } & \multicolumn{3}{|c|}{$1979-1989$} & \multicolumn{3}{|c|}{$1990-1994$} & \multicolumn{4}{|c|}{$\begin{array}{c}1995-1999 \\
\text { NIOSH REL }=1.0 \mathrm{mg} / \mathrm{m}^{3} \mathrm{MRE}\end{array}$} \\
\hline & $\begin{array}{c}\text { No. of } \\
\text { Samples }\end{array}$ & $\begin{array}{c}\mathbf{G M} \\
\left(\mathrm{mg} / \mathrm{m}^{3}\right)\end{array}$ & \begin{tabular}{|c|} 
No. of \\
Samples
\end{tabular} & $\begin{array}{c}\mathbf{G M} \\
\left(\mathrm{mg} / \mathbf{m}^{3}\right)\end{array}$ & $\begin{array}{l}\%> \\
\text { PEL }\end{array}$ & \begin{tabular}{|c|} 
No. of \\
Samples
\end{tabular} & $\begin{array}{c}\text { GM } \\
\left(\mathrm{mg} / \mathrm{m}^{3}\right)\end{array}$ & $\begin{array}{l}\%> \\
\text { PEL }\end{array}$ & $\begin{array}{c}\text { No. of } \\
\text { Samples }\end{array}$ & $\begin{array}{c}\mathbf{G M} \\
\left(\mathrm{mg} / \mathbf{m}^{3}\right)\end{array}$ & $\begin{array}{l}\%> \\
\text { PEL }\end{array}$ & $\begin{array}{l}\%> \\
\text { REL }\end{array}$ \\
\hline District 10 (Western Kentucky) & 77,891 & 0.676 & $\mathbf{5 0 , 9 2 3}$ & 0.603 & 12.1 & 14,282 & 0.847 & 14.8 & 12,686 & 0.829 & 14.2 & 48.4 \\
\hline District 11 & 85,776 & 0.686 & 52,600 & 0.714 & 15.7 & 18,307 & 0.724 & 10.7 & 14,869 & 0.556 & 6.6 & 29.8 \\
\hline Alabama & 85,776 & 0.686 & 52,600 & 0.714 & 15.7 & 18,307 & 0.724 & 10.7 & 14,869 & 0.556 & 6.6 & 29.8 \\
\hline Central and Southern Georgia & 0 & - & 0 & - & - & 0 & - & - & 0 & - & - & - \\
\hline Florida & 0 & - & 0 & - & - & 0 & - & - & 0 & - & - & - \\
\hline Mississippi & 0 & - & 0 & - & - & 0 & - & - & 0 & - & - & - \\
\hline Puerto Rico & 0 & - & 0 & - & - & 0 & - & - & 0 & - & - & - \\
\hline Virgin Islands & 0 & - & 0 & - & - & 0 & - & - & 0 & - & - & - \\
\hline TOTAL & $2,287,031$ & 0.520 & $1,492,394$ & 0.526 & 11.6 & 420,658 & 0.545 & 9.5 & 373,979 & 0.472 & 7.5 & 26.2 \\
\hline
\end{tabular}

- indicates incalculable field

PEL - permissible exposure limit

REL - recommended exposure limit

GM - geometric mean

$\mathrm{mg} / \mathrm{m}^{3}$ - milligrams per cubic meter

NOTE: In coal mining, for respirable dust containing less than 5\% quartz, the MSHA PEL is $2 \mathrm{mg} / \mathrm{m}^{3} \mathrm{MRE}$; for respirable dust containing greater than $5 \%$ quartz, the MSHA PEL is [(10 $\left.\mathrm{mg} / \mathrm{m}^{3} \mathrm{MRE}\right) /\left(\%\right.$ quartz)]. The NIOSH REL of $1 \mathrm{mg} / \mathrm{m}^{3}$ MRE for respirable coal mine dust was adopted in September of 1995. Geometric means are reported in MRE equivalent. All

samples are compared to the MSHA PEL for respirable coal mine dust containing less than 5\% quartz, regardless of actual quartz content. See appendices for source description, methods, and agents.

SOURCE: Mine Safety and Health Administration (MSHA) coal mine inspector and mine operator dust data. 
Figure 2-8. Respirable coal mine dust: Geometric mean exposures by state, MSHA inspector and mine operator samples, 1979-1999

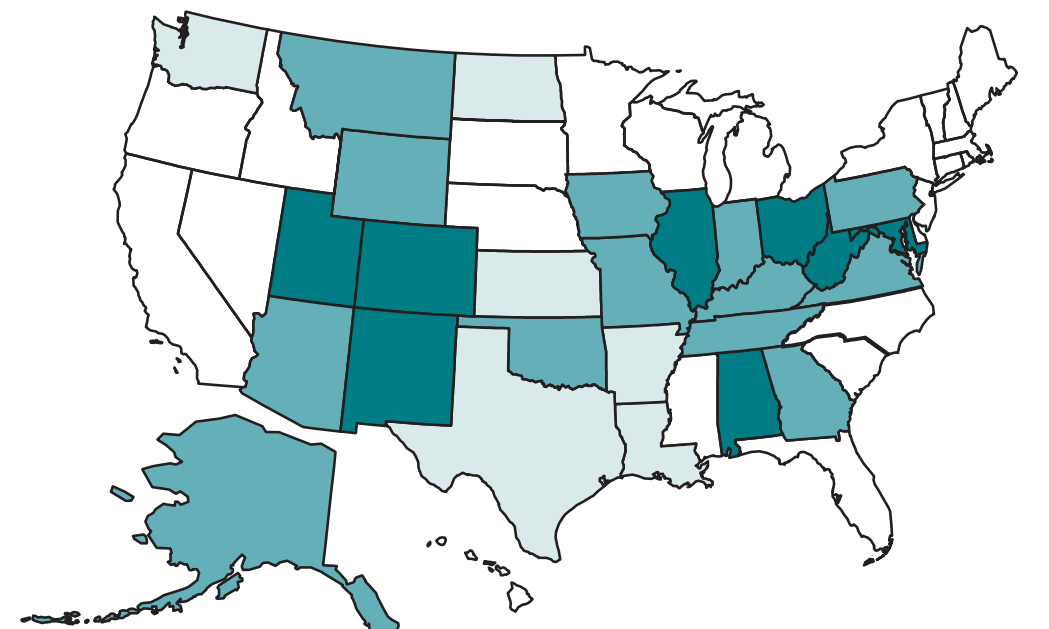

\begin{tabular}{|cc|}
\hline \multicolumn{2}{|c|}{$\mathbf{1 9 7 9}$ - 1989} \\
\hline mg/m ${ }^{3}$ MRE & No. of States \\
$\square>0.5$ & 8 \\
$\square>0.25$ to 0.5 & 13 \\
$\square 0$ to 0.25 & 6 \\
$\square<10$ samples & 24 \\
\hline
\end{tabular}

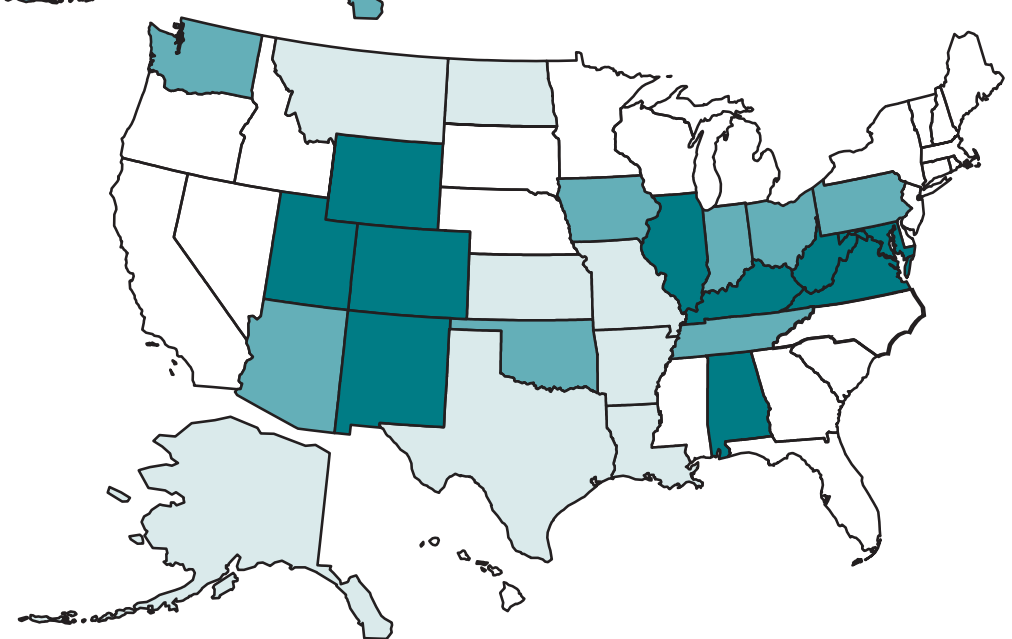

\begin{tabular}{|cc|}
\hline \multicolumn{1}{|c|}{$\mathbf{1 9 9 0} \mathbf{- 1 9 9 4}$} \\
\hline mg/m ${ }^{3}$ MRE & No. of States \\
$\square>0.5$ & 10 \\
$\square>0.25$ to 0.5 & 8 \\
$\square 0$ to 0.25 & 8 \\
$\square<10$ samples & 25 \\
\hline
\end{tabular}

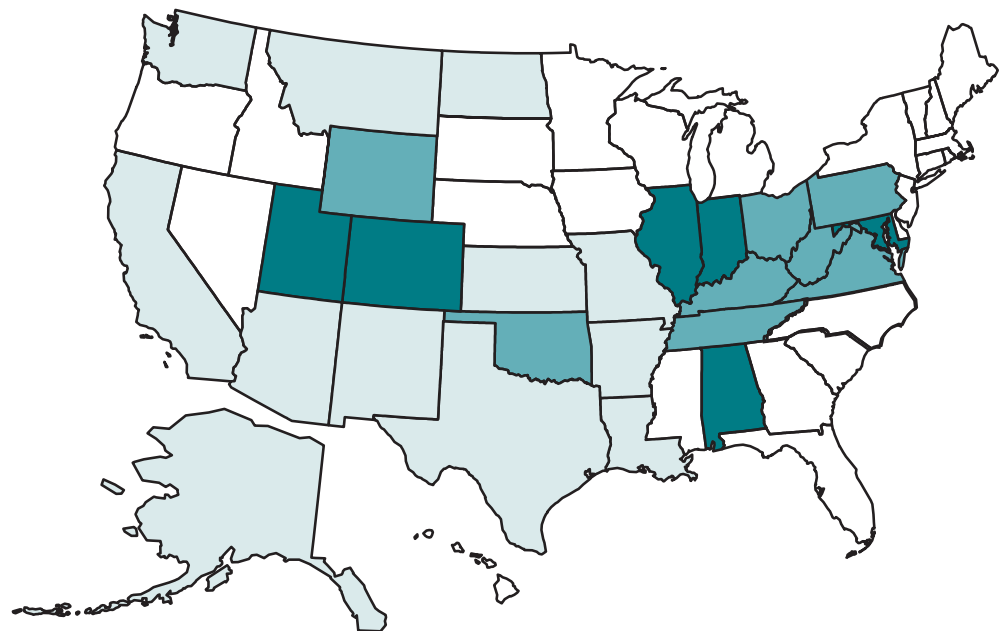

\begin{tabular}{|cc|}
\hline \multicolumn{2}{|c|}{$\mathbf{1 9 9 5}-\mathbf{1 9 9 9}$} \\
NIOSH REL $=1.0 \mathrm{mg} / \mathrm{m}^{3}$ \\
\hline mg/m $\mathrm{m}^{3}$ MRE & No. of States \\
$\square>0.5$ & 6 \\
$\square>0.25$ to 0.5 & 8 \\
$\square 0$ to 0.25 & 12 \\
$\square<10$ samples & 25 \\
\hline
\end{tabular}

PEL - permissible exposure limit REL - recommended exposure limit $\mathrm{mg} / \mathrm{m}^{3}$ - milligrams per cubic meter MRE - Mining Research Establishment NOTE: In coal mining, for respirable dust containing less than $5 \%$ quartz, the MSHA PEL is $2 \mathrm{mg} / \mathrm{m}^{3} \mathrm{MRE}$; for respirable dust containing greater than $5 \%$ quartz, the MSHA PEL is [(10 mg/m $\left.\mathrm{m}^{3} \mathrm{MRE}\right) /\left(\%\right.$ quartz)]. The NIOSH REL of $1 \mathrm{mg} / \mathrm{m}^{3}$ MRE for respirable coal mine dust was adopted in September of 1995. Geometric means are reported in MRE equivalent. See appendices for source description, methods, and agents.

SOURCE: Mine Safety and Health Administration (MSHA) coal mine inspector and mine operator dust data. 



\section{Section 2}

Coal Workers'

Pneumoconiosis

and

Related Exposures 

Figure 2-1. Coal workers' pneumoconiosis: Number of deaths, crude and ageadjusted mortality rates, U.S. residents age 15 and over, 1968-1999

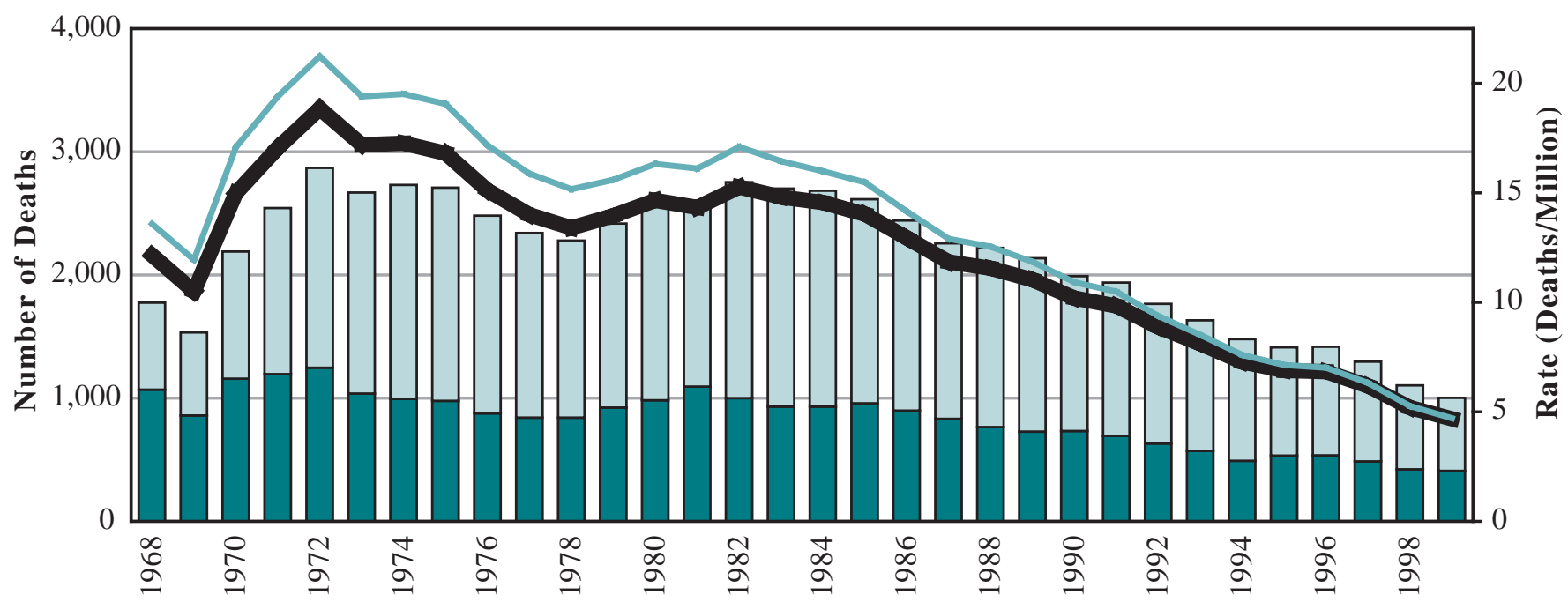

Figure 2-2. Coal workers' pneumoconiosis: Age-adjusted mortality rates by state, U.S. residents age 15 and over, 1990-1999

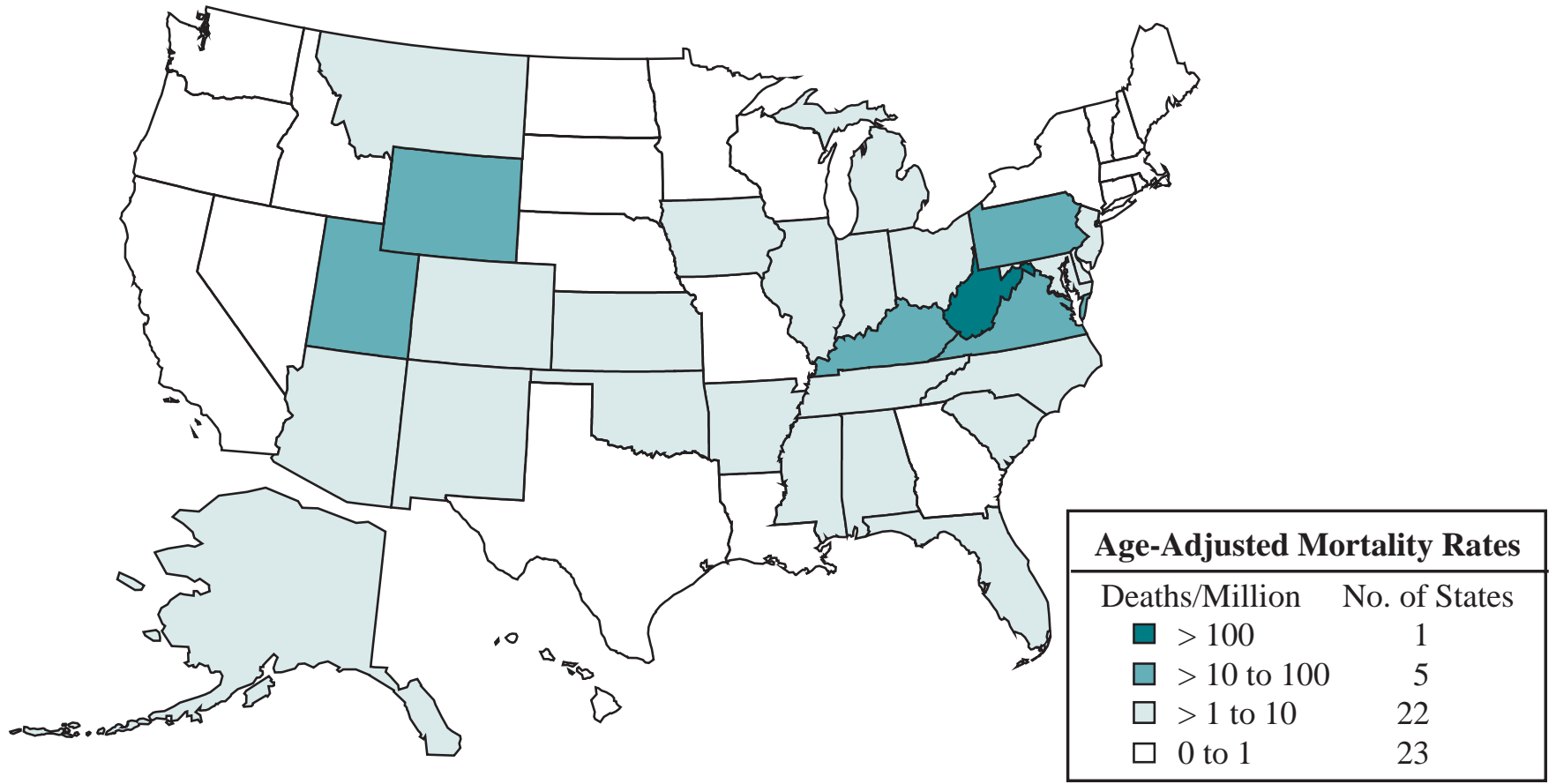

NOTE: See appendices for source description, methods, and ICD codes.

SOURCE: National Center for Health Statistics multiple cause of death data. Population estimates from U.S. Bureau of the Census. 
Table 2-1. Coal workers' pneumoconiosis: Number of deaths by sex, race, and age, and median age at death, U.S. residents age 15 and over, 1990-1999

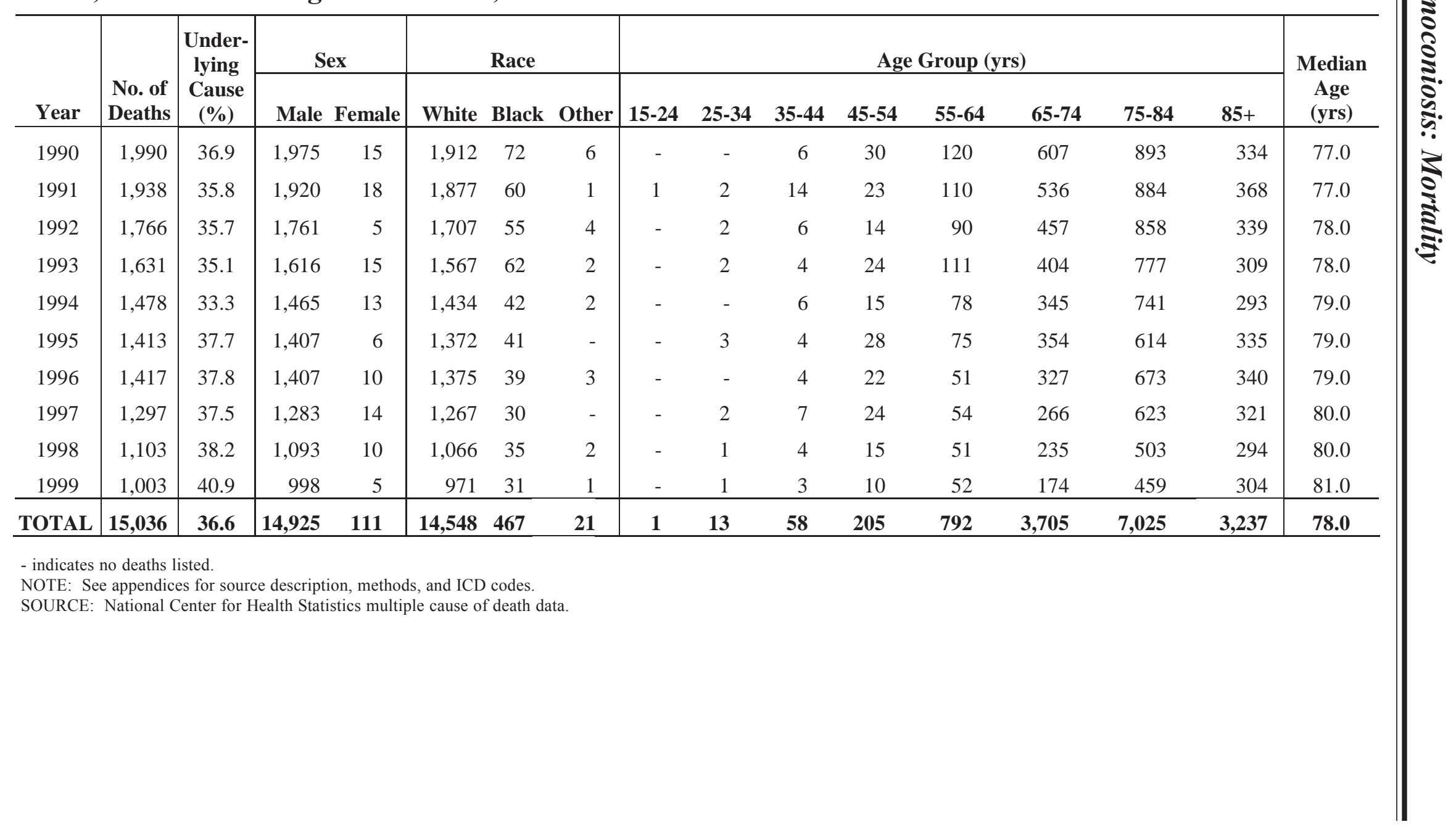


Table 2-2. Coal workers' pneumoconiosis: Mortality rates (per million population) by race and sex, U.S. residents age 15 and over, 1990-1999

\begin{tabular}{|c|c|c|c|c|c|c|c|}
\hline \multirow[b]{2}{*}{ Year } & \multirow[b]{2}{*}{ Overall } & \multicolumn{2}{|c|}{ White } & \multicolumn{2}{|c|}{ Black } & \multicolumn{2}{|c|}{ Other } \\
\hline & & Male & Female & Male & Female & Male & Female \\
\hline & \multicolumn{7}{|c|}{ Crude Mortality Rate } \\
\hline 1990 & 10.19 & 23.70 & 0.14 & 6.82 & 0.17 & 1.44 & 0.27 \\
\hline 1991 & 9.84 & 23.03 & 0.21 & 5.76 & - & 0.28 & - \\
\hline 1992 & 8.87 & 20.89 & 0.06 & 5.19 & - & 1.06 & - \\
\hline 1993 & 8.11 & 18.90 & 0.17 & 5.75 & - & 0.52 & - \\
\hline 1994 & 7.28 & 17.16 & 0.15 & 3.83 & - & 0.50 & - \\
\hline 1995 & 6.89 & 16.35 & 0.07 & 3.68 & - & - & - \\
\hline 1996 & 6.83 & 16.15 & 0.10 & 3.43 & - & 0.46 & 0.21 \\
\hline 1997 & 6.18 & 14.71 & 0.12 & 2.33 & 0.22 & - & - \\
\hline 1998 & 5.20 & 12.31 & 0.09 & 2.89 & 0.07 & 0.22 & 0.20 \\
\hline 1999 & 4.68 & 11.14 & 0.05 & 2.59 & - & 0.21 & - \\
\hline \multirow[t]{2}{*}{ 1990-1999 } & 7.33 & 17.29 & 0.11 & 4.13 & 0.05 & 0.44 & 0.07 \\
\hline & \multicolumn{7}{|c|}{ Age-Adjusted Mortality Rate } \\
\hline 1990 & 10.91 & 31.16 & 0.12 & 14.22 & 0.21 & 3.68 & 0.66 \\
\hline 1991 & 10.48 & 30.29 & 0.19 & 12.48 & - & 0.39 & - \\
\hline 1992 & 9.38 & 27.29 & 0.05 & 10.84 & - & 2.69 & - \\
\hline 1993 & 8.51 & 24.18 & 0.14 & 11.78 & - & 0.67 & - \\
\hline 1994 & 7.60 & 21.82 & 0.12 & 8.58 & - & 1.08 & - \\
\hline 1995 & 7.14 & 20.72 & 0.05 & 7.87 & - & - & - \\
\hline 1996 & 7.02 & 20.30 & 0.09 & 7.25 & - & 1.30 & 0.42 \\
\hline 1997 & 6.32 & 18.82 & 0.11 & 4.96 & 0.31 & - & - \\
\hline 1998 & 5.26 & 14.98 & 0.07 & 5.96 & 0.09 & 0.49 & 0.40 \\
\hline 1999 & 4.71 & 13.57 & 0.05 & 5.41 & - & 0.46 & - \\
\hline 1990-1999 & 7.59 & 21.75 & 0.10 & 8.72 & 0.06 & 1.04 & 0.16 \\
\hline
\end{tabular}

- indicates no deaths listed.

NOTE: See appendices for source description, methods, and ICD codes.

SOURCE: National Center for Health Statistics multiple cause of death data. Population estimates from U.S. Bureau of the Census. 
Table 2-3. Coal workers' pneumoconiosis: Years of potential life lost to age 65 and to life expectancy by race and sex, U.S. residents age 15 and over, 1990-1999

\begin{tabular}{|c|c|c|c|c|c|c|c|}
\hline \multirow[b]{2}{*}{ Year } & \multicolumn{2}{|c|}{ White } & \multicolumn{2}{|c|}{ Black } & \multicolumn{2}{|c|}{ Other } & \multirow[b]{2}{*}{ Total } \\
\hline & Male & Female & Male & Female & Male & Female & \\
\hline & \multicolumn{7}{|c|}{ Years of Potential Life Lost to Age 65} \\
\hline 1990 & 1,145 & - & 25 & 5 & 25 & - & 1,200 \\
\hline 1991 & 1,205 & 115 & 35 & - & 5 & - & 1,360 \\
\hline 1992 & 810 & 20 & 50 & - & - & - & 880 \\
\hline 1993 & 990 & 5 & 65 & - & 25 & - & 1,085 \\
\hline 1994 & 745 & 5 & 15 & - & - & - & 765 \\
\hline 1995 & 960 & 5 & 35 & - & - & - & 1,000 \\
\hline 1996 & 650 & 30 & 5 & - & - & - & 685 \\
\hline 1997 & 815 & 55 & 5 & - & - & - & 875 \\
\hline 1998 & 580 & - & 35 & - & - & - & 615 \\
\hline 1999 & 410 & 50 & 60 & - & - & - & 520 \\
\hline \multirow[t]{2}{*}{ TOTAL } & 8,310 & 285 & 330 & 5 & 55 & - & 8,985 \\
\hline & \multicolumn{7}{|c|}{ Years of Potential Life Lost to Life Expectancy } \\
\hline 1990 & 17,883 & 126 & 548 & 29 & 75 & 8 & 18,669 \\
\hline 1991 & 17,780 & 336 & 472 & - & 21 & - & 18,609 \\
\hline 1992 & 15,428 & 89 & 463 & - & 43 & - & 16,023 \\
\hline 1993 & 14,298 & 157 & 540 & - & 52 & - & 15,047 \\
\hline 1994 & 12,828 & 137 & 306 & - & 22 & - & 13,293 \\
\hline 1995 & 12,663 & 66 & 331 & - & - & - & 13,060 \\
\hline 1996 & 12,135 & 124 & 296 & - & 15 & 8 & 12,578 \\
\hline 1997 & 11,389 & 201 & 208 & 25 & - & - & 11,823 \\
\hline 1998 & 9,563 & 94 & 296 & 14 & 9 & 6 & 9,982 \\
\hline 1999 & 8,403 & 126 & 285 & - & 9 & - & 8,823 \\
\hline TOTAL & 132,370 & 1,456 & 3,745 & 68 & 246 & 22 & 137,907 \\
\hline
\end{tabular}

- indicates no deaths listed.

NOTE: See appendices for source description, methods, and ICD codes.

SOURCE: National Center for Health Statistics multiple cause of death data. 
Table 2-4. Coal workers' pneumoconiosis: Number of deaths by state, U.S. residents age 15 and over, 1990-1999

\begin{tabular}{|c|c|c|c|c|c|c|c|c|c|c|c|}
\hline State & 1990 & 1991 & 1992 & 1993 & 1994 & 1995 & 1996 & 1997 & 1998 & 1999 & Total \\
\hline Alabama & 29 & 28 & 23 & 19 & 21 & 15 & 14 & 15 & 13 & 8 & 185 \\
\hline Alaska & - & - & 1 & - & 1 & - & - & - & - & 1 & 3 \\
\hline Arizona & 5 & 4 & 2 & 4 & 6 & 6 & 7 & 2 & 1 & 4 & 41 \\
\hline Arkansas & 9 & 7 & 6 & 7 & 3 & 3 & 5 & 4 & 3 & 7 & 54 \\
\hline California & 28 & 41 & 22 & 27 & 28 & 13 & 12 & 4 & 17 & 8 & 200 \\
\hline Colorado & 15 & 15 & 19 & 18 & 6 & 10 & 7 & 11 & 8 & 9 & 118 \\
\hline Connecticut & 2 & 1 & 3 & 1 & 7 & 1 & - & - & 1 & 1 & 17 \\
\hline Delaware & 4 & 4 & 1 & 2 & 3 & - & - & 2 & - & - & 16 \\
\hline District of Columbia & - & - & - & - & - & 1 & - & - & - & - & 1 \\
\hline Florida & 25 & 33 & 23 & 25 & 15 & 21 & 28 & 23 & 14 & 16 & 223 \\
\hline Georgia & 4 & 3 & 2 & 7 & 3 & 5 & 3 & 3 & 1 & 2 & 33 \\
\hline Hawaii & - & - & - & - & - & - & - & - & - & - & - \\
\hline Idaho & - & - & 1 & 1 & - & - & 1 & 2 & 2 & 1 & 8 \\
\hline Illinois & 49 & 43 & 46 & 30 & 24 & 36 & 41 & 39 & 26 & 24 & 358 \\
\hline Indiana & 19 & 33 & 19 & 15 & 16 & 19 & 16 & 22 & 22 & 12 & 193 \\
\hline Iowa & 6 & 6 & 6 & 7 & 1 & 4 & 7 & 4 & 3 & 4 & 48 \\
\hline Kansas & 2 & 2 & 2 & 4 & 3 & 6 & 4 & 1 & 2 & 1 & 27 \\
\hline Kentucky & 115 & 112 & 116 & 114 & 81 & 84 & 129 & 99 & 90 & 90 & 1,030 \\
\hline Louisiana & 1 & 3 & - & 2 & 2 & 1 & 2 & 2 & 1 & 1 & 15 \\
\hline Maine & - & - & - & 1 & - & - & - & - & 11 & - & 12 \\
\hline Maryland & 12 & 5 & 13 & 6 & 10 & 7 & 8 & 7 & - & 7 & 75 \\
\hline Massachusetts & 2 & 2 & 2 & - & - & - & - & - & 1 & - & 7 \\
\hline Michigan & 21 & 15 & 10 & 11 & 11 & 6 & 10 & 9 & 7 & 6 & 106 \\
\hline Minnesota & - & - & - & - & - & - & - & - & - & - & - \\
\hline Mississippi & 2 & 3 & 1 & 1 & 2 & 6 & 1 & 7 & 6 & 6 & 35 \\
\hline Missouri & 4 & 6 & 11 & 7 & 5 & 3 & 1 & 2 & 2 & 3 & 44 \\
\hline Montana & - & - & - & - & 1 & 3 & 2 & 2 & - & - & 8 \\
\hline Nebraska & - & - & - & - & - & - & - & 1 & 1 & - & 2 \\
\hline Nevada & 1 & 1 & 1 & 1 & 1 & - & 1 & - & - & 1 & 7 \\
\hline New Hampshire & - & - & - & 1 & - & - & - & 1 & - & - & 2 \\
\hline New Jersey & 11 & 8 & 14 & 7 & 3 & 8 & 8 & 4 & 5 & 4 & 72 \\
\hline New Mexico & 3 & 3 & 4 & 4 & 4 & 5 & 7 & 5 & 5 & 4 & 44 \\
\hline New York & 9 & 4 & 2 & 9 & 11 & 1 & 4 & 4 & 2 & 6 & 52 \\
\hline North Carolina & 12 & 13 & 10 & 7 & 8 & 15 & 9 & 4 & 6 & 9 & 93 \\
\hline North Dakota & 1 & - & - & - & 1 & - & - & 1 & - & - & 3 \\
\hline Ohio & 79 & 83 & 74 & 64 & 57 & 71 & 64 & 41 & 52 & 31 & 616 \\
\hline Oklahoma & 5 & 4 & 1 & 4 & 3 & 4 & 1 & 5 & 2 & 2 & 31 \\
\hline Oregon & 1 & 1 & 1 & 2 & - & 1 & 1 & 1 & 1 & 1 & 10 \\
\hline Pennsylvania & 1,030 & 961 & 836 & 730 & 681 & 592 & 622 & 550 & 464 & 409 & 6,875 \\
\hline Rhode Island & - & - & - & 1 & - & - & 1 & - & - & - & 2 \\
\hline South Carolina & 1 & - & 5 & 3 & 5 & 4 & 3 & 1 & 5 & 2 & 29 \\
\hline South Dakota & - & - & - & - & - & - & - & 1 & - & - & 1 \\
\hline Tennessee & 37 & 23 & 30 & 25 & 28 & 30 & 25 & 19 & 23 & 26 & 266 \\
\hline Texas & 5 & 7 & 9 & 4 & 6 & 2 & 8 & 6 & 2 & 7 & 56 \\
\hline Utah & 18 & 12 & 13 & 12 & 16 & 13 & 6 & 8 & 9 & 5 & 112 \\
\hline Vermont & - & 1 & - & - & - & 1 & - & 129 & - & - & 131 \\
\hline Virginia & 131 & 134 & 147 & 151 & 100 & 118 & 124 & - & 82 & 86 & 1,073 \\
\hline Washington & 6 & 1 & 1 & 6 & 4 & 3 & 5 & 6 & 4 & - & 36 \\
\hline West Virginia & 279 & 308 & 283 & 287 & 289 & 291 & 228 & 245 & 207 & 196 & 2,613 \\
\hline Wisconsin & 3 & 1 & - & 1 & 1 & 1 & - & 3 & 1 & 1 & 12 \\
\hline Wyoming & 4 & 7 & 6 & 3 & 11 & 3 & 2 & 2 & 1 & 2 & 41 \\
\hline TOTAL & 1,990 & 1,938 & 1,766 & 1,631 & 1,478 & 1,413 & 1,417 & 1,297 & 1,103 & 1,003 & 15,036 \\
\hline
\end{tabular}

- indicates no deaths listed.

NOTE: See appendices for source description, methods, and ICD codes.

SOURCE: National Center for Health Statistics multiple cause of death data. 
Table 2-5. Coal workers' pneumoconiosis: Number of deaths, mortality rates (per million population), and years of potential life lost (YPLL) by state, U.S. residents age 15 and over, 1990-1999

\begin{tabular}{|c|c|c|c|c|c|c|c|c|c|c|}
\hline \multirow[b]{2}{*}{ State } & \multirow{2}{*}{$\begin{array}{l}\text { No. of } \\
\text { Deaths }\end{array}$} & \multirow[b]{2}{*}{ Rank } & \multicolumn{2}{|c|}{ Crude Mortality } & \multicolumn{2}{|c|}{ Age-Adjusted Mortality } & \multicolumn{4}{|c|}{ YPLL to Life Expectancy } \\
\hline & & & Rate & Rank & Rate & Rank & Total & Rank & YPLL/death & Rank \\
\hline Alabama & 185 & 11 & 5.58 & 9 & 5.75 & 9 & 1,756 & 11 & 9.5 & 31 \\
\hline Alaska & 3 & 41 & 0.69 & 32 & 2.59 & 16 & 23 & 42 & 7.7 & 44 \\
\hline Arizona & 41 & 24 & 1.26 & 23 & 1.35 & 23 & 363 & 26 & 8.9 & 34 \\
\hline Arkansas & 54 & 19 & 2.79 & 15 & 2.39 & 17 & 487 & 21 & 9.0 & 32 \\
\hline California & 200 & 9 & 0.82 & 31 & 0.97 & 30 & 3,063 & 7 & 15.3 & 5 \\
\hline Colorado & 118 & 12 & 4.07 & 11 & 5.34 & 10 & 1,009 & 14 & 8.5 & 38 \\
\hline Connecticut & 17 & 32 & 0.65 & 33 & 0.63 & 35 & 142 & 33 & 8.3 & 41 \\
\hline Delaware & 16 & 33 & 2.83 & 14 & 3.25 & 14 & 142 & 33 & 8.9 & 34 \\
\hline District of Columbia & 1 & 47 & 0.22 & 44 & 0.24 & 44 & 8 & 48 & 8.3 & 41 \\
\hline Florida & 223 & 8 & 1.94 & 18 & 1.50 & 22 & 2,312 & 9 & 10.4 & 18 \\
\hline Georgia & 33 & 28 & 0.59 & 35 & 0.76 & 34 & 357 & 27 & 10.8 & 16 \\
\hline Hawaii & - & - & - & - & - & - & - & - & - & - \\
\hline Idaho & 8 & 37 & 0.93 & 29 & 1.00 & 29 & 99 & 37 & 12.3 & 9 \\
\hline Illinois & 358 & 6 & 3.89 & 12 & 4.01 & 13 & 3,711 & 6 & 10.4 & 18 \\
\hline Indiana & 193 & 10 & 4.28 & 10 & 4.33 & 11 & 1,931 & 10 & 10.0 & 24 \\
\hline Iowa & 48 & 21 & 2.17 & 16 & 1.68 & 20 & 405 & 24 & 8.4 & 39 \\
\hline Kansas & 27 & 31 & 1.37 & 22 & 1.29 & 24 & 418 & 23 & 15.5 & 4 \\
\hline Kentucky & 1,030 & 4 & 34.13 & 3 & 34.39 & 3 & 11,919 & 4 & 11.6 & 12 \\
\hline Louisiana & 15 & 34 & 0.46 & 37 & 0.53 & 36 & 263 & 32 & 17.6 & 3 \\
\hline Maine & 1 & 47 & 0.10 & 49 & 0.10 & 49 & 8 & 48 & 8.3 & 41 \\
\hline Maryland & 86 & 16 & 2.16 & 17 & 2.79 & 15 & 842 & 17 & 9.8 & 25 \\
\hline Massachusetts & 7 & 39 & 0.14 & 48 & 0.14 & 47 & 95 & 38 & 13.5 & 6 \\
\hline Michigan & 106 & 14 & 1.43 & 21 & 1.56 & 21 & 1,131 & 12 & 10.7 & 17 \\
\hline Minnesota & - & - & - & - & - & - & - & - & - & - \\
\hline Mississippi & 35 & 27 & 1.72 & 19 & 1.80 & 19 & 914 & 16 & 26.1 & 2 \\
\hline Missouri & 44 & 22 & 1.06 & 27 & 0.95 & 31 & 459 & 22 & 10.4 & 18 \\
\hline Montana & 8 & 37 & 1.20 & 25 & 1.17 & 26 & 58 & 40 & 7.3 & 47 \\
\hline Nebraska & 2 & 43 & 0.16 & 47 & 0.13 & 48 & 15 & 45 & 7.4 & 46 \\
\hline Nevada & 7 & 39 & 0.58 & 36 & 0.82 & 33 & 68 & 39 & 9.7 & 27 \\
\hline New Hampshire & 2 & 43 & 0.22 & 44 & 0.25 & 43 & 17 & 44 & 8.4 & 39 \\
\hline New Jersey & 72 & 17 & 1.13 & 26 & 1.19 & 25 & 702 & 18 & 9.8 & 25 \\
\hline New Mexico & 44 & 22 & 3.49 & 13 & 4.23 & 12 & 384 & 25 & 8.7 & 36 \\
\hline New York & 52 & 20 & 0.36 & 41 & 0.37 & 40 & 537 & 20 & 10.3 & 21 \\
\hline North Carolina & 93 & 15 & 1.62 & 20 & 1.83 & 18 & 951 & 15 & 10.2 & 22 \\
\hline North Dakota & 3 & 41 & 0.61 & 34 & 0.50 & 37 & 23 & 42 & 7.6 & 45 \\
\hline Ohio & 616 & 5 & 7.08 & 7 & 7.13 & 7 & 5,968 & 5 & 9.7 & 27 \\
\hline Oklahoma & 31 & 29 & 1.22 & 24 & 1.16 & 27 & 355 & 28 & 11.5 & 13 \\
\hline Oregon & 10 & 36 & 0.41 & 39 & 0.37 & 40 & 135 & 36 & 13.5 & 6 \\
\hline Pennsylvania & 6,875 & 1 & 71.22 & 2 & 62.73 & 2 & 66,262 & 1 & 9.6 & 29 \\
\hline Rhode Island & 2 & 43 & 0.25 & 43 & 0.23 & 45 & 14 & 47 & 7.2 & 49 \\
\hline South Carolina & 29 & 30 & 1.02 & 28 & 1.14 & 28 & 341 & 30 & 11.8 & 10 \\
\hline South Dakota & 1 & 47 & 0.18 & 46 & 0.19 & 46 & 39 & 41 & 38.7 & 1 \\
\hline Tennessee & 266 & 7 & 6.41 & 8 & 6.65 & 8 & 2,677 & 8 & 10.1 & 23 \\
\hline Texas & 56 & 18 & 0.40 & 40 & 0.49 & 38 & 693 & 19 & 12.4 & 8 \\
\hline Utah & 112 & 13 & 8.37 & 6 & 10.74 & 6 & 1,073 & 13 & 9.6 & 29 \\
\hline Vermont & 2 & 43 & 0.44 & 38 & 0.46 & 39 & 15 & 45 & 7.3 & 47 \\
\hline Virginia & 1,202 & 3 & 22.96 & 4 & 28.26 & 4 & 14,223 & 3 & 11.8 & 10 \\
\hline Washington & 36 & 26 & 0.86 & 30 & 0.94 & 32 & 323 & 31 & 9.0 & 32 \\
\hline West Virginia & 2,613 & 2 & 179.64 & 1 & 157.33 & 1 & 29,547 & 2 & 11.3 & 14 \\
\hline Wisconsin & 12 & 35 & 0.30 & 42 & 0.29 & 42 & 136 & 35 & 11.3 & 14 \\
\hline Wyoming & 41 & 24 & 11.45 & 5 & 13.91 & 5 & 351 & 29 & 8.6 & 37 \\
\hline
\end{tabular}

- indicates no deaths listed.

NOTE: See appendices for source description, methods, and ICD codes.

SOURCE: National Center for Health Statistics multiple cause of death data. Population estimates from U.S. Bureau of the Census. 
Table 2-6. Coal workers' pneumoconiosis: Most frequently recorded industries on death certificate, U.S. residents age 15 and over, selected states and years, 1990-1999

\begin{tabular}{llrc}
\hline CIC & Industry & Number of Deaths & Percent \\
\hline 041 & Coal mining & 3,765 & 77.0 \\
060 & Construction & 188 & 3.8 \\
270 & Blast furnaces, steelworks, rolling and finishing mills & 56 & 1.1 \\
392 & Not specified manufacturing industries & 48 & 1.0 \\
400 & Railroads & 39 & 0.8 \\
010 & Agricultural production, crops & 35 & 0.7 \\
351 & Motor vehicles and motor vehicle equipment & 30 & 0.6 \\
410 & Trucking service & 29 & 0.6 \\
040 & Metal mining & 25 & 0.5 \\
961 & Non-paid worker or non-worker or own home/at home & 24 & 0.5 \\
& All other industries & 461 & 9.4 \\
& Industry not reported & 193 & 3.9 \\
& TOTAL & $\mathbf{4 , 8 9 3}$ & $\mathbf{1 0 0 . 0}$ \\
\hline
\end{tabular}

CIC - Census Industry Code

n.e.c. - not elsewhere classified

NOTE: Percentages may not total to $100 \%$ due to rounding. See appendices for source description, methods, and ICD codes, industry and occupation codes, and list of selected states and years.

SOURCE: National Center for Health Statistics multiple cause of death data.

Table 2-7. Coal workers' pneumoconiosis: Most frequently recorded occupations on death certificate, U.S. residents age 15 and over, selected states and years, 1990-1999

\begin{tabular}{clrc}
\hline COC & Occupation & Number of Deaths & Percent \\
\hline 616 & Mining machine operators & 3,440 & 70.3 \\
889 & Laborers, except construction & 147 & 3.0 \\
575 & Electricians & 64 & 1.3 \\
804 & Truck drivers & 62 & 1.3 \\
019 & Managers and administrators, n.e.c. & 59 & 1.2 \\
613 & Supervisors, extractive occupations & 49 & 1.0 \\
567 & Carpenters & 48 & 1.0 \\
453 & Janitors and cleaners & 47 & 1.0 \\
869 & Construction laborers & 45 & 0.9 \\
473 & Farmers, except horticulture & 39 & 0.8 \\
844 & Operating engineers & 39 & 0.8 \\
& All other occupations & 655 & 13.4 \\
& Occupation not reported & 199 & 4.1 \\
& TOTAL & $\mathbf{4 , 8 9 3}$ & $\mathbf{1 0 0 . 0}$ \\
\hline
\end{tabular}


Table 2-8. Coal workers' pneumoconiosis: Proportionate mortality ratio (PMR) adjusted for age, sex, and race by usual industry, U.S. residents age 15 and over, selected states and years, 1990-1999

\begin{tabular}{llrrrr}
\hline & & \multicolumn{2}{c}{$\begin{array}{c}\text { Number } \\
\text { CIC }\end{array}$} & Industry & \multicolumn{2}{c}{ 95\% Confidence Interval } \\
\cline { 5 - 6 } of Deaths & PMR & LCL & UCL \\
\hline 041 & Coal mining & 3,765 & 53.18 & 51.50 & 54.91 \\
040 & Metal mining & 25 & 1.98 & 1.28 & 2.92 \\
\hline
\end{tabular}

CIC - Census Industry Code n.e.c. - not elsewhere classified LCL - lower confidence limit UCL - upper confidence limit
NOTE: See appendices for source description, methods, and ICD codes, industry and occupation codes, and list of selected states and years.

SOURCE: National Center for Health Statistics multiple cause of death data. 
Table 2-9. Coal workers' pneumoconiosis: Proportionate mortality ratio (PMR) adjusted for age, sex, and race by usual occupation, U.S. residents age 15 and over, selected states and years, 1990-1999

\begin{tabular}{|c|c|c|c|c|c|}
\hline \multirow[b]{2}{*}{$\mathrm{COC}$} & \multirow[b]{2}{*}{ Occupation } & \multirow{2}{*}{$\begin{array}{c}\text { Number } \\
\text { of Deaths }\end{array}$} & \multirow[b]{2}{*}{ PMR } & \multicolumn{2}{|c|}{ 95\% Confidence Interval } \\
\hline & & & & LCL & UCL \\
\hline 616 & Mining machine operators & 3,440 & 51.67 & 49.97 & 53.44 \\
\hline 613 & Supervisors, extractive occupations & 49 & 14.36 & 10.64 & 18.99 \\
\hline 046 & Mining engineers & 8 & 6.03 & 2.60 & 11.86 \\
\hline 617 & Mining occupations, n.e.c. & 14 & 4.45 & 2.43 & 7.46 \\
\hline 859 & Miscellaneous material moving equipment operators & 12 & 2.27 & 1.17 & 3.96 \\
\hline 824 & Locomotive operating occupations & 23 & 2.03 & 1.29 & 3.05 \\
\hline
\end{tabular}

COC - Census Occupation Code n.e.c. - not elsewhere classified LCL - lower confidence limit UCL - upper confidence limit NOTE: See appendices for source description, methods, and ICD codes, industry and occupation codes, and list of selected states and years. SOURCE: National Center for Health Statistics multiple cause of death data. 
ఒ Figure 2-3. Coal workers' pneumoconiosis: Age-adjusted mortality rates by county, U.S. residents age 15 and over, 1970-1999

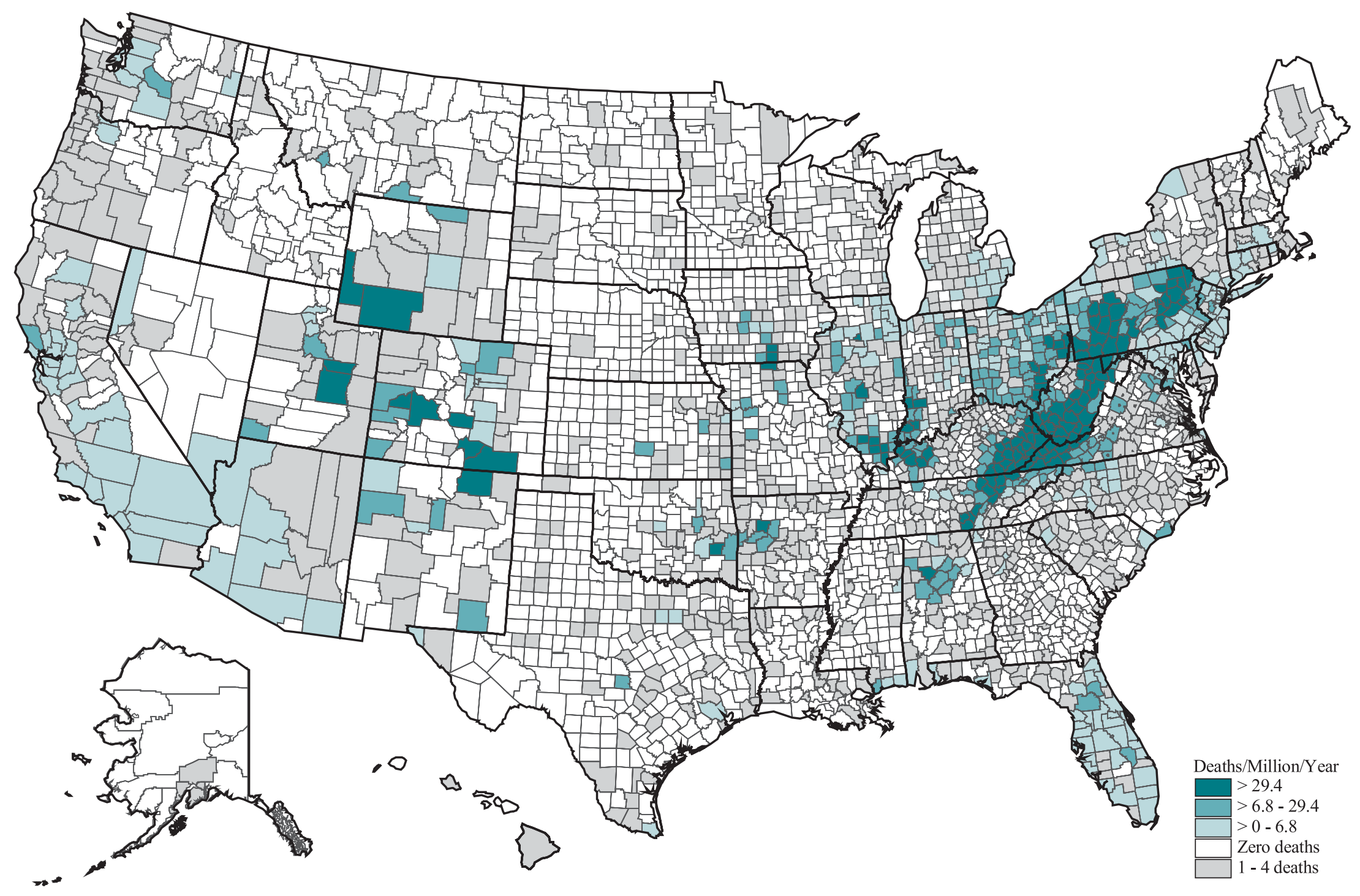

NOTE: Age-adjusted rates are not calculated for those counties with 1-4 deaths. See appendices for source description, methods, and ICD codes. SOURCE: National Center for Health Statistics multiple cause of death data. Population estimates from U.S. Bureau of the Census. 
Figure 2-4. Coal workers' pneumoconiosis: Age-adjusted mortality rates by county, U.S. residents age 15 and over, 1970-1984 and 1985-1999

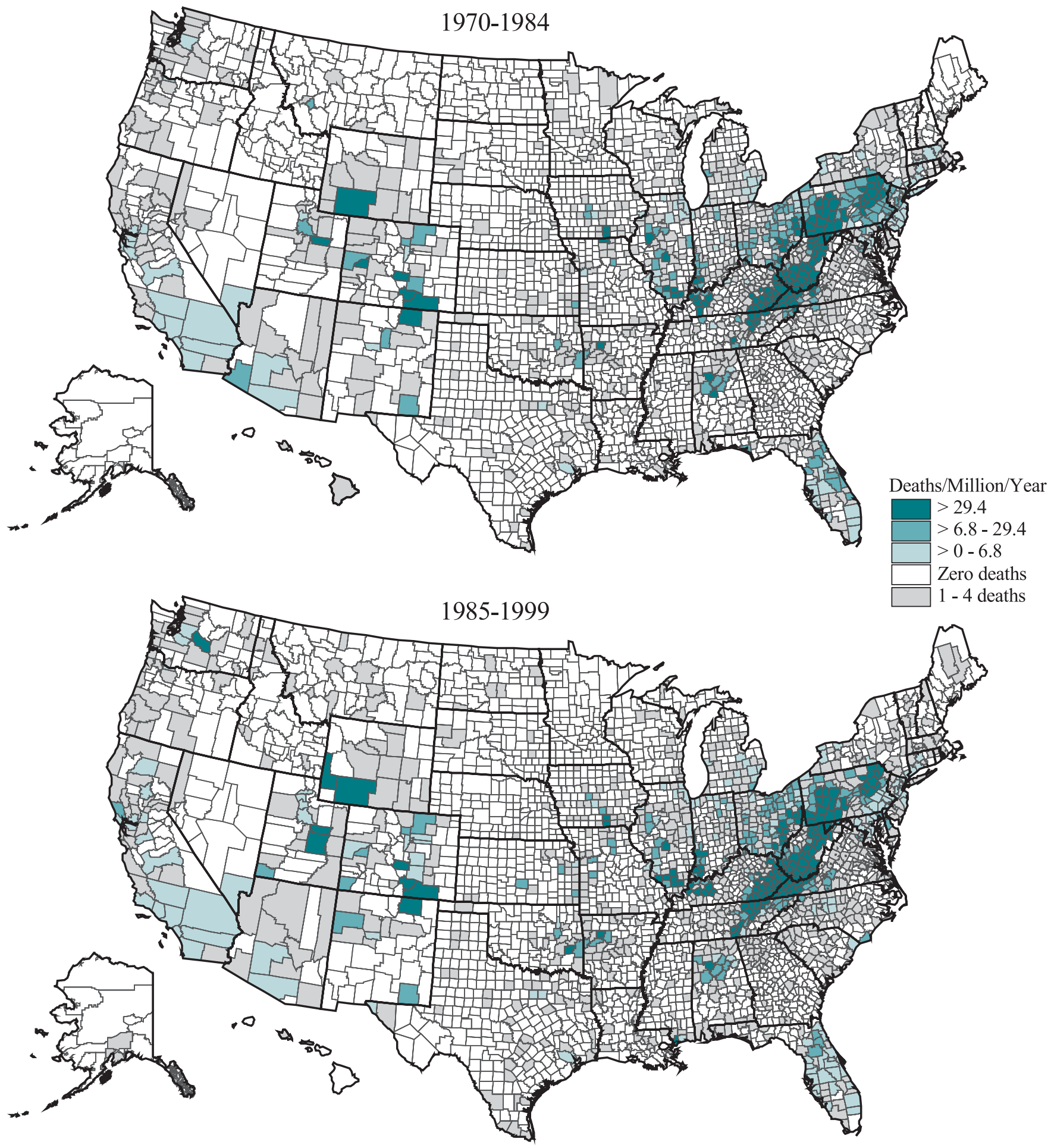

NOTE: Age-adjusted rates are not calculated for those counties with 1-4 deaths. See appendices for source description, methods, and ICD codes. SOURCE: National Center for Health Statistics multiple cause of death data. Population estimates from U.S. Bureau of the Census. 
Table 2-10. Coal workers' pneumoconiosis: Counties with highest age-adjusted mortality rates (per million population), U.S. residents age 15 and over, 1985-1999

\begin{tabular}{|c|c|c|c|c|c|}
\hline County & State & Age-Adjusted Rate & Crude Rate & Number of Deaths & \% Female \\
\hline Buchanan County & Virginia & $1,659.8$ & $1,170.4$ & 430 & 0.2 \\
\hline Schuylkill County & Pennsylvania & $1,042.4$ & $1,454.2$ & 2,744 & 0.1 \\
\hline McDowell County & West Virginia & $1,019.1$ & $1,141.7$ & 447 & 0.0 \\
\hline Raleigh County & West Virginia & 999.2 & $1,134.7$ & 1,035 & 0.0 \\
\hline Wyoming County & West Virginia & 922.0 & 769.3 & 258 & 0.0 \\
\hline Floyd County & Kentucky & 890.2 & 799.2 & 401 & 0.0 \\
\hline Tazewell County & Virginia & 741.0 & 763.6 & 426 & 0.2 \\
\hline Wise County & Virginia & 739.4 & 746.4 & 349 & 0.3 \\
\hline Dickenson County & Virginia & 716.8 & 703.8 & 146 & 0.7 \\
\hline Norton City & Virginia & 660.8 & 655.7 & 33 & 0.0 \\
\hline Luzerne County & Pennsylvania & 652.4 & 916.8 & 3,718 & 0.3 \\
\hline Boone County & West Virginia & 581.3 & 557.2 & 173 & 0.0 \\
\hline Fayette County & West Virginia & 536.8 & 677.8 & 388 & 0.0 \\
\hline Letcher County & Kentucky & 524.8 & 492.4 & 152 & 0.7 \\
\hline Logan County & West Virginia & 514.9 & 495.4 & 247 & 0.4 \\
\hline Harlan County & Kentucky & 477.8 & 471.6 & 196 & 0.0 \\
\hline Russell County & Virginia & 468.3 & 442.0 & 152 & 0.0 \\
\hline Northumberland County & Pennsylvania & 458.2 & 640.6 & 744 & 0.1 \\
\hline Carbon County & Utah & 408.8 & 449.4 & 97 & 0.0 \\
\hline Cambria County & Pennsylvania & 388.3 & 512.4 & 1,008 & 0.1 \\
\hline Knott County & Kentucky & 370.5 & 299.6 & 62 & 0.0 \\
\hline Somerset County & Pennsylvania & 360.0 & 444.3 & 415 & 0.0 \\
\hline Lee County & Virginia & 352.6 & 413.3 & 119 & 0.8 \\
\hline Mingo County & West Virginia & 350.5 & 290.5 & 109 & 0.0 \\
\hline Webster County & West Virginia & 341.7 & 409.2 & 50 & 0.0 \\
\hline Carbon County & Pennsylvania & 332.6 & 437.4 & 308 & 0.3 \\
\hline Bell County & Kentucky & 323.9 & 320.0 & 115 & 0.0 \\
\hline Lackawanna County & Pennsylvania & 313.8 & 439.7 & 1,173 & 0.3 \\
\hline Fayette County & Pennsylvania & 307.1 & 391.7 & 686 & 0.0 \\
\hline Nicholas County & West Virginia & 300.2 & 322.6 & 101 & 1.0 \\
\hline Mercer County & West Virginia & 288.3 & 353.4 & 277 & 0.4 \\
\hline Johnson County & Kentucky & 287.1 & 240.9 & 66 & 0.0 \\
\hline Emery County & Utah & 284.9 & 226.5 & 22 & 0.0 \\
\hline Pike County & Kentucky & 253.1 & 209.2 & 177 & 0.6 \\
\hline Greene County & Pennsylvania & 244.2 & 306.8 & 144 & 0.0 \\
\hline Franklin County & Illinois & 241.0 & 347.9 & 168 & 0.0 \\
\hline Muhlenberg County & Kentucky & 213.3 & 241.4 & 89 & 0.0 \\
\hline Knox County & Kentucky & 203.6 & 206.8 & 72 & 1.4 \\
\hline Leslie County & Kentucky & 201.0 & 162.6 & 25 & 0.0 \\
\hline Campbell County & Tennessee & 198.1 & 213.1 & 90 & 0.0 \\
\hline Indiana County & Pennsylvania & 194.2 & 196.6 & 214 & 0.0 \\
\hline Martin County & Kentucky & 193.8 & 149.2 & 21 & 0.0 \\
\hline Perry County & Kentucky & 187.1 & 157.9 & 56 & 0.0 \\
\hline Greenbrier County & West Virginia & 184.9 & 237.4 & 101 & 0.0 \\
\hline Clay County & West Virginia & 180.9 & 194.9 & 22 & 0.0 \\
\hline Preston County & West Virginia & 176.3 & 196.2 & 67 & 0.0 \\
\hline Marion County & West Virginia & 155.9 & 207.6 & 146 & 0.0 \\
\hline Whitley County & Kentucky & 155.4 & 156.8 & 62 & 0.0 \\
\hline Grundy County & Tennessee & 142.8 & 154.1 & 24 & 0.0 \\
\hline Sweetwater County & Wyoming & 137.2 & 79.0 & 34 & 0.0 \\
\hline Overall United States & & 9.4 & 8.9 & 26,706 & 0.7 \\
\hline
\end{tabular}

NOTE: Only counties with at least 5 deaths from the disease of interest are included. See appendices for source description, methods, and ICD codes. SOURCE: National Center for Health Statistics multiple cause of death data. Population estimates from U.S. Bureau of the Census. 


\section{Table 2-11. Coal workers' pneumoconiosis: Estimated number of discharges from} short-stay nonfederal hospitals, 1970-2000

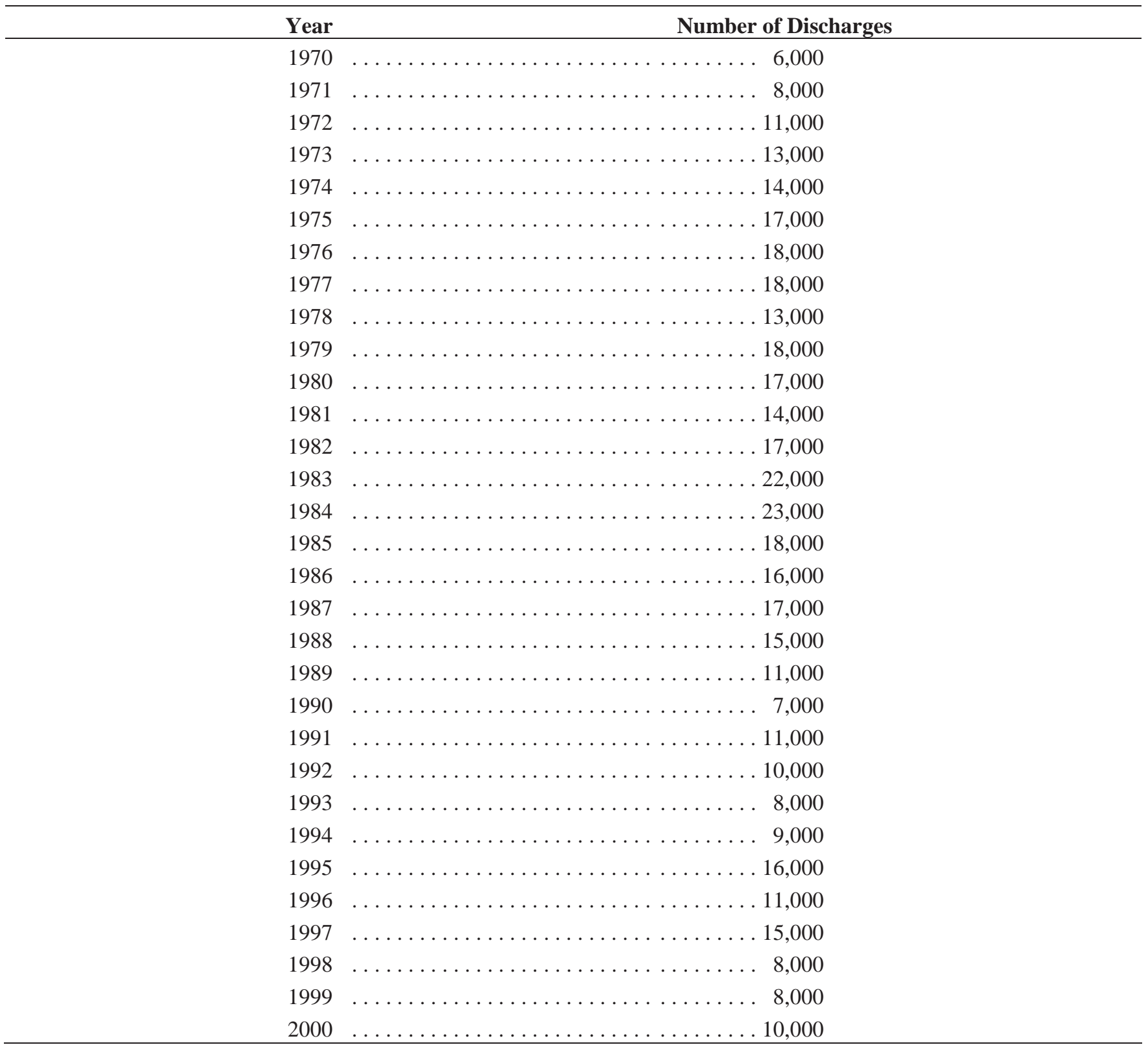

NOTE: Number of discharges has been rounded. NCHS recommends that, in statistical comparisons, estimates of less than 5,000 not be used and that estimates of 5,000 to 10,000 be used with caution. See appendices for source description and methods.

SOURCE: National Center for Health Statistics National Hospital Discharge Survey. 
Figure 2-5. CWXSP: Percentage of examined miners with coal workers' pneumoconiosis (category 1/0+) by tenure in mining, 1970-1999

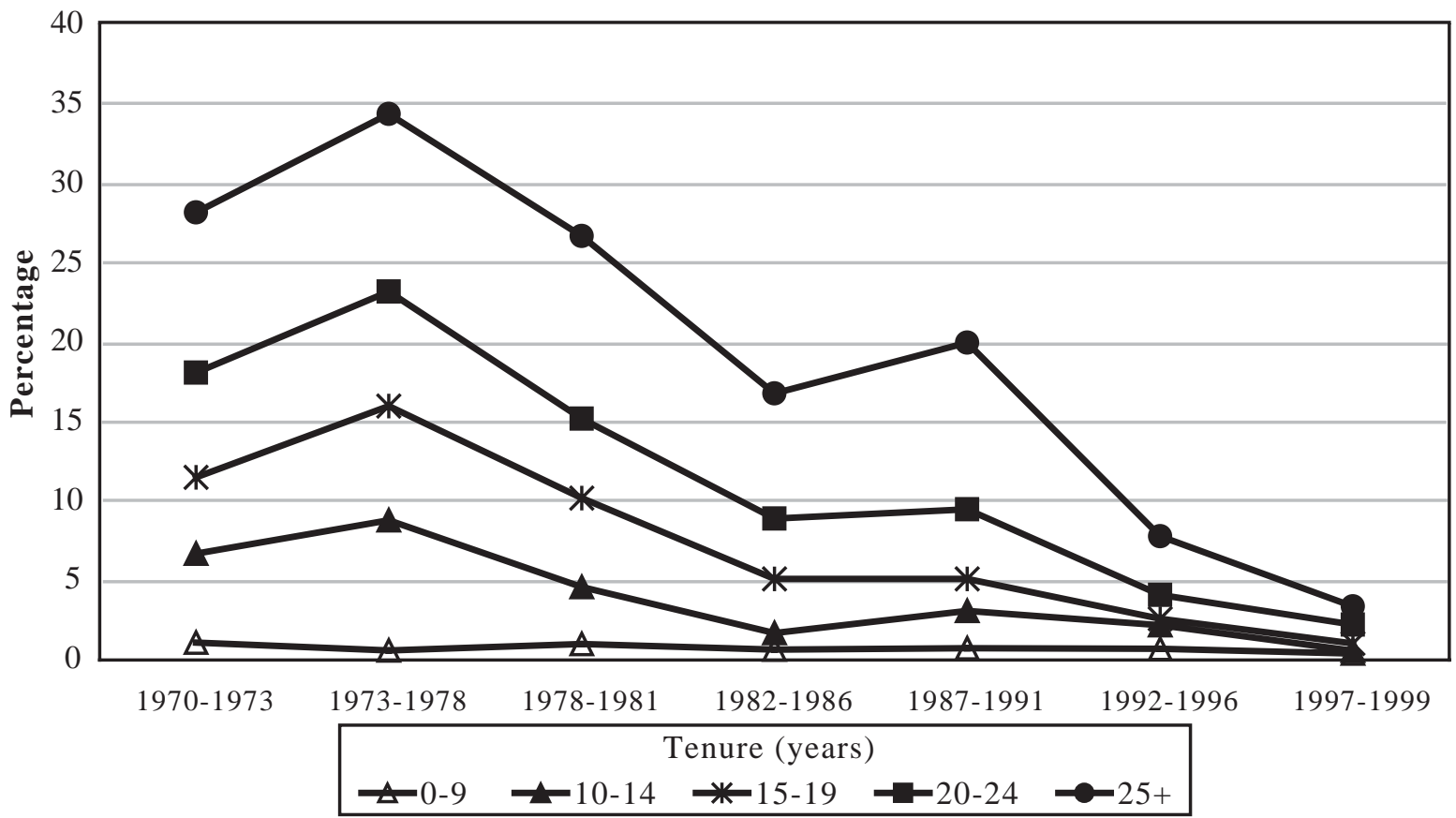

NOTE: 1997-1999 represents a partial round. See appendices for source description. SOURCE: NIOSH Coal Workers' X-ray Surveillance Program.

Figure 2-6. CWXSP: Estimated number of actively employed underground coal miners and number examined, 1970-1999

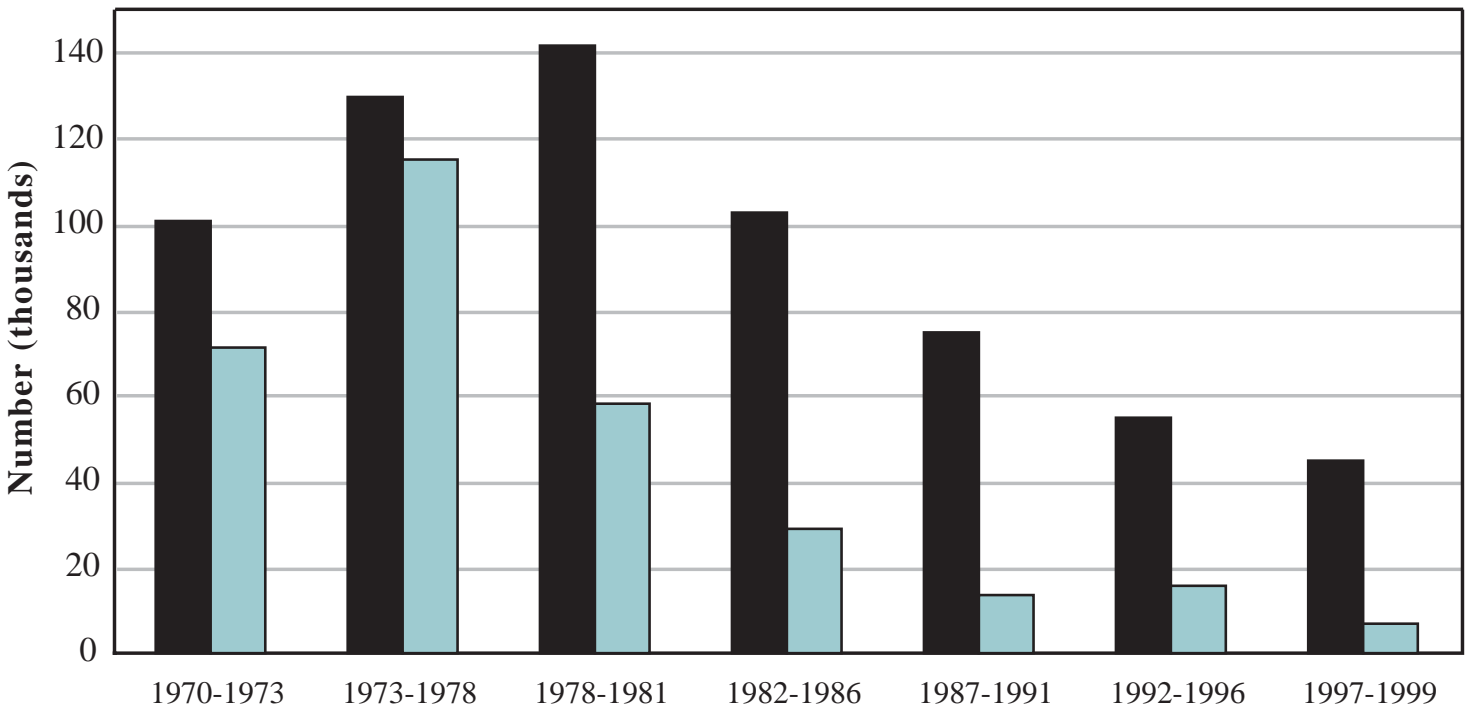

number employed $\quad$ Number x-rayed

NOTE: 1997-1999 represents a partial round. See appendices for source description.

SOURCE: NIOSH Coal Workers' X-ray Surveillance Program. Mine Safety and Health Administration (MSHA) coal mine employment data 
Table 2-12. CWXSP: Number and percentage of examined miners with coal workers' pneumoconiosis (category 1/0+), by round and tenure, 1970-1999

\begin{tabular}{|c|c|c|c|c|c|c|c|c|c|c|c|c|c|c|c|c|c|c|c|c|c|}
\hline \multirow{3}{*}{$\begin{array}{c}\text { Tenure } \\
\text { Years } \\
\text { in } \\
\text { Mining }\end{array}$} & \multicolumn{3}{|c|}{$\begin{array}{c}\text { Round 1 } \\
1970-1973\end{array}$} & \multicolumn{3}{|c|}{$\begin{array}{c}\text { Round 2 } \\
1973-1978\end{array}$} & \multicolumn{3}{|c|}{$\begin{array}{c}\text { Round 3 } \\
\text { 1978-1981 }\end{array}$} & \multicolumn{3}{|c|}{$\begin{array}{c}\text { Round } 4 \\
1982-1986\end{array}$} & \multicolumn{3}{|c|}{$\begin{array}{c}\text { Round 5 } \\
1987-1991 \\
\end{array}$} & \multicolumn{3}{|c|}{$\begin{array}{c}\text { Round } 6 \\
1992-1996 \\
\end{array}$} & \multicolumn{3}{|c|}{$\begin{array}{c}\text { Round } 7 \\
1997-1999\end{array}$} \\
\hline & \multirow{2}{*}{\begin{tabular}{|c|} 
Miners \\
examined \\
No. \\
\end{tabular}} & \multicolumn{2}{|c|}{ Cat. 1/0+ } & \multirow{2}{*}{\begin{tabular}{|c|} 
Miners \\
examined \\
No. \\
\end{tabular}} & \multicolumn{2}{|c|}{ Cat. 1/0+ } & \multirow{2}{*}{\begin{tabular}{|c|} 
Miners \\
examined \\
No.
\end{tabular}} & \multicolumn{2}{|c|}{ Cat. 1/0+ } & \multirow{2}{*}{$\begin{array}{c}\text { Miners } \\
\text { examined } \\
\text { No. }\end{array}$} & \multicolumn{2}{|c|}{ Cat. 1/0+ } & \multirow{2}{*}{\begin{tabular}{|c|} 
Miners \\
examined \\
No. \\
\end{tabular}} & \multicolumn{2}{|c|}{ Cat. 1/0+ } & \multirow{2}{*}{$\begin{array}{c}\text { Miners } \\
\text { examined } \\
\text { No. }\end{array}$} & \multicolumn{2}{|c|}{ Cat. 1/0+ } & \multirow{2}{*}{\begin{tabular}{|c|} 
Miners \\
examined \\
No.
\end{tabular}} & \multicolumn{2}{|c|}{ Cat. $1 / 0+$} \\
\hline & & No. & $\%$ & & No. & $\%$ & & No. & $\%$ & & No. & $\%$ & & No. & $\%$ & & No. & $\%$ & & No. & $\%$ \\
\hline 0 & 15,844 & 100 & 0.6 & 50,341 & 31 & 0.1 & 14,528 & 94 & 0.6 & 3,577 & 18 & 0.5 & 2,007 & 10 & 0.5 & 1,812 & 13 & 0.7 & 969 & 6 & 0.6 \\
\hline 1 & 5,287 & 49 & 0.9 & 9,579 & 13 & 0.1 & 3,719 & 18 & 0.5 & 742 & 1 & 0.1 & 356 & 0 & 0.0 & 238 & 2 & 0.8 & 163 & 0 & 0.0 \\
\hline $2-4$ & 8,274 & 73 & 0.9 & 18,432 & 137 & 0.7 & 12,059 & 103 & 0.8 & 3,786 & 25 & 0.7 & 1,057 & 6 & 0.6 & 791 & 2 & 0.3 & 388 & 2 & 0.5 \\
\hline $5-9$ & 6,706 & 182 & 2.7 & 13,528 & 386 & 2.8 & 14,157 & 215 & 1.5 & 7,434 & 57 & 0.8 & 2,763 & 30 & 1.1 & 1,235 & 12 & 1.0 & 418 & 0 & 0.0 \\
\hline $10-14$ & 4,451 & 298 & 6.7 & 5,282 & 466 & 8.8 & 5,318 & 246 & 4.6 & 5,435 & 93 & 1.7 & 4,120 & 123 & 3.0 & 2,522 & 56 & 2.2 & 511 & 3 & 0.6 \\
\hline $15-19$ & 4,743 & 546 & 11.5 & 3,380 & 542 & 16.0 & 2,168 & 221 & 10.2 & 1,824 & 93 & 5.1 & 2,279 & 114 & 5.0 & 4,646 & 119 & 2.6 & 1,148 & 12 & 1.0 \\
\hline 20-24 & 7,279 & 1,316 & 18.1 & 3,214 & 745 & 23.2 & 1,505 & 228 & 15.2 & 711 & 63 & 8.9 & 769 & 71 & 9.2 & 3,220 & 132 & 4.1 & 1,983 & 44 & 2.2 \\
\hline $25-29$ & 6,260 & 1,368 & 21.8 & 4,437 & 1,279 & 28.8 & 1,294 & 257 & 19.9 & 491 & 64 & 13.0 & 257 & 52 & 20.2 & 938 & 51 & 5.4 & 1,057 & 28 & 2.7 \\
\hline 30+ & 12,602 & 3,947 & 31.3 & 7,193 & 2,722 & 37.8 & 3,546 & 1,034 & 29.2 & 1,154 & 213 & 18.5 & 312 & 61 & 19.6 & 365 & 51 & 14.0 & 277 & 17 & 6.1 \\
\hline TOTAL & 71,446 & $\mathbf{7 , 8 9 7}$ & 11.0 & 115,386 & 6,321 & 5.5 & $\mathbf{5 8 , 2 9 4}$ & 2,416 & 4.1 & 25,154 & 627 & 2.5 & 13,920 & 467 & 3.4 & 15,767 & 438 & 2.8 & 6,914 & 112 & 1.6 \\
\hline
\end{tabular}

NOTE: Tabulations are based on one chest $\mathrm{x}$-ray per round for each participating miner

Round 1: Jan. 1970 - July 1973

Round 2: Aug. 1973 - July 1978

Round 3: Aug. 1978 - Dec. 1981

Round 4: Jan. 1982 - Dec. 1986

Round 5: Jan. 1987 - Dec. 1991

Round 6: Jan. 1992 - Dec. 1996

Round 7: Jan. 1997 - Dec. 1999

NOTE: 1997-1999 represents a partial round. See appendices for source description.

SOURCE: NIOSH Coal Workers' X-ray Surveillance Program. 
Table 2-13. Federal Black Lung Program: Number of beneficiaries and total payments by the Social Security Administration and Department of Labor, 1980-1999

\begin{tabular}{|c|c|c|c|c|c|c|}
\hline \multirow[b]{2}{*}{ Year } & \multicolumn{2}{|c|}{ Social Security Administration (SSA) } & \multicolumn{2}{|c|}{ Department of Labor (DOL) } & \multicolumn{2}{|c|}{ SSA and DOL } \\
\hline & Beneficiaries & $\begin{array}{l}\text { Amount } \\
\text { (dollars) }\end{array}$ & Beneficiaries & $\begin{array}{l}\text { Amount } \\
\text { (dollars) }\end{array}$ & $\begin{array}{c}\text { Total } \\
\text { Beneficiaries } \\
\end{array}$ & $\begin{array}{c}\text { Total Amount } \\
\text { (dollars) }\end{array}$ \\
\hline 1980 & 399,477 & $1,032,000,000$ & 139,073 & $813,205,000$ & 538,550 & $1,845,205,000$ \\
\hline 1981 & 376,505 & $1,081,300,000$ & 163,401 & $805,627,000$ & 539,906 & $1,886,927,000$ \\
\hline 1982 & 354,569 & $1,076,000,000$ & 173,972 & $784,085,000$ & 528,541 & $1,860,085,000$ \\
\hline 1983 & 333,358 & $1,055,800,000$ & 166,043 & $858,854,000$ & 499,401 & $1,914,654,000$ \\
\hline 1984 & 313,822 & $1,038,000,000$ & 163,166 & $873,932,000$ & 476,988 & $1,911,932,000$ \\
\hline 1985 & 294,846 & $1,025,000,000$ & 160,441 & $905,517,000$ & 455,287 & $1,930,517,000$ \\
\hline 1986 & 275,783 & $971,000,000$ & 156,892 & $629,075,000$ & 432,675 & $1,600,075,000$ \\
\hline 1987 & 258,988 & $940,000,000$ & 153,769 & $655,290,000$ & 412,757 & $1,595,290,000$ \\
\hline 1988 & 241,626 & $904,000,000$ & 150,123 & $656,689,000$ & 391,749 & $1,560,689,000$ \\
\hline 1989 & 225,764 & $882,000,000$ & 145,289 & $650,123,000$ & 371,053 & $1,532,123,000$ \\
\hline 1990 & 210,678 & $863,400,000$ & 139,854 & $626,521,000$ & 350,532 & $1,489,921,000$ \\
\hline 1991 & 196,419 & $844,400,000$ & 134,205 & $942,428,000$ & 330,624 & $1,786,828,000$ \\
\hline 1992 & 182,396 & $822,500,000$ & 128,761 & $973,636,000$ & 311,157 & $1,796,136,000$ \\
\hline 1993 & 168,365 & $794,300,000$ & 123,213 & $984,666,000$ & 291,578 & $1,778,966,000$ \\
\hline 1994 & 155,122 & $751,900,000$ & 117,569 & $994,655,000$ & 272,691 & $1,746,555,000$ \\
\hline 1995 & 143,011 & $696,700,000$ & 111,769 & $995,722,000$ & 254,780 & $1,692,422,000$ \\
\hline 1996 & 131,143 & $654,600,000$ & 105,923 & $992,128,000$ & 237,066 & $1,646,728,000$ \\
\hline 1997 & 119,233 & $614,888,000$ & 100,352 & $1,004,672,000$ & 219,585 & $1,619,560,000$ \\
\hline 1998 & 109,271 & $576,389,000$ & 94,488 & $999,822,000$ & 203,759 & $1,576,211,000$ \\
\hline 1999 & 98,977 & $541,200,000$ & 88,716 & $1,005,246,000$ & 187,693 & $1,546,446,000$ \\
\hline
\end{tabular}

NOTE: The Social Security Administration (SSA) was assigned initial responsibility for administering the Black Lung benefits program. The Department of Labor (DOL) assumed responsibility for processing and paying claims on July 1, 1973. Most claims filed prior to July 1 , 1973 remain within the jurisdiction of SSA, which also continues to be responsible for processing and paying claims filed by the survivors of these miners. The dollar amounts from the Department of Labor are for fiscal years. See appendices for source description.

SOURCE: Social Security Bulletin Annual Statistical Supplement (annual reports) and Black Lung Benefits Act Annual Report to Congress (annual reports). 
Figure 2-7. Respirable coal mine dust: Geometric mean exposures by type of mine, MSHA inspector and mine operator samples, 1979-1999

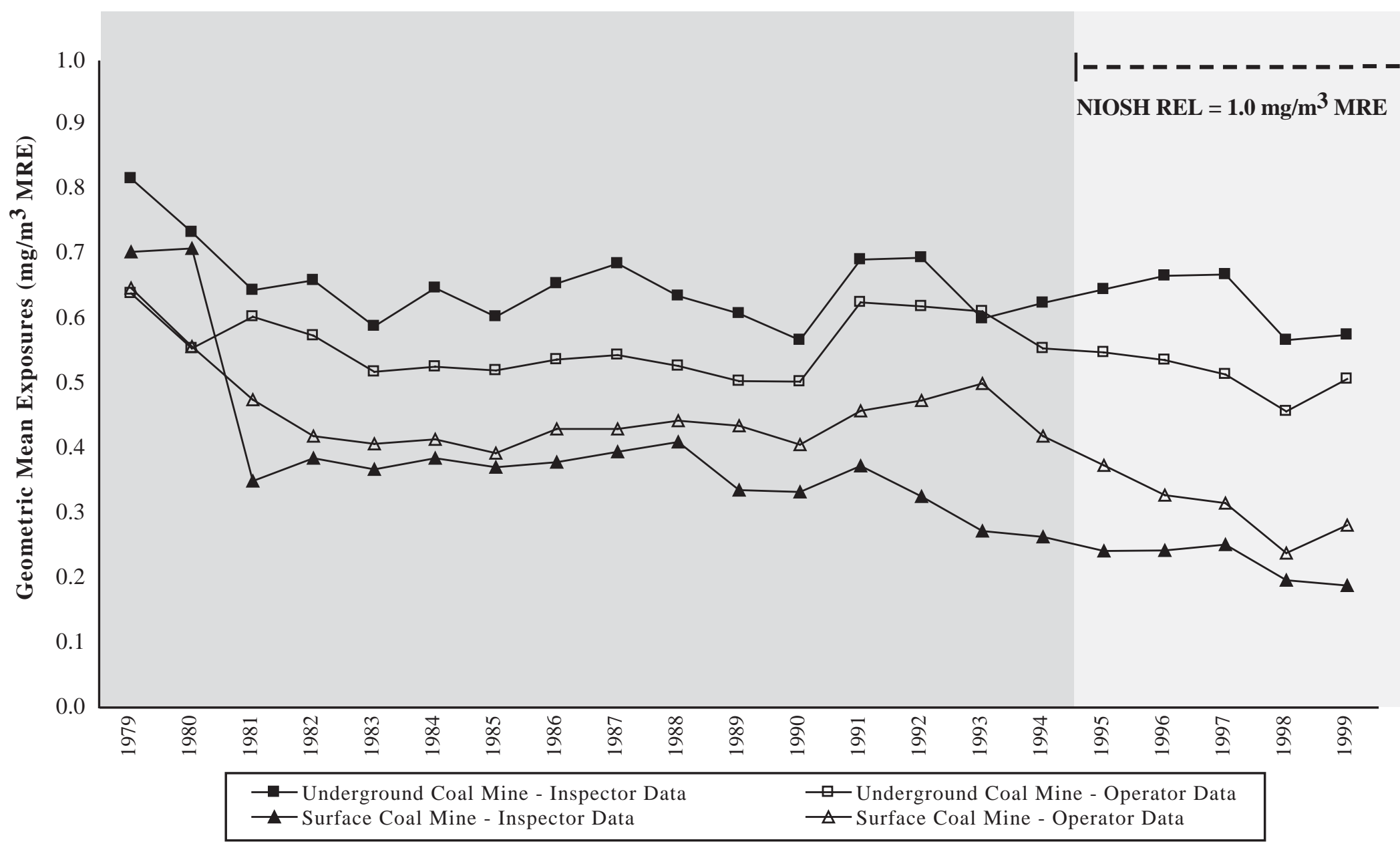

PEL - permissible exposure limit $\quad$ REL - recommended exposure limit $\quad \mathrm{mg} / \mathrm{m}^{3}$ - milligrams per cubic meter $\quad$ MRE - Mining Research Establishment

NOTE: In coal mining, for respirable dust containing less than $5 \%$ quartz, the MSHA PEL is $2 \mathrm{mg} / \mathrm{m}^{3} \mathrm{MRE}$; for respirable dust containing greater than $5 \%$ quartz, the MSHA PEL is [( 10 $\left.\mathrm{mg} / \mathrm{m}^{3} \mathrm{MRE}\right) /\left(\%\right.$ quartz)]. The NIOSH REL of $1 \mathrm{mg} / \mathrm{m}^{3}$ MRE for respirable coal mine dust was adopted in September of 1995. Geometric means are reported in MRE equivalent. See appendices for source description, methods, and agents.

SOURCE: Mine Safety and Health Administration (MSHA) coal mine inspector and mine operator dust data. 
Table 2-14. Respirable coal mine dust: Geometric mean exposures and percent exceeding designated occupational exposure limits by type of facility, MSHA inspector and mine operator samples, 1979-1999

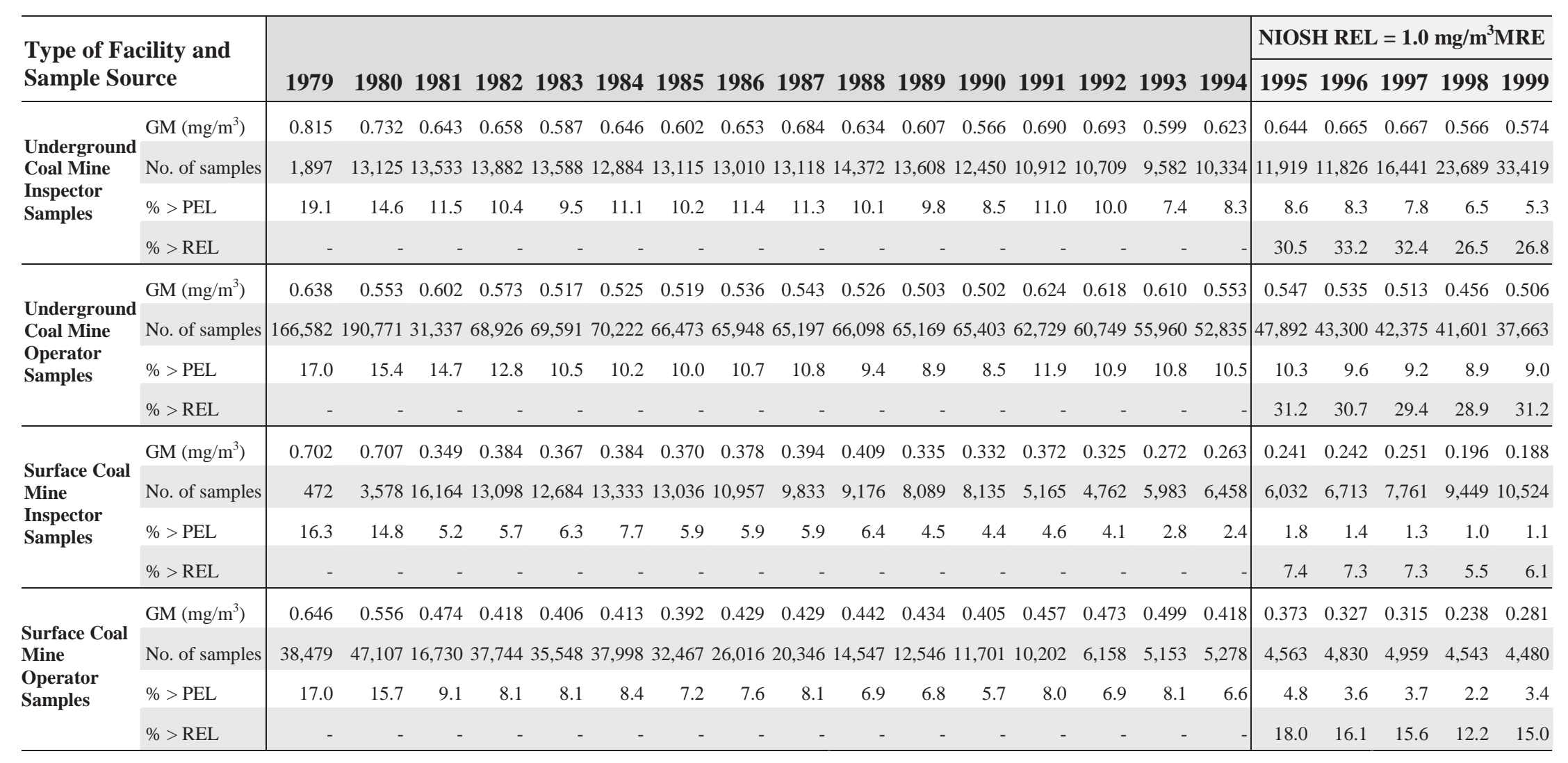

- indicates incalculable field

PEL - permissible exposure limit

MRE - Mining Research Establishment

NOTE: In coal mining, for respirable dust containing less than $5 \%$ quartz, the MSHA PEL is $2 \mathrm{mg} / \mathrm{m}^{3} \mathrm{MRE}$; for respirable dust containing greater than $5 \%$ quartz, the MSHA PEL is [(10 $\left.\mathrm{mg} / \mathrm{m}^{3} \mathrm{MRE}\right) /\left(\%\right.$ quartz)]. The NIOSH REL of $1 \mathrm{mg} / \mathrm{m}^{3}$ MRE for respirable coal mine dust was adopted in September of 1995. Geometric means are reported in MRE equivalent. All samples are compared to the MSHA PEL of $2 \mathrm{mg} / \mathrm{m}^{3}$ MRE for respirable coal mine dust containing less than $5 \%$ quartz, regardless of actual quartz content. See appendices for source description, methods, and agents.

SOURCE: Mine Safety and Health Administration (MSHA) coal mine inspector and mine operator dust data. 


\section{Table 2-15. Respirable coal mine dust: Number of samples, geometric mean} exposures, and percent exceeding designated occupational exposure limits by industries with elevated coal workers' pneumoconiosis mortality, MSHA inspector and mine operator samples, 1990-1999

\begin{tabular}{|c|c|c|c|c|c|c|c|}
\hline \multicolumn{4}{|c|}{$\begin{array}{l}\text { Coal Workers' Pneumoconiosis Mortality, } \\
\text { Selected States and Years, 1990-1999 }\end{array}$} & \multirow[b]{2}{*}{$\begin{array}{l}\text { Number of } \\
\text { Samples }\end{array}$} & \multirow[b]{2}{*}{$\begin{array}{c}\text { GM } \\
\left(\mathbf{m g} / \mathbf{m}^{3}\right)\end{array}$} & \multirow[b]{2}{*}{$\begin{array}{l}\%> \\
\text { PEL }\end{array}$} & \multirow[b]{2}{*}{$\begin{array}{c}\%> \\
\text { REL } \\
1995-1999\end{array}$} \\
\hline CIC & $\begin{array}{l}\text { Industries with elevated PMRs and most } \\
\text { frequently recorded on death certificates }\end{array}$ & $\begin{array}{l}\text { Number of } \\
\text { Deaths }\end{array}$ & PMR & & & & \\
\hline 041 & Coal mining & 3,765 & 53.18 & 794,637 & 0.509 & 8.6 & 26.2 \\
\hline & All other industries & 935 & & 0 & - & - & - \\
\hline & TOTAL & & & 794,637 & 0.509 & 8.6 & 26.2 \\
\hline
\end{tabular}

- indicates incalculable field

CIC - Census Industry Code

GM - geometric mean

PEL - permissible exposure limit

REL - recommended exposure limit

PMR - proportionate mortality ratio MRE - Mining Research Establishment $5 \%$ quartz, the MSHA PEL is $\left[\left(10 \mathrm{mg} / \mathrm{m}^{3} \mathrm{MRE}\right) /(\%\right.$ quartz $\left.)\right]$. The NIOSH REL of $1 \mathrm{mg} / \mathrm{m}^{3} \mathrm{MRE}$ for respirable coal mine dust was adopted in September of 1995. Geometric means are reported in MRE equivalent. All samples are compared to the MSHA PEL of $2 \mathrm{mg} / \mathrm{m}^{3} \mathrm{MRE}$ for respirable coal mine dust containing less than $5 \%$ quartz, regardless of actual quartz content. See appendices for source description, methods, ICD codes, industry codes, agents, and list of selected states and years for which usual industry has been reported.

SOURCE: Mine Safety and Health Administration (MSHA) coal mine inspector and mine operator dust data. National Center for Health Statistics multiple cause of death data. 
th Table 2-16 (page 1 of 3). Respirable coal mine dust: Geometric mean exposures and percent exceeding designated occupational exposure limits by MSHA coal mine district and state, MSHA inspector and mine operator samples, 1979-1999

\begin{tabular}{|c|c|c|c|c|c|c|c|c|c|c|c|c|}
\hline \multirow[b]{2}{*}{ MSHA Coal Mine District } & \multicolumn{2}{|c|}{ All years } & \multicolumn{3}{|c|}{$1979-1989$} & \multicolumn{3}{|c|}{$1990-1994$} & \multicolumn{4}{|c|}{$\begin{array}{c}1995-1999 \\
\text { NIOSH REL }=1.0 \mathrm{mg} / \mathrm{m}^{3} \text { MRE }\end{array}$} \\
\hline & $\begin{array}{c}\text { No. of } \\
\text { Samples }\end{array}$ & $\begin{array}{c}\mathbf{G M} \\
\left(\mathbf{m g} / \mathbf{m}^{3}\right)\end{array}$ & $\begin{array}{c}\text { No. of } \\
\text { Samples }\end{array}$ & $\underset{\left(\mathbf{m g} / \mathbf{m}^{3}\right)}{\mathbf{G M}}$ & $\begin{array}{l}\%> \\
\text { PEL }\end{array}$ & $\begin{array}{c}\text { No. of } \\
\text { Samples }\end{array}$ & $\begin{array}{c}\mathbf{G M} \\
\left(\mathrm{mg} / \mathbf{m}^{3}\right)\end{array}$ & $\begin{array}{l}\%> \\
\text { PEL }\end{array}$ & $\begin{array}{c}\text { No. of } \\
\text { Samples }\end{array}$ & $\underset{\left(\mathbf{m g} / \mathbf{m}^{3}\right)}{\mathbf{G M}}$ & $\begin{array}{l}\%> \\
\text { PEL }\end{array}$ & $\begin{array}{l}\%> \\
\text { REL }\end{array}$ \\
\hline $\begin{array}{l}\text { District } 1 \text { (Anthracite coal mining regions in } \\
\text { Pennsylvania) }\end{array}$ & 40,660 & 0.203 & 22,717 & 0.219 & 2.7 & 9,277 & 0.184 & 2.4 & 8,666 & 0.186 & 2.6 & 8.9 \\
\hline $\begin{array}{l}\text { District } 2 \text { (Bituminous coal mining regions in } \\
\text { Pennsylvania) }\end{array}$ & 289,781 & 0.512 & 208,709 & 0.515 & 9.8 & 40,164 & 0.564 & 9.5 & 40,908 & 0.451 & 5.8 & 23.9 \\
\hline District 3 & 258,453 & 0.558 & 184,655 & 0.590 & 12.7 & 38,816 & 0.531 & 7.5 & 34,982 & 0.436 & 5.2 & 23.3 \\
\hline Maryland & 9,818 & 0.621 & 5,668 & 0.674 & 13.1 & 1,501 & 0.614 & 6.9 & 2,649 & 0.523 & 6.1 & 32.3 \\
\hline Ohio & 85,691 & 0.606 & 66,440 & 0.672 & 16.3 & 9,792 & 0.479 & 6.0 & 9,459 & 0.372 & 4.7 & 22.5 \\
\hline Northern West Virginia & 162,944 & 0.531 & 112,547 & 0.543 & 10.5 & 27,523 & 0.547 & 8.1 & 22,874 & 0.457 & 5.4 & 22.5 \\
\hline District 4 (Southern West Virginia) & 451,111 & 0.551 & 306,083 & 0.572 & 14.6 & 76,284 & 0.531 & 10.6 & 68,744 & 0.485 & 9.0 & 27.4 \\
\hline District 5 (Virginia) & 287,317 & 0.467 & 189,909 & 0.465 & 9.3 & 55,143 & 0.512 & 8.4 & 42,265 & 0.421 & 5.9 & 20.7 \\
\hline District 6 (Eastern Kentucky) & 266,705 & 0.415 & 154,093 & 0.392 & 7.0 & 56,560 & 0.467 & 8.3 & 56,052 & 0.433 & 6.6 & 22.4 \\
\hline District 7 & 262,223 & 0.449 & 143,987 & 0.444 & 7.2 & 63,635 & 0.483 & 7.0 & 54,601 & 0.423 & 6.2 & 21.2 \\
\hline Central Kentucky & 216,315 & 0.457 & 112,157 & 0.453 & 7.5 & 54,830 & 0.492 & 7.2 & 49,328 & 0.430 & 6.3 & 21.4 \\
\hline North Carolina & 9 & 0.147 & 6 & 0.159 & 0.0 & 3 & 0.126 & 0.0 & 0 & - & - & - \\
\hline South Carolina & 0 & - & 0 & - & - & 0 & - & - & 0 & - & - & - \\
\hline Tennessee & 45,870 & 0.411 & 31,795 & 0.413 & 6.5 & 8,802 & 0.432 & 6.0 & 5,273 & 0.366 & 6.1 & 19.2 \\
\hline Northern Georgia & 29 & 0.328 & 29 & 0.328 & 0.0 & 0 & - & - & 0 & - & - & - \\
\hline District 8 & 146,515 & 0.743 & 99,969 & 0.681 & 14.7 & 26,146 & 0.899 & 14.3 & 20,400 & 0.891 & 15.5 & 50.5 \\
\hline Illinois & 131,785 & 0.794 & 90,886 & 0.731 & 15.6 & 23,477 & 0.974 & 15.0 & 17,422 & 0.934 & 15.4 & 52.0 \\
\hline Indiana & 13,033 & 0.424 & 7,575 & 0.342 & 5.2 & 2,514 & 0.459 & 8.6 & 2,944 & 0.687 & 16.6 & 42.8 \\
\hline Iowa & 886 & 0.384 & 813 & 0.388 & 5.3 & 73 & 0.344 & 0.0 & 0 & - & - & - \\
\hline Michigan & 0 & - & 0 & - & - & 0 & - & - & 0 & - & - & - \\
\hline Minnesota & 0 & - & 0 & - & - & 0 & - & - & 0 & - & - & - \\
\hline Northern Missouri & 811 & 0.249 & 695 & 0.260 & 1.7 & 82 & 0.240 & 2.4 & 34 & 0.107 & 0.0 & 0.0 \\
\hline Wisconsin & 0 & & 0 & - & - & 0 & - & - & 0 & - & - & - \\
\hline
\end{tabular}

See footnotes at end of table. 
Table 2-16 (page 2 of 3). Respirable coal mine dust: Geometric mean exposures and percent exceeding designated occupational exposure limits by MSHA coal mine district and state, MSHA inspector and mine operator samples, 1979-1999

\begin{tabular}{|c|c|c|c|c|c|c|c|c|c|c|c|c|}
\hline \multirow[b]{2}{*}{ MSHA Coal Mine District } & \multicolumn{2}{|c|}{ All years } & \multicolumn{3}{|c|}{$1979-1989$} & \multicolumn{3}{|c|}{1990 - 1994} & \multicolumn{4}{|c|}{$\begin{array}{c}1995-1999 \\
\text { NIOSH REL }=1.0 \mathrm{mg} / \mathrm{m}^{3} \text { MRE }\end{array}$} \\
\hline & $\begin{array}{c}\text { No. of } \\
\text { Samples }\end{array}$ & $\begin{array}{c}\mathbf{G M} \\
\left(\mathrm{mg} / \mathrm{m}^{3}\right)\end{array}$ & \begin{tabular}{|c|} 
No. of \\
Samples
\end{tabular} & $\begin{array}{c}\mathbf{G M} \\
\left(\mathrm{mg} / \mathbf{m}^{3}\right)\end{array}$ & $\begin{array}{l}\%> \\
\text { PEL }\end{array}$ & $\begin{array}{c}\text { No. of } \\
\text { Samples }\end{array}$ & $\begin{array}{c}\mathbf{G M} \\
\left(\mathrm{mg} / \mathbf{m}^{3}\right)\end{array}$ & $\begin{array}{l}\%> \\
\text { PEL }\end{array}$ & \begin{tabular}{|c|} 
No. of \\
Samples
\end{tabular} & $\begin{array}{c}\mathbf{G M} \\
\left(\mathrm{mg} / \mathbf{m}^{3}\right)\end{array}$ & $\begin{array}{l}\%> \\
\text { PEL }\end{array}$ & $\begin{array}{l}\%> \\
\text { REL }\end{array}$ \\
\hline District 9 & 120,599 & 0.683 & 78,749 & 0.696 & 19.8 & 22,044 & 0.759 & 15.0 & 19,806 & 0.565 & 9.3 & 33.8 \\
\hline Alaska & 246 & 0.338 & 181 & 0.408 & 6.6 & 48 & 0.237 & 2.1 & 17 & 0.123 & 0.0 & 0.0 \\
\hline Arizona & 913 & 0.252 & 484 & 0.310 & 5.0 & 201 & 0.308 & 4.5 & 228 & 0.136 & 1.3 & 1.9 \\
\hline Arkansas & 311 & 0.172 & 238 & 0.205 & 2.5 & 37 & 0.149 & 2.7 & 36 & 0.064 & 0.0 & 0.0 \\
\hline California & 12 & 0.218 & 0 & - & - & 1 & 0.400 & 0.0 & 11 & 0.206 & 0.0 & 9.1 \\
\hline Colorado & 38,040 & 0.844 & 25,744 & 0.845 & 24.1 & 6,616 & 0.915 & 16.4 & 5,680 & 0.765 & 10.4 & 40.7 \\
\hline Hawaii & 0 & - & 0 & - & - & 0 & - & - & 0 & - & - & - \\
\hline Idaho & 0 & - & 0 & - & - & 0 & - & - & 0 & - & - & - \\
\hline Kansas & 510 & 0.216 & 383 & 0.235 & 2.6 & 49 & 0.190 & 0.0 & 78 & 0.152 & 1.3 & 1.4 \\
\hline Louisiana & 105 & 0.186 & 14 & 0.163 & 0.0 & 39 & 0.228 & 0.0 & 52 & 0.165 & 0.0 & 0.0 \\
\hline Southern Missouri & 647 & 0.236 & 453 & 0.255 & 2.0 & 56 & 0.160 & 0.0 & 138 & 0.216 & 1.4 & 7.0 \\
\hline Montana & 1,720 & 0.296 & 1,096 & 0.368 & 7.2 & 213 & 0.250 & 2.8 & 411 & 0.181 & 2.7 & 5.6 \\
\hline Nebraska & 0 & - & 0 & - & - & 0 & - & - & 0 & - & - & - \\
\hline Nevada & 0 & - & 0 & - & - & 0 & - & - & 0 & - & - & - \\
\hline New Mexico & 5,222 & 0.615 & 3,535 & 0.767 & 23.7 & 876 & 0.753 & 20.2 & 811 & 0.188 & 3.5 & 6.2 \\
\hline North Dakota & 1,345 & 0.187 & 1,029 & 0.221 & 1.9 & 163 & 0.157 & 0.6 & 153 & 0.073 & 0.0 & 0.0 \\
\hline Oklahoma & 4,857 & 0.331 & 2,972 & 0.345 & 6.9 & 985 & 0.325 & 6.7 & 900 & 0.297 & 3.4 & 9.8 \\
\hline Oregon & 0 & - & 0 & - & - & 0 & - & - & 0 & - & - & \\
\hline Texas & 4,005 & 0.179 & 2,740 & 0.194 & 1.4 & 664 & 0.198 & 3.9 & 601 & 0.109 & 1.0 & 3.5 \\
\hline Utah & 52,952 & 0.852 & 34,017 & 0.834 & 21.8 & 10,277 & 0.928 & 16.4 & 8,658 & 0.834 & 12.1 & 43.6 \\
\hline Washington & 346 & 0.178 & 124 & 0.166 & 0.0 & 74 & 0.257 & 2.7 & 148 & 0.158 & 0.7 & 4.3 \\
\hline Wyoming & 9,368 & 0.463 & 5,739 & 0.479 & 12.5 & 1,745 & 0.569 & 14.1 & 1,884 & 0.345 & 7.0 & 21.9 \\
\hline
\end{tabular}

See footnotes at end of table. 
Table 2-16 (page 3 of 3). Respirable coal mine dust: Geometric mean exposures and percent exceeding designated occupational exposure limits by MSHA coal mine district and state, MSHA inspector and mine operator samples, 1979-1999

\begin{tabular}{|c|c|c|c|c|c|c|c|c|c|c|c|c|}
\hline \multirow[b]{2}{*}{ MSHA Coal Mine District } & \multicolumn{2}{|c|}{ All years } & \multicolumn{3}{|c|}{$1979-1989$} & \multicolumn{3}{|c|}{$1990-1994$} & \multicolumn{4}{|c|}{$\begin{array}{c}1995-1999 \\
\text { NIOSH REL }=1.0 \mathrm{mg} / \mathrm{m}^{3} \mathrm{MRE}\end{array}$} \\
\hline & $\begin{array}{c}\text { No. of } \\
\text { Samples }\end{array}$ & $\begin{array}{c}\mathbf{G M} \\
\left(\mathrm{mg} / \mathrm{m}^{3}\right)\end{array}$ & \begin{tabular}{|c|} 
No. of \\
Samples
\end{tabular} & $\begin{array}{c}\mathbf{G M} \\
\left(\mathrm{mg} / \mathbf{m}^{3}\right)\end{array}$ & $\begin{array}{l}\%> \\
\text { PEL }\end{array}$ & \begin{tabular}{|c|} 
No. of \\
Samples
\end{tabular} & $\begin{array}{c}\text { GM } \\
\left(\mathrm{mg} / \mathrm{m}^{3}\right)\end{array}$ & $\begin{array}{l}\%> \\
\text { PEL }\end{array}$ & $\begin{array}{c}\text { No. of } \\
\text { Samples }\end{array}$ & $\begin{array}{c}\mathbf{G M} \\
\left(\mathrm{mg} / \mathbf{m}^{3}\right)\end{array}$ & $\begin{array}{l}\%> \\
\text { PEL }\end{array}$ & $\begin{array}{l}\%> \\
\text { REL }\end{array}$ \\
\hline District 10 (Western Kentucky) & 77,891 & 0.676 & $\mathbf{5 0 , 9 2 3}$ & 0.603 & 12.1 & 14,282 & 0.847 & 14.8 & 12,686 & 0.829 & 14.2 & 48.4 \\
\hline District 11 & 85,776 & 0.686 & 52,600 & 0.714 & 15.7 & 18,307 & 0.724 & 10.7 & 14,869 & 0.556 & 6.6 & 29.8 \\
\hline Alabama & 85,776 & 0.686 & 52,600 & 0.714 & 15.7 & 18,307 & 0.724 & 10.7 & 14,869 & 0.556 & 6.6 & 29.8 \\
\hline Central and Southern Georgia & 0 & - & 0 & - & - & 0 & - & - & 0 & - & - & - \\
\hline Florida & 0 & - & 0 & - & - & 0 & - & - & 0 & - & - & - \\
\hline Mississippi & 0 & - & 0 & - & - & 0 & - & - & 0 & - & - & - \\
\hline Puerto Rico & 0 & - & 0 & - & - & 0 & - & - & 0 & - & - & - \\
\hline Virgin Islands & 0 & - & 0 & - & - & 0 & - & - & 0 & - & - & - \\
\hline TOTAL & $2,287,031$ & 0.520 & $1,492,394$ & 0.526 & 11.6 & 420,658 & 0.545 & 9.5 & 373,979 & 0.472 & 7.5 & 26.2 \\
\hline
\end{tabular}

- indicates incalculable field

PEL - permissible exposure limit

REL - recommended exposure limit

GM - geometric mean

$\mathrm{mg} / \mathrm{m}^{3}$ - milligrams per cubic meter

NOTE: In coal mining, for respirable dust containing less than 5\% quartz, the MSHA PEL is $2 \mathrm{mg} / \mathrm{m}^{3} \mathrm{MRE}$; for respirable dust containing greater than $5 \%$ quartz, the MSHA PEL is [(10 $\left.\mathrm{mg} / \mathrm{m}^{3} \mathrm{MRE}\right) /\left(\%\right.$ quartz)]. The NIOSH REL of $1 \mathrm{mg} / \mathrm{m}^{3}$ MRE for respirable coal mine dust was adopted in September of 1995. Geometric means are reported in MRE equivalent. All

samples are compared to the MSHA PEL for respirable coal mine dust containing less than 5\% quartz, regardless of actual quartz content. See appendices for source description, methods, and agents.

SOURCE: Mine Safety and Health Administration (MSHA) coal mine inspector and mine operator dust data. 
Figure 2-8. Respirable coal mine dust: Geometric mean exposures by state, MSHA inspector and mine operator samples, 1979-1999

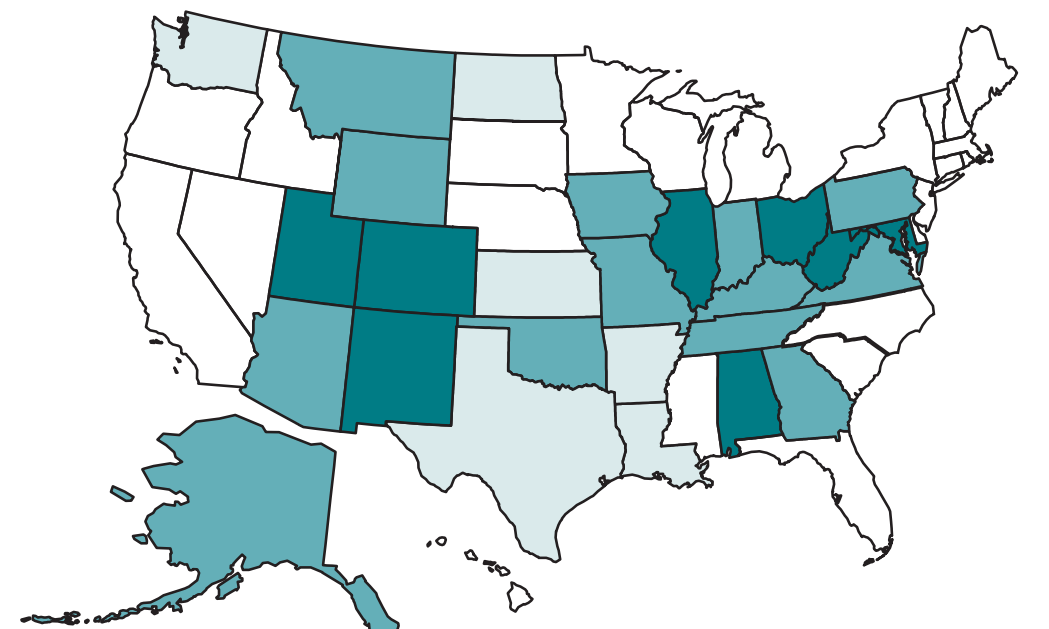

\begin{tabular}{|cc|}
\hline \multicolumn{2}{|c|}{$\mathbf{1 9 7 9}$ - 1989} \\
\hline mg/m ${ }^{3}$ MRE & No. of States \\
$\square>0.5$ & 8 \\
$\square>0.25$ to 0.5 & 13 \\
$\square 0$ to 0.25 & 6 \\
$\square<10$ samples & 24 \\
\hline
\end{tabular}

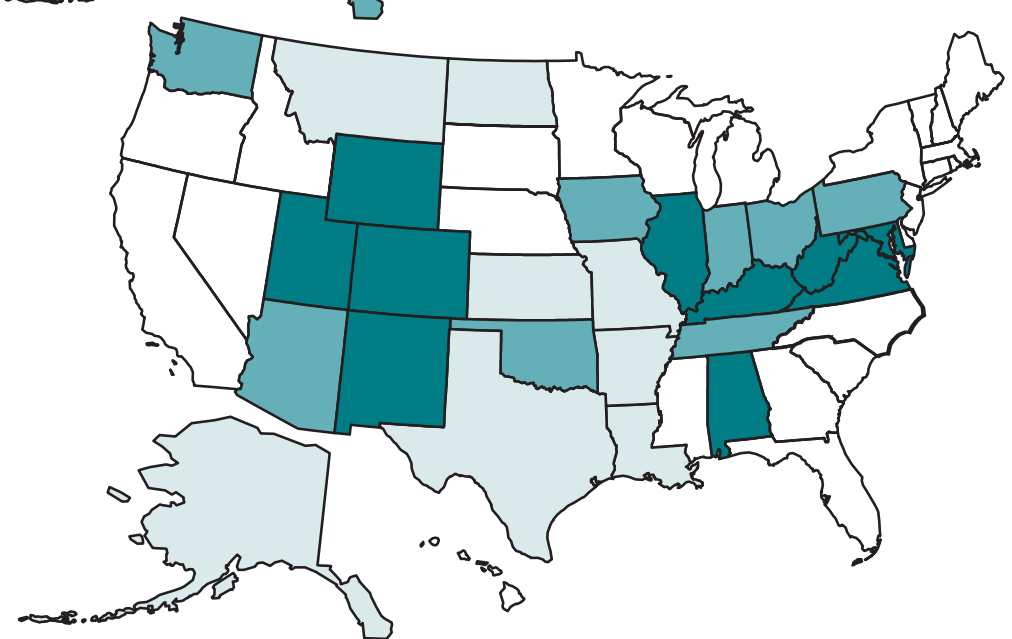

\begin{tabular}{|cc|}
\hline \multicolumn{1}{|c|}{$\mathbf{1 9 9 0} \mathbf{- 1 9 9 4}$} \\
\hline mg/m ${ }^{3}$ MRE & No. of States \\
$\square>0.5$ & 10 \\
$\square>0.25$ to 0.5 & 8 \\
$\square 0$ to 0.25 & 8 \\
$\square<10$ samples & 25 \\
\hline
\end{tabular}

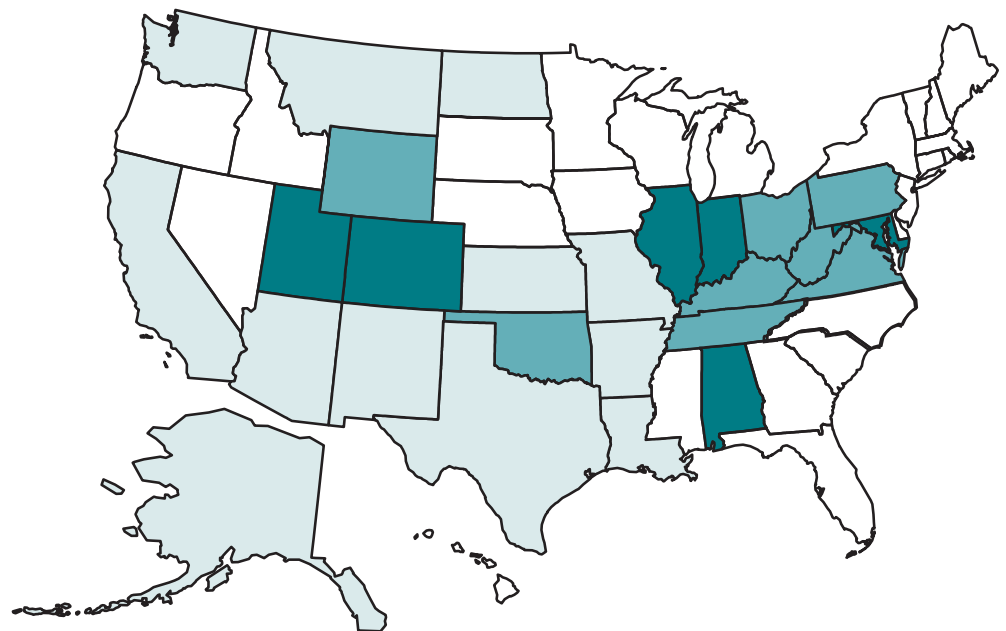

\begin{tabular}{|cc|}
\hline \multicolumn{2}{|c|}{$\mathbf{1 9 9 5}-\mathbf{1 9 9 9}$} \\
NIOSH REL $=1.0 \mathrm{mg} / \mathrm{m}^{3}$ \\
\hline mg/m $\mathrm{m}^{3}$ MRE & No. of States \\
$\square>0.5$ & 6 \\
$\square>0.25$ to 0.5 & 8 \\
$\square 0$ to 0.25 & 12 \\
$\square<10$ samples & 25 \\
\hline
\end{tabular}

PEL - permissible exposure limit REL - recommended exposure limit $\mathrm{mg} / \mathrm{m}^{3}$ - milligrams per cubic meter MRE - Mining Research Establishment NOTE: In coal mining, for respirable dust containing less than $5 \%$ quartz, the MSHA PEL is $2 \mathrm{mg} / \mathrm{m}^{3} \mathrm{MRE}$; for respirable dust containing greater than $5 \%$ quartz, the MSHA PEL is [(10 mg/m $\left.\mathrm{m}^{3} \mathrm{MRE}\right) /\left(\%\right.$ quartz)]. The NIOSH REL of $1 \mathrm{mg} / \mathrm{m}^{3}$ MRE for respirable coal mine dust was adopted in September of 1995. Geometric means are reported in MRE equivalent. See appendices for source description, methods, and agents.

SOURCE: Mine Safety and Health Administration (MSHA) coal mine inspector and mine operator dust data. 



\section{Section 3}

Silicosis

and

Related Exposures 

Figure 3-1. Silicosis: Number of deaths, crude and age-adjusted mortality rates, U.S. residents age 15 and over, 1968-1999

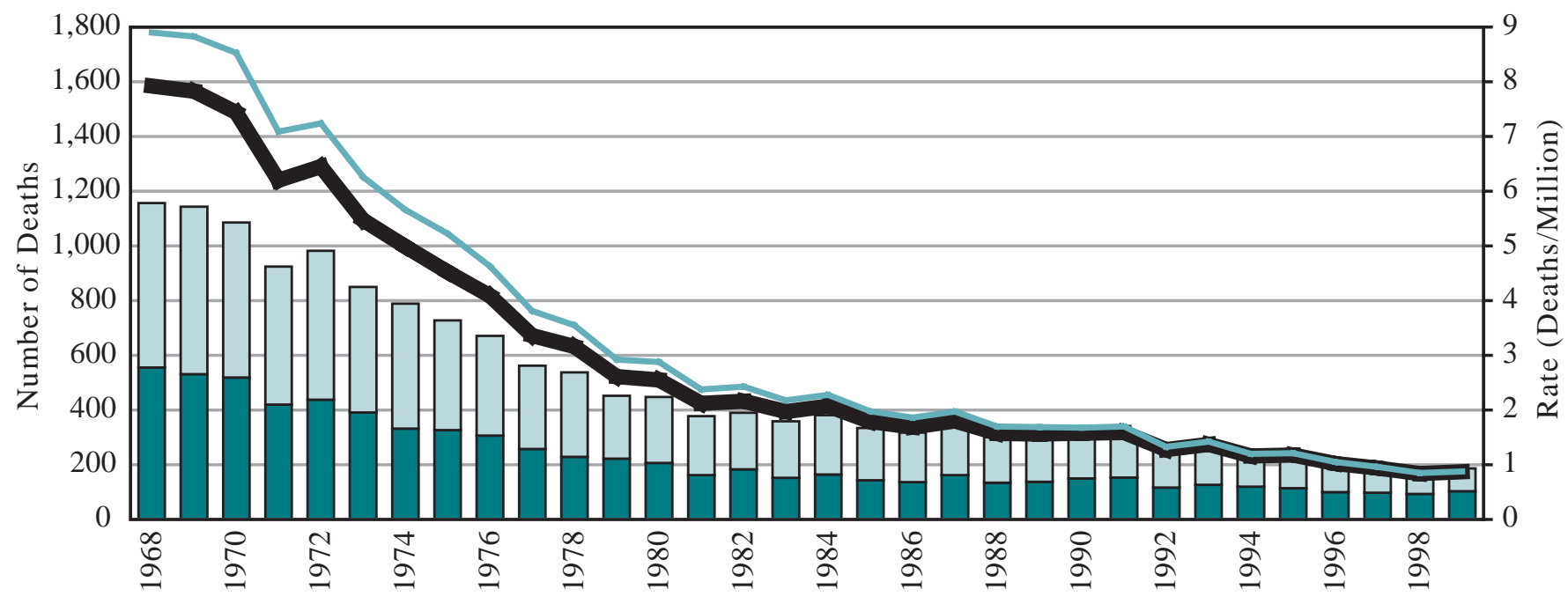

NOTE: See appendices for source description, methods, and ICD codes.

SOURCE: National Center for Health Statistics multiple cause of death data. Population estimates from U.S. Bureau of the Census.

Figure 3-2. Silicosis: Age-adjusted mortality rates by state, U.S. residents age 15 and over, 1990-1999

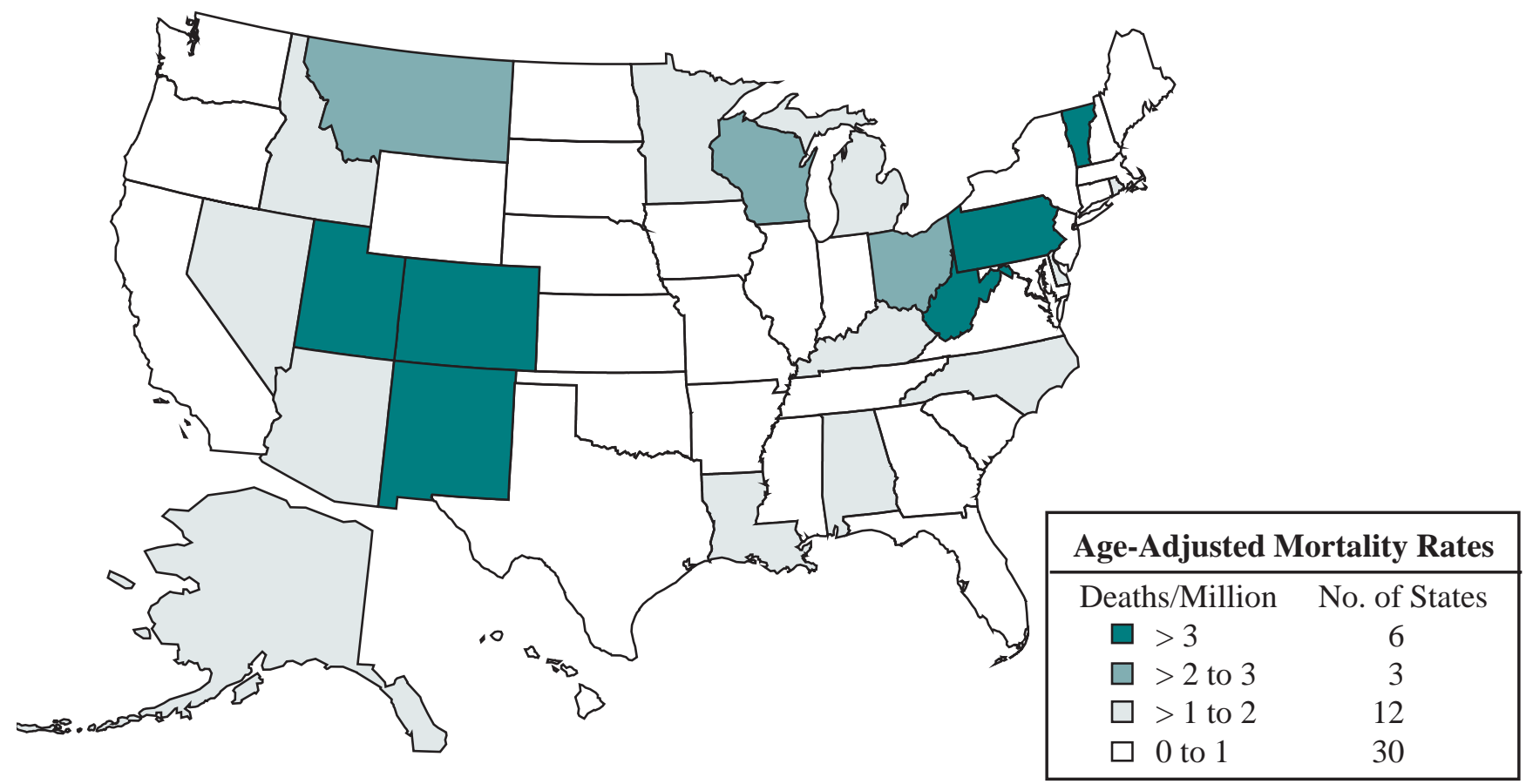

NOTE: See appendices for source description, methods, and ICD codes.

SOURCE: National Center for Health Statistics multiple cause of death data. Population estimates from U.S. Bureau of the Census. 
Table 3-1. Silicosis: Number of deaths by sex, race, age, and median age at death, U.S. residents age 15 and over, 1990-1999

\begin{tabular}{|c|c|c|c|c|c|c|c|c|c|c|c|c|c|c|c|c|}
\hline \multirow[b]{2}{*}{ Year } & \multirow{2}{*}{\begin{tabular}{|c|} 
No. of \\
Deaths
\end{tabular}} & \multirow{2}{*}{\begin{tabular}{|c|} 
Under- \\
lying \\
Cause \\
$(\%)$ \\
\end{tabular}} & \multicolumn{2}{|c|}{ Sex } & \multicolumn{3}{|c|}{ Race } & \multicolumn{8}{|c|}{ Age Group (yrs) } & \multirow{2}{*}{$\begin{array}{c}\text { Median } \\
\text { Age } \\
\text { (yrs) }\end{array}$} \\
\hline & & & Male & Female & White & Black & Other & $15-24$ & $25-34$ & $35-44$ & $45-54$ & $55-64$ & $65-74$ & $75-84$ & $85+$ & \\
\hline 1990 & 308 & 48.7 & 298 & 10 & 261 & 46 & 1 & - & 2 & 6 & 11 & 46 & 81 & 125 & 37 & 75.0 \\
\hline 1991 & 314 & 48.7 & 305 & 9 & 257 & 56 & 1 & 1 & 1 & 4 & 12 & 40 & 78 & 135 & 43 & 76.0 \\
\hline 1992 & 255 & 45.9 & 240 & 15 & 212 & 39 & 4 & - & 1 & 3 & 8 & 36 & 79 & 96 & 32 & 75.0 \\
\hline 1993 & 276 & 46.0 & 268 & 8 & 240 & 32 & 4 & - & 1 & 5 & 10 & 32 & 88 & 96 & 44 & 75.0 \\
\hline 1994 & 235 & 51.1 & 222 & 13 & 206 & 27 & 2 & - & - & 1 & 7 & 35 & 59 & 100 & 33 & 77.0 \\
\hline 1995 & 242 & 47.1 & 232 & 10 & 198 & 42 & 2 & - & - & 2 & 8 & 28 & 70 & 98 & 36 & 76.0 \\
\hline 1996 & 212 & 47.2 & 206 & 6 & 184 & 26 & 2 & - & 1 & 2 & 7 & 25 & 54 & 83 & 40 & 76.0 \\
\hline 1997 & 198 & 49.5 & 189 & 9 & 163 & 32 & 3 & - & - & 4 & 7 & 26 & 67 & 58 & 36 & 74.0 \\
\hline 1998 & 178 & 52.2 & 174 & 4 & 158 & 18 & 2 & - & - & 5 & 9 & 17 & 47 & 68 & 32 & 76.0 \\
\hline 1999 & 187 & 55.1 & 181 & 6 & 154 & 30 & 3 & - & - & 3 & 9 & 21 & 58 & 61 & 35 & 75.0 \\
\hline TOTAL & 2,405 & 48.9 & 2,315 & 90 & 2,033 & 348 & 24 & 1 & 6 & 35 & 88 & 306 & 681 & 920 & 368 & 76.0 \\
\hline
\end{tabular}

- indicates no deaths listed.

NOTE: See appendices for source description, methods, and ICD codes.

SOURCE: National Center for Health Statistics multiple cause of death data. 
Table 3-2. Silicosis: Mortality rates (per million population) by race and sex, U.S. residents age 15 and over, 1990-1999

\begin{tabular}{|c|c|c|c|c|c|c|c|}
\hline \multirow[b]{2}{*}{ Year } & \multirow[b]{2}{*}{ Overall } & \multicolumn{2}{|c|}{ White } & \multicolumn{2}{|c|}{ Black } & \multicolumn{2}{|c|}{ Other } \\
\hline & & Male & Female & Male & Female & Male & Female \\
\hline & \multicolumn{7}{|c|}{ Crude Mortality Rate } \\
\hline 1990 & 1.58 & 3.14 & 0.11 & 4.38 & 0.08 & 0.29 & - \\
\hline 1991 & 1.59 & 3.08 & 0.09 & 5.28 & 0.08 & 0.28 & - \\
\hline 1992 & 1.28 & 2.46 & 0.14 & 3.49 & 0.16 & 0.80 & 0.25 \\
\hline 1993 & 1.37 & 2.85 & 0.07 & 2.78 & 0.16 & 1.04 & - \\
\hline 1994 & 1.16 & 2.34 & 0.14 & 2.37 & 0.08 & 0.50 & - \\
\hline 1995 & 1.18 & 2.27 & 0.09 & 3.59 & 0.15 & 0.49 & - \\
\hline 1996 & 1.02 & 2.10 & 0.07 & 2.29 & - & 0.46 & - \\
\hline 1997 & 0.94 & 1.82 & 0.09 & 2.68 & 0.07 & 0.68 & - \\
\hline 1998 & 0.84 & 1.80 & 0.03 & 1.44 & 0.07 & 0.44 & - \\
\hline 1999 & 0.87 & 1.72 & 0.05 & 2.42 & 0.07 & 0.64 & - \\
\hline \multirow[t]{2}{*}{ 1990-1999 } & 1.17 & 2.34 & 0.09 & 3.01 & 0.09 & 0.56 & 0.02 \\
\hline & \multicolumn{7}{|c|}{ Age-Adjusted Mortality Rate } \\
\hline 1990 & 1.68 & 4.01 & 0.09 & 7.15 & 0.09 & 0.50 & - \\
\hline 1991 & 1.70 & 3.92 & 0.08 & 9.60 & 0.12 & 0.39 & - \\
\hline 1992 & 1.33 & 2.99 & 0.12 & 6.74 & 0.19 & 1.59 & 0.55 \\
\hline 1993 & 1.42 & 3.45 & 0.06 & 4.98 & 0.19 & 3.42 & - \\
\hline 1994 & 1.20 & 2.87 & 0.11 & 4.00 & 0.08 & 1.02 & - \\
\hline 1995 & 1.21 & 2.73 & 0.07 & 6.60 & 0.21 & 0.72 & - \\
\hline 1996 & 1.05 & 2.57 & 0.05 & 3.86 & - & 1.00 & - \\
\hline 1997 & 0.96 & 2.14 & 0.08 & 4.57 & 0.10 & 1.10 & - \\
\hline 1998 & 0.85 & 2.10 & 0.03 & 2.44 & 0.07 & 0.84 & - \\
\hline 1999 & 0.88 & 1.97 & 0.04 & 3.93 & 0.07 & 1.25 & - \\
\hline 1990-1999 & 1.21 & 2.81 & 0.07 & 5.27 & 0.11 & 1.23 & 0.05 \\
\hline
\end{tabular}

- indicates no deaths listed.

NOTE: See appendices for source description, methods, and ICD codes.

SOURCE: National Center for Health Statistics multiple cause of death data. Population estimates from U.S. Bureau of the Census. 
Table 3-3. Silicosis: Years of potential life lost to age 65 and to life expectancy by race and sex, U.S. residents age 15 and over, 1990-1999

\begin{tabular}{|c|c|c|c|c|c|c|c|}
\hline \multirow[b]{2}{*}{ Year } & \multicolumn{2}{|c|}{ White } & \multicolumn{2}{|c|}{ Black } & \multicolumn{2}{|c|}{ Other } & \multirow[b]{2}{*}{ Total } \\
\hline & Male & Female & Male & Female & Male & Female & \\
\hline & \multicolumn{7}{|c|}{ Years of Potential Life Lost to Age 65} \\
\hline 1990 & 430 & - & 160 & 25 & - & - & 615 \\
\hline 1991 & 415 & 50 & 90 & - & 5 & - & 560 \\
\hline 1992 & 325 & 15 & 45 & 25 & - & - & 410 \\
\hline 1993 & 325 & 5 & 115 & 25 & - & - & 470 \\
\hline 1994 & 230 & 5 & 40 & 25 & 5 & - & 305 \\
\hline 1995 & 220 & 5 & 65 & 15 & 5 & - & 310 \\
\hline 1996 & 240 & - & 60 & - & 15 & - & 315 \\
\hline 1997 & 225 & 35 & 75 & - & - & - & 335 \\
\hline 1998 & 260 & 5 & 55 & 25 & - & - & 345 \\
\hline 1999 & 180 & - & 110 & 25 & - & - & 315 \\
\hline \multirow[t]{2}{*}{ TOTAL } & 2,850 & 120 & 815 & 165 & 30 & - & 3,980 \\
\hline & \multicolumn{7}{|c|}{ Years of Potential Life Lost to Life Expectancy } \\
\hline 1990 & 2,758 & 92 & 557 & 37 & 14 & - & 3,458 \\
\hline 1991 & 2,732 & 136 & 571 & 6 & 21 & - & 3,466 \\
\hline 1992 & 2,278 & 188 & 348 & 46 & 37 & 9 & 2,906 \\
\hline 1993 & 2,545 & 69 & 365 & 45 & 34 & - & 3,058 \\
\hline 1994 & 2,044 & 127 & 279 & 37 & 29 & - & 2,516 \\
\hline 1995 & 1,995 & 100 & 405 & 37 & 35 & - & 2,572 \\
\hline 1996 & 1,875 & 57 & 301 & - & 36 & - & 2,269 \\
\hline 1997 & 1,758 & 132 & 371 & 9 & 43 & - & 2,313 \\
\hline 1998 & 1,737 & 39 & 204 & 38 & 23 & - & 2,041 \\
\hline 1999 & 1,643 & 41 & 385 & 37 & 35 & - & 2,141 \\
\hline TOTAL & 21,365 & 981 & 3,786 & 292 & 307 & 9 & 26,740 \\
\hline
\end{tabular}

- indicates no deaths listed.

NOTE: See appendices for source description, methods, and ICD codes.

SOURCE: National Center for Health Statistics multiple cause of death data. 
Table 3-4. Silicosis: Number of deaths by state, U.S. residents age 15 and over, 1990-1999

\begin{tabular}{|c|c|c|c|c|c|c|c|c|c|c|c|}
\hline State & 1990 & 1991 & 1992 & 1993 & 1994 & 1995 & 1996 & 1997 & 1998 & 1999 & Total \\
\hline Alabama & 3 & 9 & 3 & 1 & 3 & 4 & 3 & 1 & 8 & 4 & 39 \\
\hline Alaska & 1 & - & - & 1 & - & - & - & - & - & - & 2 \\
\hline Arizona & 5 & 2 & 1 & 4 & 7 & 6 & 3 & 3 & 3 & 4 & 38 \\
\hline Arkansas & - & 2 & 1 & 1 & - & 3 & 1 & 2 & 2 & 3 & 15 \\
\hline California & 15 & 16 & 12 & 13 & 9 & 14 & 13 & 5 & 4 & 6 & 107 \\
\hline Colorado & 10 & 15 & 9 & 11 & 6 & 9 & 10 & 7 & 6 & 7 & 90 \\
\hline Connecticut & 2 & 2 & 3 & 5 & 4 & 2 & 3 & 1 & 4 & 1 & 27 \\
\hline Delaware & 1 & - & 1 & - & - & 1 & 1 & 1 & - & 1 & 6 \\
\hline District of Columbia & 1 & - & 1 & - & - & 1 & - & 1 & - & - & 4 \\
\hline Florida & 12 & 7 & 4 & 5 & 6 & 6 & 5 & 6 & 6 & 5 & 62 \\
\hline Georgia & 4 & 3 & 3 & 3 & 3 & 6 & 4 & 4 & 3 & 2 & 35 \\
\hline Hawaii & - & - & - & - & - & - & - & - & - & - & - \\
\hline Idaho & 2 & - & 2 & 3 & - & 2 & 3 & 2 & 1 & - & 15 \\
\hline Illinois & 10 & 8 & 11 & 9 & 6 & 5 & 7 & 8 & 7 & 5 & 76 \\
\hline Indiana & 8 & 4 & 4 & 4 & 4 & 1 & 2 & 5 & 5 & 5 & 42 \\
\hline Iowa & 2 & 2 & 2 & 4 & 1 & 2 & 1 & 4 & 4 & 1 & 23 \\
\hline Kansas & - & 1 & 1 & 1 & 2 & 3 & - & 1 & 1 & 1 & 11 \\
\hline Kentucky & 6 & 2 & 6 & 3 & 5 & 5 & 3 & 4 & 5 & 2 & 41 \\
\hline Louisiana & 6 & 5 & 2 & 8 & 2 & 2 & 2 & 2 & 1 & 5 & 35 \\
\hline Maine & 2 & 2 & - & 2 & 2 & - & 1 & - & - & 1 & 10 \\
\hline Maryland & 3 & 4 & 1 & 2 & 2 & 2 & 4 & 1 & 3 & 5 & 27 \\
\hline Massachusetts & 4 & 3 & 1 & 1 & 4 & 2 & 2 & 5 & 3 & 1 & 26 \\
\hline Michigan & 12 & 9 & 14 & 15 & 7 & 12 & 16 & 5 & 9 & 6 & 105 \\
\hline Minnesota & 5 & 3 & 9 & 5 & 5 & 6 & 6 & 5 & - & 4 & 48 \\
\hline Mississippi & 3 & 1 & 1 & - & - & 1 & 1 & 3 & 1 & 2 & 13 \\
\hline Missouri & 5 & 7 & 4 & 2 & 7 & 6 & 3 & 5 & 3 & 3 & 45 \\
\hline Montana & 2 & - & 3 & 2 & - & 2 & 2 & 1 & 1 & 2 & 15 \\
\hline Nebraska & - & 3 & 1 & - & - & - & 1 & - & - & 1 & 6 \\
\hline Nevada & 3 & - & - & 3 & 4 & 2 & - & 1 & - & - & 13 \\
\hline New Hampshire & 1 & 2 & 1 & - & 1 & - & - & 2 & - & - & 7 \\
\hline New Jersey & 10 & 11 & 5 & 5 & 5 & 3 & 3 & 4 & 4 & 3 & 53 \\
\hline New Mexico & 6 & 4 & 5 & 3 & 8 & 4 & 1 & 5 & 2 & 2 & 40 \\
\hline New York & 15 & 18 & 6 & 13 & 12 & 9 & 10 & 6 & 8 & 8 & 105 \\
\hline North Carolina & 5 & 14 & 8 & 11 & 7 & 12 & 6 & 9 & 5 & 6 & 83 \\
\hline North Dakota & - & - & - & - & - & - & 1 & - & - & - & 1 \\
\hline Ohio & 33 & 35 & 24 & 23 & 21 & 20 & 16 & 12 & 16 & 23 & 223 \\
\hline Oklahoma & 4 & 4 & 5 & 1 & 3 & - & - & 1 & - & 5 & 23 \\
\hline Oregon & 3 & 3 & 3 & 2 & 2 & 2 & 2 & - & 1 & 5 & 23 \\
\hline Pennsylvania & 61 & 53 & 53 & 54 & 44 & 43 & 38 & 33 & 24 & 20 & 423 \\
\hline Rhode Island & 1 & - & 2 & - & - & - & 2 & 3 & - & 2 & 10 \\
\hline South Carolina & 5 & 3 & 1 & 3 & 3 & - & 3 & 2 & 1 & 2 & 23 \\
\hline South Dakota & 1 & - & - & - & 1 & - & 1 & 1 & - & 1 & 5 \\
\hline Tennessee & 3 & 3 & 2 & 6 & 3 & 2 & 2 & 3 & 5 & 3 & 32 \\
\hline Texas & 7 & 11 & 11 & 12 & 8 & 11 & 9 & 11 & 8 & 12 & 100 \\
\hline Utah & 2 & 5 & 4 & 10 & 4 & 4 & - & 3 & 3 & 1 & 36 \\
\hline Vermont & 5 & 1 & 4 & 1 & 1 & - & 1 & 2 & 2 & 1 & 18 \\
\hline Virginia & 3 & 2 & 3 & 5 & 4 & 3 & 2 & 3 & 6 & 1 & 32 \\
\hline Washington & - & 7 & 5 & 3 & 2 & 2 & 2 & 2 & 4 & 3 & 30 \\
\hline West Virginia & 6 & 12 & 4 & 6 & 10 & 8 & 6 & 6 & 5 & 6 & 69 \\
\hline Wisconsin & 10 & 16 & 9 & 10 & 7 & 14 & 10 & 6 & 4 & 6 & 92 \\
\hline Wyoming & - & - & - & - & - & - & - & 1 & - & - & 1 \\
\hline TOTAL & 308 & 314 & 255 & 276 & 235 & 242 & 212 & 198 & 178 & 187 & 2,405 \\
\hline
\end{tabular}

- indicates no deaths listed.

NOTE: See appendices for source description, methods, and ICD codes.

SOURCE: National Center for Health Statistics multiple cause of death data. 
Table 3-5. Silicosis: Number of deaths, mortality rates (per million population), and years of potential life lost (YPLL) by state, U.S. residents age 15 and over, 1990-1999

\begin{tabular}{|c|c|c|c|c|c|c|c|c|c|c|}
\hline \multirow[b]{2}{*}{ State } & \multirow{2}{*}{$\begin{array}{l}\text { No. of } \\
\text { Deaths }\end{array}$} & \multirow[b]{2}{*}{ Rank } & \multicolumn{2}{|c|}{ Crude Mortality } & \multicolumn{2}{|c|}{ Age-Adjusted Mortality } & \multicolumn{4}{|c|}{ YPLL to Life Expectancy } \\
\hline & & & Rate & Rank & Rate & Rank & Total & Rank & YPLL/death & Rank \\
\hline Alabama & 39 & 19 & 1.18 & 16 & 1.20 & 19 & 515 & 19 & 13.2 & 15 \\
\hline Alaska & 2 & 48 & 0.46 & 47 & 1.29 & 16 & 22 & 48 & 11.2 & 38 \\
\hline Arizona & 38 & 20 & 1.17 & 17 & 1.23 & 17 & 439 & 23 & 11.6 & 31 \\
\hline Arkansas & 15 & 35 & 0.78 & 33 & 0.72 & 42 & 308 & 29 & 20.5 & 1 \\
\hline California & 107 & 3 & 0.44 & 48 & 0.54 & 44 & 1,284 & 5 & 12.0 & 27 \\
\hline Colorado & 90 & 8 & 3.11 & 5 & 3.92 & 3 & 1,054 & 10 & 11.7 & 30 \\
\hline Connecticut & 27 & 27 & 1.03 & 23 & 1.00 & 22 & 319 & 27 & 11.8 & 29 \\
\hline Delaware & 6 & 44 & 1.06 & 21 & 1.23 & 17 & 52 & 47 & 8.6 & 49 \\
\hline District of Columbia & 4 & 47 & 0.86 & 29 & 0.96 & 25 & 73 & 44 & 18.3 & 2 \\
\hline Florida & 62 & 12 & 0.54 & 44 & 0.43 & 47 & 781 & 12 & 12.6 & 21 \\
\hline Georgia & 35 & 22 & 0.63 & 41 & 0.80 & 36 & 404 & 25 & 11.5 & 34 \\
\hline Hawaii & - & - & - & - & - & - & - & - & - & - \\
\hline Idaho & 15 & 35 & 1.74 & 10 & 1.74 & 10 & 136 & 39 & 9.1 & 48 \\
\hline Illinois & 76 & 10 & 0.82 & 31 & 0.84 & 32 & 1,086 & 8 & 14.3 & 11 \\
\hline Indiana & 42 & 16 & 0.93 & 26 & 0.94 & 26 & 616 & 14 & 14.7 & 9 \\
\hline Iowa & 23 & 30 & 1.04 & 22 & 0.89 & 28 & 296 & 31 & 12.9 & 19 \\
\hline Kansas & 11 & 40 & 0.56 & 43 & 0.53 & 45 & 159 & 37 & 14.5 & 10 \\
\hline Kentucky & 41 & 17 & 1.36 & 13 & 1.38 & 14 & 622 & 13 & 15.2 & 7 \\
\hline Louisiana & 35 & 22 & 1.07 & 20 & 1.14 & 21 & 601 & 15 & 17.2 & 4 \\
\hline Maine & 10 & 41 & 1.03 & 23 & 0.97 & 24 & 130 & 41 & 13.0 & 17 \\
\hline Maryland & 27 & 27 & 0.68 & 39 & 0.83 & 33 & 299 & 30 & 11.1 & 39 \\
\hline Massachusetts & 26 & 29 & 0.53 & 45 & 0.51 & 46 & 265 & 32 & 10.2 & 43 \\
\hline Michigan & 105 & 4 & 1.42 & 12 & 1.52 & 12 & 1,222 & 6 & 11.6 & 31 \\
\hline Minnesota & 48 & 14 & 1.36 & 13 & 1.34 & 15 & 581 & 18 & 12.1 & 26 \\
\hline Mississippi & 13 & 38 & 0.64 & 40 & 0.64 & 43 & 232 & 34 & 17.8 & 3 \\
\hline Missouri & 45 & 15 & 1.08 & 18 & 0.99 & 23 & 592 & 16 & 13.2 & 15 \\
\hline Montana & 15 & 35 & 2.26 & 9 & 2.11 & 9 & 139 & 38 & 9.2 & 47 \\
\hline Nebraska & 6 & 44 & 0.48 & 46 & 0.40 & 48 & 57 & 45 & 9.6 & 45 \\
\hline Nevada & 13 & 38 & 1.08 & 18 & 1.42 & 13 & 135 & 40 & 10.4 & 42 \\
\hline New Hampshire & 7 & 43 & 0.78 & 33 & 0.87 & 30 & 81 & 43 & 11.6 & 31 \\
\hline New Jersey & 53 & 13 & 0.83 & 30 & 0.85 & 31 & 587 & 17 & 11.1 & 39 \\
\hline New Mexico & 40 & 18 & 3.17 & 4 & 3.65 & 5 & 457 & 21 & 11.4 & 35 \\
\hline New York & 105 & 4 & 0.73 & 36 & 0.73 & 40 & 1,307 & 4 & 12.4 & 24 \\
\hline North Carolina & 83 & 9 & 1.45 & 11 & 1.57 & 11 & 1,035 & 11 & 12.5 & 23 \\
\hline North Dakota & 1 & 49 & 0.20 & 50 & 0.16 & 50 & 6 & 50 & 6.1 & 50 \\
\hline Ohio & 223 & 2 & 2.56 & 7 & 2.53 & 7 & 2,816 & 2 & 12.6 & 21 \\
\hline Oklahoma & 23 & 30 & 0.91 & 27 & 0.83 & 33 & 252 & 33 & 11.0 & 41 \\
\hline Oregon & 23 & 30 & 0.94 & 25 & 0.88 & 29 & 224 & 35 & 9.7 & 44 \\
\hline Pennsylvania & 423 & 1 & 4.38 & 2 & 3.85 & 4 & 4,835 & 1 & 11.4 & 35 \\
\hline Rhode Island & 10 & 41 & 1.25 & 15 & 1.17 & 20 & 128 & 42 & 12.8 & 20 \\
\hline South Carolina & 23 & 30 & 0.81 & 32 & 0.94 & 26 & 318 & 28 & 13.8 & 14 \\
\hline South Dakota & 5 & 46 & 0.91 & 27 & 0.73 & 40 & 57 & 45 & 11.4 & 35 \\
\hline Tennessee & 32 & 24 & 0.77 & 35 & 0.78 & 37 & 474 & 20 & 14.8 & 8 \\
\hline Texas & 100 & 6 & 0.71 & 37 & 0.83 & 33 & 1,636 & 3 & 16.4 & 5 \\
\hline Utah & 36 & 21 & 2.69 & 6 & 3.44 & 6 & 434 & 24 & 12.0 & 27 \\
\hline Vermont & 18 & 34 & 3.94 & 3 & 4.16 & 2 & 174 & 36 & 9.6 & 45 \\
\hline Virginia & 32 & 24 & 0.61 & 42 & 0.74 & 39 & 452 & 22 & 14.1 & 13 \\
\hline Washington & 30 & 26 & 0.71 & 37 & 0.76 & 38 & 369 & 26 & 12.3 & 25 \\
\hline West Virginia & 69 & 11 & 4.74 & 1 & 4.23 & 1 & 1,061 & 9 & 15.4 & 6 \\
\hline Wisconsin & 92 & 7 & 2.32 & 8 & 2.23 & 8 & 1,193 & 7 & 13.0 & 17 \\
\hline Wyoming & 1 & 49 & 0.28 & 49 & 0.29 & 49 & 14 & 49 & 14.3 & 11 \\
\hline
\end{tabular}

- indicates no deaths listed.

NOTE: See appendices for source description, methods, and ICD codes.

SOURCE: National Center for Health Statistics multiple cause of death data. Population estimates from U.S. Bureau of the Census. 
Table 3-6. Silicosis: Most frequently recorded industries on death certificate, U.S. residents age 15 and over, selected states and years, 1990-1999

\begin{tabular}{clrc}
\hline CIC & Industry & Number of Deaths & Percent \\
\hline 060 & Construction & 118 & 13.4 \\
040 & Metal mining & 86 & 9.8 \\
041 & Coal mining & 69 & 7.8 \\
270 & Blast furnaces, steelworks, rolling and finishing mills & 51 & 5.8 \\
050 & Nonmetallic mining and quarrying, except fuel & 48 & 5.5 \\
271 & Iron and steel foundries & 48 & 5.5 \\
262 & Miscellaneous nonmetallic mineral and stone products & 44 & 5.0 \\
392 & Not specified manufacturing industries & 33 & 3.8 \\
331 & Machinery, except electrical, n.e.c. & 23 & 2.6 \\
252 & Structural clay products & 20 & 2.3 \\
& All other industries & 317 & 36.0 \\
& Industry not reported & 23 & 2.6 \\
& TOTAL & $\mathbf{8 8 0}$ & $\mathbf{1 0 0 . 0}$ \\
\hline
\end{tabular}

CIC - Census Industry Code

n.e.c. - not elsewhere classified

NOTE: Percentages may not total to $100 \%$ due to rounding. See appendices for source description, methods, and ICD codes, industry and occupation codes, and list of selected states and years.

SOURCE: National Center for Health Statistics multiple cause of death data.

Table 3-7. Silicosis: Most frequently recorded occupations on death certificate, U.S. residents age 15 and over, selected states and years, 1990-1999

\begin{tabular}{clrc}
\hline COC & Occupation & Number of Deaths & Percent \\
\hline 616 & Mining machine operators & 138 & 15.7 \\
889 & Laborers, except construction & 84 & 9.6 \\
019 & Managers and administrators, n.e.c. & 34 & 3.9 \\
633 & Supervisors, production occupations & 32 & 3.6 \\
453 & Janitors and cleaners & 30 & 3.4 \\
779 & Machine operators, not specified & 30 & 3.4 \\
869 & Construction laborers & 26 & 3.0 \\
719 & Molding and casting machine operators & 25 & 2.8 \\
243 & Supervisors and proprietors, sales occupations & 18 & 2.1 \\
804 & Truck drivers & 18 & 2.1 \\
& All other occupations & 423 & 48.1 \\
& Occupation not reported & 22 & 2.5 \\
& TOTAL & $\mathbf{8 8 0}$ & $\mathbf{1 0 0 . 0}$ \\
\hline
\end{tabular}

COC - Census Occupation Code n.e.c. - not elsewhere classified

NOTE: Percentages may not total to $100 \%$ due to rounding. See appendices for source description, methods, and ICD codes, industry and occupation codes, and list of selected states and years.

SOURCE: National Center for Health Statistics multiple cause of death data. 
Table 3-8. Silicosis: Proportionate mortality ratio (PMR) adjusted for age, sex, and race by usual industry, U.S. residents age 15 and over, selected states and years, 1990-1999

\begin{tabular}{llrrrr}
\hline & & & 95\% Confidence Interval \\
\cline { 5 - 6 } CIC & Industry & Number & LCL & \multicolumn{2}{c}{ UCL } \\
\hline 040 & Metal mining & PMR & & & \\
262 & Miscellaneous nonmetallic mineral and stone products & 44 & 30.72 & 22.26 & 41.23 \\
261 & Pottery and related products & 17 & 29.35 & 17.06 & 46.96 \\
050 & Nonmetallic mining and quarrying, except fuel & 48 & 29.27 & 21.52 & 38.82 \\
271 & Iron and steel foundries & 48 & 21.14 & 15.55 & 28.04 \\
252 & Structural clay products & 20 & 19.72 & 12.02 & 30.47 \\
041 & Coal mining & 69 & 6.17 & 4.84 & 7.86 \\
300 & Miscellaneous fabricated metal products & 18 & 5.71 & 3.38 & 9.02 \\
251 & Cement, concrete, gypsum, and plaster products & 8 & 4.24 & 1.83 & 8.35 \\
280 & Other primary metal industries & 9 & 3.48 & 1.59 & 6.60 \\
270 & Blast furnaces, steelworks, rolling and finishing mills & 51 & 3.19 & 2.41 & 4.25 \\
682 & Miscellaneous retail stores & 7 & 3.15 & 1.27 & 6.50 \\
250 & Glass and glass products & 10 & 3.00 & 1.44 & 5.51 \\
331 & Machinery, except electrical, n.e.c. & 23 & 2.48 & 1.57 & 3.73 \\
392 & Not specified manufacturing industries & 33 & 1.55 & 1.07 & 2.18 \\
060 & Construction & 118 & 1.26 & 1.05 & 1.51 \\
\hline
\end{tabular}

CIC - Census Industry Code $\quad$ n.e.c. - not elsewhere classified $\quad$ LCL - lower confidence limit $\quad$ UCL - upper confidence limit NOTE: See appendices for source description, methods, and ICD codes, industry and occupation codes, and list of selected states and years. SOURCE: National Center for Health Statistics multiple cause of death data. 
Table 3-9. Silicosis: Proportionate mortality ratio (PMR) adjusted for age, sex, and race by usual occupation, U.S. residents age 15 and over, selected states and years, 1990-1999

\begin{tabular}{llrrrr}
\hline & & & 95\% Confidence Interval \\
\cline { 4 - 6 } COC & Occupation & Number & Deaths & PMR & UCL \\
\hline 725 & Miscellaneous metal and plastic processing machine operators & 10 & 92.71 & 44.57 & 170.42 \\
787 & Hand molding, casting, and forming occupations & 8 & 41.97 & 18.09 & 82.61 \\
675 & Hand molders and shapers, except jewelers & 14 & 38.91 & 21.26 & 65.28 \\
768 Crushing and grinding machine operators & 16 & 31.12 & 17.78 & 50.52 \\
719 & Molding and casting machine operators & 25 & 19.36 & 12.49 & 28.55 \\
617 & Mining occupations, n.e.c. & 8 & 15.86 & 6.84 & 31.22 \\
616 & Mining machine operators & 138 & 13.08 & 11.04 & 15.51 \\
613 & Supervisors, extractive occupations & 7 & 12.86 & 5.17 & 26.52 \\
599 & Construction trades, n.e.c. & 8 & 6.77 & 2.92 & 13.33 \\
766 & Furnace, kiln, and oven operators, except food & 11 & 5.23 & 2.62 & 9.36 \\
709 & Grinding, abrading, buffing, and polishing machine operators & 7 & 4.53 & 1.82 & 9.34 \\
849 & Crane and tower operators & 9 & 3.87 & 1.78 & 7.35 \\
544 & Millwrights & 8 & 3.49 & 1.50 & 6.87 \\
779 & Machine operators, not specified & 30 & 2.94 & 1.99 & 4.20 \\
844 & Operating engineers & 15 & 2.31 & 1.29 & 3.82 \\
889 & Laborers, except construction & 84 & 2.19 & 1.76 & 2.73 \\
579 & Painters, construction and maintenance & 14 & 2.16 & 1.18 & 3.62 \\
633 & Supervisors, production occupations & 32 & 1.77 & 1.21 & 2.50 \\
869 & Construction laborers & 26 & 1.56 & 1.02 & 2.28 \\
\hline
\end{tabular}

COC - Census Occupation Code n.e.c. - not elsewhere classified LCL - lower confidence limit UCL - upper confidence limit NOTE: See appendices for source description, methods, and ICD codes, industry and occupation codes, and list of selected states and years. SOURCE: National Center for Health Statistics multiple cause of death data. 
공 Figure 3-3. Silicosis: Age-adjusted mortality rates by county, U.S. residents age 15 and over, 1970-1999

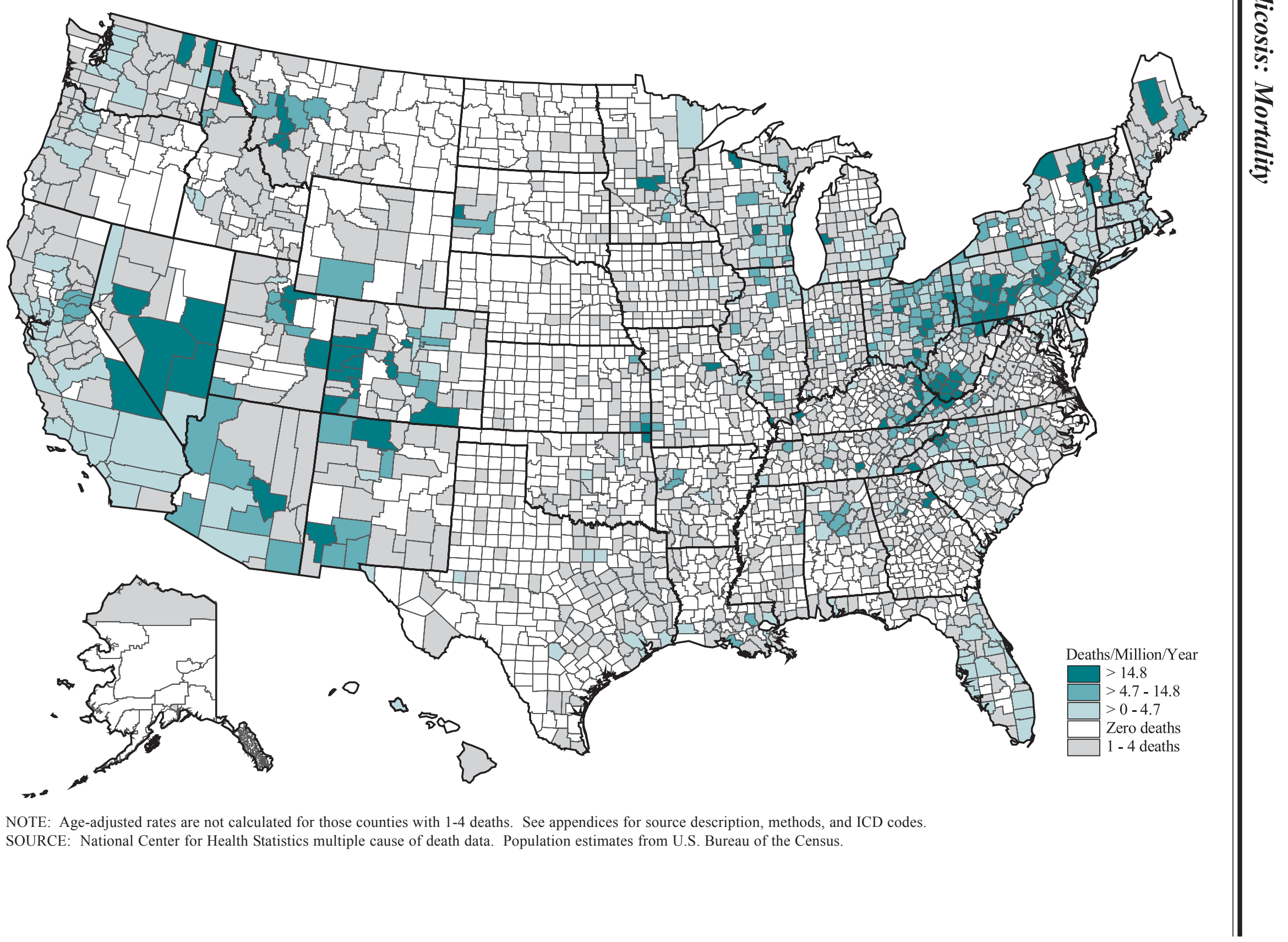


Figure 3-4. Silicosis: Age-adjusted mortality rates by county, U.S. residents age 15 and over, 1970-1984 and 1985-1999

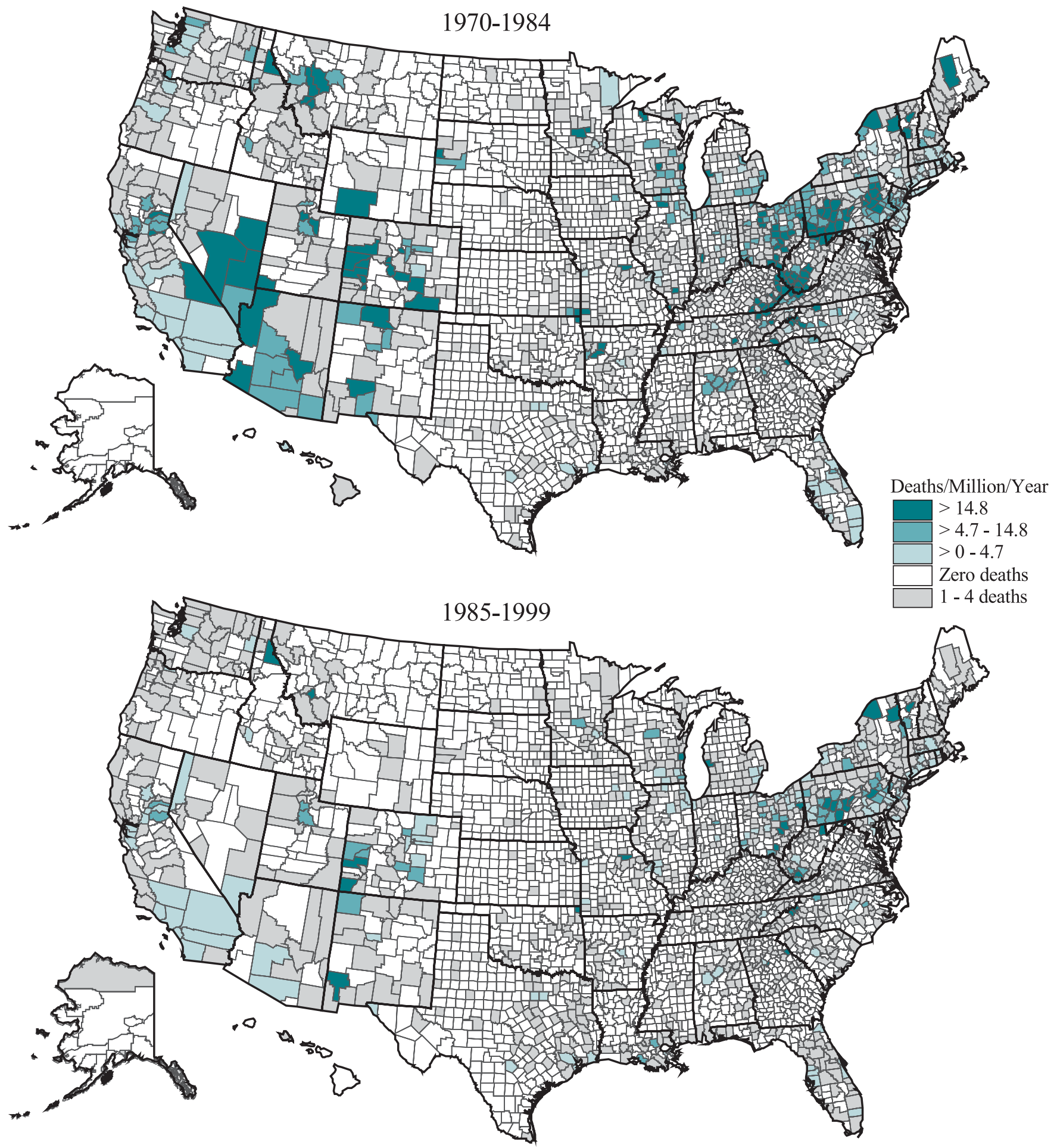

NOTE: Age-adjusted rates are not calculated for those counties with 1-4 deaths. See appendices for source description, methods, and ICD codes. SOURCE: National Center for Health Statistics multiple cause of death data. Population estimates from U.S. Bureau of the Census. 
Table 3-10. Silicosis: Counties with highest age-adjusted mortality rates (per million population), U.S. residents age 15 and over, 1985-1999

\begin{tabular}{|c|c|c|c|c|c|}
\hline County & State & Age-Adjusted Rate & Crude Rate & Number of Deaths & \% Female \\
\hline Mitchell County & North Carolina & 62.5 & 84.5 & 15 & 0.0 \\
\hline Silver Bow County & Montana & 44.7 & 56.7 & 23 & 0.0 \\
\hline Shoshone County & Idaho & 41.8 & 55.9 & 9 & 0.0 \\
\hline Yancey County & North Carolina & 40.3 & 51.9 & 10 & 0.0 \\
\hline Montrose County & Colorado & 35.3 & 44.4 & 13 & 0.0 \\
\hline Elbert County & Georgia & 35.1 & 41.1 & 9 & 0.0 \\
\hline Huntingdon County & Pennsylvania & 33.9 & 35.4 & 19 & 5.3 \\
\hline Avery County & North Carolina & 32.1 & 33.0 & 6 & 0.0 \\
\hline Perry County & Ohio & 31.6 & 32.8 & 12 & 25.0 \\
\hline Montezuma County & Colorado & 29.8 & 32.6 & 7 & 0.0 \\
\hline Washington County & Vermont & 26.8 & 27.6 & 18 & 0.0 \\
\hline Grant County & New Mexico & 26.0 & 28.2 & 9 & 0.0 \\
\hline Audrain County & Missouri & 25.7 & 36.3 & 10 & 0.0 \\
\hline Muskegon County & Michigan & 24.4 & 24.4 & 45 & 0.0 \\
\hline Morgan County & West Virginia & 23.1 & 32.3 & 5 & 20.0 \\
\hline Essex County & New York & 22.3 & 24.7 & 11 & 0.0 \\
\hline Jackson County & Ohio & 21.8 & 24.9 & 9 & 0.0 \\
\hline Preston County & West Virginia & 21.0 & 20.5 & 7 & 0.0 \\
\hline Schuylkill County & Pennsylvania & 19.9 & 28.1 & 53 & 0.0 \\
\hline Cambria County & Pennsylvania & 18.9 & 25.9 & 51 & 0.0 \\
\hline Sheboygan County & Wisconsin & 18.4 & 21.3 & 26 & 0.0 \\
\hline Rockdale County & Georgia & 17.9 & 10.4 & 7 & 0.0 \\
\hline Columbiana County & Ohio & 16.8 & 19.4 & 25 & 12.0 \\
\hline St. Lawrence County & New York & 16.6 & 15.6 & 21 & 0.0 \\
\hline Muskingum County & Ohio & 16.5 & 17.7 & 17 & 29.4 \\
\hline Indiana County & Pennsylvania & 16.2 & 16.5 & 18 & 0.0 \\
\hline Ottawa County & Oklahoma & 15.8 & 22.0 & 8 & 0.0 \\
\hline Bedford County & Pennsylvania & 15.5 & 17.4 & 10 & 0.0 \\
\hline Mifflin County & Pennsylvania & 15.4 & 18.1 & 10 & 0.0 \\
\hline Wyoming County & West Virginia & 15.0 & 14.9 & 5 & 0.0 \\
\hline Stearns County & Minnesota & 14.7 & 12.3 & 17 & 5.9 \\
\hline San Juan County & New Mexico & 14.5 & 10.3 & 10 & 0.0 \\
\hline Somerset County & Pennsylvania & 14.2 & 15.0 & 14 & 7.1 \\
\hline Armstrong County & Pennsylvania & 13.7 & 18.1 & 16 & 0.0 \\
\hline Delta County & Colorado & 12.6 & 18.9 & 5 & 0.0 \\
\hline Mesa County & Colorado & 11.6 & 13.2 & 15 & 0.0 \\
\hline Fremont County & Colorado & 11.0 & 15.0 & 6 & 0.0 \\
\hline Marion County & Ohio & 11.0 & 10.7 & 8 & 0.0 \\
\hline McDowell County & West Virginia & 11.0 & 12.8 & 5 & 0.0 \\
\hline St. Mary Parish & Louisiana & 10.8 & 9.6 & 6 & 0.0 \\
\hline Coshocton County & Ohio & 10.5 & 12.2 & 5 & 0.0 \\
\hline Logan County & West Virginia & 10.0 & 10.0 & 5 & 0.0 \\
\hline Surry County & North Carolina & 9.9 & 10.4 & 8 & 0.0 \\
\hline Rutland County & Vermont & 9.7 & 10.7 & 8 & 0.0 \\
\hline Clearfield County & Pennsylvania & 9.2 & 11.8 & 11 & 0.0 \\
\hline Carbon County & Pennsylvania & 9.0 & 11.4 & 8 & 0.0 \\
\hline Steuben County & New York & 8.4 & 8.7 & 10 & 0.0 \\
\hline Knox County & Ohio & 8.4 & 8.7 & 5 & 0.0 \\
\hline Northumberland County & Pennsylvania & 8.3 & 11.2 & 13 & 0.0 \\
\hline Nevada County & California & 7.9 & 9.1 & 9 & 0.0 \\
\hline Overall United States & & 1.4 & 1.3 & 4,002 & 3.7 \\
\hline
\end{tabular}

NOTE: Only counties with at least 5 deaths from the disease of interest are included. See appendices for source description, methods, and ICD codes. SOURCE: National Center for Health Statistics multiple cause of death data. Population estimates from U.S. Bureau of the Census. 
Table 3-11. Silicosis: Estimated number of discharges from short-stay nonfederal hospitals, 1970-2000

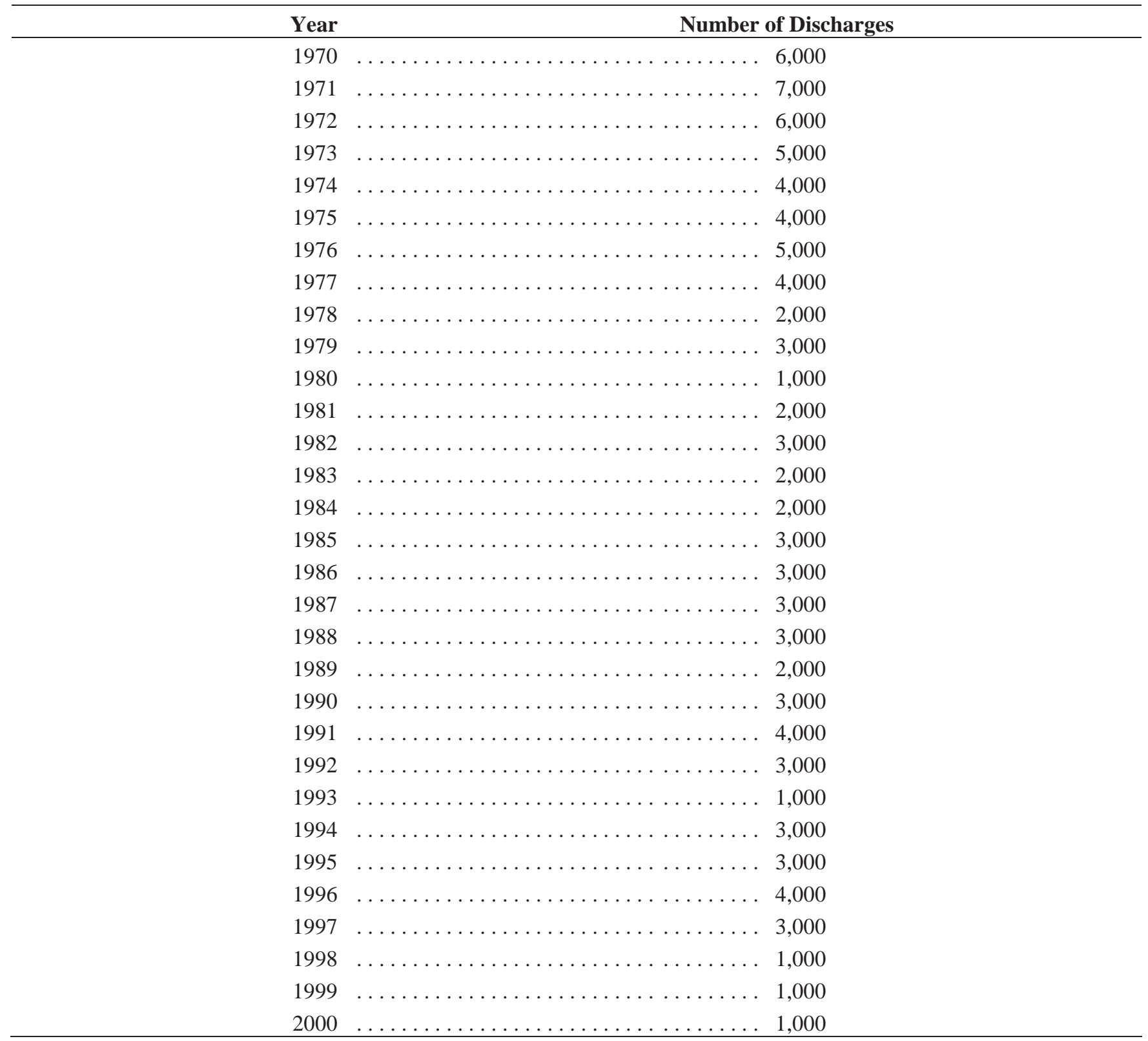

NOTE: Number of discharges has been rounded. NCHS recommends that, in statistical comparisons, estimates of less than 5,000 not be used and that estimates of 5,000 to 10,000 be used with caution. See appendices for source description and methods.

SOURCE: National Center for Health Statistics National Hospital Discharge Survey. 
Table 3-12. Silicosis: Number of cases by ascertainment source and state, 1989-1998

\begin{tabular}{|c|c|c|c|c|c|c|c|c|}
\hline \multirow[b]{2}{*}{ Source } & \multicolumn{2}{|c|}{ Michigan } & \multicolumn{2}{|c|}{ New Jersey } & \multicolumn{2}{|c|}{ Ohio } & \multicolumn{2}{|c|}{ Total } \\
\hline & No. & $\%$ & No. & $\%$ & No. & $\%$ & No. & $\%$ \\
\hline Health care professional report & 123 & 20.9 & 18 & 9.4 & 22 & 5.5 & 163 & 13.8 \\
\hline Hospital discharge data & 387 & 65.7 & 164 & 85.9 & 327 & 81.8 & 878 & 74.4 \\
\hline Death certificate data & 23 & 3.9 & 5 & 2.6 & 19 & 4.8 & 47 & 4.0 \\
\hline Workers' compensation files & 52 & 8.8 & - & - & 21 & 5.3 & 73 & 6.2 \\
\hline Other & 4 & 0.7 & 4 & 2.1 & 11 & 2.8 & 19 & 1.6 \\
\hline TOTAL & 589 & 100.0 & 191 & 100.0 & 400 & 100.0 & 1,180 & 100.0 \\
\hline
\end{tabular}

- indicates no cases reported.

NOTE: Percentages may not sum to $100 \%$ due to rounding. See appendices for source description.

SOURCE: Provisional SENSOR surveillance data as of September 2002, aggregated by reporting source years, and reported by K Rosenman, MJ Reilly, and D Kalinowski (Michigan); D Valiante and D Schill (New Jersey); and E Socie and A Migliozzi (Ohio). 
Table 3-13. Silicosis: Number of cases by duration of occupational exposure to silica and by state, 1989-1998

\begin{tabular}{|c|c|c|c|c|c|c|c|c|}
\hline \multirow[b]{2}{*}{$\begin{array}{l}\text { Years of Employment in Jobs } \\
\text { with Potential Silica Exposure }\end{array}$} & \multicolumn{2}{|c|}{ Michigan } & \multicolumn{2}{|c|}{ New Jersey } & \multicolumn{2}{|c|}{ Ohio } & \multicolumn{2}{|c|}{ Total } \\
\hline & No. & $\%$ & No. & $\%$ & No. & $\%$ & No. & $\%$ \\
\hline$<10$ & 41 & 7.0 & 19 & 9.9 & 23 & 5.8 & 83 & 7.0 \\
\hline 10 to 20 & 112 & 19.0 & 44 & 23.0 & 71 & 17.8 & 227 & 19.2 \\
\hline $21-30$ & 194 & 32.9 & 46 & 24.1 & 84 & 21.0 & 324 & 27.5 \\
\hline$>30$ & 215 & 36.5 & 56 & 29.3 & 110 & 27.5 & 381 & 32.3 \\
\hline Unknown & 27 & 4.6 & 26 & 13.6 & 112 & 28.0 & 165 & 14.0 \\
\hline TOTAL & 589 & 100.0 & 191 & 100.0 & 400 & 100.0 & 1,180 & 100.0 \\
\hline
\end{tabular}

NOTE: Percentages may not sum to $100 \%$ due to rounding. See appendices for source description.

SOURCE: Provisional SENSOR surveillance data as of September 2002, aggregated by reporting source years, and reported by K Rosenman, MJ Reilly, and D Kalinowski (Michigan); D Valiante and D Schill (New Jersey); and E Socie and A Migliozzi (Ohio). 


\section{Figure 3-5. Silicosis: Decade of first exposure for 986 confirmed cases, 1989-1998}

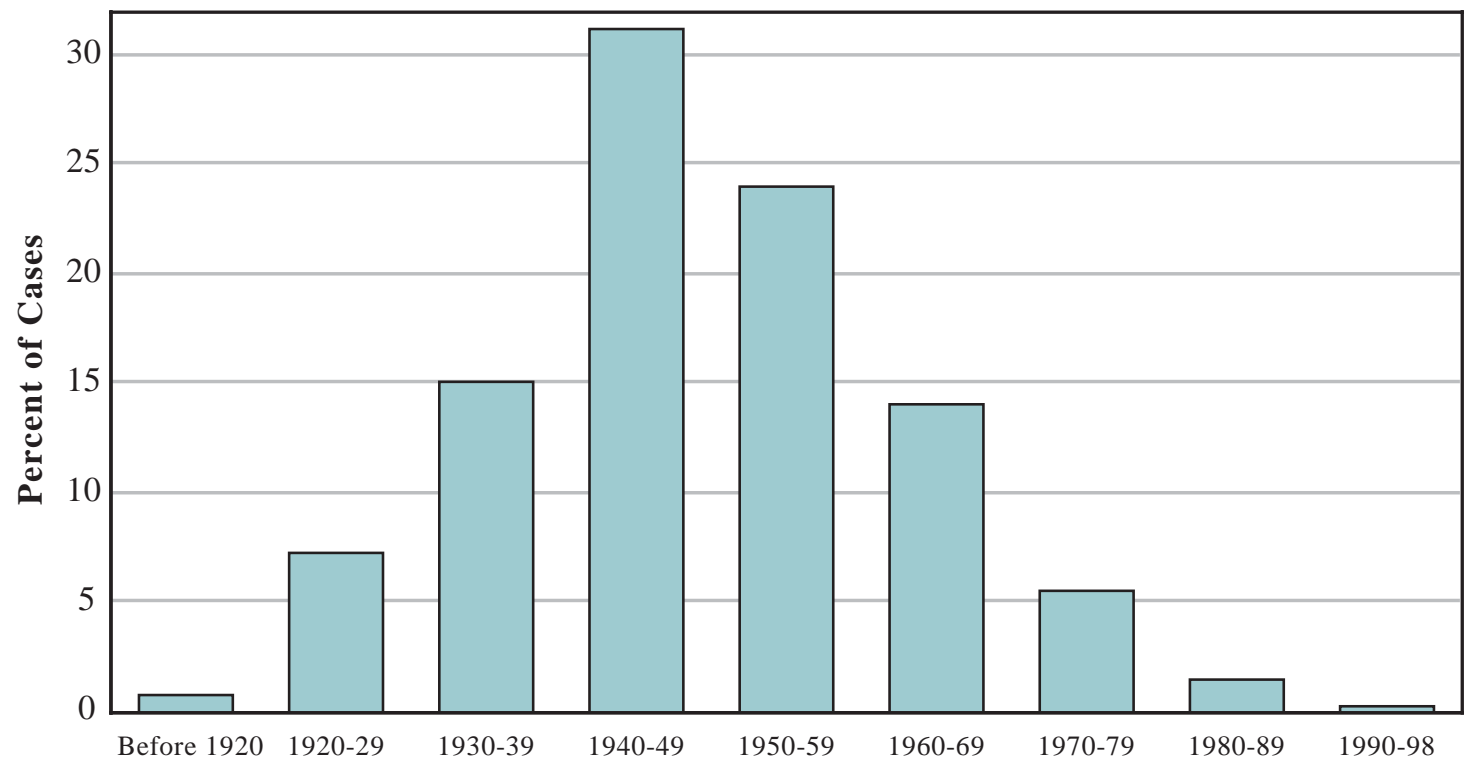

NOTE: Cases with unknown or missing values are excluded.

SOURCE: Provisional SENSOR surveillance data as of September, 2002, aggregated by reporting source years, and reported by K Rosenman, MJ Reilly, and D Kalinowski (Michigan); D Valiante and D Schill (New Jersey); and E Socie and A Migliozzi (Ohio). 
Table 3-14. Silicosis: Primary industries associated with silica exposure of silicosis cases by state, 1989-1998

\begin{tabular}{|c|c|c|c|c|c|c|c|c|}
\hline \multirow[b]{2}{*}{ Industry (SIC Code) } & \multicolumn{2}{|c|}{ Michigan } & \multicolumn{2}{|c|}{ New Jersey } & \multicolumn{2}{|c|}{ Ohio } & \multicolumn{2}{|c|}{ Total } \\
\hline & No. & $\%$ & No. & $\%$ & No. & $\%$ & No. & $\%$ \\
\hline Mining & 17 & 2.9 & 22 & 11.5 & 20 & 5.0 & 59 & 5.0 \\
\hline Mining and quarry of nonmetal minerals except fuel (14) & 1 & 0.2 & 17 & 8.9 & 15 & 3.8 & 33 & 2.8 \\
\hline Metal mining (10) & 15 & 2.5 & 4 & 2.1 & - & - & 19 & 1.6 \\
\hline All others $(12,13)$ & 1 & 0.2 & 1 & 0.5 & 5 & 1.3 & 7 & 0.6 \\
\hline Construction & 33 & 5.7 & 16 & 8.4 & 20 & 5.0 & 69 & 5.9 \\
\hline Construction, special trade contractors (17) & 24 & 4.1 & 8 & 4.2 & 16 & 4.0 & 48 & 4.1 \\
\hline Heavy construction other than building construction (16) & 4 & 0.7 & 8 & 4.2 & 4 & 1.0 & 16 & 1.4 \\
\hline All others (15) & 5 & 0.8 & - & - & - & - & 5 & 0.4 \\
\hline Manufacturing & 526 & 90.2 & 149 & 78.0 & 351 & 88.0 & 1,026 & 87.5 \\
\hline Primary metal industries (33) & 454 & 77.1 & 45 & 23.6 & 187 & 46.8 & 686 & 58.1 \\
\hline Stone, clay, glass, and concrete products (32) & 28 & 4.8 & 82 & 42.9 & 88 & 22.0 & 198 & 16.8 \\
\hline $\begin{array}{l}\text { Fabricated metal products except machinery and } \\
\text { transportation equipment }(34)\end{array}$ & 9 & 1.5 & 6 & 3.1 & 31 & 7.8 & 46 & 3.9 \\
\hline Transportation equipment (37) & 20 & 3.4 & 4 & 2.1 & 10 & 2.5 & 34 & 2.9 \\
\hline $\begin{array}{l}\text { Industrial and commercial machinery and computer } \\
\text { equipment (35) }\end{array}$ & 5 & 0.8 & 1 & 0.5 & 20 & 5.0 & 26 & 2.2 \\
\hline Chemicals and allied products (28) & 2 & 0.3 & 5 & 2.6 & 5 & 1.3 & 12 & 1.0 \\
\hline All others $(22,25,26,29,30,36,38,39)$ & 8 & 1.4 & 6 & 3.1 & 10 & 2.5 & 24 & 2.0 \\
\hline Transportation $(\mathbf{4 0 , 4 1 , 4 2 , 4 6 , 4 9 )}$ & 4 & 0.7 & 2 & 1.0 & - & - & 6 & 0.5 \\
\hline Wholesale trade (50) & 1 & 0.2 & - & - & 1 & 0.3 & 2 & 0.2 \\
\hline Finance, insurance, and real estate (65) & - & - & - & - & 1 & 0.3 & 1 & 0.1 \\
\hline Services $(75,76,80)$ & 2 & 0.3 & 2 & 1.0 & 2 & 0.5 & 6 & 0.5 \\
\hline Public administration (92) & - & - & - & - & 1 & 0.3 & $\mathbf{1}$ & 0.1 \\
\hline Nonclassifiable (99) & 6 & 1.0 & - & - & 3 & 0.8 & 9 & 0.8 \\
\hline TOTAL & 589 & 100.0 & 191 & 100.0 & 400 & 100.0 & 1,180 & 100.0 \\
\hline
\end{tabular}

- indicates no cases reported.

SIC - 1987 Standard Industrial Classification

NOTE: Percentages may not sum to $100 \%$ due to rounding. See appendices for source description.

SOURCE: Provisional SENSOR surveillance data as of September 2002, aggregated by reporting source years, and reported by K Rosenman, MJ Reilly, and D Kalinowski (Michigan); D Valiante and D Schill (New Jersey); and E Socie and A Migliozzi (Ohio). 
Table 3-15. Silicosis: Primary occupations associated with silica exposure of silicosis cases by state, 1989-1998

\begin{tabular}{|c|c|c|c|c|c|c|c|c|}
\hline \multirow[b]{2}{*}{ Occupation (COC) } & \multicolumn{2}{|c|}{ Michigan } & \multicolumn{2}{|c|}{ New Jersey } & \multicolumn{2}{|c|}{ Ohio } & \multicolumn{2}{|c|}{ Total } \\
\hline & No. & $\%$ & No. & $\%$ & No. & $\%$ & No. & $\%$ \\
\hline Managerial and professional specialty & 1 & 0.2 & 4 & 2.1 & 3 & 0.8 & 8 & 0.7 \\
\hline Technical, sales, and administrative & 5 & 0.8 & 2 & 1.0 & 1 & 0.3 & 8 & 0.7 \\
\hline Service & 4 & 0.7 & 1 & 0.5 & 3 & 0.8 & 8 & 0.7 \\
\hline Precision production, craft, and repair & 142 & 24.1 & 50 & 26.2 & 73 & 18.3 & 265 & 22.5 \\
\hline Hand molders and shapers, except jewelers (675) & 55 & 9.3 & 3 & 1.6 & 5 & 1.3 & 63 & 5.3 \\
\hline Supervisors, production (628) & 20 & 3.4 & 9 & 4.7 & 13 & 3.3 & 42 & 3.6 \\
\hline Brickmasons and stonemasons (563) & 12 & 2.0 & 7 & 3.7 & 12 & 3.0 & 31 & 2.6 \\
\hline Construction trades, n.e.c. (599) & 6 & 1.0 & 4 & 2.1 & 12 & 3.0 & 22 & 1.9 \\
\hline Mining machine operators (616) & 6 & 1.0 & 6 & 3.1 & 3 & 0.8 & 15 & 1.3 \\
\hline Millwrights (544) & 9 & 1.5 & - & - & 2 & 0.5 & 11 & 0.9 \\
\hline Mining occupations, n.e.c. (617) & 5 & 0.8 & 2 & 1.0 & 4 & 1.0 & 11 & 0.9 \\
\hline All others & 29 & 4.9 & 19 & 9.9 & 22 & 5.5 & 70 & 5.9 \\
\hline Operators, fabricators, and laborers & 360 & 61.1 & 107 & 56.0 & 301 & 75.3 & 768 & 65.1 \\
\hline Laborers, except construction (889) & 123 & 20.9 & 15 & 7.9 & 66 & 16.5 & 204 & 17.3 \\
\hline Molding and casting machine operators (719) & 59 & 10.0 & 7 & 3.7 & 39 & 9.8 & 105 & 8.9 \\
\hline Grinding, abrading, buffing, and polishing machine op. (709) & 49 & 8.3 & 3 & 1.6 & 24 & 6.0 & 76 & 6.4 \\
\hline Crushing and grinding machine operators (768) & 9 & 1.5 & 10 & 5.2 & 35 & 8.8 & 54 & 4.6 \\
\hline Miscellaneous metal and plastic processing machine op. (725) & 19 & 3.2 & 8 & 4.2 & 22 & 5.5 & 49 & 4.2 \\
\hline Furnace, kiln, and oven operators, excluding food (766) & 11 & 1.9 & 5 & 2.6 & 18 & 4.5 & 34 & 2.9 \\
\hline Miscellaneous machine operators, n.e.c. (777) & 10 & 1.7 & 4 & 2.1 & 17 & 4.3 & 31 & 2.6 \\
\hline Hand molding, casting, forming occupations (787) & 3 & 0.5 & 14 & 7.3 & 12 & 3.0 & 29 & 2.5 \\
\hline Miscellaneous hand working occupations (795) & 19 & 3.2 & 1 & 0.5 & 1 & 0.3 & 21 & 1.8 \\
\hline Mixing and blending machine operators (756) & 4 & 0.7 & 5 & 2.6 & 8 & 2.0 & 17 & 1.4 \\
\hline Machine operators, not specified (779) & 3 & 0.5 & 2 & 1.0 & 12 & 3.0 & 17 & 1.4 \\
\hline Welders and cutters (783) & 9 & 1.5 & 3 & 1.6 & 4 & 1.0 & 16 & 1.4 \\
\hline Construction laborers (869) & 2 & 0.3 & 6 & 3.1 & 4 & 1.0 & 12 & 1.0 \\
\hline Production inspectors, checkers, and examiners (796) & 5 & 0.8 & 2 & 1.0 & 4 & 1.0 & 11 & 0.9 \\
\hline Crane and tower operators (849) & 5 & 0.8 & 2 & 1.0 & 4 & 1.0 & 11 & 0.9 \\
\hline Painting and paint spraying machine operators (759) & - & - & 7 & 3.7 & 3 & 0.8 & 10 & 0.8 \\
\hline All others & 30 & 5.1 & 13 & 6.8 & 28 & 7.0 & 71 & 6.0 \\
\hline Unclassifiable and miscellaneous unemployed & 77 & 13.1 & 27 & 14.1 & 19 & 4.8 & 123 & 10.4 \\
\hline Occupation not reported & 77 & 13.1 & 27 & 14.1 & 19 & 4.8 & 123 & 10.4 \\
\hline TOTAL & 589 & 100.0 & 191 & 100.0 & 400 & 100.0 & 1,180 & 100.0 \\
\hline
\end{tabular}

- indicates no cases reported. $\quad$ COC - 1990 Census Occupation Code

n.e.c. - not elsewhere classified

NOTE: Percentages may not sum to $100 \%$ due to rounding. See appendices for source description.

SOURCE: Provisional SENSOR surveillance data as of September 2002, aggregated by reporting source years, and reported by K Rosenman, MJ Reilly, and D Kalinowski (Michigan); D Valiante and D Schill (New Jersey); and E Socie and A Migliozzi (Ohio). 
Figure 3-6a. Respirable quartz: Geometric mean exposures in coal mining, MSHA inspector and mine operator samples, 1982-1999

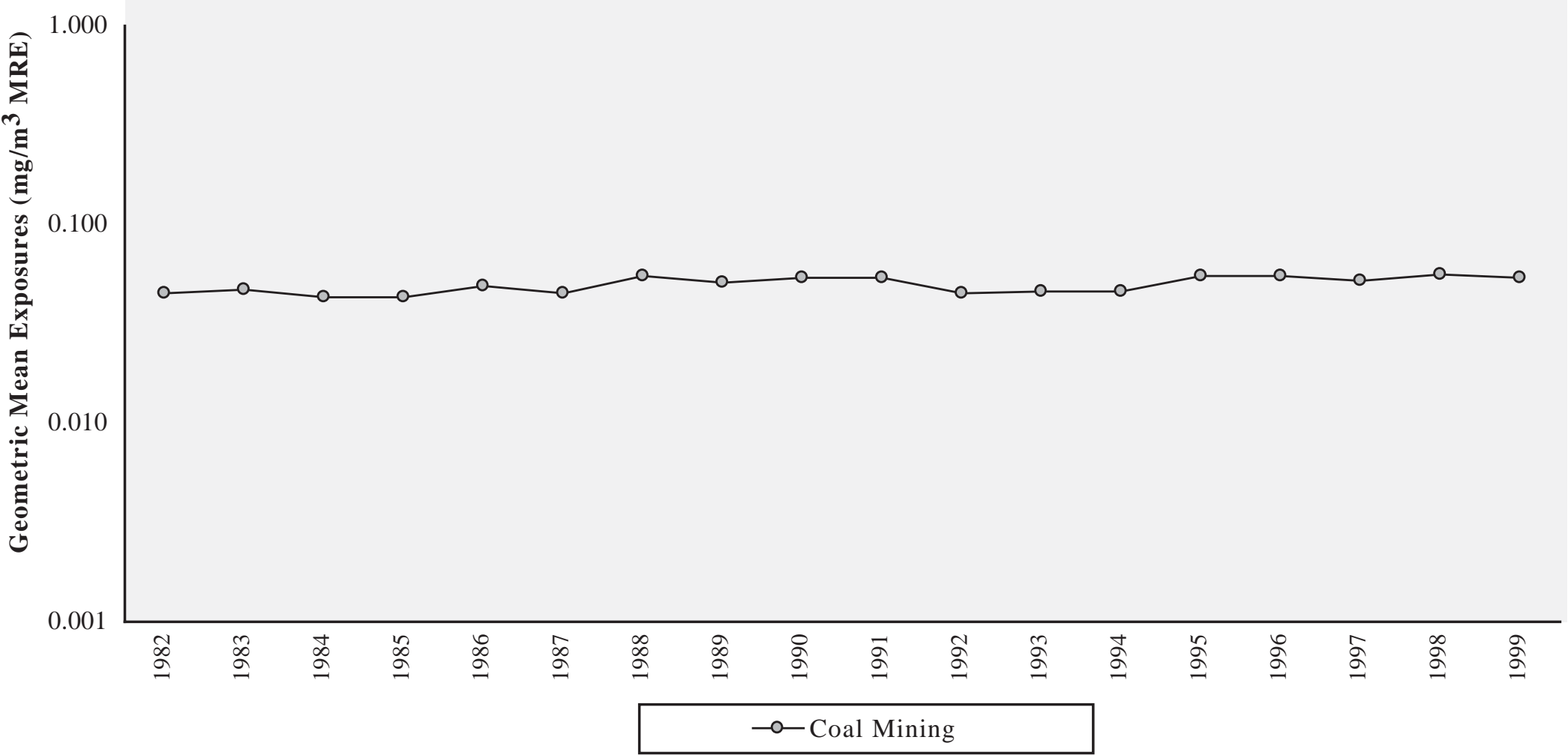

PEL - permissible exposure limit REL - recommended exposure limit $\mathrm{mg} / \mathrm{m}^{3}$ - milligrams per cubic meter MRE - Mining Research Establishment NOTE: For coal mining, the MSHA PEL is $\left[\left(10 \mathrm{mg} / \mathrm{m}^{3} \mathrm{MRE}\right) /(\%\right.$ quartz)] for respirable dust containing greater than 5 percent quartz. The MSHA respirable coal mine quartz exposure data and the NIOSH REL for respirable quartz cannot be compared to each other because they are based on different sampling criteria. See appendices for source description, methods, and agents.

SOURCE: Mine Safety and Health Administration (MSHA) coal mine inspector and mine operator quartz data. 
Figure 3-6b. Respirable quartz: Geometric mean exposures by major industry division, MSHA and OSHA samples, 1979-1999

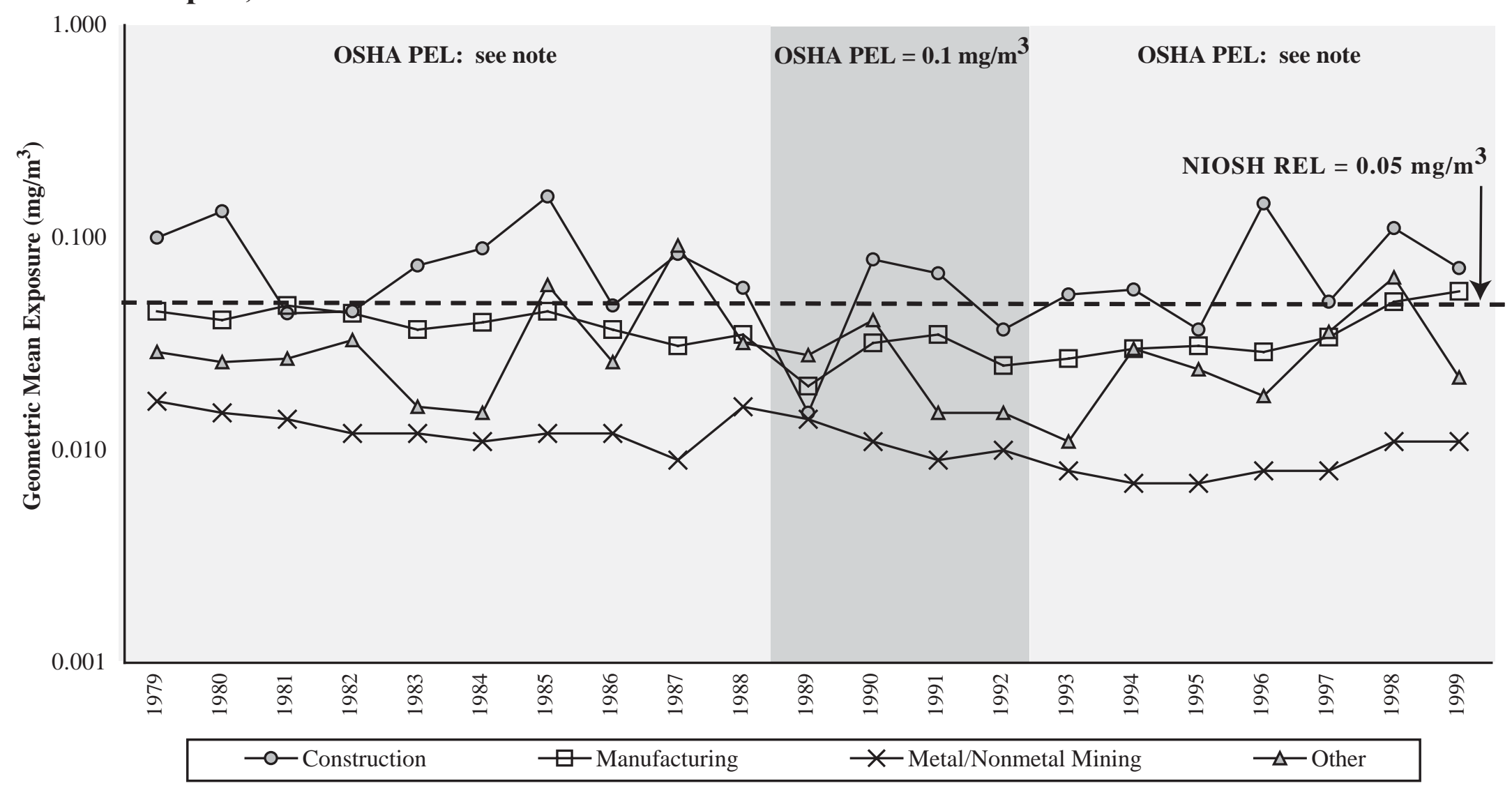

PEL - permissible exposure limit $\quad$ REL - recommended exposure limit $\quad \mathrm{mg} / \mathrm{m}^{3}$ - milligrams per cubic meter

NOTE: Before March 1, 1989 and after March 22, 1993, the OSHA PEL is [(10 mg/m $\left.\mathrm{m}^{3}\right) /(\%$ quartz + 2)], for respirable dust containing at least 1 percent quartz. From March 1, 1989 to March 22, 1993, the OSHA PEL was $0.1 \mathrm{mg} / \mathrm{m}^{3}$ for respirable quartz. For metal $/$ nonmetal mining, the MSHA PEL is $\left[\left(10 \mathrm{mg} / \mathrm{m}^{3}\right) /(\% \mathrm{quartz}+2)\right]$ for respirable dust containing at least 1 percent quartz. See appendices for source description, methods, and agents.

SOURCE: Mine Safety and Health Administration (MSHA) metal/nonmetal data. Occupational Safety and Health Administration (OSHA) Integrated Management Information System. 
Table 3-16a. Respirable quartz: Geometric mean exposures and percent exceeding designated occupational exposure limits in coal mining, MSHA inspector and mine operator samples, 1982-1999

\begin{tabular}{|c|c|c|c|c|c|c|c|c|c|c|c|c|c|c|c|c|c|c|c|}
\hline \multicolumn{2}{|c|}{ Industry Division } & 1982 & 1983 & 1984 & 1985 & 1986 & 1987 & 1988 & 1989 & 1990 & 1991 & 1992 & 1993 & 1994 & 1995 & 1996 & 1997 & 1998 & 1999 \\
\hline \multirow{3}{*}{$\begin{array}{l}\text { Coal Mining } \\
\text { SIC 11, 12 }\end{array}$} & $\begin{array}{l}\mathrm{GM} \\
\left(\mathrm{mg} / \mathrm{m}^{3} \mathrm{MRE}\right)\end{array}$ & 0.045 & 0.047 & 0.043 & 0.043 & 0.049 & 0.045 & 0.055 & 0.051 & 0.054 & 0.054 & 0.045 & 0.046 & 0.046 & 0.055 & 0.055 & 0.052 & 0.056 & 0.054 \\
\hline & No. of samples & 2,682 & 4,962 & 4,613 & 4,242 & 4,731 & 4,556 & 5,238 & 4,566 & 4,524 & 5,816 & 8,692 & 7,668 & 7,557 & 8,090 & 6,332 & 8,560 & 10,613 & 12,790 \\
\hline & $\%>$ PEL & 38.5 & 40.1 & 40.8 & 36.8 & 39.7 & 37.9 & 38.1 & 39.0 & 36.9 & 35.4 & 28.4 & 27.9 & 29.3 & 32.3 & 30.7 & 29.1 & 29.7 & 27.4 \\
\hline
\end{tabular}

- indicates incalculable field

SIC - Standard Industrial Classification

PEL - permissible exposure limit

REL - recommended exposure limit

GM - geometric mean

$\mathrm{mg} / \mathrm{m}^{3}$ - milligams per cubic meter

MRE - Mining Research Establishment

NOTE: For coal mining, the MSHA PEL is $\left[\left(10 \mathrm{mg} / \mathrm{m}^{3} \mathrm{MRE}\right) /(\%\right.$ quartz $\left.)\right]$ for respirable dust containing greater than 5 percent quartz. The MSHA respirable coal mine quartz exposure data and the NIOSH REL for respirable quartz cannot be compared to each other because they are based on different sampling criteria. See appendices for source description, methods, and agents. SOURCE: Mine Safety and Health Administration (MSHA) coal mine inspector and mine operator quartz data. 
Table 3-16b. Respirable quartz: Geometric mean exposures and percent exceeding designated occupational exposure limits by major industry division, MSHA and OSHA samples, 1979-1999

\begin{tabular}{|c|c|c|c|c|c|c|c|c|c|c|c|c|c|c|c|c|c|c|c|c|c|c|}
\hline \multirow{2}{*}{\multicolumn{2}{|c|}{ Industry Division }} & \multicolumn{10}{|c|}{ OSHA PEL: see note } & \multicolumn{4}{|c|}{ OSHA PEL $=0.1 \mathrm{mg} / \mathrm{m}^{3}$} & \multicolumn{7}{|c|}{ OSHA PEL: see note } \\
\hline & & 1979 & 1980 & 1981 & 1982 & 1983 & 1984 & 1985 & 1986 & 1987 & 1988 & 1989 & 1990 & 1991 & 1992 & 1993 & 1994 & 1995 & 1996 & 1997 & 1998 & 1999 \\
\hline \multirow{4}{*}{$\begin{array}{l}\text { Construction } \\
\text { SIC 15-17 }\end{array}$} & $\mathrm{GM}\left(\mathrm{mg} / \mathrm{m}^{3}\right)$ & 0.100 & 0.133 & 0.044 & 0.045 & 0.074 & 0.089 & 0.156 & 0.048 & 0.084 & 0.058 & 0.015 & 0.079 & 0.068 & 0.037 & 0.054 & 0.057 & 0.037 & 0.145 & 0.050 & 0.111 & 0.072 \\
\hline & No. of samples & 24 & 71 & 26 & 36 & 56 & 44 & 57 & 33 & 42 & 35 & 15 & 36 & 43 & 65 & 29 & 21 & 31 & 56 & 103 & 138 & 165 \\
\hline & $\%>$ PEL & 50.0 & 59.2 & 38.5 & 27.8 & 41.1 & 45.5 & 59.6 & 36.4 & 47.6 & 45.7 & 13.3 & 33.3 & 32.6 & 23.1 & 34.5 & 38.1 & 25.8 & 50.0 & 34.0 & 51.4 & 47.3 \\
\hline & $\%>$ REL & 58.3 & 67.6 & 50.0 & 44.4 & 64.3 & 52.3 & 64.9 & 39.4 & 64.3 & 51.4 & 20.0 & 52.8 & 39.5 & 35.4 & 55.2 & 61.9 & 29.0 & 62.5 & 47.6 & 63.0 & 58.2 \\
\hline \multirow{4}{*}{$\begin{array}{l}\text { Manufacturing } \\
\text { SIC 20-39 }\end{array}$} & $\mathrm{GM}\left(\mathrm{mg} / \mathrm{m}^{3}\right)$ & 0.045 & 0.041 & 0.048 & 0.044 & 0.037 & 0.040 & 0.045 & 0.037 & 0.031 & 0.035 & 0.020 & 0.032 & 0.035 & 0.025 & 0.027 & 0.030 & 0.031 & 0.029 & 0.034 & 0.050 & 0.056 \\
\hline & No. of samples & 745 & 1,394 & 1,269 & 1,298 & 1,065 & 852 & 1,075 & 715 & 744 & 644 & 527 & 641 & 595 & 606 & 422 & 346 & 299 & 277 & 400 & 606 & 653 \\
\hline & $\%>$ PEL & 30.3 & 30.8 & 33.2 & 30.5 & 26.9 & 31.9 & 34.3 & 27.3 & 26.5 & 30.7 & 16.1 & 20.1 & 22.2 & 13.5 & 18.2 & 24.3 & 24.7 & 23.8 & 26.0 & 37.8 & 38.3 \\
\hline & $\%>$ REL & 49.1 & 47.5 & 50.9 & 48.7 & 42.4 & 46.8 & 50.3 & 39.9 & 38.4 & 42.9 & 28.1 & 39.2 & 42.0 & 32.2 & 30.1 & 39.0 & 36.8 & 30.7 & 36.0 & 52.1 & 56.4 \\
\hline \multirow{4}{*}{$\begin{array}{l}\text { Metal/ } \\
\text { Nonmetal } \\
\text { Mining } \\
\text { SIC 10, } 14\end{array}$} & $\mathrm{GM}\left(\mathrm{mg} / \mathrm{m}^{3}\right)$ & 0.017 & 0.015 & 0.014 & 0.012 & 0.012 & 0.011 & 0.012 & 0.012 & 0.009 & 0.016 & 0.014 & 0.011 & 0.009 & 0.010 & 0.008 & 0.007 & 0.007 & 0.008 & 0.008 & 0.011 & 0.011 \\
\hline & No. of samples & 9,038 & 6,001 & 6,299 & 2,553 & 6,048 & 7,118 & 6,002 & 6,625 & 6,069 & 7,062 & 8,307 & 10,512 & 12,495 & 12,244 & 10,382 & 15,940 & 12,594 & 16,253 & 7,893 & 4,615 & 4,974 \\
\hline & $\%>$ PEL & 11.7 & 11.1 & 11.7 & 11.1 & 10.5 & 10.2 & 10.2 & 10.3 & 7.7 & 17.7 & 14.7 & 11.1 & 9.4 & 9.2 & 7.3 & 5.6 & 5.5 & 6.2 & 6.4 & 6.7 & 8.0 \\
\hline & $\%>\mathrm{REL}$ & 17.3 & 17.7 & 17.8 & 16.8 & 16.5 & 15.4 & 15.4 & 16.5 & 11.5 & 26.6 & 22.1 & 16.7 & 14.2 & 14.7 & 11.3 & 8.6 & 8.4 & 9.2 & 10.2 & 12.7 & 15.9 \\
\hline \multirow{4}{*}{$\begin{array}{l}\text { Other } \\
\text { SIC 1-9, 13, } \\
\quad 40-99\end{array}$} & $\mathrm{GM}\left(\mathrm{mg} / \mathrm{m}^{3}\right)$ & 0.029 & 0.026 & 0.027 & 0.033 & 0.016 & 0.015 & 0.060 & 0.026 & 0.092 & 0.032 & 0.028 & 0.041 & 0.015 & 0.015 & 0.011 & 0.030 & 0.024 & 0.018 & 0.036 & 0.065 & 0.022 \\
\hline & No. of samples & 36 & 36 & 15 & 15 & 30 & 4 & 26 & 39 & 71 & 62 & 56 & 31 & 48 & 40 & 22 & 33 & 26 & 41 & 29 & 26 & 49 \\
\hline & $\%>\mathrm{PEL}$ & 13.9 & 16.7 & 13.3 & 20.0 & 10.0 & 13.0 & 38.5 & 28.2 & 52.1 & 30.6 & 28.6 & 38.7 & 6.3 & 7.5 & 4.5 & 15.2 & 26.9 & 12.2 & 20.7 & 30.8 & 20.4 \\
\hline & $\%>$ REL & 36.1 & 22.2 & 26.7 & 26.7 & 20.0 & 21.7 & 61.5 & 35.9 & 60.6 & 40.3 & 33.9 & 41.9 & 22.9 & 12.5 & 9.1 & 27.3 & 26.9 & 19.5 & 27.6 & 57.7 & 24.5 \\
\hline
\end{tabular}

SIC - Standard Industrial Classification $\quad$ PEL - permissible exposure limit $\quad$ REL - recommended exposure limit $\quad$ GM - geometric mean $\mathrm{mg} / \mathrm{m}^{3}$ - milligams per cubic meter NOTE: Before March 1, 1989 and after March 22, 1993, the OSHA PEL is [(10 mg/m $) /(\%$ quartz + 2)], for respirable dust containing at least 1 percent quartz. From March 1, 1989 to March 22, 1993, the OSHA PEL was $0.1 \mathrm{mg} / \mathrm{m}^{3}$ for respirable quartz. For metal $/$ nonmetal mining, the MSHA PEL is $\left[\left(10 \mathrm{mg} / \mathrm{m}^{3}\right) /(\% \mathrm{quartz}+2)\right]$ for respirable dust containing at least 1 percent quartz. The NIOSH REL is $0.05 \mathrm{mg} / \mathrm{m}^{3}$. See appendices for source description, methods, and agents.

SOURCE: Mine Safety and Health Administration (MSHA) metal/nonmetal mine data. Occupational Safety and Health Administration (OSHA) Integrated Management Information System. 
Table 3-17. Respirable quartz: Number of samples, geometric mean exposures, and percent exceeding designated occupational exposure limits by industries with elevated silicosis mortality, MSHA inspector and mine operator and OSHA samples, 1990-1999

\begin{tabular}{|c|c|c|c|c|c|c|c|}
\hline \multicolumn{4}{|c|}{$\begin{array}{c}\text { Silicosis Mortality, } \\
\text { Selected States and Years, 1990-1999 }\end{array}$} & \multirow[b]{2}{*}{$\begin{array}{c}\text { Number of } \\
\text { Samples }\end{array}$} & \multirow[b]{2}{*}{$\begin{array}{c}\text { GM } \\
\left(\mathbf{m g} / \mathbf{m}^{3}\right)\end{array}$} & \multirow[b]{2}{*}{$\begin{array}{l}\%> \\
\text { PEL }\end{array}$} & \multirow[b]{2}{*}{$\begin{array}{l}\%> \\
\text { REL }\end{array}$} \\
\hline CIC & $\begin{array}{l}\text { Industries with elevated PMRs and most } \\
\text { frequently recorded on death certificates }\end{array}$ & $\begin{array}{l}\text { Number of } \\
\text { Deaths }\end{array}$ & PMR & & & & \\
\hline 040 & Metal mining & 86 & 41.70 & 8,382 & 0.013 & 12.4 & 19.4 \\
\hline 262 & $\begin{array}{l}\text { Miscellaneous nonmetallic mineral and stone } \\
\text { products }\end{array}$ & 44 & 30.72 & 391 & 0.041 & 30.2 & 47.3 \\
\hline 050 & $\begin{array}{l}\text { Nonmetallic mining and quarrying, except } \\
\text { fuel }\end{array}$ & 48 & 29.27 & 99,529 & 0.008 & 7.0 & 11.1 \\
\hline 271 & Iron and steel foundries & 48 & 21.14 & 1,766 & 0.047 & 29.3 & 50.8 \\
\hline 252 & Structural clay products & 20 & 19.72 & 213 & 0.045 & 32.9 & 51.6 \\
\hline 041 & Coal mining & 69 & 6.17 & 80,642 & $0.052 *$ & 30.1 & $-\AA^{\dagger}$ \\
\hline 270 & $\begin{array}{l}\text { Blast furnaces, steelworks, rolling and } \\
\text { finishing mills }\end{array}$ & 51 & 3.19 & 44 & 0.011 & 2.3 & 6.8 \\
\hline 331 & Machinery, except electrical, n.e.c. & 23 & 2.48 & 204 & 0.046 & 32.4 & 48.0 \\
\hline 392 & Not specified manufacturing industries & 33 & 1.55 & 0 & - & - & - \\
\hline 060 & Construction & 118 & 1.26 & 687 & 0.070 & 40.6 & 53.0 \\
\hline & All other industries & 317 & & 2,562 & 0.026 & 19.9 & 30.2 \\
\hline & TOTAL (excluding CIC 041) & & & 113,778 & 0.009 & 8.4 & 13.2 \\
\hline
\end{tabular}

- indicates incalculable field

* MRE equivalent

$\dagger$ The MSHA respirable coal mine quartz exposure data and the NIOSH REL for respirable quartz cannot be compared to each other because they are based on different sampling criteria.

CIC - Census Industry Code PEL - permissible exposure limit

$\mathrm{GM}$ - geometric mean $\mathrm{mg} / \mathrm{m}^{3}$ - milligrams per cubic meter

REL - recommended exposure limit

PMR - proportionate mortality ratio

classified

NOTE: Before March 1, 1989 and after March 22, 1993, the OSHA PEL is [(10 mg/m $\left.\mathrm{m}^{3}\right) /(\%$ quartz +2$\left.)\right]$, for respirable dust containing at least 1 percent quartz. From March 1, 1989 to March 22, 1993, the OSHA PEL was $0.1 \mathrm{mg} / \mathrm{m}^{3}$ for respirable quartz. For coal mining, the MSHA PEL is [(10 mg/ $\left.\mathrm{m}^{3} \mathrm{MRE}\right) /(\%$ quartz) $]$ for respirable dust containing greater than 5 percent quartz; for metal $/$ nonmetal mining, the MSHA PEL is $\left[\left(10 \mathrm{mg} / \mathrm{m}^{3}\right) /(\%\right.$ quartz $+2)$ ] for respirable dust containing at least 1 percent quartz. The NIOSH REL is $0.05 \mathrm{mg} / \mathrm{m}^{3}$. See appendices for source description, methods, ICD codes, industry codes, agents, and list of selected states and years for which usual industry has been reported.

SOURCE: Mine Safety and Health Administration (MSHA) metal/nonmetal and coal mine inspector and mine operator quartz data. Occupational Safety and Health Administration (OSHA) Integrated Management Information System. National Center for Health Statistics: multiple cause of death data. 
Table 3-18 (page 1 of 2). Respirable quartz: Geometric mean exposures and percent exceeding designated occupational exposure limits by MSHA coal mine district and state, MSHA inspector and mine operator samples, 1982-1999

\begin{tabular}{|c|c|c|c|c|c|c|c|c|c|c|c|}
\hline \multirow[b]{2}{*}{ MSHA Coal Mine District } & \multicolumn{2}{|c|}{ All years } & \multicolumn{3}{|c|}{1982 - 1988} & \multicolumn{3}{|c|}{1989 - 1992} & \multicolumn{3}{|c|}{$1993-1999$} \\
\hline & $\begin{array}{c}\text { No. of } \\
\text { Samples }\end{array}$ & $\begin{array}{c}\mathbf{G M} \\
\left(\mathrm{mg} / \mathbf{m}^{3}\right) \\
\end{array}$ & $\begin{array}{c}\text { No. of } \\
\text { Samples } \\
\end{array}$ & $\begin{array}{c}\text { GM } \\
\left(\mathbf{m g} / \mathbf{m}^{3}\right)\end{array}$ & $\begin{array}{l}\%> \\
\text { PEL }\end{array}$ & $\begin{array}{c}\text { No. of } \\
\text { Samples }\end{array}$ & $\begin{array}{c}\text { GM } \\
\left(\mathrm{mg} / \mathrm{m}^{3}\right)\end{array}$ & $\begin{array}{l}\%> \\
\text { PEL }\end{array}$ & \begin{tabular}{|c|}
$\begin{array}{c}\text { No. of } \\
\text { Samples }\end{array}$ \\
\end{tabular} & $\begin{array}{c}\mathbf{G M} \\
\left(\mathrm{mg} / \mathbf{m}^{3}\right)\end{array}$ & $\begin{array}{l}\%>> \\
\text { PEL }\end{array}$ \\
\hline District 1 (Anthracite coal mining regions in Pennsylvania) & 1,274 & 0.027 & 321 & 0.025 & 41.4 & 184 & 0.024 & 39.7 & 769 & 0.028 & 26.4 \\
\hline District 2 (Bituminous coal mining regions in Pennsylvania) & 13,802 & 0.043 & 4,123 & 0.043 & 34.7 & 2,564 & 0.041 & 25.7 & 7,115 & 0.044 & 20.8 \\
\hline District 3 & 12,214 & 0.036 & 3,867 & 0.032 & 28.8 & 1,923 & 0.040 & 23.5 & 6,424 & 0.036 & 19.6 \\
\hline Maryland & 761 & 0.042 & 128 & 0.056 & 39.8 & 46 & 0.037 & 4.3 & 587 & 0.040 & 16.9 \\
\hline Ohio & 4,334 & 0.040 & 1,962 & 0.040 & 33.3 & 823 & 0.040 & 24.5 & 1,549 & 0.042 & 21.8 \\
\hline Northern West Virginia & 7,119 & 0.032 & 1,777 & 0.025 & 23.1 & 1,054 & 0.040 & 23.5 & 4,288 & 0.034 & 19.1 \\
\hline District 4 (Southern West Virginia) & 21,177 & 0.060 & 7,130 & 0.053 & 40.3 & 4,338 & 0.058 & 38.1 & 9,709 & 0.068 & 36.9 \\
\hline District 5 (Virginia) & 14,293 & 0.062 & 4,492 & 0.057 & 45.1 & 3,341 & 0.061 & 39.0 & 6,460 & 0.066 & 34.4 \\
\hline District 6 (Eastern Kentucky) & 15,255 & 0.061 & 2,845 & 0.050 & 40.7 & 2,748 & 0.066 & 41.5 & 9,662 & 0.064 & 33.6 \\
\hline District 7 & 17,116 & 0.059 & 2,991 & 0.066 & 49.7 & 3,762 & 0.055 & 36.9 & 10,363 & 0.058 & 32.4 \\
\hline Central Kentucky & 15,102 & 0.059 & 2,463 & 0.068 & 51.2 & 3,152 & 0.056 & 37.3 & 9,487 & 0.058 & 32.4 \\
\hline North Carolina & 0 & - & 0 & - & - & 0 & - & - & 0 & - & - \\
\hline South Carolina & 0 & - & 0 & - & - & 0 & - & - & 0 & - & - \\
\hline Tennessee & 2,014 & 0.057 & 528 & 0.057 & 42.4 & 610 & 0.053 & 34.8 & 876 & 0.060 & 32.3 \\
\hline Northern Georgia & 0 & - & 0 & - & - & 0 & - & - & 0 & - & - \\
\hline District 8 & 7,297 & 0.046 & 2,122 & 0.047 & 33.8 & 1,567 & 0.042 & 22.0 & 3,608 & 0.048 & 27.1 \\
\hline Illinois & 6,160 & 0.045 & 1,719 & 0.046 & 32.8 & 1,399 & 0.041 & 20.9 & 3,042 & 0.045 & 24.5 \\
\hline Indiana & 1,042 & 0.061 & 342 & 0.051 & 38.0 & 156 & 0.056 & 32.1 & 544 & 0.070 & 41.5 \\
\hline Iowa & 54 & 0.024 & 39 & 0.023 & 38.5 & 9 & 0.027 & 22.2 & 6 & 0.022 & 0.0 \\
\hline Michigan & 0 & - & 0 & - & - & 0 & - & - & 0 & - & - \\
\hline Minnesota & 0 & - & 0 & - & - & 0 & - & - & 0 & - & - \\
\hline Northern Missouri & 41 & 0.051 & 22 & 0.071 & 40.9 & 3 & 0.031 & 0.0 & 16 & 0.036 & 31.3 \\
\hline Wisconsin & 0 & - & 0 & - & - & 0 & - & - & 0 & - & - \\
\hline
\end{tabular}

See footnotes at end of table. 
Table 3-18 (page 2 of 2). Respirable quartz: Geometric mean exposures and percent exceeding designated occupational exposure limits by MSHA coal mine district and state, MSHA inspector and mine operator samples, 1982-1999

\begin{tabular}{|c|c|c|c|c|c|c|c|c|c|c|c|}
\hline \multirow[b]{2}{*}{ MSHA Coal Mine District } & \multicolumn{2}{|c|}{ All years } & \multicolumn{3}{|c|}{$1982-1988$} & \multicolumn{3}{|c|}{1989 - 1992} & \multicolumn{3}{|c|}{1993 - 1999} \\
\hline & \begin{tabular}{|c|} 
No. of \\
Samples
\end{tabular} & $\begin{array}{c}\mathbf{G M} \\
\left(\mathrm{mg} / \mathbf{m}^{3}\right)\end{array}$ & \begin{tabular}{|c|} 
No. of \\
Samples
\end{tabular} & $\begin{array}{c}\mathbf{G M} \\
\left(\mathrm{mg} / \mathbf{m}^{3}\right)\end{array}$ & $\begin{array}{l}\%> \\
\text { PEL }\end{array}$ & $\begin{array}{c}\text { No. of } \\
\text { Samples }\end{array}$ & $\begin{array}{c}\text { GM } \\
\left(\mathrm{mg} / \mathbf{m}^{3}\right)\end{array}$ & $\begin{array}{l}\%> \\
\text { PEL }\end{array}$ & $\begin{array}{c}\text { No. of } \\
\text { Samples }\end{array}$ & $\begin{array}{c}\text { GM } \\
\left(\mathrm{mg} / \mathrm{m}^{3}\right)\end{array}$ & $\begin{array}{l}\%> \\
\text { PEL }\end{array}$ \\
\hline District 9 & 5,372 & 0.036 & 1,403 & 0.041 & 42.3 & 1,357 & 0.031 & 33.8 & 2,612 & 0.036 & 23.9 \\
\hline Alaska & 12 & 0.017 & 10 & 0.014 & 10.0 & 2 & 0.051 & 50.0 & 0 & - & - \\
\hline Arizona & 42 & 0.056 & 6 & 0.023 & 33.3 & 12 & 0.056 & 41.7 & 24 & 0.070 & 20.8 \\
\hline Arkansas & 9 & 0.082 & 6 & 0.120 & 66.7 & 1 & 0.009 & 100.0 & 2 & 0.077 & 50.0 \\
\hline California & 1 & 0.025 & 0 & - & - & 0 & - & - & 1 & 0.025 & 0.0 \\
\hline Colorado & 1,678 & 0.037 & 447 & 0.041 & 45.0 & 425 & 0.033 & 36.0 & 806 & 0.036 & 21.5 \\
\hline Hawaii & 0 & - & 0 & - & - & 0 & - & - & 0 & - & - \\
\hline Idaho & 0 & - & 0 & - & - & 0 & - & - & 0 & - & - \\
\hline Kansas & 24 & 0.061 & 9 & 0.115 & 66.7 & 7 & 0.027 & 0.0 & 8 & 0.061 & 12.5 \\
\hline Louisiana & 10 & 0.009 & 0 & - & - & 2 & 0.038 & 0.0 & 8 & 0.006 & 0.0 \\
\hline Southern Missouri & 42 & 0.068 & 16 & 0.114 & 62.5 & 4 & 0.077 & 25.0 & 22 & 0.046 & 18.2 \\
\hline Montana & 97 & 0.042 & 36 & 0.038 & 38.9 & 14 & 0.051 & 42.9 & 47 & 0.042 & 29.8 \\
\hline Nebraska & 0 & - & 0 & - & - & 0 & - & - & 0 & - & - \\
\hline Nevada & 0 & - & 0 & - & - & 0 & - & - & 0 & - & - \\
\hline New Mexico & 299 & 0.048 & 117 & 0.050 & 34.2 & 48 & 0.050 & 35.4 & 134 & 0.046 & 23.1 \\
\hline North Dakota & 47 & 0.012 & 29 & 0.012 & 13.8 & 15 & 0.010 & 20.0 & 3 & 0.026 & 0.0 \\
\hline Oklahoma & 382 & 0.080 & 162 & 0.121 & 62.3 & 84 & 0.068 & 39.3 & 136 & 0.055 & 29.4 \\
\hline Oregon & 0 & - & 0 & - & - & 0 & - & - & 0 & - & - \\
\hline Texas & 133 & 0.041 & 52 & 0.098 & 57.7 & 25 & 0.016 & 24.0 & 56 & 0.028 & 23.2 \\
\hline Utah & 2,031 & 0.033 & 338 & 0.033 & 38.8 & 608 & 0.028 & 32.9 & 1,085 & 0.036 & 25.2 \\
\hline Washington & 21 & 0.028 & 2 & 0.016 & 0.0 & 9 & 0.019 & 0.0 & 10 & 0.044 & 20.0 \\
\hline Wyoming & 544 & 0.021 & 173 & 0.017 & 28.3 & 101 & 0.020 & 31.7 & 270 & 0.025 & 25.2 \\
\hline District 10 (Western Kentucky) & 4,515 & 0.023 & 1,156 & 0.021 & 26.0 & 951 & 0.023 & 19.6 & 2,408 & 0.025 & 17.4 \\
\hline District 11 & 3,917 & 0.056 & 574 & 0.058 & 40.2 & 863 & 0.063 & 36.8 & 2,480 & 0.053 & 27.5 \\
\hline Alabama & 3,917 & 0.056 & 574 & 0.058 & 40.2 & 863 & 0.063 & 36.8 & 2,480 & 0.053 & 27.5 \\
\hline Central and Southern Georgia & 0 & - & 0 & - & - & 0 & - & - & 0 & - & - \\
\hline Florida & 0 & - & 0 & - & - & 0 & - & - & 0 & - & - \\
\hline Mississippi & 0 & - & 0 & - & - & 0 & - & - & 0 & - & - \\
\hline Puerto Rico & 0 & - & 0 & - & - & 0 & - & - & 0 & - & - \\
\hline Virgin Islands & 0 & - & 0 & - & - & 0 & - & - & 0 & - & - \\
\hline TOTAL & 116,232 & 0.050 & 31,024 & 0.047 & 38.9 & 23,598 & 0.050 & 33.8 & 61,610 & 0.052 & 29.3 \\
\hline
\end{tabular}


¿ Table 3-19 (page 1 of 2). Respirable quartz: Geometric mean exposures and percent exceeding designated occupational exposure limits by MSHA metal/nonmetal mine district and state, MSHA samples, 1979-1999

\begin{tabular}{|c|c|c|c|c|c|c|c|c|c|c|c|c|c|c|}
\hline \multirow[b]{2}{*}{$\begin{array}{l}\text { MSHA Metal/Nonmetal } \\
\text { Mine District }\end{array}$} & \multicolumn{2}{|c|}{ All years } & \multicolumn{4}{|c|}{1979 - 1988} & \multicolumn{4}{|c|}{1989 - 1992} & \multicolumn{4}{|c|}{1993 - 1999} \\
\hline & $\begin{array}{c}\text { No. of } \\
\text { Samples }\end{array}$ & $\begin{array}{c}\mathbf{G M} \\
\left(\mathbf{m g} / \mathbf{m}^{3}\right) \\
\end{array}$ & \begin{tabular}{|c|} 
No. of \\
Samples
\end{tabular} & $\begin{array}{c}\mathbf{G M} \\
\left(\mathbf{m g} / \mathbf{m}^{3}\right)\end{array}$ & $\begin{array}{l}\%> \\
\text { PEL }\end{array}$ & $\begin{array}{l}\%> \\
\text { REL }\end{array}$ & $\begin{array}{c}\begin{array}{c}\text { No. of } \\
\text { Samples }\end{array} \\
\end{array}$ & $\begin{array}{c}\mathbf{G M} \\
\left(\mathbf{m g} / \mathbf{m}^{3}\right)\end{array}$ & $\begin{array}{l}\%> \\
\text { PEL }\end{array}$ & $\begin{array}{l}\%> \\
\text { REL }\end{array}$ & $\begin{array}{c}\text { No. of } \\
\text { Samples }\end{array}$ & $\begin{array}{c}\mathbf{G M} \\
\left(\mathbf{m g} / \mathbf{m}^{3}\right)\end{array}$ & $\begin{array}{l}\%> \\
\text { PEL }\end{array}$ & $\begin{array}{l}\%> \\
\text { REL }\end{array}$ \\
\hline Northeast & 26,788 & 0.011 & 8,979 & 0.015 & 12.4 & 19.4 & 6,060 & 0.011 & 9.9 & 16.1 & 11,749 & 0.009 & 6.5 & 10.9 \\
\hline Connecticut & 718 & 0.013 & 285 & 0.027 & 26.3 & 38.2 & 156 & 0.013 & 13.5 & 23.1 & 277 & 0.006 & 2.5 & 5.1 \\
\hline Delaware & 91 & 0.005 & 17 & 0.006 & 0.0 & 0.0 & 33 & 0.005 & 0.0 & 3.0 & 41 & 0.004 & 4.9 & 0.0 \\
\hline District of Columbia & 0 & - & 0 & - & - & - & 0 & - & - & - & 0 & - & - & - \\
\hline Maine & 623 & 0.012 & 199 & 0.025 & 16.6 & 24.6 & 169 & 0.009 & 3.0 & 7.7 & 255 & 0.009 & 4.3 & 9.8 \\
\hline Massachusetts & 1,280 & 0.013 & 476 & 0.025 & 17.4 & 28.6 & 250 & 0.010 & 9.6 & 16.0 & 554 & 0.008 & 3.6 & 7.0 \\
\hline New Hampshire & 497 & 0.014 & 131 & 0.028 & 15.3 & 33.6 & 133 & 0.010 & 7.5 & 16.5 & 233 & 0.012 & 7.7 & 12.9 \\
\hline New Jersey & 2,524 & 0.012 & 992 & 0.020 & 17.5 & 27.5 & 623 & 0.012 & 12.4 & 20.1 & 909 & 0.007 & 4.2 & 8.0 \\
\hline New York & 6,153 & 0.010 & 1,875 & 0.014 & 11.4 & 17.0 & 1,649 & 0.009 & 6.2 & 11.8 & 2,629 & 0.008 & 5.6 & 9.7 \\
\hline Pennsylvania & 6,344 & 0.012 & 2,324 & 0.014 & 9.3 & 15.2 & 1,212 & 0.014 & 13.8 & 20.5 & 2,808 & 0.010 & 8.1 & 12.5 \\
\hline Rhode Island & 201 & 0.018 & 57 & 0.030 & 22.8 & 36.8 & 35 & 0.014 & 8.6 & 25.7 & 109 & 0.015 & 16.5 & 25.7 \\
\hline Vermont & 1,435 & 0.016 & 372 & 0.019 & 22.0 & 31.5 & 334 & 0.013 & 19.2 & 26.3 & 729 & 0.016 & 19.5 & 29.8 \\
\hline Virginia & 4,009 & 0.008 & 1,126 & 0.011 & 6.6 & 12.0 & 808 & 0.009 & 8.5 & 13.2 & 2,075 & 0.007 & 4.2 & 7.4 \\
\hline Southeast & 37,427 & 0.008 & 13,902 & 0.010 & 7.5 & 12.4 & 9,250 & 0.008 & 8.2 & 12.8 & 14,275 & 0.007 & 4.9 & 8.2 \\
\hline Alabama & 3,246 & 0.007 & 1,034 & 0.007 & 4.2 & 6.6 & 876 & 0.007 & 8.0 & 11.9 & 1,336 & 0.007 & 6.3 & 9.6 \\
\hline Florida & 2,998 & 0.005 & 830 & 0.006 & 3.7 & 7.1 & 827 & 0.005 & 2.2 & 5.0 & 1,341 & 0.004 & 1.3 & 2.3 \\
\hline Georgia & 6,163 & 0.010 & 2,464 & 0.014 & 11.0 & 19.6 & 1,567 & 0.010 & 10.6 & 15.3 & 2,132 & 0.007 & 5.3 & 7.7 \\
\hline Kentucky & 2,424 & 0.008 & 943 & 0.009 & 7.5 & 10.4 & 480 & 0.008 & 9.4 & 11.0 & 1,001 & 0.006 & 3.7 & 5.7 \\
\hline Mississippi & 1,690 & 0.008 & 273 & 0.007 & 4.4 & 6.6 & 476 & 0.007 & 5.5 & 8.2 & 941 & 0.009 & 7.0 & 11.8 \\
\hline North Carolina & 8,090 & 0.008 & 3,347 & 0.010 & 6.4 & 11.4 & 2,166 & 0.008 & 6.7 & 11.1 & 2,577 & 0.006 & 3.8 & 6.9 \\
\hline Puerto Rico & 1,237 & 0.005 & 586 & 0.004 & 0.9 & 0.2 & 29 & 0.007 & 3.4 & 10.3 & 622 & 0.005 & 3.1 & 3.1 \\
\hline South Carolina & 4,011 & 0.009 & 1,054 & 0.016 & 17.3 & 27.6 & 1,130 & 0.009 & 9.7 & 16.1 & 1,827 & 0.007 & 3.6 & 7.7 \\
\hline Tennessee & 7,490 & 0.009 & 3,327 & 0.009 & 6.3 & 9.9 & 1,698 & 0.010 & 10.5 & 16.3 & 2,465 & 0.009 & 7.8 & 13.8 \\
\hline Virgin Islands & 78 & 0.006 & 44 & 0.004 & 0.0 & 0.0 & 1 & 0.010 & 0.0 & 0.0 & 33 & 0.011 & 3.0 & 9.1 \\
\hline North Central & 35,491 & 0.009 & 12,515 & 0.011 & 9.3 & 13.8 & 8,549 & 0.011 & 10.8 & 15.8 & 14,427 & 0.008 & 5.2 & 8.9 \\
\hline Illinois & 6,542 & 0.011 & 2,796 & 0.014 & 16.3 & 21.0 & 1,387 & 0.011 & 13.0 & 19.3 & 2,359 & 0.007 & 4.7 & 8.3 \\
\hline Indiana & 4,021 & 0.007 & 1,174 & 0.007 & 2.9 & 3.3 & 917 & 0.007 & 4.5 & 6.0 & 1,930 & 0.006 & 3.0 & 5.0 \\
\hline Iowa & 2,118 & 0.006 & 675 & 0.007 & 4.9 & 5.0 & 570 & 0.006 & 5.3 & 4.9 & 873 & 0.005 & 1.5 & 1.8 \\
\hline Michigan & 5,112 & 0.011 & 1,397 & 0.012 & 8.4 & 15.3 & 1,459 & 0.013 & 14.3 & 19.9 & 2,256 & 0.009 & 6.8 & 11.3 \\
\hline Wisconsin & 4,682 & 0.010 & 1,710 & 0.011 & 8.8 & 13.3 & 1,031 & 0.013 & 14.9 & 20.5 & 1,941 & 0.008 & 5.6 & 9.9 \\
\hline
\end{tabular}

See footnotes at end of table. 
Table 3-19 (page 2 of 2). Respirable quartz: Geometric mean exposures and percent exceeding designated occupational exposure limits by MSHA metal/nonmetal mine district and state, MSHA samples, 1979-1999

\begin{tabular}{|c|c|c|c|c|c|c|c|c|c|c|c|c|c|c|}
\hline \multirow[b]{2}{*}{$\begin{array}{l}\text { MSHA Metal/Nonmetal } \\
\text { Mine District }\end{array}$} & \multicolumn{2}{|c|}{ All years } & \multicolumn{4}{|c|}{1979 - 1988} & \multicolumn{4}{|c|}{1989 - 1992} & \multicolumn{4}{|c|}{1993 - 1999} \\
\hline & $\begin{array}{c}\text { No. of } \\
\text { Samples } \\
\end{array}$ & $\begin{array}{c}\mathbf{G M} \\
\left(\mathbf{m g} / \mathbf{m}^{3}\right)\end{array}$ & \begin{tabular}{|c|} 
No. of \\
Samples
\end{tabular} & $\begin{array}{c}\text { GM } \\
\left(\mathbf{m g} / \mathbf{m}^{3}\right)\end{array}$ & $\begin{array}{l}\%> \\
\text { PEL }\end{array}$ & $\begin{array}{l}\%> \\
\text { REL }\end{array}$ & \begin{tabular}{|c|} 
No. of \\
Samples \\
\end{tabular} & $\begin{array}{c}\mathbf{G M} \\
\left(\mathbf{m g} / \mathbf{m}^{3}\right)\end{array}$ & $\begin{array}{l}\%> \\
\text { PEL }\end{array}$ & $\begin{array}{l}\%> \\
\text { REL }\end{array}$ & \begin{tabular}{|c|} 
No. of \\
Samples
\end{tabular} & $\begin{array}{c}\mathbf{G M} \\
\left(\mathbf{m g} / \mathbf{m}^{3}\right)\end{array}$ & $\begin{array}{l}\%> \\
\text { PEL }\end{array}$ & $\begin{array}{l}\%> \\
\text { REL }\end{array}$ \\
\hline South Central & 31,305 & 0.009 & 7,465 & 0.013 & 13.0 & 17.6 & 9,403 & 0.009 & 9.5 & 13.7 & 14,437 & 0.007 & 5.5 & 7.9 \\
\hline Arkansas & 2,396 & 0.015 & 544 & 0.038 & 31.6 & 45.8 & 660 & 0.017 & 16.7 & 28.5 & 1,192 & 0.009 & 7.9 & 13.8 \\
\hline Louisiana & 2,421 & 0.006 & 279 & 0.007 & 7.2 & 11.8 & 716 & 0.005 & 4.2 & 6.3 & 1,426 & 0.006 & 4.3 & 7.6 \\
\hline Missouri & 7,486 & 0.010 & 2,347 & 0.013 & 13.0 & 16.4 & 2,008 & 0.011 & 11.6 & 15.1 & 3,131 & 0.007 & 5.0 & 7.5 \\
\hline New Mexico & 3,729 & 0.013 & 1,013 & 0.014 & 14.4 & 19.7 & 1,059 & 0.015 & 13.1 & 21.1 & 1,657 & 0.011 & 11.9 & 16.0 \\
\hline Oklahoma & 4,890 & 0.009 & 1,570 & 0.011 & 9.9 & 13.2 & 1,340 & 0.011 & 10.9 & 16.6 & 1,980 & 0.007 & 4.3 & 7.7 \\
\hline Texas & 10,383 & 0.007 & 1,712 & 0.011 & 10.0 & 14.1 & 3,620 & 0.007 & 6.4 & 8.4 & 5,051 & 0.006 & 4.0 & 4.4 \\
\hline Rocky Mountain & 31,645 & 0.015 & 15,364 & 0.017 & 14.5 & 22.2 & 5,927 & 0.017 & 16.7 & 26.2 & 10,354 & 0.012 & 10.5 & 16.6 \\
\hline Arizona & 5,176 & 0.017 & 2,380 & 0.023 & 18.0 & 27.1 & 717 & 0.019 & 18.8 & 29.0 & 2,079 & 0.011 & 9.8 & 15.3 \\
\hline Colorado & 6,090 & 0.015 & 3,459 & 0.014 & 12.5 & 19.9 & 1,168 & 0.019 & 17.4 & 27.6 & 1,463 & 0.017 & 12.5 & 23.3 \\
\hline Kansas & 2,713 & 0.011 & 1,280 & 0.013 & 12.3 & 14.1 & 542 & 0.011 & 9.8 & 12.5 & 891 & 0.009 & 7.2 & 8.3 \\
\hline Montana & 2,930 & 0.014 & 1,370 & 0.012 & 10.4 & 15.3 & 667 & 0.023 & 22.5 & 33.7 & 893 & 0.013 & 11.8 & 19.6 \\
\hline Nebraska & 780 & 0.006 & 53 & 0.006 & 5.7 & 5.7 & 254 & 0.006 & 2.8 & 4.7 & 473 & 0.005 & 1.9 & 2.5 \\
\hline Nevada & 3,649 & 0.020 & 1,224 & 0.025 & 24.4 & 33.7 & 1,032 & 0.021 & 20.4 & 31.8 & 1,393 & 0.017 & 15.9 & 25.8 \\
\hline North Dakota & 527 & 0.010 & 194 & 0.014 & 12.9 & 18.6 & 72 & 0.009 & 1.4 & 11.1 & 261 & 0.008 & 2.3 & 7.7 \\
\hline South Dakota & 2,536 & 0.015 & 1,597 & 0.018 & 13.2 & 23.0 & 281 & 0.013 & 9.3 & 16.0 & 658 & 0.010 & 8.1 & 12.8 \\
\hline Utah & 4,183 & 0.015 & 2,083 & 0.014 & 12.6 & 20.0 & 889 & 0.020 & 18.1 & 30.4 & 1,211 & 0.013 & 9.7 & 18.3 \\
\hline Wyoming & 3,061 & 0.014 & 1,724 & 0.020 & 15.1 & 26.4 & 305 & 0.013 & 13.8 & 21.0 & 1,032 & 0.008 & 12.5 & 11.0 \\
\hline Western & 16,368 & 0.011 & 4,590 & 0.016 & 13.5 & 20.7 & 4,369 & 0.013 & 12.1 & 19.1 & 7,409 & 0.008 & 6.0 & 9.2 \\
\hline Alaska & 326 & 0.016 & 88 & 0.024 & 21.6 & 28.4 & 33 & 0.053 & 24.2 & 39.4 & 205 & 0.011 & 8.3 & 15.6 \\
\hline California & 7,453 & 0.013 & 2,326 & 0.018 & 15.2 & 23.3 & 2,425 & 0.014 & 12.0 & 21.0 & 2,702 & 0.009 & 7.9 & 11.7 \\
\hline Hawaii & 265 & 0.004 & 11 & 0.005 & 0.0 & 0.0 & 57 & 0.005 & 3.5 & 1.8 & 197 & 0.004 & 1.0 & 1.0 \\
\hline Idaho & 2,609 & 0.015 & 912 & 0.022 & 15.9 & 26.1 & 534 & 0.017 & 17.0 & 27.2 & 1,163 & 0.010 & 7.6 & 14.3 \\
\hline Oregon & 2,685 & 0.006 & 413 & 0.007 & 4.1 & 6.3 & 698 & 0.007 & 7.4 & 8.0 & 1,574 & 0.005 & 1.9 & 1.8 \\
\hline Washington & 3,030 & 0.009 & 840 & 0.011 & 10.1 & 13.8 & 622 & 0.011 & 13.3 & 17.8 & 1,568 & 0.007 & 6.0 & 8.5 \\
\hline TOTAL & 179,024 & 0.010 & 62,815 & 0.013 & 11.3 & 17.3 & 43,558 & 0.011 & 10.8 & 16.5 & 72,651 & 0.008 & 6.3 & 10.0 \\
\hline
\end{tabular}

- indicates incalculable field

PEL - permissible exposure limit

REL - recommended exposure limit

GM - geometric mean

$\mathrm{mg} / \mathrm{m}^{3}$ - milligrams per cubic meter

NOTE: For metal/nonmetal mining, the MSHA PEL is $\left[\left(10 \mathrm{mg} / \mathrm{m}^{3}\right) /(\%\right.$ quartz +2$\left.)\right]$ for respirable dust containing at least 1 percent quartz. The NIOSH REL is $0.05 \mathrm{mg} / \mathrm{m}^{3}$. See appendices for source description, methods, and agents.

SOURCE: Mine Safety and Health Administration (MSHA) metal/nonmetal mine data. 
Table 3-20 (page 1 of 3). Respirable quartz: Geometric mean exposures and percent exceeding designated occupational exposure limits by OSHA region and state, OSHA samples, 1979-1999

\begin{tabular}{|c|c|c|c|c|c|c|c|c|c|c|c|c|c|c|}
\hline \multirow[b]{2}{*}{ OSHA Region } & \multicolumn{2}{|c|}{ All years } & \multicolumn{4}{|c|}{$\begin{array}{c}1979 \text { - } 1988 \\
\text { OSHA PEL: see note }\end{array}$} & \multicolumn{4}{|c|}{$\begin{array}{c}1989-1992 \\
\text { OSHA PEL }=0.1 \mathrm{mg} / \mathrm{m}^{3}\end{array}$} & \multicolumn{4}{|c|}{$\begin{array}{c}1993 \text { - } 1999 \\
\text { OSHA PEL: see note }\end{array}$} \\
\hline & \begin{tabular}{|c|} 
No. of \\
Samples
\end{tabular} & $\begin{array}{c}\mathbf{G M} \\
\left(\mathbf{m g} / \mathbf{m}^{3}\right)\end{array}$ & $\begin{array}{c}\text { No. of } \\
\text { Samples }\end{array}$ & $\begin{array}{c}\mathbf{G M} \\
\left(\mathbf{m g} / \mathbf{m}^{3}\right)\end{array}$ & $\begin{array}{l}\%> \\
\text { PEL }\end{array}$ & $\begin{array}{l}\%> \\
\text { REL }\end{array}$ & \begin{tabular}{|c|}
$\begin{array}{c}\text { No. of } \\
\text { Samples }\end{array}$ \\
\end{tabular} & $\begin{array}{c}\mathbf{G M} \\
\left(\mathbf{m g} / \mathbf{m}^{3}\right)\end{array}$ & $\begin{array}{l}\%> \\
\text { PEL }\end{array}$ & $\begin{array}{l}\%> \\
\text { REL }\end{array}$ & \begin{tabular}{|c|} 
No. of \\
Samples
\end{tabular} & $\begin{array}{c}\mathbf{G M} \\
\left(\mathbf{m g} / \mathbf{m}^{3}\right)\end{array}$ & $\begin{array}{l}\%> \\
\text { PEL }\end{array}$ & $\begin{array}{l}\%> \\
\text { REL }\end{array}$ \\
\hline Region 1 & 1,376 & 0.032 & 980 & 0.034 & 25.8 & 40.2 & 144 & 0.018 & 9.7 & 22.2 & 252 & 0.034 & 26.6 & 40.9 \\
\hline Connecticut & 446 & 0.023 & 344 & 0.027 & 19.2 & 33.7 & 55 & 0.014 & 3.6 & 16.4 & 47 & 0.015 & 8.5 & 21.3 \\
\hline Maine & 22 & 0.034 & 19 & 0.033 & 36.8 & 42.1 & 0 & - & - & - & 3 & 0.038 & 33.3 & 33.3 \\
\hline Massachusetts & 468 & 0.038 & 296 & 0.041 & 31.4 & 46.3 & 58 & 0.022 & 15.5 & 24.1 & 114 & 0.041 & 30.7 & 46.5 \\
\hline New Hampshire & 227 & 0.029 & 143 & 0.033 & 23.8 & 39.9 & 30 & 0.020 & 10.0 & 30.0 & 54 & 0.027 & 24.1 & 37.0 \\
\hline Rhode Island & 213 & 0.044 & 178 & 0.039 & 29.8 & 42.7 & 1 & 0.007 & 0.0 & 0.0 & 34 & 0.084 & 41.2 & 55.9 \\
\hline Vermont & 0 & - & 0 & - & - & - & 0 & - & - & - & 0 & - & - & - \\
\hline Region 2 & 1,587 & 0.041 & 904 & 0.043 & 33.2 & 48.5 & 232 & 0.025 & 15.9 & 32.8 & 451 & 0.047 & 36.4 & 49.2 \\
\hline New Jersey & 443 & 0.035 & 283 & 0.041 & 32.5 & 45.9 & 103 & 0.027 & 13.6 & 34.0 & 57 & 0.027 & 19.3 & 26.3 \\
\hline New York & 1,131 & 0.044 & 619 & 0.044 & 33.3 & 49.4 & 125 & 0.025 & 18.4 & 32.8 & 387 & 0.052 & 39.5 & 53.5 \\
\hline Puerto Rico & 9 & 0.021 & 2 & 0.914 & 100.0 & 100.0 & 3 & 0.007 & 0.0 & 0.0 & 4 & 0.007 & 0.0 & 0.0 \\
\hline U.S. Virgin Islands & 4 & 0.007 & 0 & - & - & - & 1 & 0.007 & 0.0 & 0.0 & 3 & 0.007 & 0.0 & 0.0 \\
\hline Region 3 & 2,622 & 0.047 & 1,996 & 0.047 & 30.1 & 49.2 & 259 & 0.034 & 22.8 & 39.8 & 367 & 0.058 & 37.3 & 52.0 \\
\hline Delaware & 20 & 0.020 & 17 & 0.014 & 5.9 & 11.8 & 0 & - & - & - & 3 & 0.117 & 66.7 & 66.7 \\
\hline District of Columbia & 0 & - & 0 & - & - & - & 0 & - & - & - & 0 & - & - & - \\
\hline Maryland & 215 & 0.038 & 123 & 0.034 & 29.3 & 37.4 & 60 & 0.037 & 36.7 & 51.7 & 32 & 0.059 & 28.1 & 43.8 \\
\hline Pennsylvania & 1,912 & 0.048 & 1,406 & 0.048 & 29.2 & 51.1 & 180 & 0.032 & 17.8 & 36.1 & 326 & 0.057 & 37.4 & 52.5 \\
\hline Virginia & 247 & 0.041 & 246 & 0.040 & 27.6 & 41.5 & 0 & - & - & - & 1 & 0.412 & 100.0 & 100.0 \\
\hline West Virginia & 228 & 0.061 & 204 & 0.062 & 42.2 & 55.9 & 19 & 0.049 & 26.3 & 36.8 & 5 & 0.051 & 60.0 & 60.0 \\
\hline Region 4 & 2,523 & 0.034 & 1,449 & 0.039 & 30.2 & 43.8 & 419 & 0.030 & 21.0 & 35.1 & 655 & 0.028 & 20.3 & 33.3 \\
\hline Alabama & 479 & 0.033 & 323 & 0.037 & 32.2 & 45.8 & 98 & 0.037 & 23.5 & 44.9 & 58 & 0.015 & 12.1 & 20.7 \\
\hline Florida & 88 & 0.037 & 58 & 0.034 & 29.3 & 37.9 & 22 & 0.027 & 22.7 & 27.3 & 8 & 0.148 & 62.5 & 62.5 \\
\hline Georgia & 636 & 0.045 & 470 & 0.044 & 34.5 & 49.1 & 73 & 0.026 & 19.2 & 30.1 & 93 & 0.072 & 29.0 & 53.8 \\
\hline Kentucky & 338 & 0.033 & 157 & 0.044 & 33.1 & 44.6 & 64 & 0.047 & 29.7 & 43.8 & 117 & 0.018 & 15.4 & 17.9 \\
\hline Mississippi & 106 & 0.050 & 74 & 0.046 & 33.8 & 48.6 & 22 & 0.030 & 13.6 & 22.7 & 10 & 0.295 & 60.0 & 90.0 \\
\hline North Carolina & 360 & 0.025 & 155 & 0.026 & 12.3 & 26.5 & 25 & 0.023 & 16.0 & 28.0 & 180 & 0.024 & 20.0 & 31.7 \\
\hline South Carolina & 144 & 0.022 & 49 & 0.021 & 26.5 & 26.5 & 40 & 0.017 & 15.0 & 22.5 & 55 & 0.027 & 23.6 & 29.1 \\
\hline Tennessee & 372 & 0.033 & 163 & 0.040 & 27.6 & 44.8 & 75 & 0.028 & 18.7 & 34.7 & 134 & 0.027 & 15.7 & 35.8 \\
\hline
\end{tabular}

See footnotes at end of table. 
Table 3-20 (page 2 of 3). Respirable quartz: Geometric mean exposures and percent exceeding designated occupational exposure limits by OSHA region and state, OSHA samples, 1979-1999

\begin{tabular}{|c|c|c|c|c|c|c|c|c|c|c|c|c|c|c|}
\hline \multirow[b]{2}{*}{ OSHA Region } & \multicolumn{2}{|c|}{ All years } & \multicolumn{4}{|c|}{$\begin{array}{c}1979 \text { - } 1988 \\
\text { OSHA PEL: see note } \\
\end{array}$} & \multicolumn{4}{|c|}{$\begin{array}{c}1989-1992 \\
\text { OSHA PEL }=0.1 \mathrm{mg} / \mathrm{m}^{3}\end{array}$} & \multicolumn{4}{|c|}{$\begin{array}{c}1993 \text { - } 1999 \\
\text { OSHA PEL: see note }\end{array}$} \\
\hline & $\begin{array}{c}\text { No. of } \\
\text { Samples }\end{array}$ & $\begin{array}{c}\text { GM } \\
\left(\mathrm{mg} / \mathrm{m}^{3}\right)\end{array}$ & $\begin{array}{c}\text { No. of } \\
\text { Samples }\end{array}$ & $\begin{array}{c}\mathbf{G M} \\
\left(\mathbf{m g} / \mathbf{m}^{3}\right)\end{array}$ & $\begin{array}{l}\%> \\
\text { PEL }\end{array}$ & $\begin{array}{l}\%> \\
\text { REL }\end{array}$ & $\begin{array}{c}\text { No. of } \\
\text { Samples }\end{array}$ & $\underset{\left(\mathbf{m g} / \mathbf{m}^{3}\right)}{\mathbf{G M}}$ & $\begin{array}{l}\%> \\
\text { PEL }\end{array}$ & $\begin{array}{l}\%> \\
\text { REL }\end{array}$ & $\begin{array}{c}\text { No. of } \\
\text { Samples }\end{array}$ & $\begin{array}{c}\mathbf{G M} \\
\left(\mathrm{mg} / \mathbf{m}^{3}\right)\end{array}$ & $\begin{array}{l}\%> \\
\text { PEL }\end{array}$ & $\begin{array}{l}\%> \\
\text { REL }\end{array}$ \\
\hline Region 5 & 5,121 & 0.041 & 2,819 & 0.048 & 35.3 & 52.7 & 1,013 & 0.027 & 15.8 & 35.7 & 1,289 & 0.039 & 30.6 & 42.9 \\
\hline Illinois & 947 & 0.041 & 538 & 0.043 & 34.2 & 47.8 & 147 & 0.030 & 17.7 & 33.3 & 262 & 0.045 & 35.1 & 46.9 \\
\hline Indiana & 410 & 0.032 & 262 & 0.037 & 31.3 & 45.0 & 84 & 0.018 & 8.3 & 23.8 & 64 & 0.039 & 37.5 & 43.8 \\
\hline Michigan & 765 & 0.016 & 27 & 0.014 & 0.0 & 3.7 & 262 & 0.012 & 2.7 & 12.6 & 476 & 0.019 & 10.3 & 20.6 \\
\hline Minnesota & 93 & 0.051 & 71 & 0.048 & 50.7 & 54.9 & 9 & 0.029 & 0.0 & 44.4 & 13 & 0.098 & 46.2 & 46.2 \\
\hline Ohio & 1,638 & 0.047 & 1,191 & 0.047 & 32.3 & 50.1 & 229 & 0.034 & 20.5 & 39.7 & 218 & 0.069 & 42.2 & 57.3 \\
\hline Wisconsin & 1,268 & 0.061 & 730 & 0.061 & 42.3 & 64.9 & 282 & 0.052 & 25.9 & 58.5 & 256 & 0.074 & 51.6 & 67.6 \\
\hline Region 6 & 1,215 & 0.040 & 885 & 0.036 & 29.8 & 41.4 & 171 & $\mathbf{0 . 0 3 7}$ & 28.7 & 43.3 & 159 & 0.087 & 44.0 & 53.5 \\
\hline Arkansas & 118 & 0.031 & 93 & 0.023 & 23.7 & 31.2 & 14 & 0.084 & 64.3 & 64.3 & 11 & 0.098 & 63.6 & 63.6 \\
\hline Louisiana & 91 & 0.064 & 68 & 0.056 & 41.2 & 64.7 & 11 & 0.032 & 27.3 & 36.4 & 12 & 0.240 & 41.7 & 58.3 \\
\hline New Mexico & 18 & 0.073 & 11 & 0.041 & 54.5 & 54.5 & 1 & 0.007 & 0.0 & 0.0 & 6 & 0.306 & 100.0 & 100.0 \\
\hline Oklahoma & 198 & 0.036 & 153 & 0.034 & 19.6 & 37.3 & 10 & 0.044 & 30.0 & 50.0 & 35 & 0.045 & 28.6 & 48.6 \\
\hline Texas & 790 & 0.040 & 560 & 0.037 & 31.8 & 41.1 & 135 & 0.034 & 25.2 & 41.5 & 95 & 0.089 & 44.2 & 50.5 \\
\hline Region 7 & 1,050 & 0.033 & 720 & 0.035 & 25.3 & 38.9 & 181 & 0.023 & 14.4 & 33.1 & 149 & 0.040 & 33.6 & 42.3 \\
\hline Iowa & 579 & 0.030 & 336 & 0.031 & 22.6 & 32.7 & 116 & 0.022 & 14.7 & 30.2 & 127 & 0.038 & 34.6 & 41.7 \\
\hline Kansas & 105 & 0.038 & 75 & 0.033 & 25.3 & 36.0 & 19 & 0.051 & 31.6 & 68.4 & 11 & 0.049 & 27.3 & 63.6 \\
\hline Missouri & 289 & 0.040 & 242 & 0.046 & 30.6 & 50.0 & 43 & 0.017 & 7.0 & 23.3 & 4 & 0.021 & 25.0 & 25.0 \\
\hline Nebraska & 77 & 0.027 & 67 & 0.023 & 19.4 & 32.8 & 3 & 0.055 & 0.0 & 66.7 & 7 & 0.076 & 28.6 & 28.6 \\
\hline Region 8 & 737 & 0.039 & 493 & 0.032 & 23.7 & 35.5 & 122 & 0.032 & 25.4 & 39.3 & 122 & 0.107 & 54.9 & 70.5 \\
\hline Colorado & 458 & 0.030 & 321 & 0.026 & 20.9 & 30.8 & 74 & 0.025 & 17.6 & 32.4 & 63 & 0.079 & 46.0 & 63.5 \\
\hline Montana & 78 & 0.059 & 64 & 0.044 & 25.0 & 35.9 & 11 & 0.250 & 72.7 & 81.8 & 3 & 0.141 & 66.7 & 66.7 \\
\hline North Dakota & 94 & 0.070 & 56 & 0.041 & 23.2 & 50.0 & 2 & 0.069 & 50.0 & 50.0 & 36 & 0.161 & 69.4 & 80.6 \\
\hline South Dakota & 57 & 0.065 & 27 & 0.033 & 33.3 & 44.4 & 10 & 0.102 & 70.0 & 70.0 & 20 & 0.132 & 55.0 & 75.0 \\
\hline Utah & 45 & 0.043 & 20 & 0.142 & 60.0 & 60.0 & 25 & 0.017 & 8.0 & 28.0 & 0 & - & - & - \\
\hline Wyoming & 5 & 0.022 & 5 & 0.022 & 0.0 & 20.0 & 0 & - & - & - & 0 & - & - & - \\
\hline
\end{tabular}

See footnotes at end of table. 
Table 3-20 (page 3 of 3). Respirable quartz: Geometric mean exposures and percent exceeding designated occupational exposure limits by OSHA region and state, OSHA samples, 1979-1999

\begin{tabular}{|c|c|c|c|c|c|c|c|c|c|c|c|c|c|c|}
\hline \multirow[b]{2}{*}{ OSHA Region } & \multicolumn{2}{|c|}{ All years } & \multicolumn{4}{|c|}{$\begin{array}{c}1979 \text { - } 1988 \\
\text { OSHA PEL: see note }\end{array}$} & \multicolumn{4}{|c|}{$\begin{array}{c}1989-1992 \\
\text { OSHA PEL }=0.1 \mathrm{mg} / \mathrm{m}^{3}\end{array}$} & \multicolumn{4}{|c|}{$\begin{array}{c}1993 \text { - } 1999 \\
\text { OSHA PEL: see note }\end{array}$} \\
\hline & $\begin{array}{c}\text { No. of } \\
\text { Samples }\end{array}$ & $\begin{array}{c}\mathrm{GM} \\
\left(\mathrm{mg} / \mathrm{m}^{3}\right)\end{array}$ & $\begin{array}{c}\text { No. of } \\
\text { Samples }\end{array}$ & $\begin{array}{c}\mathrm{GM} \\
\left(\mathrm{mg} / \mathrm{m}^{3}\right)\end{array}$ & $\begin{array}{l}\%> \\
\text { PEL }\end{array}$ & $\begin{array}{l}\%> \\
\text { REL }\end{array}$ & \begin{tabular}{|c|} 
No. of \\
Samples
\end{tabular} & $\begin{array}{c}\text { GM } \\
\left(\mathrm{mg} / \mathrm{m}^{3}\right)\end{array}$ & $\begin{array}{l}\%> \\
\text { PEL }\end{array}$ & $\begin{array}{l}\%>> \\
\text { REL }\end{array}$ & \begin{tabular}{|c|} 
No. of \\
Samples
\end{tabular} & $\begin{array}{c}\mathrm{GM} \\
\left(\mathrm{mg} / \mathrm{m}^{3}\right)\end{array}$ & $\begin{array}{l}\%> \\
\text { PEL }\end{array}$ & $\begin{array}{l}\%> \\
\text { REL }\end{array}$ \\
\hline Region 9 & 374 & 0.045 & 235 & 0.057 & 43.4 & 55.3 & 77 & 0.028 & 29.9 & 32.5 & 62 & 0.031 & 27.4 & 32.3 \\
\hline American Samoa & 0 & - & 0 & - & - & - & 0 & - & - & - & 0 & - & - & - \\
\hline Arizona & 49 & 0.036 & 31 & 0.060 & 45.2 & 61.3 & 10 & 0.010 & 0.0 & 10.0 & 8 & 0.024 & 25.0 & 25.0 \\
\hline California & 186 & 0.026 & 115 & 0.025 & 22.6 & 36.5 & 30 & 0.017 & 13.3 & 16.7 & 41 & 0.036 & 29.3 & 36.6 \\
\hline Hawaii & 8 & 0.008 & 5 & 0.008 & 0.0 & 0.0 & 0 & - & - & - & 3 & 0.007 & 0.0 & 0.0 \\
\hline Nevada & 131 & 0.117 & 84 & 0.191 & 73.8 & 82.1 & 37 & 0.056 & 51.4 & 51.4 & 10 & 0.030 & 30.0 & 30.0 \\
\hline Region 10 & 501 & 0.041 & 130 & 0.046 & 33.1 & 40.8 & 85 & 0.034 & 21.2 & 31.8 & 286 & 0.041 & 25.9 & 42.0 \\
\hline Alaska & 7 & 0.100 & 2 & 0.152 & 50.0 & 50.0 & 5 & 0.085 & 60.0 & 80.0 & 0 & - & - & - \\
\hline Idaho & 42 & 0.041 & 18 & 0.055 & 50.0 & 55.6 & 21 & 0.040 & 14.3 & 42.9 & 3 & 0.007 & 0.0 & 0.0 \\
\hline Oregon & 175 & 0.029 & 107 & 0.046 & 30.8 & 39.3 & 4 & 0.010 & 0.0 & 0.0 & 64 & 0.015 & 12.5 & 18.8 \\
\hline Washington & 277 & 0.050 & 3 & 0.007 & 0.0 & 0.0 & 55 & 0.032 & 21.8 & 25.5 & 219 & 0.057 & 30.1 & 49.3 \\
\hline
\end{tabular}

- indicates incalculable field

PEL - permissible exposure limit

REL - recommended exposure limit

GM - geometric mean

$\mathrm{mg} / \mathrm{m}^{3}$ - milligrams per cubic meter

NOTE: Before March 1, 1989 and after March 22, 1993, the OSHA PEL is [(10 mg/m³)/(\% quartz + 2)] for respirable dust containing at least 1 percent quartz. From March 1, 1989 to March 22, 1993, the OSHA PEL was $0.1 \mathrm{mg} / \mathrm{m}^{3}$ for respirable quartz. The NIOSH REL is $0.05 \mathrm{mg} / \mathrm{m}^{3}$. See appendices for source description, methods, and agents.

SOURCE: Occupational Safety and Health Administation (OSHA) Integrated Management Information System. 
Figure 3-7. Respirable quartz: Geometric mean coal mining exposures by state, MSHA inspector and mine operator samples, 1982-1999

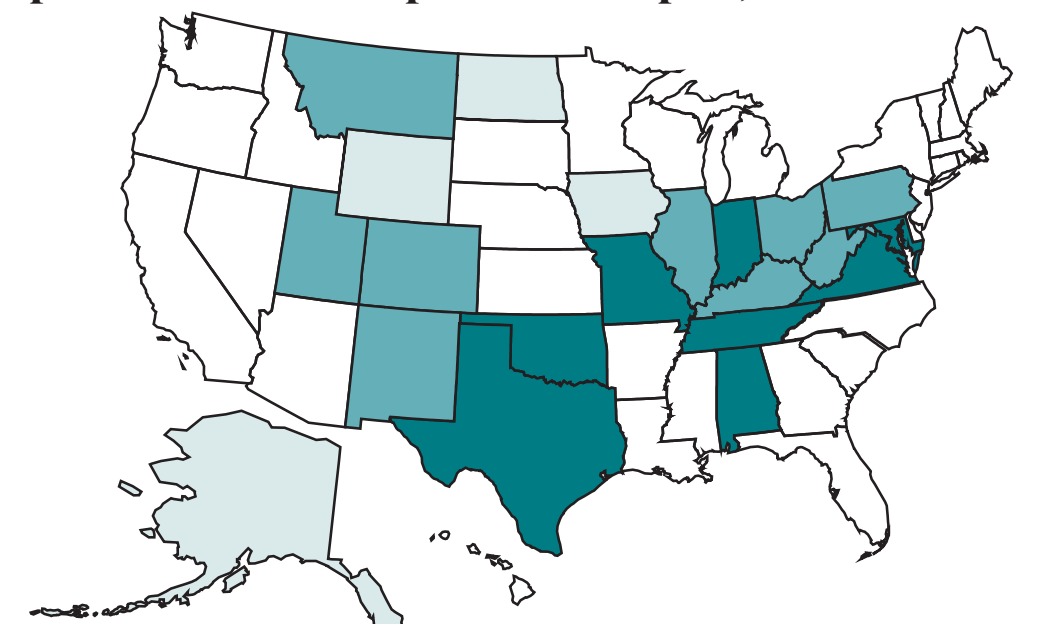

\begin{tabular}{|cc|}
\hline $\mathbf{1 9 8 2}$ - 1988 \\
\hline mg/m ${ }^{3}$ MRE & No. of States \\
$\square>0.05$ & 8 \\
$\square>0.025$ to 0.05 & 9 \\
$\square$ to 0.025 & 4 \\
$\square<10$ samples & 30 \\
\hline
\end{tabular}

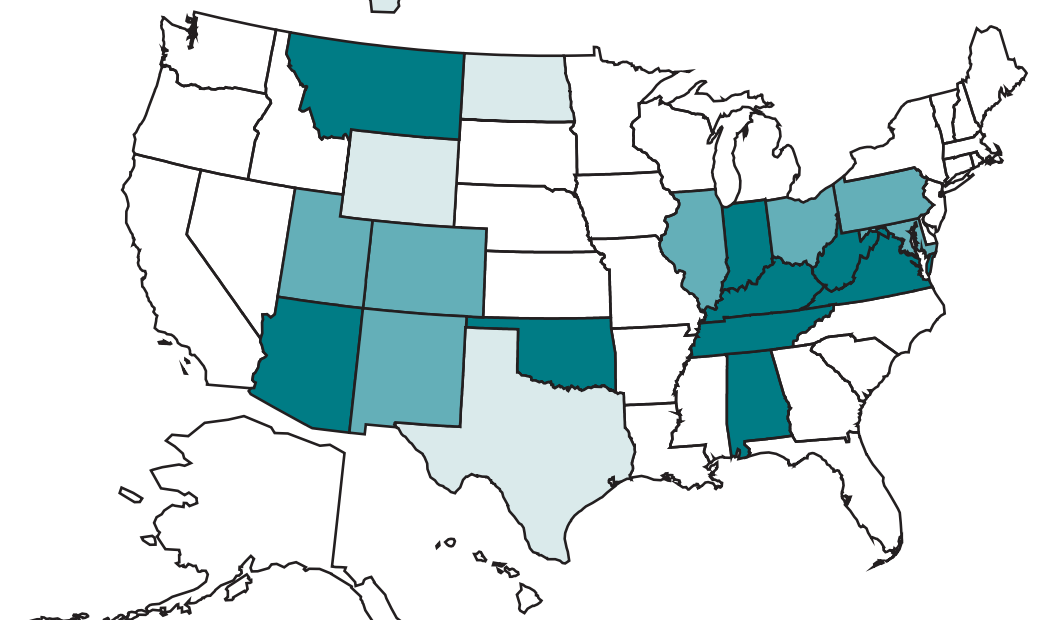

\begin{tabular}{|cc|}
\hline \multicolumn{1}{|c|}{$\mathbf{1 9 8 9} \mathbf{- 1 9 9 2}$} \\
\hline $\mathrm{mg} / \mathrm{m}^{3}$ MRE & No. of States \\
$\square>0.05$ & 9 \\
$\square>0.025$ to 0.05 & 7 \\
$\square 0$ to 0.025 & 3 \\
$\square<10$ samples & 32 \\
\hline
\end{tabular}

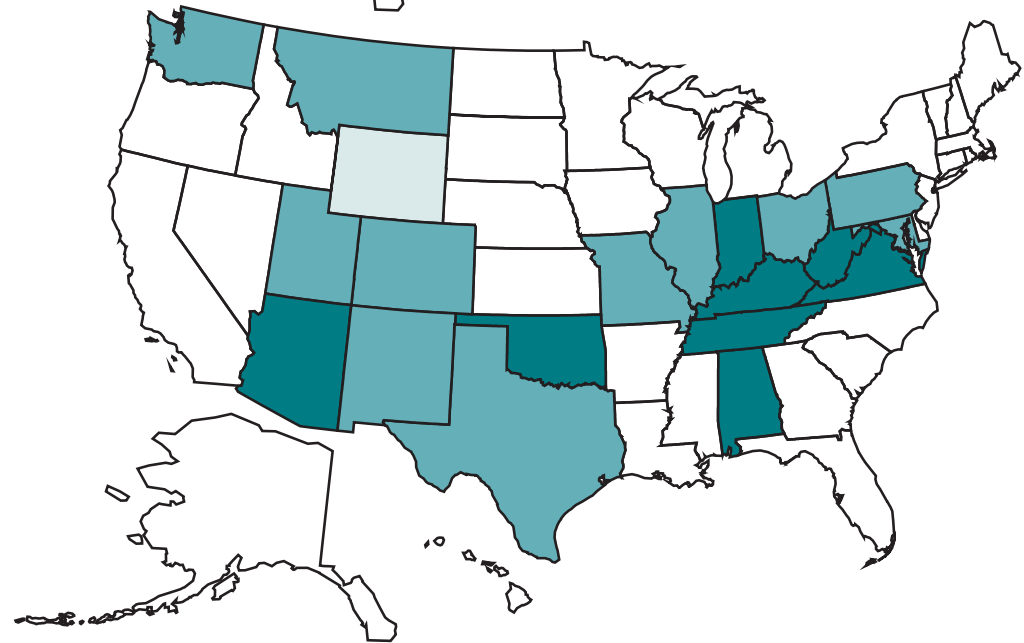

\begin{tabular}{|cc|}
\hline $\mathbf{1 9 9 3}$ - 1999 \\
\hline mg/m $\mathrm{m}^{3}$ MRE & No. of States \\
$\square>0.05$ & 8 \\
$\square>0.025$ to 0.05 & 11 \\
$\square$ to 0.025 & 1 \\
$\square<10$ samples & 31 \\
\hline
\end{tabular}

PEL - permissible exposure limit

REL - recommended exposure limit MRE - Mining Research Establishment, not comparable to NIOSH REL

NOTE: For coal mining, the MSHA PEL is $\left[\left(10 \mathrm{mg} / \mathrm{m}^{3} \mathrm{MRE}\right) /(\%\right.$ quartz) $]$ for respirable dust containing greater than 5 percent quartz. The MSHA respirable coal mine quartz exposure data and the NIOSH REL for respirable quartz cannot be compared to each other because they are based on different sampling criteria. See appendices for source description, methods, and agents.

SOURCE: Mine Safety and Health Administration (MSHA) coal mine inspector and operator quartz data. 
Figure 3-8. Respirable quartz: Geometric mean metal/nonmetal mining exposures by state, MSHA samples, 1979-1999

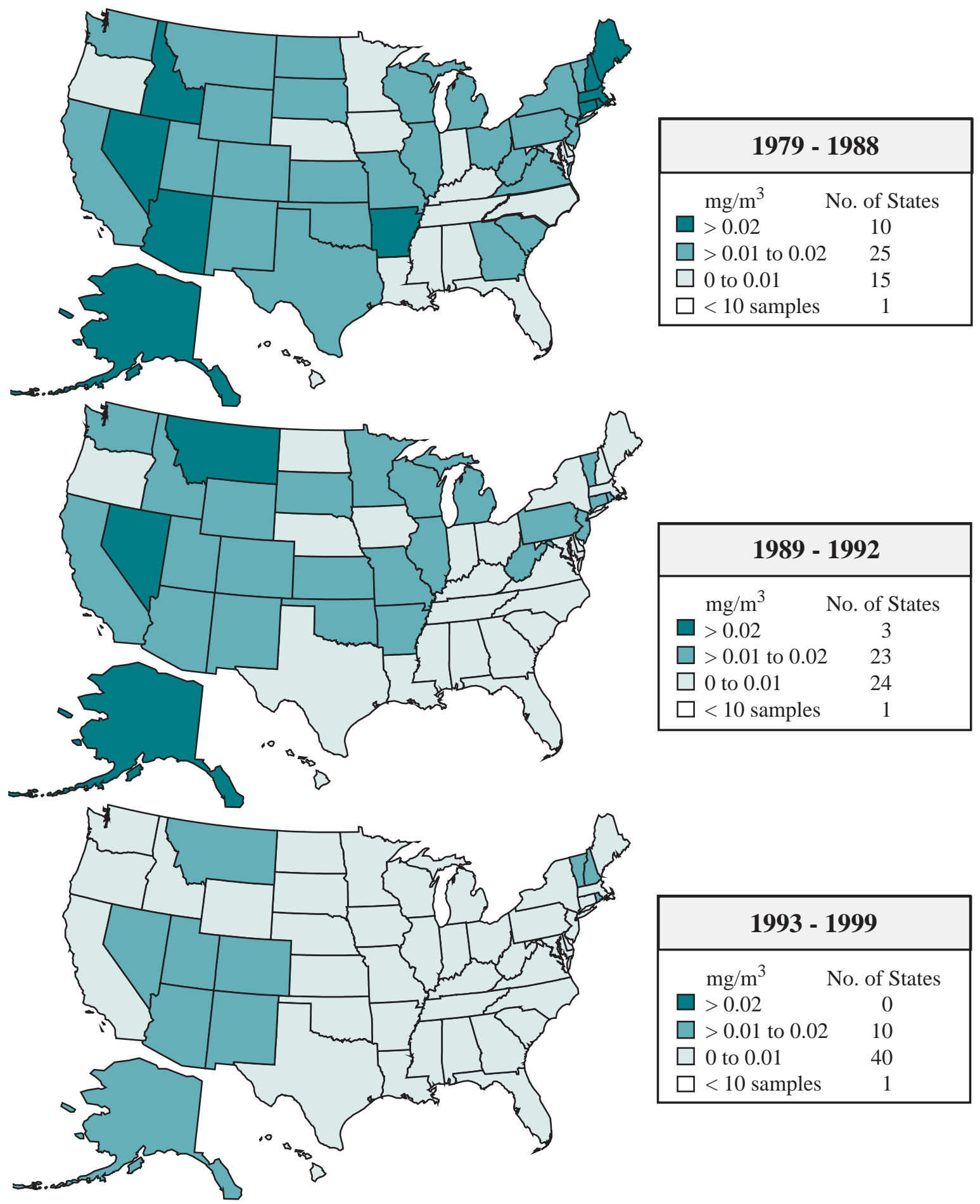

PEL - permissible exposure limit REL - recommended exposure limit $\quad \mathrm{mg} / \mathrm{m}^{3}$ - milligrams per cubic meter

NOTE: For metal/nonmetal mining, the MSHA PEL is $\left[\left(10 \mathrm{mg} / \mathrm{m}^{3}\right) /(\%\right.$ quartz +2$\left.)\right]$ for respirable dust containing at least 1 percent quartz. The NIOSH REL is $0.05 \mathrm{mg} / \mathrm{m}^{3}$. See appendices for source description, methods, and agents.

SOURCE: Mine Safety and Health Administration (MSHA) metal/nonmetal mine data. 


\section{Figure 3-9. Respirable quartz: Geometric mean exposures by state, OSHA} samples, 1979-1999

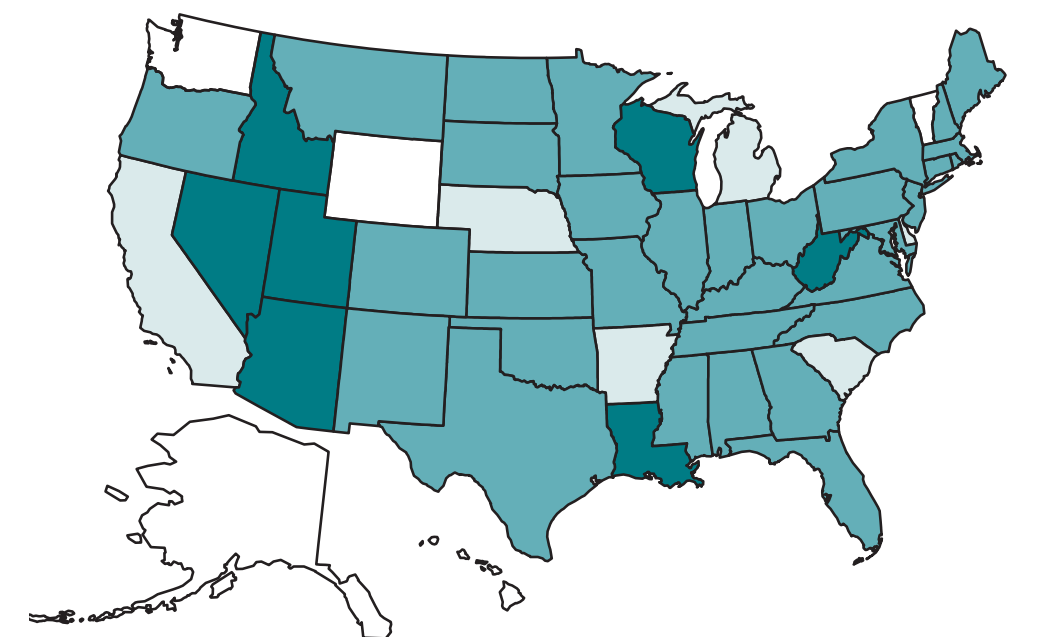

\begin{tabular}{|cc|}
\hline \multicolumn{2}{|c|}{$\mathbf{1 9 7 9}$ - 1988} \\
OSHA PEL: see note \\
\hline $\mathrm{mg} / \mathrm{m}^{3}$ & No. of States \\
$\square>0.05$ & 7 \\
$\square>0.025$ to 0.05 & 32 \\
$\square 0$ to 0.025 & 6 \\
$\square<10$ samples & 6 \\
\hline
\end{tabular}

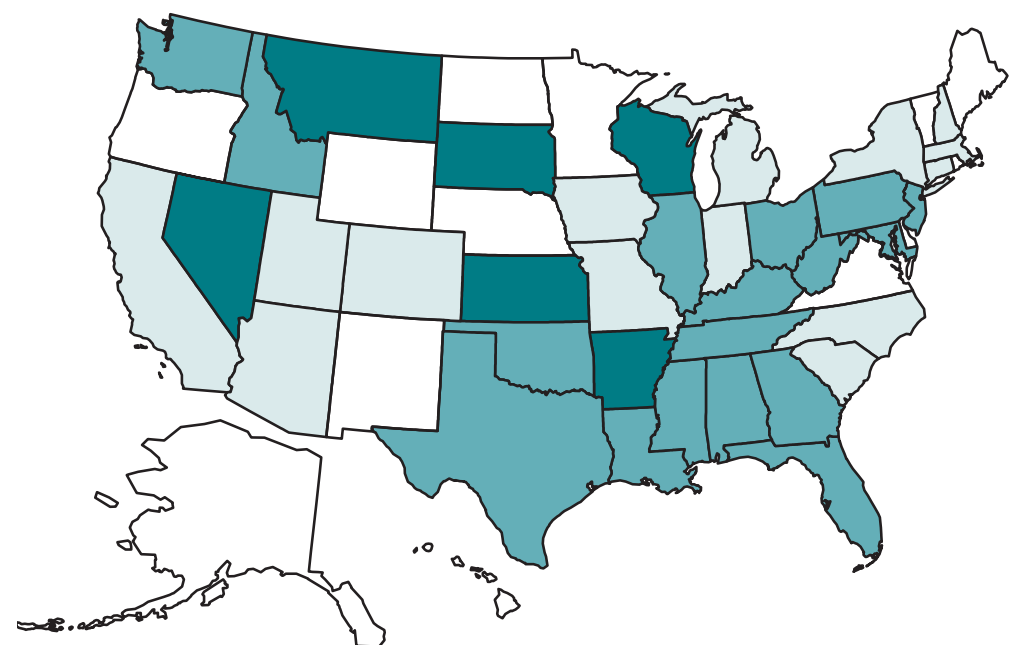

\begin{tabular}{|cc|}
\hline \multicolumn{1}{|c|}{$\mathbf{1 9 8 9} \mathbf{- 1 9 9 2}$} \\
OSHA PEL $=0.1 \mathrm{mg} / \mathrm{m}^{3}$ \\
\hline $\mathrm{mg} / \mathrm{m}^{3}$ & No. of States \\
$\square>0.05$ & 6 \\
$\square>0.025$ to 0.05 & 17 \\
$\square$ 0 to 0.025 & 14 \\
$\square<10$ samples & 14 \\
\hline
\end{tabular}

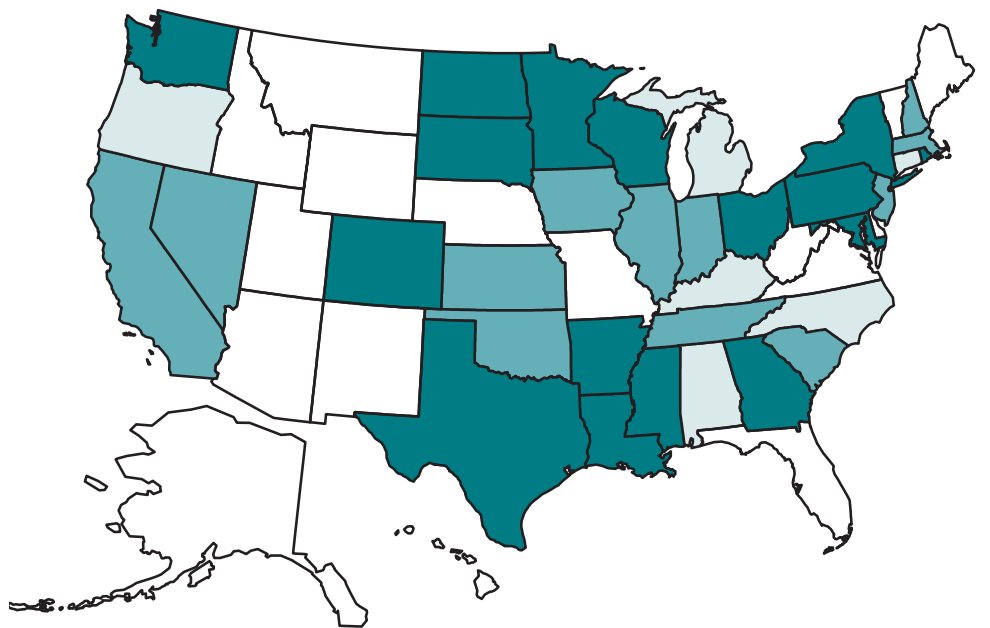

\begin{tabular}{|cc|}
\hline \multicolumn{2}{|c|}{1993 - 1999} \\
OSHA PEL: see note \\
\hline mg/m & No. of States \\
$\square>0.05$ & 16 \\
$\square>0.025$ to 0.05 & 12 \\
$\square 0$ to 0.025 & 6 \\
$\square<10$ samples & 17 \\
\hline
\end{tabular}

PEL - permissible exposure limit REL - recommended exposure limit

$\mathrm{mg} / \mathrm{m}^{3}$ - milligrams per cubic meter

NOTE: Before March 1, 1989 and after March 22, 1993, the OSHA PEL is $\left[\left(10 \mathrm{mg} / \mathrm{m}^{3}\right) /(\%\right.$ quartz +2$\left.)\right]$ for respirable dust containing at least 1 percent quartz. From March 1, 1989 to March 22, 1993, the OSHA PEL was $0.1 \mathrm{mg} / \mathrm{m}^{3}$ for respirable quartz. The NIOSH REL is $0.05 \mathrm{mg} / \mathrm{m}^{3}$. See appendices for source description, methods, and agents.

SOURCE: Occupational Safety and Health Administration (OSHA) Integrated Management Information System. 
Table 3-21. Respirable cristobalite: Geometric mean exposures and percent exceeding designated occupational exposure limits, MSHA and OSHA samples, 1979-1999

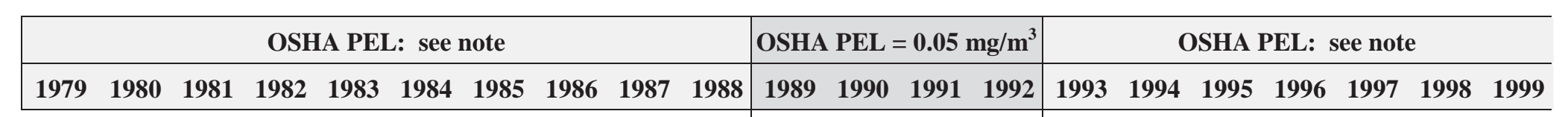

Cristobalite, respirable - MSHA

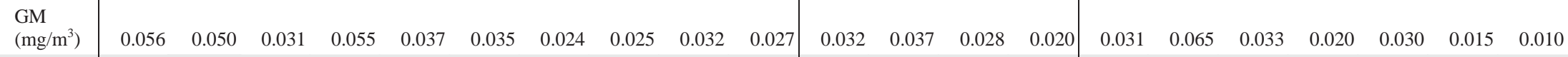

No. of

samples

$\%>$ PEL

\begin{tabular}{|r|rrrrrrrrrr|rrrrrrrrrrr}
\hline & 58.3 & 54.7 & 42.9 & 100.0 & 44.1 & 23.1 & 26.1 & 10.0 & 14.3 & 31.8 & 28.6 & 52.6 & 46.2 & 9.1 & 30.4 & 66.7 & 0.0 & 8.3 & 28.6 & 0.0 & 0.0 \\
\hline & 50.0 & 43.4 & 28.6 & 60.0 & 23.5 & 15.4 & 13.0 & 10.0 & 14.3 & 22.7 & 21.4 & 36.8 & 30.8 & 0.0 & 26.1 & 50.0 & 0.0 & 8.3 & 28.6 & 0.0 & 0.0 \\
\hline
\end{tabular}

Cristobalite, respirable - OSHA

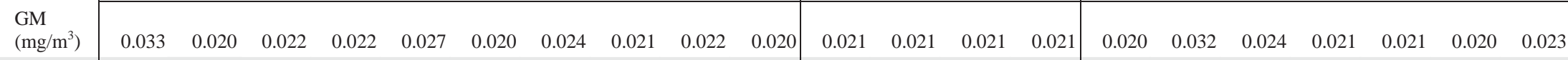

No. of

samples

$\%>$ PEL

$\%>$ REL

$\begin{array}{rrrrrrrrr}26 & 193 & 101 & 84 & 26 & 62 & 112 & 78 & 52 \\ 19.2 & 0.0 & 3.0 & 4.8 & 7.7 & 0.0 & 8.0 & 2.6 & 1.9 \\ 19.2 & 0.0 & 3.0 & 4.8 & 7.7 & 0.0 & 7.1 & 2.6 & 1.9\end{array}$

- indicates incalculable field

PEL - permissible exposure limit

REL - recommended exposure limit

GM - geometric mean

mg mer cubic meter

NOTE: Before March 1, 1989 and after March 22, 1993, the OSHA PEL is $\left[\left(5 \mathrm{mg} / \mathrm{m}^{3}\right) /(\%\right.$ cristobalite +2$\left.)\right]$, for respirable dust containing at least 1 percent cristobalite. From March 1,

1989 to March 22, 1993, the OSHA PEL was $0.05 \mathrm{mg} / \mathrm{m}^{3}$ for respirable cristobalite. For metal $/$ nonmetal mining, the MSHA PEL is [(5 mg/m $\left.) /(\% \mathrm{cristobalite}+2)\right]$ for respirable dust

containing at least 1 percent cristobalite. The NIOSH REL is $0.05 \mathrm{mg} / \mathrm{m}^{3}$. See appendices for source description, methods, and agents.

SOURCE: Mine Safety and Health Administration (MSHA) metal/nonmetal mine data. Occupational Safety and Health Administration (OSHA) Integrated Management Information System. 


\section{Section 4}

Byssinosis

and

Related Exposures 

Figure 4-1. Byssinosis: Number of deaths, crude and age-adjusted mortality rates, U.S. residents age 15 and over, 1979-1999

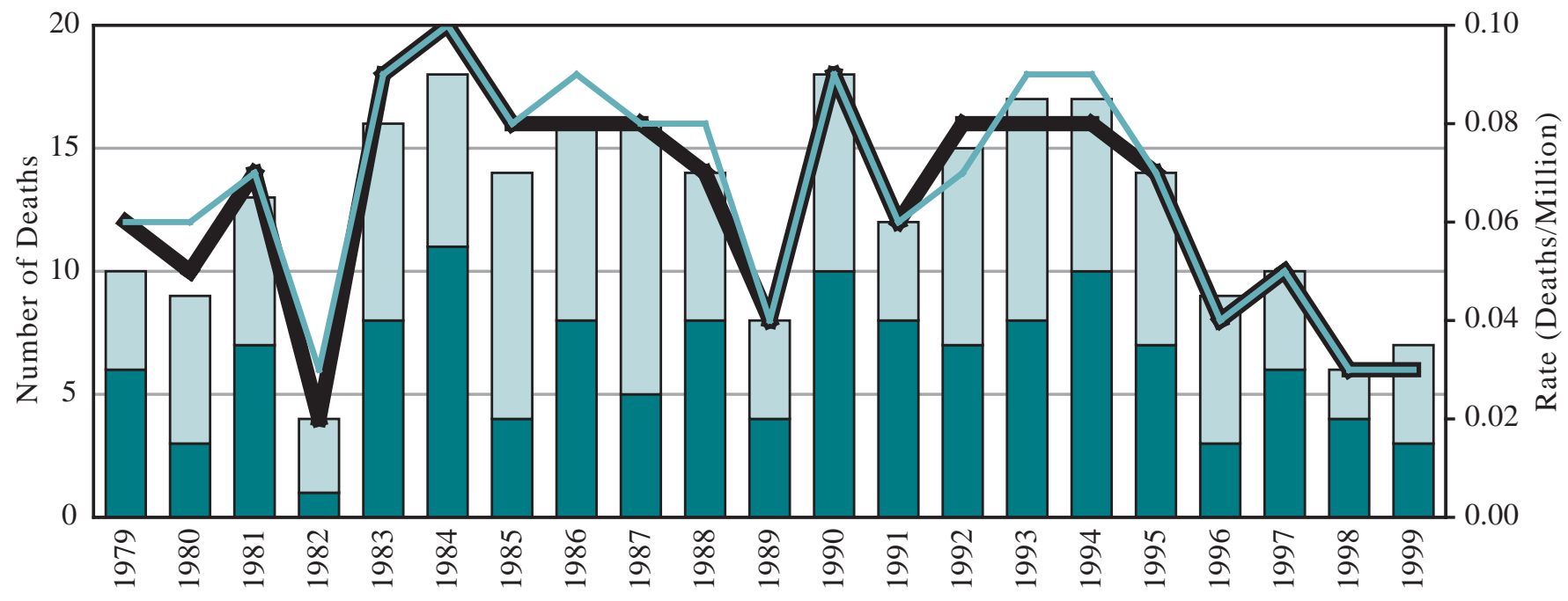

$\square$ Number of deaths, underlying cause

U.S. Crude Rate

$\square$ Number of deaths, contributing cause

U.S. Age-adjusted Rate

NOTE: See appendices for source description, methods, and ICD codes.

SOURCE: National Center for Health Statistics multiple cause of death data. Population estimates from U.S. Bureau of the Census.

Figure 4-2. Byssinosis: Age-adjusted mortality rates by state, U.S. residents age 15 and over, 1990-1999

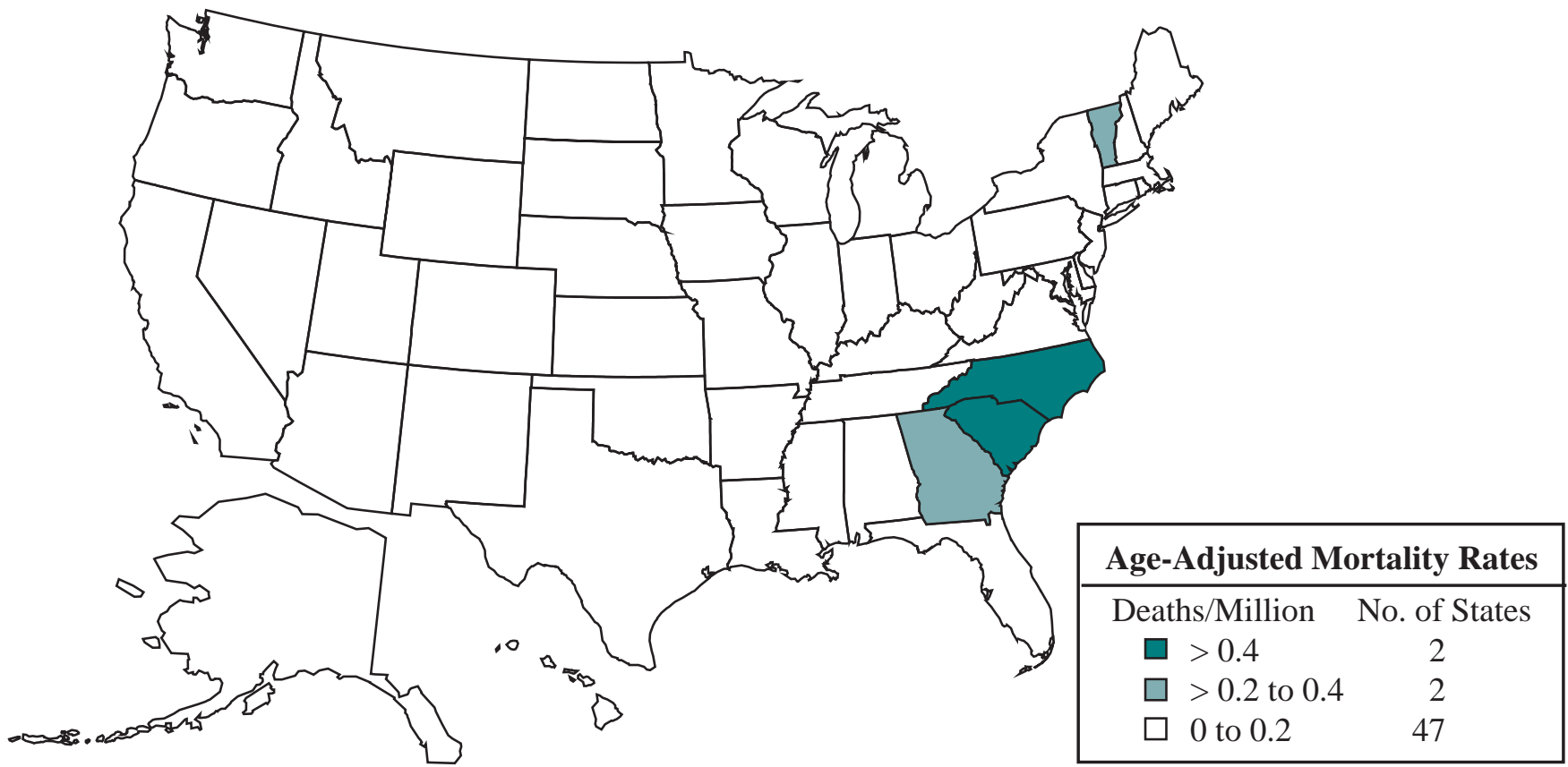

NOTE: See appendices for source description, methods, and ICD codes.

SOURCE: National Center for Health Statistics multiple cause of death data. Population estimates from U.S. Bureau of the Census. 
Table 4-1. Byssinosis: Number of deaths by sex, race, and age, and median age at death, U.S. residents age 15 and over, 1990-1999

\begin{tabular}{|c|c|c|c|c|c|c|c|c|c|c|c|c|c|c|c|c|}
\hline \multirow[b]{2}{*}{ Year } & \multirow{2}{*}{$\begin{array}{l}\text { No. of } \\
\text { Deaths }\end{array}$} & \multirow{2}{*}{\begin{tabular}{|c} 
Under- \\
lying \\
Cause \\
$(\%)$
\end{tabular}} & \multicolumn{2}{|c|}{ Sex } & \multicolumn{3}{|c|}{ Race } & \multicolumn{8}{|c|}{ Age Group (yrs) } & \multirow{2}{*}{$\begin{array}{c}\text { Median } \\
\text { Age } \\
\text { (yrs) }\end{array}$} \\
\hline & & & Male & Female & White & Black & Other & $15-24$ & 25-34 & $35-44$ & 45-54 & $55-64$ & $65-74$ & 75-84 & $85+$ & \\
\hline 1990 & 18 & 55.6 & 13 & 5 & 17 & 1 & - & - & 1 & - & - & 4 & 7 & 4 & 2 & 71.0 \\
\hline 1991 & 12 & 66.7 & 8 & 4 & 11 & 1 & - & - & - & - & 1 & 1 & 4 & 4 & 2 & 75.0 \\
\hline 1992 & 15 & 46.7 & 9 & 6 & 10 & 5 & - & 3 & 1 & - & - & 1 & 8 & 2 & - & 67.0 \\
\hline 1993 & 17 & 47.1 & 11 & 6 & 12 & 5 & - & - & 1 & 1 & 1 & 4 & 3 & 3 & 4 & 73.0 \\
\hline 1994 & 17 & 58.8 & 11 & 6 & 15 & 2 & - & - & - & 1 & 1 & 3 & 4 & 5 & 3 & 72.0 \\
\hline 1995 & 14 & 50.0 & 11 & 3 & 14 & - & - & - & 1 & 1 & - & - & 6 & 6 & - & 74.0 \\
\hline 1996 & 9 & 33.3 & 4 & 5 & 9 & - & - & - & - & - & 1 & 2 & 2 & 4 & - & 71.0 \\
\hline 1997 & 10 & 60.0 & 9 & 1 & 9 & 1 & - & - & - & - & - & 1 & 3 & 4 & 2 & 76.5 \\
\hline 1998 & 6 & 66.7 & 5 & 1 & 6 & - & - & - & - & - & - & 1 & 1 & 3 & 1 & 77.0 \\
\hline 1999 & 7 & 42.9 & 3 & 4 & 7 & - & - & - & - & 1 & - & 1 & 2 & 2 & 1 & 68.0 \\
\hline TOTAL & 125 & 52.8 & 84 & 41 & 110 & 15 & 0 & 3 & 4 & 4 & 4 & 18 & 40 & 37 & 15 & 72.0 \\
\hline
\end{tabular}

- indicates no deaths listed.

NOTE: See appendices for source description, methods, and ICD codes.

SOURCE: National Center for Health Statistics multiple cause of death data 
Byssinosis: Mortality

Table 4-2. Byssinosis: Mortality rates (per million population) by race and sex, U.S. residents age 15 and over, 1990-1999

\begin{tabular}{|c|c|c|c|c|c|c|c|}
\hline \multirow[b]{2}{*}{ Year } & \multirow[b]{2}{*}{ Overall } & \multicolumn{2}{|c|}{ White } & \multicolumn{2}{|c|}{ Black } & \multicolumn{2}{|c|}{ Other } \\
\hline & & Male & Female & Male & Female & Male & Female \\
\hline & \multicolumn{7}{|c|}{ Crude Mortality Rate } \\
\hline 1990 & 0.09 & 0.15 & 0.06 & 0.10 & - & - & - \\
\hline 1991 & 0.06 & 0.09 & 0.05 & 0.10 & - & - & - \\
\hline 1992 & 0.08 & 0.06 & 0.06 & 0.38 & 0.08 & - & - \\
\hline 1993 & 0.08 & 0.09 & 0.06 & 0.37 & 0.08 & - & - \\
\hline 1994 & 0.08 & 0.11 & 0.07 & 0.18 & - & - & - \\
\hline 1995 & 0.07 & 0.13 & 0.03 & - & - & - & - \\
\hline 1996 & 0.04 & 0.05 & 0.06 & - & - & - & - \\
\hline 1997 & 0.05 & 0.09 & 0.01 & 0.09 & - & - & - \\
\hline 1998 & 0.03 & 0.06 & 0.01 & - & - & - & - \\
\hline 1999 & 0.03 & 0.03 & 0.04 & - & - & - & - \\
\hline \multirow[t]{2}{*}{ 1990-1999 } & 0.06 & 0.08 & 0.04 & 0.12 & 0.02 & - & - \\
\hline & \multicolumn{7}{|c|}{ Age-Adjusted Mortality Rate } \\
\hline 1990 & 0.09 & 0.17 & 0.05 & 0.14 & - & - & - \\
\hline 1991 & 0.06 & 0.12 & 0.05 & 0.20 & - & - & - \\
\hline 1992 & 0.07 & 0.06 & 0.05 & 0.46 & 0.11 & - & - \\
\hline 1993 & 0.09 & 0.10 & 0.05 & 0.47 & 0.11 & - & - \\
\hline 1994 & 0.09 & 0.13 & 0.06 & 0.39 & - & - & - \\
\hline 1995 & 0.07 & 0.14 & 0.03 & - & - & - & - \\
\hline 1996 & 0.04 & 0.05 & 0.05 & - & - & - & - \\
\hline 1997 & 0.05 & 0.11 & 0.01 & 0.18 & - & - & - \\
\hline 1998 & 0.03 & 0.07 & 0.01 & - & - & - & - \\
\hline 1999 & 0.03 & 0.03 & 0.03 & - & - & - & - \\
\hline 1990-1999 & 0.06 & 0.10 & 0.04 & 0.18 & 0.02 & - & - \\
\hline
\end{tabular}

- indicates no deaths listed.

NOTE: See appendices for source description, methods, and ICD codes.

SOURCE: National Center for Health Statistics multiple cause of death data. Population estimates from U.S. Bureau of the Census. 
Table 4-3. Byssinosis: Years of potential life lost to age 65 and to life expectancy by race and sex, U.S. residents age 15 and over, 1990-1999

\begin{tabular}{|c|c|c|c|c|c|c|c|}
\hline \multirow[b]{2}{*}{ Year } & \multicolumn{2}{|c|}{ White } & \multicolumn{2}{|c|}{ Black } & \multicolumn{2}{|c|}{ Other } & \multirow[b]{2}{*}{ Total } \\
\hline & Male & Female & Male & Female & Male & Female & \\
\hline & \multicolumn{7}{|c|}{ Years of Potential Life Lost to Age 65} \\
\hline 1990 & 55 & - & - & - & - & - & 55 \\
\hline 1991 & - & 20 & - & - & - & - & 20 \\
\hline 1992 & 50 & 35 & 90 & - & - & - & 175 \\
\hline 1993 & 45 & 5 & 45 & - & - & - & 95 \\
\hline 1994 & 25 & 30 & - & - & - & - & 55 \\
\hline 1995 & 60 & - & - & - & - & - & 60 \\
\hline 1996 & 5 & 20 & - & - & - & - & 25 \\
\hline 1997 & 5 & - & - & - & - & - & 5 \\
\hline 1998 & 5 & - & - & - & - & - & 5 \\
\hline 1999 & 30 & - & - & - & - & - & 30 \\
\hline \multirow[t]{2}{*}{ TOTAL } & 280 & 110 & 135 & - & - & - & 525 \\
\hline & \multicolumn{7}{|c|}{ Years of Potential Life Lost to Life Expectancy } \\
\hline 1990 & 187 & 55 & 11 & - & - & - & 253 \\
\hline 1991 & 64 & 79 & 7 & - & - & - & 150 \\
\hline 1992 & 111 & 113 & 112 & 9 & - & - & 345 \\
\hline 1993 & 120 & 54 & 80 & 6 & - & - & 260 \\
\hline 1994 & 117 & 104 & 16 & - & - & - & 237 \\
\hline 1995 & 167 & 40 & - & - & - & - & 207 \\
\hline 1996 & 47 & 88 & - & - & - & - & 135 \\
\hline 1997 & 85 & 7 & 7 & - & - & - & 99 \\
\hline 1998 & 48 & 16 & - & - & - & - & 64 \\
\hline 1999 & 70 & 40 & - & - & - & - & 110 \\
\hline TOTAL & 1,016 & 596 & 233 & 15 & - & - & 1,860 \\
\hline
\end{tabular}

- indicates no deaths listed.

NOTE: See appendices for source description, methods, and ICD codes.

SOURCE: National Center for Health Statistics multiple cause of death data. 
Table 4-4. Byssinosis: Number of deaths by state, U.S. residents age 15 and over, 1990-1999

\begin{tabular}{|c|c|c|c|c|c|c|c|c|c|c|c|}
\hline State & 1990 & 1991 & 1992 & 1993 & 1994 & 1995 & 1996 & 1997 & 1998 & 1999 & Total \\
\hline Alabama & 1 & 1 & - & - & - & - & - & 1 & - & - & 3 \\
\hline Alaska & - & - & - & - & - & - & - & - & - & - & - \\
\hline Arizona & 1 & - & - & - & - & - & - & - & - & - & 1 \\
\hline Arkansas & - & - & - & - & - & - & 1 & - & - & - & 1 \\
\hline California & - & - & 1 & 2 & 2 & 1 & - & 1 & - & 1 & 8 \\
\hline Colorado & - & - & - & - & 1 & - & - & - & - & 1 & 2 \\
\hline Connecticut & - & - & - & - & - & - & - & - & - & - & - \\
\hline Delaware & - & - & - & - & - & - & - & - & - & - & - \\
\hline District of Columbia & - & - & - & - & - & - & - & - & - & - & - \\
\hline Florida & 1 & - & 1 & - & 1 & - & - & 1 & - & - & 4 \\
\hline Georgia & - & 1 & - & 3 & 1 & 1 & 1 & 1 & 2 & 1 & 11 \\
\hline Hawaii & - & - & - & - & - & - & - & - & - & - & - \\
\hline Idaho & - & - & - & - & - & - & - & - & - & - & - \\
\hline Illinois & 1 & - & 1 & - & - & - & - & - & - & - & 2 \\
\hline Indiana & - & 1 & - & - & - & - & - & - & - & - & 1 \\
\hline Iowa & - & - & - & - & 1 & - & - & - & - & - & 1 \\
\hline Kansas & 1 & - & - & - & - & - & - & - & - & - & 1 \\
\hline Kentucky & - & - & - & - & - & 1 & - & - & - & - & 1 \\
\hline Louisiana & - & - & 1 & - & - & - & - & - & - & - & 1 \\
\hline Maine & - & - & - & - & - & - & - & - & - & - & - \\
\hline Maryland & - & - & - & - & - & 1 & - & - & - & - & 1 \\
\hline Massachusetts & 1 & - & 1 & 1 & - & - & - & - & - & - & 3 \\
\hline Michigan & 1 & - & - & 2 & - & - & - & - & - & - & 3 \\
\hline Minnesota & 1 & - & - & - & - & - & - & 2 & - & - & 3 \\
\hline Mississippi & - & - & - & - & - & - & - & - & - & - & - \\
\hline Missouri & - & - & - & - & - & 1 & - & - & - & - & 1 \\
\hline Montana & - & - & - & - & - & - & - & - & - & - & - \\
\hline Nebraska & - & - & - & - & - & - & - & - & - & - & - \\
\hline Nevada & - & - & - & - & - & - & - & - & - & - & - \\
\hline New Hampshire & - & 1 & - & - & - & - & - & - & - & - & 1 \\
\hline New Jersey & - & - & - & - & - & - & - & - & - & - & - \\
\hline New Mexico & - & - & - & - & - & - & - & - & - & - & - \\
\hline New York & - & - & - & - & 1 & - & - & - & - & - & 1 \\
\hline North Carolina & 5 & 2 & 8 & 4 & 8 & 8 & 4 & - & 3 & 1 & 43 \\
\hline North Dakota & - & - & - & - & - & - & - & - & - & - & - \\
\hline Ohio & 1 & - & - & - & - & - & - & - & - & - & 1 \\
\hline Oklahoma & - & 1 & - & 1 & - & - & - & - & - & - & 2 \\
\hline Oregon & - & - & - & - & - & - & - & - & - & - & - \\
\hline Pennsylvania & 1 & 1 & - & - & 1 & - & - & - & - & 1 & 4 \\
\hline Rhode Island & - & - & - & - & - & - & 1 & - & - & - & 1 \\
\hline South Carolina & 1 & 3 & 2 & - & - & 1 & 1 & 1 & 1 & 1 & 11 \\
\hline South Dakota & - & - & - & - & - & - & - & - & - & - & - \\
\hline Tennessee & - & - & - & - & - & - & - & - & - & - & - \\
\hline Texas & - & - & - & 2 & - & - & 1 & 1 & - & - & 4 \\
\hline Utah & - & - & - & - & - & - & - & - & - & - & - \\
\hline Vermont & - & - & - & - & - & - & - & - & - & 1 & 1 \\
\hline Virginia & 2 & - & - & 1 & - & - & - & - & - & - & 3 \\
\hline Washington & - & - & - & - & - & - & - & - & - & - & - \\
\hline West Virginia & - & 1 & - & 1 & - & - & - & - & - & - & 2 \\
\hline Wisconsin & - & - & - & - & 1 & - & - & 2 & - & - & 3 \\
\hline Wyoming & - & - & - & - & - & - & - & - & - & - & - \\
\hline TOTAL & 18 & 12 & 15 & 17 & 17 & 14 & 9 & 10 & 6 & 7 & 125 \\
\hline
\end{tabular}

- indicates no deaths listed. 
Table 4-5. Byssinosis: Number of deaths, mortality rates (per million population), and years of potential life lost (YPLL) by state, U.S. residents age 15 and over, 1990-1999

\begin{tabular}{|c|c|c|c|c|c|c|c|c|c|c|}
\hline \multirow[b]{2}{*}{ State } & \multirow{2}{*}{$\begin{array}{l}\text { No. of } \\
\text { Deaths }\end{array}$} & \multirow[b]{2}{*}{ Rank } & \multicolumn{2}{|c|}{ Crude Mortality } & \multicolumn{2}{|c|}{ Age-Adjusted Mortality } & \multicolumn{4}{|c|}{ YPLL to Life Expectancy } \\
\hline & & & Rate & Rank & Rate & Rank & Total & Rank & YPLL/death & Rank \\
\hline Alabama & 3 & 8 & 0.09 & 8 & 0.09 & 8 & 31 & 17 & 10.3 & 25 \\
\hline Alaska & - & - & - & - & - & - & - & - & - & - \\
\hline Arizona & 1 & 18 & 0.03 & 20 & 0.03 & 21 & 21 & 20 & 20.8 & 9 \\
\hline Arkansas & 1 & 18 & 0.05 & 15 & 0.04 & 16 & 8 & 28 & 8.4 & 27 \\
\hline California & 8 & 4 & 0.03 & 20 & 0.04 & 16 & 134 & 4 & 16.7 & 13 \\
\hline Colorado & 2 & 14 & 0.07 & 12 & 0.08 & 10 & 47 & 13 & 23.6 & 6 \\
\hline Connecticut & - & - & - & - & - & - & - & - & - & - \\
\hline Delaware & - & - & - & - & - & - & - & - & - & - \\
\hline District of Columbia & - & - & - & - & - & - & - & - & - & - \\
\hline Florida & 4 & 5 & 0.03 & 20 & 0.03 & 21 & 85 & 6 & 21.4 & 7 \\
\hline Georgia & 11 & 2 & 0.20 & 4 & 0.26 & 3 & 151 & 3 & 13.7 & 20 \\
\hline Hawaii & - & - & - & - & - & - & - & - & - & - \\
\hline Idaho & - & - & - & - & - & - & - & - & - & - \\
\hline Illinois & 2 & 14 & 0.02 & 27 & 0.02 & 27 & 104 & 5 & 52.1 & 1 \\
\hline Indiana & 1 & 18 & 0.02 & 27 & 0.02 & 27 & 14 & 23 & 14 & 19 \\
\hline Iowa & 1 & 18 & 0.05 & 15 & 0.04 & 16 & 14 & 23 & 14.1 & 17 \\
\hline Kansas & 1 & 18 & 0.05 & 15 & 0.05 & 15 & 8 & 28 & 8.3 & 29 \\
\hline Kentucky & 1 & 18 & 0.03 & 20 & 0.03 & 21 & 48 & 11 & 47.5 & 2 \\
\hline Louisiana & 1 & 18 & 0.03 & 20 & 0.03 & 21 & 48 & 11 & 47.5 & 2 \\
\hline Maine & - & - & - & - & - & - & - & - & - & - \\
\hline Maryland & 1 & 18 & 0.03 & 20 & 0.03 & 21 & 14 & 23 & 14.1 & 17 \\
\hline Massachusetts & 3 & 8 & 0.06 & 13 & 0.06 & 14 & 34 & 16 & 11.4 & 24 \\
\hline Michigan & 3 & 8 & 0.04 & 18 & 0.04 & 16 & 83 & 7 & 27.5 & 5 \\
\hline Minnesota & 3 & 8 & 0.08 & 9 & 0.09 & 8 & 57 & 8 & 18.8 & 11 \\
\hline Mississippi & - & - & - & - & - & - & - & - & - & - \\
\hline Missouri & 1 & 18 & 0.02 & 27 & 0.02 & 27 & 8 & 28 & 8.3 & 29 \\
\hline Montana & - & - & - & - & - & - & - & - & - & - \\
\hline Nebraska & - & - & - & - & - & - & - & - & - & - \\
\hline Nevada & - & - & - & - & - & - & - & - & - & - \\
\hline New Hampshire & 1 & 18 & 0.11 & 7 & 0.13 & 5 & 29 & 18 & 29.2 & 4 \\
\hline New Jersey & - & - & - & - & - & - & - & - & - & - \\
\hline New Mexico & - & - & - & - & - & - & - & - & - & - \\
\hline New York & 1 & 18 & 0.01 & 30 & 0.01 & 30 & 21 & 20 & 21.1 & 8 \\
\hline North Carolina & 43 & 1 & 0.75 & 1 & 0.78 & 1 & 577 & 1 & 13.4 & 21 \\
\hline North Dakota & - & - & - & - & - & - & - & - & - & - \\
\hline Ohio & 1 & 18 & 0.01 & 30 & 0.01 & 30 & 21 & 20 & 20.8 & 9 \\
\hline Oklahoma & 2 & 14 & 0.08 & 9 & 0.07 & 12 & 14 & 23 & 7.2 & 31 \\
\hline Oregon & - & - & - & - & - & - & - & - & - & - \\
\hline Pennsylvania & 4 & 5 & 0.04 & 18 & 0.04 & 16 & 50 & 9 & 12.5 & 22 \\
\hline Rhode Island & 1 & 18 & 0.13 & 6 & 0.11 & 7 & 8 & 28 & 8.4 & 27 \\
\hline South Carolina & 11 & 2 & 0.39 & 2 & 0.43 & 2 & 174 & 2 & 15.8 & 14 \\
\hline South Dakota & - & - & - & - & - & - & - & - & - & - \\
\hline Tennessee & - & - & - & - & - & - & - & - & - & - \\
\hline Texas & 4 & 5 & 0.03 & 20 & 0.03 & 21 & 50 & 9 & 12.5 & 22 \\
\hline Utah & - & - & - & - & - & - & - & - & - & - \\
\hline Vermont & 1 & 18 & 0.22 & 3 & 0.22 & 4 & 14 & 23 & 14.3 & 16 \\
\hline Virginia & 3 & 8 & 0.06 & 13 & 0.07 & 12 & 28 & 19 & 9.4 & 26 \\
\hline Washington & - & - & - & - & - & - & - & - & - & - \\
\hline West Virginia & 2 & 14 & 0.14 & 5 & 0.12 & 6 & 35 & 15 & 17.5 & 12 \\
\hline Wisconsin & 3 & 8 & 0.08 & 9 & 0.08 & 10 & 46 & 14 & 15.4 & 15 \\
\hline Wyoming & - & - & - & - & - & - & - & - & - & - \\
\hline
\end{tabular}

- indicates no deaths listed.

NOTE: See appendices for source description, methods, and ICD codes.

SOURCE: National Center for Health Statistics multiple cause of death data. Population estimates from U.S. Bureau of the Census. 
Table 4-6. Byssinosis: Most frequently recorded industries on death certificate, U.S. residents age 15 and over, selected states and years, 1990-1999

\begin{tabular}{clrr}
\hline CIC & Industry & Number of Deaths & Percent \\
\hline 142 & Yarn, thread, and fabric mills & 39 & 48.2 \\
961 & Non-paid worker or non-worker or own home/at home & 7 & 8.6 \\
060 & Construction & 4 & 4.9 \\
041 & Coal mining & 3 & 3.7 \\
010 & Agricultural production, crops & 2 & 2.5 \\
151 & Apparel and accessories, except knit & 2 & 2.5 \\
351 & Motor vehicles and motor vehicle equipment & 2 & 2.5 \\
392 & Not specified manufacturing industries & 2 & 2.5 \\
831 & Hospitals & 2 & 2.5 \\
901 & General government, n.e.c. & 2 & 2.5 \\
& All other industries & 15 & 18.5 \\
& Industry not reported & 1 & 1.2 \\
& TOTAL & $\mathbf{8 1}$ & $\mathbf{1 0 0 . 0}$ \\
\hline
\end{tabular}

CIC - Census Industry Code

n.e.c. - not elsewhere classified

NOTE: Percentages may not total to $100 \%$ due to rounding. See appendices for source description, methods, and ICD codes, industry and occupation codes, and list of selected states and years.

SOURCE: National Center for Health Statistics multiple cause of death data.

Table 4-7. Byssinosis: Most frequently recorded occupations on death certificate, U.S. residents age 15 and over, selected states and years, 1990-1999

\begin{tabular}{clrr}
\hline COC & Occupation & Number of Deaths & Percent \\
\hline 749 & Miscellaneous textile machine operators & 11 & 13.6 \\
738 & Winding and twisting machine operators & 8 & 9.9 \\
518 & Industrial machinery repairers & 7 & 8.6 \\
914 & Housewife/Homemaker & 7 & 8.6 \\
616 & Mining machine operators & 4 & 4.9 \\
744 & Textile sewing machine operators & 4 & 4.9 \\
473 & Farmers, except horticulture & 2 & 2.5 \\
633 & Supervisors, production occupations & 2 & 2.5 \\
637 & Machinists & 2 & 2.5 \\
739 & Knitting, looping, taping, and weaving machine operators & 2 & 2.5 \\
754 & Packaging and filling machine operators & 2 & 2.5 \\
889 & Laborers, except construction & 2 & 2.5 \\
& All other occupations & 26 & 32.1 \\
& Occupation not reported & 2 & 2.5 \\
\hline
\end{tabular}


Table 4-8. Byssinosis: Proportionate mortality ratio (PMR) adjusted for age, sex, and race by usual industry, U.S. residents age 15 and over, selected states and years, 1990-1999

\begin{tabular}{llcccc}
\hline & & Number & & \multicolumn{2}{c}{ 95\% Confidence Interval } \\
\cline { 5 - 6 } CIC & Industry & of Deaths & PMR & LCL & UCL \\
\hline 142 & Yarn, thread, and fabric mills & 39 & 22.26 & 15.79 & 30.41 \\
\hline
\end{tabular}

CIC - Census Industry Code $\quad$ n.e.c. - not elsewhere classified $\quad$ LCL - lower confidence limit $\quad$ UCL - upper confidence limit

NOTE: See appendices for source description, methods, and ICD codes, industry and occupation codes, and list of selected states and years.

SOURCE: National Center for Health Statistics multiple cause of death data. 
Table 4-9. Byssinosis: Proportionate mortality ratio (PMR) adjusted for age, sex, and race by usual occupation, U.S. residents age 15 and over, selected states and years, 1990-1999

\begin{tabular}{llrrrr}
\hline COC & Occupation & $\begin{array}{c}\text { Number } \\
\text { of Deaths }\end{array}$ & PMR & \multicolumn{2}{c}{ \%5\% Confidence Interval } \\
\cline { 5 - 6 } 749 & Miscellaneous textile machine operators & 11 & 35.76 & 17.88 & UCL \\
518 & Industrial machinery repairers & 7 & 28.07 & 11.28 & 57.97 \\
738 & Winding and twisting machine operators & 8 & 27.50 & 11.85 & 54.14 \\
\hline
\end{tabular}

COC - Census Occupation Code n.e.c. - not elsewhere classified LCL - lower confidence limit UCL - upper confidence limit NOTE: See appendices for source description, methods, and ICD codes, industry and occupation codes, and list of selected states and years. SOURCE: National Center for Health Statistics multiple cause of death data. 
$\lesssim$ Figure 4-3. Byssinosis: Age-adjusted mortality rates by county, U.S. residents age 15 and over, 1980-1999

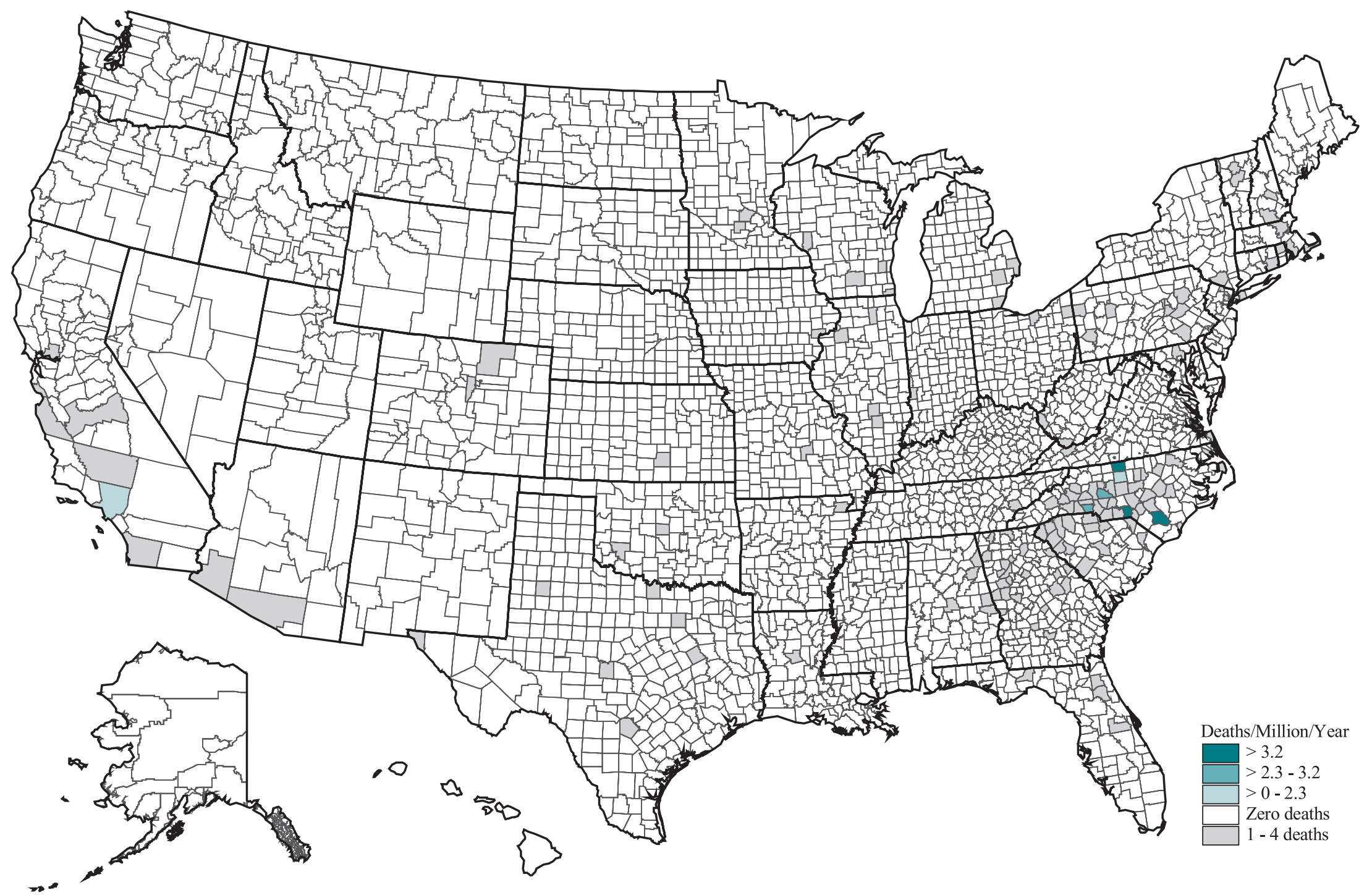

NOTE: Age-adjusted rates are not calculated for those counties with 1-4 deaths. See appendices for source description, methods, and ICD codes. SOURCE: National Center for Health Statistics multiple cause of death data. Population estimates from U.S. Bureau of the Census. 


\section{Table 4-10. Byssinosis: Counties with highest age-adjusted mortality rates (per million population), U.S. residents age 15 and over, 1985-1999}

\begin{tabular}{|c|c|c|c|c|c|}
\hline County & State & Age-Adjusted Rate & Crude Rate & Number of Deaths & \% Female \\
\hline Richmond County & North Carolina & 18.95 & 20.87 & 11 & 54.5 \\
\hline Bladen County & North Carolina & 12.38 & 14.69 & 5 & 60.0 \\
\hline Rockingham County & North Carolina & 4.25 & 4.78 & 5 & 60.0 \\
\hline Gaston County & North Carolina & 2.98 & 2.84 & 6 & 16.7 \\
\hline Guilford County & North Carolina & 1.97 & 1.84 & 8 & 12.5 \\
\hline Los Angeles County & California & 0.06 & 0.05 & 5 & 20.0 \\
\hline Overall United States & & 0.07 & 0.06 & 193 & 29.0 \\
\hline
\end{tabular}

NOTE: Only counties with at least 5 deaths from the disease of interest are included. See appendices for source description, methods, and ICD codes. SOURCE: National Center for Health Statistics multiple cause of death data. Population estimates from U.S. Bureau of the Census. 
Figure 4-4. Cotton dust: Geometric mean exposures by cotton process, OSHA samples, 1980-1999

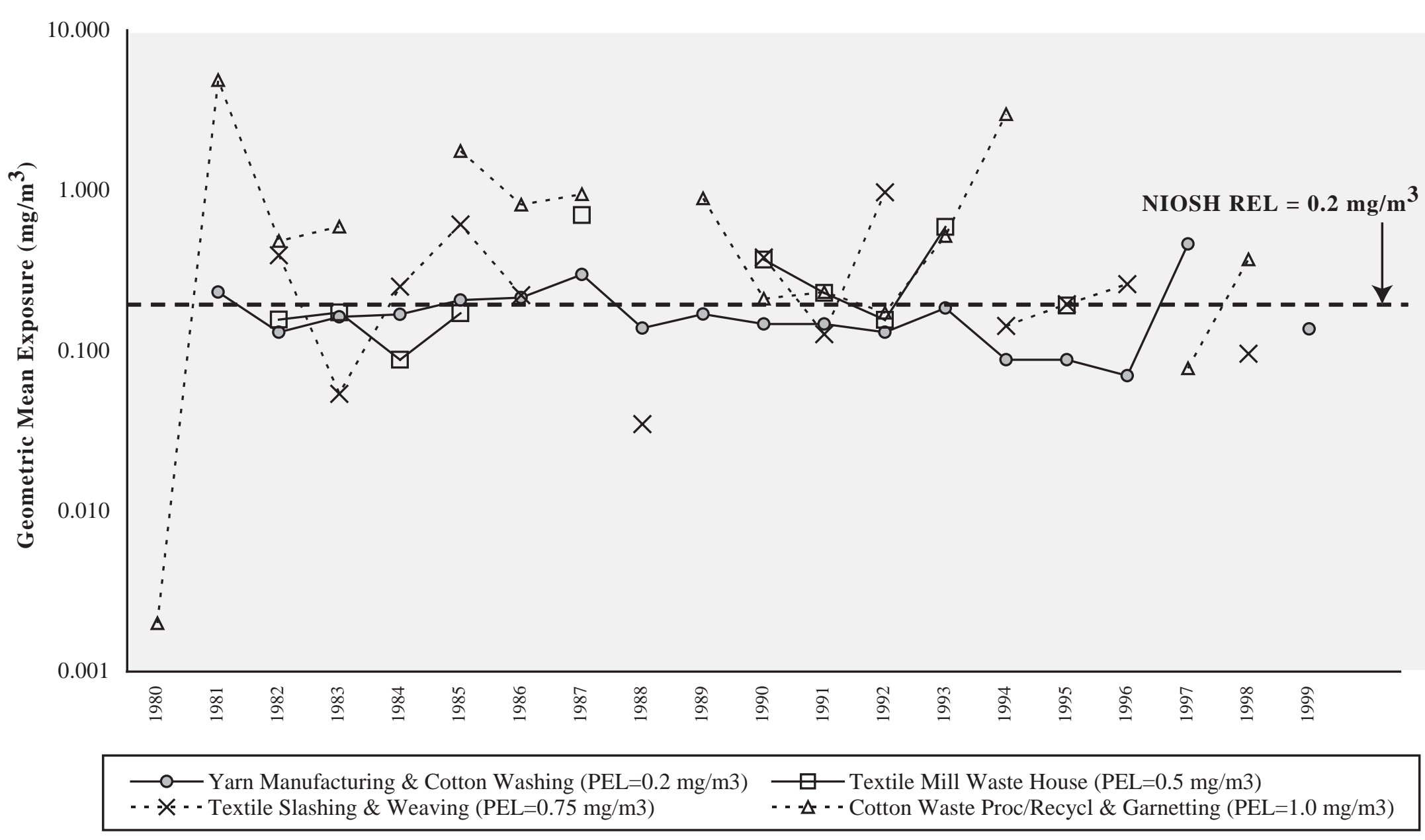

PEL - permissible exposure limit REL - recommended exposure limit $\quad \mathrm{mg} / \mathrm{m}^{3}$ - milligrams per cubic meter

NOTE: The OSHA PEL is $0.2 \mathrm{mg} / \mathrm{m}^{3}$ for yarn manufacturing and cotton washing operations, $0.5 \mathrm{mg} / \mathrm{m}^{3}$ for textile mill waste house operations or for dust from lower grade washed cotton used during yarn manufacturing, $0.75 \mathrm{mg} / \mathrm{m}^{3}$ for textile slashing and weaving operations, and $1.0 \mathrm{mg} / \mathrm{m}^{3}$ for cotton waste processing operations or waste recycling (i.e., sorting, blending, cleaning, and willowing) and garnetting. See appendices for source description, methods, and agents.

SOURCE: Occupational Safety and Health Administration (OSHA) Integrated Management Information System. 
Table 4-11. Cotton dust: Geometric mean exposures and percent exceeding designated occupational exposure limits by cotton process, OSHA samples, 1980-1999

\begin{tabular}{|c|c|c|c|c|c|c|c|c|c|c|c|c|c|c|c|c|c|c|c|c|c|}
\hline Process & & 1980 & 1981 & 1982 & 1983 & 1984 & 1985 & 1986 & 1987 & 1988 & 1989 & 1990 & 1991 & 1992 & 1993 & 1994 & 1995 & 1996 & 1997 & 1998 & 199 \\
\hline \multirow{4}{*}{$\begin{array}{l}\text { Yarn } \\
\text { Manufacturing \& } \\
\text { Cotton Washing } \\
\left(\mathrm{PEL}=0.2 \mathrm{mg} / \mathrm{m}^{3}\right)\end{array}$} & $\mathrm{GM}\left(\mathrm{mg} / \mathrm{m}^{3}\right)$ & - & 0.233 & 0.131 & 0.163 & 0.169 & 0.208 & 0.215 & 0.300 & 0.139 & 0.170 & 0.147 & 0.147 & 0.131 & 0.185 & 0.088 & 0.088 & 0.070 & 0.465 & - & 0.13 \\
\hline & No. of samples & 0 & 7 & 67 & 130 & 54 & 70 & 46 & 17 & 35 & 8 & 17 & 88 & 104 & 39 & 36 & 31 & 6 & 4 & 0 & \\
\hline & $\%>$ PEL & - & 57.1 & 28.4 & 28.5 & 40.7 & 52.9 & 45.7 & 70.6 & 28.6 & 37.5 & 23.5 & 25.0 & 28.8 & 35.9 & 13.9 & 12.9 & 0.0 & 100.0 & - & 14 \\
\hline & $\%>$ REL & - & 57.1 & 28.4 & 28.5 & 40.7 & 52.9 & 45.7 & 70.6 & 28.6 & 37.5 & 23.5 & 25.0 & 28.8 & 35.9 & 13.9 & 12.9 & 0.0 & 100.0 & - & 14 \\
\hline \multirow{4}{*}{$\begin{array}{l}\text { Textile Mill Waste } \\
\text { House or Dust from } \\
\text { Lower-Grade } \\
\text { Cotton Washing } \\
\text { During Yarn } \\
\text { Manufacturing } \\
\left(\mathrm{PEL}=0.5 \mathrm{mg} / \mathrm{m}^{3}\right)\end{array}$} & $\mathrm{GM}\left(\mathrm{mg} / \mathrm{m}^{3}\right)$ & - & - & 0.157 & 0.174 & 0.088 & 0.172 & - & 0.703 & - & - & 0.371 & 0.230 & 0.156 & 0.593 & - & 0.192 & - & & - & \\
\hline & No. of samples & 0 & 0 & 5 & 10 & 8 & 7 & 0 & 4 & 0 & 0 & 1 & 7 & 8 & 17 & 0 & 4 & 0 & 0 & 0 & \\
\hline & $\%>$ PEL & - & - & 0.0 & 20.0 & 0.0 & 14.3 & - & 100.0 & - & - & 0.0 & 14.3 & 0.0 & 70.6 & - & 50.0 & - & - & - & \\
\hline & $\%>$ REL & - & - & 40.0 & 20.0 & 25.0 & 28.6 & - & 100.0 & - & - & 100.0 & 28.6 & 25.0 & 94.1 & - & 50.0 & - & - & - & \\
\hline \multirow{4}{*}{$\begin{array}{l}\text { Textile Slashing } \\
\text { and Weaving } \\
\left(\mathrm{PEL}=0.75 \mathrm{mg} / \mathrm{m}^{3}\right)\end{array}$} & $\mathrm{GM}\left(\mathrm{mg} / \mathrm{m}^{3}\right)$ & - & - & 0.396 & 0.054 & 0.252 & 0.616 & 0.223 & - & 0.035 & - & 0.383 & 0.127 & 0.978 & - & 0.143 & 0.196 & 0.261 & - & 0.096 & \\
\hline & No. of samples & 0 & 0 & 12 & 16 & 7 & 9 & 4 & 0 & 2 & 0 & 3 & 11 & 4 & 0 & 3 & 4 & 5 & 0 & 6 & \\
\hline & $\%>$ PEL & - & - & 25.0 & 6.3 & 14.3 & 11.1 & 0.0 & - & 0.0 & - & 0.0 & 0.0 & 100.0 & - & 0.0 & 0.0 & 0.0 & - & 0.0 & \\
\hline & $\%>$ REL & - & - & 83.3 & 18.8 & 71.4 & 100.0 & 50.0 & - & 0.0 & - & 100.0 & 45.5 & 100.0 & - & 66.7 & 75.0 & 80.0 & - & 33.3 & \\
\hline \multirow{4}{*}{$\begin{array}{l}\text { Cotton Waste } \\
\text { Processing/ } \\
\text { Recycling \& } \\
\text { Garnetting } \\
\left(\mathrm{PEL}=1.0 \mathrm{mg} / \mathrm{m}^{3}\right)\end{array}$} & $\mathrm{GM}\left(\mathrm{mg} / \mathrm{m}^{3}\right)$ & 0.002 & 4.90 & 0.485 & 0.597 & - & 1.77 & 0.818 & 0.949 & - & 0.896 & 0.212 & 0.233 & 0.173 & 0.521 & 3.00 & - & - & 0.078 & 0.372 & \\
\hline & No. of samples & 1 & 3 & 4 & 8 & 0 & 4 & 13 & 7 & 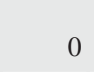 & 2 & 2 & 16 & 10 & 5 & 1 & 0 & 0 & 2 & 2 & \\
\hline & $\%>$ PEL & 0.0 & 100.0 & 25.0 & 37.5 & - & 50.0 & 23.1 & 42.9 & - & 0.0 & 0.0 & 0.0 & 0.0 & 20.0 & 100.0 & - & - & 0.0 & 0.0 & \\
\hline & $\%>$ REL & 0.0 & 100.0 & 100.0 & 50.0 & - & 75.0 & 100.0 & 100.0 & - & 100.0 & 50.0 & 50.0 & 30.0 & 80.0 & 100.0 & - & - & 0.0 & 100.0 & \\
\hline
\end{tabular}

- indicates incalculable field

PEL - permissible exposure limit $\quad$ REL - recommended exposure limit $\quad$ GM - geometric mean $\quad \mathrm{mg} / \mathrm{m}^{3}$ - milligrams per cubic meter

NOTE: The NIOSH REL is $0.2 \mathrm{mg} / \mathrm{m}^{3}$. See appendices for source description, methods, and agents.

SOURCE: Occupational Safety and Health Administration (OSHA) Integrated Management Information System. 
Table 4-12. Cotton Dust: Number of samples, geometric mean exposures, and percent exceeding designated occupational exposure limits by industries with elevated byssinosis mortality, OSHA samples, 1990-1999

\begin{tabular}{|c|c|c|c|c|c|c|c|}
\hline \multicolumn{4}{|c|}{$\begin{array}{c}\text { Byssinosis Mortality, } \\
\text { Selected States and Years, 1990-1999 }\end{array}$} & \multirow[b]{2}{*}{$\begin{array}{l}\text { Number of } \\
\text { Samples }\end{array}$} & \multirow[b]{2}{*}{$\underset{\left(\mathbf{m g} / \mathbf{m}^{3}\right)}{\mathbf{G M}}$} & \multirow[b]{2}{*}{$\begin{array}{l}\%> \\
\text { PEL }\end{array}$} & \multirow[b]{2}{*}{$\begin{array}{l}\%> \\
\text { REL }\end{array}$} \\
\hline CIC & $\begin{array}{l}\text { Industries with elevated PMRs and most } \\
\text { frequently recorded on death certificates }\end{array}$ & $\begin{array}{l}\text { Number of } \\
\text { Deaths }\end{array}$ & PMR & & & & \\
\hline \multirow[t]{3}{*}{142} & Yarn, thread, and fabric mills & 39 & 22.26 & 404 & 0.133 & 23.3 & 31.9 \\
\hline & All other industries & 41 & & 64 & 0.086 & 17.2 & 31.3 \\
\hline & TOTAL & & & 468 & 0.125 & 22.4 & 31.8 \\
\hline \multicolumn{8}{|c|}{$\begin{array}{l}\text { CIC - Census Industry Code PEL - permissible exposure limit REL - recommended exposure limit PMR - proportionate mortality ratio } \\
\text { GM - geometric mean } \mathrm{mg} / \mathrm{m}^{3} \text { - milligrams per cubic meter } \\
\text { NOTE: The OSHA PEL is } 0.2 \mathrm{mg} / \mathrm{m}^{3} \text { for yarn manufacturing and cotton washing operations, } 0.5 \mathrm{mg} / \mathrm{m}^{3} \text { for textile mill waste house operations or for dust } \\
\text { from lower grade washed cotton used during yarn manufacturing, } 0.75 \mathrm{mg} / \mathrm{m}^{3} \text { for textile slashing and weaving operations, and } 1.0 \mathrm{mg} / \mathrm{m}^{3} \text { for cotton waste } \\
\text { processing operations or waste recycling (i.e., sorting, blending, cleaning, and willowing) and garnetting. The NIOSH REL is } 0.2 \mathrm{mg} / \mathrm{m}^{3} \text {. See appendices } \\
\text { for source description, methods, ICD codes, industry codes, agents, and list of selected states (and years) for which usual industry has been reported. } \\
\text { SOURCE: Occupational Safety and Health Administration (OSHA) Integrated Management Information System. National Center for Health Statistics } \\
\text { multiple cause of death data. }\end{array}$} \\
\hline
\end{tabular}


Table 4-13 (page 1 of 3). Cotton dust: Geometric mean exposures and percent exceeding designated occupational exposure limits by OSHA region, state and process, OSHA samples, 1980-1999

\begin{tabular}{|c|c|c|c|c|c|c|c|c|c|c|c|c|c|c|}
\hline \multirow[b]{2}{*}{ OSHA Region } & \multicolumn{2}{|c|}{ All years } & \multicolumn{4}{|c|}{1980 - 1989} & \multicolumn{4}{|c|}{1990 - 1994} & \multicolumn{4}{|c|}{$1995-1999$} \\
\hline & \begin{tabular}{|c|} 
No. of \\
Samples
\end{tabular} & $\begin{array}{c}\mathbf{G M} \\
\left(\mathbf{m g} / \mathbf{m}^{3}\right)\end{array}$ & $\begin{array}{c}\text { No. of } \\
\text { Samples }\end{array}$ & $\begin{array}{c}\text { GM } \\
\left(\mathbf{m g} / \mathbf{m}^{3}\right)\end{array}$ & $\begin{array}{l}\%> \\
\text { PEL }\end{array}$ & $\begin{array}{l}\%> \\
\text { REL }\end{array}$ & $\begin{array}{c}\text { No. of } \\
\text { Samples }\end{array}$ & $\begin{array}{c}\mathbf{G M} \\
\left(\mathbf{m g} / \mathbf{m}^{3}\right)\end{array}$ & $\begin{array}{l}\%> \\
\text { PEL }\end{array}$ & $\begin{array}{c}\%> \\
\text { REL }\end{array}$ & \begin{tabular}{|c|}
$\begin{array}{c}\text { No. of } \\
\text { Samples }\end{array}$ \\
\end{tabular} & $\begin{array}{c}\mathbf{G M} \\
\left(\mathbf{m g} / \mathbf{m}^{3}\right)\end{array}$ & $\begin{array}{l}\%> \\
\text { PEL }\end{array}$ & $\begin{array}{l}\%> \\
\text { REL }\end{array}$ \\
\hline Region 1 & 7 & 0.044 & $\mathbf{0}$ & - & - & - & 3 & 0.139 & 0.0 & 0.0 & 4 & 0.019 & 0.0 & 0.0 \\
\hline Massachusetts & 3 & 0.139 & $\mathbf{0}$ & - & - & - & 3 & 0.139 & 0.0 & 0.0 & $\mathbf{0}$ & - & - & - \\
\hline Cotton Waste Proc/Recycl \& Garnetting & 3 & 0.139 & 0 & - & - & - & 3 & 0.139 & 0.0 & 0.0 & 0 & - & - & - \\
\hline New Hampshire & 4 & 0.019 & $\mathbf{0}$ & - & - & - & $\mathbf{0}$ & - & - & - & 4 & 0.019 & 0.0 & 0.0 \\
\hline Yarn Manufacturing \& Cotton Washing & 2 & 0.121 & 0 & - & - & - & 0 & - & - & - & 2 & 0.121 & 0.0 & 0.0 \\
\hline Undetermined Process & 2 & 0.003 & 0 & - & - & - & 0 & - & - & - & 2 & 0.003 & - & 0.0 \\
\hline Region 2 & 10 & 1.093 & 10 & 1.093 & $\mathbf{5 0 . 0}$ & 90.0 & $\mathbf{0}$ & - & - & - & $\mathbf{0}$ & - & - & - \\
\hline New Jersey & 9 & 2.119 & 9 & 2.119 & 55.6 & 100.0 & $\mathbf{0}$ & - & - & - & $\mathbf{0}$ & - & - & - \\
\hline Textile Mill Waste House & 1 & 0.965 & 1 & 0.965 & 100.0 & 100.0 & 0 & - & - & - & 0 & - & - & - \\
\hline Cotton Waste Proc/Recycl \& Garnetting & 8 & 2.338 & 8 & 2.338 & 50.0 & 100.0 & 0 & - & - & - & 0 & - & - & - \\
\hline Puerto Rico & 1 & 0.003 & 1 & 0.003 & - & 0.0 & $\mathbf{0}$ & - & - & - & $\mathbf{0}$ & - & - & - \\
\hline Undetermined Process & 1 & 0.003 & 1 & 0.003 & - & 0.0 & 0 & - & - & - & 0 & - & - & - \\
\hline Region 3 & 4 & 0.029 & 4 & 0.029 & 0.0 & 25.0 & $\mathbf{0}$ & - & - & - & $\mathbf{0}$ & - & - & - \\
\hline Maryland & 1 & 0.014 & 1 & 0.014 & - & 0.0 & $\mathbf{0}$ & - & - & - & $\mathbf{0}$ & - & - & - \\
\hline Undetermined Process & 1 & 0.014 & 1 & 0.014 & - & 0.0 & 0 & - & - & - & 0 & - & - & - \\
\hline Pennsylvania & 3 & 0.038 & 3 & 0.038 & 0.0 & 33.3 & $\mathbf{0}$ & - & - & - & $\mathbf{0}$ & - & - & - \\
\hline Textile Mill Waste House & 1 & 0.260 & 1 & 0.260 & 0.0 & 100.0 & 0 & - & - & - & 0 & - & - & - \\
\hline Cotton Waste Proc/Recycl \& Garnetting & 1 & 0.072 & 1 & 0.072 & 0.0 & 0.0 & 0 & - & - & - & 0 & - & - & - \\
\hline Undetermined Process & 1 & 0.003 & 1 & 0.003 & - & 0.0 & 0 & - & - & - & 0 & - & - & - \\
\hline \multicolumn{15}{|l|}{ See footnotes at end of table. } \\
\hline
\end{tabular}


Table 4-13 (page 2 of 3). Cotton dust: Geometric mean exposures and percent exceeding designated occupational exposure limits by OSHA region, state and process, OSHA samples, 1980-1999

\begin{tabular}{|c|c|c|c|c|c|c|c|c|c|c|c|c|c|c|}
\hline \multirow[b]{2}{*}{ OSHA Region } & \multicolumn{2}{|c|}{ All years } & \multicolumn{4}{|c|}{1980 - 1989} & \multicolumn{4}{|c|}{1990 - 1994} & \multicolumn{4}{|c|}{1995 - 1999} \\
\hline & \begin{tabular}{|c|}
$\begin{array}{c}\text { No. of } \\
\text { Samples }\end{array}$ \\
\end{tabular} & $\begin{array}{c}\mathbf{G M} \\
\left(\mathbf{m g} / \mathbf{m}^{3}\right) \\
\end{array}$ & $\begin{array}{c}\text { No. of } \\
\text { Samples }\end{array}$ & $\begin{array}{c}\mathbf{G M} \\
\left(\mathbf{m g} / \mathbf{m}^{3}\right) \\
\end{array}$ & $\begin{array}{l}\%> \\
\text { PEL }\end{array}$ & $\begin{array}{l}\%> \\
\text { REL }\end{array}$ & \begin{tabular}{|c|c|} 
No. of \\
Samples
\end{tabular} & $\begin{array}{c}\mathbf{G M} \\
\left(\mathbf{m g} / \mathbf{m}^{3}\right) \\
\end{array}$ & $\begin{array}{l}\%> \\
\text { PEL }\end{array}$ & $\begin{array}{l}\%> \\
\text { REL }\end{array}$ & \begin{tabular}{|c|} 
No. of \\
Samples
\end{tabular} & $\begin{array}{c}\mathbf{G M} \\
\left(\mathbf{m g} / \mathbf{m}^{3}\right) \\
\end{array}$ & $\begin{array}{l}\%> \\
\text { PEL }\end{array}$ & $\begin{array}{l}\%> \\
\text { REL }\end{array}$ \\
\hline Region 4 & 909 & 0.136 & 470 & 0.154 & 30.2 & 37.2 & 367 & 0.123 & 22.1 & 29.7 & 72 & 0.096 & 13.9 & 29.2 \\
\hline Alabama & 83 & 0.230 & 37 & 0.282 & 56.8 & 56.8 & 46 & 0.196 & 19.6 & 41.3 & $\mathbf{0}$ & - & - & - \\
\hline Yarn Manufacturing \& Cotton Washing & 62 & 0.196 & 33 & 0.223 & 54.5 & 54.5 & 29 & 0.168 & 31.0 & 31.0 & 0 & - & - & - \\
\hline Textile Mill Waste House & 3 & 0.154 & 1 & 0.115 & 0.0 & 0.0 & 2 & 0.179 & 0.0 & 50.0 & 0 & - & - & - \\
\hline Textile Slashing \& Weaving & 1 & 0.449 & 0 & - & - & - & 1 & 0.449 & 0.0 & 100.0 & 0 & - & - & - \\
\hline Cotton Waste Proc/Recycl \& Garnetting & 17 & 0.431 & 3 & 4.898 & 100.0 & 100.0 & 14 & 0.256 & 0.0 & 57.1 & 0 & - & - & - \\
\hline Georgia & 196 & 0.156 & 131 & 0.160 & 43.5 & 46.6 & 53 & 0.146 & 24.5 & 28.3 & 12 & 0.156 & 41.7 & 41.7 \\
\hline Yarn Manufacturing \& Cotton Washing & 177 & 0.168 & 120 & 0.175 & 47.5 & 47.5 & 46 & 0.147 & 28.3 & 28.3 & 11 & 0.184 & 45.5 & 45.5 \\
\hline Textile Mill Waste House & 4 & 0.159 & 1 & 0.098 & 0.0 & 0.0 & 3 & 0.187 & 0.0 & 0.0 & 0 & - & - & - \\
\hline Textile Slashing \& Weaving & 11 & 0.086 & 6 & 0.089 & 0.0 & 33.3 & 4 & 0.111 & 0.0 & 50.0 & 1 & 0.026 & 0.0 & 0.0 \\
\hline Cotton Waste Proc/Recycl \& Garnetting & 2 & 0.376 & 2 & 0.376 & 0.0 & 100.0 & 0 & - & - & - & 0 & - & - & - \\
\hline Undetermined Process & 2 & 0.003 & 2 & 0.003 & - & 0.0 & 0 & - & - & - & 0 & - & - & - \\
\hline Mississippi & 1 & 0.050 & 1 & 0.050 & 0.0 & $\mathbf{0 . 0}$ & $\mathbf{0}$ & - & - & - & $\mathbf{0}$ & - & - & - \\
\hline Yarn Manufacturing \& Cotton Washing & 1 & 0.050 & 1 & 0.050 & 0.0 & 0.0 & 0 & - & - & - & 0 & - & - & - \\
\hline North Carolina & 495 & 0.124 & 250 & 0.129 & 21.2 & 28.0 & 208 & 0.128 & 21.6 & 27.9 & 37 & 0.076 & 8.1 & 18.9 \\
\hline Yarn Manufacturing \& Cotton Washing & 380 & 0.132 & 189 & 0.144 & 24.9 & 24.9 & 166 & 0.131 & 24.1 & 24.1 & 25 & 0.074 & 12.0 & 12.0 \\
\hline Textile Mill Waste House & 27 & 0.118 & 15 & 0.087 & 0.0 & 13.3 & 11 & 0.201 & 9.1 & 36.4 & 1 & 0.037 & 0.0 & 0.0 \\
\hline Textile Slashing \& Weaving & 53 & 0.161 & 31 & 0.141 & 12.9 & 51.6 & 16 & 0.255 & 25.0 & 68.8 & 6 & 0.096 & 0.0 & 33.3 \\
\hline Cotton Waste Proc/Recycl \& Garnetting & 22 & 0.202 & 9 & 0.255 & 22.2 & 55.6 & 9 & 0.172 & 0.0 & 33.3 & 4 & 0.170 & 0.0 & 50.0 \\
\hline Undetermined Process & 13 & 0.003 & 6 & 0.003 & - & 0.0 & 6 & 0.003 & - & 0.0 & 1 & 0.003 & - & 0.0 \\
\hline South Carolina & 118 & 0.156 & 47 & 0.202 & 21.3 & 40.4 & 51 & 0.115 & 27.5 & 33.3 & 20 & 0.184 & 10.0 & 45.0 \\
\hline Yarn Manufacturing \& Cotton Washing & 78 & 0.123 & 26 & 0.130 & 15.4 & 15.4 & 43 & 0.125 & 30.2 & 30.2 & 9 & 0.098 & 0.0 & 0.0 \\
\hline Textile Mill Waste House & 12 & 0.247 & 9 & 0.224 & 44.4 & 55.6 & 0 & - & - & - & 3 & 0.332 & 66.7 & 66.7 \\
\hline Textile Slashing \& Weaving & 20 & 0.403 & 12 & 0.489 & 16.7 & 83.3 & 0 & - & - & - & 8 & 0.302 & 0.0 & 87.5 \\
\hline Cotton Waste Proc/Recycl \& Garnetting & 5 & 0.521 & 0 & - & - & - & 5 & 0.521 & 20.0 & 80.0 & 0 & - & - & - \\
\hline Undetermined Process & 3 & 0.003 & 0 & - & - & - & 3 & 0.003 & - & 0.0 & 0 & - & - & - \\
\hline Tennessee & 16 & 0.011 & 4 & 0.645 & 25.0 & 100.0 & 9 & 0.003 & 0.0 & 0.0 & 3 & 0.003 & 0.0 & $\mathbf{0 . 0}$ \\
\hline Yarn Manufacturing \& Cotton Washing & 1 & 7.400 & 1 & 7.400 & 100.0 & 100.0 & 0 & - & - & - & 0 & - & - & - \\
\hline Cotton Waste Proc/Recycl \& Garnetting & 3 & 0.286 & 3 & 0.286 & 0.0 & 100.0 & 0 & - & - & - & 0 & - & - & - \\
\hline Undetermined Process & 12 & 0.003 & 0 & - & - & - & 9 & 0.003 & - & 0.0 & 3 & 0.003 & - & 0.0 \\
\hline
\end{tabular}

See footnotes at end of table. 
Table 4-13 (page 3 of 3). Cotton dust: Geometric mean exposures and percent exceeding designated occupational exposure limits by OSHA region, state and process, OSHA samples, 1980-1999

\begin{tabular}{|c|c|c|c|c|c|c|c|c|c|c|c|c|c|c|}
\hline \multirow[b]{2}{*}{ OSHA Region } & \multicolumn{2}{|c|}{ All years } & \multicolumn{4}{|c|}{1980 - 1989} & \multicolumn{4}{|c|}{$1990-1994$} & \multicolumn{4}{|c|}{1995 - 1999} \\
\hline & \begin{tabular}{|c|}
$\begin{array}{c}\text { No. of } \\
\text { Samples }\end{array}$ \\
\end{tabular} & $\begin{array}{c}\text { GM } \\
\left(\mathrm{mg} / \mathrm{m}^{3}\right)\end{array}$ & $\begin{array}{c}\text { No. of } \\
\text { Samples }\end{array}$ & $\begin{array}{c}\mathbf{G M} \\
\left(\mathbf{m g} / \mathbf{m}^{3}\right) \\
\end{array}$ & $\begin{array}{l}\%> \\
\text { PEL }\end{array}$ & $\begin{array}{l}\%> \\
\text { REL }\end{array}$ & $\begin{array}{c}\begin{array}{c}\text { No. of } \\
\text { Samples }\end{array} \\
\end{array}$ & $\begin{array}{c}\mathbf{G M} \\
\left(\mathrm{mg} / \mathbf{m}^{3}\right)\end{array}$ & $\begin{array}{l}\%> \\
\text { PEL }\end{array}$ & $\begin{array}{l}\%> \\
\text { REL }\end{array}$ & \begin{tabular}{|c|} 
No. of \\
Samples
\end{tabular} & $\begin{array}{c}\mathbf{G M} \\
\left(\mathbf{m g} / \mathbf{m}^{3}\right)\end{array}$ & $\begin{array}{l}\%> \\
\text { PEL }\end{array}$ & $\begin{array}{l}\%> \\
\text { REL }\end{array}$ \\
\hline Region 5 & 8 & 3.415 & 7 & 3.479 & 71.4 & 71.4 & 1 & 3.000 & 100.0 & 100.0 & $\mathbf{0}$ & - & - & - \\
\hline Illinois & 7 & 3.479 & 7 & 3.479 & 71.4 & 71.4 & $\mathbf{0}$ & - & - & - & $\mathbf{0}$ & - & - & - \\
\hline Yarn Manufacturing \& Cotton Washing & 2 & 0.200 & 2 & 0.200 & 0.0 & 0.0 & 0 & - & - & - & 0 & - & - & - \\
\hline Textile Mill Waste House & 2 & 7.421 & 2 & 7.421 & 100.0 & 100.0 & 0 & - & - & - & 0 & - & - & - \\
\hline Cotton Waste Proc/Recycl \& Garnetting & 3 & 14.092 & 3 & 14.092 & 100.0 & 100.0 & 0 & - & - & - & 0 & - & - & - \\
\hline Michigan & 1 & 3.000 & $\mathbf{0}$ & - & - & - & 1 & 3.000 & 100.0 & 100.0 & $\mathbf{0}$ & - & - & - \\
\hline Cotton Waste Proc/Recycl \& Garnetting & 1 & 3.000 & 0 & - & - & - & 1 & 3.000 & 100.0 & 100.0 & 0 & - & - & - \\
\hline Region 6 & 77 & 0.281 & 75 & 0.283 & 54.7 & 62.7 & 2 & 0.212 & 0.0 & 50.0 & $\mathbf{0}$ & - & - & - \\
\hline Texas & 77 & 0.281 & 75 & 0.283 & 54.7 & 62.7 & 2 & 0.212 & $\mathbf{0 . 0}$ & 50.0 & $\mathbf{0}$ & - & - & - \\
\hline Yarn Manufacturing \& Cotton Washing & 62 & 0.278 & 62 & 0.278 & 61.3 & 61.3 & 0 & - & - & - & 0 & - & - & - \\
\hline Textile Mill Waste House & 4 & 0.139 & 4 & 0.139 & 0.0 & 25.0 & 0 & - & - & - & 0 & - & - & - \\
\hline Textile Slashing \& Weaving & 1 & 0.410 & 1 & 0.410 & 0.0 & 100.0 & 0 & - & - & - & 0 & - & - & - \\
\hline Cotton Waste Proc/Recycl \& Garnetting & 10 & 0.380 & 8 & 0.439 & 37.5 & 87.5 & 2 & 0.212 & 0.0 & 50.0 & 0 & - & - & - \\
\hline Region 7 & 19 & 0.493 & 1 & 0.740 & 0.0 & 100.0 & 18 & 0.482 & 66.7 & 88.9 & $\mathbf{0}$ & - & - & - \\
\hline Kansas & 18 & 0.482 & $\mathbf{0}$ & - & - & - & 18 & 0.482 & 66.7 & 88.9 & $\mathbf{0}$ & - & - & - \\
\hline Textile Mill Waste House & 17 & 0.593 & 0 & - & - & - & 17 & 0.593 & 70.6 & 94.1 & 0 & - & - & - \\
\hline Undetermined Process & 1 & 0.014 & 0 & - & - & - & 1 & 0.014 & - & 0.0 & 0 & - & - & - \\
\hline Missouri & 1 & 0.740 & 1 & 0.740 & 0.0 & 100.0 & $\mathbf{0}$ & - & - & - & $\mathbf{0}$ & - & - & - \\
\hline Cotton Waste Proc/Recycl \& Garnetting & 1 & 0.740 & 1 & 0.740 & 0.0 & 100.0 & 0 & - & - & - & 0 & - & - & - \\
\hline Region 8 & 4 & 0.714 & 4 & 0.714 & 0.0 & 100.0 & $\mathbf{0}$ & - & - & - & $\mathbf{0}$ & - & - & - \\
\hline Colorado & 2 & 0.570 & 2 & 0.570 & 0.0 & 100.0 & $\mathbf{0}$ & - & - & - & $\mathbf{0}$ & - & - & - \\
\hline Cotton Waste Proc/Recycl \& Garnetting & 2 & 0.570 & 2 & 0.570 & 0.0 & 100.0 & 0 & & & - & 0 & - & & - \\
\hline Utah & 2 & 0.896 & 2 & 0.896 & 0.0 & 100.0 & $\mathbf{0}$ & - & - & - & $\mathbf{0}$ & - & - & - \\
\hline Cotton Waste Proc/Recycl \& Garnetting & 2 & 0.896 & 2 & 0.896 & 0.0 & 100.0 & 0 & - & - & - & 0 & - & - & - \\
\hline Region 9 & 1 & 1.958 & $\mathbf{0}$ & - & - & - & $\mathbf{0}$ & - & - & - & 1 & 1.958 & 100.0 & 100.0 \\
\hline Hawaii & 1 & 1.958 & $\mathbf{0}$ & - & - & - & $\mathbf{0}$ & - & - & - & 1 & 1.958 & 100.0 & 100.0 \\
\hline Yarn Manufacturing \& Cotton Washing & 1 & 1.958 & 0 & - & - & - & 0 & - & - & - & 1 & 1.958 & 100.0 & 100.0 \\
\hline TOTAL & 1,039 & 0.153 & 571 & 0.180 & 33.8 & 42.4 & 391 & 0.133 & 24.0 & 32.5 & 77 & 0.091 & 14.3 & 28.6 \\
\hline $\begin{array}{l}\text { - indicates incalculable field PEL } \\
\text { NOTE: The OSHA PEL is } 0.2 \mathrm{mg} / \mathrm{m}^{3} \text { for y } \\
\text { used during yarn manufacturing, } 0.75 \mathrm{mg} / \mathrm{r} \\
\text { cleaning, and willowing) and garnetting. T } \\
\text { SOURCE: Occupational Safety and Healt }\end{array}$ & $\begin{array}{l}\text { - permissibl } \\
\text { yarn manufa } \\
\mathrm{m}^{3} \text { for textil } \\
\text { he NIOSH }\end{array}$ & $\begin{array}{l}\text { le exposure } \\
\text { acturing anc } \\
\text { le slashing } \\
\text { REL is } 0.2\end{array}$ & $\begin{array}{l}\text { mit } \\
\text { otton w: } \\
\text { weavir } \\
\mathrm{g} / \mathrm{m}^{3} . \mathrm{S}\end{array}$ & $\begin{array}{l}\text { REL - reco } \\
\text { hing operatic } \\
\text { operations, } \\
\text { appendices }\end{array}$ & ommend & $\begin{array}{l}\text { exposur } \\
\mathrm{g} / \mathrm{m}^{3} \text { for } \\
\mathrm{g} / \mathrm{m}^{3} \text { for }\end{array}$ & $\begin{array}{l}\text { ce limit } \\
\text { textile m } \\
\text { cotton } \mathrm{x} \\
\text { tion, met }\end{array}$ & $\begin{array}{r}\text { GM - } \\
\text { waste hou } \\
\text { e process }\end{array}$ & $\begin{array}{l}\text { metr } \\
\text { oper } \\
\text { oper }\end{array}$ & $\begin{array}{l}\text { ean } \\
\text { s or }\end{array}$ & $\begin{array}{l}\mathrm{mg} / \mathrm{m}^{3} \\
\text { dust from } 1 \\
\text { te recyclin }\end{array}$ & $\begin{array}{l}\text { - milligram } \\
\text { lower grade } \\
\text { a (i.e., sort }\end{array}$ & $\begin{array}{l}\text { s per cub } \\
\text { washed } \\
\text { ing, blen }\end{array}$ & $\begin{array}{l}\text { ic meter } \\
\text { cotton } \\
\text { ding, }\end{array}$ \\
\hline
\end{tabular}


Figure 4-5. Cotton dust: Percent of exposures exceeding the NIOSH recommended exposure limit by state, OSHA samples, 1980-1999



\begin{tabular}{|cc|}
\hline \multicolumn{2}{|c|}{$\mathbf{1 9 8 0}$ - 1989} \\
\hline$\%>$ REL & No. of States \\
$\square>50$ & 2 \\
$\square>25$ to 50 & 3 \\
$\square 0$ to 25 & 0 \\
$\square<10$ samples & 46 \\
\hline
\end{tabular}

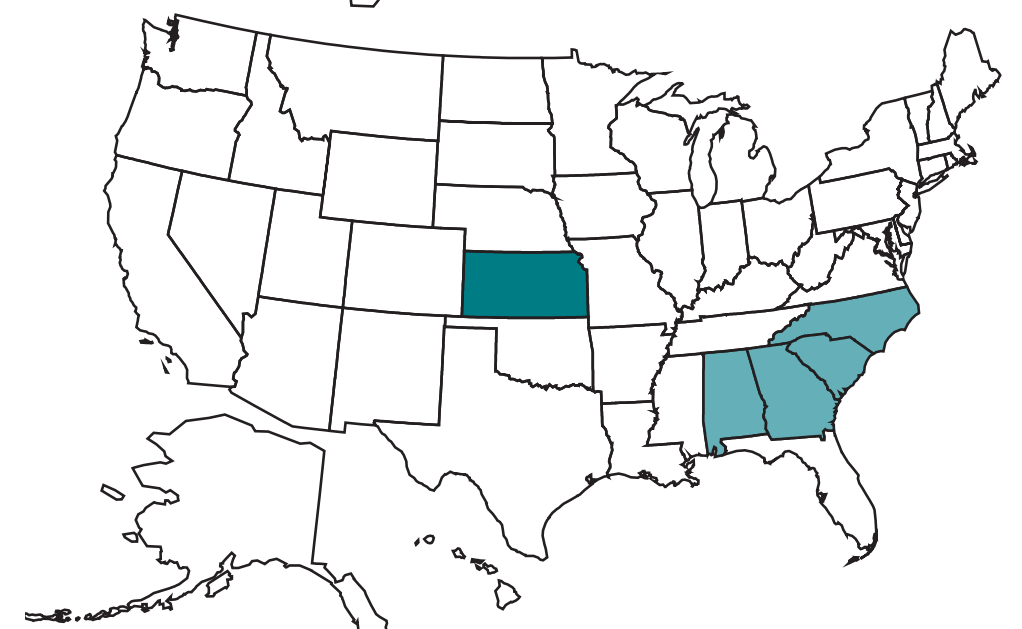

\begin{tabular}{|lc|}
\hline \multicolumn{2}{|c|}{$\mathbf{1 9 9 0}$ - 1994} \\
\hline$\%>$ REL & No. of States \\
$\square>50$ & 1 \\
$\square>25$ to 50 & 4 \\
$\square 0$ to 25 & 0 \\
$\square<10$ samples & 46 \\
\hline
\end{tabular}

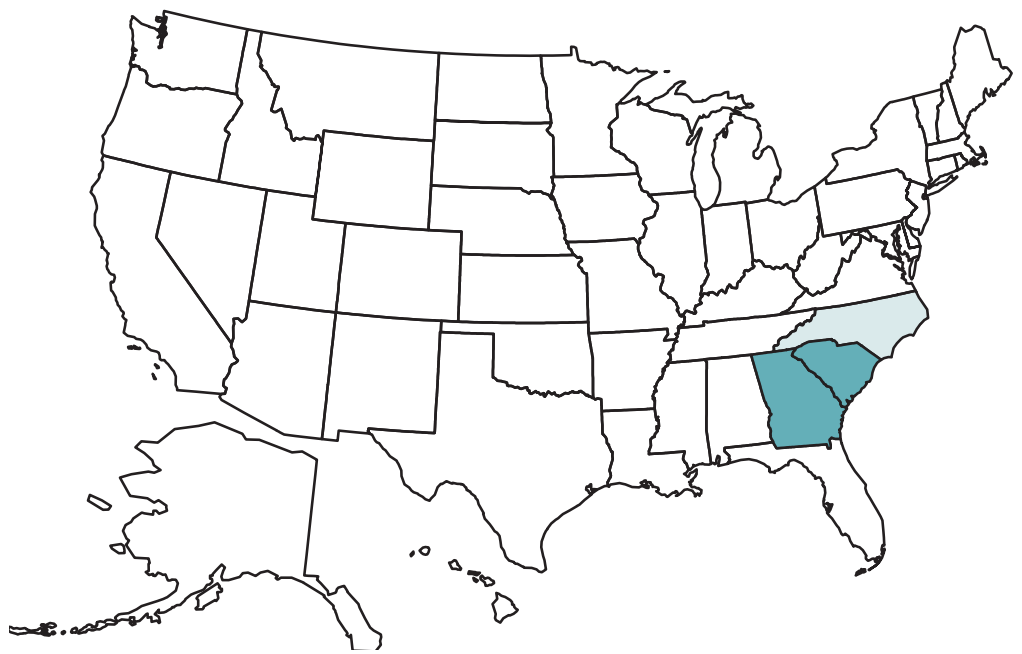

\begin{tabular}{|c|c|}
\hline \multicolumn{2}{|c|}{1995 - 1999} \\
\hline$\%>$ REL & No. of States \\
\hline$\square>50$ & 0 \\
\hline$\square>25$ to 50 & 2 \\
\hline$\square 0$ to 25 & 1 \\
\hline$\square<10$ samples & 48 \\
\hline
\end{tabular}

REL - recommended exposure limit

NOTE: The NIOSH REL is $0.2 \mathrm{mg} / \mathrm{m}^{3}$. See appendices for source description, methods, and agents.

SOURCE: Occupational Safety and Health Administration (OSHA) Integrated Management Information System. 


\section{Section 5}

Unspecified

and

Other Pneumoconioses

and

Selected Agent Exposures 

Figure 5-1. Unspecified and other pneumoconioses: Number of deaths, crude and age-adjusted mortality rates, U.S. residents age 15 and over, 1968-1999

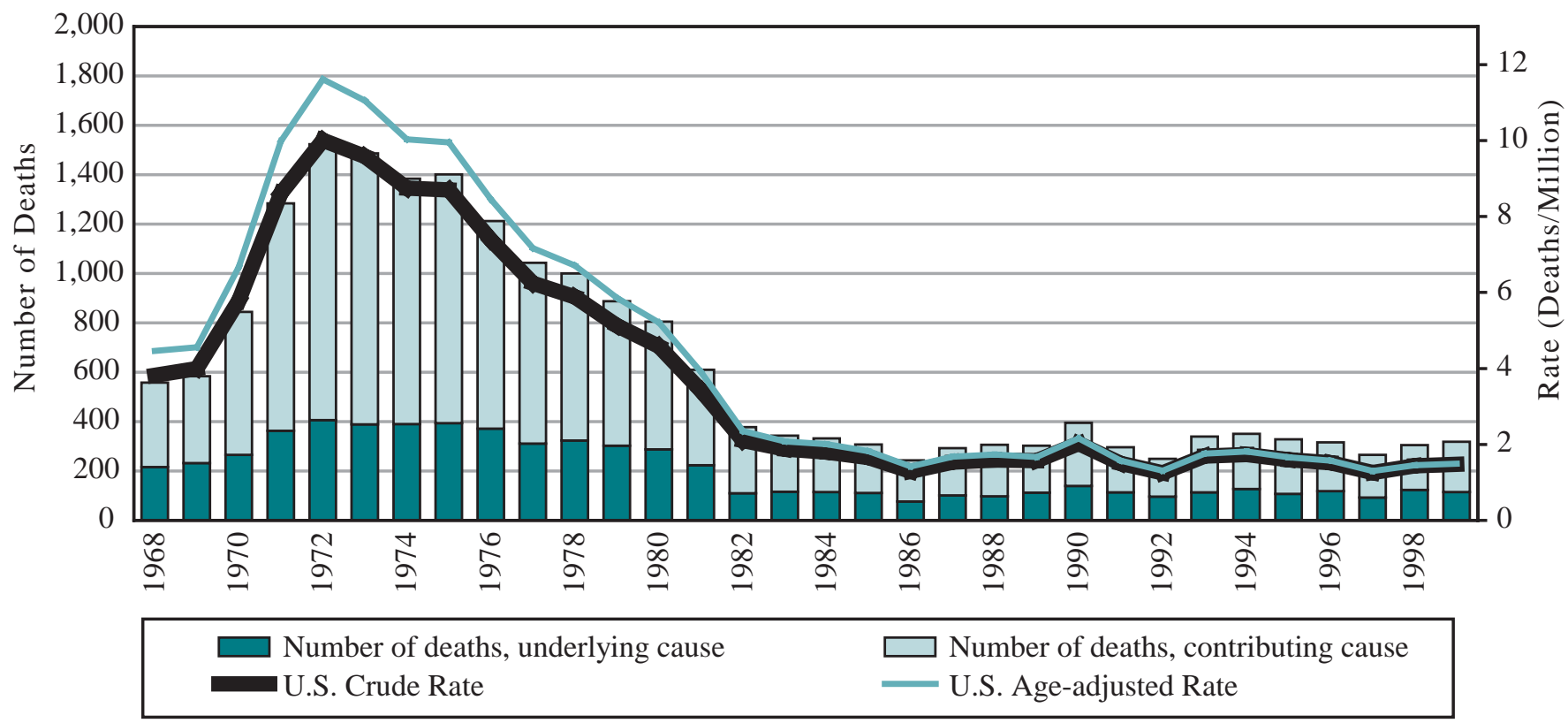

NOTE: See appendices for source description, methods, and ICD codes.

SOURCE: National Center for Health Statistics multiple cause of death data. Population estimates from U.S. Bureau of the Census.

Figure 5-2. Unspecified and other pneumoconioses: Age-adjusted mortality rates by state, U.S. residents age 15 and over, 1990-1999

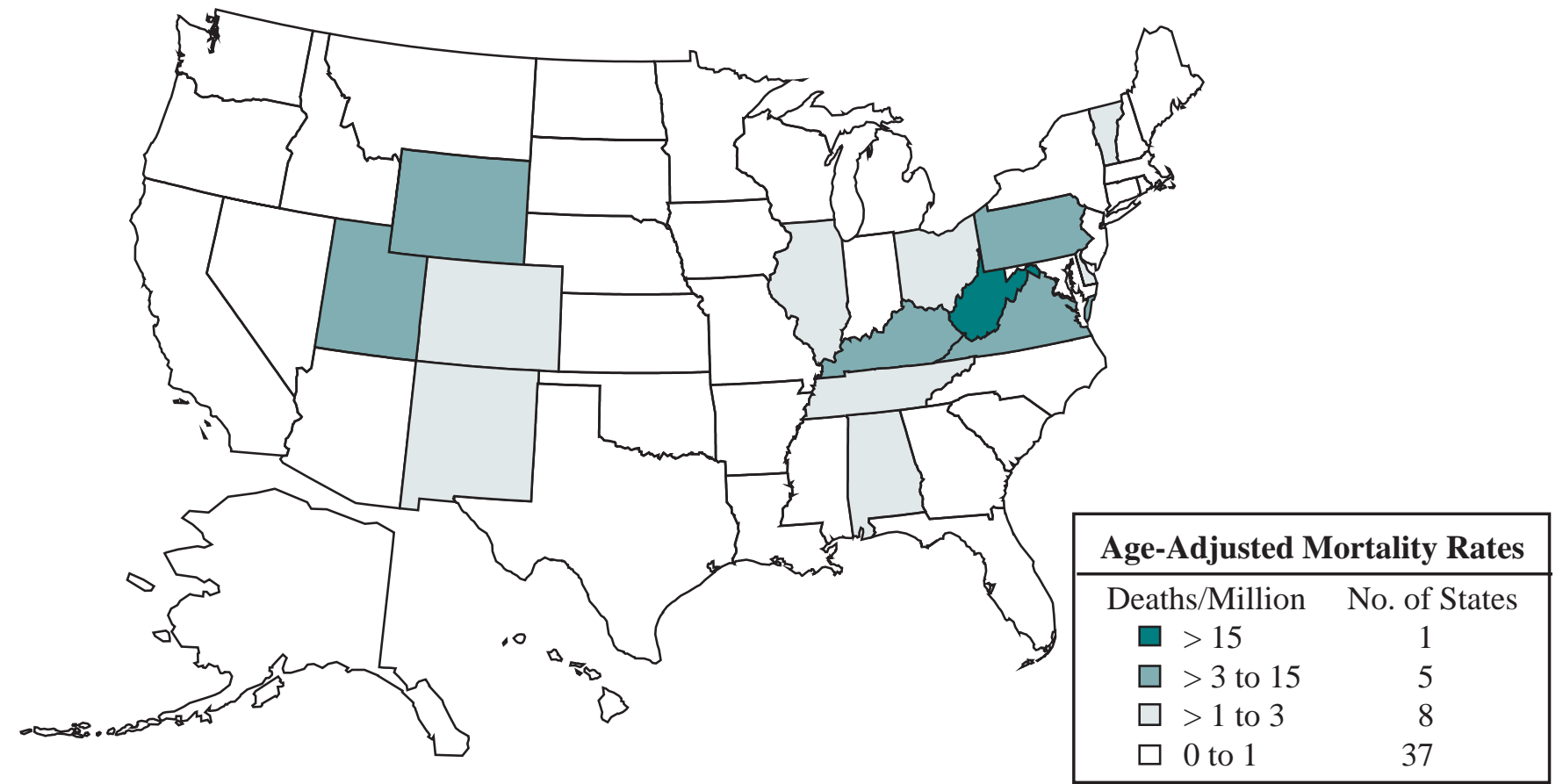

NOTE: See appendices for source description, methods, and ICD codes.

SOURCE: National Center for Health Statistics multiple cause of death data. Population estimates from U.S. Bureau of the Census. 
Table 5-1. Unspecified and other pneumoconioses: Number of deaths by sex, race, and age, and median age at death, U.S. residents age 15 and over, 1990-1999

\begin{tabular}{|c|c|c|c|c|c|c|c|c|c|c|c|c|c|c|c|c|}
\hline \multirow[b]{2}{*}{ Year } & \multirow{2}{*}{\begin{tabular}{|l|} 
No. of \\
Deaths
\end{tabular}} & \multirow{2}{*}{$\begin{array}{c}\text { Under- } \\
\text { lying } \\
\text { Cause } \\
(\%)\end{array}$} & \multicolumn{2}{|c|}{ Sex } & \multicolumn{3}{|c|}{ Race } & \multicolumn{8}{|c|}{ Age Group (yrs) } & \multirow{2}{*}{$\begin{array}{c}\text { Median } \\
\text { Age } \\
\text { (yrs) }\end{array}$} \\
\hline & & & Male & Female & White & Black & Other & 15-24 & 25-34 & $35-44$ & $45-54$ & $55-64$ & $65-74$ & $75-84$ & $85+$ & \\
\hline 1990 & 395 & 35.2 & 385 & 10 & 367 & 26 & 2 & - & 2 & 1 & 7 & 48 & 114 & 152 & 71 & 76.0 \\
\hline 1991 & 296 & 38.5 & 282 & 14 & 271 & 24 & 1 & - & 1 & 6 & 3 & 22 & 103 & 110 & 51 & 76.0 \\
\hline 1992 & 249 & 38.6 & 234 & 15 & 231 & 18 & - & 1 & - & - & 8 & 19 & 76 & 104 & 41 & 76.0 \\
\hline 1993 & 340 & 33.5 & 331 & 9 & 319 & 18 & 3 & - & 1 & 3 & 1 & 21 & 101 & 151 & 62 & 77.0 \\
\hline 1994 & 351 & 36.2 & 343 & 8 & 330 & 21 & - & - & - & - & 1 & 25 & 81 & 173 & 71 & 78.0 \\
\hline 1995 & 329 & 32.8 & 320 & 9 & 318 & 10 & 1 & - & - & - & 9 & 26 & 80 & 144 & 70 & 78.0 \\
\hline 1996 & 316 & 37.7 & 306 & 10 & 295 & 16 & 5 & - & - & 2 & 5 & 28 & 76 & 132 & 73 & 78.0 \\
\hline 1997 & 265 & 34.7 & 255 & 10 & 248 & 16 & 1 & - & - & 1 & 3 & 17 & 53 & 119 & 72 & 80.0 \\
\hline 1998 & 305 & 40.3 & 293 & 12 & 288 & 14 & 3 & - & 1 & 1 & 6 & 24 & 65 & 137 & 71 & 79.0 \\
\hline 1999 & 318 & 36.2 & 311 & 7 & 300 & 16 & 2 & - & - & 2 & 4 & 19 & 70 & 138 & 85 & 79.0 \\
\hline TOTAL & 3,164 & 36.3 & 3,060 & 104 & 2,967 & 179 & 18 & 1 & 5 & 16 & 47 & 249 & 819 & $\mathbf{1 , 3 6 0}$ & 667 & 78.0 \\
\hline
\end{tabular}

- indicates no deaths listed.

NOTE: See appendices for source description, methods, and ICD codes.

SOURCE: National Center for Health Statistics multiple cause of death data. 
Table 5-2. Unspecified and other pneumoconioses: Mortality rates (per million population) by race and sex, U.S. residents age 15 and over, 1990-1999

\begin{tabular}{|c|c|c|c|c|c|c|c|}
\hline \multirow[b]{2}{*}{ Year } & \multirow[b]{2}{*}{ Overall } & \multicolumn{2}{|c|}{ White } & \multicolumn{2}{|c|}{ Black } & \multicolumn{2}{|c|}{ Other } \\
\hline & & Male & Female & Male & Female & Male & Female \\
\hline & \multicolumn{7}{|c|}{ Crude Mortality Rate } \\
\hline 1990 & 2.02 & 4.47 & 0.11 & 2.43 & 0.08 & 0.58 & - \\
\hline 1991 & 1.50 & 3.20 & 0.15 & 2.21 & 0.08 & 0.28 & - \\
\hline 1992 & 1.25 & 2.66 & 0.16 & 1.60 & 0.08 & - & - \\
\hline 1993 & 1.69 & 3.77 & 0.10 & 1.67 & - & 0.78 & - \\
\hline 1994 & 1.73 & 3.89 & 0.09 & 1.92 & - & - & - \\
\hline 1995 & 1.60 & 3.70 & 0.10 & 0.90 & - & 0.25 & - \\
\hline 1996 & 1.52 & 3.37 & 0.11 & 1.41 & - & 1.16 & - \\
\hline 1997 & 1.26 & 2.79 & 0.11 & 1.38 & - & 0.23 & - \\
\hline 1998 & 1.44 & 3.25 & 0.10 & 1.10 & 0.07 & 0.22 & 0.39 \\
\hline 1999 & 1.48 & 3.38 & 0.08 & 1.34 & - & 0.42 & - \\
\hline \multirow[t]{2}{*}{ 1990-1999 } & 1.54 & 3.43 & 0.11 & 1.57 & 0.03 & 0.39 & 0.04 \\
\hline & \multicolumn{7}{|c|}{ Age-Adjusted Mortality Rate } \\
\hline 1990 & 2.16 & 5.80 & 0.09 & 4.60 & 0.09 & 2.10 & - \\
\hline 1991 & 1.57 & 4.06 & 0.13 & 4.13 & 0.09 & 0.47 & - \\
\hline 1992 & 1.31 & 3.37 & 0.14 & 3.12 & 0.09 & - & - \\
\hline 1993 & 1.76 & 4.76 & 0.09 & 3.40 & - & 1.55 & - \\
\hline 1994 & 1.81 & 4.98 & 0.07 & 3.85 & - & - & - \\
\hline 1995 & 1.66 & 4.65 & 0.09 & 1.82 & - & 0.29 & - \\
\hline 1996 & 1.57 & 4.22 & 0.10 & 2.72 & - & 2.13 & - \\
\hline 1997 & 1.30 & 3.50 & 0.09 & 2.88 & - & 0.37 & - \\
\hline 1998 & 1.45 & 3.91 & 0.09 & 2.09 & 0.09 & 0.62 & 0.50 \\
\hline 1999 & 1.49 & 4.06 & 0.06 & 2.33 & - & 0.72 & - \\
\hline 1990-1999 & 1.59 & 4.29 & 0.09 & 3.05 & 0.04 & 0.87 & 0.06 \\
\hline
\end{tabular}

- indicates no deaths listed.

NOTE: See appendices for source description, methods, and ICD codes.

SOURCE: National Center for Health Statistics multiple cause of death data. Population estimates from U.S. Bureau of the Census. 
Table 5-3. Unspecified and other pneumoconioses: Years of potential life lost to age 65 and to life expectancy by race and sex, U.S. residents age 15 and over, 1990-1999

\begin{tabular}{|c|c|c|c|c|c|c|c|}
\hline \multirow[b]{2}{*}{ Year } & \multicolumn{2}{|c|}{ White } & \multicolumn{2}{|c|}{ Black } & \multicolumn{2}{|c|}{ Other } & \multirow[b]{2}{*}{ Total } \\
\hline & Male & Female & Male & Female & Male & Female & \\
\hline & \multicolumn{7}{|c|}{ Years of Potential Life Lost to Age 65} \\
\hline 1990 & 365 & 10 & 65 & - & - & - & 440 \\
\hline 1991 & 220 & 30 & 90 & - & - & - & 340 \\
\hline 1992 & 235 & 15 & 10 & - & - & - & 260 \\
\hline 1993 & 210 & 10 & 10 & - & - & - & 230 \\
\hline 1994 & 125 & - & 15 & - & - & - & 140 \\
\hline 1995 & 220 & 20 & 10 & - & 15 & - & 265 \\
\hline 1996 & 205 & 40 & 5 & - & 15 & - & 265 \\
\hline 1997 & 130 & 15 & 10 & - & - & - & 155 \\
\hline 1998 & 220 & 10 & 30 & 5 & - & 5 & 270 \\
\hline 1999 & 155 & - & 45 & - & 5 & - & 205 \\
\hline \multirow[t]{2}{*}{ TOTAL } & 2,085 & 150 & 290 & 5 & 35 & 5 & 2,570 \\
\hline & \multicolumn{7}{|c|}{ Years of Potential Life Lost to Life Expectancy } \\
\hline 1990 & 3,635 & 100 & 263 & 14 & 14 & - & 4,026 \\
\hline 1991 & 2,652 & 182 & 269 & 14 & 14 & - & 3,131 \\
\hline 1992 & 2,226 & 176 & 148 & 14 & - & - & 2,564 \\
\hline 1993 & 2,901 & 135 & 154 & - & 36 & - & 3,226 \\
\hline 1994 & 2,860 & 89 & 183 & - & - & - & 3,132 \\
\hline 1995 & 2,921 & 127 & 86 & - & 29 & - & 3,163 \\
\hline 1996 & 2,700 & 164 & 140 & - & 78 & - & 3,082 \\
\hline 1997 & 2,143 & 117 & 137 & - & 14 & - & 2,411 \\
\hline 1998 & 2,639 & 140 & 139 & 21 & 6 & 36 & 2,981 \\
\hline 1999 & 2,683 & 59 & 189 & - & 30 & - & 2,961 \\
\hline TOTAL & 27,360 & 1,289 & 1,708 & 63 & 221 & 36 & 30,677 \\
\hline
\end{tabular}

- indicates no deaths listed.

NOTE: See appendices for source description, methods, and ICD codes.

SOURCE: National Center for Health Statistics multiple cause of death data. 
Table 5-4. Unspecified and other pneumoconioses: Number of deaths by state, U.S. residents age 15 and over, 1990-1999

\begin{tabular}{|c|c|c|c|c|c|c|c|c|c|c|c|}
\hline State & 1990 & 1991 & 1992 & 1993 & 1994 & 1995 & 1996 & 1997 & 1998 & 1999 & Total \\
\hline Alabama & 7 & 10 & 5 & 8 & 1 & 3 & 5 & 7 & 3 & 5 & 54 \\
\hline Alaska & - & - & - & - & - & - & - & - & - & - & - \\
\hline Arizona & 3 & 1 & 2 & 3 & - & 3 & 2 & 1 & - & 1 & 16 \\
\hline Arkansas & 1 & 2 & 1 & - & 1 & - & 1 & - & - & - & 6 \\
\hline California & 7 & 4 & 4 & 8 & 7 & 5 & 3 & 5 & 6 & 7 & 56 \\
\hline Colorado & 1 & 3 & 3 & 7 & 4 & 5 & 7 & 4 & 6 & 3 & 43 \\
\hline Connecticut & 2 & 2 & 4 & 4 & - & - & 1 & 1 & 2 & - & 16 \\
\hline Delaware & - & - & - & 1 & - & 4 & 1 & - & 2 & 1 & 9 \\
\hline District of Columbia & - & 1 & - & - & - & - & 1 & - & - & - & 2 \\
\hline Florida & 5 & 10 & 11 & 5 & 7 & 8 & 11 & 5 & 8 & 1 & 71 \\
\hline Georgia & 2 & 1 & 2 & 3 & 1 & 3 & 5 & 3 & 2 & 1 & 23 \\
\hline Hawaii & - & - & - & - & - & - & 1 & - & - & - & 1 \\
\hline Idaho & - & - & - & - & - & - & 1 & - & - & - & 1 \\
\hline Illinois & 16 & 19 & 13 & 14 & 13 & 10 & 20 & 10 & 7 & 14 & 136 \\
\hline Indiana & 6 & 4 & 4 & 3 & 1 & 5 & 6 & 6 & - & 8 & 43 \\
\hline Iowa & - & - & 4 & 1 & 1 & 4 & - & - & 1 & 1 & 12 \\
\hline Kansas & - & 3 & - & 1 & 1 & - & 1 & - & - & - & 6 \\
\hline Kentucky & 47 & 32 & 34 & 14 & 26 & 29 & 14 & 24 & 25 & 38 & 283 \\
\hline Louisiana & 3 & 4 & - & - & 1 & - & 1 & - & 2 & - & 11 \\
\hline Maine & - & - & - & - & - & - & 2 & - & - & - & 2 \\
\hline Maryland & 4 & 4 & 1 & 1 & 5 & 6 & 3 & 2 & 3 & 3 & 32 \\
\hline Massachusetts & - & 2 & 3 & 1 & 1 & 2 & 4 & - & 2 & 1 & 16 \\
\hline Michigan & 8 & 3 & 7 & 5 & 4 & 3 & 4 & 3 & 5 & 6 & 48 \\
\hline Minnesota & 1 & 1 & 1 & 1 & - & - & - & 1 & 1 & - & 6 \\
\hline Mississippi & - & - & - & - & 2 & 1 & - & 1 & - & - & 4 \\
\hline Missouri & 6 & 3 & 1 & 4 & 2 & 3 & - & 2 & 2 & 1 & 24 \\
\hline Montana & 1 & - & - & 2 & - & - & - & - & 1 & - & 4 \\
\hline Nebraska & - & 1 & 1 & - & - & - & - & - & - & - & 2 \\
\hline Nevada & - & - & - & 3 & - & - & - & - & 1 & 1 & 5 \\
\hline New Hampshire & - & - & 1 & - & - & 1 & - & - & - & 1 & 3 \\
\hline New Jersey & 9 & 7 & 7 & 2 & 6 & 9 & 1 & 3 & 3 & 2 & 49 \\
\hline New Mexico & 4 & - & 1 & 3 & 3 & 2 & 1 & 4 & 2 & - & 20 \\
\hline New York & 10 & 3 & 9 & 7 & 6 & 3 & 8 & 4 & 1 & 5 & 56 \\
\hline North Carolina & 4 & 2 & 4 & 2 & 3 & 1 & 2 & 1 & 4 & 5 & 28 \\
\hline North Dakota & 1 & - & - & - & - & - & - & - & - & 1 & 2 \\
\hline Ohio & 29 & 42 & 27 & 15 & 24 & 16 & 20 & 8 & 29 & 28 & 238 \\
\hline Oklahoma & 2 & 1 & 2 & 2 & 1 & 1 & - & - & - & 2 & 11 \\
\hline Oregon & 1 & 1 & - & - & - & 1 & - & - & 1 & - & 4 \\
\hline Pennsylvania & 51 & 49 & 33 & 159 & 130 & 98 & 94 & 83 & 64 & 75 & 836 \\
\hline Rhode Island & - & - & - & - & - & - & - & - & - & - & - \\
\hline South Carolina & - & 3 & - & - & - & - & - & - & - & - & 3 \\
\hline South Dakota & 1 & - & - & - & - & - & - & - & - & - & 1 \\
\hline Tennessee & 11 & 8 & 3 & 5 & 5 & 6 & - & 2 & 7 & 3 & 50 \\
\hline Texas & 6 & 1 & 3 & 4 & - & 3 & 6 & 3 & 2 & 6 & 34 \\
\hline Utah & 5 & 1 & 2 & 3 & 7 & 6 & 7 & 12 & 2 & 6 & 51 \\
\hline Vermont & - & - & - & 1 & - & 1 & 1 & 2 & - & 1 & 6 \\
\hline Virginia & 17 & 7 & 4 & 10 & 36 & 32 & 15 & 9 & 36 & 26 & 192 \\
\hline Washington & 4 & 3 & 3 & 1 & 1 & - & 1 & - & - & - & 13 \\
\hline West Virginia & 118 & 58 & 44 & 36 & 49 & 50 & 60 & 54 & 68 & 58 & 595 \\
\hline Wisconsin & 2 & - & 2 & - & - & 1 & 1 & 1 & 1 & 1 & 9 \\
\hline Wyoming & - & - & 3 & 1 & 2 & 4 & 5 & 4 & 6 & 6 & 31 \\
\hline TOTAL & 395 & 296 & 249 & 340 & 351 & 329 & 316 & 265 & 305 & 318 & 3,164 \\
\hline
\end{tabular}

- indicates no deaths listed.

NOTE: See appendices for source description, methods, and ICD codes.

SOURCE: National Center for Health Statistics multiple cause of death data. 
Table 5-5. Unspecified and other pneumoconioses: Number of deaths, mortality rates (per million population), and years of potential life lost (YPLL) by state, U.S. residents age 15 and over, 1990-1999

\begin{tabular}{|c|c|c|c|c|c|c|c|c|c|c|}
\hline \multirow[b]{2}{*}{ State } & \multirow{2}{*}{$\begin{array}{l}\text { No. of } \\
\text { Deaths }\end{array}$} & \multirow[b]{2}{*}{ Rank } & \multicolumn{2}{|c|}{ Crude Mortality } & \multicolumn{2}{|c|}{ Age-Adjusted Mortality } & \multicolumn{4}{|c|}{ YPLL to Life Expectancy } \\
\hline & & & Rate & Rank & Rate & Rank & Total & Rank & YPLL/death & Rank \\
\hline Alabama & 54 & 10 & 1.63 & 8 & 1.71 & 11 & 520 & 13 & 9.6 & 40 \\
\hline Alaska & - & - & - & - & - & - & - & - & - & - \\
\hline Arizona & 16 & 24 & 0.49 & 24 & 0.47 & 24 & 185 & 26 & 11.5 & 23 \\
\hline Arkansas & 6 & 33 & 0.31 & 35 & 0.25 & 39 & 62 & 37 & 10.3 & 36 \\
\hline California & 56 & 8 & 0.23 & 39 & 0.28 & 36 & 736 & 8 & 13.2 & 10 \\
\hline Colorado & 43 & 15 & 1.48 & 11 & 1.95 & 8 & 360 & 19 & 8.4 & 45 \\
\hline Connecticut & 16 & 24 & 0.61 & 20 & 0.58 & 19 & 192 & 25 & 12.0 & 21 \\
\hline Delaware & 9 & 31 & 1.59 & 9 & 1.78 & 10 & 95 & 32 & 10.6 & 34 \\
\hline District of Columbia & 2 & 43 & 0.43 & 26 & 0.44 & 27 & 77 & 35 & 38.3 & 1 \\
\hline Florida & 71 & 7 & 0.62 & 19 & 0.47 & 24 & 761 & 7 & 10.7 & 33 \\
\hline Georgia & 23 & 22 & 0.41 & 29 & 0.53 & 21 & 301 & 21 & 13.1 & 13 \\
\hline Hawaii & 1 & 47 & 0.11 & 48 & 0.09 & 49 & 14 & 47 & 14.1 & 5 \\
\hline Idaho & 1 & 47 & 0.12 & 47 & 0.12 & 48 & 8 & 48 & 8.4 & 45 \\
\hline Illinois & 136 & 6 & 1.48 & 11 & 1.51 & 12 & 1,349 & 6 & 9.9 & 37 \\
\hline Indiana & 43 & 15 & 0.95 & 15 & 0.96 & 16 & 464 & 16 & 10.8 & 32 \\
\hline Iowa & 12 & 28 & 0.54 & 23 & 0.42 & 28 & 112 & 31 & 9.4 & 42 \\
\hline Kansas & 6 & 33 & 0.30 & 37 & 0.27 & 38 & 68 & 36 & 11.3 & 25 \\
\hline Kentucky & 283 & 3 & 9.38 & 2 & 9.46 & 3 & 3,141 & 3 & 11.1 & 28 \\
\hline Louisiana & 11 & 29 & 0.34 & 32 & 0.37 & 31 & 151 & 28 & 13.7 & 9 \\
\hline Maine & 2 & 43 & 0.21 & 41 & 0.19 & 42 & 23 & 44 & 11.3 & 25 \\
\hline Maryland & 32 & 18 & 0.80 & 16 & 0.99 & 15 & 400 & 17 & 12.5 & 17 \\
\hline Massachusetts & 16 & 24 & 0.33 & 33 & 0.32 & 35 & 206 & 24 & 12.9 & 14 \\
\hline Michigan & 48 & 14 & 0.65 & 18 & 0.69 & 18 & 610 & 11 & 12.7 & 15 \\
\hline Minnesota & 6 & 33 & 0.17 & 44 & 0.17 & 43 & 94 & 33 & 15.6 & 3 \\
\hline Mississippi & 4 & 38 & 0.20 & 42 & 0.20 & 41 & 44 & 40 & 11.0 & 29 \\
\hline Missouri & 24 & 21 & 0.58 & 22 & 0.52 & 22 & 339 & 20 & 14.1 & 5 \\
\hline Montana & 4 & 38 & 0.60 & 21 & 0.57 & 20 & 35 & 41 & 8.7 & 44 \\
\hline Nebraska & 2 & 43 & 0.16 & 45 & 0.14 & 46 & 28 & 42 & 14.1 & 5 \\
\hline Nevada & 5 & 37 & 0.42 & 28 & 0.47 & 24 & 93 & 34 & 18.6 & 2 \\
\hline New Hampshire & 3 & 41 & 0.33 & 33 & 0.37 & 31 & 27 & 43 & 8.9 & 43 \\
\hline New Jersey & 49 & 13 & 0.77 & 17 & 0.78 & 17 & 624 & 10 & 12.7 & 15 \\
\hline New Mexico & 20 & 23 & 1.59 & 9 & 1.84 & 9 & 213 & 23 & 10.6 & 34 \\
\hline New York & 56 & 8 & 0.39 & 31 & 0.39 & 30 & 641 & 9 & 11.4 & 24 \\
\hline North Carolina & 28 & 20 & 0.49 & 24 & 0.51 & 23 & 388 & 18 & 13.8 & 8 \\
\hline North Dakota & 2 & 43 & 0.41 & 29 & 0.35 & 33 & 22 & 46 & 11.2 & 27 \\
\hline Ohio & 238 & 4 & 2.74 & 7 & 2.74 & 7 & 2,618 & 4 & 11.0 & 29 \\
\hline Oklahoma & 11 & 29 & 0.43 & 26 & 0.41 & 29 & 134 & 29 & 12.2 & 19 \\
\hline Oregon & 4 & 38 & 0.16 & 45 & 0.16 & 44 & 50 & 39 & 12.5 & 17 \\
\hline Pennsylvania & 836 & 1 & 8.66 & 3 & 7.70 & 4 & 8,131 & 1 & 9.7 & 39 \\
\hline Rhode Island & - & - & - & - & - & - & - & - & - & - \\
\hline South Carolina & 3 & 41 & 0.11 & 48 & 0.14 & 46 & 23 & 44 & 7.7 & 49 \\
\hline South Dakota & 1 & 47 & 0.18 & 43 & 0.15 & 45 & 8 & 48 & 8.3 & 47 \\
\hline Tennessee & 50 & 12 & 1.21 & 14 & 1.24 & 14 & 610 & 11 & 12.2 & 19 \\
\hline Texas & 34 & 17 & 0.24 & 38 & 0.28 & 36 & 519 & 14 & 15.3 & 4 \\
\hline Utah & 51 & 11 & 3.81 & 5 & 4.92 & 5 & 505 & 15 & 9.9 & 37 \\
\hline Vermont & 6 & 33 & 1.31 & 13 & 1.37 & 13 & 57 & 38 & 9.6 & 40 \\
\hline Virginia & 192 & 5 & 3.67 & 6 & 4.59 & 6 & 2,119 & 5 & 11.0 & 29 \\
\hline Washington & 13 & 27 & 0.31 & 35 & 0.33 & 34 & 172 & 27 & 13.2 & 10 \\
\hline West Virginia & 595 & 2 & 40.91 & 1 & 35.63 & 1 & 6,938 & 2 & 11.7 & 22 \\
\hline Wisconsin & 9 & 31 & 0.23 & 39 & 0.22 & 40 & 119 & 30 & 13.2 & 10 \\
\hline Wyoming & 31 & 19 & 8.66 & 3 & 10.61 & 2 & 249 & 22 & 8.0 & 48 \\
\hline
\end{tabular}

- indicates no deaths listed.

NOTE: See appendices for source description, methods, and ICD codes.

SOURCE: National Center for Health Statistics multiple cause of death data. Population estimates from U.S. Bureau of the Census. 
Table 5-6. Unspecified and other pneumoconioses: Most frequently recorded industries on death certificate, U.S. residents age 15 and over, selected states and years, 1990-1999

\begin{tabular}{|c|c|c|c|}
\hline CIC & Industry & Number of Deaths & Percent \\
\hline 041 & Coal mining & 801 & 58.9 \\
\hline 060 & Construction & 81 & 6.0 \\
\hline 270 & Blast furnaces, steelworks, rolling and finishing mills & 31 & 2.3 \\
\hline 392 & Not specified manufacturing industries & 27 & 2.0 \\
\hline 040 & Metal mining & 18 & 1.3 \\
\hline 351 & Motor vehicles and motor vehicle equipment & 18 & 1.3 \\
\hline 010 & Agricultural production, crops & 16 & 1.2 \\
\hline 842 & Elementary and secondary schools & 16 & 1.2 \\
\hline 400 & Railroads & 15 & 1.1 \\
\hline \multirow[t]{4}{*}{961} & Non-paid worker or non-worker or own home/at home & 14 & 1.0 \\
\hline & All other industries & 291 & 21.4 \\
\hline & Industry not reported & 33 & 2.4 \\
\hline & TOTAL & $\mathbf{1 , 3 6 1}$ & 100.0 \\
\hline
\end{tabular}

CIC - Census Industry Code

n.e.c. - not elsewhere classified

NOTE: Percentages may not total to $100 \%$ due to rounding. See appendices for source description, methods, and ICD codes, industry and occupation codes, and list of selected states and years.

SOURCE: National Center for Health Statistics multiple cause of death data.

Table 5-7. Unspecified and other pneumoconioses: Most frequently recorded occupations on death certificate, U.S. residents age 15 and over, selected states and years, 1990-1999

\begin{tabular}{clrc}
\hline COC & Occupation & Number of Deaths & Percent \\
\hline 616 & Mining machine operators & 727 & 53.4 \\
889 & Laborers, except construction & 63 & 4.6 \\
453 & Janitors and cleaners & 28 & 2.1 \\
019 & Managers and administrators, n.e.c. & 27 & 2.0 \\
575 & Electricians & 24 & 1.8 \\
783 & Welders and cutters & 22 & 1.6 \\
633 & Supervisors, production occupations & 21 & 1.5 \\
869 & Construction laborers & 19 & 1.4 \\
473 & Farmers, except horticulture & 18 & 1.3 \\
804 & Truck drivers & 18 & 1.3 \\
& All other occupations & 365 & 26.8 \\
& Occupation not reported & 29 & 2.1 \\
& TOTAL & $\mathbf{1 , 3 6 1}$ & $\mathbf{1 0 0 . 0}$ \\
\hline
\end{tabular}

COC - Census Occupation Code n.e.c. - not elsewhere classified

NOTE: Percentages may not total to $100 \%$ due to rounding. See appendices for source description, methods, and ICD codes, industry and occupation codes, and list of selected states and years.

SOURCE: National Center for Health Statistics multiple cause of death data. 
Table 5-8. Unspecified and other pneumoconioses: Proportionate mortality ratio (PMR) adjusted for age, sex, and race by usual industry, U.S. residents age 15 and over, selected states and years, 1990-1999

\begin{tabular}{llrrrr}
\hline & & & & & 95\% Confidence Interval \\
\cline { 4 - 6 } CIC & Industry & Number & of Deaths & PMR & UCL \\
\hline 041 & Coal mining & 801 & 42.37 & 39.51 & 45.43 \\
040 & Metal mining & 18 & 5.19 & 3.07 & 8.20 \\
252 & Structural clay products & 7 & 4.71 & 1.89 & 9.71 \\
262 & Miscellaneous nonmetallic mineral and stone products & 7 & 3.22 & 1.29 & 6.63 \\
271 & Iron and steel foundries & 8 & 2.36 & 1.02 & 4.64 \\
250 & Glass and glass products & 11 & 2.02 & 1.01 & 3.62 \\
\hline
\end{tabular}

CIC - Census Industry Code $\quad$ n.e.c. - not elsewhere classified $\quad$ LCL - lower confidence limit $\quad$ UCL - upper confidence limit NOTE: See appendices for source description, methods, and ICD codes, industry and occupation codes, and list of selected states and years. SOURCE: National Center for Health Statistics multiple cause of death data. 
Table 5-9. Unspecified and other pneumoconioses: Proportionate mortality ratio (PMR) adjusted for age, sex, and race by usual occupation, U.S. residents age 15 and over, selected states and years, 1990-1999

\begin{tabular}{llrrrr}
\hline COC & Occupation & Number & & \multicolumn{2}{c}{ 95\% Confidence Interval } \\
\cline { 5 - 6 } & of Deaths & PMR & LCL & UCL \\
\hline 16 & Mining machine operators & 727 & 40.86 & 37.97 & 43.98 \\
613 & Supervisors, extractive occupations & 17 & 18.40 & 10.70 & 29.44 \\
768 & Crushing and grinding machine operators & 5 & 6.41 & 2.07 & 14.98 \\
783 & Welders and cutters & 22 & 1.86 & 1.16 & 2.81 \\
575 & Electricians & 24 & 1.66 & 1.06 & 2.47 \\
\hline
\end{tabular}

COC - Census Occupation Code n.e.c. - not elsewhere classified LCL - lower confidence limit UCL - upper confidence limit NOTE: See appendices for source description, methods, and ICD codes, industry and occupation codes, and list of selected states and years. SOURCE: National Center for Health Statistics multiple cause of death data. 
$\succsim_{\infty}$ Figure 5-3. Unspecified and other pneumoconioses: Age-adjusted mortality rates by county, U.S. residents age 15 and over, 1970-1999

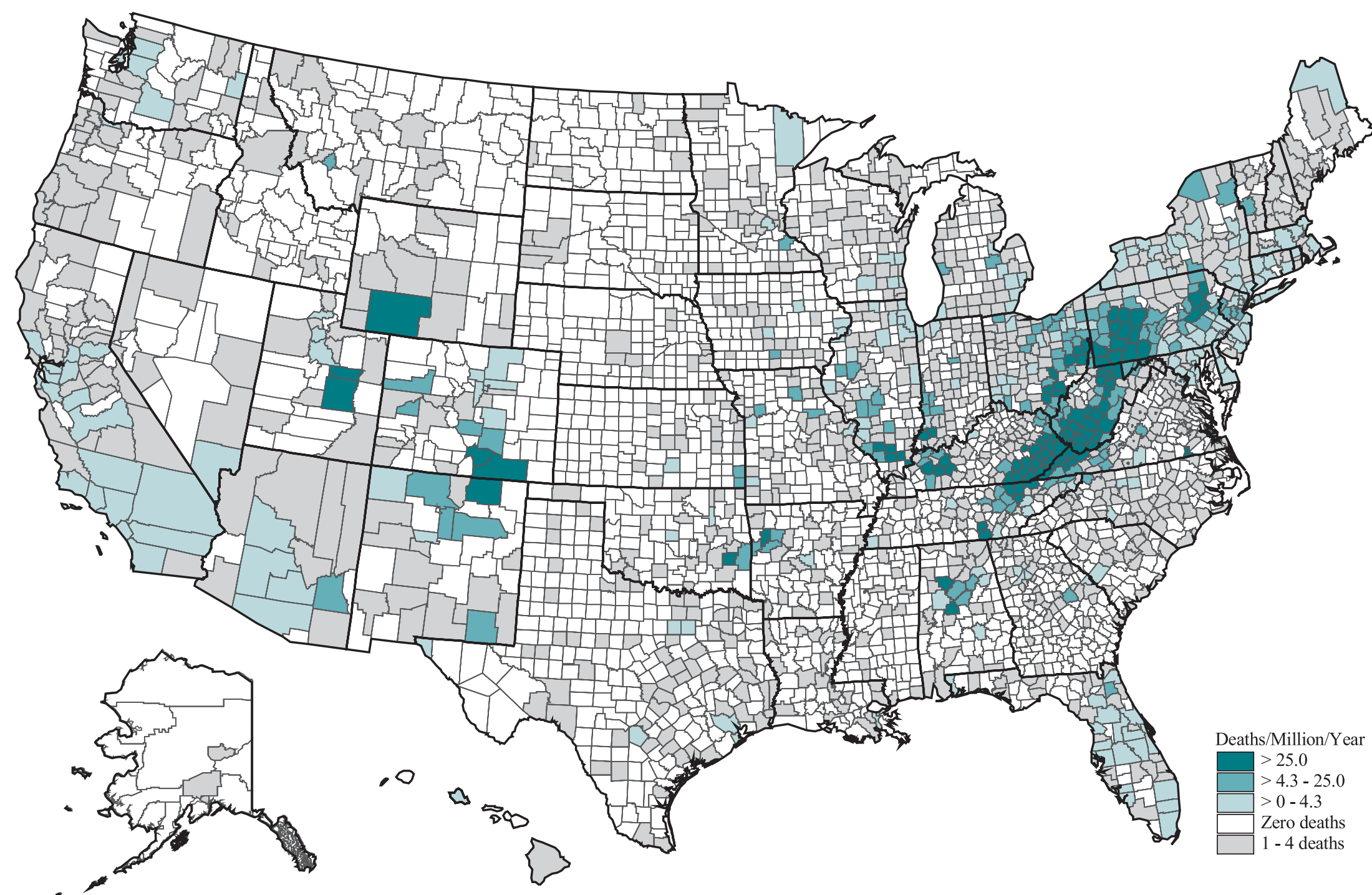

NOTE: Age-adjusted rates are not calculated for those counties with 1-4 deaths. See appendices for source description, methods, and ICD codes. SOURCE: National Center for Health Statistics multiple cause of death data. Population estimates from U.S. Bureau of the Census. 
Figure 5-4. Unspecified and other pneumoconioses: Age-adjusted mortality rates by county, U.S. residents age 15 and over, 1970-1984 and 1985-1999


NOTE: Age-adjusted rates are not calculated for those counties with 1-4 deaths. See appendices for source description, methods, and ICD codes. SOURCE: National Center for Health Statistics multiple cause of death data. Population estimates from U.S. Bureau of the Census. 


\section{Table 5-10. Unspecified and other pneumoconioses: Counties with highest age- adjusted mortality rates (per million population), U.S. residents age 15 and over, 1985-1999}

\begin{tabular}{|c|c|c|c|c|c|}
\hline County & State & Age-Adjusted Rate & Crude Rate & Number of Deaths & $\%$ Female \\
\hline Carbon County & Utah & 178.9 & 185.3 & 40 & 0.0 \\
\hline Harlan County & Kentucky & 167.9 & 168.4 & 70 & 0.0 \\
\hline Boone County & West Virginia & 160.6 & 148.1 & 46 & 0.0 \\
\hline Dickenson County & Virginia & 155.8 & 139.8 & 29 & 0.0 \\
\hline Norton City & Virginia & 143.7 & 119.2 & 6 & 0.0 \\
\hline Wyoming County & West Virginia & 140.1 & 128.2 & 43 & 0.0 \\
\hline Sweetwater County & Wyoming & 139.7 & 79.0 & 34 & 0.0 \\
\hline Logan County & West Virginia & 133.5 & 130.4 & 65 & 0.0 \\
\hline Mingo County & West Virginia & 125.3 & 103.9 & 39 & 0.0 \\
\hline Raleigh County & West Virginia & 121.2 & 138.1 & 126 & 0.0 \\
\hline Wise County & Virginia & 120.7 & 117.6 & 55 & 0.0 \\
\hline Fayette County & West Virginia & 119.1 & 152.0 & 87 & 1.1 \\
\hline Las Animas County & Colorado & 111.3 & 179.7 & 29 & 3.4 \\
\hline Bell County & Kentucky & 110.2 & 114.1 & 41 & 0.0 \\
\hline Muhlenberg County & Kentucky & 107.8 & 124.7 & 46 & 0.0 \\
\hline Leslie County & Kentucky & 101.9 & 78.0 & 12 & 0.0 \\
\hline McDowell County & West Virginia & 101.8 & 114.9 & 45 & 0.0 \\
\hline Franklin County & Illinois & 96.4 & 145.0 & 70 & 0.0 \\
\hline Tazewell County & Virginia & 93.5 & 93.2 & 52 & 0.0 \\
\hline Nicholas County & West Virginia & 85.6 & 92.6 & 29 & 0.0 \\
\hline Butler County & Kentucky & 80.4 & 90.4 & 12 & 0.0 \\
\hline Cambria County & Pennsylvania & 78.5 & 102.7 & 202 & 1.0 \\
\hline Russell County & Virginia & 76.7 & 69.8 & 24 & 0.0 \\
\hline Buchanan County & Virginia & 69.8 & 54.4 & 20 & 0.0 \\
\hline Floyd County & Kentucky & 64.9 & 59.8 & 30 & 0.0 \\
\hline Letcher County & Kentucky & 62.5 & 58.3 & 18 & 5.6 \\
\hline Knox County & Kentucky & 61.9 & 63.2 & 22 & 0.0 \\
\hline Saline County & Illinois & 60.1 & 94.1 & 30 & 0.0 \\
\hline Pike County & Kentucky & 60.1 & 49.6 & 42 & 0.0 \\
\hline Clay County & West Virginia & 58.1 & 62.0 & 7 & 14.3 \\
\hline Mercer County & West Virginia & 57.8 & 71.4 & 56 & 0.0 \\
\hline Webster County & West Virginia & 54.4 & 65.5 & 8 & 0.0 \\
\hline Marion County & West Virginia & 52.6 & 69.7 & 49 & 2.0 \\
\hline Pike County & Indiana & 50.5 & 60.7 & 9 & 0.0 \\
\hline Meigs County & Ohio & 50.3 & 58.7 & 16 & 0.0 \\
\hline Whitley County & Kentucky & 44.9 & 45.5 & 18 & 0.0 \\
\hline Somerset County & Pennsylvania & 44.6 & 52.5 & 49 & 0.0 \\
\hline Taylor County & West Virginia & 40.5 & 50.3 & 9 & 0.0 \\
\hline Lee County & Virginia & 38.2 & 45.2 & 13 & 0.0 \\
\hline Indiana County & Pennsylvania & 37.6 & 35.8 & 39 & 0.0 \\
\hline Clearfield County & Pennsylvania & 34.9 & 42.0 & 39 & 0.0 \\
\hline Upshur County & West Virginia & 33.8 & 40.3 & 11 & 0.0 \\
\hline Perry County & Kentucky & 33.2 & 28.2 & 10 & 0.0 \\
\hline Fayette County & Pennsylvania & 31.8 & 38.8 & 68 & 0.0 \\
\hline Carbon County & Pennsylvania & 30.4 & 39.8 & 28 & 3.6 \\
\hline Perry County & Illinois & 29.7 & 40.1 & 10 & 0.0 \\
\hline Gibson County & Indiana & 29.6 & 37.5 & 14 & 0.0 \\
\hline Schuylkill County & Pennsylvania & 27.7 & 37.6 & 71 & 0.0 \\
\hline Armstrong County & Pennsylvania & 26.5 & 32.7 & 29 & 0.0 \\
\hline Lincoln County & West Virginia & 26.5 & 24.0 & 6 & 0.0 \\
\hline Overall United States & & 1.6 & 1.5 & 4,615 & 3.4 \\
\hline
\end{tabular}

NOTE: Only counties with at least 5 deaths from the disease of interest are included. See appendices for source description, methods, and ICD codes. SOURCE: National Center for Health Statistics multiple cause of death data. Population estimates from U.S. Bureau of the Census. 
Table 5-11 (page 1 of 4). Selected pneumoconiotic agents: Geometric mean exposures and percent exceeding designated occupational exposure limits, MSHA and OSHA samples, 1979-1999

\begin{tabular}{|c|c|c|c|c|c|c|c|c|c|c|c|c|c|c|c|c|c|c|c|c|c|}
\hline Agent & 1979 & 1980 & 1981 & 1982 & 1983 & 1984 & 1985 & 1986 & 1987 & 1988 & 1989 & 1990 & 1991 & 1992 & 1993 & 1994 & 1995 & 1996 & 1997 & 1998 & 1999 \\
\hline \multicolumn{22}{|c|}{ Aluminum Oxide Fume and Dust - MSHA } \\
\hline $\begin{array}{l}\mathrm{GM} \\
\left(\mathrm{mg} / \mathrm{m}^{3}\right)\end{array}$ & 0.115 & 0.025 & 0.017 & 0.010 & 0.017 & 0.017 & 0.020 & 0.019 & 0.031 & 0.032 & 0.025 & 0.030 & 0.022 & 0.028 & 0.009 & 0.011 & 0.011 & 0.006 & 0.008 & 0.005 & 0.002 \\
\hline $\begin{array}{l}\text { No. of } \\
\text { samples }\end{array}$ & 145 & 152 & 170 & 85 & 148 & 160 & 177 & 121 & 139 & 112 & 133 & 182 & 143 & 120 & 140 & 241 & 206 & 765 & 227 & 67 & 101 \\
\hline$\%>$ PEL & 0.0 & 0.0 & 0.6 & 1.2 & 0.0 & 0.0 & 0.0 & 0.0 & 0.7 & 1.8 & 0.0 & 1.6 & 0.7 & 1.7 & 0.0 & 0.0 & 0.0 & 0.0 & 0.0 & 0.0 & 0.0 \\
\hline$\%>$ REL & - & - & - & - & - & - & - & - & - & & - & - & - & & - & - & - & - & - & - & - \\
\hline \multicolumn{22}{|c|}{ Aluminum Oxide and Alpha Aluminum - OSHA } \\
\hline $\begin{array}{l}\mathrm{GM} \\
\left(\mathrm{mg} / \mathrm{m}^{3}\right)\end{array}$ & 0.209 & 1.16 & 0.196 & 1.235 & 0.396 & 0.035 & 0.007 & 0.016 & 0.004 & 0.034 & 0.031 & 0.050 & 0.003 & 0.019 & 0.013 & 0.035 & 0.049 & 0.031 & 0.002 & 0.008 & 0.022 \\
\hline $\begin{array}{l}\text { No. of } \\
\text { samples }\end{array}$ & 22 & 15 & 6 & 27 & 11 & 70 & 96 & 28 & 24 & 50 & 47 & 41 & 44 & 16 & 42 & 41 & 35 & 13 & 145 & 85 & 65 \\
\hline$\%>$ PEL & 18.2 & 0.0 & 0.0 & 18.5 & 18.2 & 0.0 & 4.2 & 3.6 & 0.0 & 2.0 & 2.1 & 0.0 & 0.0 & 0.0 & 2.4 & 0.0 & 5.7 & 7.7 & 0.0 & 0.0 & 0.0 \\
\hline$\%>$ REL & - & - & - & - & - & - & - & - & - & & - & - & - & & - & - & - & - & - & - & - \\
\hline \multicolumn{22}{|c|}{ Antimony and Compounds - OSHA } \\
\hline $\begin{array}{l}\mathrm{GM} \\
\left(\mathrm{mg} / \mathrm{m}^{3}\right)\end{array}$ & 0.013 & 0.010 & 0.016 & 0.010 & 0.009 & 0.009 & 0.009 & 0.009 & 0.009 & 0.009 & 0.008 & 0.009 & 0.008 & 0.009 & 0.009 & 0.009 & 0.007 & 0.008 & 0.009 & 0.010 & 0.009 \\
\hline $\begin{array}{l}\text { No. of } \\
\text { samples }\end{array}$ & 96 & 213 & 145 & 158 & 104 & 714 & 756 & 640 & 910 & 901 & 650 & 915 & 733 & 672 & 561 & 432 & 502 & 411 & 557 & 370 & 346 \\
\hline$\%>$ PEL & 5.2 & 0.9 & 4.8 & 1.9 & 0.0 & 0.6 & 0.8 & 0.3 & 0.8 & 0.3 & 0.5 & 0.3 & 0.8 & 0.3 & 0.5 & 0.2 & 1.0 & 0.7 & 0.2 & 2.4 & 0.6 \\
\hline$\%>$ REL & 5.2 & 0.9 & 4.8 & 1.9 & 0.0 & 0.6 & 0.8 & 0.3 & 0.8 & 0.3 & 0.5 & 0.3 & 0.8 & 0.3 & 0.5 & 0.2 & 1.0 & 0.7 & 0.2 & 2.4 & 0.6 \\
\hline \multicolumn{22}{|c|}{ Beryllium Fume and Dust - MSHA } \\
\hline $\begin{array}{l}\mathrm{GM} \\
\left(\mu \mathrm{g} / \mathrm{m}^{3}\right)\end{array}$ & 0.334 & 0.164 & 0.176 & 0.183 & 0.189 & 0.161 & 0.164 & 0.215 & 0.172 & 0.189 & 0.166 & 0.160 & 0.169 & 0.159 & 0.150 & 0.152 & 0.152 & 0.153 & 0.162 & 0.166 & 0.169 \\
\hline $\begin{array}{l}\text { No. of } \\
\text { samples }\end{array}$ & 65 & 53 & 83 & 72 & 171 & 155 & 168 & 81 & 81 & 97 & 75 & 64 & 48 & 39 & 107 & 217 & 183 & 582 & 113 & 44 & 88 \\
\hline$\%>$ PEL & 13.8 & 0.0 & 1.2 & 4.2 & 2.9 & 0.0 & 0.0 & 1.2 & 0.0 & 0.0 & 1.3 & 0.0 & 0.0 & 0.0 & 0.0 & 0.0 & 0.0 & 0.2 & 0.0 & 0.0 & 0.0 \\
\hline$\%>$ REL & - & - & - & - & - & - & - & - & - & -1 & - & - & - & -1 & - & - & - & - & - & - & - \\
\hline
\end{tabular}


Table 5-11 (page 2 of 4). Selected pneumoconiotic agents: Geometric mean exposures and percent exceeding designated occupational exposure limits, MSHA and OSHA samples, 1979-1999

\begin{tabular}{|c|c|c|c|c|c|c|c|c|c|c|c|c|c|c|c|c|c|c|c|c|c|}
\hline Agent & 1979 & 1980 & 1981 & 1982 & 1983 & 1984 & 1985 & 1986 & 1987 & 1988 & 1989 & 1990 & 1991 & 1992 & 1993 & 1994 & 1995 & 1996 & 1997 & 1998 & 1999 \\
\hline \multicolumn{22}{|c|}{ Beryllium and Compounds - OSHA } \\
\hline $\begin{array}{l}\mathrm{GM} \\
\left(\mu \mathrm{g} / \mathrm{m}^{3}\right)\end{array}$ & 0.043 & 0.079 & 0.037 & 0.031 & 0.044 & 0.031 & 0.035 & 0.026 & 0.034 & 0.039 & 0.031 & 0.033 & 0.032 & 0.031 & 0.034 & 0.029 & 0.027 & 0.029 & 0.029 & 0.027 & 0.032 \\
\hline $\begin{array}{l}\text { No. of } \\
\text { samples }\end{array}$ & 31 & 71 & 110 & 151 & 102 & 698 & 683 & 579 & 864 & 991 & 711 & 911 & 707 & 676 & 595 & 452 & 408 & 349 & 558 & 372 & 410 \\
\hline$\%>$ PEL & 6.5 & 7.0 & 2.7 & 0.7 & 2.9 & 1.4 & 2.9 & 0.3 & 1.9 & 1.2 & 0.8 & 1.4 & 0.3 & 0.6 & 1.3 & 0.4 & 0.0 & 0.9 & 0.5 & 0.8 & 1.2 \\
\hline$\%>$ REL & - & - & - & - & - & - & - & - & - & & - & - & - & . & - & - & - & - & - & - & - \\
\hline \multicolumn{22}{|c|}{ Carbon Black - OSHA } \\
\hline $\begin{array}{l}\mathrm{GM} \\
\left(\mathrm{mg} / \mathrm{m}^{3}\right)\end{array}$ & 0.460 & 1.53 & 1.38 & 1.01 & 1.14 & 1.16 & 1.71 & 0.976 & 0.692 & 0.659 & 0.716 & 1.05 & 0.712 & 0.556 & 0.686 & 0.705 & 0.918 & 0.513 & 0.798 & 0.620 & 0.325 \\
\hline $\begin{array}{l}\text { No. of } \\
\text { samples }\end{array}$ & 16 & 30 & 30 & 29 & 29 & 26 & 29 & 28 & 22 & 27 & 20 & 27 & 17 & 32 & 28 & 18 & 39 & 21 & 35 & 26 & 23 \\
\hline$\%>$ PEL & 0.0 & 20.0 & 16.7 & 10.3 & 10.3 & 23.1 & 24.1 & 10.7 & 13.6 & 3.7 & 15.0 & 18.5 & 17.6 & 6.3 & 7.1 & 11.1 & 10.3 & 14.3 & 17.1 & 7.7 & 0.0 \\
\hline$\%>$ REL & 0.0 & 20.0 & 16.7 & 10.3 & 10.3 & 23.1 & 24.1 & 10.7 & 13.6 & 3.7 & 15.0 & 18.5 & 17.6 & 6.3 & 7.1 & 11.1 & 10.3 & 14.3 & 17.1 & 7.7 & 0.0 \\
\hline \multicolumn{22}{|c|}{ Cobalt Fume and Dust - MSHA } \\
\hline $\begin{array}{l}\mathrm{GM} \\
\left(\mu \mathrm{g} / \mathrm{m}^{3}\right)\end{array}$ & 1.20 & 26.0 & 1.44 & - & 0.485 & 0.313 & 0.382 & 0.426 & 0.410 & 0.389 & 0.366 & 0.375 & 0.367 & 0.393 & 0.286 & 0.329 & 0.306 & 0.270 & 0.309 & 0.287 & 0.308 \\
\hline $\begin{array}{l}\text { No. of } \\
\text { samples }\end{array}$ & 2 & 3 & 4 & 0 & 111 & 124 & 123 & 55 & 89 & 60 & 102 & 105 & 72 & 41 & 105 & 212 & 187 & 580 & 102 & 45 & 90 \\
\hline$\%>$ PEL & 100.0 & 33.3 & 0.0 & - & 0.9 & 0.0 & 0.0 & 0.0 & 0.0 & 0.0 & 0.0 & 0.0 & 0.0 & 0.0 & 0.0 & 0.9 & 0.0 & 0.2 & 0.0 & 0.0 & 0.0 \\
\hline$\%>\mathrm{REL}$ & 100.0 & 33.3 & 0.0 & - & 1.8 & 0.0 & 0.8 & 0.0 & 0.0 & 0.0 & 0.0 & 1.0 & 1.4 & 0.0 & 0.0 & 1.9 & 0.0 & 0.2 & 1.0 & 0.0 & 0.0 \\
\hline \multicolumn{22}{|c|}{ Cobalt, Metal, Fume and Dust - OSHA } \\
\hline $\begin{array}{l}\mathrm{GM} \\
\left(\mu \mathrm{g} / \mathrm{m}^{3}\right)\end{array}$ & 4.32 & 7.41 & 5.74 & 6.19 & 7.52 & 3.19 & 3.83 & 3.59 & 3.07 & 3.10 & 2.61 & 3.60 & 4.01 & 3.29 & 2.96 & 2.53 & 2.96 & 1.87 & 1.84 & 2.13 & 1.97 \\
\hline $\begin{array}{l}\text { No. of } \\
\text { samples }\end{array}$ & 51 & 71 & 168 & 288 & 222 & 829 & 785 & 809 & 990 & 1069 & 760 & 987 & 836 & 823 & 653 & 512 & 595 & 582 & 748 & 511 & 476 \\
\hline$\%>$ PEL & 3.9 & 4.2 & 3.6 & 8.3 & 13.1 & 2.5 & 3.3 & 3.6 & 2.7 & 3.5 & 2.1 & 5.3 & 6.1 & 3.9 & 1.1 & 0.8 & 3.5 & 1.0 & 1.6 & 1.6 & 0.0 \\
\hline$\%>$ REL & 5.9 & 12.7 & 6.5 & 14.2 & 15.8 & 4.0 & 5.6 & 4.9 & 4.4 & 5.1 & 2.1 & 5.3 & 6.1 & 3.9 & 3.5 & 1.8 & 5.0 & 2.6 & 2.8 & 2.2 & 0.0 \\
\hline
\end{tabular}

See footnotes at end of table. 
Table 5-11 (page 3 of 4). Selected pneumoconiotic agents: Geometric mean exposures and percent exceeding designated occupational exposure limits, MSHA and OSHA samples, 1979-1999

\begin{tabular}{|c|c|c|c|c|c|c|c|c|c|c|c|c|c|c|c|c|c|c|c|c|c|}
\hline Agent & 1979 & 1980 & 1981 & 1982 & 1983 & 1984 & 1985 & 1986 & 1987 & 1988 & 1989 & 1990 & 1991 & 1992 & 1993 & 1994 & 1995 & 1996 & 1997 & 1998 & 1999 \\
\hline \multicolumn{22}{|c|}{ Iron Oxide Fume - MSHA } \\
\hline $\begin{array}{l}\mathrm{GM} \\
\left(\mathrm{mg} / \mathrm{m}^{3}\right)\end{array}$ & 0.430 & 0.480 & 0.333 & 0.394 & 0.210 & 0.279 & 0.301 & 0.238 & 0.214 & 0.327 & 0.282 & 0.198 & 0.199 & 0.198 & 0.097 & 0.109 & 0.110 & 0.062 & 0.059 & 0.130 & 0.027 \\
\hline $\begin{array}{l}\text { No. of } \\
\text { samples }\end{array}$ & 155 & 155 & 226 & 119 & 152 & 182 & 211 & 171 & 181 & 136 & 163 & 213 & 177 & 136 & 136 & 200 & 173 & 381 & 155 & 59 & 40 \\
\hline$\%>$ PEL & 1.9 & 0.6 & 0.9 & 2.5 & 1.3 & 1.6 & 0.5 & 0.6 & 1.1 & 0.7 & 2.5 & 2.3 & 1.1 & 1.5 & 1.5 & 3.0 & 0.0 & 1.0 & 0.0 & 0.0 & 0.0 \\
\hline$\%>$ REL & 3.2 & 5.2 & 2.2 & 4.2 & 4.6 & 3.3 & 4.7 & 2.3 & 5.0 & 3.7 & 5.5 & 5.2 & 3.4 & 5.9 & 4.4 & 4.0 & 2.9 & 3.4 & 2.6 & 0.0 & 0.0 \\
\hline \multicolumn{22}{|c|}{ Iron Oxide Fume - OSHA } \\
\hline $\begin{array}{l}\mathrm{GM} \\
\left(\mathrm{mg} / \mathrm{m}^{3}\right)\end{array}$ & 1.33 & 1.10 & 0.974 & 0.924 & 0.514 & 0.421 & 0.456 & 0.356 & 0.245 & 0.277 & 0.502 & 0.360 & 0.264 & 0.261 & 0.280 & 0.301 & 0.406 & 0.238 & 0.315 & 0.333 & 0.279 \\
\hline $\begin{array}{l}\text { No. of } \\
\text { samples }\end{array}$ & 575 & 901 & 1204 & 1347 & 1303 & 1315 & 1759 & 1586 & 1540 & 1818 & 1674 & 1760 & 1338 & 1258 & 1146 & 925 & 859 & 968 & 986 & 833 & 668 \\
\hline$\%>$ PEL & 6.3 & 5.4 & 5.7 & 5.3 & 3.4 & 3.2 & 4.3 & 1.3 & 2.4 & 2.7 & 5.0 & 3.4 & 2.5 & 2.0 & 1.9 & 3.7 & 2.9 & 3.9 & 2.7 & 3.4 & 1.8 \\
\hline$\%>$ REL & 18.3 & 16.5 & 17.1 & 13.8 & 9.7 & 10.7 & 12.5 & 6.6 & 6.9 & 8.0 & 13.4 & 10.1 & 6.3 & 6.1 & 5.9 & 7.4 & 6.8 & 6.9 & 5.5 & 7.9 & 5.5 \\
\hline \multicolumn{22}{|c|}{ Talc, Nonfibrous, < 1\% quartz - MSHA } \\
\hline $\left.\begin{array}{l}\text { GM } \\
(\mathrm{mppcf})\end{array}\right]$ & 7.98 & 5.39 & 3.93 & 5.97 & 1.70 & 2.08 & 2.87 & 4.75 & 5.41 & 4.90 & 5.66 & 8.11 & 3.41 & 2.55 & 6.50 & - & - & 26.0 & 3.00 & - & 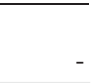 \\
\hline $\begin{array}{l}\text { No. of } \\
\text { samples }\end{array}$ & 56 & 35 & 61 & 33 & 68 & 22 & 14 & 29 & 18 & 30 & 24 & 9 & 6 & 13 & 11 & 0 & 0 & 5 & 1 & 0 & 0 \\
\hline$\%>$ PEL & 14.3 & 8.6 & 4.9 & 6.1 & 7.4 & 9.1 & 7.1 & 3.4 & 11.1 & 6.7 & 8.3 & 33.3 & 0.0 & 0.0 & 18.2 & - & - & 40.0 & 0.0 & - & - \\
\hline$\%>$ REL & - & - & - & - & - & - & - & - & - & - & - & - & - & - & - & - & - & - & - & - & - \\
\hline \multicolumn{22}{|c|}{ Tin, inorganic compounds except oxide, as Sn - OSHA } \\
\hline $\begin{array}{l}\mathrm{GM} \\
\left(\mu \mathrm{g} / \mathrm{m}^{3}\right)\end{array}$ & 6.76 & 5.84 & 8.66 & 5.25 & 8.64 & 4.88 & 5.03 & 10.4 & 6.70 & 5.80 & 4.94 & 5.02 & 7.32 & 7.36 & 10.8 & 4.58 & 11.3 & 3.60 & 9.25 & 4.22 & 4.40 \\
\hline $\begin{array}{l}\text { No. of } \\
\text { samples }\end{array}$ & 124 & 130 & 63 & 46 & 27 & 57 & 99 & 62 & 69 & 88 & 46 & 182 & 111 & 79 & 51 & 75 & 52 & 125 & 37 & 66 & 45 \\
\hline$\%>$ PEL & 1.6 & 0.8 & 1.6 & 0.0 & 0.0 & 0.0 & 0.0 & 0.0 & 0.0 & 0.0 & 0.0 & 0.0 & 0.0 & 0.0 & 3.9 & 0.0 & 0.0 & 0.0 & 0.0 & 0.0 & 0.0 \\
\hline$\%>$ REL & 1.6 & 0.8 & 1.6 & 0.0 & 0.0 & 0.0 & 0.0 & 0.0 & 0.0 & 0.0 & 0.0 & 0.0 & 0.0 & 0.0 & 3.9 & 0.0 & 0.0 & 0.0 & 0.0 & 0.0 & 0.0 \\
\hline
\end{tabular}


Table 5-11 (page 4 of 4). Selected pneumoconiotic agents: Geometric mean exposures and percent exceeding designated occupational exposure limits, MSHA and OSHA samples, 1979-1999

\begin{tabular}{|c|c|c|c|c|c|c|c|c|c|c|c|c|c|c|c|c|c|c|c|c|c|}
\hline Agent & 1979 & 1980 & 1981 & 1982 & 1983 & 1984 & 19851 & 1986 & 1987 & 1988 & 1989 & 1990 & 1991 & 1992 & 1993 & 1994 & 1995 & 1996 & 1997 & 1998 & 1999 \\
\hline \multicolumn{22}{|c|}{ Titanium Dioxide Fume and Dust - MSHA } \\
\hline $\begin{array}{l}\mathrm{GM} \\
\left(\mu \mathrm{g} / \mathrm{m}^{3}\right)\end{array}$ & 1.48 & 4.11 & 6.49 & 2.57 & 3.70 & 2.42 & 3.43 & 3.22 & 4.95 & 7.22 & 7.27 & 4.24 & 6.48 & 3.73 & 1.29 & 1.37 & 1.06 & 0.677 & 0.822 & 1.55 & 0.488 \\
\hline $\begin{array}{l}\text { No. of } \\
\text { samples }\end{array}$ & 111 & 56 & 116 & 97 & 124 & 129 & 145 & 100 & 123 & 104 & 106 & 145 & 108 & 55 & 119 & 215 & 188 & 578 & 128 & 49 & 86 \\
\hline$\%>$ PEL & 0.0 & 0.0 & 0.0 & 0.0 & 0.0 & 0.0 & 0.0 & 0.0 & 0.0 & 0.0 & 0.9 & 0.0 & 0.0 & 0.0 & 0.0 & 0.0 & 0.0 & 0.0 & 0.0 & 0.0 & 0.0 \\
\hline$\%>$ REL & - & - & - & - & - & - & - & - & - & & - & - & - & & - & - & - & - & - & - & - \\
\hline \multicolumn{22}{|c|}{ Welding Fumes (total particulate) - MSHA } \\
\hline $\begin{array}{l}\mathrm{GM} \\
\left(\mathrm{mg} / \mathrm{m}^{3}\right)\end{array}$ & 2.39 & 1.59 & 1.07 & 0.931 & 0.907 & 0.931 & 1.57 & 1.34 & 1.98 & 1.77 & 1.53 & 0.169 & 1.95 & 0.070 & 1.00 & - & - & - & - & - & - \\
\hline $\begin{array}{l}\text { No. of } \\
\text { samples }\end{array}$ & 165 & 175 & 212 & 117 & 94 & 47 & 79 & 45 & 31 & 44 & 20 & 5 & 2 & 1 & 1 & 0 & 0 & 0 & 0 & 0 & 0 \\
\hline$\%>$ PEL & 13.3 & 9.1 & 4.7 & 2.6 & 3.2 & 4.3 & 11.4 & 0.0 & 16.1 & 6.8 & 0.0 & 0.0 & 0.0 & 0.0 & 0.0 & - & - & - & - & - & - \\
\hline$\%>$ REL & - & - & - & - & - & - & - & - & - & & - & - & - & & - & - & - & - & - & - & - \\
\hline \multicolumn{22}{|c|}{ Welding Fumes (total particulate) - OSHA } \\
\hline $\begin{array}{l}\mathrm{GM} \\
\left(\mathrm{mg} / \mathrm{m}^{3}\right)\end{array}$ & 7.05 & 3.34 & 3.05 & 2.33 & 2.75 & 4.12 & 2.75 & 1.09 & 2.36 & 2.12 & 3.55 & 2.18 & 1.67 & 2.22 & 1.79 & 1.46 & 1.20 & 1.00 & 0.978 & 1.20 & 0.975 \\
\hline $\begin{array}{l}\text { No. of } \\
\text { samples }\end{array}$ & 7 & 35 & 35 & 128 & 48 & 27 & 38 & 32 & 146 & 33 & 230 & 435 & 629 & 585 & 457 & 261 & 230 & 480 & 216 & 304 & 203 \\
\hline$\%>$ PEL & - & - & - & - & - & - & - & - & - & - & 52.5 & 34.7 & 25.4 & 24.4 & 36.8 & - & - & - & - & - & - \\
\hline$\%>$ REL & - & - & - & - & - & - & - & - & - & & - & - & - & & - & - & - & - & - & - & - \\
\hline \multicolumn{22}{|c|}{$\begin{array}{l}\text { - indicates incalculable field } \\
\text { PEL - permissible exposure limit REL - recommended exposure limit } \quad \text { GM - geometric mean } \quad \mathrm{mg} / \mathrm{m}^{3}-\text { milligrams per cubic meter } \\
\text { mppcf - millions of particles per cubic foot } \\
\text { NOTE: Pneumoconiotic agents with at least } 300 \text { total samples in MSHA or OSHA are presented. From March 1, } 1989 \text { to March } 22,1993 \text {, the OSHA PELs in force differed from those } \\
\text { employed before and after those dates. NIOSH has designated beryllium and titanium dioxide as potential occupational carcinogens, and recommends reducing exposures to as low as } \\
\text { feasible. The } \%>\text { REL cannot be calculated for talc because the REL is in units of } \mathrm{mg} / \mathrm{m}^{3} \text {, but MSHA samples and analyzes for talc using units of millions of particles per cubic foot }\end{array}$} \\
\hline
\end{tabular}




\section{Section 6}

All Pneumoconioses

and

Related Exposures 

Figure 6-1. All pneumoconioses: Number of deaths, crude and age-adjusted mortality rates, U.S. residents age 15 and over, 1968-1999

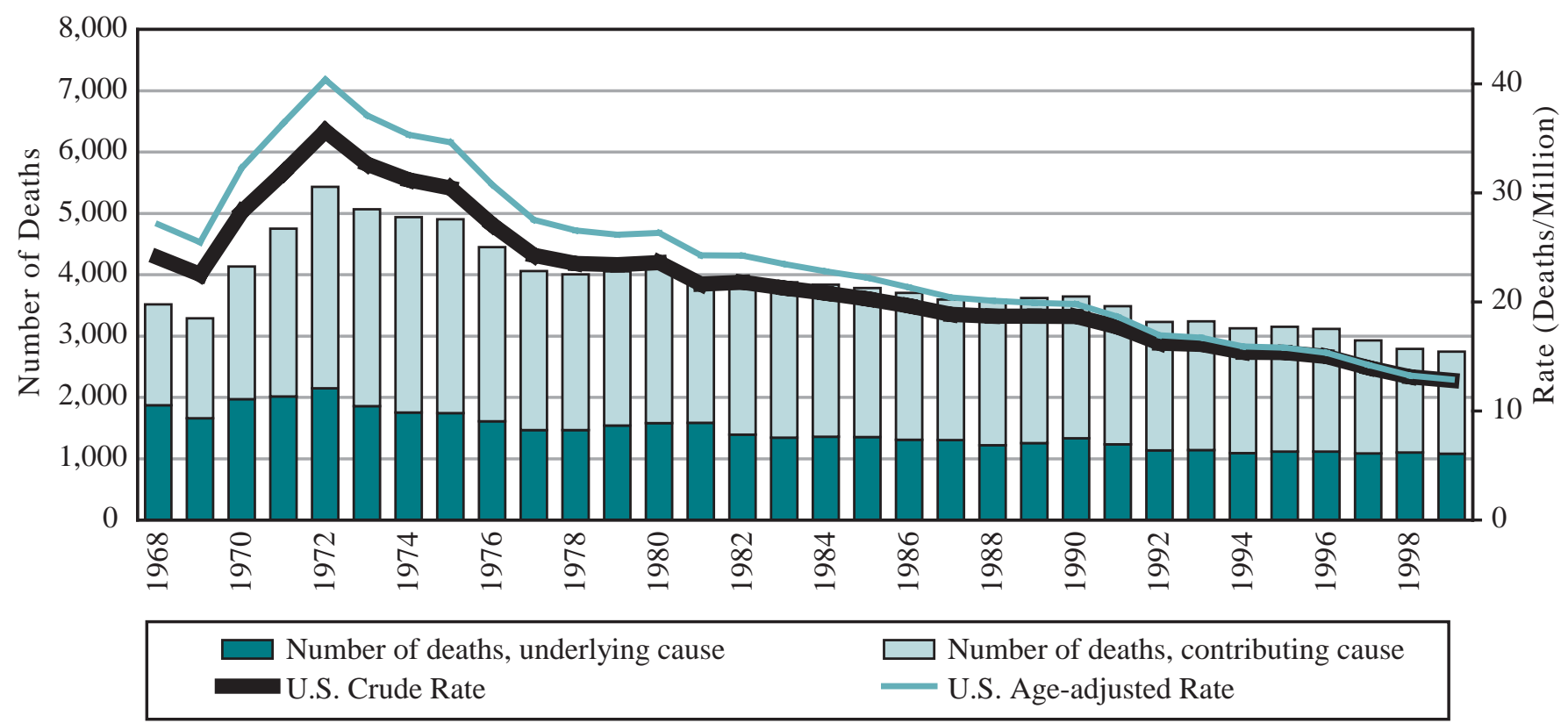

NOTE: See appendices for source description, methods, and ICD codes.

SOURCE: National Center for Health Statistics multiple cause of death data. Population estimates from U.S. Bureau of the Census.

Figure 6-2. All pneumoconioses: Age-adjusted mortality rates by state, U.S. residents age 15 and over, 1990-1999

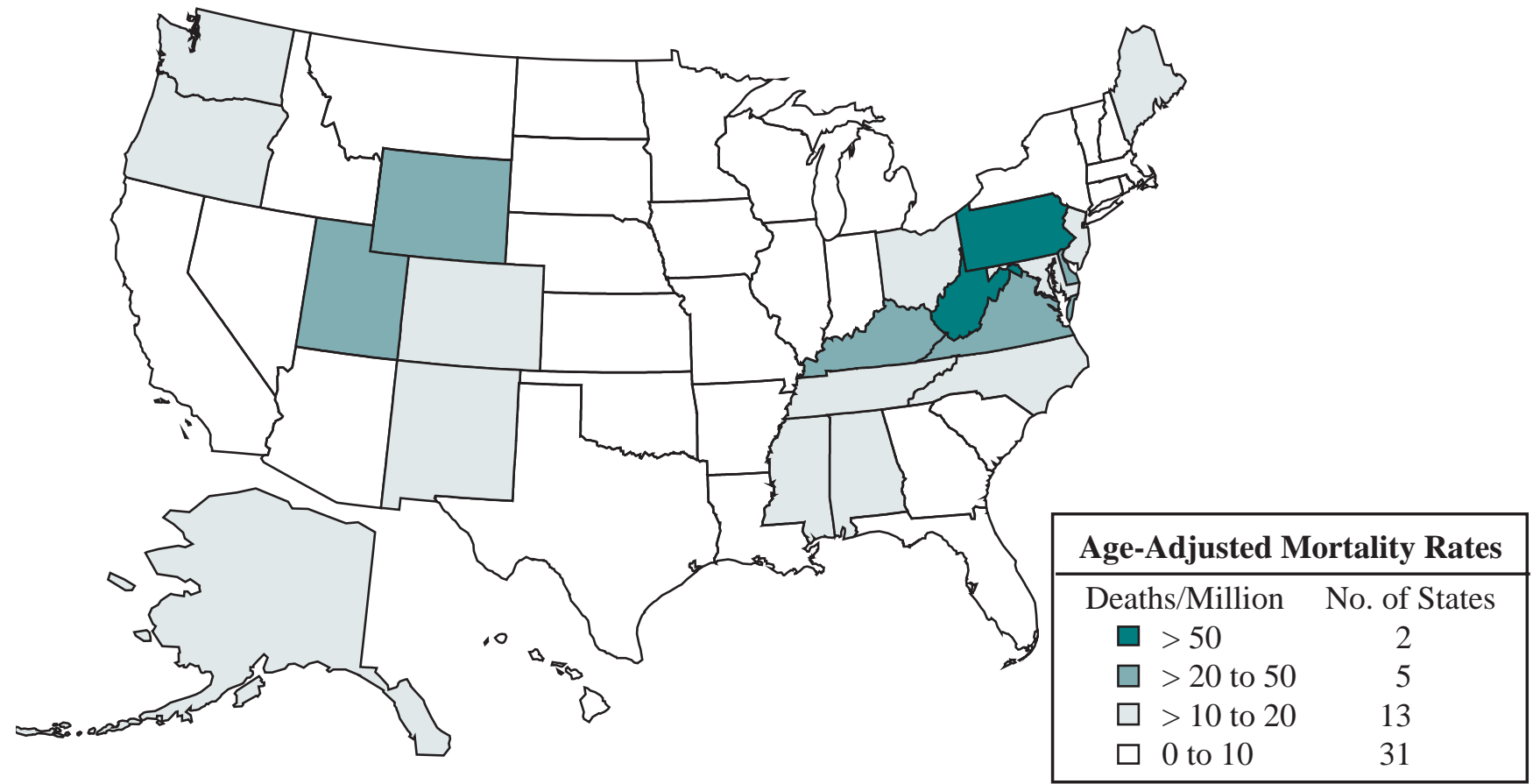

NOTE: See appendices for source description, methods, and ICD codes.

SOURCE: National Center for Health Statistics multiple cause of death data. Population estimates from U.S. Bureau of the Census. 
Table 6-1. All pneumoconioses: Number of deaths by sex, race, and age, and median age at death, U.S. residents age 15 and over, 1990-1999

\begin{tabular}{|c|c|c|c|c|c|c|c|c|c|c|c|c|c|c|c|c|}
\hline \multirow[b]{2}{*}{ Year } & \multirow{2}{*}{$\begin{array}{l}\text { No. of } \\
\text { Deaths }\end{array}$} & \multirow{2}{*}{\begin{tabular}{|c|} 
Under- \\
lying \\
Cause \\
$(\%)$
\end{tabular}} & \multicolumn{2}{|c|}{ Sex } & \multicolumn{3}{|c|}{ Race } & \multicolumn{8}{|c|}{ Age Group (yrs) } & \multirow{2}{*}{$\begin{array}{c}\text { Median } \\
\text { Age } \\
\text { (yrs) }\end{array}$} \\
\hline & & & Male & Female & White & Black & Other & $15-24$ & $25-34$ & $35-44$ & $45-54$ & $55-64$ & $65-74$ & $75-84$ & $85+$ & \\
\hline 1990 & 3,644 & 36.6 & 3,557 & 87 & 3,407 & 222 & 15 & - & 5 & 17 & 66 & 358 & 1,162 & 1,503 & 533 & 76.0 \\
\hline 1991 & 3,486 & 35.5 & 3,403 & 83 & 3,275 & 202 & 9 & 3 & 4 & 25 & 63 & 283 & 1,084 & 1,484 & 540 & 76.0 \\
\hline 1992 & 3,230 & 35.1 & 3,153 & 77 & 3,044 & 174 & 12 & 4 & 4 & 12 & 42 & 265 & 989 & 1,409 & 505 & 77.0 \\
\hline 1993 & 3,238 & 35.3 & 3,170 & 68 & 3,049 & 173 & 16 & - & 4 & 14 & 56 & 277 & 953 & 1,410 & 524 & 77.0 \\
\hline 1994 & 3,126 & 34.9 & 3,052 & 74 & 2,963 & 154 & 9 & - & - & 10 & 45 & 233 & 893 & 1,434 & 511 & 77.0 \\
\hline 1995 & 3,151 & 35.4 & 3,092 & 59 & 2,983 & 160 & 8 & - & 4 & 10 & 68 & 245 & 913 & 1,337 & 574 & 77.0 \\
\hline 1996 & 3,114 & 35.8 & 3,030 & 84 & 2,936 & 164 & 14 & - & 1 & 11 & 51 & 210 & 883 & 1,364 & 594 & 78.0 \\
\hline 1997 & 2,928 & 37.1 & 2,851 & 77 & 2,780 & 139 & 9 & - & 2 & 12 & 48 & 191 & 751 & 1,313 & 611 & 78.0 \\
\hline 1998 & 2,790 & 39.4 & 2,719 & 71 & 2,650 & 127 & 13 & - & 3 & 11 & 41 & 189 & 733 & 1,225 & 588 & 78.0 \\
\hline 1999 & 2,745 & 39.5 & 2,683 & 62 & 2,592 & 136 & 17 & - & 1 & 9 & 38 & 177 & 683 & 1,210 & 627 & 78.0 \\
\hline TOTAL & 31,452 & 36.4 & 30,710 & 742 & 29,679 & 1,651 & 122 & 7 & 28 & 131 & 518 & 2,428 & 9,044 & 13,689 & 5,607 & 77.0 \\
\hline
\end{tabular}

- indicates no deaths listed.

NOTE: See appendices for source description, methods, and ICD codes.

SOURCE: National Center for Health Statistics multiple cause of death data. 
Table 6-2. All pneumoconioses: Mortality rates (per million population) by race and sex, U.S. residents age 15 and over, 1990-1999

\begin{tabular}{|c|c|c|c|c|c|c|c|}
\hline \multirow[b]{2}{*}{ Year } & \multirow[b]{2}{*}{ Overall } & \multicolumn{2}{|c|}{ White } & \multicolumn{2}{|c|}{ Black } & \multicolumn{2}{|c|}{ Other } \\
\hline & & Male & Female & Male & Female & Male & Female \\
\hline & \multicolumn{7}{|c|}{ Crude Mortality Rate } \\
\hline 1990 & 18.66 & 41.52 & 0.92 & 21.03 & 0.50 & 3.74 & 0.54 \\
\hline 1991 & 17.69 & 39.59 & 0.92 & 19.02 & 0.33 & 2.49 & - \\
\hline 1992 & 16.22 & 36.53 & 0.78 & 15.66 & 0.65 & 2.92 & 0.25 \\
\hline 1993 & 16.11 & 36.36 & 0.72 & 15.57 & 0.40 & 4.15 & - \\
\hline 1994 & 15.40 & 34.94 & 0.79 & 13.68 & 0.31 & 2.27 & - \\
\hline 1995 & 15.37 & 35.03 & 0.64 & 14.17 & 0.15 & 1.96 & - \\
\hline 1996 & 15.00 & 33.75 & 0.91 & 14.26 & 0.15 & 3.02 & 0.21 \\
\hline 1997 & 13.96 & 31.74 & 0.78 & 11.41 & 0.52 & 2.04 & - \\
\hline 1998 & 13.15 & 30.11 & 0.68 & 10.36 & 0.37 & 1.96 & 0.79 \\
\hline 1999 & 12.81 & 29.21 & 0.65 & 11.19 & 0.14 & 3.61 & - \\
\hline \multirow[t]{2}{*}{ 1990-1999 } & 15.34 & 34.71 & 0.78 & 14.40 & 0.35 & 2.80 & 0.18 \\
\hline & \multicolumn{7}{|c|}{ Age-Adjusted Mortality Rate } \\
\hline 1990 & 19.81 & 53.28 & 0.77 & 38.57 & 0.58 & 9.71 & 1.06 \\
\hline 1991 & 18.65 & 50.44 & 0.80 & 36.33 & 0.40 & 4.49 & - \\
\hline 1992 & 16.95 & 46.05 & 0.66 & 29.67 & 0.78 & 6.23 & 0.55 \\
\hline 1993 & 16.73 & 45.21 & 0.60 & 29.67 & 0.52 & 9.18 & - \\
\hline 1994 & 15.92 & 43.17 & 0.65 & 25.77 & 0.38 & 4.68 & - \\
\hline 1995 & 15.80 & 42.82 & 0.53 & 27.71 & 0.21 & 3.67 & - \\
\hline 1996 & 15.33 & 41.12 & 0.75 & 26.01 & 0.18 & 6.32 & 0.42 \\
\hline 1997 & 14.22 & 38.57 & 0.64 & 21.18 & 0.70 & 3.82 & - \\
\hline 1998 & 13.26 & 35.66 & 0.55 & 19.28 & 0.43 & 3.81 & 1.25 \\
\hline 1999 & 12.88 & 34.41 & 0.52 & 20.91 & 0.16 & 6.27 & - \\
\hline 1990-1999 & 15.76 & 42.46 & 0.64 & 27.08 & 0.43 & 5.92 & 0.34 \\
\hline
\end{tabular}

- indicates no deaths listed.

NOTE: See appendices for source description, methods, and ICD codes.

SOURCE: National Center for Health Statistics multiple cause of death data. Population estimates from U.S. Bureau of the Census. 
Table 6-3. All pneumoconioses: Years of potential life lost to age 65 and to life expectancy by race and sex, U.S. residents age 15 and over, 1990-1999

\begin{tabular}{|c|c|c|c|c|c|c|c|}
\hline \multirow[b]{2}{*}{ Year } & \multicolumn{2}{|c|}{ White } & \multicolumn{2}{|c|}{ Black } & \multicolumn{2}{|c|}{ Other } & \multirow[b]{2}{*}{ Total } \\
\hline & Male & Female & Male & Female & Male & Female & \\
\hline & \multicolumn{7}{|c|}{ Years of Potential Life Lost to Age 65} \\
\hline 1990 & 2,915 & 35 & 340 & 55 & 35 & - & 3,380 \\
\hline 1991 & 2,655 & 245 & 340 & - & 20 & - & 3,260 \\
\hline 1992 & 2,160 & 100 & 245 & 55 & 15 & - & 2,575 \\
\hline 1993 & 2,350 & 45 & 270 & 25 & 25 & - & 2,715 \\
\hline 1994 & 1,825 & 55 & 180 & 25 & 5 & - & 2,090 \\
\hline 1995 & 2,365 & 40 & 165 & 15 & 50 & - & 2,635 \\
\hline 1996 & 1,815 & 115 & 140 & - & 55 & - & 2,125 \\
\hline 1997 & 1,810 & 105 & 130 & - & - & - & 2,045 \\
\hline 1998 & 1,665 & 60 & 170 & 30 & 10 & 5 & 1,940 \\
\hline 1999 & 1,340 & 60 & 260 & 25 & 30 & - & 1,715 \\
\hline \multirow[t]{2}{*}{ TOTAL } & 20,900 & 860 & 2,240 & 230 & 245 & 5 & 24,480 \\
\hline & \multicolumn{7}{|c|}{ Years of Potential Life Lost to Life Expectancy } \\
\hline 1990 & 33,353 & 931 & 2,139 & 131 & 173 & 22 & 36,749 \\
\hline 1991 & 32,303 & 1,199 & 1,959 & 49 & 143 & - & 35,653 \\
\hline 1992 & 29,270 & 959 & 1,616 & 149 & 146 & 9 & 32,149 \\
\hline 1993 & 29,159 & 772 & 1,651 & 69 & 209 & - & 31,860 \\
\hline 1994 & 27,761 & 810 & 1,441 & 66 & 111 & - & 30,189 \\
\hline 1995 & 28,815 & 684 & 1,440 & 37 & 152 & - & 31,128 \\
\hline 1996 & 27,380 & 1,004 & 1,566 & 28 & 195 & 8 & 30,181 \\
\hline 1997 & 25,884 & 865 & 1,305 & 63 & 111 & - & 28,228 \\
\hline 1998 & 24,969 & 746 & 1,213 & 95 & 112 & 51 & 27,186 \\
\hline 1999 & 23,921 & 673 & 1,397 & 51 & 243 & - & 26,285 \\
\hline TOTAL & 282,815 & 8,643 & 15,727 & 738 & 1,595 & 90 & 309,608 \\
\hline
\end{tabular}

- indicates no deaths listed.

NOTE: See appendices for source description, methods, and ICD codes.

SOURCE: National Center for Health Statistics multiple cause of death data. 
Table 6-4. All pneumoconioses: Number of deaths by state, U.S. residents age 15 and over, 1990-1999



- indicates no deaths listed.

NOTE: See appendices for source description, methods, and ICD codes.

SOURCE: National Center for Health Statistics multiple cause of death data. 
Table 6-5. All pneumoconioses: Number of deaths, mortality rates (per million population), and years of potential life lost (YPLL) by state, U.S. residents age 15 and over, 1990-1999

\begin{tabular}{|c|c|c|c|c|c|c|c|c|c|c|}
\hline \multirow[b]{2}{*}{ State } & \multirow{2}{*}{$\begin{array}{l}\text { No. of } \\
\text { Deaths }\end{array}$} & \multirow[b]{2}{*}{ Rank } & \multicolumn{2}{|c|}{ Crude Mortality } & \multicolumn{2}{|c|}{ Age-Adjusted Mortality } & \multicolumn{4}{|c|}{ YPLL to Life Expectancy } \\
\hline & & & Rate & Rank & Rate & Rank & Total & Rank & YPLL/death & Rank \\
\hline Alabama & 596 & 13 & 17.98 & 7 & 17.95 & 8 & 6,839 & 12 & 11.5 & 27 \\
\hline Alaska & 23 & 49 & 5.27 & 43 & 14.49 & 14 & 287 & 49 & 12.5 & 7 \\
\hline Arizona & 210 & 29 & 6.47 & 34 & 6.53 & 35 & 2,352 & 30 & 11.2 & 31 \\
\hline Arkansas & 137 & 33 & 7.09 & 32 & 6.18 & 38 & 1,663 & 32 & 12.1 & 18 \\
\hline California & 1,365 & 6 & 5.59 & 38 & 6.69 & 33 & 16,647 & 4 & 12.2 & 14 \\
\hline Colorado & 325 & 20 & 11.22 & 16 & 14.34 & 15 & 3,367 & 22 & 10.4 & 46 \\
\hline Connecticut & 187 & 31 & 7.11 & 31 & 6.85 & 32 & 2,186 & 31 & 11.7 & 23 \\
\hline Delaware & 139 & 32 & 24.61 & 6 & 26.69 & 6 & 1,592 & 33 & 11.5 & 27 \\
\hline District of Columbia & 12 & 50 & 2.59 & 49 & 2.76 & 50 & 215 & 50 & 17.9 & 1 \\
\hline Florida & 972 & 9 & 8.47 & 24 & 6.43 & 37 & 10,928 & 9 & 11.2 & 31 \\
\hline Georgia & 225 & 27 & 4.05 & 47 & 5.07 & 42 & 2,818 & 26 & 12.5 & 7 \\
\hline Hawaii & 24 & 48 & 2.59 & 49 & 2.98 & 49 & 299 & 48 & 12.5 & 7 \\
\hline Idaho & 64 & 41 & 7.44 & 28 & 7.45 & 30 & 696 & 42 & 10.9 & 40 \\
\hline Illinois & 784 & 10 & 8.51 & 23 & 8.68 & 24 & 8,775 & 10 & 11.2 & 31 \\
\hline Indiana & 341 & 19 & 7.56 & 27 & 7.62 & 29 & 3,775 & 20 & 11.1 & 37 \\
\hline Iowa & 137 & 33 & 6.19 & 35 & 5.00 & 43 & 1,473 & 34 & 10.7 & 41 \\
\hline Kansas & 101 & 38 & 5.11 & 44 & 4.68 & 44 & 1,302 & 38 & 12.9 & 5 \\
\hline Kentucky & 1,417 & 4 & 46.96 & 3 & 47.30 & 3 & 16,462 & 5 & 11.6 & 25 \\
\hline Louisiana & 255 & 23 & 7.83 & 26 & 8.57 & 26 & 3,520 & 21 & 13.8 & 3 \\
\hline Maine & 124 & 36 & 12.71 & 14 & 12.01 & 17 & 1,376 & 37 & 11.1 & 37 \\
\hline Maryland & 553 & 14 & 13.90 & 13 & 16.89 & 10 & 6,729 & 13 & 12.2 & 14 \\
\hline Massachusetts & 430 & 18 & 8.78 & 22 & 8.45 & 27 & 4,743 & 18 & 11.0 & 39 \\
\hline Michigan & 456 & 17 & 6.16 & 36 & 6.52 & 36 & 5,575 & 16 & 12.2 & 14 \\
\hline Minnesota & 196 & 30 & 5.54 & 39 & 5.53 & 39 & 2,448 & 28 & 12.5 & 7 \\
\hline Mississippi & 312 & 21 & 15.31 & 11 & 15.29 & 13 & 4,566 & 19 & 14.6 & 2 \\
\hline Missouri & 236 & 24 & 5.68 & 37 & 5.13 & 40 & 2,834 & 24 & 12.0 & 20 \\
\hline Montana & 61 & 43 & 9.18 & 21 & 8.73 & 23 & 638 & 44 & 10.5 & 43 \\
\hline Nebraska & 46 & 45 & 3.66 & 48 & 3.13 & 48 & 467 & 45 & 10.2 & 47 \\
\hline Nevada & 66 & 40 & 5.49 & 40 & 6.88 & 31 & 810 & 40 & 12.3 & 13 \\
\hline New Hampshire & 62 & 42 & 6.90 & 33 & 7.65 & 28 & 712 & 41 & 11.5 & 27 \\
\hline New Jersey & 1,082 & 7 & 17.01 & 9 & 17.01 & 9 & 12,068 & 8 & 11.2 & 31 \\
\hline New Mexico & 135 & 35 & 10.72 & 18 & 12.45 & 16 & 1,419 & 36 & 10.5 & 43 \\
\hline New York & 611 & 11 & 4.23 & 46 & 4.25 & 47 & 7,183 & 11 & 11.8 & 21 \\
\hline North Carolina & 539 & 15 & 9.42 & 19 & 10.04 & 20 & 6,564 & 14 & 12.2 & 14 \\
\hline North Dakota & 26 & 47 & 5.30 & 42 & 4.64 & 45 & 322 & 47 & 12.4 & 12 \\
\hline Ohio & 1,403 & 5 & 16.12 & 10 & 16.07 & 11 & 15,701 & 6 & 11.2 & 31 \\
\hline Oklahoma & 123 & 37 & 4.85 & 45 & 4.52 & 46 & 1,423 & 35 & 11.6 & 25 \\
\hline Oregon & 279 & 22 & 11.37 & 15 & 10.66 & 19 & 2,990 & 23 & 10.7 & 41 \\
\hline Pennsylvania & 9,050 & 1 & 93.76 & 2 & 82.40 & 2 & 90,030 & 1 & 9.9 & 50 \\
\hline Rhode Island & 59 & 44 & 7.40 & 29 & 6.61 & 34 & 690 & 43 & 11.7 & 23 \\
\hline South Carolina & 233 & 25 & 8.17 & 25 & 9.27 & 22 & 2,823 & 25 & 12.1 & 18 \\
\hline South Dakota & 11 & 51 & 2.00 & 51 & 1.68 & 51 & 149 & 51 & 13.5 & 4 \\
\hline Tennessee & 458 & 16 & 11.04 & 17 & 11.34 & 18 & 5,151 & 17 & 11.2 & 31 \\
\hline Texas & 1,019 & 8 & 7.20 & 30 & 8.63 & 25 & 12,856 & 7 & 12.6 & 6 \\
\hline Utah & 233 & 25 & 17.42 & 8 & 22.32 & 7 & 2,377 & 29 & 10.2 & 47 \\
\hline Vermont & 43 & 46 & 9.40 & 20 & 9.94 & 21 & 434 & 46 & 10.1 & 49 \\
\hline Virginia & 1,872 & 3 & 35.76 & 4 & 44.04 & 4 & 22,032 & 3 & 11.8 & 21 \\
\hline Washington & 602 & 12 & 14.31 & 12 & 15.45 & 12 & 6,323 & 15 & 10.5 & 43 \\
\hline West Virginia & 3,513 & 2 & 241.51 & 1 & 211.31 & 1 & 40,366 & 2 & 11.5 & 27 \\
\hline Wisconsin & 213 & 28 & 5.38 & 41 & 5.11 & 41 & 2,659 & 27 & 12.5 & 7 \\
\hline Wyoming & 92 & 39 & 25.69 & 5 & 31.08 & 5 & 812 & 39 & 8.8 & 51 \\
\hline
\end{tabular}

- indicates no deaths listed.

NOTE: See appendices for source description, methods, and ICD codes.

SOURCE: National Center for Health Statistics multiple cause of death data. Population estimates from U.S. Bureau of the Census. 
Table 6-6. All pneumoconioses: Percent of deaths by condition and state, U.S. residents age 15 and over, 1990-1999

\begin{tabular}{|c|c|c|c|c|c|}
\hline State & Asbestosis & Silicosis & $\begin{array}{l}\text { Coal Workers' } \\
\text { Pneumoconiosis }\end{array}$ & Byssinosis & $\begin{array}{c}\text { Unspecified/Other } \\
\text { Pneumoconioses }\end{array}$ \\
\hline Alabama & 53.4 & 6.5 & 31.0 & 0.5 & 9.1 \\
\hline Alaska & 78.3 & 8.7 & 13.0 & - & - \\
\hline Arizona & 54.8 & 18.1 & 19.5 & 0.5 & 7.6 \\
\hline Arkansas & 45.3 & 10.9 & 39.4 & 0.7 & 4.4 \\
\hline California & 73.3 & 7.8 & 14.7 & 0.6 & 4.1 \\
\hline Colorado & 22.5 & 27.7 & 36.3 & 0.6 & 13.2 \\
\hline Connecticut & 68.4 & 14.4 & 9.1 & - & 8.6 \\
\hline Delaware & 78.4 & 4.3 & 11.5 & - & 6.5 \\
\hline District of Columbia & 41.7 & 33.3 & 8.3 & - & 16.7 \\
\hline Florida & 63.8 & 6.4 & 22.9 & 0.4 & 7.3 \\
\hline Georgia & 55.6 & 15.6 & 14.7 & 4.9 & 10.2 \\
\hline Hawaii & 95.8 & - & - & - & 4.2 \\
\hline Idaho & 62.5 & 23.4 & 12.5 & - & 1.6 \\
\hline Illinois & 27.8 & 9.7 & 45.7 & 0.3 & 17.3 \\
\hline Indiana & 18.8 & 12.3 & 56.6 & 0.3 & 12.6 \\
\hline Iowa & 39.4 & 16.8 & 35.0 & 0.7 & 8.8 \\
\hline Kansas & 55.4 & 10.9 & 26.7 & 1.0 & 5.9 \\
\hline Kentucky & 4.6 & 2.9 & 72.7 & 0.1 & 20.0 \\
\hline Louisiana & 76.1 & 13.7 & 5.9 & 0.4 & 4.3 \\
\hline Maine & 89.5 & 8.1 & 0.8 & - & 1.6 \\
\hline Maryland & 74.5 & 4.9 & 15.6 & 0.2 & 5.8 \\
\hline Massachusetts & 88.4 & 6.0 & 1.6 & 0.7 & 3.7 \\
\hline Michigan & 43.6 & 23.0 & 23.2 & 0.7 & 10.5 \\
\hline Minnesota & 72.4 & 24.5 & - & 1.5 & 3.1 \\
\hline Mississippi & 83.7 & 4.2 & 11.2 & - & 1.3 \\
\hline Missouri & 52.5 & 19.1 & 18.6 & 0.4 & 10.2 \\
\hline Montana & 55.7 & 24.6 & 13.1 & - & 6.6 \\
\hline Nebraska & 78.3 & 13.0 & 4.3 & - & 4.3 \\
\hline Nevada & 63.6 & 19.7 & 10.6 & - & 7.6 \\
\hline New Hampshire & 80.6 & 11.3 & 3.2 & 1.6 & 4.8 \\
\hline New Jersey & 84.6 & 4.9 & 6.7 & - & 4.5 \\
\hline New Mexico & 23.0 & 29.6 & 32.6 & - & 14.8 \\
\hline New York & 66.0 & 17.2 & 8.5 & 0.2 & 9.2 \\
\hline North Carolina & 55.3 & 15.4 & 17.3 & 8.0 & 5.2 \\
\hline North Dakota & 76.9 & 3.8 & 11.5 & - & 7.7 \\
\hline Ohio & 24.2 & 15.9 & 43.9 & 0.1 & 17.0 \\
\hline Oklahoma & 45.5 & 18.7 & 25.2 & 1.6 & 8.9 \\
\hline Oregon & 87.5 & 8.2 & 3.6 & - & 1.4 \\
\hline Pennsylvania & 10.6 & 4.7 & 76.0 & - & 9.2 \\
\hline Rhode Island & 79.7 & 16.9 & 3.4 & 1.7 & - \\
\hline South Carolina & 71.7 & 9.9 & 12.4 & 4.7 & 1.3 \\
\hline South Dakota & 36.4 & 45.5 & 9.1 & - & 9.1 \\
\hline Tennessee & 24.2 & 7.0 & 58.1 & - & 10.9 \\
\hline Texas & 82.2 & 9.8 & 5.5 & 0.4 & 3.3 \\
\hline Utah & 15.5 & 15.5 & 48.1 & - & 21.9 \\
\hline Vermont & 37.2 & 41.9 & 4.7 & 2.3 & 14.0 \\
\hline Virginia & 23.9 & 1.7 & 64.2 & 0.2 & 10.3 \\
\hline Washington & 87.4 & 5.0 & 6.0 & - & 2.2 \\
\hline West Virginia & 7.3 & 2.0 & 74.4 & 0.1 & 16.9 \\
\hline Wisconsin & 46.5 & 43.2 & 5.6 & 1.4 & 4.2 \\
\hline Wyoming & 20.7 & 1.1 & 44.6 & - & 33.7 \\
\hline TOTAL & 34.7 & 7.6 & 47.8 & 0.4 & 10.1 \\
\hline
\end{tabular}

- indicates no deaths listed.

NOTE: Percentages may total more than $100 \%$ due to deaths with multiple pneumoconioses. See appendices for source description, methods, and ICD codes. SOURCE: National Center for Health Statistics multiple cause of death data. 
Table 6-7. All pneumoconioses: Most frequently recorded industries on death certificate, U.S. residents age 15 and over, selected states and years, 1990-1999

\begin{tabular}{clrc}
\hline CIC & Industry & Number of Deaths & Percent \\
\hline 041 & Coal mining & 4,623 & 46.2 \\
060 & Construction & 1,078 & 10.8 \\
270 & Blast furnaces, steelworks, rolling and finishing mills & 203 & 2.0 \\
360 & Ship and boat building and repairing & 176 & 1.8 \\
392 & Not specified manufacturing industries & 170 & 1.7 \\
192 & Industrial and miscellaneous chemicals & 159 & 1.6 \\
400 & Railroads & 146 & 1.5 \\
040 & Metal mining & 131 & 1.3 \\
262 & Miscellaneous nonmetallic mineral and stone products & 127 & 1.3 \\
901 & General government, n.e.c. & 105 & 1.1 \\
& All other industries & 2,741 & 27.4 \\
& Industry not reported & 354 & 3.5 \\
& TOTAL & $\mathbf{1 0 , 0 1 3}$ & $\mathbf{1 0 0 . 0}$ \\
\hline
\end{tabular}

CIC - Census Industry Code

n.e.c. - not elsewhere classified

NOTE: Percentages may not total to $100 \%$ due to rounding. See appendices for source description, methods, and ICD codes, industry and occupation codes, and list of selected states and years.

SOURCE: National Center for Health Statistics multiple cause of death data.

Table 6-8. All pneumoconioses: Most frequently recorded occupations on death certificate, U.S. residents age 15 and over, selected states and years, 1990-1999

\begin{tabular}{clrc}
\hline COC & Occupation & Number of Deaths & Percent \\
\hline 616 & Mining machine operators & 4,299 & 42.9 \\
889 & Laborers, except construction & 389 & 3.9 \\
585 & Plumbers, pipefitters, and steamfitters & 257 & 2.6 \\
019 & Managers and administrators, n.e.c. & 248 & 2.5 \\
575 & Electricians & 220 & 2.2 \\
567 & Carpenters & 198 & 2.0 \\
453 & Janitors and cleaners & 179 & 1.8 \\
633 & Supervisors, production occupations & 167 & 1.7 \\
804 & Truck drivers & 163 & 1.6 \\
869 & Construction laborers & 142 & 1.4 \\
& All other occupations & 3,397 & 33.9 \\
& Occupation not reported & 354 & 3.5 \\
& TOTAL & $\mathbf{1 0 , 0 1 3}$ & $\mathbf{1 0 0 . 0}$ \\
\hline
\end{tabular}

COC - Census Occupation Code n.e.c. - not elsewhere classified

NOTE: Percentages may not total to $100 \%$ due to rounding. See appendices for source description, methods, and ICD codes, industry and occupation codes, and list of selected states and years.

SOURCE: National Center for Health Statistics multiple cause of death data. 
Table 6-9. All pneumoconioses: Proportionate mortality ratio (PMR) adjusted for age, sex, and race by usual industry, U.S. residents age 15 and over, selected states and years, 1990-1999

\begin{tabular}{llrrrr}
\hline & & & 95\% Confidence Interval \\
\cline { 4 - 6 } CIC & Industry & Number & Of Deaths & PMR & UCL \\
\hline & Coal mining & 4,623 & 33.13 & 32.19 & 34.10 \\
262 & Miscellaneous nonmetallic mineral and stone products & 127 & 7.86 & 6.58 & 9.39 \\
040 & Metal mining & 131 & 5.19 & 4.36 & 6.18 \\
360 & Ship and boat building and repairing & 176 & 4.48 & 3.86 & 5.21 \\
261 & Pottery and related products & 27 & 3.81 & 2.51 & 5.55 \\
050 & Nonmetallic mining and quarrying, except fuel & 69 & 3.58 & 2.81 & 4.57 \\
271 & Iron and steel foundries & 70 & 2.77 & 2.18 & 3.53 \\
361 & Railroad locomotives and equipment & 7 & 2.66 & 1.07 & 5.48 \\
252 & Structural clay products & 29 & 2.62 & 1.76 & 3.77 \\
502 & Lumber and construction materials & 23 & 2.26 & 1.43 & 3.40 \\
192 & Industrial and miscellaneous chemicals & 159 & 1.75 & 1.50 & 2.05 \\
211 & Other rubber products, and plastics footwear and belting & 48 & 1.45 & 1.07 & 1.93 \\
250 & Glass and glass products & 55 & 1.37 & 1.04 & 1.80 \\
\hline
\end{tabular}

CIC - Census Industry Code $\quad$ n.e.c. - not elsewhere classified $\quad$ LCL - lower confidence limit $\quad$ UCL - upper confidence limit

NOTE: See appendices for source description, methods, and ICD codes, industry and occupation codes, and list of selected states and years.

SOURCE: National Center for Health Statistics multiple cause of death data. 
Table 6-10. All pneumoconioses: Proportionate mortality ratio (PMR) adjusted for age, sex, and race by usual occupation, U.S. residents age 15 and over, selected states and years, 1990-1999

\begin{tabular}{|c|c|c|c|c|c|}
\hline \multirow[b]{2}{*}{$\mathrm{COC}$} & \multirow[b]{2}{*}{ Occupation } & \multirow{2}{*}{$\begin{array}{c}\text { Number } \\
\text { of Deaths }\end{array}$} & \multirow[b]{2}{*}{ PMR } & \multicolumn{2}{|c|}{ 95\% Confidence Interval } \\
\hline & & & & $\mathbf{L C L}$ & UCL \\
\hline 616 & Mining machine operators & 4,299 & 32.79 & 31.82 & 33.79 \\
\hline 593 & Insulation workers & 109 & 25.52 & 21.07 & 30.94 \\
\hline 613 & Supervisors, extractive occupations & 73 & 10.74 & 8.49 & 13.61 \\
\hline 725 & Miscellaneous metal and plastic processing machine operators & 11 & 10.47 & 5.23 & 18.72 \\
\hline 046 & Mining engineers & 16 & 6.11 & 3.49 & 9.93 \\
\hline 643 & Boilermakers & 61 & 5.91 & 4.57 & 7.67 \\
\hline 615 & Explosives workers & 6 & 5.03 & 1.84 & 10.96 \\
\hline 787 & Hand molding, casting, and forming occupations & 10 & 4.58 & 2.20 & 8.42 \\
\hline 768 & Crushing and grinding machine operators & 26 & 4.51 & 2.95 & 6.61 \\
\hline 617 & Mining occupations, n.e.c. & 26 & 4.16 & 2.72 & 6.11 \\
\hline 675 & Hand molders and shapers, except jewelers & 16 & 3.67 & 2.10 & 5.95 \\
\hline 585 & Plumbers, pipefitters, and steamfitters & 257 & 2.89 & 2.55 & 3.27 \\
\hline 719 & Molding and casting machine operators & 38 & 2.70 & 1.92 & 3.71 \\
\hline 843 & Supervisors, material moving equipment operators & 9 & 2.39 & 1.10 & 4.53 \\
\hline 859 & Miscellaneous material moving equipment operators & 23 & 2.17 & 1.37 & 3.26 \\
\hline 584 & Plasterers & 11 & 2.12 & 1.06 & 3.80 \\
\hline 575 & Electricians & 220 & 2.07 & 1.81 & 2.37 \\
\hline 653 & Sheet metal workers & 58 & 1.95 & 1.50 & 2.54 \\
\hline 544 & Millwrights & 52 & 1.81 & 1.37 & 2.39 \\
\hline 824 & Locomotive operating occupations & 36 & 1.63 & 1.14 & 2.25 \\
\hline 783 & Welders and cutters & 139 & 1.58 & 1.34 & 1.87 \\
\hline
\end{tabular}

COC - Census Occupation Code n.e.c. - not elsewhere classified $\quad$ LCL - lower confidence limit UCL - upper confidence limit NOTE: See appendices for source description, methods, and ICD codes, industry and occupation codes, and list of selected states and years. SOURCE: National Center for Health Statistics multiple cause of death data. 
Figure 6-3. All pneumoconioses: Age-adjusted mortality rates by county, U.S. residents age 15 and over, 1970-1999

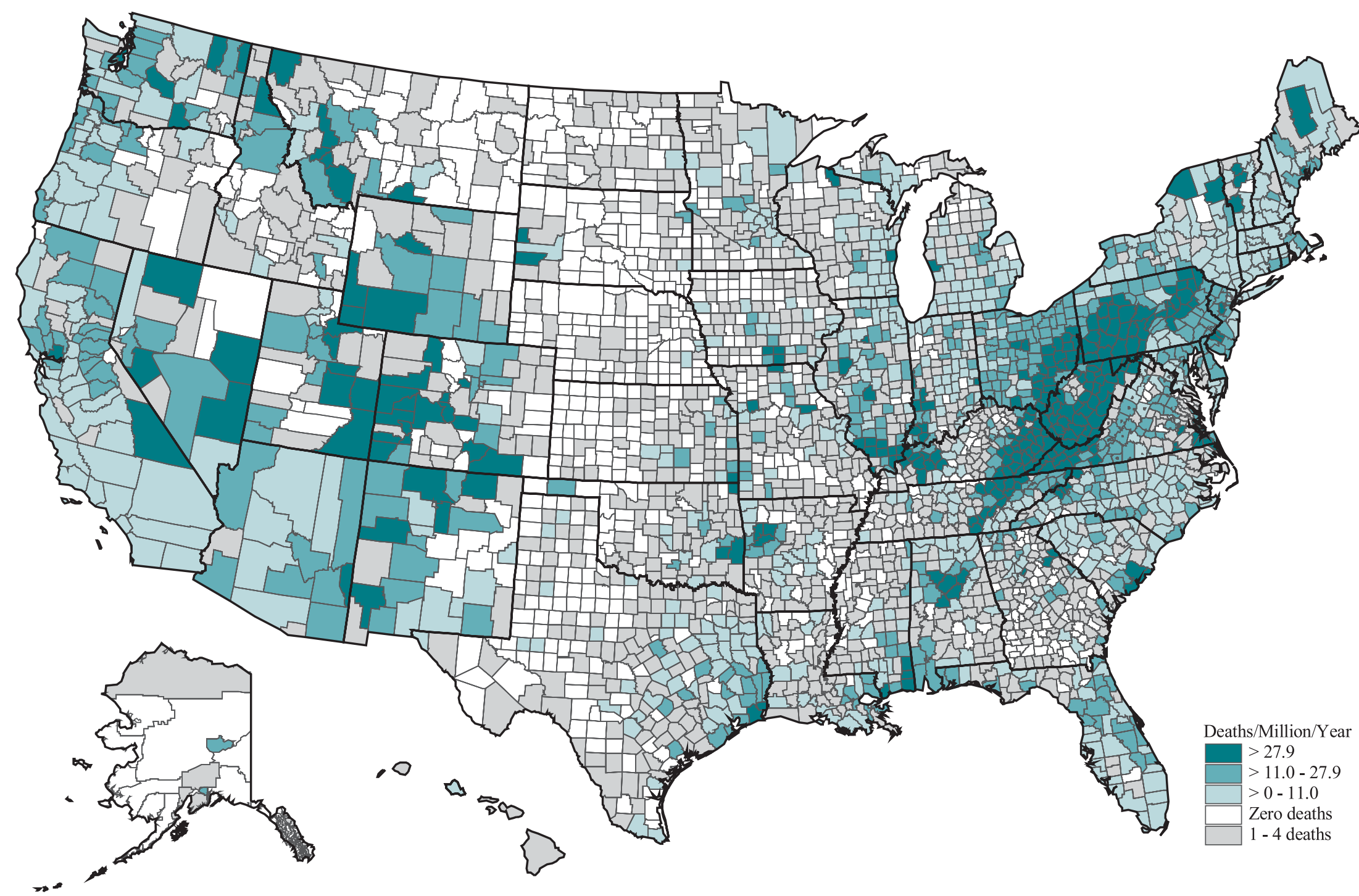

NOTE: Age-adjusted rates are not calculated for those counties with 1-4 deaths. See appendices for source description, methods, and ICD codes. SOURCE: National Center for Health Statistics multiple cause of death data. Population estimates from U.S. Bureau of the Census. 
Figure 6-4. All pneumoconioses: Age-adjusted mortality rates by county, U.S. residents age 15 and over, 1970-1984 and 1985-1999
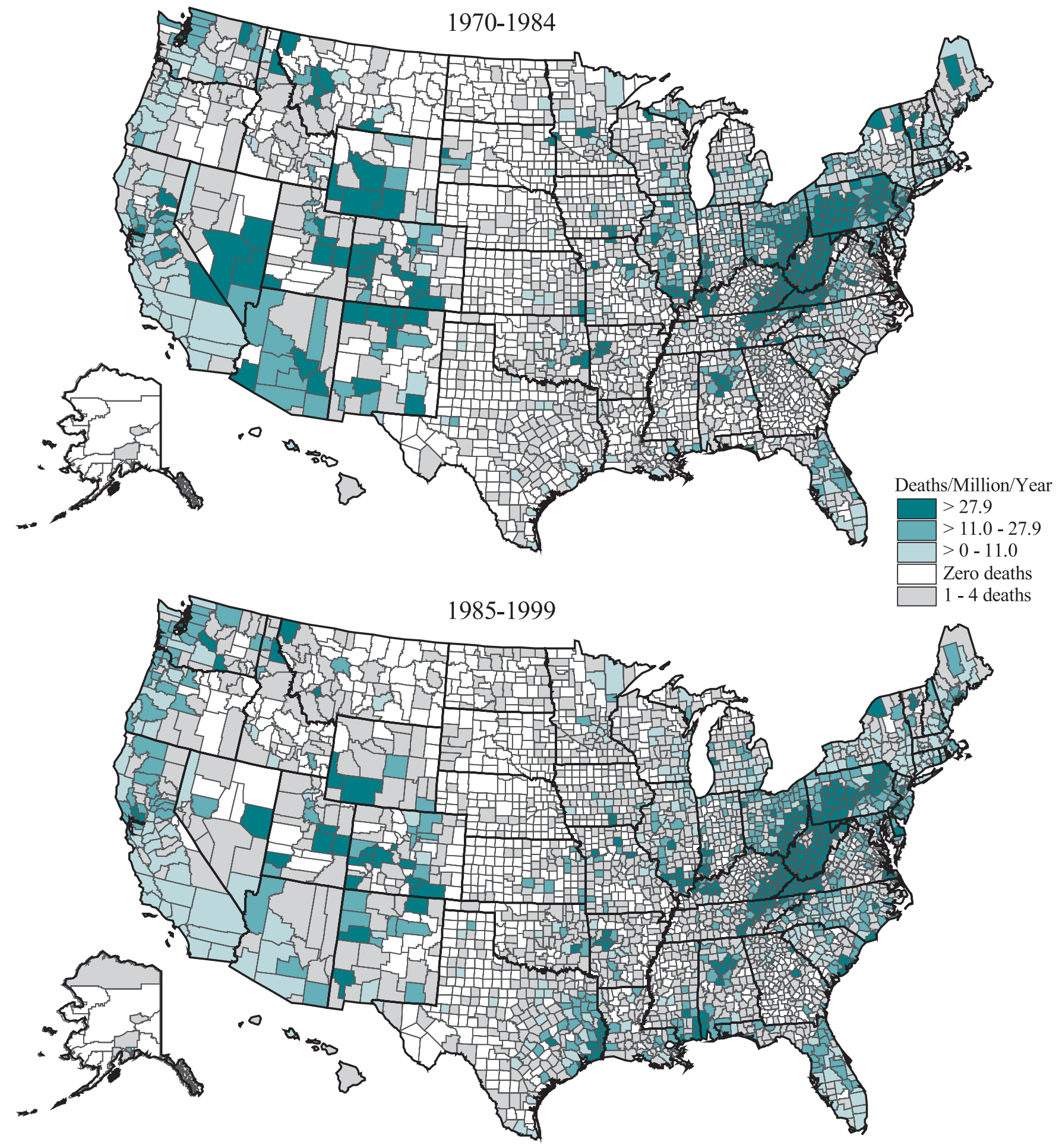

NOTE: Age-adjusted rates are not calculated for those counties with 1-4 deaths. See appendices for source description, methods, and ICD codes. SOURCE: National Center for Health Statistics multiple cause of death data. Population estimates from U.S. Bureau of the Census. 


\section{Table 6-11. All pneumoconioses: Counties with highest age-adjusted mortality rates (per million population), U.S. residents age 15 and over, 1985-1999}

\begin{tabular}{|c|c|c|c|c|c|}
\hline County & State & Age-Adjusted Rate & Crude Rate & Number of Deaths & \% Female \\
\hline Buchanan County & Virginia & $1,732.5$ & $1,227.6$ & 451 & 0.2 \\
\hline Raleigh County & West Virginia & $1,128.7$ & $1,281.6$ & 1,169 & 0.0 \\
\hline McDowell County & West Virginia & $1,127.8$ & $1,264.3$ & 495 & 0.2 \\
\hline Schuylkill County & Pennsylvania & $1,100.9$ & $1,535.8$ & 2,898 & 0.2 \\
\hline Wyoming County & West Virginia & $1,067.5$ & 903.5 & 303 & 0.0 \\
\hline Floyd County & Kentucky & 961.3 & 864.9 & 434 & 0.0 \\
\hline Dickenson County & Virginia & 884.3 & 853.2 & 177 & 0.6 \\
\hline Wise County & Virginia & 864.1 & 868.3 & 406 & 0.2 \\
\hline Tazewell County & Virginia & 846.9 & 869.4 & 485 & 0.4 \\
\hline Norton City & Virginia & 841.7 & 814.7 & 41 & 0.0 \\
\hline Boone County & West Virginia & 774.8 & 734.3 & 228 & 0.4 \\
\hline Luzerne County & Pennsylvania & 684.1 & 960.7 & 3,896 & 0.4 \\
\hline Fayette County & West Virginia & 669.8 & 847.3 & 485 & 0.2 \\
\hline Logan County & West Virginia & 658.1 & 635.9 & 317 & 0.3 \\
\hline Harlan County & Kentucky & 648.7 & 642.4 & 267 & 0.0 \\
\hline Carbon County & Utah & 595.2 & 644.1 & 139 & 0.0 \\
\hline Letcher County & Kentucky & 590.7 & 553.9 & 171 & 1.2 \\
\hline Russell County & Virginia & 548.2 & 514.7 & 177 & 0.0 \\
\hline Cambria County & Pennsylvania & 494.1 & 653.2 & 1,285 & 0.2 \\
\hline Northumberland County & Pennsylvania & 478.7 & 669.0 & 777 & 0.3 \\
\hline Mingo County & West Virginia & 478.5 & 397.1 & 149 & 0.0 \\
\hline Bell County & Kentucky & 436.8 & 436.8 & 157 & 0.0 \\
\hline Somerset County & Pennsylvania & 426.9 & 522.4 & 488 & 0.2 \\
\hline Webster County & West Virginia & 402.3 & 482.9 & 59 & 0.0 \\
\hline Knott County & Kentucky & 401.9 & 323.8 & 67 & 0.0 \\
\hline Lee County & Virginia & 393.6 & 462.0 & 133 & 0.8 \\
\hline Nicholas County & West Virginia & 391.3 & 421.6 & 132 & 0.8 \\
\hline Carbon County & Pennsylvania & 386.5 & 508.4 & 358 & 0.6 \\
\hline Mercer County & West Virginia & 352.1 & 432.5 & 339 & 0.3 \\
\hline Fayette County & Pennsylvania & 348.2 & 443.0 & 776 & 0.0 \\
\hline Franklin County & Illinois & 343.7 & 503.3 & 243 & 0.0 \\
\hline Emery County & Utah & 339.0 & 267.7 & 26 & 0.0 \\
\hline Lackawanna County & Pennsylvania & 336.4 & 470.5 & 1,255 & 0.2 \\
\hline Muhlenberg County & Kentucky & 327.6 & 374.2 & 138 & 0.0 \\
\hline Pike County & Kentucky & 318.3 & 263.6 & 223 & 0.4 \\
\hline Johnson County & Kentucky & 306.1 & 259.2 & 71 & 0.0 \\
\hline Leslie County & Kentucky & 302.9 & 240.6 & 37 & 0.0 \\
\hline Sweetwater County & Wyoming & 277.0 & 158.0 & 68 & 0.0 \\
\hline Greene County & Pennsylvania & 274.6 & 343.1 & 161 & 0.0 \\
\hline Knox County & Kentucky & 265.5 & 270.0 & 94 & 1.1 \\
\hline Indiana County & Pennsylvania & 249.8 & 250.8 & 273 & 0.0 \\
\hline Clay County & West Virginia & 239.0 & 256.9 & 29 & 3.4 \\
\hline Martin County & Kentucky & 231.7 & 177.6 & 25 & 0.0 \\
\hline Preston County & West Virginia & 229.0 & 251.8 & 86 & 0.0 \\
\hline Las Animas County & Colorado & 227.0 & 365.6 & 59 & 1.7 \\
\hline Perry County & Kentucky & 223.4 & 188.9 & 67 & 0.0 \\
\hline Campbell County & Tennessee & 220.5 & 236.8 & 100 & 1.0 \\
\hline Greenbrier County & West Virginia & 216.3 & 275.0 & 117 & 0.0 \\
\hline Marion County & West Virginia & 213.6 & 284.4 & 200 & 0.5 \\
\hline Whitley County & Kentucky & 210.7 & 212.4 & 84 & 0.0 \\
\hline Overall United States & & 17.4 & 16.7 & 49,740 & 2.2 \\
\hline
\end{tabular}

NOTE: Only counties with at least 5 deaths from the disease of interest are included. See appendices for source description, methods, and ICD codes. SOURCE: National Center for Health Statistics multiple cause of death data. Population estimates from U.S. Bureau of the Census. 
Table 6-12. Occupational dust diseases of the lungs: Estimated number of cases reported by employers, by industry division, U.S. private sector, 1973-2000

\begin{tabular}{|c|c|c|c|c|c|c|c|c|c|}
\hline Year & Agriculture & Mining & Construction & Manufacturing & $\begin{array}{c}\text { Transportation } \\
\text { \& Public } \\
\text { Utilities }\end{array}$ & $\begin{array}{c}\text { Wholesale } \\
\text { \& Retail } \\
\text { Trade }\end{array}$ & Finance & Services & Total \\
\hline 1973 & 100 & - & 100 & 700 & 200 & 200 & - & 100 & 1,500 \\
\hline 1974 & 100 & 300 & 100 & 900 & - & 300 & - & 100 & 1,700 \\
\hline 1975 & - & - & 200 & 600 & - & 100 & - & - & 1,000 \\
\hline 1976 & - & - & 200 & 800 & 100 & - & - & - & 1,200 \\
\hline 1977 & 100 & 200 & 800 & 700 & 100 & 100 & 100 & 100 & 2,000 \\
\hline 1978 & - & 300 & 200 & 800 & 100 & 200 & - & 100 & 1,600 \\
\hline 1979 & - & 300 & 200 & 900 & 100 & 100 & - & 100 & 1,700 \\
\hline 1980 & - & 300 & 200 & 1,300 & 100 & 100 & - & 200 & 2,300 \\
\hline 1981 & - & 300 & 200 & 1,500 & - & - & - & 100 & 2,100 \\
\hline 1982 & - & 300 & 100 & 1,200 & 100 & 100 & - & 100 & 2,000 \\
\hline 1983 & - & 200 & 100 & 900 & - & 200 & - & 200 & 1,700 \\
\hline 1984 & - & 200 & 200 & 1,000 & 100 & 100 & - & 100 & 1,800 \\
\hline 1985 & - & 200 & 100 & 800 & 100 & 200 & - & 200 & 1,700 \\
\hline 1986 & 100 & 600 & 100 & - & - & - & 100 & 300 & 3,200 \\
\hline 1987 & - & 900 & 500 & 1,200 & 200 & - & - & 400 & 3,400 \\
\hline 1988 & - & 700 & 200 & 1,200 & 300 & - & - & 300 & 2,900 \\
\hline 1989 & - & 500 & 200 & 1,300 & 100 & 100 & - & 200 & 2,600 \\
\hline 1990 & 100 & 300 & 300 & 1,600 & 400 & 100 & - & 300 & 3,000 \\
\hline 1991 & 100 & 500 & 200 & 1,000 & 200 & - & - & 300 & 2,500 \\
\hline 1992 & - & 600 & 100 & 1,000 & - & 200 & - & 500 & 2,600 \\
\hline 1993 & 100 & 600 & 200 & 900 & 300 & - & - & 600 & 2,700 \\
\hline 1994 & - & 400 & 200 & 900 & 300 & 300 & - & 500 & 2,700 \\
\hline 1995 & - & 200 & 100 & 700 & 200 & 200 & - & 1,100 & 2,700 \\
\hline 1996 & - & 100 & 100 & 2,600 & 200 & 100 & - & 400 & 3,500 \\
\hline 1997 & - & 100 & 300 & 900 & 300 & 200 & 300 & 800 & 2,900 \\
\hline 1998 & - & 300 & 200 & 800 & 300 & 200 & - & 400 & 2,100 \\
\hline 1999 & - & 100 & 100 & 900 & 300 & 100 & 100 & 500 & 2,200 \\
\hline 2000 & - & 100 & 100 & 500 & 200 & 300 & 100 & 400 & 1,700 \\
\hline
\end{tabular}

- indicates data do not meet BLS publication guidelines.

NOTE: Dust diseases of the lungs (pneumoconioses) include silicosis, asbestosis, coal workers' pneumoconiosis, byssinosis, siderosis, and other pneumoconioses. The sum of industry divisions may not equal the total due to rounding. See appendices for source description.

SOURCE: Bureau of Labor Statistics annual reports of occupational injuries and illnesses. 
Table 6-13. Occupational dust diseases of the lungs: Estimated rate (based on cases reported by employers, per 10,000 full-time workers) by industry division, U.S. private sector, 1973-2000

\begin{tabular}{|c|c|c|c|c|c|c|c|c|c|}
\hline Year & Agriculture & Mining & Construction & Manufacturing & $\begin{array}{c}\text { Transportation } \\
\text { \& Public } \\
\text { Utilities } \\
\end{array}$ & $\begin{array}{c}\text { Wholesale } \\
\text { \& Retail } \\
\text { Trade } \\
\end{array}$ & Finance & Services & Overall \\
\hline 1973 & 1.3 & 0.5 & 0.4 & 0.4 & 0.3 & 0.2 & 0.1 & 0.1 & 0.3 \\
\hline 1974 & 0.8 & 4.8 & 0.3 & 0.4 & - & 0.2 & 0.0 & 0.1 & 0.3 \\
\hline 1975 & 0.4 & 0.2 & 0.6 & 0.4 & 0.1 & - & - & - & 0.2 \\
\hline 1976 & 0.2 & 0.1 & 0.5 & 0.4 & 0.2 & - & - & - & 0.2 \\
\hline 1977 & 1.3 & 2.0 & 2.5 & 0.4 & 0.1 & - & 0.1 & 0.1 & 0.3 \\
\hline 1978 & 0.3 & 4.0 & 0.6 & 0.4 & 0.1 & 0.1 & - & - & 0.3 \\
\hline 1979 & 0.1 & 3.4 & 0.5 & 0.4 & 0.1 & 0.1 & - & 0.1 & 0.3 \\
\hline 1980 & 0.4 & 3.3 & 0.6 & 0.7 & 0.1 & 0.1 & - & 0.1 & 0.4 \\
\hline 1981 & 0.3 & 2.5 & 0.5 & 0.8 & 0.1 & - & - & 0.1 & 0.3 \\
\hline 1982 & 0.4 & 3.2 & 0.3 & 0.7 & 0.2 & 0.1 & - & 0.1 & 0.3 \\
\hline 1983 & 0.4 & 1.9 & 0.4 & 0.5 & 0.1 & 0.1 & - & 0.1 & 0.3 \\
\hline 1984 & 0.4 & 1.7 & 0.5 & 0.5 & 0.2 & 0.1 & - & 0.1 & 0.3 \\
\hline 1985 & 0.5 & 2.7 & 0.3 & 0.4 & 0.2 & 0.1 & - & 0.1 & 0.2 \\
\hline 1986 & 1.0 & 8.4 & 0.3 & 0.9 & - & - & - & 0.1 & 0.5 \\
\hline 1987 & 0.5 & 12.9 & 1.2 & 0.6 & 0.3 & - & - & 0.2 & 0.5 \\
\hline 1988 & - & 10.2 & 0.5 & 0.6 & 0.6 & - & - & 0.1 & 0.4 \\
\hline 1989 & 0.2 & 7.5 & 0.5 & 0.7 & 0.2 & - & - & 0.1 & 0.3 \\
\hline 1990 & 0.6 & 4.4 & 0.6 & 0.9 & 0.7 & - & - & 0.1 & 0.4 \\
\hline 1991 & 0.5 & 7.3 & 0.4 & 0.5 & 0.3 & - & - & 0.1 & 0.3 \\
\hline 1992 & 0.2 & 9.0 & 0.3 & 0.6 & - & 0.1 & - & 0.2 & 0.3 \\
\hline 1993 & 0.5 & 8.7 & 0.4 & 0.5 & 0.5 & - & - & 0.3 & 0.3 \\
\hline 1994 & 0.3 & 6.6 & 0.5 & 0.5 & 0.5 & 0.1 & 0.1 & 0.2 & 0.3 \\
\hline 1995 & 0.1 & 3.4 & 0.3 & 0.4 & 0.4 & 0.1 & 0.1 & 0.5 & 0.3 \\
\hline 1996 & 0.1 & 1.6 & 0.1 & 1.4 & 0.3 & - & 0.1 & 0.2 & 0.4 \\
\hline 1997 & 0.3 & 1.7 & 0.5 & 0.5 & 0.5 & 0.1 & 0.5 & 0.3 & 0.3 \\
\hline 1998 & 0.2 & 4.3 & 0.3 & 0.4 & 0.4 & 0.1 & 0.1 & 0.2 & 0.2 \\
\hline 1999 & 0.2 & 2.2 & 0.1 & 0.5 & 0.5 & 0.1 & 0.1 & 0.2 & 0.2 \\
\hline 2000 & 0.2 & 1.7 & 0.1 & 0.3 & 0.3 & 0.1 & 0.1 & 0.2 & 0.2 \\
\hline
\end{tabular}

- indicates no data reported or data that do not meet BLS publication guidelines.

NOTE: Dust diseases of the lungs (pneumoconioses) include silicosis, asbestosis, coal workers' pneumoconiosis, byssinosis, siderosis, and other pneumoconioses. See appendices for source description.

SOURCE: Bureau of Labor Statistics annual reports of occupational injuries and illnesses. 
Table 6-14 (page 1 of 2). Occupational dust diseases of the lungs: Industries with highest estimated incidence rates (based on cases reported by employers, per 10,000 full-time workers) by industry division, U.S. private sector, 1996-2000

\begin{tabular}{|c|c|c|c|}
\hline Year/Industry & SIC & $\begin{array}{c}\text { Estimated } \\
\text { Number of } \\
\text { Cases }\end{array}$ & $\begin{array}{c}\text { Rate } \\
\text { (per 10,000 full- } \\
\text { time workers) }\end{array}$ \\
\hline \multicolumn{4}{|l|}{1996} \\
\hline$\overline{\text { Coal mining }}$ & 12 & 100 & 8.6 \\
\hline Primary metal industries & 33 & 200 & 2.2 \\
\hline Local and interurban passenger transit & 41 & 100 & 1.7 \\
\hline Chemical and allied products & 28 & 100 & 1.1 \\
\hline Transportation equipment & 37 & 200 & 1.0 \\
\hline Electric, gas, and sanitary services & 49 & 100 & 0.9 \\
\hline Lumber and wood products & 24 & 100 & 0.9 \\
\hline Nonmetallic minerals, except fuels & 14 & - & 0.9 \\
\hline Stone, clay, and glass products & 32 & - & 0.9 \\
\hline Transportation by air & 45 & 100 & 0.6 \\
\hline ALL INDUSTRIES & & 3,500 & 0.4 \\
\hline \multicolumn{4}{|l|}{1997} \\
\hline$\overline{\text { Coal mining }}$ & 12 & 100 & 9.5 \\
\hline Fishing, hunting, and trapping & 09 & - & 7.8 \\
\hline Local and interurban passenger transit & 41 & 100 & 3.7 \\
\hline Primary metal products & 33 & 100 & 1.8 \\
\hline Insurance carriers & 63 & 200 & 1.4 \\
\hline Metal mining & 10 & - & 0.9 \\
\hline Nonmetallic minerals, except fuels & 14 & - & 0.9 \\
\hline General building contractors & 15 & 100 & 0.9 \\
\hline Fabricated metal products & 34 & 100 & 0.9 \\
\hline Electric, gas, and sanitary services & 49 & 100 & 0.9 \\
\hline ALL INDUSTRIES & & 2,900 & 0.3 \\
\hline \multicolumn{4}{|l|}{$\underline{1998}$} \\
\hline$\overline{\text { Coal mining }}$ & 12 & 200 & 25.3 \\
\hline Museums, botanical, zoological gardens & 84 & - & 4.7 \\
\hline Nonmetallic minerals, except fuels & 14 & - & 2.3 \\
\hline Electric, gas, and sanitary services & 49 & 200 & 2.3 \\
\hline Primary metal industries & 33 & 100 & 2.0 \\
\hline Transportation equipment & 37 & 300 & 1.4 \\
\hline Agricultural production livestock & 02 & - & 0.9 \\
\hline Local and interurban passenger transit & 41 & - & 0.9 \\
\hline Stone, clay, and glass products & 32 & - & 0.8 \\
\hline Leather and leather products & 31 & - & 0.8 \\
\hline ALL INDUSTRIES & & 2,100 & 0.2 \\
\hline
\end{tabular}

See footnotes at end of table. 
Table 6-14 (page 2 of 2). Occupational dust diseases of the lungs: Industries with highest estimated incidence rates (based on cases reported by employers, per 10,000 full-time workers) by industry division, U.S. private sector, 1996-2000

\begin{tabular}{lccc}
\hline Year/Industry & SIC & $\begin{array}{c}\text { Estimated } \\
\text { Number of } \\
\text { Cases }\end{array}$ & $\begin{array}{c}\text { Rate } \\
\text { (per 10,000 full- } \\
\text { time workers) }\end{array}$ \\
\hline $\mathbf{1 9 9 9}$ & 12 & 100 & 13.0 \\
Coal mining & 33 & 200 & 3.2 \\
Primary metal industries & 44 & - & 1.9 \\
Water transportation & 49 & 100 & 1.7 \\
Electric, gas, and sanitary services & 28 & 200 & 1.7 \\
Chemicals and allied products & 81 & 200 & 1.7 \\
Legal services & 31 & - & 1.2 \\
Leather and leather products & 37 & 200 & 1.0 \\
Transportation equipment & 22 & - & 0.7 \\
Textile mill products & 42 & 100 & 0.7 \\
Trucking and warehousing & & $\mathbf{2 , 2 0 0}$ & $\mathbf{0 . 2}$ \\
ALL INDUSTRIES & & & 10.4 \\
2000 & & & 10.2 \\
Coal mining & 12 & 100 & 1.2 \\
Forestry & 08 & - & 1.2 \\
Metal mining & 10 & - & 1.2 \\
Tobacco products & 21 & - & 0.9 \\
Water transportation & 44 & - & 0.8 \\
Nonmetallic minerals, except fuels & 14 & - & 0.6 \\
Electric, gas, and sanitary services & 49 & 100 & 0.5 \\
Primary metal industries & 33 & - & $\mathbf{0 . 2}$ \\
Transportation equipment & 37 & 100 & - \\
Petroleum and coal products & 29 & $\mathbf{1 , 7 0 0}$ & \\
ALL INDUSTRIES & & & \\
\hline
\end{tabular}

- indicates no data reported or data that do not meet BLS publication guidelines.

SIC - 1987 Standard Industial Classification NOTE: Dust diseases of the lungs (pneumoconioses) include silicosis, asbestosis, coal workers' pneumoconiosis, byssinosis, siderosis, and other pneumoconioses. See appendices for source description.

SOURCE: Bureau of Labor Statistics annual reports of occupational injuries and illnesses. 
Figure 6-5. Pneumoconiotic agents: Percent of exposures exceeding the NIOSH recommended exposure limits by major industry division, MSHA inspector and mine operator and OSHA samples, 1979-1999

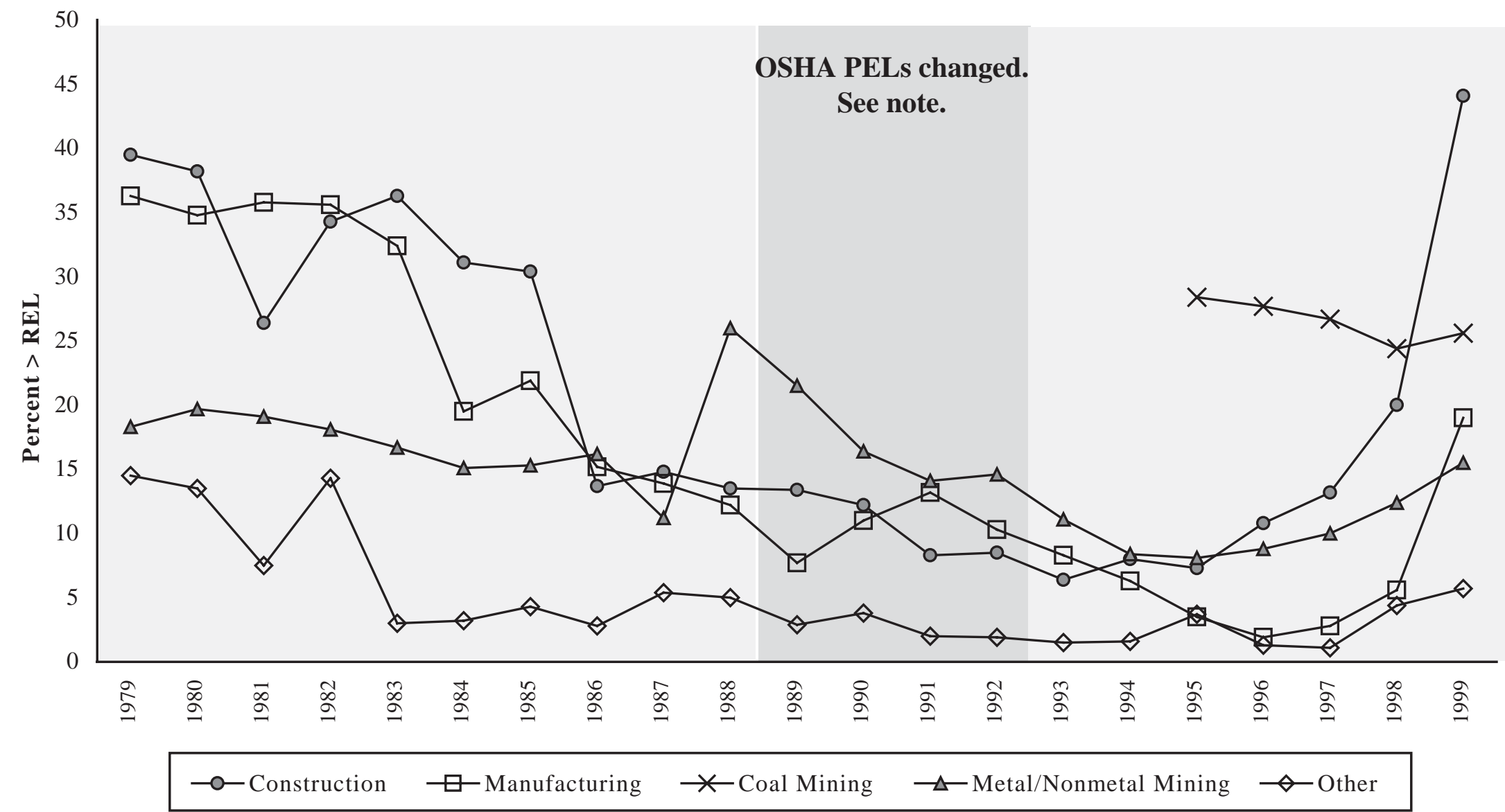

REL - recommended exposure limit

NOTE: From March 1, 1989 to March 22, 1993, the OSHA PELs in force differed from those employed before and after those dates. The NIOSH REL for respirable coal mine dust of 1.0 $\mathrm{mg} / \mathrm{m}^{3}$ was adopted in September 1995. The MSHA respirable coal mine quartz exposure data and the NIOSH REL for respirable quartz cannot be compared to each other because they are based on different sampling criteria. See appendices for source description, methods, and agents.

SOURCE: Mine Safety and Health Administration (MSHA) metal/nonmetal mine and coal mine inspector and mine operator dust and quartz data. Occupational Safety and Health Administation (OSHA) Integrated Management Information System. 
Table 6-15a. Pneumoconiotic agents: Percent of exposures exceeding designated occupational exposure limits by type of mine, MSHA inspector and mine operator samples, 1979-1999

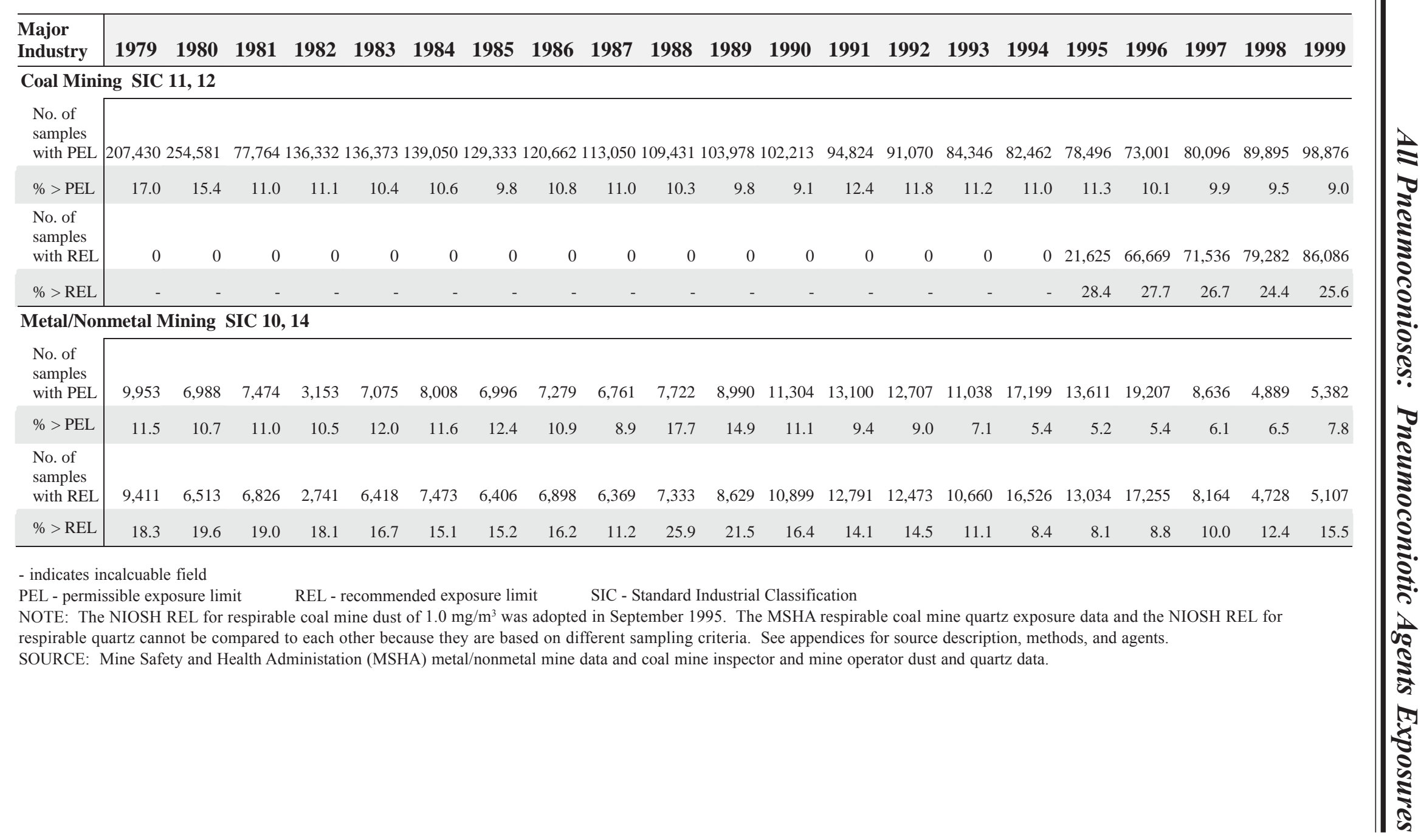


Table 6-15b. Pneumoconiotic agents: Percent of exposures exceeding designated occupational exposure limits by major industry division, OSHA samples, 1979-1999

\begin{tabular}{|c|c|c|c|c|c|c|c|c|c|c|c|c|c|c|c|c|c|c|c|c|c|}
\hline \multirow{2}{*}{$\begin{array}{l}\text { Major } \\
\text { Industry }\end{array}$} & \multirow[b]{2}{*}{1979} & \multirow[b]{2}{*}{1980} & \multirow[b]{2}{*}{1981} & \multirow[b]{2}{*}{1982} & \multirow[b]{2}{*}{1983} & \multirow[b]{2}{*}{1984} & \multirow[b]{2}{*}{1985} & \multirow[b]{2}{*}{1986} & \multirow[b]{2}{*}{1987} & \multirow[b]{2}{*}{1988} & \multicolumn{4}{|c|}{$\begin{array}{c}\text { OSHA PELs changed. } \\
\text { See note. }\end{array}$} & \multirow[b]{2}{*}{1993} & \multirow[b]{2}{*}{1994} & \multirow[b]{2}{*}{1995} & \multirow[b]{2}{*}{1996} & \multirow[b]{2}{*}{1997} & \multirow[b]{2}{*}{1998} & \multirow[b]{2}{*}{1999} \\
\hline & & & & & & & & & & & 1989 & 1990 & 1991 & 1992 & & & & & & & \\
\hline \multicolumn{22}{|c|}{ Construction SIC 15-17 } \\
\hline $\begin{array}{l}\text { No. of } \\
\text { samples } \\
\text { with PEL }\end{array}$ & 132 & 309 & 194 & 218 & 599 & 796 & 658 & 435 & 396 & 435 & 405 & 523 & 526 & 655 & 521 & 296 & 195 & 433 & 498 & 368 & 294 \\
\hline$\%>$ PEL & 11.4 & 16.2 & 6.2 & 11.0 & 7.0 & 7.4 & 9.1 & 5.1 & 8.3 & 9.2 & 10.9 & 8.6 & 7.2 & 5.5 & 4.8 & 7.1 & 10.8 & 13.2 & 8.4 & 22.8 & 29.6 \\
\hline $\begin{array}{l}\text { No. of } \\
\text { samples } \\
\text { with REL }\end{array}$ & 124 & 262 & 144 & 204 & 498 & 731 & 596 & 357 & 337 & 363 & 328 & 362 & 374 & 472 & 359 & 250 & 165 & 334 & 395 & 454 & 238 \\
\hline$\%>$ REL & 39.5 & 38.2 & 26.4 & 34.3 & 36.3 & 31.1 & 30.4 & 13.7 & 14.8 & 13.5 & 13.4 & 12.2 & 8.3 & 8.5 & 6.4 & 7.6 & 7.3 & 10.8 & 12.9 & 19.8 & 43.3 \\
\hline \multicolumn{22}{|c|}{ Manufacturing SIC 20-39 } \\
\hline $\begin{array}{l}\text { No. of } \\
\text { samples } \\
\text { with PEL }\end{array}$ & 1,944 & 3,212 & 3,194 & 3,665 & 3,192 & 4,768 & 5,678 & 4,391 & 5,512 & 5,682 & 4,827 & 5,664 & 4,981 & 4,788 & 3,686 & 2,998 & 3,612 & 3,558 & 4,087 & 2,893 & 2,721 \\
\hline$\%>$ PEL & 15.0 & 16.1 & 16.2 & 14.5 & 13.3 & 7.9 & 10.0 & 6.4 & 6.8 & 5.7 & 5.8 & 7.1 & 8.1 & 6.6 & 5.4 & 4.1 & 3.2 & 2.6 & 3.7 & 9.1 & 9.9 \\
\hline $\begin{array}{l}\text { No. of } \\
\text { samples } \\
\text { with REL }\end{array}$ & 1,395 & 2,322 & 1,959 & 2,189 & 1,962 & 3,005 & 3,455 & 2,630 & 3,346 & 3,262 & 2,644 & 3,099 & 2,809 & 2,761 & 2,447 & 2,713 & 3,947 & 5,903 & 6,510 & 6,090 & 1,974 \\
\hline$\%>$ REL & 36.3 & 34.8 & 35.8 & 35.6 & 32.4 & 19.5 & 21.7 & 15.1 & 13.9 & 12.2 & 7.7 & 11.0 & 13.2 & 10.3 & 8.3 & 6.3 & 3.5 & 1.9 & 2.7 & 5.6 & 18.9 \\
\hline \multicolumn{22}{|c|}{ Other SIC 1-9, 13, 40-99 } \\
\hline $\begin{array}{l}\text { No. of } \\
\text { samples } \\
\text { with PEL }\end{array}$ & 219 & 239 & 144 & 92 & 591 & 1,188 & 962 & 1,023 & 1,168 & 1,068 & 1,104 & 1,089 & 1,056 & 769 & 657 & 615 & 656 & 773 & 663 & 340 & 260 \\
\hline$\%>$ PEL & 3.7 & 4.6 & 5.6 & 4.3 & 1.2 & 0.9 & 1.7 & 2.0 & 4.3 & 3.5 & 2.7 & 3.7 & 1.4 & 1.7 & 0.6 & 2.6 & 3.5 & 1.3 & 1.2 & 3.2 & 4.2 \\
\hline $\begin{array}{l}\text { No. of } \\
\text { samples } \\
\text { with REL }\end{array}$ & 138 & 185 & 93 & 77 & 530 & 1,033 & 870 & 798 & 1,040 & 818 & 899 & 783 & 793 & 577 & 538 & 613 & 794 & 798 & 833 & 362 & 209 \\
\hline$\%>\mathrm{REL}$ & 14.5 & 13.5 & 7.5 & 14.3 & 3.0 & 3.2 & 4.3 & 2.8 & 5.4 & 5.0 & 2.9 & 3.8 & 2.0 & 1.9 & 1.5 & 1.6 & 3.7 & 1.3 & 1.1 & 4.4 & 5.7 \\
\hline
\end{tabular}

PEL - permissible exposure limit $\quad$ REL - recommended exposure limit $\quad$ SIC - Standard Industrial Classification

NOTE: From March 1, 1989 to March 22, 1993, the OSHA PELs in force differed from those employed before and after those dates. See appendices for source description, methods, and agents.

SOURCE: Occupational Safety and Health Administation (OSHA) Integrated Management Information System. 
Table 6-16. Pneumoconiotic agents: Number of samples and percent of exposures exceeding designated occupational exposure limits by industries with elevated pneumoconiosis mortality, MSHA inspector and mine operator and OSHA samples, 1990-1999

\begin{tabular}{|c|c|c|c|c|c|c|c|}
\hline \multicolumn{4}{|c|}{$\begin{array}{l}\text { All Pneumoconioses Mortality, } \\
\text { Selected States and Years, 1990-1999 }\end{array}$} & \multirow[b]{2}{*}{$\begin{array}{l}\text { No. of } \\
\text { Samples } \\
\text { with PEL }\end{array}$} & \multirow[b]{2}{*}{$\begin{array}{l}\%> \\
\text { PEL }\end{array}$} & \multirow[b]{2}{*}{$\begin{array}{l}\text { No. of } \\
\text { Samples } \\
\text { with REL }\end{array}$} & \multirow[b]{2}{*}{$\begin{array}{l}\%> \\
\text { REL }\end{array}$} \\
\hline CIC & $\begin{array}{l}\text { Industries with elevated PMRs and most } \\
\text { frequently recorded on death certificates }\end{array}$ & $\begin{array}{l}\text { Number of } \\
\text { Deaths }\end{array}$ & PMR & & & & \\
\hline 041 & Coal mining & 4,623 & 33.13 & 875,279 & 10.5 & 325,198 & $26.2^{*}$ \\
\hline 262 & $\begin{array}{l}\text { Miscellaneous nonmetallic mineral and } \\
\text { stone products }\end{array}$ & 127 & 7.86 & 709 & 22.4 & 643 & 37.3 \\
\hline 040 & Metal mining & 131 & 5.19 & 13,439 & 9.0 & 10,362 & 16.1 \\
\hline 360 & Ship and boat building and repairing & 176 & 4.48 & 540 & 8.7 & 393 & 3.6 \\
\hline 050 & $\begin{array}{l}\text { Nonmetallic mining and quarrying, except } \\
\text { fuel }\end{array}$ & 69 & 3.58 & 103,665 & 6.9 & 101,292 & 10.9 \\
\hline 271 & Iron and steel foundries & 70 & 2.77 & 4,022 & 14.6 & 3,348 & 27.3 \\
\hline 252 & Structural clay products & 29 & 2.62 & 314 & 22.3 & 307 & 35.8 \\
\hline 192 & Industrial and miscellaneous chemicals & 159 & 1.75 & 541 & 7.0 & 413 & 11.1 \\
\hline 211 & $\begin{array}{l}\text { Other rubber products, and plastics } \\
\text { footwear and belting }\end{array}$ & 48 & 1.45 & 245 & 8.2 & 310 & 7.7 \\
\hline 250 & Glass and glass products & 55 & 1.37 & 348 & 0.3 & 289 & 2.4 \\
\hline & All other industries & 2,741 & & 43,383 & 4.6 & 42,171 & 4.2 \\
\hline & TOTAL & & & $1,042,485$ & 9.9 & 484,726 & 20.8 \\
\hline
\end{tabular}

CIC - Census Industry Code PEL - permissible exposure limit REL - recommended exposure limit PMR - proportionate mortality ratio *This number is based solely on the the NIOSH REL for respirable coal mine dust of $1.0 \mathrm{mg} / \mathrm{m}^{3}$ which was adopted in September 1995 . The MSHA respirable coal mine quartz exposure data and the NIOSH REL for respirable quartz cannot be compared to each other because they are based on different sampling criteria.

NOTE: See appendices for source description, methods, ICD codes, industry codes, agents, and list of selected states (and years) for which usual industry has been reported.

SOURCE: Mine Safety and Health Administration (MSHA) coal mine inspector and mine operator dust and quartz data and metal/nonmetal data. Occupational Safety and Health Administration (OSHA) Integrated Management Information System. National Center for Health Statistics multiple cause of death data. 
Table 6-17 (page 1 of 2). Pneumoconiotic agents: Percent of exposures exceeding designated occupational exposure limits by MSHA coal mine district and states, MSHA inspector and mine operator samples, 1979-1999

\begin{tabular}{|c|c|c|c|c|c|c|c|c|c|c|c|c|}
\hline \multirow[b]{2}{*}{ MSHA Coal Mine District } & \multicolumn{4}{|c|}{ All Years } & \multicolumn{2}{|c|}{1979 - 1988} & \multicolumn{2}{|c|}{1989 - 1992} & \multicolumn{4}{|c|}{1993 - 1999} \\
\hline & $\begin{array}{c}\text { No. of } \\
\text { Samples } \\
\text { with PEL } \\
\end{array}$ & $\begin{array}{l}\%> \\
\text { PEL }\end{array}$ & $\begin{array}{c}\text { No. of } \\
\text { Samples } \\
\text { with REL } \\
\end{array}$ & $\begin{array}{l}\%> \\
\text { REL }\end{array}$ & $\begin{array}{c}\text { No. of } \\
\text { Samples } \\
\text { with PEL }\end{array}$ & $\begin{array}{l}\%> \\
\text { PEL }\end{array}$ & $\begin{array}{c}\text { No. of } \\
\text { Samples } \\
\text { with PEL }\end{array}$ & $\begin{array}{l}\%> \\
\text { PEL }\end{array}$ & $\begin{array}{c}\text { No. of } \\
\text { Samples } \\
\text { with PEL }\end{array}$ & $\begin{array}{l}\%> \\
\text { PEL }\end{array}$ & $\begin{array}{c}\text { No. of } \\
\text { Samples } \\
\text { with REL }\end{array}$ & $\begin{array}{l}\%> \\
\text { REL }\end{array}$ \\
\hline $\begin{array}{l}\text { District } 1 \text { (Anthracite coal mining } \\
\text { regions in Pennsylvania) }\end{array}$ & 41,934 & 3.5 & 7,576 & 8.9 & 20,987 & 3.4 & 7,975 & 2.8 & 12,972 & 4.0 & 7,576 & 8.9 \\
\hline $\begin{array}{l}\text { District } 2 \text { (Bituminous coal mining } \\
\text { regions in Pennsylvania) }\end{array}$ & 303,583 & 9.9 & 35,457 & 23.9 & 202,334 & 10.3 & 38,816 & 11.0 & 62,433 & 8.0 & 35,457 & 23.9 \\
\hline District 3 & 270,667 & 11.5 & 30,269 & 23.3 & 178,203 & 13.3 & 37,109 & 8.8 & 55,355 & 7.3 & 30,269 & 23.3 \\
\hline Maryland & 10,579 & 11.0 & 2,323 & 32.3 & 5,405 & 13.9 & 1,179 & 8.7 & 3,995 & 7.7 & 2,323 & 32.3 \\
\hline Ohio & 90,025 & 14.5 & 8,242 & 22.5 & 65,421 & 17.1 & 10,168 & 9.0 & 14,436 & 6.5 & 8,242 & 22.5 \\
\hline Northern West Virginia & 170,063 & 9.9 & 19,704 & 22.5 & 107,377 & 10.9 & 25,762 & 8.7 & 36,924 & 7.6 & 19,704 & 22.5 \\
\hline District 4 (Southern West Virginia) & 472,288 & 14.2 & 60,324 & 27.4 & 294,243 & 15.6 & 74,563 & 11.7 & 103,482 & 12.1 & 60,324 & 27.4 \\
\hline District 5 (Virginia) & 301,610 & 10.1 & 36,399 & 20.7 & 181,204 & 10.4 & 52,466 & 9.9 & 67,940 & 9.4 & 36,399 & 20.7 \\
\hline District 6 (Eastern Kentucky) & 281,960 & 8.8 & 49,022 & 22.4 & 144,798 & 7.8 & 49,971 & 9.1 & 87,191 & 10.1 & 49,022 & 22.4 \\
\hline District 7 & 279,339 & 8.8 & 47,325 & 21.2 & 133,482 & 8.3 & 57,363 & 8.6 & 88,494 & 9.6 & 47,325 & 21.2 \\
\hline Central Kentucky & 231,417 & 9.0 & 42,746 & 21.4 & 103,604 & 8.7 & 47,796 & 8.6 & 80,017 & 9.8 & 42,746 & 21.4 \\
\hline North Carolina & 9 & 0.0 & 0 & - & 2 & 0.0 & 7 & 0.0 & 0 & - & 0 & - \\
\hline South Carolina & 0 & - & 0 & - & 0 & - & 0 & - & 0 & - & 0 & - \\
\hline Tennessee & 47,884 & 7.6 & 4,579 & 19.2 & 29,847 & 7.1 & 9,560 & 8.2 & 8,477 & 8.5 & 4,579 & 19.2 \\
\hline Northern Georgia & 29 & 0.0 & 0 & - & 29 & 0.0 & 0 & - & 0 & - & 0 & - \\
\hline District 8 & 153,812 & 15.3 & 17,574 & 50.5 & 95,380 & 15.3 & 25,822 & 14.0 & 32,610 & 16.5 & 17,574 & $\mathbf{5 0 . 5}$ \\
\hline Illinois & 137,945 & 15.9 & 14,857 & 52.0 & 86,573 & 16.2 & 23,347 & 14.7 & 28,025 & 16.2 & 14,857 & 52.0 \\
\hline Indiana & 14,075 & 10.7 & 2,696 & 42.8 & 7,312 & 6.9 & 2,298 & 7.5 & 4,465 & 18.5 & 2,696 & 42.8 \\
\hline Iowa & 940 & 6.4 & 0 & - & 794 & 6.5 & 127 & 6.3 & 19 & 0.0 & 0 & - \\
\hline Michigan & 0 & - & 0 & - & 0 & - & 0 & - & 0 & - & 0 & - \\
\hline Minnesota & 0 & - & 0 & - & 0 & - & 0 & - & 0 & - & 0 & - \\
\hline Northern Missouri & 852 & 3.3 & 21 & 0.0 & 701 & 3.0 & 50 & 0.0 & 101 & 6.9 & 21 & 0.0 \\
\hline Wisconsin & 0 & - & 0 & - & 0 & - & 0 & - & 0 & - & 0 & - \\
\hline
\end{tabular}

See footnotes at end of table. 
Table 6-17 (page 2 of 2). Pneumoconiotic agents: Percent of exposures exceeding designated occupational exposure limits by MSHA coal mine district and states, MSHA inspector and mine operator samples, 1979-1999

\begin{tabular}{|c|c|c|c|c|c|c|c|c|c|c|c|c|}
\hline \multirow[b]{2}{*}{ MSHA Coal Mine District } & \multicolumn{4}{|c|}{ All Years } & \multicolumn{2}{|c|}{$1979-1988$} & \multicolumn{2}{|c|}{$1989-1992$} & \multicolumn{4}{|c|}{1993 - 1999} \\
\hline & $\begin{array}{c}\text { No. of } \\
\text { Samples } \\
\text { with PEL }\end{array}$ & $\begin{array}{l}\%> \\
\text { PEL }\end{array}$ & $\begin{array}{c}\text { No. of } \\
\text { Samples } \\
\text { with REL } \\
\end{array}$ & $\begin{array}{c}\%> \\
\text { REL } \\
\end{array}$ & $\begin{array}{c}\text { No. of } \\
\text { Samples } \\
\text { with PEL }\end{array}$ & $\begin{array}{c}\%> \\
\text { PEL }\end{array}$ & $\begin{array}{c}\text { No. of } \\
\text { Samples } \\
\text { with PEL } \\
\end{array}$ & $\begin{array}{l}\%> \\
\text { PEL }\end{array}$ & \begin{tabular}{|c|} 
No. of \\
Samples \\
with PEL \\
\end{tabular} & $\begin{array}{l}\%> \\
\text { PEL } \\
\end{array}$ & $\begin{array}{c}\text { No. of } \\
\text { Samples } \\
\text { with REL }\end{array}$ & $\begin{array}{l}\%> \\
\text { REL }\end{array}$ \\
\hline District 9 & 125,971 & 17.8 & 17,367 & 33.8 & 74,282 & 20.4 & 21,315 & 17.2 & 30,374 & 11.8 & 17,367 & 33.8 \\
\hline Alaska & 258 & 5.8 & 14 & 0.0 & 179 & 7.3 & 54 & 3.7 & 25 & 0.0 & 14 & 0.0 \\
\hline Arizona & 955 & 5.0 & 207 & 1.9 & 424 & 6.1 & 212 & 3.3 & 319 & 4.7 & 207 & 1.9 \\
\hline Arkansas & 320 & 4.1 & 36 & 0.0 & 232 & 4.3 & 33 & 6.1 & 55 & 1.8 & 36 & 0.0 \\
\hline California & 13 & 0.0 & 11 & 9.1 & 0 & - & 0 & - & 13 & 0.0 & 11 & 9.1 \\
\hline Colorado & 39,718 & 21.2 & 4,937 & 40.7 & 24,351 & 24.8 & 6,438 & 19.0 & 8,929 & 12.6 & 4,937 & 40.7 \\
\hline Hawaii & 0 & - & 0 & - & 0 & - & 0 & - & 0 & - & 0 & - \\
\hline Idaho & 0 & - & 0 & - & 0 & - & 0 & - & 0 & - & 0 & - \\
\hline Kansas & 534 & 3.4 & 73 & 1.4 & 368 & 4.3 & 70 & 0.0 & 96 & 2.1 & 73 & 1.4 \\
\hline Louisiana & 115 & 0.0 & 44 & 0.0 & 12 & 0.0 & 23 & 0.0 & 80 & 0.0 & 44 & 0.0 \\
\hline Southern Missouri & 689 & 3.8 & 114 & 7.0 & 439 & 4.3 & 59 & 1.7 & 191 & 3.1 & 114 & 7.0 \\
\hline Montana & 1,817 & 7.2 & 391 & 5.6 & 1,087 & 8.6 & 160 & 5.6 & 570 & 4.9 & 391 & 5.6 \\
\hline Nebraska & 0 & - & 0 & - & 0 & - & 0 & - & 0 & - & 0 & - \\
\hline Nevada & 0 & - & 0 & - & 0 & - & 0 & - & 0 & - & 0 & - \\
\hline New Mexico & 5,521 & 20.5 & 696 & 6.2 & 3,551 & 24.3 & 622 & 18.2 & 1,348 & 11.6 & 696 & 6.2 \\
\hline North Dakota & 1,392 & 2.0 & 144 & 0.0 & 975 & 2.3 & 227 & 2.6 & 190 & 0.0 & 144 & 0.0 \\
\hline Oklahoma & 5,239 & 9.1 & 799 & 9.8 & 2,835 & 10.0 & 1,025 & 10.7 & 1,379 & 6.0 & 799 & 9.8 \\
\hline Oregon & 0 & - & 0 & - & 0 & - & 0 & - & 0 & - & 0 & - \\
\hline Texas & 4,138 & 2.9 & 515 & 3.5 & 2,613 & 2.4 & 609 & 5.6 & 916 & 2.5 & 515 & 3.5 \\
\hline Utah & 54,983 & 19.5 & 7,585 & 43.6 & 31,598 & 22.1 & 10,247 & 18.8 & 13,138 & 13.8 & 7,585 & 43.6 \\
\hline Washington & 367 & 1.4 & 115 & 4.3 & 124 & 0.0 & 41 & 4.9 & 202 & 1.5 & 115 & 4.3 \\
\hline Wyoming & 9,912 & 12.5 & 1,686 & 21.9 & 5,494 & 12.9 & 1,495 & 15.2 & 2,923 & 10.5 & 1,686 & 21.9 \\
\hline District 10 (Western Kentucky) & 82,406 & 13.3 & 11,047 & 48.4 & 49,001 & 12.6 & 13,128 & 14.1 & 20,277 & 14.7 & 11,047 & 48.4 \\
\hline District 11 & 89,693 & 13.9 & 12,838 & 29.8 & 50,092 & 16.1 & 13,557 & 13.4 & 26,044 & 9.9 & 12,838 & 29.8 \\
\hline Alabama & 89,693 & 13.9 & 12,838 & 29.8 & 50,092 & 16.1 & 13,557 & 13.4 & 26,044 & 9.9 & 12,838 & 29.8 \\
\hline Central and Southern Georgia & 0 & - & 0 & - & 0 & - & 0 & - & 0 & - & 0 & - \\
\hline Florida & 0 & - & 0 & - & 0 & - & 0 & - & 0 & - & 0 & - \\
\hline Mississippi & 0 & - & 0 & - & 0 & - & 0 & - & 0 & - & 0 & - \\
\hline Puerto Rico & 0 & - & 0 & - & 0 & - & 0 & - & 0 & - & 0 & - \\
\hline \multirow[t]{2}{*}{ Virgin Islands } & 0 & - & 0 & - & 0 & - & 0 & - & 0 & - & 0 & - \\
\hline & 0 & - & 0 & - & 0 & - & 0 & - & 0 & - & 0 & - \\
\hline TOTAL & $2,403,263$ & 11.6 & 325,198 & 26.2 & $1,424,006$ & 12.4 & 392,085 & 10.7 & 587,172 & 10.3 & 325,198 & 26.2 \\
\hline
\end{tabular}


ك̌̆ Table 6-18 (page 1 of 2). Pneumoconiotic agents: Percent of exposures exceeding designated occupational exposure limits by MSHA metal/nonmetal district and state, MSHA samples, 1979-1999

\begin{tabular}{|c|c|c|c|c|c|c|c|c|c|c|c|c|}
\hline \multirow[b]{2}{*}{$\begin{array}{l}\text { MSHA Metal/Nonmetal } \\
\text { Mine District }\end{array}$} & \multicolumn{4}{|c|}{1979 - 1988} & \multicolumn{4}{|c|}{$1989-1992$} & \multicolumn{4}{|c|}{1993 - 1999} \\
\hline & $\begin{array}{c}\text { No. of } \\
\text { Samples } \\
\text { with PEL }\end{array}$ & $\begin{array}{l}\%> \\
\text { PEL }\end{array}$ & $\begin{array}{c}\text { No. of } \\
\text { Samples } \\
\text { with REL }\end{array}$ & $\begin{array}{c}\%> \\
\text { REL }\end{array}$ & $\begin{array}{c}\text { No. of } \\
\text { Samples } \\
\text { with PEL }\end{array}$ & $\begin{array}{l}\%> \\
\text { PEL }\end{array}$ & $\begin{array}{c}\text { No. of } \\
\text { Samples } \\
\text { with REL }\end{array}$ & $\begin{array}{c}\%> \\
\text { REL }\end{array}$ & $\begin{array}{c}\text { No. of } \\
\text { Samples } \\
\text { with PEL }\end{array}$ & $\begin{array}{l}\%> \\
\text { PEL } \\
\end{array}$ & $\begin{array}{c}\text { No. of } \\
\text { Samples } \\
\text { with REL }\end{array}$ & $\begin{array}{l}\%> \\
\text { REL }\end{array}$ \\
\hline Connecticut & 351 & 26.2 & 308 & 36.0 & 167 & 13.2 & 162 & 22.2 & 342 & 5.3 & 303 & 5.3 \\
\hline Delaware & 30 & 33.3 & 23 & 0.0 & 33 & 0.0 & 33 & 3.0 & 41 & 4.9 & 41 & 0.0 \\
\hline District of Columbia & 0 & - & 0 & - & 0 & - & 0 & - & 0 & - & 0 & - \\
\hline Maine & 207 & 15.9 & 201 & 24.4 & 169 & 3.0 & 169 & 7.7 & 255 & 4.3 & 255 & 9.8 \\
\hline Maryland & 563 & 4.1 & 556 & 10.1 & 364 & 3.3 & 341 & 6.7 & 645 & 2.5 & 624 & 6.1 \\
\hline Massachusetts & 497 & 17.5 & 483 & 28.2 & 263 & 9.9 & 256 & 15.6 & 554 & 3.6 & 554 & 7.0 \\
\hline New Hampshire & 132 & 15.2 & 132 & 33.3 & 133 & 7.5 & 133 & 16.5 & 248 & 7.3 & 239 & 12.6 \\
\hline New Jersey & 1,055 & 17.0 & 1,013 & 28.1 & 625 & 12.3 & 623 & 20.1 & 914 & 4.2 & 911 & 8.0 \\
\hline New York & 2,221 & 11.5 & 1,970 & 17.9 & 1,733 & 6.6 & 1,679 & 11.6 & 2,973 & 5.3 & 2,776 & 9.3 \\
\hline Pennsylvania & 2,413 & 9.5 & 2,351 & 15.1 & 1,371 & 13.4 & 1,286 & 19.4 & 2,988 & 7.7 & 2,884 & 12.2 \\
\hline Rhode Island & 64 & 23.4 & 60 & 35.0 & 35 & 8.6 & 35 & 25.7 & 114 & 15.8 & 111 & 25.2 \\
\hline Vermont & 708 & 15.7 & 549 & 47.5 & 350 & 20.0 & 348 & 27.9 & 729 & 19.5 & 729 & 29.8 \\
\hline Virginia & 1,312 & 8.6 & 1,215 & 13.2 & 939 & 9.1 & 869 & 12.7 & 2,188 & 4.2 & 2,115 & 7.4 \\
\hline Southeast & 15,562 & 7.9 & 14,577 & 12.2 & 9,936 & 8.2 & 9,560 & 12.4 & 15,192 & 4.6 & 14,647 & 8.0 \\
\hline Alabama & 1,097 & 4.4 & 1,055 & 6.5 & 876 & 8.0 & 876 & 11.9 & 1,351 & 6.2 & 1,342 & 9.5 \\
\hline Florida & 1,215 & 5.3 & 966 & 6.4 & 841 & 2.1 & 836 & 5.0 & 1,481 & 1.3 & 1,397 & 2.4 \\
\hline Georgia & 2,526 & 11.0 & 2,494 & 19.6 & 1,585 & 10.5 & 1,576 & 15.2 & 2,149 & 5.3 & 2,140 & 7.7 \\
\hline Kentucky & 1,055 & 7.8 & 998 & 10.1 & 534 & 9.4 & 501 & 11.0 & 1,001 & 3.7 & 1,001 & 5.7 \\
\hline Mississippi & 301 & 4.3 & 286 & 6.3 & 480 & 5.4 & 478 & 8.2 & 941 & 7.0 & 941 & 11.8 \\
\hline North Carolina & 3,480 & 6.6 & 3,385 & 11.3 & 2,223 & 6.7 & 2,199 & 11.0 & 2,668 & 3.7 & 2,614 & 6.9 \\
\hline Puerto Rico & 1,106 & 5.9 & 788 & 0.3 & 230 & 10.0 & 114 & 3.5 & 829 & 2.7 & 704 & 2.7 \\
\hline South Carolina & 1,176 & 16.2 & 1,145 & 27.5 & 1,371 & 8.8 & 1,235 & 14.8 & 2,195 & 3.0 & 1,978 & 7.1 \\
\hline Tennessee & 3,517 & 7.0 & 3,401 & 9.8 & 1,774 & 10.5 & 1,735 & 16.0 & 2,516 & 7.7 & 2,486 & 13.6 \\
\hline Virgin Islands & 89 & 6.7 & 59 & 0.0 & 22 & 9.1 & 10 & 0.0 & 61 & 1.6 & 44 & 6.8 \\
\hline North Central & 14,704 & 10.0 & 13,332 & 13.5 & 8,922 & 11.0 & 8,755 & 15.6 & 15,428 & 5.2 & 14,928 & 8.7 \\
\hline Illinois & 3,046 & 17.6 & 2,912 & 20.4 & 1,414 & 13.0 & 1,396 & 19.2 & 2,564 & 4.8 & 2,426 & 8.1 \\
\hline Indiana & 1,247 & 4.2 & 1,204 & 3.2 & 929 & 4.7 & 925 & 5.9 & 2,001 & 2.9 & 1,958 & 5.0 \\
\hline Iowa & 692 & 5.5 & 681 & 5.1 & 573 & 5.2 & 572 & 4.9 & 883 & 1.6 & 877 & 1.8 \\
\hline Michigan & 2,062 & 8.0 & 1,577 & 13.8 & 1,559 & 14.6 & 1,506 & 19.5 & 2,664 & 6.2 & 2,520 & 10.4 \\
\hline
\end{tabular}

See footnotes at end of table. 
Table 6-18 (page 2 of 2). Pneumoconiotic agents: Percent of exposures exceeding designated occupational exposure limits by MSHA metal/nonmetal district and state, MSHA samples, 1979-1999

\begin{tabular}{|c|c|c|c|c|c|c|c|c|c|c|c|c|}
\hline \multirow[b]{2}{*}{$\begin{array}{l}\text { MSHA Metal/Nonmetal } \\
\text { Mine District }\end{array}$} & \multicolumn{4}{|c|}{1979 - 1988} & \multicolumn{4}{|c|}{1989 - 1992} & \multicolumn{4}{|c|}{1993 - 1999} \\
\hline & $\begin{array}{c}\text { No. of } \\
\text { Samples } \\
\text { with PEL }\end{array}$ & $\begin{array}{l}\%> \\
\text { PEL }\end{array}$ & $\begin{array}{c}\text { No. of } \\
\text { Samples } \\
\text { with REL }\end{array}$ & $\begin{array}{l}\%> \\
\text { REL }\end{array}$ & $\begin{array}{c}\text { No. of } \\
\text { Samples } \\
\text { with PEL }\end{array}$ & $\begin{array}{l}\%> \\
\text { PEL }\end{array}$ & $\begin{array}{c}\text { No. of } \\
\text { Samples } \\
\text { with REL }\end{array}$ & $\begin{array}{l}\%> \\
\text { REL }\end{array}$ & $\begin{array}{c}\text { No. of } \\
\text { Samples } \\
\text { with PEL }\end{array}$ & $\begin{array}{l}\%> \\
\text { PEL }\end{array}$ & $\begin{array}{c}\text { No. of } \\
\text { Samples } \\
\text { with REL }\end{array}$ & $\begin{array}{l}\%> \\
\text { REL }\end{array}$ \\
\hline South Central & 8,315 & 13.4 & 7,860 & 17.9 & 9,558 & 9.4 & 9,483 & 13.6 & 15,513 & 5.3 & 14,775 & 7.8 \\
\hline Arkansas & 596 & 32.4 & 556 & 44.8 & 672 & 16.4 & 664 & 28.3 & 1,231 & 7.6 & 1,207 & 13.6 \\
\hline Louisiana & 319 & 7.8 & 288 & 11.5 & 748 & 4.0 & 748 & 6.1 & 1,475 & 4.2 & 1,451 & 7.5 \\
\hline Missouri & 2,532 & 12.9 & 2,393 & 16.0 & 2,037 & 11.6 & 2,021 & 15.0 & 3,511 & 4.8 & 3,219 & 7.3 \\
\hline New Mexico & 1,331 & 13.7 & 1,189 & 20.9 & 1,095 & 13.0 & 1,072 & 20.9 & 2,054 & 10.0 & 1,764 & 15.1 \\
\hline Oklahoma & 1,628 & 9.9 & 1,600 & 13.7 & 1,340 & 10.9 & 1,340 & 16.6 & 2,067 & 4.2 & 2,016 & 7.7 \\
\hline Texas & 1,909 & 11.8 & 1,834 & 14.9 & 3,666 & 6.4 & 3,638 & 8.4 & 5,175 & 4.0 & 5,118 & 4.3 \\
\hline Rocky Mountain & 17,435 & 15.3 & 16,211 & 22.7 & 6,434 & 16.5 & 6,164 & 25.3 & 13,136 & 8.7 & 11,310 & 15.3 \\
\hline Arizona & 2,709 & 17.6 & 2,548 & 28.5 & 745 & 18.8 & 733 & 28.4 & 3,098 & 6.8 & 2,348 & 13.7 \\
\hline Colorado & 3,872 & 13.2 & 3,609 & 19.8 & 1,255 & 16.9 & 1,211 & 26.7 & 1,496 & 12.2 & 1,472 & 23.2 \\
\hline Kansas & 1,316 & 12.6 & 1,289 & 14.0 & 553 & 9.8 & 544 & 12.5 & 1,036 & 6.2 & 949 & 7.8 \\
\hline Montana & 1,915 & 10.7 & 1,568 & 17.6 & 842 & 20.3 & 733 & 31.2 & 1,071 & 10.2 & 958 & 18.3 \\
\hline Nebraska & 54 & 5.6 & 53 & 5.7 & 260 & 2.7 & 257 & 4.7 & 490 & 1.8 & 480 & 2.5 \\
\hline Nevada & 1,357 & 25.8 & 1,331 & 33.1 & 1,073 & 19.9 & 1,053 & 31.1 & 1,816 & 12.4 & 1,535 & 23.6 \\
\hline North Dakota & 194 & 12.9 & 194 & 18.6 & 72 & 1.4 & 72 & 11.1 & 266 & 2.3 & 263 & 7.6 \\
\hline South Dakota & 1,814 & 14.4 & 1,698 & 24.5 & 356 & 9.8 & 321 & 14.6 & 1,075 & 5.5 & 835 & 10.4 \\
\hline Utah & 2,433 & 16.2 & 2,165 & 19.5 & 957 & 19.1 & 919 & 29.4 & 1,627 & 8.8 & 1,384 & 16.2 \\
\hline Wyoming & 1,771 & 15.2 & 1,756 & 26.1 & 321 & 15.0 & 321 & 20.9 & 1,161 & 11.6 & 1,086 & 10.9 \\
\hline Western & 5,217 & 13.8 & 4,937 & 21.8 & 4,639 & 11.7 & 4,519 & 19.1 & 8,086 & 5.7 & 7,713 & 9.0 \\
\hline Alaska & 93 & 20.4 & 91 & 27.5 & 88 & 10.2 & 56 & 25.0 & 399 & 4.3 & 282 & 11.3 \\
\hline California & 2,511 & 15.1 & 2,482 & 26.5 & 2,571 & 11.7 & 2,513 & 21.2 & 2,880 & 7.6 & 2,799 & 11.6 \\
\hline Hawaii & 11 & 0.0 & 11 & 0.0 & 57 & 3.5 & 57 & 1.8 & 208 & 1.0 & 202 & 1.0 \\
\hline Idaho & 1,230 & 16.4 & 1,034 & 23.4 & 590 & 15.9 & 560 & 26.1 & 1,374 & 6.7 & 1,244 & 13.5 \\
\hline Oregon & 422 & 4.3 & 422 & 6.4 & 705 & 7.4 & 705 & 7.9 & 1,595 & 1.9 & 1,586 & 1.8 \\
\hline Washington & 950 & 10.6 & 897 & 13.8 & 628 & 13.7 & 628 & 18.0 & 1,630 & 6.1 & 1,600 & 8.7 \\
\hline TOTAL & 71,409 & 11.8 & 66,388 & 17.6 & 46,101 & 10.8 & 44,792 & 16.2 & 79,962 & 5.9 & 75,474 & 9.7 \\
\hline
\end{tabular}

- indicates incalculable field

PEL - permissible exposure limit $\quad$ REL - recommended exposure limit

NOTE: See appendices for source description, methods, and agents.

SOURCE: Mine Safety and Health Administration (MSHA) metal/nonmetal mine data. 
Table 6-19 (page 1 of 3). Pneumoconiotic agents: Percent of exposures exceeding designated occupational exposure limits by OSHA region and state, OSHA samples, 1979-1999

\begin{tabular}{|c|c|c|c|c|c|c|c|c|c|c|c|c|}
\hline \multirow[b]{2}{*}{ OSHA Region } & \multicolumn{4}{|c|}{$1979-1988$} & \multicolumn{4}{|c|}{$\begin{array}{c}\quad 1989-1992 \\
\text { OSHA PELs changed. See note. }\end{array}$} & \multicolumn{4}{|c|}{$1993-1999$} \\
\hline & $\begin{array}{c}\text { No. of } \\
\text { Samples } \\
\text { with PEL } \\
\end{array}$ & $\begin{array}{l}\%> \\
\text { PEL }\end{array}$ & $\begin{array}{c}\text { No. of } \\
\text { Samples } \\
\text { with REL }\end{array}$ & $\begin{array}{l}\%> \\
\text { REL }\end{array}$ & $\begin{array}{c}\text { No. of } \\
\text { Samples } \\
\text { with PEL }\end{array}$ & $\begin{array}{l}\%> \\
\text { PEL }\end{array}$ & $\begin{array}{c}\text { No. of } \\
\text { Samples } \\
\text { with REL }\end{array}$ & $\begin{array}{l}\%> \\
\text { REL }\end{array}$ & \begin{tabular}{|c|} 
No. of \\
Samples \\
with PEL \\
\end{tabular} & $\begin{array}{l}\%> \\
\text { PEL }\end{array}$ & $\begin{array}{c}\text { No. of } \\
\text { Samples } \\
\text { with REL }\end{array}$ & $\begin{array}{l}\%> \\
\text { REL }\end{array}$ \\
\hline Region 1 & 4,523 & 8.6 & 3,332 & 20.6 & 2,460 & 2.3 & 1,526 & 3.4 & 2,917 & 3.3 & 3,822 & 3.7 \\
\hline Connecticut & 1,159 & 7.2 & 987 & 17.7 & 785 & 2.4 & 542 & 2.8 & 1,037 & 0.5 & 1,191 & 0.9 \\
\hline Maine & 186 & 16.1 & 135 & 23.7 & 94 & 0.0 & 62 & 0.0 & 90 & 12.2 & 57 & 1.8 \\
\hline Massachusetts & 1,889 & 8.3 & 1,280 & 22.4 & 1,206 & 2.4 & 693 & 3.2 & 1,345 & 3.9 & 1,704 & 5.3 \\
\hline New Hampshire & 637 & 6.3 & 459 & 20.7 & 342 & 1.5 & 209 & 5.3 & 394 & 3.6 & 488 & 4.1 \\
\hline Rhode Island & 650 & 11.8 & 469 & 21.1 & 31 & 12.9 & 19 & 21.1 & 51 & 27.5 & 376 & 5.1 \\
\hline Vermont & 2 & 0.0 & 2 & 0.0 & 2 & 0.0 & 1 & 0.0 & 0 & - & 6 & 0.0 \\
\hline Region 2 & 4,520 & 9.8 & 3,653 & 20.6 & 2,267 & 3.2 & 1,646 & 7.2 & 3,309 & 7.0 & 2,760 & 8.7 \\
\hline New Jersey & 1,576 & 12.4 & 1,236 & 25.0 & 846 & 3.5 & 568 & 8.8 & 1,069 & 3.4 & 912 & 2.6 \\
\hline New York & 2,872 & 8.5 & 2,353 & 18.5 & 1,330 & 3.1 & 998 & 6.6 & 2,194 & 8.8 & 1,763 & 12.3 \\
\hline Puerto Rico & 67 & 4.5 & 59 & 11.9 & 66 & 0.0 & 58 & 0.0 & 43 & 0.0 & 82 & 0.0 \\
\hline U.S. Virgin Islands & 5 & 0.0 & 5 & 0.0 & 25 & 8.0 & 22 & 9.1 & 3 & 0.0 & 3 & 0.0 \\
\hline Delaware & 136 & 5.1 & 65 & 15.4 & 0 & - & 0 & - & 11 & 18.2 & 7 & 28.6 \\
\hline District of Columbia & 130 & 0.8 & 124 & 9.7 & 20 & 10.0 & 14 & 0.0 & 22 & 9.1 & 12 & 0.0 \\
\hline Maryland & 514 & 8.9 & 297 & 19.5 & 319 & 12.2 & 131 & 26.7 & 224 & 7.1 & 104 & 13.5 \\
\hline Pennsylvania & 4,164 & 13.0 & 3,040 & 31.3 & 1,044 & 17.0 & 663 & 19.6 & 1,422 & 11.3 & 1,257 & 14.1 \\
\hline Virginia & 849 & 12.5 & 652 & 31.0 & 211 & 26.5 & 159 & 32.7 & 236 & 1.3 & 379 & 0.3 \\
\hline West Virginia & 836 & 12.3 & 507 & 27.0 & 174 & 9.2 & 103 & 12.6 & 107 & 2.8 & 73 & 4.1 \\
\hline Region 4 & 6,433 & 12.0 & 4,586 & 24.3 & 4,276 & 5.8 & 2,752 & 10.0 & 5,666 & 4.2 & 7,937 & 4.3 \\
\hline Alabama & 998 & 17.2 & 686 & 33.2 & 550 & 7.1 & 388 & 16.5 & 836 & 1.3 & 917 & 1.5 \\
\hline Florida & 644 & 4.3 & 427 & 10.3 & 548 & 1.8 & 353 & 2.3 & 251 & 2.8 & 1,801 & 0.5 \\
\hline Georgia & 1,878 & 14.2 & 1,387 & 26.2 & 1,182 & 3.4 & 755 & 6.6 & 946 & 6.7 & 1,393 & 6.7 \\
\hline Kentucky & 733 & 8.2 & 519 & 16.0 & 414 & 8.2 & 274 & 10.9 & 938 & 3.9 & 478 & 6.9 \\
\hline Mississippi & 287 & 10.5 & 193 & 30.1 & 272 & 2.2 & 182 & 2.7 & 264 & 2.3 & 614 & 1.5 \\
\hline North Carolina & 783 & 11.9 & 621 & 26.9 & 525 & 11.4 & 403 & 17.6 & 1,494 & 3.9 & 1,574 & 5.5 \\
\hline South Carolina & 653 & 8.6 & 441 & 16.8 & 482 & 5.4 & 229 & 7.9 & 636 & 4.4 & 914 & 4.5 \\
\hline Tennessee & 457 & 14.9 & 312 & 30.8 & 303 & 11.6 & 168 & 16.7 & 301 & 8.3 & 246 & 21.5 \\
\hline
\end{tabular}

See footnotes at end of table. 
Table 6-19 (page 2 of 3). Pneumoconiotic agents: Percent of exposures exceeding designated occupational exposure limits by OSHA region and state, OSHA samples, 1979-1999

\begin{tabular}{|c|c|c|c|c|c|c|c|c|c|c|c|c|}
\hline \multirow[b]{2}{*}{ OSHA Region } & \multicolumn{4}{|c|}{$1979-1988$} & \multicolumn{4}{|c|}{$\begin{array}{c}\quad 1989-1992 \\
\text { OSHA PELs changed. See note. }\end{array}$} & \multicolumn{4}{|c|}{$1993-1999$} \\
\hline & $\begin{array}{c}\text { No. of } \\
\text { Samples } \\
\text { with PEL }\end{array}$ & $\begin{array}{l}\%> \\
\text { PEL }\end{array}$ & $\begin{array}{c}\text { No. of } \\
\text { Samples } \\
\text { with REL }\end{array}$ & $\begin{array}{l}\%> \\
\text { REL }\end{array}$ & $\begin{array}{c}\text { No. of } \\
\text { Samples } \\
\text { with PEL }\end{array}$ & $\begin{array}{l}\%> \\
\text { PEL }\end{array}$ & $\begin{array}{c}\text { No. of } \\
\text { Samples } \\
\text { with REL }\end{array}$ & $\begin{array}{l}\%> \\
\text { REL }\end{array}$ & $\begin{array}{c}\text { No. of } \\
\text { Samples } \\
\text { with PEL }\end{array}$ & $\begin{array}{l}\%> \\
\text { PEL }\end{array}$ & $\begin{array}{c}\text { No. of } \\
\text { Samples } \\
\text { with REL }\end{array}$ & $\begin{array}{l}\%> \\
\text { REL }\end{array}$ \\
\hline Region 5 & 13,020 & 9.8 & 8,134 & 22.8 & 8,564 & 6.3 & 4,776 & 8.9 & 10,161 & 4.7 & 8,643 & 6.7 \\
\hline Illinois & 3,263 & 7.6 & 2,043 & 15.9 & 2,230 & 7.2 & 1,110 & 5.6 & 3,888 & 2.9 & 3,407 & 3.7 \\
\hline Indiana & 927 & 12.6 & 591 & 36.0 & 327 & 4.6 & 229 & 11.4 & 474 & 7.0 & 298 & 11.4 \\
\hline Michigan & 214 & 0.5 & 158 & 2.5 & 1,341 & 5.1 & 798 & 6.0 & 1,855 & 3.6 & 1,106 & 9.5 \\
\hline Minnesota & 116 & 32.8 & 98 & 52.0 & 114 & 3.5 & 76 & 5.3 & 24 & 25.0 & 24 & 25.0 \\
\hline Ohio & 5,494 & 9.4 & 3,310 & 22.1 & 2,181 & 4.5 & 1,332 & 8.5 & 1,305 & 9.2 & 1,543 & 8.8 \\
\hline Wisconsin & 3,006 & 11.9 & 1,934 & 27.2 & 2,371 & 8.3 & 1,231 & 14.1 & 2,615 & 5.5 & 2,265 & 7.7 \\
\hline Region 6 & 6,303 & 6.8 & 3,686 & 15.6 & 2,360 & 4.2 & 1,376 & 9.0 & 2,043 & 4.8 & 5,421 & 2.0 \\
\hline Arkansas & 606 & 6.9 & 332 & 13.9 & 176 & 7.4 & 123 & 12.2 & 224 & 8.9 & 1,973 & 1.2 \\
\hline Louisiana & 449 & 7.6 & 265 & 19.2 & 190 & 2.1 & 87 & 4.6 & 42 & 11.9 & 38 & 18.4 \\
\hline New Mexico & 22 & 27.3 & 17 & 35.3 & 18 & 0.0 & 18 & 0.0 & 15 & 40.0 & 22 & 27.3 \\
\hline Oklahoma & 1,698 & 3.5 & 877 & 9.8 & 447 & 2.2 & 245 & 3.3 & 296 & 3.4 & 1,361 & 1.2 \\
\hline Texas & 3,528 & 8.1 & 2,195 & 17.6 & 1,529 & 4.6 & 903 & 10.7 & 1,466 & 4.0 & 2,027 & 2.6 \\
\hline Region 7 & 3,951 & 6.2 & 2,509 & 15.5 & 1,647 & 8.4 & 954 & 7.8 & 1,187 & 10.1 & 1,724 & 5.3 \\
\hline Iowa & 1,585 & 6.8 & 880 & 16.5 & 609 & 6.4 & 322 & 10.9 & 416 & 11.8 & 242 & 23.1 \\
\hline Kansas & 700 & 4.3 & 418 & 14.8 & 248 & 3.2 & 157 & 9.6 & 376 & 8.0 & 1,013 & 2.5 \\
\hline Missouri & 1,200 & 7.4 & 920 & 16.4 & 619 & 12.3 & 345 & 6.1 & 122 & 30.3 & 297 & 3.0 \\
\hline Nebraska & 466 & 4.3 & 291 & 11.0 & 171 & 8.8 & 130 & 2.3 & 273 & 1.5 & 172 & 1.2 \\
\hline Region 8 & 4,158 & 4.3 & 2,374 & 10.7 & 1,604 & 5.9 & 824 & 6.6 & 1,527 & 5.9 & 2,238 & 4.5 \\
\hline Colorado & 2,385 & 4.4 & 1,285 & 11.1 & 1,078 & 3.1 & 600 & 4.5 & 756 & 4.2 & 718 & 6.0 \\
\hline Montana & 709 & 3.0 & 471 & 11.0 & 261 & 12.6 & 117 & 8.5 & 205 & 7.3 & 209 & 6.2 \\
\hline North Dakota & 455 & 4.8 & 236 & 12.3 & 71 & 4.2 & 29 & 3.4 & 164 & 15.2 & 177 & 16.4 \\
\hline South Dakota & 484 & 2.9 & 278 & 5.0 & 126 & 18.3 & 25 & 28.0 & 203 & 8.4 & 522 & 3.1 \\
\hline Utah & 95 & 16.8 & 77 & 22.1 & 58 & 3.4 & 48 & 18.8 & 22 & 4.5 & 267 & 0.0 \\
\hline Wyoming & 30 & 0.0 & 27 & 3.7 & 10 & 0.0 & 5 & 0.0 & 177 & 0.0 & 345 & 0.0 \\
\hline
\end{tabular}

See footnotes at end of table. 
Table 6-19 (page 3 of 3). Pneumoconiotic agents: Percent of exposures exceeding designated occupational exposure limits by OSHA region and state, OSHA samples, 1979-1999

\begin{tabular}{|c|c|c|c|c|c|c|c|c|c|c|c|c|}
\hline \multirow[b]{2}{*}{ OSHA Region } & \multicolumn{4}{|c|}{$1979-1988$} & \multicolumn{4}{|c|}{$\begin{array}{l}1989-1992 \\
\text { OSHA PELs changed. See note. }\end{array}$} & \multicolumn{4}{|c|}{$1993-1999$} \\
\hline & $\begin{array}{c}\text { No. of } \\
\text { Samples } \\
\text { with PEL }\end{array}$ & $\begin{array}{l}\%> \\
\text { PEL }\end{array}$ & $\begin{array}{c}\text { No. of } \\
\text { Samples } \\
\text { with REL }\end{array}$ & $\begin{array}{l}\%> \\
\text { REL }\end{array}$ & $\begin{array}{c}\text { No. of } \\
\text { Samples } \\
\text { with PEL }\end{array}$ & $\begin{array}{l}\%> \\
\text { PEL }\end{array}$ & $\begin{array}{c}\text { No. of } \\
\text { Samples } \\
\text { with REL }\end{array}$ & $\begin{array}{l}\%> \\
\text { REL }\end{array}$ & $\begin{array}{c}\text { No. of } \\
\text { Samples } \\
\text { with PEL }\end{array}$ & $\begin{array}{l}\%> \\
\text { PEL }\end{array}$ & $\begin{array}{c}\text { No. of } \\
\text { Samples } \\
\text { with REL }\end{array}$ & $\begin{array}{l}\%> \\
\text { REL }\end{array}$ \\
\hline Region 9 & 1,707 & 8.1 & 1,150 & 16.3 & 792 & 7.1 & 570 & 10.0 & 389 & 5.1 & 789 & 2.9 \\
\hline American Samoa & 0 & - & 0 & - & 0 & - & 0 & - & 0 & - & 0 & - \\
\hline Arizona & 165 & 10.3 & 123 & 20.3 & 136 & 1.5 & 62 & 4.8 & 31 & 6.5 & 22 & 9.1 \\
\hline California & 1,306 & 4.1 & 837 & 10.3 & 492 & 5.9 & 385 & 7.8 & 307 & 3.9 & 714 & 2.1 \\
\hline Guam & 3 & 0.0 & 3 & 0.0 & 0 & - & 0 & - & 0 & - & 0 & - \\
\hline Hawaii & 47 & 0.0 & 44 & 2.3 & 8 & 0.0 & 8 & 0.0 & 22 & 13.6 & 18 & 16.7 \\
\hline Nevada & 186 & 36.6 & 143 & 53.1 & 156 & 16.0 & 115 & 20.9 & 29 & 10.3 & 35 & 8.6 \\
\hline Region 10 & 876 & 8.0 & 627 & 17.9 & 664 & 10.1 & 409 & 8.3 & 930 & 9.0 & 786 & 16.7 \\
\hline Alaska & 194 & 2.1 & 178 & 3.4 & 47 & 6.4 & 42 & 9.5 & 26 & 0.0 & 38 & 0.0 \\
\hline Idaho & 215 & 4.2 & 141 & 11.3 & 308 & 2.6 & 182 & 4.9 & 167 & 0.0 & 109 & 0.0 \\
\hline Oregon & 439 & 11.2 & 299 & 28.4 & 61 & 9.8 & 31 & 6.5 & 252 & 5.2 & 142 & 11.3 \\
\hline
\end{tabular}

- indicates incalculable field

PEL - permissible exposure limit

REL - recommended exposure limit

NOTE: From March 1, 1989 to March 22, 1993, the OSHA PELs in force differed from those employed before and after those dates. See appendices for source description, methods, and agents.

SOURCE: Occupational Safety and Health Administation (OSHA) Integrated Management Information System. 
Figure 6-6. Pneumoconiotic agents: Percent of exposures exceeding the NIOSH recommended exposure limits by state, MSHA metal/nonmetal mine samples, 1979-1999

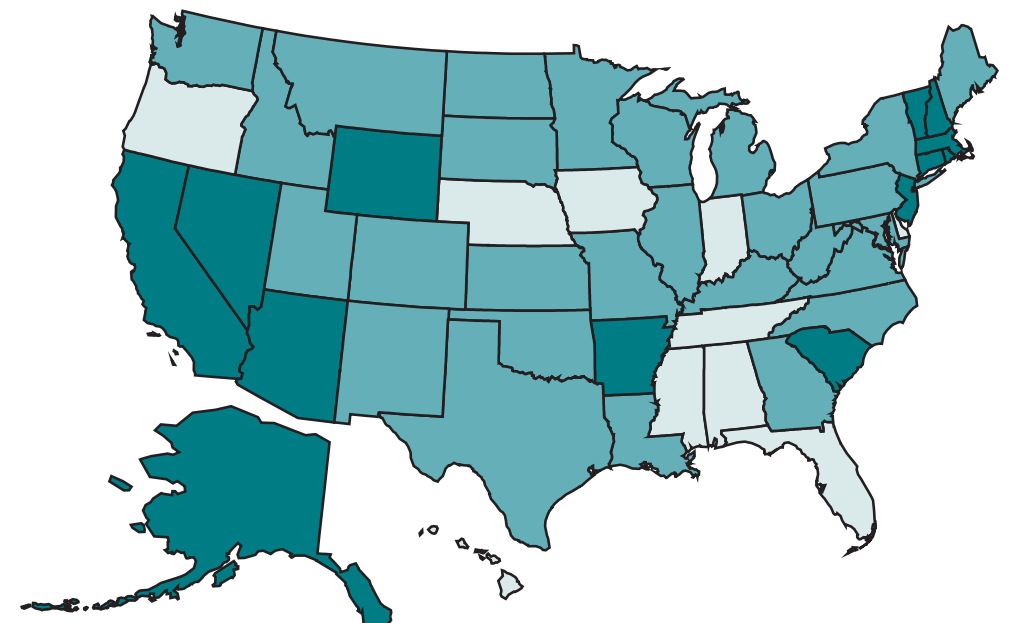

\begin{tabular}{|lc|}
\hline \multicolumn{2}{|c|}{$\mathbf{1 9 7 9} \mathbf{~ - ~} 1988$} \\
\hline$\%>$ REL & No. of States \\
$\square>25$ & 13 \\
$\square>10$ to 25 & 27 \\
$\square 0$ to 10 & 10 \\
$\square<10$ samples & 1 \\
\hline
\end{tabular}

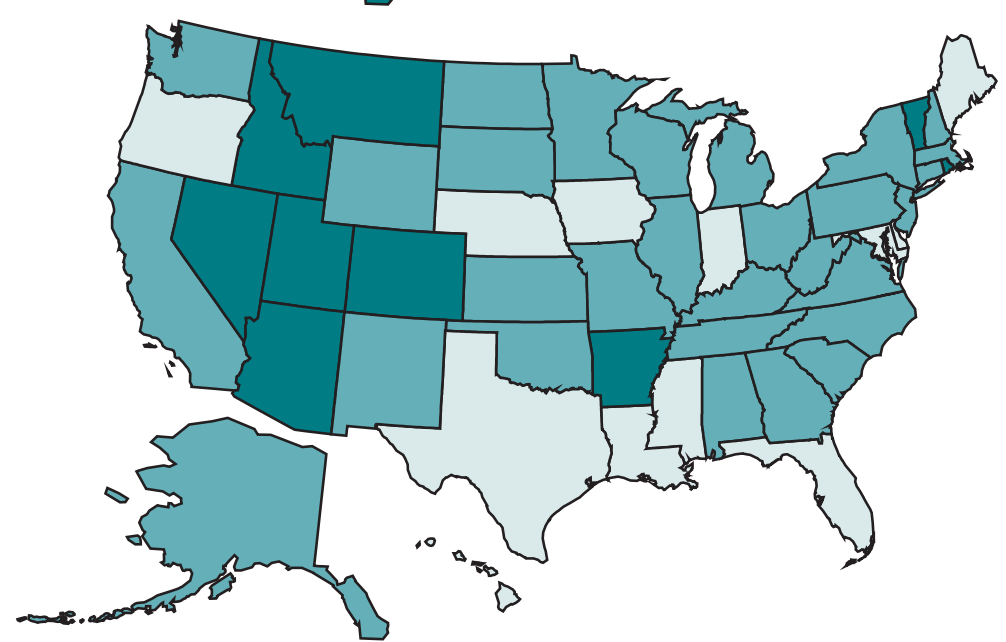

\begin{tabular}{|cc|}
\hline \multicolumn{2}{|c|}{1989 - 1992} \\
\hline \% > REL & No. of States \\
$\square>25$ & 9 \\
$\square>10$ to 25 & 29 \\
$\square$ to 10 & 12 \\
$\square<10$ samples & 1 \\
\hline
\end{tabular}

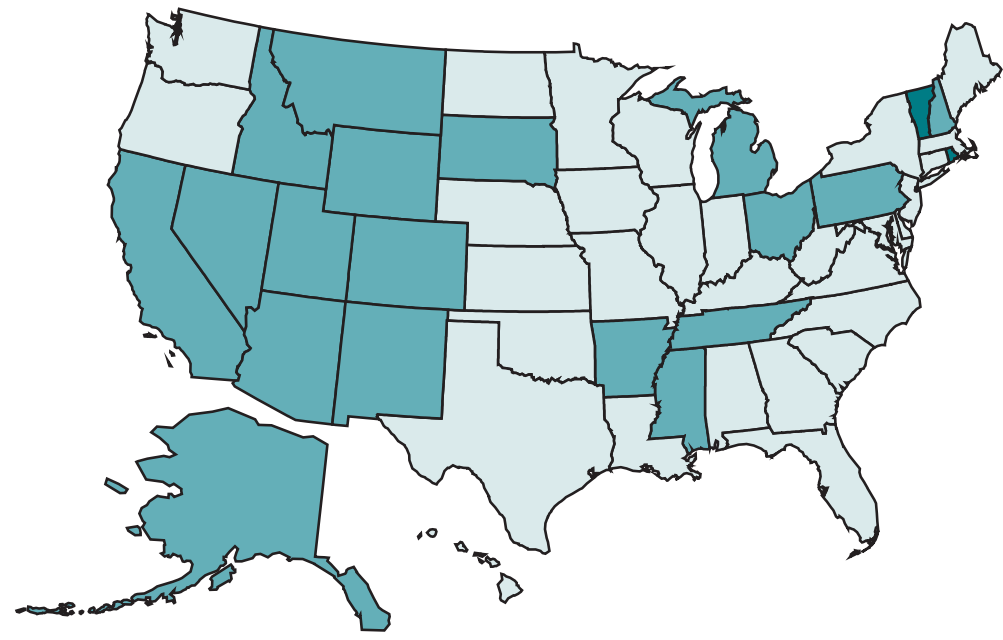

\begin{tabular}{|cc|}
\hline \multicolumn{2}{|c|}{$\mathbf{1 9 9 3} \mathbf{- 1 9 9 9}$} \\
\hline$\%>$ REL & No. of States \\
$\square>25$ & 2 \\
$\square>10$ to 25 & 18 \\
$\square$ to 10 & 30 \\
$\square<10$ samples & 1 \\
\hline
\end{tabular}

REL - recommended exposure limit

NOTE: See appendices for source description, methods, and agents.

SOURCE: Mine Safety and Health Administration (MSHA) metal/nonmetal mine data 
Figure 6-7. Pneumoconiotic agents: Percent of exposures exceeding the NIOSH recommended exposure limits by state, OSHA samples, 1979-1999

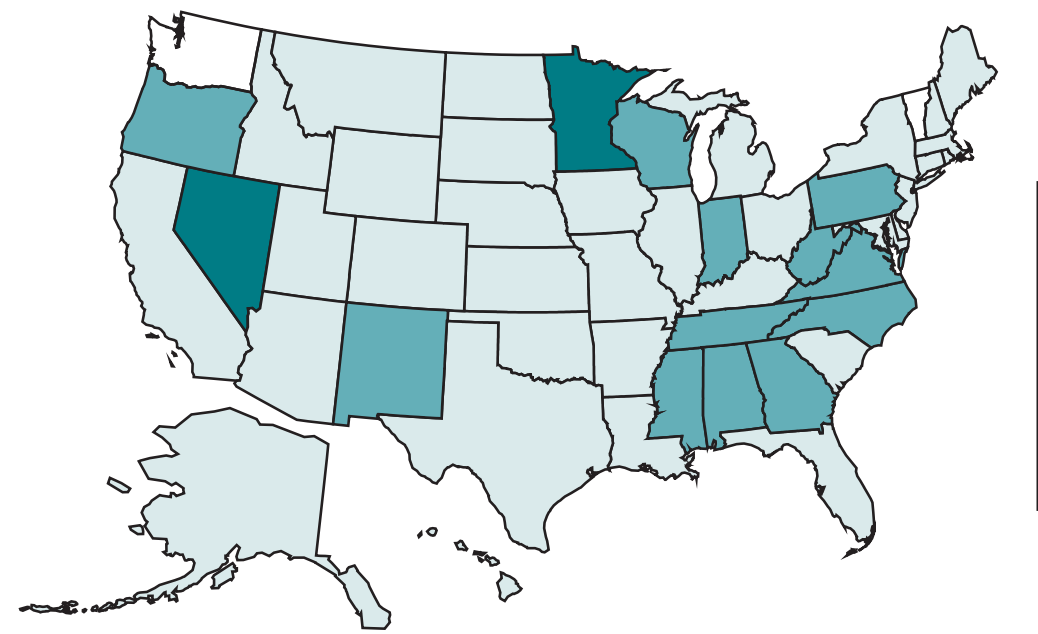

\begin{tabular}{|cc|}
\hline \multicolumn{1}{|c|}{$\mathbf{1 9 7 9} \mathbf{- 1 9 8 8}$} \\
\hline$\%>$ REL & No. of States \\
$\square>50$ & 2 \\
$\square>25$ to 50 & 12 \\
$\square$ 0 to 25 & 35 \\
$\square<10$ samples & 2 \\
\hline
\end{tabular}

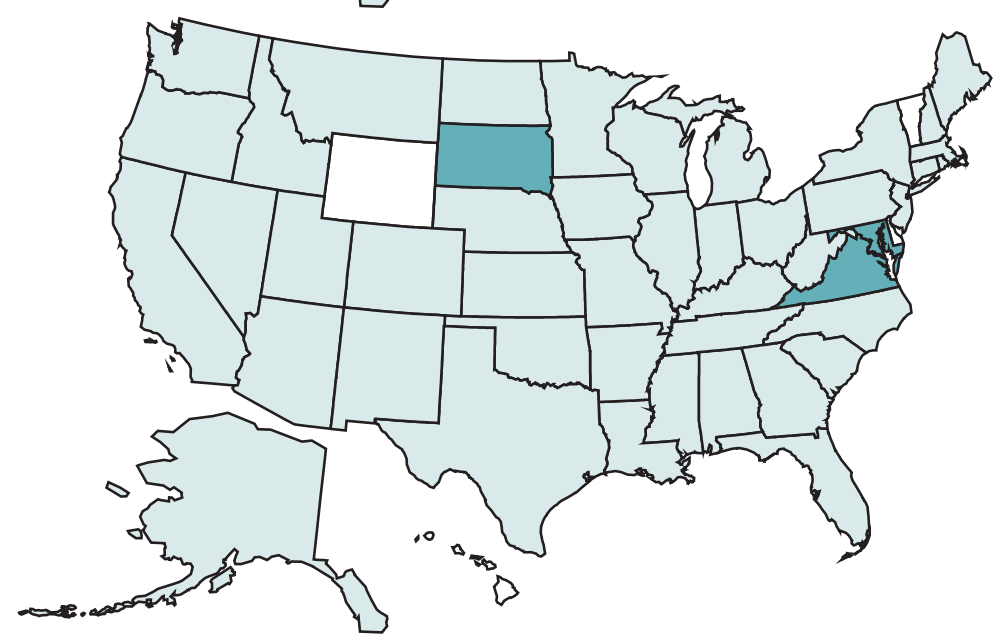

\begin{tabular}{|cc|}
\hline \multicolumn{2}{|c|}{1989 - 1992} \\
OSHA PELs changed. See note. \\
\hline$\%>$ REL & No. of States \\
$\square>50$ & 0 \\
$\square>25$ to 50 & 3 \\
$\square 0$ to 25 & 44 \\
$\square<10$ samples & 4 \\
\hline
\end{tabular}

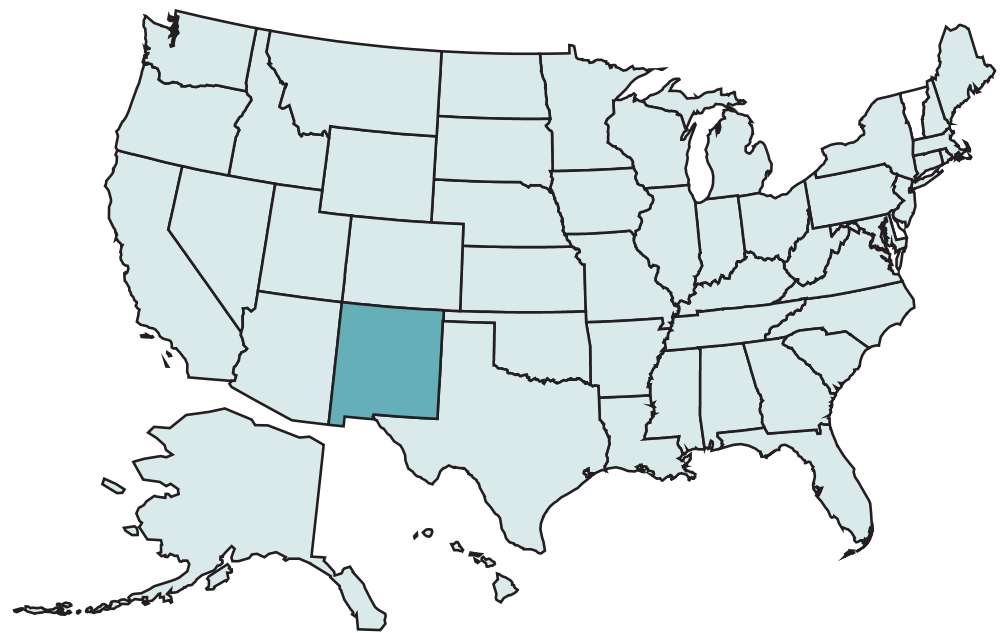

\begin{tabular}{|cc|}
\hline \multicolumn{1}{|c|}{$\mathbf{1 9 9 3}$ - 1999} \\
\hline$\%>$ REL & No. of States \\
$\square>50$ & 0 \\
$\square>25$ to 50 & 1 \\
$\square 0$ to 25 & 48 \\
$\square<10$ samples & 2 \\
\hline
\end{tabular}

REL - recommended exposure limit

PEL - permissable exposure limit

NOTE: From March 1, 1989 to March 22, 1993, the OSHA PELs in force differed from those employed before and after those dates. See appendices for source description, methods, and agents.

SOURCE: Occupational Safety and Health Administration (OSHA) Integrated Management Information System. 


\section{Section 7}

Malignant

Mesothelioma 

Figure 7-1. Malignant mesothelioma: Number of deaths by anatomical site, U.S. residents age 15 and over, 1999

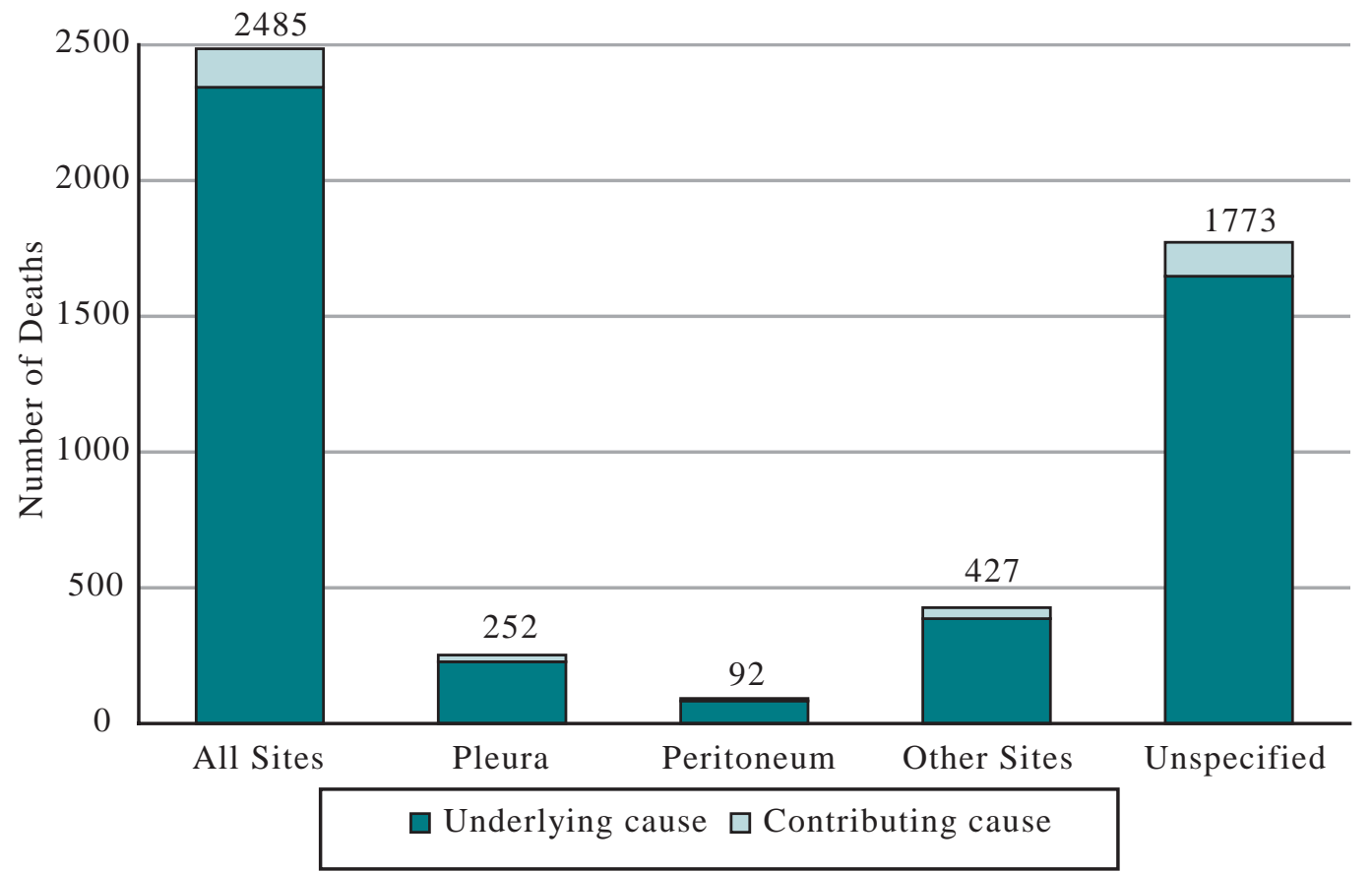

NOTE: See appendices for source description, methods, and ICD codes.

SOURCE: National Center for Health Statistics multiple cause of death data.

Figure 7-2. Malignant mesothelioma: Age-adjusted mortality rates by state, U.S. residents age 15 and over, 1999

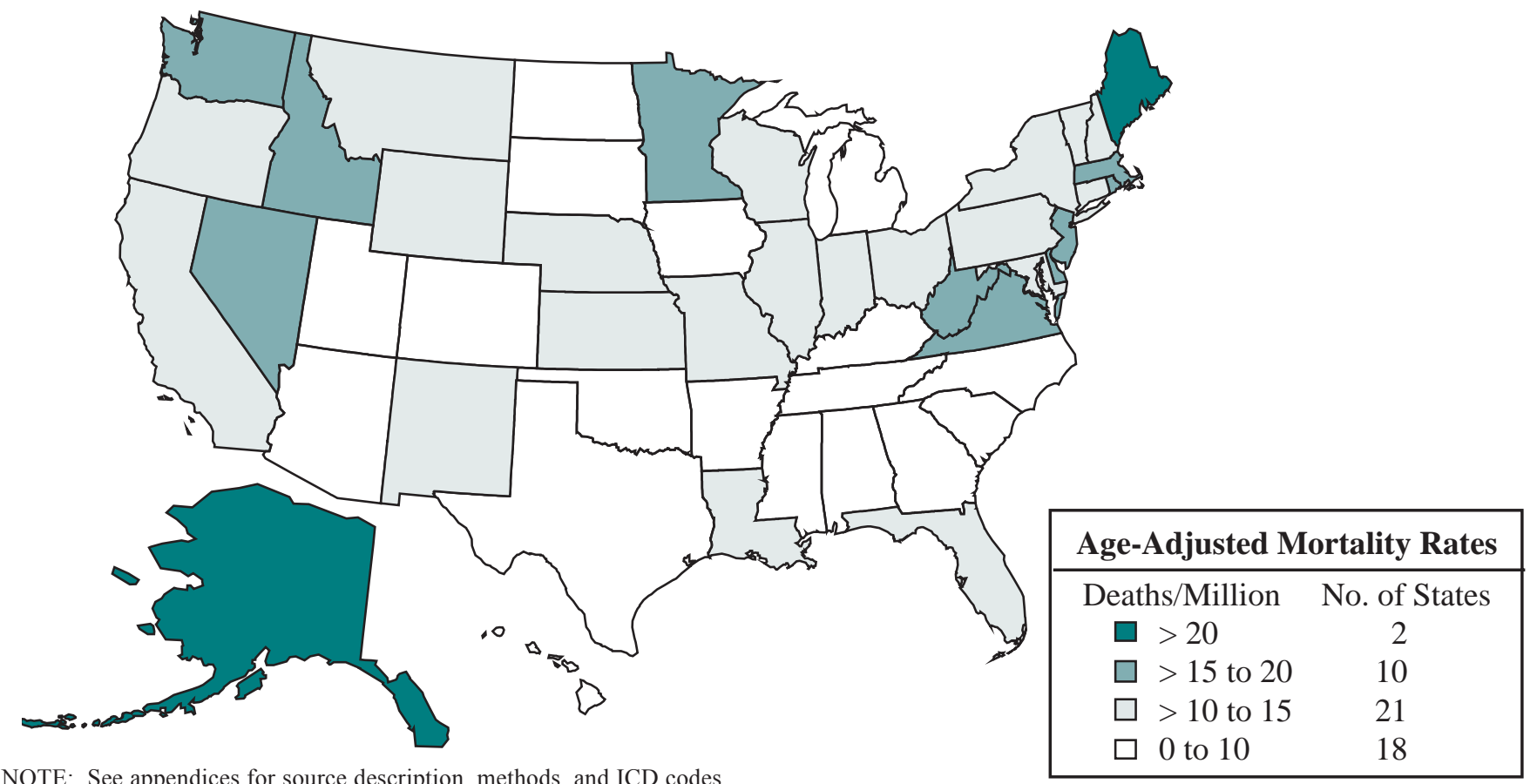

NOTE: See appendices for source description, methods, and ICD codes.

SOURCE: National Center for Health Statistics multiple cause of death data. Population estimates from U.S. Bureau of the Census. 
Table 7-1. Malignant mesothelioma: Number of deaths by sex, race, and age, and median age at death, U.S. residents age 15 and over, 1999

\begin{tabular}{|c|c|c|c|c|c|c|c|c|c|c|c|c|c|c|c|c|}
\hline \multirow[b]{2}{*}{ Site } & \multirow{2}{*}{$\begin{array}{l}\text { No. of } \\
\text { Deaths }\end{array}$} & \multirow{2}{*}{$\begin{array}{l}\text { Under- } \\
\text { lying } \\
\text { Cause } \\
(\%)\end{array}$} & \multicolumn{2}{|c|}{ Sex } & \multicolumn{3}{|c|}{ Race } & \multicolumn{8}{|c|}{ Age Group (yrs) } & \multirow{2}{*}{$\begin{array}{l}\text { Median } \\
\text { Age } \\
(\text { yrs })\end{array}$} \\
\hline & & & Male & Female & White & Black & Other & $15-24$ & $25-34$ & $35-44$ & $45-54$ & $55-64$ & $65-74$ & $75-84$ & $85+$ & \\
\hline Pleura & 252 & 90.1 & 219 & 33 & 240 & 10 & 2 & - & - & 2 & 12 & 32 & 101 & 86 & 19 & 72.0 \\
\hline Peritoneum & 92 & 90.2 & 62 & 30 & 90 & 2 & - & - & - & 2 & 10 & 23 & 31 & 20 & 6 & 69.5 \\
\hline Other Sites & 427 & 90.4 & 345 & 82 & 407 & 14 & 6 & 1 & 2 & 3 & 23 & 61 & 134 & 154 & 49 & 74.0 \\
\hline Unspecified & 1,773 & 92.9 & 1,424 & 349 & 1,673 & 83 & 17 & 1 & 2 & 26 & 94 & 279 & 572 & 654 & 145 & 73.0 \\
\hline Any Site & 2,485 & 94.3 & 1,995 & 490 & 2,355 & 105 & 25 & 2 & 4 & 33 & 138 & 389 & 818 & 888 & 213 & 73.0 \\
\hline \multicolumn{17}{|c|}{$\begin{array}{l}\text { - indicates no deaths listed. } \\
\text { NOTE: The sum of individual site death totals may be greater than the the total number of deaths for "any site" because some decedents have more than one site of mesothelioma listed on } \\
\text { their death certificates. See appendices for source description, methods, and ICD codes. } \\
\text { SOURCE: National Center for Health Statistics multiple cause of death data. }\end{array}$} \\
\hline
\end{tabular}


Table 7-2. Malignant mesothelioma: Mortality rates (per million population) by race and sex, U.S. residents age 15 and over, 1999

\begin{tabular}{|c|c|c|c|c|c|c|c|}
\hline \multirow[b]{2}{*}{ Year } & \multirow[b]{2}{*}{ Overall } & \multicolumn{2}{|c|}{ White } & \multicolumn{2}{|c|}{ Black } & \multicolumn{2}{|c|}{ Other } \\
\hline & & Male & Female & Male & Female & Male & Female \\
\hline \multirow{3}{*}{1999} & \multicolumn{7}{|c|}{ Crude Mortality Rate } \\
\hline & 11.60 & 21.93 & 4.95 & 6.43 & 2.02 & 3.61 & 1.53 \\
\hline & \multicolumn{7}{|c|}{ Age-Adjusted Mortality Rate } \\
\hline 1999 & 11.65 & 23.89 & 4.29 & 10.32 & 2.57 & 6.47 & 2.18 \\
\hline
\end{tabular}

- indicates no deaths listed.

NOTE: See appendices for source description, methods, and ICD codes.

SOURCE: National Center for Health Statistics multiple cause of death data. Population estimates from U.S. Bureau of the Census. 
Table 7-3. Malignant mesothelioma: Years of potential life lost to age 65 and to life expectancy by race and sex, U.S. residents age 15 and over, 1999

\begin{tabular}{|c|c|c|c|c|c|c|c|}
\hline \multirow[b]{2}{*}{ Year } & \multicolumn{2}{|c|}{ White } & \multicolumn{2}{|c|}{ Black } & \multicolumn{2}{|c|}{ Other } & \multirow[b]{2}{*}{ Total } \\
\hline & Male & Female & Male & Female & Male & Female & \\
\hline & & & ears of 1 & ential Lif & st to $\mathrm{Ag}$ & & \\
\hline \multirow[t]{2}{*}{1999} & 3,395 & 1,205 & 320 & 95 & 25 & 30 & 5,070 \\
\hline & \multicolumn{7}{|c|}{ Years of Potential Life Lost to Life Expectancy } \\
\hline 1999 & 23,535 & 7,120 & 1,060 & 408 & 230 & 132 & 32,485 \\
\hline
\end{tabular}

- indicates no deaths listed.

NOTE: See appendices for source description, methods, and ICD codes.

SOURCE: National Center for Health Statistics multiple cause of death data. 
Table 7-4. Malignant mesothelioma: Number of deaths, mortality rates (per million population), and years of potential life lost (YPLL) by state, U.S. residents age 15 and over, 1999

\begin{tabular}{|c|c|c|c|c|c|c|c|c|c|c|}
\hline \multirow[b]{2}{*}{ State } & \multirow{2}{*}{$\begin{array}{l}\text { No. of } \\
\text { Deaths }\end{array}$} & \multirow[b]{2}{*}{ Rank } & \multicolumn{2}{|c|}{ Crude Mortality } & \multicolumn{2}{|c|}{ Age-Adjusted Mortality } & \multicolumn{4}{|c|}{ YPLL to Life Expectancy } \\
\hline & & & Rate & Rank & Rate & Rank & Total & Rank & YPLL/death & Rank \\
\hline Alabama & 33 & 20 & 9.46 & 34 & 9.35 & 37 & 525 & 20 & 15.9 & 8 \\
\hline Alaska & 8 & 45 & 17.53 & 5 & 32.81 & 1 & 132 & 44 & 16.5 & 4 \\
\hline Arizona & 32 & 22 & 8.76 & 39 & 8.40 & 40 & 423 & 24 & 13.2 & 32 \\
\hline Arkansas & 15 & 37 & 7.47 & 45 & 6.70 & 47 & 205 & 37 & 13.7 & 25 \\
\hline California & 259 & 1 & 10.12 & 33 & 11.42 & 28 & 3,670 & 1 & 14.2 & 20 \\
\hline Colorado & 17 & 35 & 5.35 & 49 & 6.46 & 48 & 239 & 36 & 14.1 & 21 \\
\hline Connecticut & 33 & 20 & 12.77 & 22 & 11.60 & 27 & 435 & 23 & 13.2 & 32 \\
\hline Delaware & 12 & 41 & 19.96 & 2 & 19.71 & 3 & 157 & 41 & 13.1 & 36 \\
\hline District of Columbia & 5 & 47 & 11.45 & 30 & 11.15 & 29 & 51 & 51 & 10.2 & 51 \\
\hline Florida & 163 & 2 & 13.44 & 19 & 10.16 & 33 & 2,134 & 2 & 13.1 & 36 \\
\hline Georgia & 30 & 25 & 4.94 & 51 & 6.05 & 50 & 453 & 22 & 15.1 & 13 \\
\hline Hawaii & 5 & 47 & 5.30 & 50 & 4.95 & 51 & 73 & 48 & 14.6 & 16 \\
\hline Idaho & 14 & 39 & 14.48 & 14 & 15.33 & 12 & 192 & 39 & 13.7 & 25 \\
\hline Illinois & 128 & 6 & 13.52 & 18 & 13.76 & 17 & 1,844 & 6 & 14.4 & 17 \\
\hline Indiana & 58 & 14 & 12.40 & 25 & 12.53 & 22 & 916 & 13 & 15.8 & 10 \\
\hline Iowa & 21 & 33 & 9.19 & 37 & 7.92 & 42 & 258 & 34 & 12.3 & 49 \\
\hline Kansas & 27 & 28 & 12.97 & 21 & 12.25 & 24 & 336 & 30 & 12.4 & 45 \\
\hline Kentucky & 24 & 30 & 7.58 & 44 & 7.63 & 43 & 400 & 27 & 16.7 & 3 \\
\hline Louisiana & 47 & 19 & 13.83 & 16 & 14.78 & 14 & 718 & 16 & 15.3 & 11 \\
\hline Maine & 28 & 27 & 27.50 & 1 & 26.40 & 2 & 363 & 28 & 13.0 & 38 \\
\hline Maryland & 51 & 16 & 12.52 & 24 & 13.74 & 18 & 680 & 17 & 13.3 & 31 \\
\hline Massachusetts & 81 & 9 & 16.39 & 8 & 15.40 & 10 & 1,006 & 11 & 12.4 & 45 \\
\hline Michigan & 69 & 12 & 8.91 & 38 & 9.07 & 38 & 1,028 & 10 & 14.9 & 14 \\
\hline Minnesota & 61 & 13 & 16.34 & 9 & 16.82 & 7 & 875 & 14 & 14.3 & 19 \\
\hline Mississippi & 20 & 34 & 9.30 & 35 & 9.61 & 35 & 257 & 35 & 12.9 & 39 \\
\hline Missouri & 49 & 17 & 11.35 & 31 & 10.73 & 31 & 618 & 19 & 12.6 & 43 \\
\hline Montana & 10 & 43 & 14.21 & 15 & 13.38 & 20 & 124 & 46 & 12.4 & 45 \\
\hline Nebraska & 15 & 37 & 11.50 & 29 & 10.77 & 30 & 191 & 40 & 12.7 & 41 \\
\hline Nevada & 22 & 31 & 15.79 & 11 & 17.73 & 4 & 280 & 32 & 12.7 & 41 \\
\hline New Hampshire & 11 & 42 & 11.58 & 28 & 12.31 & 23 & 147 & 42 & 13.4 & 30 \\
\hline New Jersey & 111 & 8 & 17.20 & 6 & 16.30 & 8 & 1,515 & 8 & 13.6 & 27 \\
\hline New Mexico & 17 & 35 & 12.77 & 22 & 13.05 & 21 & 362 & 29 & 21.3 & 1 \\
\hline New York & 156 & 4 & 10.79 & 32 & 10.46 & 32 & 2,121 & 3 & 13.6 & 27 \\
\hline North Carolina & 56 & 15 & 9.30 & 35 & 9.45 & 36 & 773 & 15 & 13.8 & 24 \\
\hline North Dakota & 4 & 50 & 7.92 & 43 & 7.37 & 45 & 53 & 50 & 13.2 & 32 \\
\hline Ohio & 131 & 5 & 14.70 & 12 & 14.26 & 15 & 2,005 & 5 & 15.3 & 11 \\
\hline Oklahoma & 22 & 31 & 8.34 & 41 & 7.93 & 41 & 262 & 33 & 11.9 & 50 \\
\hline Oregon & 32 & 22 & 12.14 & 26 & 11.66 & 25 & 405 & 25 & 12.6 & 43 \\
\hline Pennsylvania & 162 & 3 & 16.80 & 7 & 14.14 & 16 & 2,095 & 4 & 12.9 & 39 \\
\hline Rhode Island & 14 & 39 & 17.74 & 4 & 15.56 & 9 & 202 & 38 & 14.4 & 17 \\
\hline South Carolina & 26 & 29 & 8.40 & 40 & 8.79 & 39 & 322 & 31 & 12.4 & 45 \\
\hline South Dakota & 4 & 50 & 6.98 & 47 & 6.36 & 49 & 65 & 49 & 16.2 & 6 \\
\hline Tennessee & 31 & 24 & 7.09 & 46 & 7.30 & 46 & 499 & 21 & 16.1 & 7 \\
\hline Texas & 125 & 7 & 8.19 & 42 & 9.62 & 34 & 1,832 & 7 & 14.7 & 15 \\
\hline Utah & 9 & 44 & 5.83 & 48 & 7.52 & 44 & 143 & 43 & 15.9 & 8 \\
\hline Vermont & 7 & 46 & 14.55 & 13 & 14.85 & 13 & 128 & 45 & 18.2 & 2 \\
\hline Virginia & 75 & 10 & 13.67 & 17 & 15.36 & 11 & 1,047 & 9 & 14.0 & 22 \\
\hline Washington & 72 & 11 & 15.90 & 10 & 17.29 & 6 & 947 & 12 & 13.2 & 32 \\
\hline West Virginia & 29 & 26 & 19.59 & 3 & 17.37 & 5 & 402 & 26 & 13.9 & 23 \\
\hline Wisconsin & 49 & 17 & 11.80 & 27 & 11.61 & 26 & 663 & 18 & 13.5 & 29 \\
\hline Wyoming & 5 & 47 & 13.20 & 20 & 13.73 & 19 & 83 & 47 & 16.5 & 4 \\
\hline
\end{tabular}

- indicates no deaths listed.

NOTE: See appendices for source description, methods, and ICD codes.

SOURCE: National Center for Health Statistics multiple cause of death data. Population estimates from U.S. Bureau of the Census. 
Table 7-5. Malignant mesothelioma: Most frequently recorded industries on death certificate, U.S. residents age 15 and over, selected states, 1999

\begin{tabular}{llrc}
\hline CIC & Industry & Number of Deaths & Percent \\
\hline 060 & Construction & 77 & 14.2 \\
961 & Non-paid worker or non-worker or own home/at home & 38 & 7.0 \\
842 & Elementary and secondary schools & 20 & 3.7 \\
192 & Industrial and miscellaneous chemicals & 19 & 3.5 \\
901 & General government, n.e.c. & 13 & 2.4 \\
010 & Agricultural production, crops & 10 & 1.9 \\
392 & Not specified manufacturing industries & 10 & 1.9 \\
460 & Electric light and power & 10 & 1.9 \\
400 & Railroads & 9 & 1.7 \\
831 & Hospitals & 9 & 1.7 \\
& All other industries & 303 & 56.0 \\
& Industry not reported & 23 & 4.3 \\
& TOTAL & $\mathbf{5 4 1}$ & $\mathbf{1 0 0 . 0}$ \\
\hline
\end{tabular}

CIC - Census Industry Code

n.e.c. - not elsewhere classified

NOTE: Percentages may not total to $100 \%$ due to rounding. See appendices for source description, methods, and ICD codes, industry and occupation codes, and list of selected states.

SOURCE: National Center for Health Statistics multiple cause of death data.

Table 7-6. Malignant mesothelioma: Most frequently recorded occupations on death certificate, U.S. residents age 15 and over, selected states, 1999

\begin{tabular}{clcc}
\hline COC & Occupation & Number of Deaths & Percent \\
\hline 019 & Managers and administrators, n.e.c. & 41 & 7.6 \\
914 & Housewife/Homemaker & 37 & 6.8 \\
585 & Plumbers, pipefitters, and steamfitters & 18 & 3.3 \\
453 & Janitors and cleaners & 17 & 3.1 \\
243 & Supervisors and proprietors, sales occupations & 16 & 3.0 \\
567 & Carpenters & 16 & 3.0 \\
156 & Teachers, elementary school & 13 & 2.4 \\
473 & Farmers, except horticulture & 12 & 2.2 \\
575 & Electricians & 12 & 2.2 \\
633 & Supervisors, production occupations & 12 & 2.2 \\
804 & Truck drivers & 12 & 2.2 \\
& All other occupations & 307 & 56.8 \\
& Occupation not reported & 28 & 5.2 \\
& TOTAL & $\mathbf{5 4 1}$ & $\mathbf{1 0 0 . 0}$ \\
\hline
\end{tabular}

COC - Census Occupation Code n.e.c. - not elsewhere classified

NOTE: Percentages may not total to $100 \%$ due to rounding. See appendices for source description, methods, and ICD codes, industry and occupation codes, and list of selected states.

SOURCE: National Center for Health Statistics multiple cause of death data. 


\section{Table 7-7. Malignant mesothelioma: Proportionate mortality ratio (PMR) adjusted} for age, sex, and race by usual industry, U.S. residents age 15 and over, selected states, 1999

\begin{tabular}{llrrrr}
\hline CIC & Industry & \multicolumn{2}{c}{$\begin{array}{c}\text { Number } \\
\text { of Deaths }\end{array}$} & PMR & 95\% Confidence Interval \\
\cline { 5 - 6 } 360 & Ship and boat building and repairing & 7 & 5.95 & 2.39 & UCL \\
192 & Industrial and miscellaneous chemicals & 19 & 4.81 & 2.90 & 7.27 \\
200 & Petroleum refining & 5 & 3.80 & 1.23 & 8.87 \\
460 & Electric light and power & 10 & 3.08 & 1.48 & 5.66 \\
060 & Construction & 77 & 1.55 & 1.23 & 1.94 \\
\hline
\end{tabular}

CIC - Census Industry Code $\quad$ n.e.c. - not elsewhere classified $\quad$ LCL - lower confidence limit $\quad$ UCL - upper confidence limit NOTE: See appendices for source description, methods, and ICD codes, industry and occupation codes, and list of selected states. SOURCE: National Center for Health Statistics multiple cause of death data. 
Table 7-8. Malignant mesothelioma: Proportionate mortality ratio (PMR) adjusted for age, sex, and race by usual occupation, U.S. residents age 15 and over, selected states, 1999

\begin{tabular}{llcccc}
\hline COC & Occupation & $\begin{array}{c}\text { Number } \\
\text { of Deaths }\end{array}$ & PMR & \multicolumn{2}{c}{ 95\% Confidence Interval } \\
\hline 585 & Plumbers, pipefitters, and steamfitters & 18 & 4.76 & 2.81 & UCL \\
057 & Mechanical engineers & 6 & 3.04 & 1.11 & 6.51 \\
575 & Electricians & 12 & 2.42 & 1.25 & 4.22 \\
156 & Teachers, elementary school & 13 & 2.13 & 1.13 & 3.64 \\
\hline
\end{tabular}

COC - Census Occupation Code n.e.c. - not elsewhere classified LCL - lower confidence limit UCL - upper confidence limit NOTE: See appendices for source description, methods, and ICD codes, industry and occupation codes, and list of selected states.

SOURCE: National Center for Health Statistics multiple cause of death data. 
Figure 7-3. Malignant mesothelioma: Age-adjusted mortality rates by county, U.S. residents age 15 and over, 1999

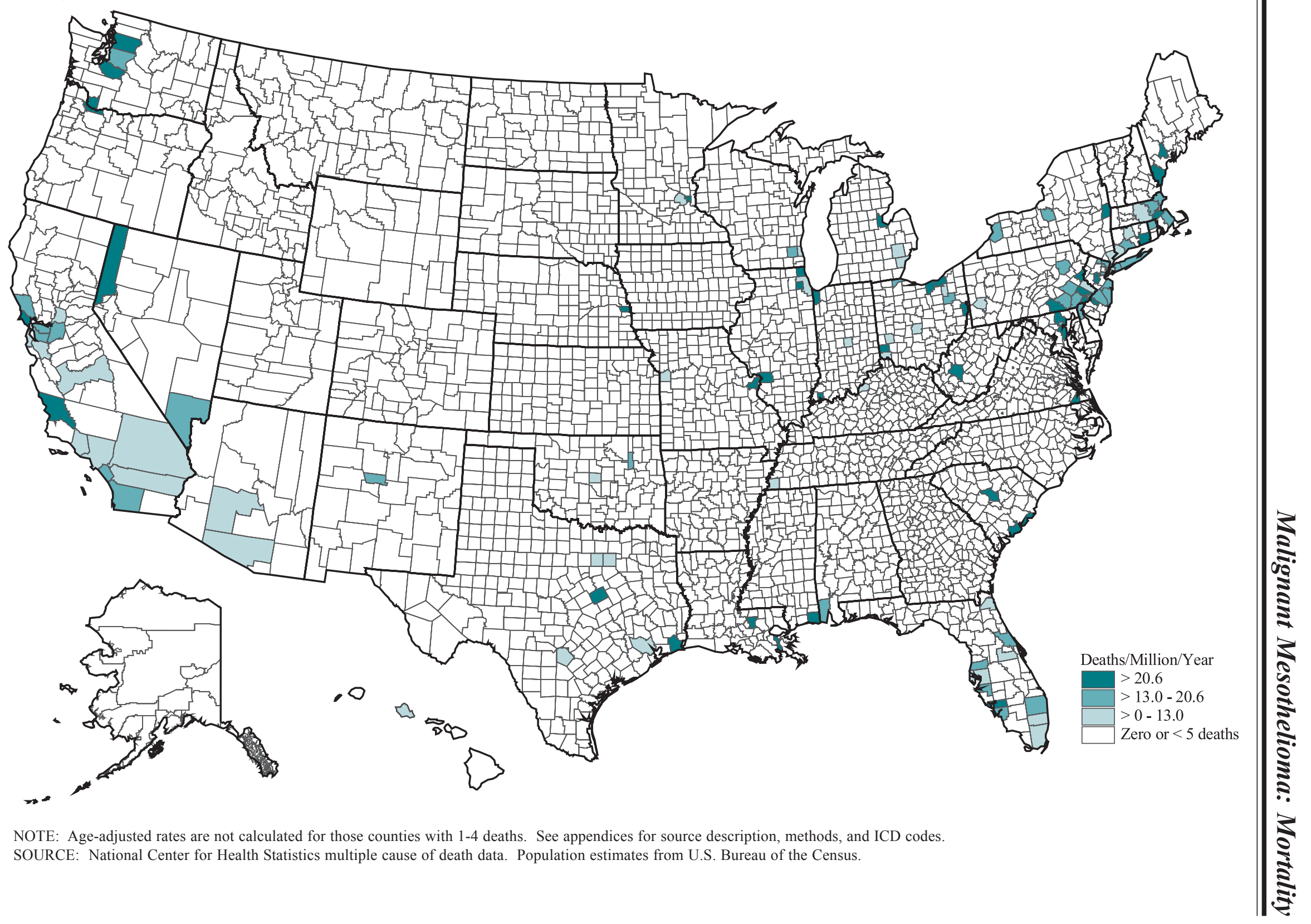


Table 7-9. Malignant mesothelioma: Counties with highest age-adjusted mortality rates (per million population), U.S. residents age 15 and over, 1999

\begin{tabular}{|c|c|c|c|c|c|}
\hline County & State & Age-Adjusted Rate & Crude Rate & Number of Deaths & $\%$ Female \\
\hline Suffolk City & Virginia & 111.7 & 99.5 & 5 & 40.0 \\
\hline Hampton City & Virginia & 95.9 & 82.6 & 9 & 0.0 \\
\hline Jefferson County & Ohio & 90.1 & 115.6 & 7 & 0.0 \\
\hline Androscoggin County & Maine & 82.2 & 85.7 & 7 & 57.1 \\
\hline Somerset County & New Jersey & 73.9 & 64.7 & 15 & 33.3 \\
\hline Jackson County & Mississippi & 70.0 & 48.4 & 5 & 20.0 \\
\hline Bay County & Michigan & 63.1 & 69.3 & 6 & 0.0 \\
\hline Kanawha County & West Virginia & 45.6 & 54.4 & 9 & 22.2 \\
\hline York County & Maine & 42.1 & 42.0 & 6 & 33.3 \\
\hline Rensselaer County & New York & 40.3 & 41.5 & 5 & 40.0 \\
\hline Jefferson Parish & Louisiana & 39.9 & 39.2 & 14 & 42.9 \\
\hline Washoe County & Nevada & 37.8 & 32.0 & 8 & 12.5 \\
\hline Lake County & Illinois & 35.8 & 27.5 & 13 & 30.8 \\
\hline Lorain County & Ohio & 34.1 & 31.7 & 7 & 42.9 \\
\hline Vanderburgh County & Indiana & 33.3 & 37.0 & 5 & 0.0 \\
\hline Norfolk County & Massachusetts & 32.5 & 36.0 & 19 & 0.0 \\
\hline McLennan County & Texas & 31.8 & 31.4 & 5 & 40.0 \\
\hline Delaware County & Pennsylvania & 31.1 & 36.7 & 16 & 25.0 \\
\hline Pierce County & Washington & 30.6 & 26.2 & 14 & 7.1 \\
\hline Douglas County & Nebraska & 30.0 & 25.7 & 9 & 33.3 \\
\hline Jefferson County & Texas & 29.6 & 31.7 & 6 & 33.3 \\
\hline Anne Arundel County & Maryland & 28.2 & 23.8 & 9 & 22.2 \\
\hline San Luis Obispo County & California & 27.2 & 31.4 & 6 & 0.0 \\
\hline Camden County & New Jersey & 26.2 & 26.2 & 10 & 30.0 \\
\hline Madison County & Illinois & 26.1 & 29.3 & 6 & 0.0 \\
\hline Clark County & Washington & 25.8 & 19.3 & 5 & 40.0 \\
\hline Northampton County & Pennsylvania & 25.6 & 28.7 & 6 & 0.0 \\
\hline New London County & Connecticut & 25.5 & 26.1 & 5 & 0.0 \\
\hline Butler County & Ohio & 25.3 & 22.8 & 6 & 33.3 \\
\hline Richland County & South Carolina & 25.0 & 20.0 & 5 & 20.0 \\
\hline Baltimore County & Maryland & 24.7 & 28.9 & 17 & 23.5 \\
\hline Marin County & California & 24.6 & 25.0 & 5 & 40.0 \\
\hline St. Louis County & Missouri & 23.4 & 25.0 & 20 & 20.0 \\
\hline York County & Pennsylvania & 22.7 & 23.3 & 7 & 0.0 \\
\hline Charleston County & South Carolina & 22.7 & 19.6 & 5 & 0.0 \\
\hline Ramsey County & Minnesota & 22.6 & 23.4 & 9 & 11.1 \\
\hline Snohomish County & Washington & 22.2 & 17.3 & 8 & 12.5 \\
\hline Du Page County & Illinois & 21.9 & 18.7 & 13 & 15.4 \\
\hline Charlotte County & Florida & 21.3 & 50.8 & 6 & 50.0 \\
\hline Multnomah County & Oregon & 21.2 & 19.5 & 10 & 0.0 \\
\hline East Baton Rouge Parish & Louisiana & 21.0 & 16.1 & 5 & 20.0 \\
\hline Cuyahoga County & Ohio & 20.7 & 23.6 & 26 & 23.1 \\
\hline Lake County & Indiana & 20.6 & 21.5 & 8 & 37.5 \\
\hline Montgomery County & Pennsylvania & 20.6 & 25.4 & 15 & 6.7 \\
\hline Union County & New Jersey & 19.9 & 22.4 & 9 & 0.0 \\
\hline Manatee County & Florida & 19.8 & 35.1 & 7 & 42.9 \\
\hline Chester County & Pennsylvania & 19.0 & 17.6 & 6 & 33.3 \\
\hline Philadelphia County & Pennsylvania & 19.0 & 21.5 & 24 & 12.5 \\
\hline Waukesha County & Wisconsin & 19.0 & 17.5 & 5 & 0.0 \\
\hline New Castle County & Delaware & 18.8 & 18.0 & 7 & 14.3 \\
\hline Overall United States & & 11.6 & 11.6 & 2,485 & 19.7 \\
\hline
\end{tabular}

NOTE: Only counties with at least 5 deaths from the disease of interest are included. See appendices for source description, methods, and ICD codes. SOURCE: National Center for Health Statistics multiple cause of death data. Population estimates from U.S. Bureau of the Census. 


\section{Section 8}

Hypersensitivity

Pneumonitis 

Figure 8-1. Hypersensitivity pneumonitis: Number of deaths, crude and ageadjusted mortality rates, U.S. residents age 15 and over, 1979-1999

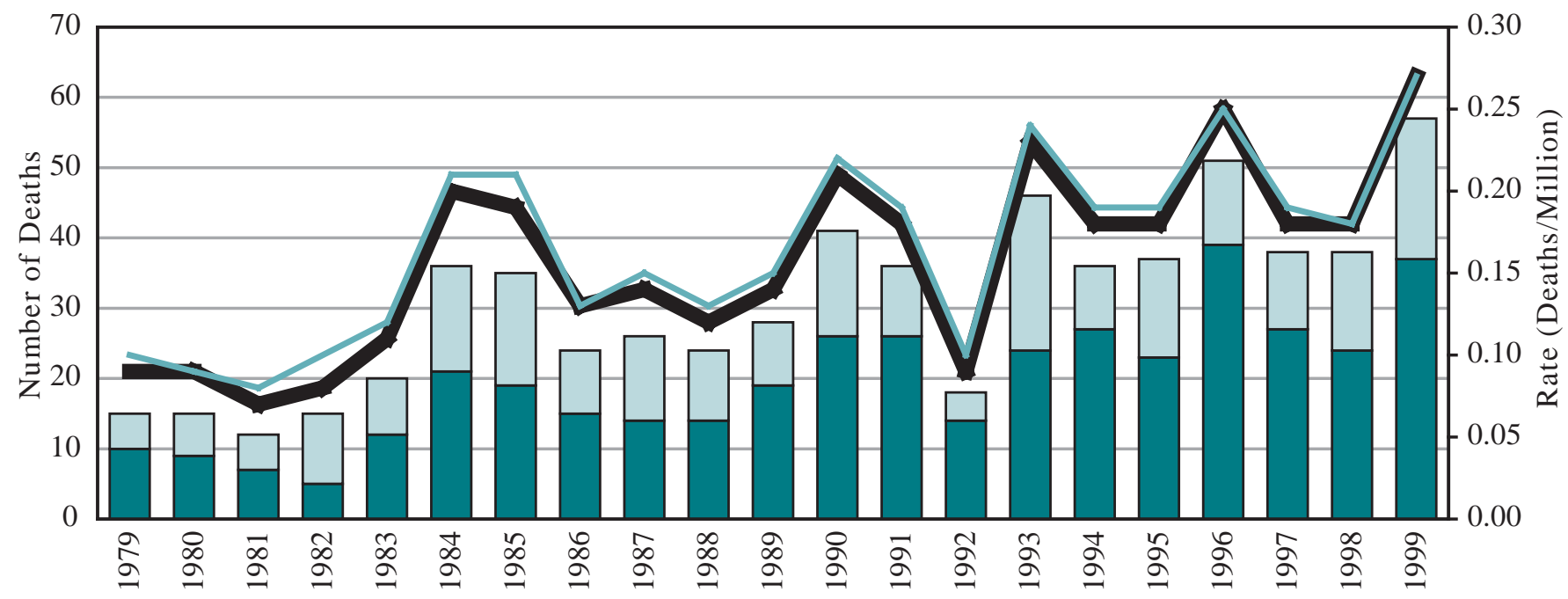

$\square$ Number of deaths, underlying cause U.S. Crude Rate

$\square$ Number of deaths, contributing cause U.S. Age-adjusted Rate

NOTE: See appendices for source description, methods, and ICD codes.

SOURCE: National Center for Health Statistics multiple cause of death data. Population estimates from U.S. Bureau of the Census.

Figure 8-2. Hypersensitivity pneumonitis: Age-adjusted mortality rates by state, U.S. residents age 15 and over, 1990-1999

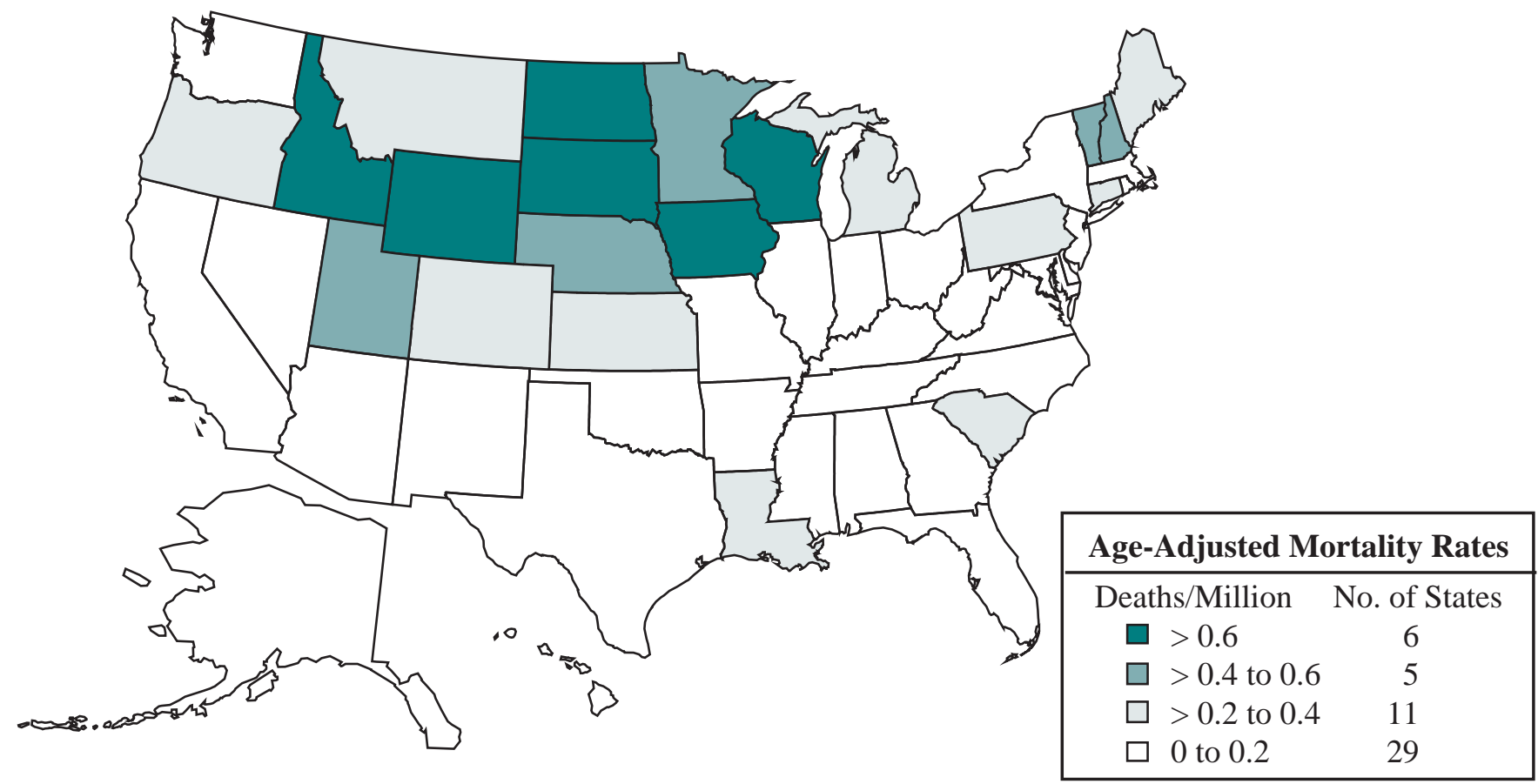

NOTE: See appendices for source description, methods, and ICD codes.

SOURCE: National Center for Health Statistics multiple cause of death data. Population estimates from U.S. Bureau of the Census. 
Table 8-1. Hypersensitivity pneumonitis: Number of deaths by sex, race, and age, and median age at death, U.S. residents age 15 and over, 1990-1999

\begin{tabular}{|c|c|c|c|c|c|c|c|c|c|c|c|c|c|c|c|c|}
\hline \multirow[b]{2}{*}{ Year } & \multirow{2}{*}{$\begin{array}{l}\text { No. of } \\
\text { Deaths }\end{array}$} & \multirow{2}{*}{$\begin{array}{c}\text { Under- } \\
\text { lying } \\
\text { Cause } \\
(\%)\end{array}$} & \multicolumn{2}{|c|}{ Sex } & \multicolumn{3}{|c|}{ Race } & \multicolumn{8}{|c|}{ Age Group (yrs) } & \multirow{2}{*}{$\begin{array}{c}\text { Median } \\
\text { Age } \\
\text { (yrs) }\end{array}$} \\
\hline & & & Male & Female & White & Black & Other & $15-24$ & 25-34 & $35-44$ & 45-54 & $55-64$ & $65-74$ & $75-84$ & $85+$ & \\
\hline 1990 & 41 & 63.4 & 31 & 10 & 38 & 1 & 2 & - & 1 & 1 & 3 & 8 & 11 & 15 & 2 & 73.0 \\
\hline 1991 & 36 & 72.2 & 28 & 8 & 34 & - & 2 & 2 & - & 1 & 2 & 4 & 11 & 11 & 5 & 73.5 \\
\hline 1992 & 18 & 77.8 & 14 & 4 & 17 & 1 & - & - & 1 & 2 & 2 & 2 & 3 & 7 & 1 & 72.5 \\
\hline 1993 & 46 & 52.2 & 31 & 15 & 44 & 1 & 1 & - & 1 & 1 & 4 & 7 & 14 & 14 & 5 & 71.5 \\
\hline 1994 & 36 & 75.0 & 28 & 8 & 36 & - & - & - & - & - & 2 & 5 & 8 & 12 & 9 & 76.5 \\
\hline 1995 & 37 & 62.2 & 24 & 13 & 32 & 5 & - & - & 1 & - & 6 & 4 & 7 & 14 & 5 & 75.0 \\
\hline 1996 & 51 & 76.5 & 35 & 16 & 49 & 2 & - & - & 3 & 3 & 5 & 7 & 11 & 16 & 6 & 73.0 \\
\hline 1997 & 38 & 71.1 & 25 & 13 & 38 & - & - & - & - & 1 & 2 & 6 & 8 & 14 & 7 & 76.0 \\
\hline 1998 & 38 & 63.2 & 31 & 7 & 37 & 1 & - & - & - & - & 2 & 3 & 10 & 17 & 6 & 78.5 \\
\hline 1999 & 57 & 64.9 & 36 & 21 & 56 & 1 & - & - & 3 & 2 & 9 & 5 & 11 & 19 & 8 & 74.0 \\
\hline TOTAL & 398 & 67.1 & 283 & 115 & 381 & 12 & 5 & 2 & 10 & 11 & 37 & 51 & 94 & 139 & 54 & 74.0 \\
\hline
\end{tabular}

- indicates no deaths listed.

NOTE: See appendices for source description, methods, and ICD codes.

SOURCE: National Center for Health Statistics multiple cause of death data. 
Table 8-2. Hypersensitivity pneumonitis: Mortality rates (per million population) by race and sex, U.S. residents age 15 and over, 1990-1999

\begin{tabular}{|c|c|c|c|c|c|c|c|}
\hline \multirow[b]{2}{*}{ Year } & \multirow[b]{2}{*}{ Overall } & \multicolumn{2}{|c|}{ White } & \multicolumn{2}{|c|}{ Black } & \multicolumn{2}{|c|}{ Other } \\
\hline & & Male & Female & Male & Female & Male & Female \\
\hline & \multicolumn{7}{|c|}{ Crude Mortality Rate } \\
\hline 1990 & 0.21 & 0.36 & 0.11 & 0.10 & - & 0.29 & 0.27 \\
\hline 1991 & 0.18 & 0.32 & 0.09 & - & - & 0.55 & - \\
\hline 1992 & 0.09 & 0.16 & 0.05 & 0.09 & - & - & - \\
\hline 1993 & 0.23 & 0.38 & 0.15 & - & 0.08 & - & 0.24 \\
\hline 1994 & 0.18 & 0.34 & 0.09 & - & - & - & - \\
\hline 1995 & 0.18 & 0.26 & 0.11 & 0.18 & 0.23 & - & - \\
\hline 1996 & 0.25 & 0.40 & 0.17 & 0.09 & 0.08 & - & - \\
\hline 1997 & 0.18 & 0.29 & 0.14 & - & - & - & - \\
\hline 1998 & 0.18 & 0.35 & 0.08 & 0.08 & - & - & - \\
\hline 1999 & 0.27 & 0.40 & 0.23 & 0.08 & - & - & - \\
\hline \multirow[t]{2}{*}{ 1990-1999 } & 0.19 & 0.33 & 0.12 & 0.06 & 0.04 & 0.07 & 0.04 \\
\hline & \multicolumn{7}{|c|}{ Age-Adjusted Mortality Rate } \\
\hline 1990 & 0.22 & 0.41 & 0.09 & 0.07 & - & 0.42 & 0.34 \\
\hline 1991 & 0.19 & 0.40 & 0.08 & - & - & 0.95 & - \\
\hline 1992 & 0.10 & 0.18 & 0.05 & 0.20 & - & - & - \\
\hline 1993 & 0.24 & 0.45 & 0.13 & - & 0.11 & - & 0.54 \\
\hline 1994 & 0.19 & 0.43 & 0.08 & - & - & - & - \\
\hline 1995 & 0.19 & 0.33 & 0.10 & 0.31 & 0.28 & - & - \\
\hline 1996 & 0.25 & 0.46 & 0.14 & 0.07 & 0.09 & - & - \\
\hline 1997 & 0.19 & 0.35 & 0.12 & - & - & - & - \\
\hline 1998 & 0.18 & 0.40 & 0.06 & 0.10 & - & - & - \\
\hline 1999 & 0.27 & 0.46 & 0.20 & 0.10 & - & - & - \\
\hline 1990-1999 & 0.20 & 0.39 & 0.11 & 0.09 & 0.05 & 0.12 & 0.08 \\
\hline
\end{tabular}

- indicates no deaths listed.

NOTE: See appendices for source description, methods, and ICD codes.

SOURCE: National Center for Health Statistics multiple cause of death data. Population estimates from U.S. Bureau of the Census. 
Table 8-3. Hypersensitivity pneumonitis: Years of potential life lost to age 65 and to life expectancy by race and sex, U.S. residents age 15 and over, 1990-1999

\begin{tabular}{|c|c|c|c|c|c|c|c|}
\hline \multirow[b]{2}{*}{ Year } & \multicolumn{2}{|c|}{ White } & \multicolumn{2}{|c|}{ Black } & \multicolumn{2}{|c|}{ Other } & \multirow[b]{2}{*}{ Total } \\
\hline & Male & Female & Male & Female & Male & Female & \\
\hline & \multicolumn{7}{|c|}{ Years of Potential Life Lost to Age 65} \\
\hline 1990 & 95 & 5 & 35 & - & 5 & 5 & 145 \\
\hline 1991 & 75 & 45 & - & - & 45 & - & 165 \\
\hline 1992 & 85 & 40 & - & - & - & - & 125 \\
\hline 1993 & 115 & 25 & - & 15 & - & - & 155 \\
\hline 1994 & 40 & 15 & - & - & - & - & 55 \\
\hline 1995 & 85 & 35 & - & 25 & - & - & 145 \\
\hline 1996 & 190 & 65 & 35 & - & - & - & 290 \\
\hline 1997 & 40 & 45 & - & - & - & - & 85 \\
\hline 1998 & 30 & - & 15 & - & - & - & 45 \\
\hline 1999 & 190 & 110 & 15 & - & - & - & 315 \\
\hline \multirow[t]{2}{*}{ TOTAL } & 945 & 385 & 100 & 40 & 50 & 5 & 1,525 \\
\hline & \multicolumn{7}{|c|}{ Years of Potential Life Lost to Life Expectancy } \\
\hline 1990 & 396 & 103 & 38 & - & 21 & 21 & 579 \\
\hline 1991 & 344 & 134 & - & - & 65 & - & 543 \\
\hline 1992 & 218 & 92 & 7 & - & - & - & 317 \\
\hline 1993 & 425 & 196 & - & 28 & - & 8 & 657 \\
\hline 1994 & 301 & 100 & - & - & - & - & 401 \\
\hline 1995 & 280 & 162 & 18 & 70 & - & - & 530 \\
\hline 1996 & 542 & 242 & 39 & 14 & - & - & 837 \\
\hline 1997 & 283 & 202 & - & - & - & - & 485 \\
\hline 1998 & 311 & 78 & 24 & - & - & - & 413 \\
\hline 1999 & 531 & 387 & 24 & - & - & - & 942 \\
\hline TOTAL & 3,631 & 1,696 & 150 & 112 & 86 & 29 & 5,704 \\
\hline
\end{tabular}

- indicates no deaths listed.

NOTE: See appendices for source description, methods, and ICD codes.

SOURCE: National Center for Health Statistics multiple cause of death data. 
Table 8-4. Hypersensitivity pneumonitis: Number of deaths by state, U.S. residents age 15 and over, 1990-1999

\begin{tabular}{|c|c|c|c|c|c|c|c|c|c|c|c|}
\hline State & 1990 & 1991 & 1992 & 1993 & 1994 & 1995 & 1996 & 1997 & 1998 & 1999 & Total \\
\hline Alabama & - & 2 & - & - & - & - & - & 1 & - & - & 3 \\
\hline Alaska & - & - & - & - & - & - & - & - & - & - & - \\
\hline Arizona & - & 1 & 1 & - & - & - & 1 & 2 & - & - & 5 \\
\hline Arkansas & - & - & - & - & 1 & - & - & - & - & - & 1 \\
\hline California & 2 & 1 & 1 & 6 & 3 & 2 & 7 & 7 & 4 & 6 & 39 \\
\hline Colorado & - & 1 & - & 1 & 1 & 1 & - & 1 & - & 1 & 6 \\
\hline Connecticut & 1 & 2 & 1 & - & - & - & 1 & 1 & - & 1 & 7 \\
\hline Delaware & - & - & - & - & 1 & - & - & - & - & - & 1 \\
\hline District of Columbia & - & - & 1 & - & - & - & - & - & - & - & 1 \\
\hline Florida & - & 1 & - & 3 & 5 & 1 & 2 & 1 & 1 & 2 & 16 \\
\hline Georgia & - & - & - & - & 1 & - & - & - & 1 & - & 2 \\
\hline Hawaii & - & - & - & - & - & - & - & - & & - & - \\
\hline Idaho & - & 1 & 1 & - & 1 & - & - & - & 4 & - & 7 \\
\hline Illinois & 1 & 1 & 1 & 2 & 1 & 2 & 1 & - & 2 & 3 & 14 \\
\hline Indiana & 1 & 1 & - & - & 1 & 1 & 1 & 1 & - & 2 & 8 \\
\hline Iowa & 1 & 1 & 1 & 2 & - & 3 & 2 & 3 & 7 & 2 & 22 \\
\hline Kansas & 1 & - & - & - & 2 & 1 & 2 & - & - & 2 & 8 \\
\hline Kentucky & - & 1 & - & 1 & - & 1 & - & - & - & - & 3 \\
\hline Louisiana & 4 & - & - & - & 1 & - & 2 & - & - & - & 7 \\
\hline Maine & - & 1 & - & - & - & - & 1 & 1 & - & 1 & 4 \\
\hline Maryland & 1 & - & 1 & - & 1 & 1 & - & - & - & 1 & 5 \\
\hline Massachusetts & 1 & - & - & 1 & - & - & - & - & - & - & 2 \\
\hline Michigan & 5 & 1 & 2 & - & 2 & 2 & 2 & 2 & 2 & 3 & 21 \\
\hline Minnesota & 2 & 3 & 2 & 1 & 3 & 1 & 2 & 1 & 1 & 3 & 19 \\
\hline Mississippi & - & - & - & - & - & - & - & - & - & - & - \\
\hline Missouri & - & 1 & 1 & 1 & - & - & 2 & - & - & 2 & 7 \\
\hline Montana & - & - & - & - & 1 & - & 1 & - & - & & 2 \\
\hline Nebraska & 1 & 1 & 1 & - & 2 & - & 2 & - & - & 1 & 8 \\
\hline Nevada & - & - & - & - & - & - & - & - & - & - & - \\
\hline New Hampshire & 1 & 1 & - & - & - & 1 & - & - & 1 & - & 4 \\
\hline New Jersey & - & 1 & 1 & - & - & - & 1 & - & - & 2 & 5 \\
\hline New Mexico & - & - & - & - & - & - & - & - & - & - & - \\
\hline New York & 3 & 1 & 1 & 4 & 2 & 3 & 3 & 1 & - & 3 & 21 \\
\hline North Carolina & - & - & - & 1 & 1 & 1 & 1 & 4 & 1 & - & 9 \\
\hline North Dakota & 1 & 1 & - & 1 & - & - & - & 1 & - & - & 4 \\
\hline Ohio & - & 2 & 1 & - & - & 2 & 2 & - & 2 & 3 & 12 \\
\hline Oklahoma & - & 1 & - & 1 & - & - & - & - & - & - & 2 \\
\hline Oregon & 1 & - & - & 1 & 1 & 2 & - & 2 & 2 & - & 9 \\
\hline Pennsylvania & 5 & 1 & - & 3 & 2 & 4 & 4 & 1 & 3 & 1 & 24 \\
\hline Rhode Island & - & - & - & 1 & - & - & - & - & - & - & 1 \\
\hline South Carolina & 1 & - & - & 2 & - & 1 & 1 & - & 1 & 1 & 7 \\
\hline South Dakota & 1 & - & - & 2 & - & - & - & - & 2 & - & 5 \\
\hline Tennessee & - & 1 & - & - & - & - & 1 & - & - & - & 2 \\
\hline Texas & - & 2 & - & 1 & 1 & 2 & 1 & 1 & 1 & 5 & 14 \\
\hline Utah & - & - & - & 1 & 1 & - & - & - & 1 & 2 & 5 \\
\hline Vermont & - & 1 & - & 1 & - & - & - & - & - & - & 2 \\
\hline Virginia & - & - & - & 2 & - & - & 1 & - & - & 1 & 4 \\
\hline Washington & - & - & - & - & - & 2 & - & 1 & - & - & 3 \\
\hline West Virginia & - & - & - & - & - & - & 1 & - & - & - & 1 \\
\hline Wisconsin & 7 & 3 & 1 & 7 & 1 & 3 & 6 & 6 & 2 & 7 & 43 \\
\hline Wyoming & - & 1 & - & - & - & - & - & - & - & 2 & $\mathbf{3}$ \\
\hline TOTAL & 41 & 36 & 18 & 46 & 36 & 37 & 51 & 38 & 38 & 57 & 398 \\
\hline
\end{tabular}

- indicates no deaths listed.

NOTE: See appendices for source description, methods, and ICD codes.

SOURCE: National Center for Health Statistics multiple cause of death data. 
Table 8-5. Hypersensitivity pneumonitis: Number of deaths, mortality rates (per million population), and years of potential life lost (YPLL) by state, U.S. residents age 15 and over, 1990-1999

\begin{tabular}{|c|c|c|c|c|c|c|c|c|c|c|}
\hline \multirow[b]{2}{*}{ State } & \multirow{2}{*}{$\begin{array}{l}\text { No. of } \\
\text { Deaths }\end{array}$} & \multirow[b]{2}{*}{ Rank } & \multicolumn{2}{|c|}{ Crude Mortality } & \multicolumn{2}{|c|}{ Age-Adjusted Mortality } & \multicolumn{4}{|c|}{ YPLL to Life Expectancy } \\
\hline & & & Rate & Rank & Rate & Rank & Total & Rank & YPLL/death & Rank \\
\hline Alabama & 3 & 32 & 0.09 & 37 & 0.10 & 36 & 53 & 30 & 17.7 & 15 \\
\hline Alaska & - & - & - & - & - & - & - & - & - & - \\
\hline Arizona & 5 & 23 & 0.15 & 28 & 0.14 & 31 & 107 & 17 & 21.4 & 4 \\
\hline Arkansas & 1 & 42 & 0.05 & 43 & 0.05 & 43 & 21 & 40 & 21.0 & 6 \\
\hline California & 39 & 2 & 0.16 & 26 & 0.18 & 23 & 751 & 1 & 19.3 & 11 \\
\hline Colorado & 6 & 22 & 0.21 & 21 & 0.25 & 19 & 103 & 19 & 17.2 & 16 \\
\hline Connecticut & 7 & 17 & 0.27 & 17 & 0.27 & 17 & 158 & 12 & 22.6 & 3 \\
\hline Delaware & 1 & 42 & 0.18 & 23 & 0.18 & 23 & 21 & 40 & 21.0 & 6 \\
\hline District of Columbia & 1 & 42 & 0.22 & 20 & 0.24 & 20 & 9 & 45 & 9.0 & 42 \\
\hline Florida & 16 & 8 & 0.14 & 31 & 0.12 & 33 & 220 & 8 & 13.8 & 24 \\
\hline Georgia & 2 & 36 & 0.04 & 45 & 0.05 & 43 & 17 & 43 & 8.5 & 44 \\
\hline Hawaii & - & - & - & - & - & - & - & - & - & - \\
\hline Idaho & 7 & 17 & 0.81 & 6 & 0.80 & 4 & 83 & 26 & 11.9 & 34 \\
\hline Illinois & 14 & 9 & 0.15 & 28 & 0.16 & 27 & 199 & 10 & 14.2 & 21 \\
\hline Indiana & 8 & 14 & 0.18 & 23 & 0.18 & 23 & 106 & 18 & 13.3 & 27 \\
\hline Iowa & 22 & 4 & 0.99 & 2 & 0.84 & 3 & 279 & 5 & 12.7 & 30 \\
\hline Kansas & 8 & 14 & 0.41 & 11 & 0.39 & 13 & 124 & 14 & 15.5 & 19 \\
\hline Kentucky & 3 & 32 & 0.10 & 35 & 0.10 & 36 & 64 & 29 & 21.3 & 5 \\
\hline Louisiana & 7 & 17 & 0.21 & 21 & 0.23 & 21 & 124 & 14 & 17.7 & 14 \\
\hline Maine & 4 & 28 & 0.41 & 11 & 0.40 & 12 & 53 & 30 & 13.3 & 27 \\
\hline Maryland & 5 & 23 & 0.13 & 33 & 0.15 & 28 & 94 & 24 & 18.8 & 13 \\
\hline Massachusetts & 2 & 36 & 0.04 & 45 & 0.04 & 46 & 28 & 36 & 14.0 & 22 \\
\hline Michigan & 21 & 5 & 0.28 & 16 & 0.31 & 15 & 332 & 3 & 15.8 & 18 \\
\hline Minnesota & 19 & 7 & 0.54 & 8 & 0.53 & 8 & 225 & 7 & 11.8 & 36 \\
\hline Mississippi & - & - & - & - & - & - & - & - & - & - \\
\hline Missouri & 7 & 17 & 0.17 & 25 & 0.15 & 28 & 83 & 26 & 11.9 & 34 \\
\hline Montana & 2 & 36 & 0.30 & 15 & 0.27 & 17 & 22 & 39 & 11.0 & 40 \\
\hline Nebraska & 8 & 14 & 0.64 & 7 & 0.57 & 7 & 97 & 22 & 12.1 & 32 \\
\hline Nevada & - & - & - & - & - & - & - & - & - & - \\
\hline New Hampshire & 4 & 28 & 0.44 & 9 & 0.47 & 9 & 48 & 33 & 12.0 & 33 \\
\hline New Jersey & 5 & 23 & 0.08 & 38 & 0.08 & 39 & 120 & 16 & 24.0 & 2 \\
\hline New Mexico & - & - & - & - & - & - & - & - & - & - \\
\hline New York & 21 & 5 & 0.15 & 28 & 0.15 & 28 & 266 & 6 & 12.7 & 31 \\
\hline North Carolina & 9 & 12 & 0.16 & 26 & 0.18 & 23 & 103 & 19 & 11.4 & 38 \\
\hline North Dakota & 4 & 28 & 0.82 & 5 & 0.78 & 5 & 139 & 13 & 34.8 & 1 \\
\hline Ohio & 12 & 11 & 0.14 & 31 & 0.14 & 31 & 165 & 11 & 13.8 & 24 \\
\hline Oklahoma & 2 & 36 & 0.08 & 38 & 0.07 & 41 & 14 & 44 & 7.0 & 46 \\
\hline Oregon & 9 & 12 & 0.37 & 13 & 0.37 & 14 & 100 & 21 & 11.1 & 39 \\
\hline Pennsylvania & 24 & 3 & 0.25 & 18 & 0.22 & 22 & 315 & 4 & 13.1 & 29 \\
\hline Rhode Island & 1 & 42 & 0.13 & 33 & 0.11 & 35 & 8 & 46 & 8.0 & 45 \\
\hline South Carolina & 7 & 17 & 0.25 & 18 & 0.28 & 16 & 93 & 25 & 13.3 & 26 \\
\hline South Dakota & 5 & 23 & 0.91 & 3 & 0.73 & 6 & 51 & 32 & 10.2 & 41 \\
\hline Tennessee & 2 & 36 & 0.05 & 43 & 0.05 & 43 & 38 & 34 & 19.0 & 12 \\
\hline Texas & 14 & 9 & 0.10 & 35 & 0.12 & 33 & 203 & 9 & 14.5 & 20 \\
\hline Utah & 5 & 23 & 0.37 & 13 & 0.44 & 10 & 97 & 22 & 19.4 & 10 \\
\hline Vermont & 2 & 36 & 0.44 & 9 & 0.44 & 10 & 28 & 36 & 14.0 & 22 \\
\hline Virginia & 4 & 28 & 0.08 & 38 & 0.09 & 38 & 82 & 28 & 20.5 & 9 \\
\hline Washington & 3 & 32 & 0.07 & 41 & 0.08 & 39 & 26 & 38 & 8.7 & 43 \\
\hline West Virginia & 1 & 42 & 0.07 & 41 & 0.07 & 41 & 21 & 40 & 21.0 & 6 \\
\hline Wisconsin & 43 & 1 & 1.09 & 1 & 1.05 & 1 & 680 & 2 & 15.8 & 17 \\
\hline Wyoming & 3 & 32 & 0.84 & 4 & 0.94 & 2 & 35 & 35 & 11.7 & 37 \\
\hline
\end{tabular}

- indicates no deaths listed.

NOTE: See appendices for source description, methods, and ICD codes.

SOURCE: National Center for Health Statistics multiple cause of death data. Population estimates from U.S. Bureau of the Census. 
Table 8-6. Hypersensitivity pneumonitis: Most frequently recorded industries on death certificate, U.S. residents age 15 and over, selected states and years, 1990-1999

\begin{tabular}{llrc}
\hline CIC & Industry & Number of Deaths & Percent \\
\hline 011 & Agricultural production, livestock & 30 & 24.6 \\
010 & Agricultural production, crops & 22 & 18.0 \\
961 & Non-paid worker or non-worker or own home/at home & 13 & 10.7 \\
060 & Construction & 4 & 3.3 \\
410 & Trucking service & 4 & 3.3 \\
831 & Hospitals & 4 & 3.3 \\
142 & Yarn, thread, and fabric mills & 3 & 2.5 \\
630 & Apparel and accessory stores, except shoe & 3 & 2.5 \\
601 & Grocery stores & 2 & 1.6 \\
842 & Elementary and secondary schools & 2 & 1.6 \\
850 & Colleges and universities & 2 & 1.6 \\
942 & Military & 2 & 1.6 \\
& All other industries & 23 & 18.9 \\
& Industry not reported & 8 & 6.6 \\
& TOTAL & $\mathbf{1 2 2}$ & $\mathbf{1 0 0 . 0}$ \\
\hline
\end{tabular}

Table 8-7. Hypersensitivity pneumonitis: Most frequently recorded occupations on death certificate, U.S. residents age 15 and over, selected states and years, 1990-1999

\begin{tabular}{clrc}
\hline COC & Occupation & Number of Deaths & Percent \\
\hline 473 & Farmers, except horticulture & 51 & 41.8 \\
914 & Housewife/Homemaker & 12 & 9.8 \\
019 & Managers and administrators, n.e.c. & 4 & 3.3 \\
633 & Supervisors, production occupations & 3 & 2.5 \\
804 & Truck drivers & 3 & 2.5 \\
095 & Registered nurses & 2 & 1.6 \\
243 & Supervisors and proprietors, sales occupations & 2 & 1.6 \\
637 & Machinists & 2 & 1.6 \\
889 & Laborers, except construction & 2 & 1.6 \\
905 & Military occupations & 2 & 1.6 \\
& All other occupations & 32 & 26.2 \\
& Occupation not reported & 7 & 5.7 \\
& TOTAL & $\mathbf{1 2 2}$ & $\mathbf{1 0 0 . 0}$ \\
\hline
\end{tabular}

COC - Census Occupation Code n.e.c. - not elsewhere classified

NOTE: Percentages may not total to $100 \%$ due to rounding. See appendices for source description, methods, and ICD codes, industry and occupation codes, and list of selected states and years.

SOURCE: National Center for Health Statistics multiple cause of death data. 
Table 8-8. Hypersensitivity pneumonitis: Proportionate mortality ratio (PMR) adjusted for age, sex, and race by usual industry, U.S. residents age 15 and over, selected states and years, 1990-1999

\begin{tabular}{llrrrr}
\hline & & Number & & \multicolumn{2}{c}{ 95\% Confidence Interval } \\
\cline { 5 - 6 } CIC & Industry & of Deaths & PMR & LCL & UCL \\
\hline 011 & Agricultural production, livestock & 30 & 17.61 & 11.90 & 25.15 \\
010 & Agricultural production, crops & 22 & 4.44 & 2.78 & 6.73 \\
\hline
\end{tabular}

CIC - Census Industry Code n.e.c. - not elsewhere classified

LCL - lower confidence limit

UCL - upper confidence limit

NOTE: See appendices for source description, methods, and ICD codes, industry and occupation codes, and list of selected states and years.

SOURCE: National Center for Health Statistics multiple cause of death data. 
Table 8-9. Hypersensitivity pneumonitis: Proportionate mortality ratio (PMR) adjusted for age, sex, and race by usual occupation, U.S. residents age 15 and over, selected states and years, 1990-1999

\begin{tabular}{llccccc}
\hline COC & Occupation & $\begin{array}{c}\text { Number } \\
\text { of Deaths }\end{array}$ & PMR & \multicolumn{2}{c}{ LCL Confidence Interval } \\
\cline { 5 - 7 } 473 & Farmers, except horticulture & 51 & 8.51 & 6.42 & UCL \\
\hline
\end{tabular}

COC - Census Occupation Code n.e.c. - not elsewhere classified $\quad$ LCL - lower confidence limit UCL - upper confidence limit NOTE: See appendices for source description, methods, and ICD codes, industry and occupation codes, and list of selected states and years. SOURCE: National Center for Health Statistics multiple cause of death data. 
$\lesssim$ Figure 8-3. Hypersensitivity pneumonitis: Age-adjusted mortality rates by county, U.S. residents age 15 and over, 1980-1999

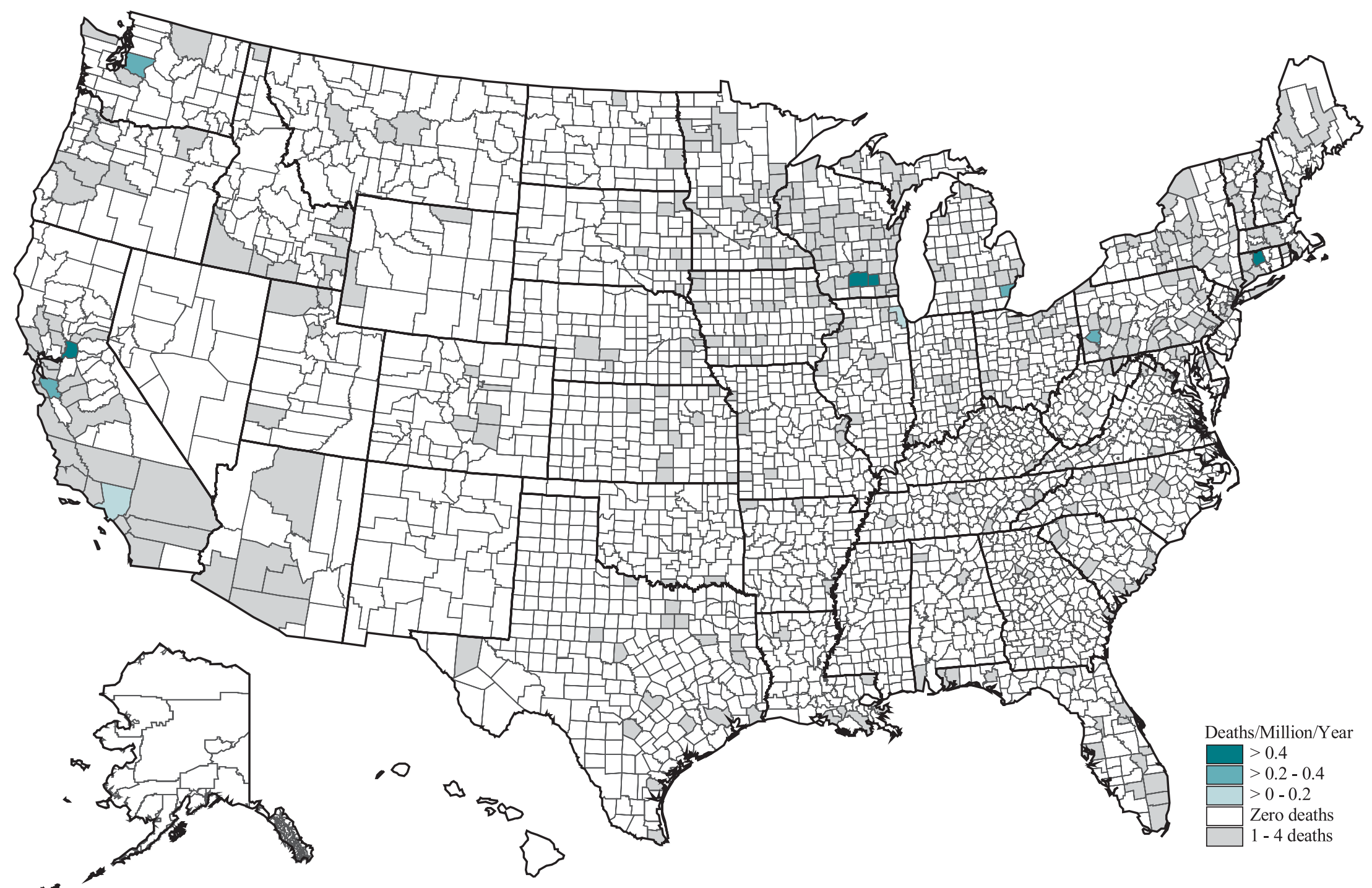

NOTE: Age-adjusted rates are not calculated for those counties with 1-4 deaths. See appendices for source description, methods, and ICD codes.

SOURCE: National Center for Health Statistics multiple cause of death data. Population estimates from U.S. Bureau of the Census. 
Table 8-10. Hypersensitivity pneumonitis: Counties with highest age-adjusted mortality rates (per million population), U.S. residents age 15 and over, 1985-1999

\begin{tabular}{llccrr}
\hline County & State & Age-Adjusted Rate & Crude Rate & Number of Deaths & \% Female \\
\hline Jefferson County & Wisconsin & 6.9 & 7.4 & 6 & 0.0 \\
Dane County & Wisconsin & 1.5 & 1.1 & 5 & 0.0 \\
Hartford County & Connecticut & 0.7 & 0.7 & 7 & 42.9 \\
Sacramento County & California & 0.5 & 0.5 & 6 & 33.3 \\
Wayne County & Michigan & 0.3 & 0.2 & 6 & 5 \\
Allegheny County & Pennsylvania & 0.2 & 0.3 & 11 & 6.0 \\
Los Angeles County & California & 0.1 & 0.1 & 54.0 & 5 \\
Cook County & Illinois & 0.1 & $\mathbf{0 . 2}$ & $\mathbf{5 3 5}$ & 50.0 \\
\hline Overall United States & & $\mathbf{0 . 2}$ & & $\mathbf{2 9 . 9}$ \\
\hline
\end{tabular}

NOTE: Only counties with at least 5 deaths from the disease of interest are included. See appendices for source description, methods, and ICD codes. SOURCE: National Center for Health Statistics multiple cause of death data. Population estimates from U.S. Bureau of the Census. 



\section{Section 9}

Asthma 

Table 9-1. Asthma: Proportionate mortality ratio (PMR) adjusted for age, sex, and race by usual industry, U.S. residents age 15 and over, selected states and years, 1990-1999

\begin{tabular}{llrrrr}
\hline & & \multicolumn{2}{c}{$\begin{array}{c}\text { Number } \\
\text { OIC }\end{array}$} & Industry & 95\% Confidence Interval \\
\cline { 4 - 6 } & Peaths & PMR & LCL & UCL \\
\hline 11 & Agricultural production, livestock & 272 & 1.51 & 1.34 & 1.71 \\
311 & Farm machinery and equipment & 28 & 1.51 & 1.01 & 2.18 \\
862 & Child day care services & 68 & 1.40 & 1.09 & 1.78 \\
642 & Drug stores & 83 & 1.32 & 1.06 & 1.64 \\
840 & Health services, n.e.c. & 228 & 1.29 & 1.13 & 1.47 \\
850 & Colleges and universities & 268 & 1.25 & 1.11 & 1.41 \\
812 & Offices and clinics of physicians & 119 & 1.21 & 1.01 & 1.45 \\
771 & Laundry, cleaning, and garment services & 177 & 1.17 & 1.01 & 1.36 \\
831 & Hospitals & 1,102 & 1.14 & 1.07 & 1.21 \\
901 & General government, n.e.c. & 498 & 1.13 & 1.03 & 1.23 \\
010 & Agricultural production, crops & 684 & 1.10 & 1.02 & 1.18 \\
961 & Non-paid worker or non-worker or own home/at home & 9,047 & 1.06 & 1.04 & 1.08 \\
\hline
\end{tabular}

CIC - Census Industry Code $\quad$ n.e.c. - not elsewhere classified $\quad$ LCL - lower confidence limit UCL - upper confidence limit NOTE: See appendices for source description, methods, and ICD codes, industry and occupation codes, and list of selected states and years. SOURCE: National Center for Health Statistics multiple cause of death data. 
Table 9-2. Asthma: Proportionate mortality ratio (PMR) adjusted for age, sex, and race by usual occupation, U.S. residents age 15 and over, selected states and years, 1990-1999

\begin{tabular}{|c|c|c|c|c|c|}
\hline \multirow[b]{2}{*}{ COC } & \multirow[b]{2}{*}{ Occupation } & \multirow{2}{*}{$\begin{array}{c}\text { Number } \\
\text { of Deaths }\end{array}$} & \multirow[b]{2}{*}{ PMR } & \multicolumn{2}{|c|}{ 95\% Confidence Interval } \\
\hline & & & & LCL & UCL \\
\hline 098 & Respiratory therapists & 13 & 3.11 & 1.65 & 5.31 \\
\hline 169 & Social scientists, n.e.c. & 8 & 2.88 & 1.24 & 5.67 \\
\hline 758 & Compressing and compacting machine operators & 9 & 2.78 & 1.28 & 5.28 \\
\hline 028 & Purchasing agents and buyers, farm products & 10 & 2.21 & 1.06 & 4.06 \\
\hline 089 & Health diagnosing practitioners, n.e.c. & 11 & 2.20 & 1.10 & 3.94 \\
\hline 197 & Public relations specialists & 17 & 2.09 & 1.21 & 3.34 \\
\hline 317 & Hotel clerks & 13 & 2.02 & 1.07 & 3.45 \\
\hline 085 & Dentists & 25 & 1.84 & 1.19 & 2.72 \\
\hline 154 & Postsecondary teachers, subject not specified & 61 & 1.59 & 1.23 & 2.06 \\
\hline 203 & Clinical laboratory technologists and technicians & 47 & 1.43 & 1.05 & 1.90 \\
\hline 186 & Musicians and composers & 47 & 1.43 & 1.05 & 1.90 \\
\hline 446 & Health aides, except nursing & 52 & 1.39 & 1.05 & 1.85 \\
\hline 015 & Managers, medicine and health & 46 & 1.37 & 1.00 & 1.83 \\
\hline 686 & Butchers and meat cutters & 64 & 1.33 & 1.04 & 1.72 \\
\hline 915 & Student & 313 & 1.29 & 1.15 & 1.44 \\
\hline 055 & Electrical and electronic engineers & 69 & 1.28 & 1.00 & 1.63 \\
\hline 157 & Teachers, secondary school & 86 & 1.26 & 1.01 & 1.56 \\
\hline 473 & Farmers, except horticulture & 791 & 1.21 & 1.13 & 1.30 \\
\hline 917 & Unemployed, never worked, disabled & 599 & 1.19 & 1.10 & 1.29 \\
\hline 207 & Licensed practical nurses & 152 & 1.18 & 1.01 & 1.39 \\
\hline 095 & Registered nurses & 455 & 1.17 & 1.07 & 1.29 \\
\hline 447 & Nursing aides, orderlies, and attendants & 461 & 1.15 & 1.05 & 1.26 \\
\hline 914 & Housewife/Homemaker & 8,145 & 1.04 & 1.02 & 1.06 \\
\hline
\end{tabular}

COC - Census Occupation Code n.e.c. - not elsewhere classified LCL - lower confidence limit UCL - upper confidence limit NOTE: See appendices for source description, methods, and ICD codes, industry and occupation codes, and list of selected states and years. SOURCE: National Center for Health Statistics multiple cause of death data. 
Work-Related Asthma: SENSOR

Table 9-3. Work-related asthma: Number of cases by classification and state, 1993-1999

\begin{tabular}{|c|c|c|c|c|c|c|c|c|c|c|}
\hline \multirow[b]{2}{*}{ Classification } & \multicolumn{2}{|c|}{ California } & \multicolumn{2}{|c|}{ Massachusetts } & \multicolumn{2}{|c|}{ Michigan } & \multicolumn{2}{|c|}{ New Jersey } & \multicolumn{2}{|c|}{ Total } \\
\hline & No. & $\%$ & No. & $\%$ & No. & $\%$ & No. & $\%$ & No. & $\%$ \\
\hline Work-aggravated asthma* & 328 & 35.7 & 34 & 9.2 & 124 & 11.7 & 22 & 12.2 & 508 & 20.1 \\
\hline New-onset asthma ${ }^{\dagger}$ & 590 & 64.2 & 334 & 90.7 & 936 & 88.3 & 158 & 87.7 & 2,018 & 79.9 \\
\hline Reactive airways dysfunction syndrome (RADS) & 60 & 6.5 & 34 & 9.2 & 97 & 9.2 & 47 & 26.1 & 238 & 9.4 \\
\hline Occupational asthma $(\mathrm{OA})$ & 530 & 57.7 & 300 & 81.5 & 839 & 79.1 & 111 & 61.6 & 1,780 & 70.5 \\
\hline Known asthma inducer ${ }^{*}$ with objective evidence & - & - & - & - & 19 & 1.8 & 2 & 1.1 & 21 & 0.8 \\
\hline Known asthma inducer with no objective evidence & 91 & 9.9 & 104 & 28.3 & 382 & 36.0 & 47 & 26.1 & 624 & 24.7 \\
\hline Other & 439 & 47.8 & 196 & 53.2 & 438 & 41.3 & 62 & 34.4 & 1,135 & 44.9 \\
\hline TOTAL & 918 & 100.0 & 368 & 100.0 & 1,060 & 100.0 & 180 & 100.0 & 2,526 & 100.0 \\
\hline
\end{tabular}

- indicates no cases reported.

* Pre-existing asthma aggravated by exposure or condition at work.

$\dagger$ Includes cases of RADS and OA.

$\$$ Known asthma inducers, defined by medical literature review, are designated in the Association of Occupational and Environmental Clinics (AOEC) exposure coding scheme (www.aoec.org/aoeccode.htm).

.NOTE: Percentages may not sum to $100 \%$ due to rounding. See appendices for source description.

SOURCE: Provisional SENSOR surveillance data as of September 2002, aggregated by reporting source years, and reported by R Harrison and J Flattery (California); L Davis, E Pechter, and B Pazos (Massachusetts); K Rosenman, MJ Reilly, and D Kalinowski (Michigan); and D Valiante and D Schill (New Jersey). 
Table 9-4. Work-related asthma: Number of cases by ascertainment source and state, 1993-1999

\begin{tabular}{|c|c|c|c|c|c|c|c|c|c|}
\hline \multirow[b]{2}{*}{ Source } & California & \multicolumn{2}{|c|}{ Massachusetts } & \multicolumn{2}{|c|}{ Michigan } & \multicolumn{2}{|c|}{ New Jersey } & \multicolumn{2}{|c|}{ Total } \\
\hline & No. & No. & $\%$ & No. & $\%$ & No. & $\%$ & No. & $\%$ \\
\hline Physicia & $918 \quad 100.0$ & 363 & 98.6 & 806 & 76.0 & 145 & 80.6 & 2,232 & 88.4 \\
\hline $\mathrm{Ho}$ & - & 5 & 1.4 & 231 & 21.8 & 34 & 18.9 & 270 & 10.7 \\
\hline (athe & - & - & & 23 & 2.2 & 1 & 0.6 & 24 & 1.0 \\
\hline Total & 918100.0 & 368 & 100.0 & 1,060 & 100.0 & 180 & 100.0 & 2,526 & 100.0 \\
\hline
\end{tabular}

- indicates no cases reported.

* Massachusetts, Michigan, and New Jersey actively solicit reports from physicians; California uses an existing administrative data source to passively identify physician reports of work-related asthma.

$\dagger$ Some cases have been identified through workers' compensation data, screening co-workers, reports from OSHA or MSHA, etc.

NOTE: Percentages may not sum to $100 \%$ due to rounding. See appendices for source description.

SOURCE: Provisional SENSOR surveillance data as of September 2002, aggregated by reporting source years, and reported by R Harrison and J Flattery (California); L Davis, E Pechter, and B Pazos (Massachusetts); K Rosenman, MJ Reilly, and D Kalinowski (Michigan); and D Valiante and D Schill (New Jersey). 
Table 9-5 (page 1 of 2). Work-related asthma: Most frequently reported putative agents associated with work-related asthma cases by state, 1993-1999

\begin{tabular}{|c|c|c|c|c|c|c|c|c|c|c|c|}
\hline \multirow[b]{2}{*}{ Code* } & \multirow[b]{2}{*}{ Agent } & \multicolumn{2}{|c|}{ California } & \multicolumn{2}{|c|}{ Massachusetts } & \multicolumn{2}{|c|}{ Michigan } & \multicolumn{2}{|c|}{ New Jersey } & \multicolumn{2}{|c|}{ Total } \\
\hline & & No. & $\%$ & No. & $\%$ & No. & $\%$ & No. & $\%$ & No. & $\%$ \\
\hline 320.06 & Chemicals, n.o.s. & 108 & 11.8 & 32 & 8.7 & 87 & 8.2 & 20 & 11.1 & 247 & 9.8 \\
\hline 320.01 & Air pollutants, indoor & 66 & 7.2 & 76 & 20.7 & 64 & 6.0 & 22 & 12.2 & 228 & 9.0 \\
\hline 010.00 & Dust, n.o.s. & 116 & 12.6 & 37 & 10.1 & 21 & 2.0 & 9 & 5.0 & 183 & 7.2 \\
\hline 322.00 & Cleaning materials, n.o.s. & 63 & 6.9 & 33 & 9.0 & 64 & 6.0 & 6 & 3.3 & 166 & 6.6 \\
\hline 170.01 & Cutting oils & - & - & 6 & 1.6 & 126 & 11.9 & 2 & 1.1 & 134 & 5.3 \\
\hline 330.03 & Smoke, n.o.s. ${ }^{\dagger}$ & 47 & 5.1 & 19 & 5.2 & 47 & 4.3 & 5 & 2.8 & 118 & 4.7 \\
\hline 171.01 & Paint & 48 & 5.2 & 14 & 3.8 & 45 & 4.2 & 3 & 1.7 & 110 & 4.4 \\
\hline 270.02 & Latex, natural rubber & 16 & 1.7 & 38 & 10.3 & 40 & 3.8 & 6 & 3.3 & 100 & 4.0 \\
\hline 221.00 & Diisocyanates, n.o.s. & 9 & 1.0 & 14 & 3.8 & 69 & 6.5 & 2 & 1.1 & 94 & 3.7 \\
\hline 390.01 & Mold, n.o.s. & 54 & 5.9 & 36 & 9.8 & - & - & 4 & 2.2 & 94 & 3.7 \\
\hline 171.00 & Solvents, n.o.s. & 11 & 1.2 & 17 & 4.6 & 36 & 3.4 & 10 & 5.6 & 74 & 2.9 \\
\hline 023.01 & Welding fume, stainless steel & 1 & 0.1 & - & - & 72 & 6.8 & - & - & 73 & 2.9 \\
\hline 120.03 & Formaldehyde & 17 & 1.9 & 19 & 5.2 & 26 & 2.5 & 5 & 2.8 & 67 & 2.7 \\
\hline 221.01 & Toluene diisocyanate & 9 & 1.0 & 4 & 1.1 & 50 & 4.7 & 2 & 1.1 & 65 & 2.6 \\
\hline 320.16 & Pesticides, n.o.s. & 42 & 4.6 & 7 & 1.9 & 5 & 0.5 & 1 & 0.6 & 55 & 2.2 \\
\hline 320.11 & Glues, n.o.s. & 24 & 2.6 & 10 & 2.7 & 14 & 1.3 & 5 & 2.8 & 53 & 2.1 \\
\hline 322.10 & Bleach & 15 & 1.6 & 7 & 1.7 & 23 & 2.2 & 3 & 1.7 & 48 & 1.9 \\
\hline 221.02 & Methylene diisocyanate & 2 & 0.2 & - & - & 41 & 3.9 & 2 & 1.1 & 45 & 1.8 \\
\hline 030.02 & Chlorine & 17 & 1.9 & 1 & 0.3 & 18 & 1.7 & 5 & 2.8 & 41 & 1.6 \\
\hline 010.09 & Man-made mineral fibers & 19 & 2.1 & 14 & 3.8 & 7 & 0.7 & - & - & 40 & 1.6 \\
\hline 330.01 & Cigarette smoke & 22 & 2.4 & 6 & 1.6 & 8 & 0.8 & 1 & 0.6 & 37 & 1.5 \\
\hline 331.01 & Diesel exhaust & 13 & 1.4 & 4 & 1.1 & 10 & 0.9 & 9 & 5.0 & 36 & 1.4 \\
\hline 120.05 & Glutaraldehyde & 9 & 1.0 & 11 & 3.0 & 4 & 0.4 & 7 & 3.9 & 31 & 1.2 \\
\hline 320.23 & Perfume, n.o.s. & 28 & 3.1 & 2 & 0.5 & - & - & - & - & 30 & 1.2 \\
\hline 373.00 & Wood dust, n.o.s. & 9 & 1.0 & 3 & 0.8 & 15 & 1.4 & 3 & 1.7 & 30 & 1.2 \\
\hline 050.00 & Acids, bases, oxidizers, n.o.s. & 1 & 0.1 & 5 & 1.4 & 21 & 2.0 & 2 & 1.1 & 29 & 1.1 \\
\hline 023.00 & Welding, n.o.s. & 9 & 1.0 & 10 & 2.7 & 2 & 0.2 & 8 & 4.4 & 28 & 1.1 \\
\hline 330.02 & Plastic smoke & 9 & 1.0 & 1 & 0.3 & 16 & 1.5 & 2 & 1.1 & 28 & 1.1 \\
\hline 010.02 & Asbestos & 13 & 1.4 & 9 & 2.4 & 4 & 0.4 & - & - & 26 & 1.0 \\
\hline 110.02 & Epoxy resins & 2 & 0.2 & 5 & 1.4 & 18 & 1.7 & 1 & 0.6 & 26 & 1.0 \\
\hline 320.33 & Air pollutants, indoor-building renovation & 25 & 2.7 & - & - & - & - & - & - & 25 & 1.0 \\
\hline 322.19 & Cleaners, disinfectant, n.o.s. & 17 & 1.9 & 6 & 1.6 & 2 & 0.2 & - & - & 25 & 1.0 \\
\hline 060.11 & 4-Phenylcyclohexene (4-PC) & 20 & 2.2 & 4 & 1.1 & - & - & - & - & 24 & 1.0 \\
\hline 142.00 & Acrylates, n.o.s. & - & - & - & - & 21 & 2.0 & - & - & 21 & 0.8 \\
\hline
\end{tabular}

See footnotes at end of table. 
Table 9-5 (page 2 of 2). Work-related asthma: Most frequently reported putative agents associated with work-related asthma cases by state, 1993-1999

\begin{tabular}{|c|c|c|c|c|c|c|c|c|c|c|c|}
\hline \multirow[b]{2}{*}{ Code* } & \multirow[b]{2}{*}{ Agent } & \multicolumn{2}{|c|}{ California } & \multicolumn{2}{|c|}{ Massachusetts } & \multicolumn{2}{|c|}{ Michigan } & \multicolumn{2}{|c|}{ New Jersey } & \multicolumn{2}{|c|}{ Total } \\
\hline & & No. & $\%$ & No. & $\%$ & No. & $\%$ & No. & $\%$ & No. & $\%$ \\
\hline 370.01 & Paper dust & 9 & 1.0 & 2 & 0.5 & 2 & 0.2 & 7 & 3.9 & 20 & 0.8 \\
\hline 010.03 & Cement dust & 11 & 1.2 & 3 & 0.8 & 5 & 0.5 & - & - & 19 & 0.8 \\
\hline 322.16 & Cleaners, carpet & 12 & 1.3 & - & - & 6 & 0.6 & 1 & 0.6 & 19 & 0.8 \\
\hline 322.21 & Cleaners, floor stripping & 5 & 0.5 & 2 & 0.5 & 9 & 0.8 & 2 & 1.1 & 18 & 0.7 \\
\hline 370.00 & Plant material, n.o.s. & 15 & 1.6 & 2 & 0.5 & 1 & 0.1 & - & - & 18 & 0.7 \\
\hline 380.00 & Animal material, n.o.s. & 8 & 0.9 & 3 & 0.8 & 6 & 0.6 & 1 & 0.6 & 18 & 0.7 \\
\hline 331.00 & Exhaust, n.o.s. & 11 & 1.2 & 4 & 1.1 & - & - & 2 & 1.1 & 17 & 0.7 \\
\hline 161.00 & Polycyclic aromatic hydrocarbons, n.o.s. & 14 & 1.5 & - & - & 2 & 0.2 & - & - & 16 & 0.6 \\
\hline 331.02 & Engine exhaust & 4 & 0.4 & 1 & 0.3 & 5 & 0.5 & 6 & 3.3 & 16 & 0.6 \\
\hline 050.24 & Sulfuric acid & 8 & 0.9 & - & - & 4 & 0.4 & 2 & 1.1 & 14 & 0.6 \\
\hline 160.02 & Toluene & 1 & 0.1 & 4 & 1.1 & 3 & 0.3 & 6 & 3.3 & 14 & 0.6 \\
\hline 322.07 & Ammonia solution, n.o.s. & 9 & 1.0 & 3 & 0.8 & 1 & 0.1 & 1 & 0.6 & 14 & 0.6 \\
\hline 350.03 & Heat & 11 & 1.2 & - & - & 3 & 0.3 & - & - & 14 & 0.6 \\
\hline 370.10 & Pollen & 14 & 1.5 & - & - & - & - & - & - & 14 & 0.6 \\
\hline 050.18 & Sodium hydroxide & 1 & 0.1 & 3 & 0.8 & 6 & 0.6 & 2 & 1.1 & 12 & 0.5 \\
\hline 052.01 & Ammonia gas & - & - & - & - & 10 & 0.9 & 2 & 1.1 & 12 & 0.5 \\
\hline 231.00 & Ethanolamines, n.o.s. & 9 & 1.0 & - & - & 3 & 0.3 & - & - & 12 & 0.5 \\
\hline 320.29 & Printing chemicals, n.o.s. & - & - & 4 & 1.1 & 8 & 0.8 & - & - & 12 & 0.5 \\
\hline 371.00 & Flour, n.o.s. & 2 & 0.2 & 4 & 1.1 & 4 & 0.4 & 2 & 1.1 & 12 & 0.5 \\
\hline 380.04 & Dander, animal & 11 & 1.2 & 1 & 0.3 & - & - & - & - & 12 & 0.5 \\
\hline 040.20 & Sulfur oxides & 4 & 0.4 & 2 & 0.5 & 3 & 0.3 & 2 & 1.1 & 11 & 0.4 \\
\hline 050.10 & Hydrochloric acid & 4 & 0.4 & 2 & 0.5 & 1 & 0.1 & 4 & 2.2 & 11 & 0.4 \\
\hline 171.06 & Paint, oil-based & 9 & 1.0 & 2 & 0.5 & - & - & - & - & 11 & 0.4 \\
\hline 221.04 & Hexamethylene diisocyanate & - & - & - & - & 11 & 1.0 & - & - & 11 & 0.4 \\
\hline 320.17 & Photo developing chemicals, n.o.s. & 6 & 0.7 & 2 & 0.5 & 2 & 0.2 & 1 & 0.6 & 11 & 0.4 \\
\hline 360.04 & Stress & 10 & 1.1 & - & - & 1 & 0.1 & - & - & 11 & 0.4 \\
\hline 010.13 & Silica, crystalline & 7 & 0.8 & 1 & 0.3 & 1 & 0.1 & 1 & 0.6 & 10 & 0.4 \\
\hline 021.00 & Metal dust, n.o.s. & 8 & 0.9 & 1 & 0.3 & - & - & 1 & 0.6 & 10 & 0.4 \\
\hline 061.00 & Petroleum fractions, n.o.s. & 3 & 0.3 & - & - & 5 & 0.5 & 2 & 1.1 & 10 & 0.4 \\
\hline 320.14 & Lubricants, n.o.s. & 2 & 0.2 & 1 & 0.3 & 5 & 0.5 & 2 & 1.1 & 10 & 0.4 \\
\hline & All others & 324 & 35.3 & 139 & 37.8 & 137 & 12.9 & 107 & 59.4 & 707 & 28.0 \\
\hline
\end{tabular}

- indicates no cases reported. n.o.s. - not otherwise specified

* Association of Occupational and Environmental Clinics (AOEC) exposure codes, 12/2000

$\dagger$ Smoke includes pyrolysis products other than incinerator fume or cigarette, plastic, marijuana, or lead-containing smoke.

NOTE: Number column sums exceed the corresponding number of cases because each case was associated with up to three putative agents. Percentages are based on the actual number of cases; refer to Table 9-3 for actual number of cases. See appendices for source description and methods.

SOURCE: Provisional SENSOR surveillance data as of September 2002, aggregated by reporting source years, and reported by R Harrison and J Flattery (California); L Davis, E Pechter, and B Pazos (Massachusetts); K Rosenman, MJ Reilly, and D Kalinowski (Michigan); and D Valiante and D Schill (New Jersey). 


\section{Figure 9-1. Work-related asthma: Most frequently reported agent categories associated with cases of work-related asthma, 1993-1999}

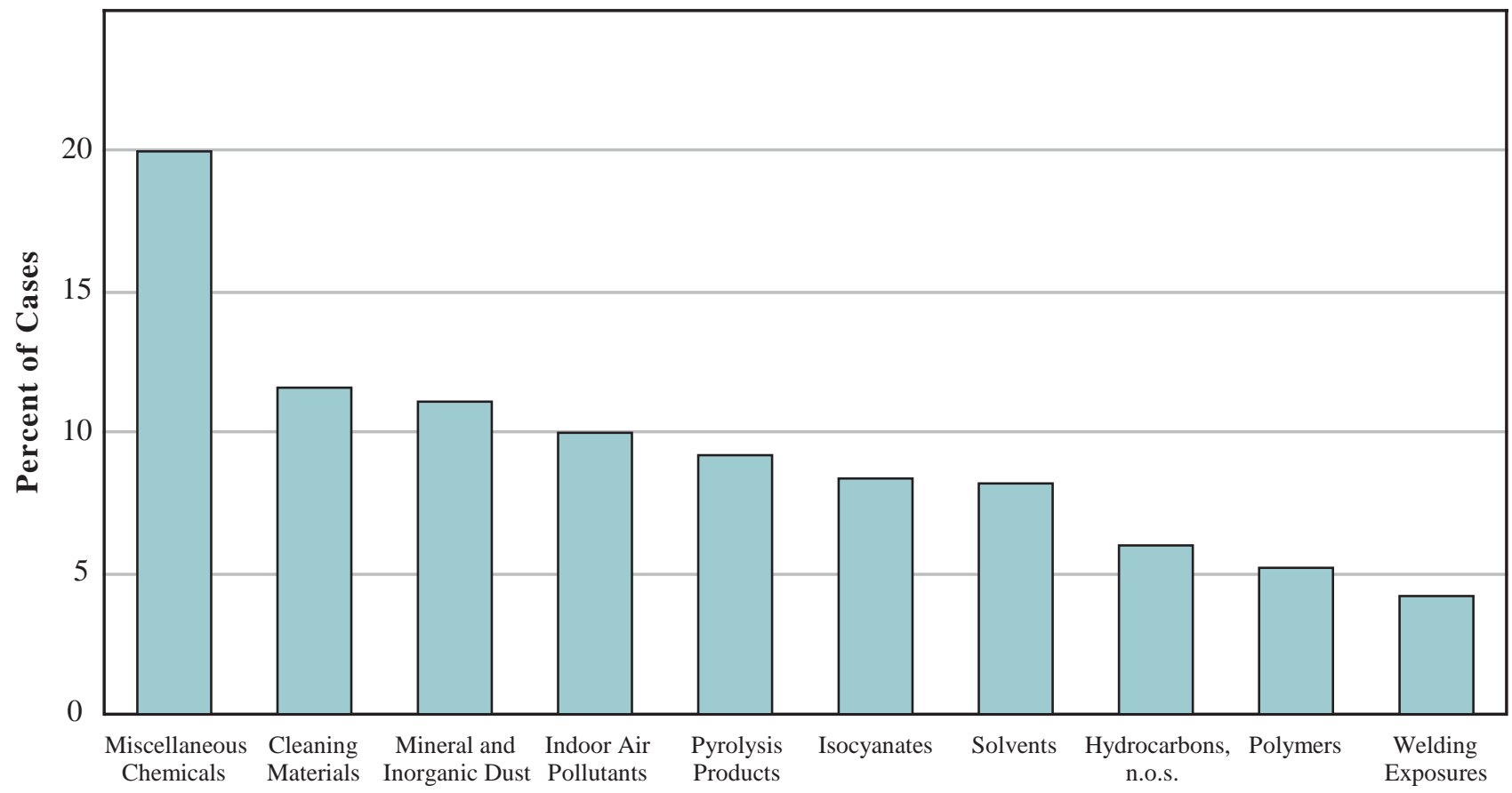

n.o.s. - not otherwise specified

NOTE: Total number of WRA cases was 2,526. The category 'Miscellaneous Chemicals' accounts for a large range of exposures including pesticides, perfume, enzymes, and odors; 'Mineral and Inorganic Dust' includes exposures such as plaster, fiberglass, and cement; 'Indoor Air Pollutants' includes pollutants from building renovation; 'Pyrolysis Products' includes smoke and diesel exhaust; 'Solvents' includes paint and paint thinners; 'Hydrocarbons' includes cutting oils; and 'Polymers' includes natural rubber latex.

SOURCE: Provisional SENSOR surveillance data as of September 2002, aggregated by reporting source years, and reported by R Harrison and J Flattery (California); L Davis, E Pechter, and B Pazos (Massachusetts); K Rosenman, MJ Reilly, and D Kalinowski (Michigan); and D Valiante and D Schill (New Jersey). 
Table 9-6 (page 1 of 2). Work-related asthma: Primary industries associated with work-related asthma cases by state, 1993-1999

\begin{tabular}{|c|c|c|c|c|c|c|c|c|c|c|}
\hline \multirow[b]{2}{*}{ Industry (SIC) } & \multicolumn{2}{|c|}{ California } & \multicolumn{2}{|c|}{ Massachusetts } & \multicolumn{2}{|c|}{ Michigan } & \multicolumn{2}{|c|}{ New Jersey } & \multicolumn{2}{|c|}{ Total } \\
\hline & No. & $\%$ & No. & $\%$ & No. & $\%$ & No. & $\%$ & No. & $\%$ \\
\hline Agriculture, forestry, fishing & 44 & 4.8 & 3 & 0.8 & 4 & 0.4 & 1 & 0.6 & 52 & 2.1 \\
\hline Agricultural production, crops $(01)$ & 20 & 2.2 & - & - & - & - & - & - & 20 & 0.8 \\
\hline Agricultural services (07) & 10 & 1.1 & 1 & 0.3 & 2 & 0.2 & 1 & 0.6 & 14 & 0.6 \\
\hline Forestry (08) & 10 & 1.1 & - & - & - & - & - & - & 10 & 0.4 \\
\hline All others $(02,09)$ & 4 & 0.4 & 2 & 0.5 & 2 & 0.2 & - & - & 8 & 0.3 \\
\hline Mining $(10,13,14)$ & 2 & 0.2 & - & - & 2 & 0.2 & - & - & 4 & 0.2 \\
\hline Construction & 23 & 2.5 & 15 & 4.1 & 24 & 2.3 & 13 & 7.2 & 75 & 3.0 \\
\hline Construction, special trade contractors (17) & 11 & 1.2 & 12 & 3.3 & 19 & 1.8 & 12 & 6.7 & 54 & 2.1 \\
\hline $\begin{array}{l}\text { Heavy construction other than building construction, } \\
\text { contractors (16) }\end{array}$ & 9 & 1.0 & 1 & 0.3 & 1 & 0.1 & - & - & 11 & 0.4 \\
\hline $\begin{array}{l}\text { Building construction, general contractors and } \\
\text { operative builders (15) }\end{array}$ & 3 & 0.3 & 2 & 0.5 & 4 & 0.4 & 1 & 0.6 & 10 & 0.4 \\
\hline Manufacturing & 144 & 15.7 & 98 & 26.6 & 728 & 68.9 & 76 & 42.2 & 1,046 & 41.5 \\
\hline Transportation equipment (37) & 26 & 2.8 & 3 & 0.8 & 450 & 42.5 & 3 & 1.7 & 482 & 19.1 \\
\hline Chemicals and allied products (28) & 12 & 1.3 & 18 & 4.9 & 37 & 3.5 & 19 & 10.6 & 86 & 3.4 \\
\hline $\begin{array}{l}\text { Fabricated metal products except machinery and } \\
\text { transportation equipment (34) }\end{array}$ & 12 & 1.3 & 9 & 2.4 & 50 & 4.7 & 1 & 0.6 & 72 & 2.9 \\
\hline Rubber and miscellaneous plastics products (30) & 3 & 0.3 & 6 & 1.6 & 44 & 4.2 & 5 & 2.8 & 58 & 2.3 \\
\hline $\begin{array}{l}\text { Industrial and commercial machinery and computer } \\
\text { equipment (35) }\end{array}$ & 5 & 0.5 & 13 & 3.5 & 37 & 3.5 & - & - & 55 & 2.2 \\
\hline Food and kindred products (20) & 21 & 2.3 & 5 & 1.4 & 12 & 1.1 & 14 & 7.8 & 52 & 2.1 \\
\hline Primary metal industries (33) & 5 & 0.5 & - & - & 33 & 3.1 & 7 & 3.9 & 45 & 1.8 \\
\hline $\begin{array}{l}\text { Electronic and other electrical equipment and } \\
\text { components except computer equipment (36) }\end{array}$ & 16 & 1.7 & 11 & 3.0 & 9 & 0.8 & 6 & 3.3 & 42 & 1.7 \\
\hline Lumber and wood products, except furniture (24) & 18 & 2.0 & 1 & 0.3 & 11 & 1.0 & - & - & 30 & 1.2 \\
\hline Miscellaneous manufacturing industries (39) & 5 & 0.5 & 7 & 1.9 & 10 & 0.9 & 2 & 1.1 & 24 & 1.0 \\
\hline Measuring, analyzing, and controlling instruments (38) & 4 & 0.4 & 8 & 2.2 & 6 & 0.6 & 2 & 1.1 & 20 & 0.8 \\
\hline Paper and allied products (26) & 1 & 0.1 & 5 & 1.4 & 6 & 0.6 & 7 & 3.9 & 19 & 0.8 \\
\hline Stone, clay, glass, and concrete products (32) & 7 & 0.8 & 2 & 0.5 & 6 & 0.6 & 2 & 1.1 & 17 & 0.7 \\
\hline Printing, publishing, and allied industries (27) & 2 & 0.2 & 3 & 0.8 & 9 & 0.8 & 2 & 1.1 & 16 & 0.6 \\
\hline All others $(22,23,25,29,31)$ & 7 & 0.8 & 7 & 1.9 & 8 & 0.8 & 6 & 3.3 & 28 & 1.1 \\
\hline Transportation & 62 & 6.8 & 7 & 1.9 & 18 & 1.7 & 9 & 5.0 & 96 & 3.8 \\
\hline Electric, gas, and sanitary services (49) & 15 & 1.6 & 4 & 1.1 & 5 & 0.5 & 1 & 0.6 & 25 & 1.0 \\
\hline $\begin{array}{l}\text { Local and suburban transit and interurban highway } \\
\text { passenger transportation (41) }\end{array}$ & 13 & 1.4 & 1 & 0.3 & 2 & 0.2 & 4 & 2.2 & 20 & 0.8 \\
\hline Motor freight transportation and warehousing (42) & 10 & 1.1 & - & - & 3 & 0.3 & 2 & 1.1 & 15 & 0.6 \\
\hline Communications (48) & 13 & 1.4 & - & - & 1 & 0.1 & - & - & 14 & 0.6 \\
\hline All others $(40,43,44,45,47)$ & 11 & 1.2 & 2 & 0.5 & 7 & 0.7 & 2 & 1.1 & 22 & 0.9 \\
\hline Wholesale trade & 13 & 1.4 & 4 & 1.1 & 14 & 1.3 & 4 & 2.2 & 35 & 1.4 \\
\hline Wholesale trade, nondurable goods (51) & 8 & 0.9 & 3 & 0.8 & 5 & 0.5 & 2 & 1.1 & 18 & 0.7 \\
\hline Wholesale trade, durable goods (50) & 5 & 0.5 & 1 & 0.3 & 9 & 0.8 & 2 & 1.1 & 17 & 0.7 \\
\hline
\end{tabular}

See footnotes at end of table. 
Table 9-6 (page 2 of 2). Work-related asthma: Primary industries associated with work-related asthma cases by state, 1993-1999

\begin{tabular}{|c|c|c|c|c|c|c|c|c|c|c|}
\hline \multirow[b]{2}{*}{ Industry (SIC) } & \multicolumn{2}{|c|}{ California } & \multicolumn{2}{|c|}{ Massachusetts } & \multicolumn{2}{|c|}{ Michigan } & \multicolumn{2}{|c|}{ New Jersey } & \multicolumn{2}{|c|}{ Total } \\
\hline & No. & $\%$ & No. & $\%$ & No. & $\%$ & No. & $\%$ & No. & $\%$ \\
\hline Retail trade & 41 & 4.5 & 12 & 3.3 & 27 & 2.6 & 4 & 2.2 & 84 & 3.3 \\
\hline Food stores (54) & 10 & 1.1 & 3 & 0.8 & 7 & 0.7 & 1 & 0.6 & 21 & 0.8 \\
\hline Eating and drinking places (58) & 8 & 0.9 & 4 & 1.1 & 4 & 0.4 & - & - & 16 & 0.6 \\
\hline Miscellaneous retail (59) & 9 & 1.0 & 2 & 0.5 & 4 & 0.4 & 1 & 0.6 & 16 & 0.6 \\
\hline Automotive dealers and gasoline service stations (55) & 4 & 0.4 & 1 & 0.3 & 6 & 0.6 & 1 & 0.6 & 12 & 0.5 \\
\hline General merchandise stores (53) & 6 & 0.7 & - & - & 4 & 0.4 & 1 & 0.6 & 11 & 0.4 \\
\hline All others $(52,56,57)$ & 4 & 0.4 & 2 & 0.5 & 2 & 0.2 & - & - & 8 & 0.3 \\
\hline Finance, Insurance, and Real Estate & 32 & 3.5 & 1 & 0.3 & 5 & 0.5 & 1 & 0.6 & 39 & 1.5 \\
\hline Insurance carriers (63) & 10 & 1.1 & 1 & 0.3 & - & - & 1 & 0.6 & 12 & 0.5 \\
\hline Real estate (65) & 10 & 1.1 & - & - & 2 & 0.2 & - & - & 12 & 0.5 \\
\hline All others $(60,61,64)$ & 12 & 1.3 & - & - & 3 & 0.3 & - & - & 15 & 0.6 \\
\hline Services & 412 & 44.9 & 190 & 51.6 & 202 & 19.1 & 59 & 32.8 & 863 & 34.2 \\
\hline Health services $(80)$ & 156 & 17.0 & 108 & 29.3 & 112 & 10.6 & 29 & 16.1 & 405 & 16.0 \\
\hline Educational services (82) & 128 & 13.9 & 49 & 13.3 & 38 & 3.6 & 14 & 7.8 & 229 & 9.1 \\
\hline Social services (83) & 37 & 4.0 & 2 & 0.5 & 6 & 0.6 & 3 & 1.7 & 48 & 1.9 \\
\hline Business services (73) & 27 & 2.9 & 3 & 0.8 & 9 & 0.8 & 1 & 0.6 & 40 & 1.6 \\
\hline Amusement and recreation services (79) & 19 & 2.1 & 3 & 0.8 & 4 & 0.4 & - & - & 26 & 1.0 \\
\hline $\begin{array}{l}\text { Engineering, accounting, research, management, and } \\
\text { related services (87) }\end{array}$ & 11 & 1.2 & 6 & 1.6 & 4 & 0.4 & 4 & 2.2 & 25 & 1.0 \\
\hline $\begin{array}{l}\text { Hotels, rooming houses, camps and other lodging } \\
\text { places (70) }\end{array}$ & 8 & 0.9 & 3 & 0.8 & 10 & 0.9 & 2 & 1.1 & 23 & 0.9 \\
\hline Personal services (72) & 5 & 0.5 & 7 & 1.9 & 6 & 0.6 & 3 & 1.7 & 21 & 0.8 \\
\hline Automotive repair, services and parking (75) & 7 & 0.8 & 6 & 1.6 & 7 & 0.7 & 1 & 0.6 & 21 & 0.8 \\
\hline All others $(76,78,81,84,86,88,89)$ & 14 & 1.5 & 3 & 0.8 & 6 & 0.6 & 2 & 1.1 & 25 & 1.0 \\
\hline Public Administration & 142 & 15.5 & 38 & 10.3 & 33 & 3.1 & 13 & 7.2 & 226 & 9.0 \\
\hline Justice, public order, and safety (92) & 69 & 7.5 & 15 & 4.1 & 14 & 1.3 & 3 & 1.7 & 101 & 4.0 \\
\hline $\begin{array}{l}\text { Executive, legislative, general government, except } \\
\text { finance (91) }\end{array}$ & 27 & 2.9 & 3 & 0.8 & 1 & 0.1 & 3 & 1.7 & 34 & 1.3 \\
\hline Administration of economic programs (96) & 10 & 1.1 & 13 & 3.5 & 6 & 0.6 & 1 & 0.6 & 30 & 1.2 \\
\hline Administration of human resource programs (94) & 17 & 1.9 & 2 & 0.5 & 5 & 0.5 & 3 & 1.7 & 27 & 1.1 \\
\hline National security and international affairs (97) & 5 & 0.5 & 3 & 0.8 & 2 & 0.2 & 2 & 1.1 & 12 & 0.5 \\
\hline Public finance, taxation, and monetary policy (93) & 9 & 1.0 & 1 & 0.3 & - & - & 1 & 0.6 & 11 & 0.4 \\
\hline $\begin{array}{l}\text { Administration of environmental quality and housing } \\
\text { programs (95) }\end{array}$ & 5 & 0.5 & 1 & 0.3 & 5 & 0.5 & - & - & 11 & 0.4 \\
\hline Nonclassifiable & 3 & 0.3 & - & - & 3 & 0.3 & - & - & 6 & 0.2 \\
\hline TOTAL & 918 & 100.0 & 368 & 100.0 & 1,060 & 100.0 & 180 & 100.0 & 2,526 & 100.0 \\
\hline
\end{tabular}

- indicates no cases reported.

SIC - 1987 Standard Industrial Classification

NOTE: Percentages may not sum to $100 \%$ due to rounding. See appendices for source description and methods.

SOURCE: Provisional SENSOR surveillance data as of September 2002, aggregated by reporting source years, and reported by R Harrison and J Flattery (California); L Davis, E Pechter, and B Pazos (Massachusetts); K Rosenman, MJ Reilly, and D Kalinowski (Michigan); and D Valiante and D Schill (New Jersey). 
Table 9-7 (page 1 of 2). Work-related asthma: Primary occupations associated with work-related asthma cases by state, 1993-1999

\begin{tabular}{|c|c|c|c|c|c|c|c|c|c|c|}
\hline \multirow[b]{2}{*}{ Occupation (COC) } & \multicolumn{2}{|c|}{ California } & \multicolumn{2}{|c|}{ Massachusetts } & \multicolumn{2}{|c|}{ Michigan } & \multicolumn{2}{|c|}{ New Jersey } & \multicolumn{2}{|c|}{ Total } \\
\hline & No. & $\%$ & No. & $\%$ & No. & $\%$ & No. & $\%$ & No. & $\%$ \\
\hline Managerial and professional specialty & 235 & 25.6 & 125 & 34.0 & 108 & 10.2 & 41 & 22.8 & 509 & 20.2 \\
\hline Registered nurses (095) & 53 & 5.8 & 59 & 16.0 & 27 & 2.5 & 10 & 5.6 & 149 & 5.9 \\
\hline Teachers, secondary school (157) & 12 & 1.3 & 14 & 3.8 & 5 & 0.5 & 3 & 1.7 & 34 & 1.3 \\
\hline Teachers, n.e.c. (159) & 8 & 0.9 & 12 & 3.3 & 10 & 0.9 & 3 & 1.7 & 33 & 1.3 \\
\hline Teachers, elementary school (156) & 22 & 2.4 & 1 & 0.3 & 2 & 0.2 & 2 & 1.1 & 27 & 1.1 \\
\hline Managers and administrators, n.e.c. (022) & 17 & 1.9 & 5 & 1.4 & 4 & 0.4 & - & - & 26 & 1.0 \\
\hline Social workers (174) & 11 & 1.2 & 3 & 0.8 & 5 & 0.5 & 2 & 1.1 & 21 & 0.8 \\
\hline Management related occupations, n.e.c. (37) & 16 & 1.7 & - & - & 3 & 0.3 & - & - & 19 & 0.8 \\
\hline Teachers, special education (158) & 9 & 1.0 & 1 & 0.3 & 1 & 0.1 & 1 & 0.6 & 12 & 0.5 \\
\hline Chemists, except biochemists (73) & 2 & 0.2 & 3 & 0.8 & 2 & 0.2 & 4 & 2.2 & 11 & 0.4 \\
\hline All others & 85 & 9.3 & 27 & 7.3 & 49 & 4.6 & 16 & 8.9 & 177 & 7.0 \\
\hline Technical, sales, and administrative support & 289 & 31.5 & 75 & 20.4 & 86 & 8.1 & 35 & 19.4 & 485 & 19.2 \\
\hline General office clerks (379) & 30 & 3.3 & 15 & 4.1 & 5 & 0.5 & 2 & 1.1 & 52 & 2.1 \\
\hline Secretaries (313) & 26 & 2.8 & 13 & 3.5 & 7 & 0.7 & 3 & 1.7 & 49 & 1.9 \\
\hline Health technologists and technicians, n.e.c. (208) & 13 & 1.4 & 2 & 0.5 & 6 & 0.6 & 4 & 2.2 & 25 & 1.0 \\
\hline Licensed practical nurses (207) & 14 & 1.5 & 4 & 1.1 & 5 & 0.5 & 1 & 0.6 & 24 & 1.0 \\
\hline Investigators and adjusters, except insurance (376) & 12 & 1.3 & 2 & 0.5 & 1 & 0.1 & 3 & 1.7 & 18 & 0.7 \\
\hline Administrative support, n.e.c. (389) & 13 & 1.4 & 2 & 0.5 & 2 & 0.2 & - & - & 17 & 0.7 \\
\hline Typists (315) & 9 & 1.0 & 4 & 1.1 & 1 & 0.1 & 1 & 0.6 & 15 & 0.6 \\
\hline Bookkeepers, accounting, and auditing clerks (337) & 12 & 1.3 & 1 & 0.3 & - & - & - & - & 13 & 0.5 \\
\hline Clinical laboratory technologists and technicians (203) & 3 & 0.3 & 6 & 1.6 & 2 & 0.2 & 1 & 0.6 & 12 & 0.5 \\
\hline Receptionists (319) & 10 & 1.1 & - & - & 1 & 0.1 & 1 & 0.6 & 12 & 0.5 \\
\hline Science technicians, n.e.c. (225) & 1 & 0.1 & - & - & 6 & 0.6 & 4 & 2.2 & 11 & 0.4 \\
\hline Cashiers (276) & 9 & 1.0 & 1 & 0.3 & 1 & 0.1 & - & - & 11 & 0.4 \\
\hline Supervisors, general office (303) & 6 & 0.7 & 2 & 0.5 & 2 & 0.2 & - & - & 10 & 0.4 \\
\hline Teachers' aides (387) & 7 & 0.8 & - & - & 2 & 0.2 & 1 & 0.6 & 10 & 0.4 \\
\hline All others & 124 & 13.5 & 23 & 6.2 & 45 & 4.2 & 14 & 7.8 & 206 & 8.2 \\
\hline Service & 136 & 14.8 & 43 & 11.7 & 98 & 9.2 & 20 & 11.1 & 297 & 11.8 \\
\hline Janitors and cleaners (453) & 27 & 2.9 & 6 & 1.6 & 32 & 3.0 & 4 & 2.2 & 69 & 2.7 \\
\hline Nursing aides, orderlies, and attendants (447) & 13 & 1.4 & 8 & 2.2 & 5 & 0.5 & 2 & 1.1 & 28 & 1.1 \\
\hline Firefighting (417) & 20 & 2.2 & 4 & 1.1 & 2 & 0.2 & 1 & 0.6 & 27 & 1.1 \\
\hline Health aides, except nursing (446) & 3 & 0.3 & 1 & 0.3 & 12 & 1.1 & 3 & 1.7 & 19 & 0.8 \\
\hline $\begin{array}{l}\text { Supervisors, cleaning and building service } \\
\text { workers (448) }\end{array}$ & 3 & 0.3 & 1 & 0.3 & 15 & 1.4 & - & - & 19 & 0.8 \\
\hline Maids and housemen (449) & 4 & 0.4 & 3 & 0.8 & 5 & 0.5 & 3 & 1.7 & 15 & 0.6 \\
\hline Cooks (436) & 4 & 0.4 & 2 & 0.5 & 4 & 0.4 & 2 & 1.1 & 12 & 0.5 \\
\hline Miscellaneous food preparation (444) & 5 & 0.5 & 2 & 0.5 & 5 & 0.5 & - & - & 12 & 0.5 \\
\hline Hairdressers and cosmetologists (458) & 4 & 0.4 & 5 & 1.4 & 2 & 0.2 & - & - & 11 & 0.4 \\
\hline All others & 53 & 5.8 & 11 & 3.0 & 16 & 1.5 & 5 & 2.8 & 85 & 3.4 \\
\hline Farming, forestry, and fishing & 42 & 4.6 & 4 & 1.1 & 6 & 0.6 & - & - & 52 & 2.1 \\
\hline Farm workers (479) & 17 & 1.9 & - & - & - & - & - & - & 17 & 0.7 \\
\hline Groundskeepers and gardeners, except farm (486) & 14 & 1.5 & 1 & 0.3 & - & - & - & - & 15 & 0.6 \\
\hline All others & 11 & 1.2 & 3 & 0.8 & 6 & 0.6 & - & - & 20 & 0.8 \\
\hline
\end{tabular}

See footnotes at end of table. 
Table 9-7 (page 2 of 2). Work-related asthma: Primary occupations associated with work-related asthma cases by state, 1993-1999

\begin{tabular}{|c|c|c|c|c|c|c|c|c|c|c|}
\hline \multirow[b]{2}{*}{ Occupation (COC) } & \multicolumn{2}{|c|}{ California } & \multicolumn{2}{|c|}{ Massachusetts } & \multicolumn{2}{|c|}{ Michigan } & \multicolumn{2}{|c|}{ New Jersey } & \multicolumn{2}{|c|}{ Total } \\
\hline & No. & $\%$ & No. & $\%$ & No. & $\%$ & No. & $\%$ & No. & $\%$ \\
\hline Precision production, craft, and repair & 75 & 8.2 & 50 & 13.6 & 156 & 14.7 & 30 & 16.7 & 311 & $\overline{12.3}$ \\
\hline Supervisors, production (628) & 7 & 0.8 & 5 & 1.4 & 31 & 2.9 & 3 & 1.7 & 46 & 1.8 \\
\hline Millwrights (544) & 1 & 0.1 & 1 & 0.3 & 20 & 1.9 & - & - & 22 & 0.9 \\
\hline Machinists (637) & 3 & 0.3 & 4 & 1.1 & 10 & 0.9 & 2 & 1.1 & 19 & 0.8 \\
\hline Specified mechanics and repairers, n.e.c. (547) & 3 & 0.3 & - & - & 11 & 1.0 & 2 & 1.1 & 16 & 0.6 \\
\hline Electricians (575) & 6 & 0.7 & - & - & 5 & 0.5 & 5 & 2.8 & 16 & 0.6 \\
\hline Optical goods workers (677) & 1 & 0.1 & - & - & 15 & 1.4 & - & - & 16 & 0.6 \\
\hline Plumbers, pipefitters, and steamfitters (585) & 3 & 0.3 & 2 & 0.5 & 9 & 0.8 & - & - & 14 & 0.6 \\
\hline Carpenters (567) & 5 & 0.5 & 5 & 1.4 & - & - & 2 & 1.1 & 12 & 0.5 \\
\hline Industrial machinery repairers (518) & 2 & 0.2 & 2 & 0.5 & 4 & 0.4 & 2 & 1.1 & 10 & 0.4 \\
\hline All others & 44 & 4.8 & 31 & 8.4 & 51 & 4.8 & 14 & 7.8 & 140 & 5.5 \\
\hline Operators, fabricators, and laborers & 137 & 14.9 & 71 & 19.3 & 568 & 53.6 & 54 & 30.0 & 830 & 32.9 \\
\hline Assemblers (785) & 9 & 1.0 & 7 & 1.9 & 139 & 13.1 & 1 & 0.6 & 156 & 6.2 \\
\hline Welders and cutters (783) & 8 & 0.9 & 6 & 1.6 & 51 & 4.8 & 1 & 0.6 & 66 & 2.6 \\
\hline Laborers, except construction (889) & 5 & 0.5 & 2 & 0.5 & 48 & 4.5 & 1 & 0.6 & 56 & 2.2 \\
\hline Miscellaneous machine operators, n.e.c. (777) & 8 & 0.9 & 5 & 1.4 & 30 & 2.8 & 5 & 2.8 & 48 & 1.9 \\
\hline $\begin{array}{l}\text { Miscellaneous metal, plastic, stone, and glass } \\
\text { working (715) }\end{array}$ & - & - & - & - & 35 & 3.3 & - & - & 35 & 1.4 \\
\hline Production inspectors, checkers and examiners (796) & 2 & 0.2 & 2 & 0.5 & 29 & 2.7 & 1 & 0.6 & 34 & 1.3 \\
\hline Painting and paint spray machine operators (759) & 5 & 0.5 & 8 & 2.2 & 13 & 1.2 & 2 & 1.1 & 28 & 1.1 \\
\hline Mixing and blending machine operators (756) & 6 & 0.7 & 6 & 1.6 & 9 & 0.8 & 1 & 0.6 & 22 & 0.9 \\
\hline Hand painting, coating, and decorating (789) & - & - & - & - & 20 & 1.9 & 1 & 0.6 & 21 & 0.8 \\
\hline $\begin{array}{l}\text { Separating, filtering, and clarifying machine } \\
\text { operators (757) }\end{array}$ & 4 & 0.4 & 3 & 0.8 & 5 & 0.5 & 8 & 4.4 & 20 & 0.8 \\
\hline Truck drivers $(804)$ & 8 & 0.9 & - & - & 5 & 0.5 & 4 & 2.2 & 17 & 0.7 \\
\hline Molding and casting machine operators (719) & 3 & 0.3 & - & - & 13 & 1.2 & - & - & 16 & 0.6 \\
\hline Pressing machine operators (747) & - & - & - & - & 16 & 1.5 & - & - & 16 & 0.6 \\
\hline Packaging and filling machine operators (754) & 2 & 0.2 & 2 & 0.5 & 4 & 0.4 & 8 & 4.4 & 16 & 0.6 \\
\hline Machine operators, not specified (779) & 5 & 0.5 & 6 & 1.6 & 0 & 0.0 & 5 & 2.8 & 16 & 0.6 \\
\hline Freight, stock, and material handlers, n.e.c. (883) & 6 & 0.7 & 1 & 0.3 & 8 & 0.8 & - & - & 15 & 0.6 \\
\hline $\begin{array}{l}\text { Grinding, abrading, buffing, and polishing machine } \\
\text { operators (709) }\end{array}$ & - & 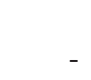 & 3 & 0.8 & 10 & 0.9 & 1 & 0.6 & 14 & 0.6 \\
\hline Machine feeders and offbearers (878) & 1 & 0.1 & - & - & 13 & 1.2 & - & - & 14 & 0.6 \\
\hline Bus drivers (808) & 11 & 1.2 & - & - & 2 & 0.2 & - & - & 13 & 0.5 \\
\hline Hand packers and packagers (888) & 3 & 0.3 & 1 & 0.3 & 7 & 0.7 & 1 & 0.6 & 12 & 0.5 \\
\hline Textile sewing machine operators (744) & 2 & 0.2 & 1 & 0.3 & 7 & 0.7 & - & - & 10 & 0.4 \\
\hline Miscellaneous hand working occupations (795) & 2 & 0.2 & - & - & 8 & 0.8 & - & - & 10 & 0.4 \\
\hline All others & 47 & 5.1 & 18 & 4.9 & 96 & 9.1 & 14 & 7.8 & 175 & 6.9 \\
\hline Military occupations & 2 & 0.2 & - & - & - & - & - & - & 2 & 0.1 \\
\hline Unclassifiable and miscellaneous unemployed & 2 & 0.2 & - & - & 38 & 3.6 & - & - & 40 & 1.6 \\
\hline TOTAL & 918 & 100.0 & 368 & 100.0 & 1,060 & 100.0 & 180 & 100.0 & 2,526 & 100.0 \\
\hline
\end{tabular}

- indicates no cases reported. 
Table 9-8. Asthma: Estimated prevalence by current industry and smoking status, U.S. residents age 18 and over, 2000

\begin{tabular}{|c|c|c|c|c|c|c|}
\hline \multirow[b]{2}{*}{ Industry } & \multicolumn{3}{|c|}{ Nonsmokers } & \multicolumn{3}{|c|}{ Current Smokers } \\
\hline & $\begin{array}{c}\text { Number of } \\
\text { Respondents }\end{array}$ & $\begin{array}{c}\text { Prevalence } \\
(\%)\end{array}$ & $\begin{array}{c}95 \% \\
\text { Confidence } \\
\text { Interval } \\
\end{array}$ & $\begin{array}{c}\text { Number of } \\
\text { Respondents }\end{array}$ & $\begin{array}{c}\text { Prevalence } \\
(\%)\end{array}$ & $\begin{array}{c}95 \% \\
\text { Confidence } \\
\text { Interval } \\
\end{array}$ \\
\hline General merchandise stores & 208 & 13.0 & $7.3-18.7$ & 106 & 14.7 & $6.9-22.5$ \\
\hline Food, bakery and dairy stores & 215 & 12.8 & $7.5-18.1$ & 154 & 11.7 & $5.8-17.6$ \\
\hline Furniture, lumber and wood & 115 & 12.5 & $4.7-20.3$ & 61 & 5.7 & $0.4-11.0$ \\
\hline Banking and credit agencies & 266 & 11.3 & $6.6-16.0$ & 89 & 11.2 & $3.4-19.0$ \\
\hline Elementary and secondary schools and colleges & 1,160 & 10.8 & $8.6-13.0$ & 211 & 6.1 & $2.8-9.4$ \\
\hline Trucking service and warehousing & 181 & 10.7 & $5.2-16.2$ & 126 & 10.1 & $4.4-15.8$ \\
\hline Health services, except hospitals & 654 & 10.2 & $7.5-12.9$ & 252 & 12.1 & $7.6-16.6$ \\
\hline Primary metal industries & 46 & 10.1 & $0.9-19.3$ & 40 & 10.5 & $0.5-20.5$ \\
\hline Utilities and sanitary & 107 & 10.1 & $4.0-16.2$ & 38 & 3.4 & $0.0-9.9$ \\
\hline Transportation equipment & 166 & 10.0 & $4.7-15.3$ & 83 & 9.9 & $2.8-17.0$ \\
\hline Other and not specified durable goods & 139 & 9.8 & $3.7-15.9$ & 60 & 1.7 & $0.0-5.0$ \\
\hline Fabricated metal industries, including ordnance & 82 & 9.7 & $1.9-17.5$ & 67 & 11.1 & $1.9-20.3$ \\
\hline Insurance, real estate, and other finance & 479 & 9.7 & $5.0-14.4$ & 194 & 11.2 & $6.3-16.1$ \\
\hline Social services, religious and membership orgs. & 491 & 9.7 & $6.2-13.2$ & 148 & 6.5 & $2.6-10.4$ \\
\hline Legal, engineering and other professional services & 422 & 9.6 & $6.5-12.7$ & 145 & 11.8 & $5.7-17.9$ \\
\hline Communications & 195 & 9.5 & $4.8-14.2$ & 79 & 6.1 & $0.0-12.2$ \\
\hline Private households & 121 & 9.5 & $2.4-16.6$ & 27 & 5.4 & $0.0-15.6$ \\
\hline Other transportation & 227 & 9.3 & $4.6-14.0$ & 110 & 7.4 & $1.1-13.7$ \\
\hline Hospitals & 510 & 8.8 & $5.7-11.9$ & 164 & 9.1 & $4.4-13.8$ \\
\hline Other and not specified retail trade & 510 & 8.1 & $5.6-10.6$ & 229 & 10.6 & $5.7-15.5$ \\
\hline Other nondurable goods & 113 & 8.0 & $1.1-14.9$ & 61 & 1.9 & $0.0-5.6$ \\
\hline Eating and drinking places & 426 & 8.0 & $5.1-10.9$ & 353 & 12.4 & $8.7-16.1$ \\
\hline Public administrations & 534 & 7.9 & $5.0-10.8$ & 194 & 8.3 & $4.0-12.6$ \\
\hline Other personal services & 296 & 7.7 & $4.0-11.4$ & 151 & 12.1 & $6.2-18.0$ \\
\hline Railroads & 22 & 7.6 & $0.0-19.9$ & 12 & 0.0 & - \\
\hline Wholesale trade & 367 & 7.4 & $4.3-10.5$ & 172 & 8.9 & $4.0-13.8$ \\
\hline Entertainment and recreation services & 200 & 7.4 & $2.7-12.1$ & 89 & 10.5 & $3.2-17.8$ \\
\hline Printing, publishing and allied industries & 132 & 7.2 & $1.5-12.9$ & 47 & 5.9 & $0.0-12.4$ \\
\hline Business services & 678 & 7.1 & $4.9-9.3$ & 350 & 13.1 & $9.0-17.2$ \\
\hline Electrical machinery, equipment and supplies & 161 & 6.3 & $2.8-9.8$ & 75 & 11.9 & $3.7-20.1$ \\
\hline Textile mill and finished textile products & 114 & 6.2 & $0.0-12.9$ & 45 & 3.5 & $0.0-8.0$ \\
\hline Construction & 547 & 5.7 & $3.3-8.1$ & 450 & 4.7 & $2.9-6.5$ \\
\hline Other educational services & 53 & 5.3 & $0.0-10.6$ & 10 & 14.7 & $0.0-36.5$ \\
\hline Repair services & 112 & 5.0 & $0.9-9.1$ & 113 & 10.9 & $4.0-17.8$ \\
\hline Agriculture & 277 & 4.4 & $2.0-6.8$ & 109 & 5.0 & $0.3-9.7$ \\
\hline Machinery, except electrical & 148 & 4.4 & $1.1-7.7$ & 93 & 11.3 & $4.1-18.6$ \\
\hline Automotive dealers and gasoline stations & 142 & 4.4 & $0.1-8.7$ & 119 & 5.9 & $1.4-10.4$ \\
\hline Mining & 28 & 4.0 & $0.0-11.6$ & 25 & 18.3 & $1.4-35.2$ \\
\hline Chemicals and allied products & 93 & 3.7 & $0.0-8.0$ & 36 & 2.8 & $0.0-8.3$ \\
\hline Food and kindred products & 121 & 1.5 & $0.0-3.1$ & 83 & 5.9 & $0.0-13.0$ \\
\hline Forestry and fisheries & 17 & 0.0 & - & 7 & 6.3 & $0.0-18.8$ \\
\hline Armed forces & 2 & 0.0 & - & 0 & - & - \\
\hline TOTAL & 11,230 & 8.4 & $7.8-9.0$ & 5,082 & 9.1 & $8.3-9.9$ \\
\hline
\end{tabular}

- No estimates due to no asthma cases or no respondents.

Nonsmokers - Those respondents who indicated that they never smoked or smoked less than 100 cigarettes in their lifetime.

NOTE: Industries were classified according to 1995 Standard Industrial Classification System and then regrouped by NCHS. See appendices for source description, methods, and ICD codes.

SOURCE: National Center for Health Statistics 2000 National Health Interview Survey. 
Table 9-9. Asthma: Estimated prevalence by current industry and smoking status, U.S. male residents age 18 and over, 2000

\begin{tabular}{|c|c|c|c|c|c|c|c|}
\hline \multirow{3}{*}{$\begin{array}{l}\text { Industry } \\
\text { Private households }\end{array}$} & \multicolumn{4}{|c|}{ Nonsmokers } & \multicolumn{3}{|c|}{ Current Smokers } \\
\hline & \multirow{2}{*}{$\begin{array}{c}\begin{array}{c}\text { Number of } \\
\text { Respondents }\end{array} \\
6\end{array}$} & \multirow{2}{*}{$\begin{array}{c}\begin{array}{c}\text { Prevalence } \\
(\%)\end{array} \\
17.0\end{array}$} & \multicolumn{2}{|c|}{$\begin{array}{c}95 \% \\
\text { Confidence } \\
\text { Interval } \\
\end{array}$} & \multirow{2}{*}{$\begin{array}{c}\begin{array}{c}\text { Number of } \\
\text { Respondents }\end{array} \\
2\end{array}$} & \multirow{2}{*}{$\begin{array}{c}\text { Prevalence } \\
(\%)\end{array}$} & $\begin{array}{c}95 \% \\
\text { Confidence } \\
\text { Interval }\end{array}$ \\
\hline & & & 0.0 & -47.6 & & & - \\
\hline Food, bakery and dairy stores & 100 & 14.4 & 5.8 & -23.0 & 57 & 12.6 & $1.2-24.0$ \\
\hline General merchandise stores & 49 & 11.4 & 0.0 & - 23.4 & 40 & 10.6 & $1.2-20.0$ \\
\hline Communications & 105 & 11.2 & 4.5 & - 17.9 & 42 & 4.0 & $0.0-9.5$ \\
\hline Primary metal industries & 31 & 10.7 & 0.0 & - 22.1 & 30 & 9.5 & $0.0-19.9$ \\
\hline Trucking service and warehousing & 136 & 10.7 & 4.4 & - 17.0 & 96 & 7.6 & $1.5-13.7$ \\
\hline Other nondurable goods & 75 & 10.5 & 1.3 & - 19.7 & 46 & 0.0 & - \\
\hline Elementary and secondary schools and colleges & 313 & 10.1 & 6.8 & -13.4 & 56 & 1.3 & $0.0-3.8$ \\
\hline Fabricated metal industries, including ordnance & 57 & 9.8 & 0.2 & - 19.4 & 54 & 11.0 & $0.8-21.2$ \\
\hline Printing, publishing and allied industries & 65 & 9.5 & 0.0 & -19.3 & 32 & 6.0 & $0.0-13.8$ \\
\hline Health services, except hospitals & 103 & 8.9 & 2.0 & -15.8 & 32 & 0.0 & - \\
\hline Furniture, lumber and wood & 81 & 8.8 & 1.9 & -15.7 & 41 & 8.6 & $0.6-16.6$ \\
\hline Insurance, real estate, and other finance & 181 & 8.7 & 4.0 & - 13.4 & 80 & 8.5 & $2.0-15.0$ \\
\hline Other and not specified durable goods & 78 & 8.5 & 1.1 & -15.9 & 47 & 2.1 & $0.0-6.0$ \\
\hline Banking and credit agencies & 55 & 8.5 & 0.3 & -16.7 & 23 & 5.7 & $0.0-14.3$ \\
\hline Transportation equipment & 114 & 8.4 & 2.9 & -13.9 & 58 & 10.7 & $2.5-18.9$ \\
\hline Legal, engineering and other professional services & 211 & 8.2 & 4.1 & -12.3 & 59 & 11.8 & $2.6-21.0$ \\
\hline Railroads & 19 & 8.0 & 0.0 & -20.9 & 12 & 0.0 & - \\
\hline Entertainment and recreation services & 116 & 8.0 & 1.5 & -14.5 & 48 & 4.8 & $0.0-12.4$ \\
\hline Wholesale trade & 218 & 7.4 & 3.3 & -11.5 & 115 & 6.6 & $1.7-11.5$ \\
\hline Public administrations & 258 & 7.4 & 3.5 & -11.3 & 91 & 12.2 & $4.6-19.8$ \\
\hline Utilities and sanitary & 83 & 7.1 & 1.4 & -12.8 & 29 & 4.1 & $0.0-12.1$ \\
\hline Other and not specified retail trade & 214 & 7.0 & 3.5 & -10.5 & 103 & 10.0 & $2.6-17.4$ \\
\hline Eating and drinking places & 195 & 6.8 & 2.5 & -11.1 & 161 & 12.3 & $6.2-18.4$ \\
\hline Business services & 350 & 6.6 & 3.7 & -9.5 & 186 & 13.6 & $7.9-19.3$ \\
\hline Social services, religious and membership orgs. & 98 & 6.5 & 1.4 & -11.6 & 21 & 3.2 & $0.0-9.3$ \\
\hline Construction & 490 & 5.8 & 3.3 & -8.3 & 423 & 5.0 & $3.0-7.0$ \\
\hline Machinery, except electrical & 105 & 5.8 & 1.5 & -10.1 & 72 & 12.2 & $3.8-20.6$ \\
\hline Hospitals & 112 & 5.8 & 0.9 & -10.7 & 26 & 2.4 & $0.0-7.1$ \\
\hline Chemicals and allied products & 54 & 5.6 & 0.0 & -12.1 & 21 & 0.0 & - \\
\hline Electrical machinery, equipment and supplies & 87 & 5.3 & 1.2 & -9.4 & 43 & 17.6 & $3.9-31.3$ \\
\hline Automotive dealers and gasoline stations & 98 & 4.8 & 0.0 & -10.3 & 82 & 6.6 & $0.7-12.5$ \\
\hline Mining & 24 & 4.4 & 0.0 & -12.6 & 21 & 14.8 & $0.0-31.1$ \\
\hline Textile mill and finished textile products & 33 & 3.5 & 0.0 & -10.8 & 23 & 0.0 & - \\
\hline Agriculture & 203 & 3.4 & 0.9 & $-\quad 5.9$ & 87 & 2.8 & $0.0-6.9$ \\
\hline Repair services & 99 & 3.0 & 0.1 & $-\quad 5.9$ & 96 & 11.4 & $3.8-19.0$ \\
\hline Other transportation & 125 & 2.7 & 0.0 & -5.4 & 69 & 6.4 & $0.0-14.0$ \\
\hline Other personal services & 75 & 2.1 & 0.0 & -4.6 & 60 & 15.4 & $5.2-25.6$ \\
\hline Food and kindred products & 62 & 1.3 & 0.0 & $-\quad 3.1$ & 56 & 7.6 & $0.0-17.0$ \\
\hline Forestry and fisheries & 12 & 0.0 & - & - & 6 & 7.9 & $0.0-23.6$ \\
\hline Other educational services & 7 & 0.0 & - & - & 3 & 0.0 & - \\
\hline Armed forces & 1 & 0.0 & - & - & 0 & - & - \\
\hline TOTAL & 5,056 & 7.2 & 6.4 & -8.0 & 2,714 & 8.0 & $6.8-9.2$ \\
\hline
\end{tabular}

-- No estimates due to no asthma cases or no respondents.

Nonsmokers - Those respondents who indicated that they never smoked or smoked less than 100 cigarettes in their lifetime.

NOTE: Industries were classified according to 1995 Standard Industrial Classification System and then regrouped by NCHS. See appendices for source description, methods, and ICD codes.

SOURCE: National Center for Health Statistics 2000 National Health Interview Survey. 
Table 9-10. Asthma: Estimated prevalence by current industry and smoking status, U.S. female residents age 18 and over, 2000

\begin{tabular}{|c|c|c|c|c|c|c|c|}
\hline \multirow{3}{*}{$\frac{\text { Industry }}{\text { Utilities and sanitary }}$} & \multicolumn{4}{|c|}{ Nonsmokers } & \multicolumn{3}{|c|}{ Current Smokers } \\
\hline & \multirow{2}{*}{$\begin{array}{c}\begin{array}{c}\text { Number of } \\
\text { Respondents }\end{array} \\
24\end{array}$} & \multirow{2}{*}{$\begin{array}{c}\begin{array}{c}\text { Prevalence } \\
(\%)\end{array} \\
28.0\end{array}$} & \multicolumn{2}{|c|}{$\begin{array}{c}95 \% \\
\text { Confidence } \\
\text { Interval }\end{array}$} & \multirow{2}{*}{$\begin{array}{c}\begin{array}{c}\text { Number of } \\
\text { Respondents }\end{array} \\
9\end{array}$} & \multirow{2}{*}{$\begin{array}{c}\begin{array}{c}\text { Prevalence } \\
(\%)\end{array} \\
0.0\end{array}$} & $\begin{array}{c}95 \% \\
\text { Confidence } \\
\text { Interval }\end{array}$ \\
\hline & & & 5.1 & -50.9 & & & - \\
\hline Repair services & 13 & 22.9 & 0.0 & -50.5 & 17 & 7.0 & $0.0-20.3$ \\
\hline Furniture, lumber and wood & 34 & 20.7 & 0.5 & -40.9 & 20 & 0.0 & - \\
\hline Other transportation & 102 & 19.1 & 8.9 & - 29.3 & 41 & 9.9 & $0.0-20.9$ \\
\hline Transportation equipment & 52 & 14.5 & 2.2 & - 26.8 & 25 & 7.3 & $0.0-19.3$ \\
\hline General merchandise stores & 159 & 13.4 & 6.9 & - 19.9 & 66 & 17.4 & $6.6-28.2$ \\
\hline Banking and credit agencies & 211 & 12.2 & 6.7 & - 17.7 & 66 & 13.5 & $3.7-23.3$ \\
\hline Other and not specified durable goods & 61 & 11.7 & 1.3 & -22.1 & 13 & 0.0 & - \\
\hline Food, bakery and dairy stores & 115 & 11.4 & 5.5 & - 17.3 & 97 & 11.2 & $4.9-17.5$ \\
\hline Legal, engineering and other professional services & 211 & 11.3 & 6.6 & - 16.0 & 86 & 11.7 & $3.3-20.1$ \\
\hline Elementary and secondary schools and colleges & 847 & 11.1 & 8.6 & - 13.6 & 155 & 8.0 & $3.7-12.3$ \\
\hline Trucking service and warehousing & 45 & 10.7 & 0.0 & -22.7 & 30 & 19.6 & $4.7-34.5$ \\
\hline Social services, religious and membership orgs. & 393 & 10.7 & 6.6 & - 14.8 & 127 & 7.2 & $2.7-11.7$ \\
\hline Insurance, real estate, and other finance & 298 & 10.5 & 3.1 & - 17.9 & 114 & 13.1 & $6.2-20.0$ \\
\hline Health services, except hospitals & 551 & 10.5 & 7.6 & - 13.4 & 220 & 13.9 & $8.6-19.2$ \\
\hline Other personal services & 221 & 10.1 & 5.0 & - 15.2 & 91 & 9.7 & $2.6-16.8$ \\
\hline Hospitals & 398 & 9.7 & 6.0 & - 13.4 & 138 & 10.4 & $4.9-15.9$ \\
\hline Eating and drinking places & 231 & 9.3 & 5.0 & - 13.6 & 192 & 12.5 & $7.4-17.6$ \\
\hline Fabricated metal industries, including ordnance & 25 & 9.2 & 0.0 & - 21.7 & 13 & 11.8 & $0.0-33.2$ \\
\hline Private households & 115 & 9.2 & 1.9 & - 16.5 & 25 & 5.8 & $0.0-16.8$ \\
\hline Other and not specified retail trade & 296 & 9.0 & 5.3 & - 12.7 & 126 & 11.1 & $5.0-17.2$ \\
\hline Public administrations & 276 & 8.4 & 4.3 & - 12.5 & 103 & 4.3 & $0.4-8.2$ \\
\hline Primary metal industries & 15 & 8.0 & 0.0 & -23.1 & 10 & 14.6 & $0.0-40.7$ \\
\hline Agriculture & 74 & 7.7 & 1.4 & - 14.0 & 22 & 14.0 & $0.0-29.7$ \\
\hline Business services & 328 & 7.7 & 4.6 & - 10.8 & 164 & 12.4 & $6.3-18.5$ \\
\hline Textile mill and finished textile products & 81 & 7.6 & 0.0 & - 16.8 & 22 & 6.1 & $0.0-13.7$ \\
\hline Electrical machinery, equipment and supplies & 74 & 7.6 & 1.5 & - 13.7 & 32 & 4.3 & $0.0-10.4$ \\
\hline Wholesale trade & 149 & 7.5 & 3.4 & - 11.6 & 57 & 15.0 & $3.2-26.8$ \\
\hline Communications & 90 & 6.6 & 0.9 & - 12.3 & 37 & 9.1 & $0.0-21.8$ \\
\hline Entertainment and recreation services & 84 & 6.4 & 0.0 & - 13.1 & 41 & 18.7 & $4.6-32.8$ \\
\hline Other educational services & 46 & 6.0 & 0.0 & - 12.1 & 7 & 18.4 & $0.0-46.0$ \\
\hline Printing, publishing and allied industries & 67 & 4.9 & 0.0 & -10.4 & 15 & 5.5 & $0.0-15.9$ \\
\hline Construction & 57 & 3.8 & 0.0 & -8.5 & 27 & 0.0 & - \\
\hline Automotive dealers and gasoline stations & 44 & 3.2 & 0.0 & 7.1 & 37 & 4.3 & $0.0-10.2$ \\
\hline Food and kindred products & 59 & 1.9 & 0.0 & 4.4 & 27 & 1.7 & $0.0-5.0$ \\
\hline Other nondurable goods & 38 & 1.3 & 0.0 & 3.8 & 15 & 8.8 & $0.0-25.1$ \\
\hline Forestry and fisheries & 5 & 0.0 & - & - & 1 & 0.0 & - \\
\hline Mining & 4 & 0.0 & - & - & 4 & 31.8 & $0.0-81.0$ \\
\hline Chemicals and allied products & 39 & 0.0 & - & - & 15 & 6.8 & $0.0-19.7$ \\
\hline Machinery, except electrical & 43 & 0.0 & - & - & 21 & 8.0 & $0.0-19.4$ \\
\hline Railroads & 3 & 0.0 & - & - & 0 & - & - \\
\hline Armed forces & 1 & 0.0 & - & - & 0 & - & - \\
\hline TOTAL & 6,174 & 9.7 & 8.7 & -10.7 & 2,368 & 10.5 & $9.1-11.9$ \\
\hline
\end{tabular}

- No estimates due to no asthma cases or no respondents.

Nonsmokers - Those respondents who indicated that they never smoked or smoked less than 100 cigarettes in their lifetime.

NOTE: Industries were classified according to 1995 Standard Industrial Classification System and then regrouped by NCHS. See appendices for source description, methods, and ICD codes.

SOURCE: National Center for Health Statistics 2000 National Health Interview Survey. 
Table 9-11. Asthma: Estimated prevalence by current occupation and smoking status, U.S. residents age 18 and over, 2000

\begin{tabular}{|c|c|c|c|c|c|c|c|c|}
\hline \multirow{3}{*}{$\begin{array}{l}\text { Occupation } \\
\text { Computer equipment operators }\end{array}$} & \multicolumn{4}{|c|}{ Nonsmokers } & \multicolumn{4}{|c|}{ Current Smokers } \\
\hline & \multirow{2}{*}{$\begin{array}{c}\begin{array}{c}\text { Number of } \\
\text { Respondents }\end{array} \\
29\end{array}$} & \multirow{2}{*}{$\begin{array}{c}\begin{array}{c}\text { Prevalence } \\
(\%)\end{array} \\
17.3\end{array}$} & \multicolumn{2}{|c|}{$\begin{array}{c}95 \% \\
\text { Confidence } \\
\text { Interval } \\
\end{array}$} & \multirow{2}{*}{$\begin{array}{c}\begin{array}{c}\text { Number of } \\
\text { Respondents }\end{array} \\
6\end{array}$} & \multirow{2}{*}{$\begin{array}{c}\begin{array}{c}\text { Prevalence } \\
(\%)\end{array} \\
0.0\end{array}$} & \multicolumn{2}{|c|}{$\begin{array}{c}95 \% \\
\text { Confidence } \\
\text { Interval } \\
\end{array}$} \\
\hline & & & 0.2 & -34.4 & & & - & - \\
\hline Financial records processing occupations & 202 & 16.5 & 6.7 & -26.3 & 79 & 10.2 & 3.3 & - 17.1 \\
\hline Mail and message distributing & 75 & 12.5 & 0.5 & -24.5 & 28 & 15.7 & 0.4 & -31.0 \\
\hline Teachers, librarians and counselors & 779 & 12.1 & 9.4 & -14.8 & 117 & 11.2 & 5.3 & -17.1 \\
\hline Other protective service occupations & 78 & 12.1 & 1.3 & -22.9 & 49 & 15.6 & 4.1 & -27.2 \\
\hline Other sales & 511 & 11.4 & 8.1 & -14.7 & 239 & 12.4 & 7.3 & -17.5 \\
\hline Writers, artists, entertainers and athletes & 200 & 10.9 & 6.0 & -15.8 & 64 & 8.5 & 0.5 & -16.5 \\
\hline Other professional specialty occupations & 266 & 10.9 & 6.4 & -15.4 & 72 & 10.5 & 2.9 & -18.1 \\
\hline Health technologists and technicians & 165 & 10.8 & 4.9 & -16.7 & 71 & 15.2 & 6.2 & -24.2 \\
\hline Forestry and fishing occupations & 12 & 10.8 & 0.0 & -30.0 & 7 & 7.6 & 0.0 & -22.5 \\
\hline Secretaries, stenographers and typists & 239 & 10.7 & 5.6 & -15.8 & 91 & 6.1 & 1.6 & -10.6 \\
\hline Supervisors and proprietors & 312 & 10.2 & 6.5 & -13.9 & 151 & 5.7 & 2.0 & -9.4 \\
\hline Private household occupations & 109 & 10.1 & 2.3 & -17.9 & 18 & 7.5 & 0.0 & -21.6 \\
\hline Personal service & 290 & 10.1 & 4.8 & -15.4 & 96 & 8.0 & 1.3 & -14.7 \\
\hline Other administrative support & 1,118 & 10.0 & 7.8 & -12.2 & 473 & 10.3 & 7.0 & -13.6 \\
\hline Health diagnosing occupations & 122 & 9.9 & 2.6 & -17.2 & 5 & 0.0 & - & - \\
\hline Technologists, technicians except health & 220 & 9.8 & 5.5 & -14.1 & 75 & 10.2 & 2.6 & -17.8 \\
\hline Natural mathematical and computer scientists & 235 & 9.5 & 5.2 & -13.8 & 65 & 10.2 & 3.5 & -16.9 \\
\hline Food service & 399 & 9.0 & 5.5 & -12.5 & 324 & 9.4 & 5.9 & -12.9 \\
\hline Freight, stock and material handlers & 310 & 8.9 & 4.8 & -13.0 & 194 & 11.0 & 5.3 & - 16.7 \\
\hline Managers and administrators, except public admin. & 984 & 8.5 & 6.3 & -10.7 & 463 & 7.0 & 4.5 & $-\quad 9.5$ \\
\hline Health service & 257 & 8.3 & 4.6 & -12.0 & 154 & 18.1 & 11.0 & -25.2 \\
\hline Construction and extractive trades & 332 & 8.3 & 4.6 & -12.0 & 325 & 4.6 & 2.4 & 6.8 \\
\hline Fabricators, assemblers, inspectors and samplers & 217 & 8.1 & 4.4 & -11.8 & 117 & 11.6 & 4.9 & -18.3 \\
\hline Health assessment and treating occupations & 324 & 7.5 & 4.6 & -10.4 & 76 & 5.3 & 0.2 & -10.4 \\
\hline Management related occupations & 476 & 6.1 & 3.9 & -8.3 & 161 & 5.4 & 2.3 & $-\quad 8.5$ \\
\hline Engineers & 199 & 5.9 & 2.4 & $-\quad 9.4$ & 41 & 13.3 & 1.3 & -25.3 \\
\hline Machine operators and tenderers, except precision & 370 & 5.9 & 3.0 & -8.8 & 239 & 5.7 & 2.4 & $-\quad 9.0$ \\
\hline Sales representatives, commodities and finance & 346 & 5.5 & 2.8 & -8.2 & 131 & 14.0 & 6.9 & -21.1 \\
\hline Police and firefighters & 120 & 5.5 & 0.0 & -11.0 & 33 & 14.3 & 0.0 & -29.0 \\
\hline Cleaning and building service & 289 & 4.8 & 1.7 & -7.9 & 166 & 9.5 & 5.0 & -14.0 \\
\hline Motor vehicle operators & 268 & 4.6 & 2.4 & -6.8 & 207 & 6.4 & 2.9 & -9.9 \\
\hline Precision production occupations & 240 & 4.3 & 1.8 & -6.8 & 185 & 13.2 & 7.1 & -19.3 \\
\hline Construction laborers & 80 & 3.9 & 0.0 & -8.8 & 52 & 4.3 & 0.0 & 9.4 \\
\hline Farm workers and other agricultural workers & 195 & 3.7 & 1.2 & -6.2 & 97 & 10.4 & 2.6 & -18.2 \\
\hline Architects and surveyors & 17 & 3.5 & 0.0 & -10.4 & 6 & 14.5 & 0.0 & -42.5 \\
\hline Material moving equipment operators & 64 & 3.2 & 0.0 & -8.1 & 56 & 7.3 & 0.0 & -15.3 \\
\hline Mechanics and repairers & 283 & 3.1 & 1.1 & -5.1 & 200 & 9.3 & 4.8 & -13.8 \\
\hline Farm operators and managers & 69 & 2.5 & 0.0 & -5.8 & 19 & 0.0 & - & - \\
\hline Officials and administrators, public administration & 56 & 1.3 & 0.0 & 3.8 & 25 & 2.9 & 0.0 & -7.6 \\
\hline Other transportation, except motor vehicles & 15 & 0.0 & - & - & 11 & 22.1 & 0.0 & -59.1 \\
\hline Military & 3 & 0.0 & - & - & 0 & - & - & - \\
\hline TOTAL & 11,230 & 8.4 & 7.8 & 9.0 & 5,082 & 9.1 & 8.3 & 9.9 \\
\hline
\end{tabular}

- No estimates due to no asthma cases or no respondents.

Nonsmokers - Those respondents who indicated that they never smoked or smoked less than 100 cigarettes in their lifetime.

NOTE: Industries were classified according to 1995 Standard Industrial Classification System and then regrouped by NCHS. See appendices for source description, methods, and ICD codes.

SOURCE: National Center for Health Statistics 2000 National Health Interview Survey. 
Table 9-12. Asthma: Estimated prevalence by current occupation and smoking status, U.S. male residents age 18 and over, 2000

\begin{tabular}{|c|c|c|c|c|c|c|}
\hline \multirow[b]{2}{*}{ Occupation } & \multicolumn{3}{|c|}{ Nonsmokers } & \multicolumn{3}{|c|}{ Current Smokers } \\
\hline & $\begin{array}{c}\text { Number of } \\
\text { Respondents }\end{array}$ & $\begin{array}{c}\begin{array}{c}\text { Prevalence } \\
(\%)\end{array} \\
\end{array}$ & $\begin{array}{c}95 \% \\
\text { Confidence } \\
\text { Interval } \\
\end{array}$ & $\begin{array}{c}\text { Number of } \\
\text { Respondents }\end{array}$ & $\begin{array}{c}\text { Prevalence } \\
(\%)\end{array}$ & $\begin{array}{c}95 \% \\
\text { Confidence } \\
\text { Interval } \\
\end{array}$ \\
\hline Private household occupations & 4 & 28.8 & $0.0-77.0$ & 1 & 0.0 & - \\
\hline Computer equipment operators & 15 & 17.3 & $0.0-42.2$ & 3 & 0.0 & - \\
\hline Other protective service occupations & 49 & 15.7 & $1.0-30.4$ & 31 & 17.0 & $1.7-32.3$ \\
\hline Writers, artists, entertainers and athletes & 107 & 12.9 & $5.6-20.2$ & 34 & 4.2 & $0.0-12.2$ \\
\hline Teachers, librarians and counselors & 193 & 11.6 & $6.9-16.3$ & 30 & 3.7 & $0.0-10.8$ \\
\hline Forestry and fishing occupations & 11 & 11.3 & $0.0-32.3$ & 6 & 8.6 & $0.0-25.6$ \\
\hline Other professional specialty occupations & 110 & 11.1 & $4.6-17.6$ & 22 & 9.1 & $0.0-22.8$ \\
\hline Mail and message distributing & 34 & 10.0 & $0.0-28.0$ & 16 & 10.9 & $0.0-30.7$ \\
\hline Freight, stock and material handlers & 200 & 10.0 & $4.9-15.1$ & 137 & 11.2 & $4.5-17.9$ \\
\hline Food service & 161 & 9.6 & $3.5-15.7$ & 133 & 8.5 & $3.0-14.0$ \\
\hline Natural mathematical and computer scientists & 147 & 9.2 & $4.1-14.3$ & 50 & 11.9 & $3.9-19.9$ \\
\hline Technologists, technicians except health & 139 & 9.1 & $3.8-14.3$ & 43 & 13.8 & $2.2-25.4$ \\
\hline Other sales & 174 & 9.1 & $3.8-14.4$ & 87 & 14.7 & $5.1-24.3$ \\
\hline Health technologists and technicians & 34 & 8.9 & $0.0-21.1$ & 7 & 0.0 & - \\
\hline Other administrative support & 241 & 8.7 & $4.6-12.8$ & 120 & 7.2 & $2.1-12.3$ \\
\hline Construction and extractive trades & 322 & 8.5 & $4.6-12.4$ & 319 & 4.7 & $2.5-6.9$ \\
\hline Machine operators and tenderers, except precision & 193 & 8.4 & $3.7-13.1$ & 167 & 6.4 & $2.3-10.5$ \\
\hline Supervisors and proprietors & 169 & 8.0 & $4.1-11.9$ & 79 & 4.5 & $0.0-9.0$ \\
\hline Managers and administrators, except public admin. & 507 & 7.4 & $4.7-10.1$ & 264 & 5.7 & $2.8-8.6$ \\
\hline Fabricators, assemblers, inspectors and samplers & 103 & 7.4 & $2.7-12.1$ & 77 & 10.8 & $2.8-18.8$ \\
\hline Management related occupations & 166 & 6.7 & $3.0-10.4$ & 57 & 3.0 & $0.0-7.3$ \\
\hline Sales representatives, commodities and finance & 186 & 6.4 & $2.5-10.3$ & 73 & 15.7 & $5.1-26.3$ \\
\hline Personal service & 38 & 6.2 & $0.0-13.5$ & 14 & 0.0 & - \\
\hline Financial records processing occupations & 20 & 6.1 & $0.0-14.9$ & 4 & 0.0 & - \\
\hline Cleaning and building service & 129 & 5.7 & $0.6-10.8$ & 70 & 5.3 & $0.6-10.0$ \\
\hline Police and firefighters & 98 & 5.5 & $0.0-11.6$ & 26 & 18.4 & $0.2-36.6$ \\
\hline Engineers & 172 & 4.7 & $1.4-8.0$ & 34 & 15.5 & $1.4-29.6$ \\
\hline Health diagnosing occupations & 77 & 4.6 & $0.0-9.9$ & 1 & 0.0 & - \\
\hline Motor vehicle operators & 226 & 4.3 & $1.6-7.0$ & 174 & 6.5 & $2.8-10.2$ \\
\hline Precision production occupations & 168 & 4.2 & $1.1-7.3$ & 131 & 12.7 & $5.4-20.0$ \\
\hline Construction laborers & 77 & 4.0 & $0.0-8.9$ & 52 & 4.3 & $0.0-9.4$ \\
\hline Material moving equipment operators & 56 & 3.5 & $0.0-8.8$ & 53 & 7.8 & $0.0-16.6$ \\
\hline Health service & 16 & 3.3 & $0.0-10.2$ & 19 & 3.9 & $0.0-11.5$ \\
\hline Mechanics and repairers & 252 & 3.0 & $1.0-5.0$ & 188 & 9.3 & $4.6-14.0$ \\
\hline Health assessment and treating occupations & 38 & 2.8 & $0.0-6.7$ & 9 & 0.0 & - \\
\hline Farm workers and other agricultural workers & 152 & 2.4 & $0.2-4.6$ & 81 & 11.5 & $2.5-20.5$ \\
\hline Farm operators and managers & 54 & 1.4 & $0.0-4.1$ & 15 & 0.0 & - \\
\hline Officials and administrators, public administration & 23 & 0.0 & - & 12 & 0.0 & - \\
\hline Architects and surveyors & 13 & 0.0 & - & 6 & 14.5 & $0.0-42.5$ \\
\hline Secretaries, stenographers and typists & 4 & 0.0 & - & 3 & 0.0 & - \\
\hline Other transportation, except motor vehicles & 15 & 0.0 & - & 11 & 22.1 & $0.0-59.1$ \\
\hline Military & 1 & 0.0 & - & 0 & - & - \\
\hline TOTAL & 5,056 & 7.2 & $6.4-8.0$ & 2,714 & 8.0 & $6.8-9.2$ \\
\hline
\end{tabular}

- No estimates due to no asthma cases or no respondents.

Nonsmokers - Those respondents who indicated that they never smoked or smoked less than 100 cigarettes in their lifetime.

NOTE: Industries were classified according to 1995 Standard Industrial Classification System and then regrouped by NCHS. See appendices for source description, methods, and ICD codes.

SOURCE: National Center for Health Statistics 2000 National Health Interview Survey. 


\section{Table 9-13. Asthma: Estimated prevalence by current occupation and smoking status, U.S. female residents age 18 and over, 2000}

\begin{tabular}{|c|c|c|c|c|c|c|c|}
\hline \multirow{3}{*}{$\begin{array}{l}\text { Occupation } \\
\text { Health diagnosing occupations }\end{array}$} & \multicolumn{4}{|c|}{ Nonsmokers } & \multicolumn{3}{|c|}{ Current Smokers } \\
\hline & \multirow{2}{*}{$\begin{array}{c}\begin{array}{c}\text { Number of } \\
\text { Respondents }\end{array} \\
45\end{array}$} & \multirow{2}{*}{$\begin{array}{c}\begin{array}{c}\text { Prevalence } \\
(\%)\end{array} \\
20.1\end{array}$} & \multicolumn{2}{|c|}{$\begin{array}{c}95 \% \\
\text { Confidence } \\
\text { Interval } \\
\end{array}$} & \multirow{2}{*}{$\begin{array}{c}\begin{array}{c}\text { Number of } \\
\text { Respondents }\end{array} \\
4\end{array}$} & \multirow{2}{*}{$\begin{array}{c}\text { Prevalence } \\
(\%)\end{array}$} & $\begin{array}{c}95 \% \\
\text { Confidence } \\
\text { Interval }\end{array}$ \\
\hline & & & 3.0 & -37.2 & & & - \\
\hline Architects and surveyors & 4 & 18.9 & 0.0 & -52.8 & 0 & - & - \\
\hline Financial records processing occupations & 182 & 17.6 & 7.0 & -28.2 & 75 & 10.7 & $3.4-18.0$ \\
\hline Computer equipment operators & 14 & 17.3 & 0.0 & - 37.9 & 3 & 0.0 & - \\
\hline Engineers & 27 & 16.3 & 1.8 & -30.8 & 7 & 0.0 & - \\
\hline Mail and message distributing & 41 & 15.6 & 0.9 & -30.3 & 12 & 23.4 & $0.0-47.5$ \\
\hline Supervisors and proprietors & 143 & 13.3 & 5.9 & - 20.7 & 72 & 7.4 & $1.1-13.7$ \\
\hline Other sales & 337 & 12.8 & 8.7 & - 16.9 & 152 & 11.0 & $5.5-16.5$ \\
\hline Teachers, librarians and counselors & 586 & 12.3 & 9.0 & - 15.6 & 87 & 13.6 & $6.0-21.2$ \\
\hline Health technologists and technicians & 131 & 11.5 & 4.8 & -18.2 & 64 & 18.4 & $7.8-29.0$ \\
\hline Technologists, technicians except health & 81 & 11.3 & 4.2 & - 18.4 & 32 & 4.8 & $0.0-11.7$ \\
\hline Secretaries, stenographers and typists & 235 & 10.9 & 5.8 & - 16.0 & 88 & 6.3 & $1.8-10.8$ \\
\hline Other professional specialty occupations & 156 & 10.7 & 4.4 & - 17.0 & 50 & 11.2 & $2.0-20.4$ \\
\hline Personal service & 252 & 10.7 & 4.8 & - 16.6 & 82 & 10.0 & $2.0-18.0$ \\
\hline Other administrative support & 877 & 10.5 & 8.0 & - 13.0 & 353 & 11.5 & $7.6-15.4$ \\
\hline Natural mathematical and computer scientists & 88 & 10.2 & 3.3 & - 17.1 & 15 & 4.0 & $0.0-12.6$ \\
\hline Farm workers and other agricultural workers & 43 & 10.1 & 0.0 & -20.3 & 16 & 4.5 & $0.0-13.3$ \\
\hline Managers and administrators, except public admin. & 477 & 10.0 & 6.9 & -13.1 & 199 & 8.9 & $4.8-13.0$ \\
\hline Private household occupations & 105 & 9.5 & 1.5 & - 17.5 & 17 & 8.0 & $0.0-22.9$ \\
\hline Fabricators, assemblers, inspectors and samplers & 114 & 9.1 & 2.8 & - 15.4 & 40 & 13.2 & $1.8-24.6$ \\
\hline Health service & 241 & 8.7 & 4.6 & - 12.8 & 135 & 20.1 & $12.1-28.1$ \\
\hline Writers, artists, entertainers and athletes & 93 & 8.5 & 2.2 & -14.8 & 30 & 13.7 & $0.0-27.8$ \\
\hline Food service & 238 & 8.5 & 4.6 & - 12.4 & 191 & 10.3 & $6.0-14.6$ \\
\hline Health assessment and treating occupations & 286 & 8.2 & 4.9 & - 11.5 & 67 & 6.2 & $0.5-11.9$ \\
\hline Farm operators and managers & 15 & 7.1 & 0.0 & -20.4 & 4 & 0.0 & - \\
\hline Motor vehicle operators & 42 & 6.4 & 0.0 & - 14.2 & 33 & 6.2 & $0.0-15.8$ \\
\hline Freight, stock and material handlers & 110 & 6.3 & 0.2 & - 12.4 & 57 & 10.7 & $0.9-20.5$ \\
\hline Management related occupations & 310 & 5.6 & 2.9 & -8.3 & 104 & 6.8 & $2.7-10.9$ \\
\hline Police and firefighters & 22 & 5.3 & 0.0 & - 12.2 & 7 & 0.0 & - \\
\hline Precision production occupations & 72 & 4.5 & 0.0 & $-\quad 9.2$ & 54 & 14.8 & $3.6-26.0$ \\
\hline Sales representatives, commodities and finance & 160 & 4.1 & 0.8 & - 7.4 & 58 & 11.6 & $3.2-20.0$ \\
\hline Other protective service occupations & 29 & 4.0 & 0.0 & - $\quad 9.7$ & 18 & 12.5 & $0.0-28.6$ \\
\hline Mechanics and repairers & 31 & 3.9 & 0.0 & -11.3 & 12 & 9.0 & $0.0-26.1$ \\
\hline Cleaning and building service & 160 & 3.8 & 0.3 & - 7.3 & 96 & 13.4 & $6.0-20.8$ \\
\hline Officials and administrators, public administration & 33 & 2.6 & 0.0 & - 7.7 & 13 & 7.2 & $0.0-18.8$ \\
\hline Machine operators and tenderers, except precision & 177 & 2.6 & 0.1 & -5.1 & 72 & 3.9 & $0.0-8.4$ \\
\hline Forestry and fishing occupations & 1 & 0.0 & - & - & 1 & 0.0 & - \\
\hline Construction and extractive trades & 10 & 0.0 & - & - & 6 & 0.0 & - \\
\hline Other transportation, except motor vehicles & 0 & - & - & - & 0 & - & - \\
\hline Material moving equipment operators & 8 & 0.0 & - & - & 3 & 0.0 & - \\
\hline Construction laborers & 3 & 0.0 & - & - & 0 & - & - \\
\hline Military & 1 & 0.0 & - & - & 0 & - & - \\
\hline TOTAL & 6,174 & 9.7 & 8.7 & - 10.7 & 2,368 & 10.5 & $9.1-11.9$ \\
\hline
\end{tabular}

- No estimates due to no asthma cases or no respondents.

Nonsmokers - Those respondents who indicated that they never smoked or smoked less than 100 cigarettes in their lifetime.

NOTE: Industries were classified according to 1995 Standard Industrial Classification System and then regrouped by NCHS. See appendices for source description, methods, and ICD codes.

SOURCE: National Center for Health Statistics 2000 National Health Interview Survey. 



\section{Section 10}

Chronic Obstructive Pulmonary Disease 

Table 10-1. Chronic obstructive pulmonary disease: Proportionate mortality ratio (PMR) adjusted for age, sex, and race by usual industry, U.S. residents age 15 and over, selected states, 1999

\begin{tabular}{llrrrr}
\hline & & & 95\% Confidence Interval \\
\cline { 4 - 6 } CIC & Industry & $\begin{array}{c}\text { Number } \\
\text { of Deaths }\end{array}$ & PMR & LCL & UCL \\
\hline 041 & Coal mining & 783 & 1.98 & 1.84 & 2.12 \\
410 & Trucking service & 1,281 & 1.29 & 1.22 & 1.37 \\
050 & Nonmetallic mining and quarrying, except fuel & 77 & 1.28 & 1.01 & 1.61 \\
751 & Automotive repair and related services & 627 & 1.26 & 1.17 & 1.37 \\
040 & Metal mining & 115 & 1.26 & 1.04 & 1.52 \\
230 & Logging & 172 & 1.25 & 1.07 & 1.46 \\
042 & Oil and gas extraction & 129 & 1.24 & 1.04 & 1.48 \\
641 & Eating and drinking places & 1,373 & 1.23 & 1.17 & 1.30 \\
760 & Miscellaneous repair services & 183 & 1.23 & 1.06 & 1.42 \\
060 & Construction & 4,288 & 1.19 & 1.16 & 1.23 \\
242 & Furniture and fixtures & 335 & 1.19 & 1.06 & 1.32 \\
762 & Hotels and motels & 335 & 1.18 & 1.06 & 1.32 \\
771 & Laundry, cleaning, and garment services & 257 & 1.15 & 1.01 & 1.30 \\
802 & Miscellaneous entertainment and recreation services & 291 & 1.15 & 1.02 & 1.29 \\
832 & Nursing and personal care facilities & 310 & 1.13 & 1.01 & 1.27 \\
942 & Military & 855 & 1.11 & 1.04 & 1.19 \\
142 & Yarn, thread, and fabric mills & 1,423 & 1.07 & 1.01 & 1.12 \\
831 & Hospitals & 1,380 & 1.06 & 1.01 & 1.12 \\
\hline
\end{tabular}

CIC - Census Industry Code $\quad$ n.e.c. - not elsewhere classified $\quad$ LCL - lower confidence limit $\quad$ UCL - upper confidence limit NOTE: See appendices for source description, methods, and ICD codes, industry and occupation codes, and list of selected states. SOURCE: National Center for Health Statistics multiple cause of death data. 
Table 10-2. Chronic obstructive pulmonary disease: Proportionate mortality ratio (PMR) adjusted for age, sex, and race by usual occupation, U.S. residents age 15 and over, selected states, 1999

\begin{tabular}{|c|c|c|c|c|c|}
\hline \multirow[b]{2}{*}{$\mathrm{COC}$} & \multirow[b]{2}{*}{ Occupation } & \multirow{2}{*}{$\begin{array}{c}\text { Number of } \\
\text { Deaths }\end{array}$} & \multirow[b]{2}{*}{ PMR } & \multicolumn{2}{|c|}{ 95\% Confidence Interval } \\
\hline & & & & LCL & UCL \\
\hline 764 & Washing, cleaning, and pickling machine operators & 10 & 2.35 & 1.13 & 4.33 \\
\hline 864 & Helpers, mechanics and repairers & 9 & 2.22 & 1.02 & 4.22 \\
\hline 743 & Textile cutting machine operators & 11 & 2.02 & 1.01 & 3.62 \\
\hline 616 & Mining machine operators & 768 & 2.01 & 1.87 & 2.16 \\
\hline 599 & Construction trades, n.e.c. & 69 & 1.81 & 1.42 & 2.31 \\
\hline 617 & Mining occupations, n.e.c. & 28 & 1.81 & 1.21 & 2.62 \\
\hline 853 & Excavating and loading machine operators & 28 & 1.73 & 1.15 & 2.50 \\
\hline 595 & Roofers & 71 & 1.66 & 1.31 & 2.11 \\
\hline 435 & Waiters and waitresses & 434 & 1.61 & 1.46 & 1.77 \\
\hline 573 & Drywall installers & 33 & 1.48 & 1.02 & 2.08 \\
\hline 709 & Grinding, abrading, buffing, and polishing machine operators & 53 & 1.44 & 1.09 & 1.91 \\
\hline 747 & Pressing machine operators & 70 & 1.43 & 1.12 & 1.82 \\
\hline 888 & Hand packers and packagers & 84 & 1.39 & 1.12 & 1.73 \\
\hline 885 & Garage and service station related occupations & 44 & 1.39 & 1.01 & 1.86 \\
\hline 579 & Painters, construction and maintenance & 308 & 1.37 & 1.22 & 1.53 \\
\hline 727 & Sawing machine operators & 63 & 1.32 & 1.03 & 1.71 \\
\hline 507 & Bus, truck, and stationary engine mechanic & 149 & 1.30 & 1.11 & 1.54 \\
\hline 514 & Automobile body and related repairers & 66 & 1.30 & 1.02 & 1.67 \\
\hline 567 & Carpenters & 938 & 1.30 & 1.21 & 1.38 \\
\hline 783 & Welders and cutters & 351 & 1.28 & 1.15 & 1.43 \\
\hline 496 & Timber cutting and logging occupations & 135 & 1.26 & 1.06 & 1.50 \\
\hline 804 & Truck drivers & 1,617 & 1.26 & 1.20 & 1.32 \\
\hline 869 & Construction laborers & 664 & 1.25 & 1.15 & 1.35 \\
\hline 844 & Operating engineers & 318 & 1.23 & 1.10 & 1.38 \\
\hline 505 & Automobile mechanics & 499 & 1.23 & 1.13 & 1.35 \\
\hline 585 & Plumbers, pipefitters, and steamfitters & 326 & 1.17 & 1.05 & 1.31 \\
\hline 889 & Laborers, except construction & 1,586 & 1.17 & 1.11 & 1.23 \\
\hline 447 & Nursing aides, orderlies, and attendants & 569 & 1.17 & 1.07 & 1.27 \\
\hline 449 & Maids and housemen & 176 & 1.16 & 1.00 & 1.35 \\
\hline 777 & Miscellaneous machine operators, n.e.c. & 293 & 1.16 & 1.04 & 1.31 \\
\hline 479 & Farm workers & 217 & 1.16 & 1.01 & 1.33 \\
\hline 563 & Brickmasons and stonemasons & 186 & 1.16 & 1.00 & 1.34 \\
\hline 436 & Cooks & 484 & 1.15 & 1.05 & 1.25 \\
\hline 779 & Machine operators, not specified & 632 & 1.14 & 1.05 & 1.23 \\
\hline 905 & Military occupations & 746 & 1.10 & 1.03 & 1.19 \\
\hline
\end{tabular}

COC - Census Occupation Code n.e.c. - not elsewhere classified $\quad$ LCL - lower confidence limit UCL - upper confidence limit NOTE: See appendices for source description, methods, and ICD codes, industry and occupation codes, and list of selected states.

SOURCE: National Center for Health Statistics multiple cause of death data. 


\section{Table 10-3. Chronic obstructive pulmonary disease: Estimated prevalence by current industry and smoking status, U.S. residents age 18 and over, 2000}

\begin{tabular}{|c|c|c|c|c|c|c|}
\hline \multirow[b]{2}{*}{ Industry } & \multicolumn{3}{|c|}{ Nonsmokers } & \multicolumn{3}{|c|}{ Current Smokers } \\
\hline & $\begin{array}{c}\text { Number of } \\
\text { Respondents }\end{array}$ & $\begin{array}{c}\text { Prevalence } \\
(\%)\end{array}$ & $\begin{array}{c}95 \% \\
\text { Confidence } \\
\text { Interval } \\
\end{array}$ & $\begin{array}{c}\text { Number of } \\
\text { Respondents }\end{array}$ & $\begin{array}{c}\text { Prevalence } \\
(\%)\end{array}$ & $\begin{array}{c}95 \% \\
\text { Confidence } \\
\text { Interval } \\
\end{array}$ \\
\hline Forestry and fisheries & 17 & 15.0 & $0.0-41.3$ & 7 & 0.0 & - \\
\hline General merchandise stores & 208 & 7.2 & $2.5-11.9$ & 106 & 16.3 & $8.5-24.1$ \\
\hline Furniture, lumber and wood & 115 & 5.6 & $0.0-13.2$ & 61 & 4.3 & $0.0-9.0$ \\
\hline Other transportation & 227 & 5.5 & $2.0-9.0$ & 109 & 5.9 & $2.2-9.6$ \\
\hline Banking and credit agencies & 265 & 5.1 & $2.2-8.0$ & 90 & 7.6 & $0.0-16.2$ \\
\hline Social services, religious and membership orgs. & 490 & 4.5 & $2.3-6.7$ & 148 & 6.9 & $2.0-13.7$ \\
\hline Machinery, except electrical & 148 & 4.1 & $0.4-7.8$ & 92 & 6.0 & $0.7-11.3$ \\
\hline Textile mill and finished textile products & 114 & 4.0 & $0.0-9.3$ & 45 & 5.1 & $0.0-12.2$ \\
\hline Automotive dealers and gasoline stations & 142 & 3.9 & $0.0-8.2$ & 119 & 7.6 & $2.3-12.9$ \\
\hline Health services, except hospitals & 653 & 3.7 & $2.1-5.3$ & 252 & 10.1 & $5.8-14.4$ \\
\hline Legal, engineering and other professional services & 422 & 3.7 & $1.7-5.7$ & 144 & 8.6 & $3.5-13.7$ \\
\hline Elementary and secondary schools and colleges & 1,160 & 3.6 & $2.2-5.0$ & 211 & 10.1 & $4.2-16.0$ \\
\hline Agriculture & 278 & 3.4 & $1.2-5.6$ & 109 & 6.6 & $1.1-12.1$ \\
\hline Other and not specified retail trade & 510 & 3.4 & $1.4-5.4$ & 229 & 6.4 & $2.9-9.9$ \\
\hline Other nondurable goods & 113 & 3.2 & $0.0-7.2$ & 60 & 6.3 & $0.0-12.8$ \\
\hline Primary metal industries & 46 & 3.1 & $0.0-9.0$ & 40 & 6.5 & $0.0-14.1$ \\
\hline Utilities and sanitary & 107 & 2.9 & $0.0-6.0$ & 38 & 4.2 & $0.0-12.2$ \\
\hline Insurance, real estate, and other finance & 479 & 2.8 & $1.2-4.4$ & 194 & 4.8 & $1.5-8.1$ \\
\hline Other personal services & 297 & 2.7 & $0.9-4.5$ & 151 & 4.4 & $0.9-7.9$ \\
\hline Communications & 195 & 2.6 & $0.3-5.0$ & 79 & 5.7 & $0.0-11.4$ \\
\hline Food, bakery and dairy stores & 215 & 2.4 & $0.4-4.4$ & 154 & 8.2 & $2.5-13.9$ \\
\hline Public administration & 534 & 2.3 & $1.1-3.5$ & 193 & 7.8 & $3.9-11.7$ \\
\hline Printing, publishing and allied industries & 132 & 2.1 & $0.0-6.0$ & 47 & 5.6 & $0.0-12.1$ \\
\hline Transportation equipment & 166 & 2.0 & $0.0-4.4$ & 84 & 10.2 & $3.1-17.3$ \\
\hline Wholesale trade & 367 & 2.0 & $0.4-3.6$ & 172 & 3.7 & $0.8-6.6$ \\
\hline Hospitals & 510 & 2.0 & $0.6-3.4$ & 164 & 3.8 & $1.3-6.3$ \\
\hline Business services & 679 & 1.8 & $0.8-2.8$ & 349 & 8.9 & $5.6-12.2$ \\
\hline Entertainment and recreation services & 200 & 1.8 & $0.0-3.6$ & 89 & 4.1 & $0.0-8.4$ \\
\hline Food and kindred products & 121 & 1.7 & $0.0-4.2$ & 83 & 2.7 & $0.0-6.4$ \\
\hline Eating and drinking places & 426 & 1.7 & $0.1-3.3$ & 353 & 7.9 & $4.6-11.2$ \\
\hline Other educational services & 53 & 1.7 & $0.0-4.1$ & 10 & 46.5 & $5.5-87.5$ \\
\hline Construction & 547 & 1.6 & $0.4-2.8$ & 450 & 4.1 & $2.1-6.1$ \\
\hline Electrical machinery, equipment and supplies & 161 & 1.2 & $0.0-2.8$ & 75 & 3.8 & $0.0-7.9$ \\
\hline Repair services & 112 & 1.2 & $0.0-2.8$ & 113 & 6.6 & $1.9-11.3$ \\
\hline Other and not specified durable goods & 139 & 1.1 & $0.0-2.7$ & 60 & 0.0 & - \\
\hline Trucking service and warehousing & 181 & 1.1 & $0.0-2.5$ & 126 & 6.7 & $2.6-10.8$ \\
\hline Private households & 120 & 0.9 & $0.0-2.7$ & 27 & 13.1 & $0.0-29.8$ \\
\hline Mining & 28 & 0.0 & - & 25 & 11.8 & $0.0-25.2$ \\
\hline Chemicals and allied products & 93 & 0.0 & - & 36 & 8.7 & $0.0-20.1$ \\
\hline Fabricated metal industries, including ordnance & 82 & 0.0 & - & 67 & 3.3 & $0.0-8.0$ \\
\hline Railroads & 22 & 0.0 & - & 12 & 10.2 & $0.0-29.2$ \\
\hline Armed forces & 2 & 0.0 & - & 0 & - & - \\
\hline TOTAL & 11,229 & 2.8 & $2.4-3.2$ & 5,078 & 6.8 & $6.0-7.6$ \\
\hline
\end{tabular}

- No estimates due to no chronic obstructive pulmonary disease cases or no respondents.

Nonsmokers - Those respondents who indicated that they never smoked or smoked less than 100 cigarettes in their lifetime.

NOTE: Industries were classified according to 1995 Standard Industrial Classification System and then regrouped by NCHS. See appendices for source description, methods, and ICD codes.

SOURCE: National Center for Health Statistics 2000 National Health Interview Survey. 
Table 10-4. Chronic obstructive pulmonary disease: Estimated prevalence by current industry and smoking status, U.S. male residents age 18 and over, 2000

\begin{tabular}{|c|c|c|c|c|c|c|c|c|}
\hline \multirow[b]{2}{*}{ Industry } & \multicolumn{4}{|c|}{ Nonsmokers } & \multicolumn{4}{|c|}{ Current Smokers } \\
\hline & $\begin{array}{c}\text { Number of } \\
\text { Respondents }\end{array}$ & $\begin{array}{c}\text { Prevalence } \\
(\%)\end{array}$ & $\begin{array}{r}95 \\
\text { Conf } \\
\text { Int }\end{array}$ & $\begin{array}{l}5 \% \\
\text { idence } \\
\text { erval }\end{array}$ & $\begin{array}{c}\text { Number of } \\
\text { Respondents }\end{array}$ & $\begin{array}{c}\text { Prevalence } \\
(\%)\end{array}$ & $\begin{array}{r}9 \\
\text { Conf } \\
\text { Int }\end{array}$ & $\begin{array}{l}5 \% \\
\text { fidence } \\
\text { erval }\end{array}$ \\
\hline Forestry and fisheries & 12 & 22.2 & 0.0 & -58.7 & 6 & 0.0 & - & \\
\hline Other educational services & 7 & 6.0 & 0.0 & - 18.0 & 3 & 22.6 & 0.0 & -66.9 \\
\hline Social services, religious and membership orgs. & 97 & 5.4 & 0.3 & -10.5 & 21 & 4.0 & 0.0 & -11.6 \\
\hline Banking and credit agencies & 55 & 5.2 & 0.0 & - 12.3 & 23 & 0.0 & - & - \\
\hline Textile mill and finished textile products & 33 & 4.8 & 0.0 & - 12.6 & 23 & 4.3 & 0.0 & -12.9 \\
\hline Machinery, except electrical & 105 & 4.3 & 0.0 & - $\quad 8.8$ & 71 & 6.8 & 0.3 & -13.3 \\
\hline Printing, publishing and allied industries & 65 & 4.1 & 0.0 & - 11.7 & 32 & 3.5 & 0.0 & -10.2 \\
\hline Primary metal industries & 31 & 3.9 & 0.0 & -11.5 & 30 & 3.2 & 0.0 & $-\quad 9.5$ \\
\hline Legal, engineering and other professional services & 211 & 3.8 & 1.1 & -6.5 & 59 & 3.9 & 0.0 & -8.6 \\
\hline Other transportation & 125 & 3.2 & 0.0 & - 7.1 & 68 & 3.5 & 0.0 & -8.0 \\
\hline Utilities and sanitary & 83 & 2.8 & 0.0 & -6.1 & 29 & 0.0 & - & - \\
\hline General merchandise stores & 49 & 2.5 & 0.0 & -6.8 & 40 & 13.9 & 1.7 & -26.1 \\
\hline Communications & 105 & 2.3 & 0.0 & -5.0 & 42 & 3.6 & 0.0 & -10.5 \\
\hline Automotive dealers and gasoline stations & 98 & 2.3 & 0.0 & -6.8 & 82 & 6.1 & 0.2 & -12.0 \\
\hline Transportation equipment & 114 & 2.1 & 0.0 & -5.0 & 59 & 11.5 & 3.1 & -19.9 \\
\hline Insurance, real estate, and other finance & 181 & 2.1 & 0.0 & -4.5 & 80 & 5.7 & 0.2 & -11.2 \\
\hline Eating and drinking places & 195 & 1.9 & 0.0 & - 4.4 & 161 & 2.7 & 0.0 & $-\quad 5.8$ \\
\hline Agriculture & 204 & 1.8 & 0.0 & $-\quad 3.8$ & 87 & 6.4 & 0.5 & -12.3 \\
\hline Other and not specified retail trade & 214 & 1.8 & 0.0 & - 4.3 & 103 & 2.0 & 0.0 & $-\quad 4.4$ \\
\hline Construction & 490 & 1.5 & 0.3 & -2.7 & 423 & 4.1 & 1.9 & 6.3 \\
\hline Elementary and secondary schools and colleges & 313 & 1.4 & 0.2 & -2.6 & 56 & 10.1 & 0.7 & -19.5 \\
\hline Food, bakery and dairy stores & 100 & 1.3 & 0.0 & -3.1 & 57 & 10.1 & 0.0 & -21.7 \\
\hline Repair services & 99 & 1.3 & 0.0 & $-\quad 3.1$ & 96 & 3.6 & 0.0 & 7.5 \\
\hline Public administration & 258 & 1.2 & 0.0 & -2.6 & 91 & 4.2 & 0.0 & 8.7 \\
\hline Entertainment and recreation services & 116 & 1.0 & 0.0 & $-\quad 3.0$ & 48 & 2.8 & 0.0 & 8.3 \\
\hline Food and kindred products & 62 & 0.8 & 0.0 & -2.4 & 56 & 0.0 & - & - \\
\hline Other nondurable goods & 75 & 0.8 & 0.0 & -2.4 & 45 & 3.8 & 0.0 & 9.1 \\
\hline Wholesale trade & 218 & 0.7 & 0.0 & -1.5 & 115 & 1.8 & 0.0 & 4.0 \\
\hline Trucking service and warehousing & 136 & 0.6 & 0.0 & -1.8 & 96 & 2.7 & 0.0 & 6.0 \\
\hline Business services & 351 & 0.6 & 0.0 & -1.2 & 186 & 9.0 & 4.3 & -13.7 \\
\hline Hospitals & 112 & 0.6 & 0.0 & $-\quad 1.8$ & 26 & 2.8 & 0.0 & 8.3 \\
\hline Electrical machinery, equipment and supplies & 87 & 0.5 & 0.0 & -1.5 & 43 & 0.0 & - & - \\
\hline Mining & 24 & 0.0 & - & - & 21 & 14.8 & 0.0 & -31.1 \\
\hline Chemicals and allied products & 54 & 0.0 & - & - & 21 & 7.4 & 0.0 & -21.3 \\
\hline Furniture, lumber and wood & 81 & 0.0 & - & - & 41 & 6.5 & 0.0 & -13.6 \\
\hline Fabricated metal industries, including ordnance & 57 & 0.0 & - & - & 54 & 3.9 & 0.0 & 9.4 \\
\hline Other and not specified durable goods & 78 & 0.0 & - & - & 47 & 0.0 & - & - \\
\hline Railroads & 19 & 0.0 & - & - & 12 & 10.2 & 0.0 & -29.2 \\
\hline Private households & 6 & 0.0 & - & - & 2 & 0.0 & - & 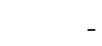 \\
\hline Other personal services & 75 & 0.0 & - & - & 60 & 5.8 & 0.0 & -12.9 \\
\hline Health services, except hospitals & 103 & 0.0 & - & - & 32 & 4.1 & 0.0 & - 11.7 \\
\hline Armed forces & 1 & 0.0 & - & - & 0 & - & - & - \\
\hline TOTAL & $\mathbf{5 , 0 5 7}$ & 1.7 & 1.3 & $-\quad 2.1$ & 2,712 & 4.6 & 3.6 & 5.6 \\
\hline
\end{tabular}

- No estimates due to no chronic obstructive pulmonary disease cases or no respondents.

Nonsmokers - Those respondents who indicated that they never smoked or smoked less than 100 cigarettes in their lifetime.

NOTE: Industries were classified according to 1995 Standard Industrial Classification System and then regrouped by NCHS. See appendices for source description, methods, and ICD codes.

SOURCE: National Center for Health Statistics 2000 National Health Interview Survey. 


\section{Table 10-5. Chronic obstructive pulmonary disease: Estimated prevalence by current industry and smoking status, U.S. female residents age 18 and over, 2000}

\begin{tabular}{|c|c|c|c|c|c|c|c|c|}
\hline \multirow[b]{2}{*}{ Industry } & \multicolumn{4}{|c|}{ Nonsmokers } & \multicolumn{4}{|c|}{ Current Smokers } \\
\hline & $\begin{array}{c}\text { Number of } \\
\text { Respondents }\end{array}$ & $\begin{array}{c}\text { Prevalence } \\
(\%)\end{array}$ & $\begin{array}{r}95 \\
\text { Confi } \\
\text { Inte } \\
\end{array}$ & $\begin{array}{l}5 \% \\
\text { iddence } \\
\text { erval }\end{array}$ & $\begin{array}{c}\text { Number of } \\
\text { Respondents }\end{array}$ & $\begin{array}{c}\text { Prevalence } \\
(\%)\end{array}$ & $\begin{array}{r}95 \\
\text { Conf } \\
\text { Inte }\end{array}$ & $\begin{array}{l}\mathbf{5 \%} \\
\text { fidence } \\
\text { erval }\end{array}$ \\
\hline Furniture, lumber and wood & 34 & 18.1 & $0.0-$ & -40.2 & 20 & 0.0 & - & \\
\hline Other nondurable goods & 38 & 9.7 & $0.0-$ & - 22.4 & 15 & 15.4 & 0.0 & -36.8 \\
\hline Other transportation & 102 & 8.8 & $2.1-$ & - 15.5 & 41 & 11.8 & 0.6 & -23.0 \\
\hline General merchandise stores & 159 & 8.6 & $2.5-$ & - 14.7 & 66 & 17.8 & 8.0 & -27.6 \\
\hline Automotive dealers and gasoline stations & 44 & 8.6 & $0.0-$ & - 19.6 & 37 & 11.2 & 0.0 & -22.6 \\
\hline Agriculture & 74 & 8.3 & $1.0-$ & - 15.6 & 22 & 7.7 & 0.0 & -22.0 \\
\hline Banking and credit agencies & 210 & 5.1 & $2.2-$ & - $\quad 8.0$ & 67 & 10.8 & 0.0 & -22.6 \\
\hline Other and not specified retail trade & 296 & 4.8 & $1.9-$ & - $\quad 7.7$ & 126 & 11.1 & 4.4 & -17.8 \\
\hline Health services, except hospitals & 550 & 4.6 & $2.6-$ & -6.6 & 220 & 11.0 & 6.1 & -15.9 \\
\hline Elementary and secondary schools and colleges & 847 & 4.5 & $2.7-$ & -6.3 & 155 & 11.5 & 4.4 & -18.6 \\
\hline Wholesale trade & 149 & 4.3 & $0.6-$ & - $\quad 8.0$ & 57 & 8.8 & 0.4 & -17.2 \\
\hline Social services, religious and membership orgs. & 393 & 4.2 & $1.8-$ & -6.6 & 127 & 7.5 & 2.0 & -13.0 \\
\hline Utilities and sanitary & 24 & 3.9 & $0.0-$ & - 11.5 & 9 & 23.6 & 0.0 & -61.8 \\
\hline Other personal services & 222 & 3.8 & $1.3-$ & -6.3 & 91 & 3.3 & 0.2 & -6.4 \\
\hline Public administration & 276 & 3.7 & $1.3-$ & -6.1 & 102 & 11.7 & 4.8 & -18.6 \\
\hline Textile mill and finished textile products & 81 & 3.6 & $0.0-$ & - 10.7 & 22 & 5.7 & 0.0 & -16.1 \\
\hline Machinery, except electrical & 43 & 3.6 & $0.0-$ & - 10.5 & 21 & 2.7 & 0.0 & -8.0 \\
\hline Legal, engineering and other professional services & 211 & 3.6 & $0.7-$ & -6.5 & 85 & 12.6 & 4.2 & -21.0 \\
\hline Business services & 328 & 3.5 & $1.3-$ & -5.7 & 163 & 8.9 & 4.0 & -13.8 \\
\hline Food, bakery and dairy stores & 115 & 3.4 & $0.3-$ & -6.5 & 97 & 6.9 & 1.6 & -12.2 \\
\hline Insurance, real estate, and other finance & 298 & 3.3 & $1.3-$ & -5.3 & 114 & 4.2 & 0.1 & -8.3 \\
\hline Trucking service and warehousing & 45 & 3.1 & $0.0-$ & -8.4 & 30 & 21.9 & 7.4 & -36.4 \\
\hline Communications & 90 & 3.1 & $0.0-$ & -7.4 & 37 & 8.8 & 0.0 & -18.4 \\
\hline Entertainment and recreation services & 84 & 3.1 & $0.0-$ & -6.8 & 41 & 6.0 & 0.0 & -13.3 \\
\hline Food and kindred products & 59 & 2.9 & $0.0-$ & -8.6 & 27 & 9.4 & 0.0 & -22.1 \\
\hline Other and not specified durable goods & 61 & 2.7 & $0.0-$ & -6.6 & 13 & 0.0 & - & - \\
\hline Construction & 57 & 2.6 & $0.0-$ & -6.7 & 27 & 4.2 & 0.0 & -10.3 \\
\hline Hospitals & 398 & 2.5 & $0.7-$ & -4.3 & 138 & 4.0 & 1.1 & -6.9 \\
\hline Electrical machinery, equipment and supplies & 74 & 2.3 & $0.0-$ & -5.8 & 32 & 8.9 & 0.0 & -18.3 \\
\hline Transportation equipment & 52 & 1.6 & $0.0-$ & -4.5 & 25 & 6.0 & 0.0 & -14.8 \\
\hline Eating and drinking places & 231 & 1.5 & $0.0-$ & $-\quad 3.1$ & 192 & 13.2 & 7.5 & -18.9 \\
\hline Other educational services & 46 & 1.1 & $0.0-$ & $-\quad 3.3$ & 7 & 52.4 & 6.3 & -98.5 \\
\hline Private households & 114 & 0.9 & $0.0-$ & $-\quad 2.7$ & 25 & 14.2 & 0.0 & -32.1 \\
\hline Forestry and fisheries & 5 & 0.0 & - & - & 1 & 0.0 & - & - \\
\hline Mining & 4 & 0.0 & - & - & 4 & 0.0 & - & - \\
\hline Printing, publishing and allied industries & 67 & 0.0 & - & - & 15 & 12.5 & 0.0 & -28.8 \\
\hline Chemicals and allied products & 39 & 0.0 & - & - & 15 & 10.4 & 0.0 & -29.4 \\
\hline Primary metal industries & 15 & 0.0 & - & - & 10 & 19.3 & 0.0 & -46.3 \\
\hline Fabricated metal industries, including ordnance & 25 & 0.0 & - & - & 13 & 0.0 & - & - \\
\hline Railroads & 3 & 0.0 & - & - & 0 & - & - & - \\
\hline Repair services & 13 & 0.0 & - & - & 17 & 29.0 & 4.5 & -53.5 \\
\hline Armed forces & 1 & 0.0 & - & - & 0 & - & - & - \\
\hline TOTAL & 6,172 & 4.0 & $3.4-$ & -4.6 & 2,366 & 9.7 & 8.1 & -11.3 \\
\hline
\end{tabular}

- No estimates due to no chronic obstructive pulmonary disease cases or no respondents.

Nonsmokers - Those respondents who indicated that they never smoked or smoked less than 100 cigarettes in their lifetime.

NOTE: Industries were classified according to 1995 Standard Industrial Classification System and then regrouped by NCHS. See appendices for source description, methods, and ICD codes.

SOURCE: National Center for Health Statistics 2000 National Health Interview Survey. 
Table 10-6. Chronic obstructive pulmonary disease: Estimated prevalence by current occupation and smoking status, U.S. residents age 18 and over, 2000

\begin{tabular}{|c|c|c|c|c|c|c|}
\hline \multirow{3}{*}{$\begin{array}{l}\text { Occupation } \\
\text { Supervisors and proprietors }\end{array}$} & \multicolumn{3}{|c|}{ Nonsmokers } & \multicolumn{3}{|c|}{ Current Smokers } \\
\hline & $\begin{array}{c}\text { Number of } \\
\text { Respondents }\end{array}$ & $\begin{array}{c}\text { Prevalence } \\
(\%)\end{array}$ & $\begin{array}{c}95 \% \\
\text { Confidence } \\
\text { Interval } \\
\end{array}$ & $\begin{array}{c}\text { Number of } \\
\text { Respondents }\end{array}$ & $\begin{array}{c}\text { Prevalence } \\
(\%)\end{array}$ & $\begin{array}{c}95 \% \\
\text { Confidence } \\
\text { Interval } \\
\end{array}$ \\
\hline & 312 & 5.5 & $2.0-9.0$ & 151 & 5.7 & $1.6-9.8$ \\
\hline Financial records processing occupations & 202 & 5.4 & $1.9-8.9$ & 78 & 10.8 & $2.6-19.0$ \\
\hline Other administrative support & 1117 & 5.2 & $3.6-6.8$ & 472 & 9.7 & $6.4-13.0$ \\
\hline Other professional specialty occupations & 265 & 5.0 & $2.1-7.9$ & 72 & 9.5 & $2.8-16.2$ \\
\hline Other protective service occupations & 78 & 4.8 & $0.0-9.9$ & 49 & 17.2 & $5.0-29.4$ \\
\hline Mail and message distributing & 75 & 4.7 & $0.0-9.4$ & 28 & 5.7 & $0.0-16.5$ \\
\hline Health service & 257 & 4.7 & $1.8-7.6$ & 154 & 8.4 & $2.5-14.3$ \\
\hline Secretaries, stenographers and typists & 239 & 4.3 & $1.4-7.2$ & 91 & 9.9 & $3.4-16.4$ \\
\hline Fabricators, assemblers, inspectors and samplers & 217 & 3.9 & $0.6-7.2$ & 117 & 6.0 & $1.1-10.9$ \\
\hline Teachers, librarians and counselors & 779 & 3.8 & $2.2-5.4$ & 117 & 10.9 & $8.9-12.9$ \\
\hline Writers, artists, entertainers and athletes & 200 & 3.4 & $0.1-6.7$ & 64 & 1.9 & $0.0-5.4$ \\
\hline Management related occupations & 475 & 3.3 & $1.3-5.3$ & 161 & 4.1 & $1.2-7.0$ \\
\hline Technologists, technicians except health & 220 & 3.2 & $0.5-5.9$ & 75 & 4.1 & $0.0-9.8$ \\
\hline Food service & 399 & 3.2 & $0.8-5.6$ & 324 & 9.0 & $5.1-12.9$ \\
\hline Other sales & 511 & 3.1 & $1.1-5.1$ & 239 & 7.3 & $3.8-10.8$ \\
\hline Farm workers and other agricultural workers & 196 & 3.1 & $0.2-6.0$ & 97 & 8.7 & $2.2-15.2$ \\
\hline Officials and administrators, public administration & 56 & 2.7 & $0.0-6.6$ & 25 & 5.4 & $0.0-13.0$ \\
\hline Sales representatives, commodities and finance & 346 & 2.6 & $1.0-4.2$ & 131 & 2.3 & $0.0-4.7$ \\
\hline Farm operators and managers & 69 & 2.6 & $0.0-5.9$ & 19 & 6.2 & $0.0-18.0$ \\
\hline Managers and administrators, except public admin. & 985 & 2.4 & $1.4-3.4$ & 463 & 5.2 & $2.3-8.1$ \\
\hline Personal service & 290 & 2.3 & $0.5-4.1$ & 96 & 8.9 & $2.0-15.8$ \\
\hline Cleaning and building service & 289 & 2.0 & $0.0-4.2$ & 167 & 8.5 & $4.0-13.0$ \\
\hline Construction laborers & 80 & 1.9 & $0.0-5.4$ & 52 & 7.2 & $0.1-14.3$ \\
\hline Health technologists and technicians & 165 & 1.8 & $0.0-4.0$ & 71 & 12.4 & $4.2-20.6$ \\
\hline Freight, stock and material handlers & 310 & 1.8 & $0.2-3.4$ & 194 & 8.0 & $3.5-12.5$ \\
\hline Engineers & 199 & 1.6 & $0.0-3.4$ & 41 & 6.9 & $0.0-14.3$ \\
\hline Machine operators and tenderers, except precision & 370 & 1.5 & $0.3-2.7$ & 237 & 3.5 & $1.0-6.0$ \\
\hline Health assessment and treating occupations & 324 & 1.4 & $0.0-2.8$ & 76 & 4.8 & $0.0-9.7$ \\
\hline Mechanics and repairers & 283 & 1.2 & $0.0-2.6$ & 200 & 5.5 & $2.2-8.8$ \\
\hline Natural mathematical and computer scientists & 236 & 1.0 & $0.0-2.2$ & 65 & 6.5 & $0.0-13.2$ \\
\hline Private household occupations & 108 & 1.0 & $0.0-3.0$ & 18 & 18.3 & $0.0-40.6$ \\
\hline Construction and extractive trades & 332 & 0.8 & $0.0-1.6$ & 325 & 4.4 & $2.0-6.8$ \\
\hline Motor vehicle operators & 268 & 0.6 & $0.0-1.6$ & 206 & 6.9 & $3.0-10.8$ \\
\hline Precision production occupations & 240 & 0.5 & $0.0-1.1$ & 185 & 6.1 & $1.8-10.4$ \\
\hline Health diagnosing occupations & 122 & 0.2 & $0.0-0.6$ & 5 & 0.0 & - \\
\hline Architects and surveyors & 17 & 0.0 & - & 6 & 0.0 & - \\
\hline Computer equipment operators & 29 & 0.0 & - & 6 & 0.0 & - \\
\hline Police and firefighters & 120 & 0.0 & - & 33 & 7.0 & $0.0-17.2$ \\
\hline Forestry and fishing occupations & 12 & 0.0 & - & 7 & 0.0 & - \\
\hline Other transportation, except motor vehicles & 15 & 0.0 & - & 11 & 18.3 & $0.0-40.1$ \\
\hline Material moving equipment operators & 64 & 0.0 & - & 56 & 2.2 & $0.0-6.3$ \\
\hline Military & 3 & 0.0 & - & 3 & 0.0 & - \\
\hline TOTAL & 11,229 & 2.8 & $2.4-3.2$ & 5,078 & 6.8 & $6.0-7.6$ \\
\hline
\end{tabular}

- No estimates due to no chronic obstructive pulmonary disease cases or no respondents.

Nonsmokers - Those respondents who indicated that they never smoked or smoked less than 100 cigarettes in their lifetime.

NOTE: Industries were classified according to 1995 Standard Industrial Classification System and then regrouped by NCHS. See appendices for source description, methods, and ICD codes.

SOURCE: National Center for Health Statistics 2000 National Health Interview Survey. 
Table 10-7. Chronic obstructive pulmonary disease: Estimated prevalence by current occupation and smoking status, U.S. male residents age 18 and over, 2000

\begin{tabular}{|c|c|c|c|c|c|c|}
\hline \multirow[b]{2}{*}{ Occupation } & \multicolumn{3}{|c|}{ Nonsmokers } & \multicolumn{3}{|c|}{ Current Smokers } \\
\hline & $\begin{array}{c}\text { Number of } \\
\text { Respondents }\end{array}$ & $\begin{array}{c}\text { Prevalence } \\
(\%)\end{array}$ & $\begin{array}{c}95 \% \\
\text { Confidence } \\
\text { Interval } \\
\end{array}$ & $\begin{array}{c}\text { Number of } \\
\text { Respondents }\end{array}$ & $\begin{array}{c}\text { Prevalence } \\
(\%)\end{array}$ & $\begin{array}{c}95 \% \\
\text { Confidence } \\
\text { Interval } \\
\end{array}$ \\
\hline Writers, artists, entertainers and athletes & 107 & 5.8 & $0.0-11.7$ & 34 & 0.0 & - \\
\hline Other protective service occupations & 49 & 5.1 & $0.0-12.0$ & 31 & 17.9 & $2.6-33.2$ \\
\hline Management related occupations & 166 & 4.5 & $0.4-8.6$ & 57 & 1.2 & $0.0-3.6$ \\
\hline Other administrative support & 241 & 4.3 & $1.6-7.0$ & 120 & 3.4 & $0.1-6.7$ \\
\hline Other professional specialty occupations & 109 & 4.1 & $0.4-7.8$ & 22 & 13.2 & $0.0-27.9$ \\
\hline Technologists, technicians except health & 139 & 3.9 & $0.2-7.6$ & 43 & 6.8 & $0.0-16.2$ \\
\hline Financial records processing occupations & 20 & 3.4 & $0.0-10.1$ & 4 & 0.0 & - \\
\hline Mail and message distributing & 34 & 3.1 & $0.0-9.2$ & 16 & 9.3 & $0.0-26.5$ \\
\hline Other sales & 174 & 2.9 & $0.0-6.4$ & 87 & 3.7 & $0.2-7.2$ \\
\hline Supervisors and proprietors & 169 & 2.3 & $0.0-5.0$ & 79 & 2.2 & $0.0-5.5$ \\
\hline Food service & 161 & 2.2 & $0.0-5.1$ & 133 & 5.0 & $0.0-10.7$ \\
\hline Farm workers and other agricultural workers & 153 & 1.9 & $0.0-4.4$ & 81 & 8.3 & $1.4-15.2$ \\
\hline Construction laborers & 77 & 1.9 & $0.0-5.6$ & 52 & 7.2 & $0.1-14.3$ \\
\hline Teachers, librarians and counselors & 193 & 1.8 & $0.0-4.0$ & 30 & 16.6 & $1.4-33.1$ \\
\hline Farm operators and managers & 54 & 1.5 & $0.0-4.2$ & 15 & 8.0 & $0.0-22.7$ \\
\hline Managers and administrators, except public admin. & 507 & 1.4 & $0.2-2.6$ & 264 & 3.9 & $1.4-6.4$ \\
\hline Engineers & 172 & 1.4 & $0.0-3.4$ & 3.4 & 3.2 & $0.0-9.3$ \\
\hline Sales representatives, commodities and finance & 186 & 1.4 & $0.0-3.0$ & 73 & 2.9 & $0.0-6.6$ \\
\hline Mechanics and repairers & 252 & 1.3 & $0.0-2.9$ & 188 & 5.0 & $1.7-8.3$ \\
\hline Fabricators, assemblers, inspectors and samplers & 103 & 1.2 & $0.0-2.8$ & 77 & 4.9 & $0.0-10.4$ \\
\hline Freight, stock and material handlers & 200 & 1.1 & $0.0-2.5$ & 137 & 5.2 & $0.9-9.5$ \\
\hline Construction and extractive trades & 322 & 0.8 & $0.0-1.6$ & 319 & 4.5 & $2.0-7.0$ \\
\hline Cleaning and building service & 129 & 0.7 & $0.0-1.7$ & 71 & 3.9 & $0.0-8.6$ \\
\hline Precision production occupations & 168 & 0.6 & $0.0-1.4$ & 131 & 5.8 & $0.7-10.9$ \\
\hline Machine operators and tenderers, except precision & 193 & 0.6 & $0.0-1.4$ & 165 & 3.1 & $0.4-5.8$ \\
\hline Health diagnosing occupations & 77 & 0.3 & $0.0-0.9$ & 1 & 0.0 & - \\
\hline Motor vehicle operators & 226 & 0.2 & $0.0-0.6$ & 173 & 4.2 & $1.1-7.3$ \\
\hline Officials and administrators, public administration & 23 & 0.0 & - & 12 & 0.0 & - \\
\hline Architects and surveyors & 13 & 0.0 & - & 6 & 0.0 & - \\
\hline Natural mathematical and computer scientists & 148 & 0.0 & - & 50 & 5.9 & $0.0-13.5$ \\
\hline Health assessment and treating occupations & 38 & 0.0 & - & 9 & 0.0 & - \\
\hline Health technologists and technicians & 34 & 0.0 & - & 7 & 10.0 & $0.0-29.4$ \\
\hline Computer equipment operators & 15 & 0.0 & - & 3 & 0.0 & - \\
\hline Secretaries, stenographers and typists & 4 & 0.0 & - & 3 & 0.0 & - \\
\hline Private household occupations & 4 & 0.0 & - & 1 & 0.0 & - \\
\hline Police and firefighters & 98 & 0.0 & - & 26 & 6.6 & $0.0-18.9$ \\
\hline Health service & 16 & 0.0 & - & 19 & 0.0 & - \\
\hline Personal service & 38 & 0.0 & - & 14 & 0.0 & - \\
\hline Forestry and fishing occupations & 11 & 0.0 & - & 6 & 0.0 & - \\
\hline Other transportation, except motor vehicles & 15 & 0.0 & - & 11 & 18.3 & $0.0-40.1$ \\
\hline Material moving equipment operators & 56 & 0.0 & - & 53 & 2.4 & $0.0-6.9$ \\
\hline Military & 2 & 0.0 & - & 0 & - & - \\
\hline TOTAL & 5,057 & 1.7 & $1.3-2.1$ & 2,712 & 4.6 & $3.6-5.6$ \\
\hline
\end{tabular}

- No estimates due to no chronic obstructive pulmonary disease cases or no respondents.

Nonsmokers - Those respondents who indicated that they never smoked or smoked less than 100 cigarettes in their lifetime.

NOTE: Industries were classified according to 1995 Standard Industrial Classification System and then regrouped by NCHS. See appendices for source description, methods, and ICD codes.

SOURCE: National Center for Health Statistics 2000 National Health Interview Survey. 
Table 10-8. Chronic obstructive pulmonary disease: Estimated prevalence by current occupation and smoking status, U.S. female residents age 18 and over, 2000

\begin{tabular}{|c|c|c|c|c|c|c|}
\hline \multirow[b]{2}{*}{ Occupation } & \multicolumn{3}{|c|}{ Nonsmokers } & \multicolumn{3}{|c|}{ Current Smokers } \\
\hline & $\begin{array}{c}\text { Number of } \\
\text { Respondents }\end{array}$ & $\begin{array}{c}\text { Prevalence } \\
(\%)\end{array}$ & $\begin{array}{c}95 \% \\
\text { Confidence } \\
\text { Interval } \\
\end{array}$ & $\begin{array}{c}\text { Number of } \\
\text { Respondents }\end{array}$ & $\begin{array}{c}\text { Prevalence } \\
(\%)\end{array}$ & $\begin{array}{c}95 \% \\
\text { Confidence } \\
\text { Interval } \\
\end{array}$ \\
\hline Supervisors and proprietors & 143 & 10.0 & $2.9-17.1$ & 72 & 10.7 & $1.9-19.5$ \\
\hline Farm workers and other agricultural workers & 43 & 9.1 & $0.0-20.1$ & 16 & 10.9 & $0.0-30.7$ \\
\hline Fabricators, assemblers, inspectors and samplers & 114 & 7.2 & $0.5-13.9$ & 40 & 8.4 & $0.0-17.8$ \\
\hline Farm operators and managers & 15 & 7.1 & $0.0-20.4$ & 4 & 0.0 & - \\
\hline Mail and message distributing & 41 & 6.6 & $0.0-14.0$ & 12 & 0.0 & - \\
\hline Other professional specialty occupations & 156 & 5.8 & $1.7-9.9$ & 50 & 7.7 & $0.6-14.8$ \\
\hline Financial records processing occupations & 182 & 5.6 & $1.7-9.5$ & 74 & 11.4 & $2.8-20.0$ \\
\hline Other administrative support & 876 & 5.5 & $3.5-7.5$ & 352 & 12.1 & $8.6-15.6$ \\
\hline Officials and administrators, public administration & 33 & 5.4 & $0.0-13.0$ & 13 & 13.3 & $0.0-31.1$ \\
\hline Health service & 241 & 5.2 & $1.9-8.5$ & 135 & 9.6 & $2.9-16.3$ \\
\hline Teachers, librarians and counselors & 586 & 4.5 & $2.5-6.5$ & 87 & 9.1 & $3.2-15.0$ \\
\hline Sales representatives, commodities and finance & 160 & 4.4 & $1.3-7.5$ & 58 & 1.3 & $0.0-3.8$ \\
\hline Secretaries, stenographers and typists & 235 & 4.4 & $1.3-7.5$ & 88 & 10.2 & $3.5-16.9$ \\
\hline Other protective service occupations & 29 & 4.2 & $0.0-10.1$ & 18 & 15.7 & $0.0-33.9$ \\
\hline Food service & 238 & 4.0 & $0.5-7.5$ & 191 & 12.6 & $6.9-18.3$ \\
\hline Managers and administrators, except public admin. & 478 & 3.8 & $1.8-5.8$ & 199 & 7.3 & $3.6-11.0$ \\
\hline Freight, stock and material handlers & 110 & 3.6 & $0.0-7.7$ & 57 & 14.9 & $4.1-25.7$ \\
\hline Engineers & 27 & 3.4 & $0.0-10.1$ & 7 & 29.2 & $0.0-65.3$ \\
\hline Cleaning and building service & 160 & 3.4 & $0.0-7.5$ & 96 & 12.8 & $5.2-20.4$ \\
\hline Other sales & 337 & 3.2 & $1.2-5.2$ & 152 & 9.7 & $4.6-14.8$ \\
\hline Natural mathematical and computer scientists & 88 & 3.0 & $0.0-6.5$ & 15 & 8.6 & $0.0-21.7$ \\
\hline Motor vehicle operators & 42 & 3.0 & $0.0-8.7$ & 33 & 22.8 & $5.4-40.2$ \\
\hline Machine operators and tenderers, except precision & 177 & 2.7 & $0.2-5.2$ & 72 & 4.7 & $0.0-10.6$ \\
\hline Management related occupations & 309 & 2.6 & $0.8-4.4$ & 104 & 5.9 & $1.6-10.2$ \\
\hline Personal service & 252 & 2.6 & $0.6-4.6$ & 82 & 11.1 & $2.7-19.5$ \\
\hline Health technologists and technicians & 131 & 2.3 & $0.0-5.0$ & 64 & 12.9 & $3.9-21.9$ \\
\hline Technologists, technicians except health & 81 & 1.8 & $0.0-4.3$ & 32 & 0.0 & - \\
\hline Health assessment and treating occupations & 286 & 1.6 & $0.0-3.2$ & 67 & 5.5 & $0.0-11.2$ \\
\hline Private household occupations & 104 & 1.0 & $0.0-3.0$ & 17 & 19.5 & $0.0-43.2$ \\
\hline Writers, artists, entertainers and athletes & 93 & 0.6 & $0.0-1.6$ & 30 & 4.1 & $0.0-11.9$ \\
\hline Architects and surveyors & 4 & 0.0 & - & 0 & - & - \\
\hline Health diagnosing occupations & 45 & 0.0 & - & 4 & 0.0 & - \\
\hline Computer equipment operators & 14 & 0.0 & - & 3 & 0.0 & - \\
\hline Police and firefighters & 22 & 0.0 & - & 7 & 8.4 & $0.0-24.9$ \\
\hline Forestry and fishing occupations & 1 & 0.0 & - & 1 & 0.0 & - \\
\hline Mechanics and repairers & 31 & 0.0 & - & 12 & 17.1 & $0.0-39.6$ \\
\hline Construction and extractive trades & 10 & 0.0 & - & 6 & 0.0 & - \\
\hline Precision production occupations & 72 & 0.0 & - & 54 & 6.9 & $0.0-14.0$ \\
\hline Other transportation, except motor vehicles & 0 & - & - & 0 & - & - \\
\hline Material moving equipment operators & 8 & 0.0 & - & 3 & 0.0 & - \\
\hline Construction laborers & 3 & 0.0 & - & 0 & - & - \\
\hline Military & 1 & 0.0 & - & 0 & - & - \\
\hline TOTAL & 6,172 & 4.0 & $3.4-4.6$ & 2,366 & 9.7 & $8.1-11.3$ \\
\hline
\end{tabular}

- No estimates due to no chronic obstructive pulmonary disease cases or no respondents.

Nonsmokers - Those respondents who indicated that they never smoked or smoked less than 100 cigarettes in their lifetime.

NOTE: Industries were classified according to 1995 Standard Industrial Classification System and then regrouped by NCHS. See appendices for source description, methods, and ICD codes.

SOURCE: National Center for Health Statistics 2000 National Health Interview Survey. 


\section{Section 11}

Respiratory Conditions Due to Toxic Agents 

Table 11-1. Occupational respiratory conditions due to toxic agents: Estimated number of cases reported by employers, by industry division, U.S. private sector, 1973-2000

\begin{tabular}{|c|c|c|c|c|c|c|c|c|c|}
\hline Year & Agriculture & Mining & Construction & Manufacturing & $\begin{array}{c}\text { Transportation } \\
\text { \& Public } \\
\text { Utilities } \\
\end{array}$ & $\begin{array}{c}\text { Wholesale } \\
\text { \& Retail } \\
\text { Trade } \\
\end{array}$ & Finance & Services & Total \\
\hline 1973 & 100 & - & 1,000 & 7,300 & 700 & 1,100 & 100 & 1,100 & 11,500 \\
\hline 1974 & 200 & 100 & 900 & 8,500 & 700 & 1,200 & 100 & 1,000 & 12,700 \\
\hline 1975 & 200 & 100 & 900 & 7,100 & 900 & 1,400 & 300 & 1,100 & 11,900 \\
\hline 1976 & 200 & 100 & 1,100 & 7,700 & 1,100 & 1,000 & 200 & 1,600 & 13,100 \\
\hline 1977 & 100 & - & 1,100 & 7,500 & 1,100 & 1,400 & 100 & 1,700 & 13,100 \\
\hline 1978 & 100 & 100 & 1,100 & 7,900 & 1,100 & 1,600 & 200 & 1,600 & 13,600 \\
\hline 1979 & 100 & 100 & 1,100 & 7,800 & 900 & 1,300 & 200 & 1,700 & 13,100 \\
\hline 1980 & 100 & 100 & 700 & 6,700 & 1,000 & 1,300 & 100 & 1,300 & 11,400 \\
\hline 1981 & 100 & 100 & 1,000 & 5,900 & 800 & 1,100 & 100 & 1,600 & 10,800 \\
\hline 1982 & 100 & 100 & 600 & 4,700 & 700 & 700 & 100 & 1,600 & 8,800 \\
\hline 1983 & 100 & 100 & 700 & 4,000 & 600 & 700 & 100 & 1,700 & 7,900 \\
\hline 1984 & 100 & 100 & 700 & 5,500 & 700 & 1,200 & 200 & 2,100 & 10,600 \\
\hline 1985 & 200 & 100 & 800 & 6,000 & 900 & 1,400 & 400 & 1,800 & 11,600 \\
\hline 1986 & 100 & - & 600 & 6,400 & 700 & 1,600 & 400 & 2,400 & 12,300 \\
\hline 1987 & 700 & - & 700 & 7,500 & 900 & 1,700 & 400 & 2,400 & 14,300 \\
\hline 1988 & 200 & 100 & 900 & 9,200 & 1,000 & 1,300 & 500 & 3,000 & 16,100 \\
\hline 1989 & 100 & - & 700 & 9,900 & 800 & 3,500 & 300 & 3,500 & 18,900 \\
\hline 1990 & 200 & 100 & 1,200 & 10,300 & 1,200 & 2,200 & 800 & 4,700 & 20,500 \\
\hline 1991 & 300 & - & 800 & 8,800 & 1,100 & 1,600 & 700 & 4,800 & 18,300 \\
\hline 1992 & 400 & 100 & 1,000 & 10,000 & 1,100 & 3,300 & 900 & 6,800 & 23,500 \\
\hline 1993 & 300 & 100 & 800 & 10,100 & 2,000 & 3,000 & 1,500 & 6,400 & 24,200 \\
\hline 1994 & 200 & 100 & 900 & 11,000 & 1,700 & 3,000 & 800 & 7,700 & 25,300 \\
\hline 1995 & 200 & 100 & 800 & 9,400 & 1,800 & 2,900 & 1,400 & 7,900 & 24,400 \\
\hline 1996 & 200 & - & 600 & 7,800 & 1,800 & 2,000 & 700 & 8,500 & 21,700 \\
\hline 1997 & 400 & - & 700 & 7,500 & 1,600 & 2,300 & 900 & 6,800 & 20,300 \\
\hline 1998 & 500 & - & 800 & 6,600 & 1,200 & 2,600 & 600 & 5,100 & 17,500 \\
\hline 1999 & 300 & - & 600 & 6,600 & 1,800 & 1,700 & 500 & 5,000 & 16,500 \\
\hline 2000 & 100 & - & 500 & 5,500 & 1,000 & 1,700 & 600 & 5,400 & 14,700 \\
\hline
\end{tabular}

- indicates no data reported or data that do not meet BLS publication guidelines.

NOTE: Respiratory conditions due to toxic agents include pneumonitis, pharyngitis, rhinitis or acute congestions due to chemicals, dusts, gases or fumes. The sum of industry divisions may not equal the total due to rounding. See appendices for source description.

SOURCE: Bureau of Labor Statistics annual reports of occupational injuries and illnesses. 
Table 11-2. Occupational respiratory conditions due to toxic agents: Estimated rate (based on cases reported by employers, per 10,000 full-time workers) by industry division, U.S. private sector, 1973-2000

\begin{tabular}{|c|c|c|c|c|c|c|c|c|c|}
\hline Year & Agriculture & Mining & Construction & Manufacturing & $\begin{array}{c}\text { Transportation } \\
\text { \& Public } \\
\text { Utilities } \\
\end{array}$ & $\begin{array}{c}\text { Wholesale } \\
\text { \& Retail } \\
\text { Trade } \\
\end{array}$ & Finance & Services & Overall \\
\hline 1973 & 1.8 & 1.7 & 3.2 & 3.8 & 1.7 & 0.8 & 0.2 & 1.2 & 2.1 \\
\hline 1974 & 2.4 & 0.9 & 3.0 & 4.4 & 1.6 & 0.8 & 0.2 & 0.9 & 2.2 \\
\hline 1975 & 1.7 & 0.8 & 3.1 & 4.1 & 2.1 & 1.0 & 0.7 & 1.1 & 2.2 \\
\hline 1976 & 3.1 & 1.6 & 3.7 & 4.3 & 2.6 & 0.7 & 0.5 & 1.5 & 2.3 \\
\hline 1977 & 2.0 & 0.5 & 3.3 & 4.0 & 2.5 & 0.9 & 0.2 & 1.4 & 2.2 \\
\hline 1978 & 2.2 & 0.8 & 2.9 & 4.0 & 2.4 & 1.0 & 0.6 & 1.3 & 2.2 \\
\hline 1979 & 1.1 & 0.8 & 2.8 & 3.9 & 1.9 & 0.8 & 0.5 & 1.3 & 2.0 \\
\hline 1980 & 2.0 & 0.8 & 2.0 & 3.5 & 2.0 & 0.8 & 0.2 & 1.0 & 1.8 \\
\hline 1981 & 1.1 & 1.0 & 2.9 & 3.1 & 1.7 & 0.7 & 0.2 & 1.1 & 1.7 \\
\hline 1982 & 1.7 & 0.5 & 1.9 & 2.7 & 1.5 & 0.5 & 0.3 & 1.1 & 1.4 \\
\hline 1983 & 1.4 & 0.8 & 2.0 & 2.3 & 1.4 & 0.4 & 0.2 & 1.1 & 1.2 \\
\hline 1984 & 1.5 & 0.9 & 1.8 & 2.9 & 1.4 & 0.7 & 0.5 & 1.3 & 1.6 \\
\hline 1985 & 2.4 & 1.0 & 1.9 & 3.2 & 1.8 & 0.8 & 0.8 & 1.1 & 1.7 \\
\hline 1986 & 1.3 & - & 1.5 & 3.5 & 1.5 & 0.9 & 0.6 & 1.4 & 1.7 \\
\hline 1987 & 7.9 & 0.6 & 1.6 & 4.0 & 1.7 & 0.9 & 0.7 & 1.3 & 2.0 \\
\hline 1988 & 2.1 & 0.7 & 2.0 & 4.9 & 1.9 & 0.6 & 0.9 & 1.6 & 2.2 \\
\hline 1989 & 1.5 & 0.5 & 1.5 & 5.2 & 1.6 & 1.7 & 0.5 & 1.7 & 2.5 \\
\hline 1990 & 1.6 & 0.7 & 2.6 & 5.6 & 2.2 & 1.1 & - & 2.2 & 2.7 \\
\hline 1991 & 2.7 & 0.6 & 2.1 & 5.0 & 2.1 & 0.8 & - & 2.3 & 2.4 \\
\hline 1992 & 3.8 & 1.3 & 2.4 & 5.6 & 2.1 & 1.6 & 1.4 & 3.1 & 3.1 \\
\hline 1993 & 2.5 & 0.9 & 2.0 & 5.6 & 3.7 & 1.5 & 2.5 & 2.8 & 3.1 \\
\hline 1994 & 1.8 & 1.2 & 1.9 & 6.0 & 3.0 & 1.4 & 1.2 & 3.3 & 3.1 \\
\hline 1995 & 1.4 & 1.5 & 1.7 & 5.1 & 3.2 & 1.3 & 2.3 & 3.4 & 3.0 \\
\hline 1996 & 1.7 & 0.3 & 1.2 & 4.2 & 3.1 & 0.9 & 1.2 & 3.5 & 2.6 \\
\hline 1997 & 2.7 & 0.6 & 1.4 & 4.0 & 2.7 & 1.0 & 1.4 & 2.7 & 2.4 \\
\hline 1998 & 3.7 & 0.8 & 1.4 & 3.5 & 2.0 & 1.1 & 1.0 & 1.9 & 2.0 \\
\hline 1999 & 1.8 & 0.6 & 1.0 & 3.6 & 2.8 & 0.7 & 0.8 & 1.9 & 1.8 \\
\hline 2000 & 0.8 & 0.4 & 0.9 & 3.0 & 1.5 & 0.7 & 0.9 & 1.9 & 1.6 \\
\hline
\end{tabular}

- indicates no data reported or data that do not meet BLS publication guidelines.

NOTE: Respiratory conditions due to toxic agents include pneumonitis, pharyngitis, rhinitis or acute congestions due to chemicals, dusts, gases or fumes. The sum of industry divisions may not equal the total due to rounding. See appendices for source description.

SOURCE: Bureau of Labor Statistics annual reports of occupational injuries and illnesses. 
Table 11-3 (page 1 of 2). Occupational respiratory conditions due to toxic agents: Industries with the highest estimated incidence rates (based on cases reported by employers, per 10,000 full-time workers), U.S. private sector, 1996-2000

\begin{tabular}{|c|c|c|c|}
\hline Year/Industry & SIC & $\begin{array}{l}\text { Estimated } \\
\text { Number of } \\
\text { Cases }\end{array}$ & $\begin{array}{c}\text { Rate } \\
\text { (per 10,000 full- } \\
\text { time workers) }\end{array}$ \\
\hline \multicolumn{4}{|l|}{1996} \\
\hline$\overline{\text { Transportation equipment }}$ & 37 & 3,000 & 11.2 \\
\hline Fishing, hunting, and trapping & 09 & - & 7.4 \\
\hline Health services & 80 & 5,200 & 6.9 \\
\hline Hotels and other lodging places & 70 & 900 & 6.3 \\
\hline Food and kindred products & 20 & 1,000 & 5.7 \\
\hline Primary metal industries & 33 & 400 & 4.8 \\
\hline Communications & 48 & 600 & 4.7 \\
\hline Fabricated metal products & 34 & 700 & 4.7 \\
\hline Chemicals and allied products & 28 & 500 & 4.5 \\
\hline Leather and leather products & 31 & - & 4.5 \\
\hline Rubber and miscellaneous plastic products & 30 & 400 & 4.5 \\
\hline ALL INDUSTRIES & & 21,700 & 2.6 \\
\hline \multicolumn{4}{|l|}{1997} \\
\hline$\overline{\text { Fishing, hunting, and trapping }}$ & 09 & - & 15.7 \\
\hline Leather and leather products & 31 & 100 & 15.2 \\
\hline Transportation equipment & 37 & 1,900 & 10.1 \\
\hline Museums, botanical, zoological gardens & 84 & 100 & 9.7 \\
\hline Instruments and related products & 38 & 400 & 5.0 \\
\hline Food and kindred products & 20 & 800 & 5.0 \\
\hline Electronic and other electic equipment & 36 & 800 & 4.6 \\
\hline Primary metal industries & 33 & 300 & 4.4 \\
\hline Health services & 80 & 3,400 & 4.4 \\
\hline Chemicals and allied products & 28 & 400 & 4.3 \\
\hline ALL INDUSTRIES & & 20,300 & 2.4 \\
\hline \multicolumn{4}{|l|}{1998} \\
\hline$\overline{\text { Agricultural production-livestock }}$ & 02 & 100 & 11.2 \\
\hline Transportation equipment & 37 & 1,800 & 9.1 \\
\hline Primary metal industries & 33 & 500 & 6.9 \\
\hline Food and kindred products & 20 & 100 & 5.7 \\
\hline Fishing, hunting, and trapping & 09 & - & 5.4 \\
\hline Furniture and fixtures & 25 & 200 & 4.6 \\
\hline Electronic and other electic equipment & 36 & 700 & 4.0 \\
\hline Amusement and recreation services & 79 & 400 & 3.9 \\
\hline Health services & 80 & 2,800 & 3.6 \\
\hline Transportation by air & 45 & 400 & 3.6 \\
\hline ALL INDUSTRIES & & $\mathbf{1 7 , 5 0 0}$ & 2.0 \\
\hline
\end{tabular}

See footnotes at end of table. 
Table 11-3 (page 2 of 2). Occupational respiratory conditions due to toxic agents: Industries with the highest estimated incidence rates (based on cases reported by employers, per 10,000 full-time workers), U.S. private sector, 1996-2000

\begin{tabular}{lrrr}
\hline Year/Industry & SIC & $\begin{array}{c}\text { Estimated } \\
\text { Number of } \\
\text { Cases }\end{array}$ & $\begin{array}{c}\text { Rate } \\
\text { (per 10,000 full- } \\
\text { time workers) }\end{array}$ \\
\hline 1999 & 29 & 100 & 9.8 \\
Petroleum and coal products & 37 & 1,700 & 8.7 \\
Cransportation equipment & 48 & 900 & 5.9 \\
Food and kindred products & 20 & 1,000 & 5.8 \\
Primary metal industries & 33 & 300 & 4.7 \\
Rubber and miscellaneous plastic products & 30 & 400 & 4.2 \\
Museums, botanical, zoological gardens & 84 & - & 3.8 \\
Electronic and other electric equipment & 36 & 600 & 3.5 \\
Health services & 80 & 2,800 & 3.5 \\
Chemicals and allied products & 28 & 300 & 3.3 \\
ALL INDUSTRIES & & $\mathbf{1 6 , 5 0 0}$ & $\mathbf{1 . 8}$ \\
& & & \\
2000 & & & 7.0 \\
Transportation equipment & 37 & 1,300 & 5.3 \\
Food and kindred products & 900 & 4.0 \\
Health services & 20 & 3,200 & 3.8 \\
Primary metal industries & 80 & 300 & 3.7 \\
Petroleum and coal products & 33 & - & 3.2 \\
Instruments and related products & 29 & 300 & 3.2 \\
Chemicals and allied products & 38 & 300 & 3.1 \\
Stone, clay, and glass products & 28 & 200 & 3.0 \\
Agricultural production-livestock & 32 & -500 & 3.0 \\
Electronic and other electric equipment & 02 & $\mathbf{1 4 , 7 0 0}$ & $\mathbf{1 . 6}$ \\
ALL INDUSTRIES & 36 & &
\end{tabular}

- indicates no data reported or data that do not meet BLS publication guidelines.

SIC - 1987 Standard Industial Classification

NOTE: Respiratory conditions due to toxic agents include pneumonitis, pharyngitis, rhinitis or acute congestions due to chemicals, dusts, gases or fumes. See appendices for source description.

SOURCE: Bureau of Labor Statistics annual reports of occupational injuries and illnesses. 


\section{Section 12}

Respiratory

Tuberculosis 

Table 12-1. Respiratory tuberculosis: Proportionate mortality ratio (PMR) adjusted for age, sex, and race by usual industry, U.S. residents age 15 and over, selected states and years, 1990-1999

\begin{tabular}{llrrrr}
\hline & & & & 95\% Confidence Interval \\
\cline { 4 - 6 } CIC & Industry & Number & LCL & UCL \\
\hline 830 & Offices and clinics of health practitioners, n.e.c. & 13 & 3.04 & 1.62 & 5.19 \\
141 & Carpets and rugs & 12 & 2.10 & 1.08 & 3.67 \\
332 & Not specified machinery & 10 & 2.09 & 1.01 & 3.84 \\
050 & Nonmetallic mining and quarrying, except fuel & 16 & 1.92 & 1.10 & 3.12 \\
791 & Miscellaneous personal services & 29 & 1.87 & 1.26 & 2.69 \\
040 & Metal mining & 17 & 1.86 & 1.08 & 2.97 \\
280 & Other primary metal industries & 23 & 1.64 & 1.04 & 2.46 \\
760 & Miscellaneous repair services & 35 & 1.60 & 1.11 & 2.22 \\
010 & Agricultural production, crops & 429 & 1.49 & 1.35 & 1.63 \\
041 & Coal mining & 66 & 1.41 & 1.10 & 1.81 \\
831 & Hospitals & 227 & 1.18 & 1.04 & 1.35 \\
961 & Non-paid worker or non-worker or own home/at home & 1,487 & 1.09 & 1.03 & 1.14 \\
060 & Construction & 663 & 1.08 & 1.00 & 1.17 \\
\hline
\end{tabular}

CIC - Census Industry Code $\quad$ n.e.c. - not elsewhere classified $\quad$ LCL - lower confidence limit $\quad$ UCL - upper confidence limit NOTE: See appendices for source description, methods, and ICD codes, industry and occupation codes, and list of selected states and years. SOURCE: National Center for Health Statistics multiple cause of death data. 
Table 12-2. Respiratory tuberculosis: Proportionate mortality ratio (PMR) adjusted for age, sex, and race by usual occupation, U.S. residents age 15 and over, selected states and years, 1990-1999

\begin{tabular}{|c|c|c|c|c|c|}
\hline \multirow[b]{2}{*}{ COC } & \multirow[b]{2}{*}{ Occupation } & \multirow{2}{*}{$\begin{array}{c}\text { Number } \\
\text { of Deaths }\end{array}$} & \multirow[b]{2}{*}{ PMR } & \multicolumn{2}{|c|}{ 95\% Confidence Interval } \\
\hline & & & & LCL & UCL \\
\hline 768 & Crushing and grinding machine operators & 11 & 3.50 & 1.75 & 6.26 \\
\hline 829 & Sailors and deckhands & 11 & 2.29 & 1.14 & 4.09 \\
\hline 534 & Heating, air conditioning, and refrigeration mechanics & 14 & 2.18 & 1.19 & 3.66 \\
\hline 479 & Farm workers & 96 & 1.73 & 1.41 & 2.12 \\
\hline 616 & Mining machine operators & 75 & 1.70 & 1.35 & 2.14 \\
\hline 875 & Garbage collectors & 22 & 1.64 & 1.03 & 2.49 \\
\hline 913 & Retired, with no other occupation reported & 33 & 1.53 & 1.05 & 2.14 \\
\hline 917 & Unemployed, never worked, disabled & 226 & 1.41 & 1.23 & 1.60 \\
\hline 449 & Maids and housemen & 55 & 1.40 & 1.07 & 1.84 \\
\hline 869 & Construction laborers & 216 & 1.35 & 1.18 & 1.55 \\
\hline 473 & Farmers, except horticulture & 352 & 1.26 & 1.14 & 1.40 \\
\hline 379 & General office clerks & 76 & 1.26 & 1.00 & 1.59 \\
\hline 567 & Carpenters & 131 & 1.20 & 1.01 & 1.43 \\
\hline 889 & Laborers, except construction & 370 & 1.15 & 1.04 & 1.28 \\
\hline
\end{tabular}

COC - Census Occupation Code n.e.c. - not elsewhere classified LCL - lower confidence limit UCL - upper confidence limit NOTE: See appendices for source description, methods, and ICD codes, industry and occupation codes, and list of selected states and years. SOURCE: National Center for Health Statistics multiple cause of death data. 


\section{Section 13}

Lung Cancer 

Table 13-1. Lung cancer: Proportionate mortality ratio (PMR) adjusted for age, sex, and race by usual industry, U.S. residents age 15 and over, selected states, 1999

\begin{tabular}{llrrrr}
\hline & & & 95\% Confidence Interval \\
\cline { 4 - 5 } CIC & Industry & Number & LCL & UCL \\
\hline 801 & Bowling alleys, billiard and pool parlors & 19 & 1.80 & 1.08 & 2.81 \\
371 & Scientific and controlling instruments & 55 & 1.42 & 1.08 & 1.86 \\
360 & Ship and boat building and repairing & 84 & 1.32 & 1.06 & 1.65 \\
472 & Not specified utilities & 63 & 1.31 & 1.02 & 1.70 \\
772 & Beauty shops & 211 & 1.30 & 1.14 & 1.49 \\
130 & Tobacco manufactures & 95 & 1.30 & 1.06 & 1.60 \\
271 & Iron and steel foundries & 90 & 1.29 & 1.05 & 1.60 \\
041 & Coal mining & 327 & 1.25 & 1.12 & 1.39 \\
802 & Miscellaneous entertainment and recreation services & 251 & 1.22 & 1.07 & 1.38 \\
351 & Motor vehicles and motor vehicle equipment & 493 & 1.21 & 1.11 & 1.33 \\
410 & Trucking service & 1,004 & 1.21 & 1.14 & 1.29 \\
682 & Miscellaneous retail stores & 142 & 1.20 & 1.02 & 1.42 \\
282 & Fabricated structural metal products & 124 & 1.20 & 1.00 & 1.44 \\
172 & Printing, publishing, and allied industries, except newspapers & 270 & 1.19 & 1.06 & 1.35 \\
060 & Construction & 3,336 & 1.19 & 1.15 & 1.23 \\
641 & Eating and drinking places & 907 & 1.16 & 1.08 & 1.23 \\
942 & Military & 680 & 1.14 & 1.06 & 1.23 \\
751 & Automotive repair and related services & 452 & 1.14 & 1.04 & 1.25 \\
400 & Railroads & 385 & 1.12 & 1.01 & 1.23 \\
392 & Not specified manufacturing industries & 782 & 1.11 & 1.03 & 1.19 \\
\hline
\end{tabular}

CIC - Census Industry Code $\quad$ n.e.c. - not elsewhere classified $\quad$ LCL - lower confidence limit $\quad$ UCL - upper confidence limit NOTE: See appendices for source description, methods, and ICD codes, industry and occupation codes, and list of selected states. SOURCE: National Center for Health Statistics multiple cause of death data. 
Table 13-2. Lung cancer: Proportionate mortality ratio (PMR) adjusted for age, sex, and race by usual occupation, U.S. residents age 15 and over, selected states, 1999

\begin{tabular}{|c|c|c|c|c|c|}
\hline \multirow[b]{2}{*}{$\mathrm{COC}$} & \multirow[b]{2}{*}{ Occupation } & \multirow{2}{*}{$\begin{array}{c}\text { Number } \\
\text { of Deaths }\end{array}$} & \multirow[b]{2}{*}{ PMR } & \multicolumn{2}{|c|}{ 95\% Confidence Interval } \\
\hline & & & & $\mathbf{L C L}$ & UCL \\
\hline 205 & Health record technologists and technicians & 5 & 3.13 & 1.01 & 7.32 \\
\hline 646 & Lay-out workers & 13 & 2.50 & 1.33 & 4.28 \\
\hline 636 & Precision assemblers, metal & 14 & 2.40 & 1.31 & 4.02 \\
\hline 459 & Attendants, amusement and recreation facilities & 48 & 1.73 & 1.27 & 2.30 \\
\hline 614 & Drillers, oil well & 18 & 1.71 & 1.01 & 2.69 \\
\hline 556 & Supervisors: painters, paperhangers, and plasterers & 30 & 1.52 & 1.03 & 2.18 \\
\hline 029 & Buyers, wholesale and retail trade except farm products & 38 & 1.47 & 1.04 & 2.01 \\
\hline 435 & Waiters and waitresses & 258 & 1.43 & 1.26 & 1.62 \\
\hline 756 & Mixing and blending machine operators & 48 & 1.42 & 1.04 & 1.88 \\
\hline 534 & Heating, air conditioning, and refrigeration mechanics & 57 & 1.41 & 1.08 & 1.84 \\
\hline 469 & Personal service occupations, n.e.c. & 54 & 1.40 & 1.06 & 1.84 \\
\hline 683 & Electrical and electronic equipment assemblers & 90 & 1.37 & 1.11 & 1.70 \\
\hline 823 & Railroad conductors and yardmasters & 58 & 1.36 & 1.04 & 1.77 \\
\hline 689 & Inspectors, testers, and graders & 54 & 1.35 & 1.02 & 1.78 \\
\hline 518 & Industrial machinery repairers & 205 & 1.31 & 1.14 & 1.50 \\
\hline 544 & Millwrights & 64 & 1.29 & 1.01 & 1.66 \\
\hline 653 & Sheet metal workers & 83 & 1.29 & 1.03 & 1.61 \\
\hline 616 & Mining machine operators & 317 & 1.27 & 1.13 & 1.42 \\
\hline 579 & Painters, construction and maintenance & 227 & 1.26 & 1.10 & 1.44 \\
\hline 503 & Supervisors, mechanics and repairers & 113 & 1.25 & 1.04 & 1.51 \\
\hline 458 & Hairdressers and cosmetologists & 190 & 1.25 & 1.08 & 1.44 \\
\hline 633 & Supervisors, production occupations & 632 & 1.24 & 1.14 & 1.34 \\
\hline 585 & Plumbers, pipefitters, and steamfitters & 240 & 1.21 & 1.07 & 1.38 \\
\hline 337 & Bookkeepers, accounting, and auditing clerks & 343 & 1.21 & 1.09 & 1.35 \\
\hline 783 & Welders and cutters & 253 & 1.21 & 1.07 & 1.37 \\
\hline 575 & Electricians & 307 & 1.20 & 1.08 & 1.35 \\
\hline 844 & Operating engineers & 241 & 1.18 & 1.04 & 1.34 \\
\hline 869 & Construction laborers & 557 & 1.17 & 1.08 & 1.28 \\
\hline 804 & Truck drivers & 1,258 & 1.16 & 1.10 & 1.23 \\
\hline 505 & Automobile mechanics & 364 & 1.15 & 1.03 & 1.27 \\
\hline 567 & Carpenters & 588 & 1.15 & 1.06 & 1.24 \\
\hline 905 & Military occupations & 591 & 1.14 & 1.06 & 1.24 \\
\hline 785 & Assemblers & 345 & 1.12 & 1.00 & 1.24 \\
\hline 453 & Janitors and cleaners & 756 & 1.12 & 1.04 & 1.20 \\
\hline 019 & Managers and administrators, n.e.c. & 2,074 & 1.09 & 1.04 & 1.13 \\
\hline
\end{tabular}

COC - Census Occupation Code n.e.c. - not elsewhere classified LCL - lower confidence limit UCL - upper confidence limit NOTE: See appendices for source description, methods, and ICD codes, industry and occupation codes, and list of selected states. SOURCE: National Center for Health Statistics multiple cause of death data. 


\section{Section 14}

Other Interstitial

Pulmonary Diseases 



\section{Table 14-1. Other interstitial pulmonary diseases: Proportionate mortality ratio} (PMR) adjusted for age, sex, and race by usual industry, U.S. residents age 15 and over, selected states, 1999

\begin{tabular}{llrrrr}
\hline & & & \multicolumn{2}{c}{ 95\% Confidence Interval } \\
\cline { 4 - 6 } CIC & Industry & of Deaths & PMR & LCL & UCL \\
\hline 232 & Wood buildings and mobile homes & 5 & 4.75 & 1.54 & 11.10 \\
040 & Metal mining & 15 & 2.18 & 1.22 & 3.60 \\
891 & Research, development and testing services & 15 & 2.14 & 1.19 & 3.52 \\
812 & Offices and clinics of physicians & 31 & 1.81 & 1.23 & 2.57 \\
282 & Fabricated structural metal products & 19 & 1.80 & 1.08 & 2.81 \\
460 & Electric light and power & 32 & 1.65 & 1.13 & 2.34 \\
700 & Banking & 43 & 1.44 & 1.04 & 1.94 \\
831 & Hospitals & 128 & 1.22 & 1.03 & 1.46 \\
\hline
\end{tabular}

CIC - Census Industry Code n.e.c. - not elsewhere classified

LCL - lower confidence limit

UCL - upper confidence limit

NOTE: See appendices for source description, methods, and ICD codes, industry and occupation codes, and list of selected states.

SOURCE: National Center for Health Statistics multiple cause of death data. 
Table 14-2. Other interstitial pulmonary diseases: Proportionate mortality ratio (PMR) adjusted for age, sex, and race by usual occupation, U.S. residents age 15 and over, selected states, 1999

\begin{tabular}{llrrrr}
\hline COC & Occupation & $\begin{array}{c}\text { Number } \\
\text { of Deaths }\end{array}$ & PMR & \multicolumn{2}{c}{ 95\% Confidence Interval } \\
\cline { 4 - 6 } 086 & Veterinarians & 7 & 5.71 & 2.29 & UCL \\
155 & Teachers, prekindergarten and kindergarten & 5 & 3.41 & 1.10 & 7.98 \\
508 & Aircraft engine mechanics & 8 & 2.42 & 1.04 & 4.76 \\
319 & Receptionists & 14 & 2.05 & 1.12 & 3.44 \\
084 & Physicians & 23 & 2.03 & 1.28 & 3.04 \\
095 & Registered nurses & 74 & 1.57 & 1.24 & 1.98 \\
\hline
\end{tabular}

COC - Census Occupation Code n.e.c. - not elsewhere classified LCL - lower confidence limit UCL - upper confidence limit NOTE: See appendices for source description, methods, and ICD codes, industry and occupation codes, and list of selected states.

SOURCE: National Center for Health Statistics multiple cause of death data. 


\section{Section 15}

Various

Work-Related

Respiratory Conditions 

Various Work-Related Respiratory Conditions: Morbidity

\section{Table 15-1. Work-related respiratory illnesses (with days away from work): Estimated} number, 1996-2000

\begin{tabular}{|c|c|c|c|c|c|}
\hline & 1996 & 1997 & 1998 & 1999 & 2000 \\
\hline Acute respiratory infections (including common cold) & 212 & 267 & 193 & 149 & 307 \\
\hline Other diseases of upper respiratory tract & 169 & 155 & 193 & 320 & 117 \\
\hline Allergic rhinitis & 51 & 71 & - & 121 & - \\
\hline $\begin{array}{l}\text { Chronic conditions of upper respiratory tract (including chronic } \\
\text { sinusitis, pharyngitis) }\end{array}$ & 41 & - & - & - & - \\
\hline Other diseases of upper respiratory tract, unspecified or n.e.c. & 42 & - & - & 167 & - \\
\hline Pneumonia, influenza & 79 & 115 & 144 & 197 & 95 \\
\hline Influenza & - & - & - & 63 & - \\
\hline Pneumonia & 46 & - & 72 & 99 & 46 \\
\hline Legionnaires' disease & 17 & - & - & - & - \\
\hline $\begin{array}{l}\text { Chronic obstructive pulmonary disease (COPD) and allied } \\
\text { conditions }\end{array}$ & 2,160 & 1,005 & 1,097 & 760 & 1,083 \\
\hline Bronchitis & 738 & 127 & - & 171 & 205 \\
\hline Extrinsic asthma & 403 & 606 & 154 & 282 & 618 \\
\hline COPD and allied conditions, unspecified or n.e.c. & 926 & 173 & 237 & 133 & 171 \\
\hline $\begin{array}{l}\text { Extrinsic alveolitis and pneumonitis (including farmers' lung, } \\
\text { bagassosis) }\end{array}$ & 87 & 98 & 161 & 145 & 88 \\
\hline Pneumoconioses & 45 & - & 108 & 82 & 70 \\
\hline $\begin{array}{l}\text { Coal workers' pneumoconiosis (including anthracosis, black } \\
\text { lung, miners' asthma) }\end{array}$ & - & - & 73 & - & - \\
\hline Pneumoconioses, unspecified & 18 & - & - & - & 19 \\
\hline Pneumonopathy & 39 & - & - & 19 & 101 \\
\hline Byssinosis, mill fever & - & - & - & - & . \\
\hline Metal fume fever & - & - & - & - & 80 \\
\hline Pneumonopathy, unspecified & - & - & - & - & - \\
\hline Other respiratory system diseases & 80 & 289 & 182 & 114 & 139 \\
\hline Pneumonitis, n.e.c. & - & - & - & 18 & - \\
\hline Atelectasis, collapsed lung & 18 & 103 & - & - & - \\
\hline Other respiratory system diseases, unspecified or n.e.c. & 47 & 163 & 76 & 83 & 101 \\
\hline Respiratory system diseases, unspecified & 882 & 357 & 544 & 517 & 679 \\
\hline All respiratory system diseases & 3,665 & 2,270 & 2,490 & 2,158 & 2,591 \\
\hline
\end{tabular}

- indicates no data reported or data do not meet BLS publication guidelines.

n.e.c. - not elsewhere classified.

NOTE: Numbers may not sum to totals due to rounding. See appendices for source description and methods.

SOURCE: Bureau of Labor Statistics annual reports of occupational injuries and illnesses. 
Table 15-2. Work-related respiratory illnesses (with days away from work): Median days away from work, 1996-2000

\begin{tabular}{|c|c|c|c|c|c|}
\hline & 1996 & 1997 & 1998 & 1999 & 2000 \\
\hline Acute respiratory infections (including common cold) & 5 & 2 & 2 & 5 & 3 \\
\hline Other diseases of upper respiratory tract & 3 & 3 & 3 & 2 & 2 \\
\hline Allergic rhinitis & 3 & - & - & 1 & - \\
\hline $\begin{array}{l}\text { Chronic conditions of upper respiratory tract (including chronic } \\
\text { sinusitis, pharyngitis) }\end{array}$ & - & - & - & - & - \\
\hline Other diseases of upper respiratory tract, unspecified or n.e.c. & 4 & 3 & 3 & 2 & - \\
\hline Pneumonia, influenza & 10 & 9 & 4 & 9 & 23 \\
\hline Influenza & - & - & - & 2 & - \\
\hline Pneumonia & 10 & - & 4 & 10 & 8 \\
\hline Legionnaires' disease & 12 & - & - & - & - \\
\hline $\begin{array}{l}\text { Chronic obstructive pulmonary disease (COPD) and allied } \\
\text { conditions }\end{array}$ & 6 & 3 & 2 & 5 & 3 \\
\hline Bronchitis & 2 & 3 & - & 5 & 7 \\
\hline Extrinsic asthma & 6 & 3 & 1 & 8 & 3 \\
\hline COPD and allied conditions, unspecified or n.e.c. & 9 & 5 & 3 & 5 & 15 \\
\hline $\begin{array}{l}\text { Extrinsic alveolitis and pneumonitis (including farmers' lung, } \\
\text { bagassosis) }\end{array}$ & 5 & 2 & 5 & 3 & 12 \\
\hline Pneumoconioses & 14 & - & 157 & 81 & 41 \\
\hline $\begin{array}{l}\text { Coal workers' pneumoconiosis (including anthracosis, black } \\
\text { lung, miners' asthma) }\end{array}$ & - & - & 190 & - & - \\
\hline Pneumoconioses, unspecified & 14 & - & - & - & 8 \\
\hline Pneumonopathy & 15 & - & - & 3 & 3 \\
\hline Byssinosis, mill fever & - & - & - & - & - \\
\hline Metal fume fever & - & - & - & - & 3 \\
\hline Pneumonopathy, unspecified & - & - & - & - & - \\
\hline Other respiratory system diseases & 5 & 5 & 2 & 11 & 2 \\
\hline Pneumonitis, n.e.c. & - & - & - & 3 & - \\
\hline Atelectasis, collapsed lung & 11 & 5 & - & - & - \\
\hline Other respiratory system diseases, unspecified or n.e.c. & 2 & - & 5 & 24 & 2 \\
\hline Respiratory system diseases, unspecified & 2 & 6 & 2 & 1 & 1 \\
\hline All respiratory system diseases & 5 & 5 & 2 & 3 & 4 \\
\hline
\end{tabular}

- indicates no data reported or data do not meet BLS publication guidelines.

NOTE: See appendices for source description and methods.

SOURCE: Bureau of Labor Statistics annual reports of occupational injuries and illnesses. 


\section{Table 15-3a. Annual average employment: Estimated number (in thousands) by} major industry division, 1996-2000

\begin{tabular}{|c|c|c|c|c|c|c|c|c|c|}
\hline Year & Agriculture & Mining & Construction & Manufacturing & $\begin{array}{c}\text { Transportation } \\
\text { \& Public } \\
\text { Utilities } \\
\end{array}$ & $\begin{array}{c}\text { Wholesale } \\
\text { \& Retail } \\
\text { Trade } \\
\end{array}$ & Finance & Services & Total \\
\hline 1996 & 1,717 & 578 & 5,360 & 18,461 & 5,989 & 28,027 & 6,746 & 31,895 & 98,773 \\
\hline 1997 & 1,765 & 596 & 5,637 & 18,657 & 6,171 & 28,584 & 6,952 & 33,305 & 101,667 \\
\hline 1998 & 1,815 & 589 & 5,950 & 18,807 & 6,307 & 29,087 & 7,219 & 34,624 & 104,641 \\
\hline 1999 & 1,861 & 535 & 6,337 & 18,538 & 6,578 & 29,716 & 7,400 & 36,374 & 107,612 \\
\hline 2000 & 1,912 & 536 & 6,623 & 18,425 & 6,792 & 30,305 & 7,436 & 37,686 & 110,065 \\
\hline
\end{tabular}

NOTE: The sum of individual industries may not equal yearly total due to rounding. See appendices for source description and methods. SOURCE: Bureau of Labor Statistics annual reports of occupational injuries and illnesses.

Table 15-3b. Work-related respiratory illnesses (with days away from work): Estimated number by industry division, 1996-2000

\begin{tabular}{|c|c|c|c|c|c|c|c|c|c|}
\hline Year & Agriculture & Mining & Construction & Manufacturing & $\begin{array}{c}\text { Transportation } \\
\text { \& Public } \\
\text { Utilities } \\
\end{array}$ & $\begin{array}{c}\text { Wholesale } \\
\text { \& Retail } \\
\text { Trade } \\
\end{array}$ & Finance & Services & Total \\
\hline 1996 & - & 15 & 178 & 840 & 503 & 589 & 161 & 1,365 & 3,665 \\
\hline 1997 & - & 33 & 177 & 671 & 163 & 490 & 42 & 667 & 2,270 \\
\hline 1998 & - & 91 & 67 & 681 & 244 & 423 & 162 & 798 & 2,490 \\
\hline 1999 & - & 38 & 59 & 682 & 236 & 381 & 84 & 665 & 2,158 \\
\hline 2000 & - & 37 & 80 & 527 & 222 & 705 & 247 & 750 & 2,591 \\
\hline
\end{tabular}

- indicates no data reported or data that do not meet BLS publication guidelines.

NOTE: The sum of individual industries may not equal yearly total due to rounding. See appendices for source description and methods.

SOURCE: Bureau of Labor Statistics annual reports of occupational injuries and illnesses.

Table 15-3c. Work-related respiratory illnesses (with days away from work): Estimated rate (per 100,000 workers) by industry division, 1996-2000

\begin{tabular}{|c|c|c|c|c|c|c|c|c|c|}
\hline Year & Agriculture & Mining & Construction & Manufacturing & $\begin{array}{c}\text { Transportation } \\
\text { \& Public } \\
\text { Utilities } \\
\end{array}$ & $\begin{array}{c}\text { Wholesale } \\
\text { \& Retail } \\
\text { Trade } \\
\end{array}$ & Finance & Services & Total \\
\hline 1996 & - & 2.6 & 3.3 & 4.6 & 8.4 & 2.1 & 2.4 & 4.3 & 3.7 \\
\hline 1997 & - & 5.5 & 3.1 & 3.6 & 2.6 & 1.7 & 0.6 & 2.0 & 2.2 \\
\hline 1998 & - & 15.4 & 1.1 & 3.6 & 3.8 & 1.5 & 2.2 & 2.3 & 2.4 \\
\hline 1999 & - & 7.1 & 0.9 & 3.7 & 3.6 & 1.3 & 1.1 & 1.8 & 2.0 \\
\hline 2000 & - & 6.9 & 1.2 & 2.9 & 3.3 & 2.3 & 3.3 & 2.0 & 2.4 \\
\hline
\end{tabular}

- indicates no data reported or data that do not meet BLS publication guidelines.

NOTE: See appendices for source description and methods. 
Table 15-4. Work-related respiratory conditions: Number of diagnoses related to asbestos or to other occupational exposures, based on physician's judgement, selected occupational and environmental medical clinics, 1991-2000

\begin{tabular}{|c|c|c|c|c|c|}
\hline & \multicolumn{2}{|c|}{$\begin{array}{c}\text { Asbestos-related } \\
(4,471 \text { patients })\end{array}$} & \multicolumn{3}{|r|}{$\begin{array}{c}\text { Other } \\
(2,021 \text { patients })\end{array}$} \\
\hline & No. & $\%$ & No. & $\%$ & Most frequent hazard (No. of cases) \\
\hline \multicolumn{6}{|l|}{ MALIGNANT DISEASES } \\
\hline Lung cancer & 37 & 0.8 & 9 & 0.4 & crystalline silica (3) \\
\hline Nasal/pharyngeal cancer & - & - & 2 & 0.1 & wood dust (1), smoke n.o.s. (1) \\
\hline Laryngeal cancer & 7 & 0.2 & 3 & 0.1 & various (1 each) \\
\hline Mesothelioma & 10 & 0.2 & - & - & \\
\hline \multicolumn{6}{|l|}{ OTHER CONDITIONS } \\
\hline Upper airway diseases & 4 & 0.1 & 457 & 22.1 & \\
\hline Upper respiratory irritation & 2 & 0.1 & 440 & 21.3 & indoor air pollutants (132) \\
\hline Laryngeal disorders (other than irritation or cancer) & 2 & 0.1 & 12 & 0.6 & indoor air pollutants (4), \\
\hline Nasal septum perforation & - & - & 3 & 0.1 & $\mathrm{NaOH}(1), \mathrm{NH}_{3}$ gas (1), $\mathrm{Cr}(1)$ \\
\hline Nasal polyps & - & - & 2 & 0.1 & petroleum (1), solvents n.o.s. (1) \\
\hline Lower airway diseases & 60 & 1.3 & 1,075 & 52.0 & \\
\hline Asthma \& reactive airways dysfunction syndrome & 7 & 0.2 & 847 & 41.0 & indoor air pollutants (134) \\
\hline COPD \& emphysema \& chronic bronchitis & 53 & 1.1 & 128 & 6.2 & smoke n.o.s. (14), dust n.o.s. (8) \\
\hline Bronchitis, acute & - & - & 16 & 0.8 & welding fume n.o.s. (2), smoke n.o.s. (2) \\
\hline Bronchitis, n.o.s. & - & - & 82 & 4.0 & indoor air pollutants (9) \\
\hline Bronchiectasis \& pneumonia & - & - & 2 & 0.2 & $\begin{array}{l}\text { indoor air pollutants (1), late effects of } \\
\text { traumatic injury (1) }\end{array}$ \\
\hline Interstitial inflammatory/fibrotic diseases & 3,930 & 86.5 & 273 & 13.2 & \\
\hline Asbestosis & 3,921 & 86.3 & - & - & \\
\hline Silicosis & - & - & 145 & 7.0 & crystalline silica (145) \\
\hline Coal workers' pneumoconiosis & - & - & 58 & 2.8 & $\operatorname{coal}(58)$ \\
\hline Pneumoconiosis due to other or mixed dust & 7 & 0.2 & 18 & 0.9 & crystalline silica (4) \\
\hline Interstitial pulmonary fibrosis & 2 & 0.1 & 19 & 0.9 & methyl chloroform (3), paint (3) \\
\hline Chemical pneumonitis & - & - & 15 & 0.7 & chlorine (3), solvents n.o.s. (3) \\
\hline Hypersensitivity pneumonitis & - & - & 18 & 0.9 & cutting oils (3), mold n.o.s. (3) \\
\hline Pleural disease (without apparent lung disease) & 463 & 10.1 & 2 & 0.1 & crystalline silica (1), latex gloves n.o.s. (1) \\
\hline Lung function abnormalities (n.o.s.) & 10 & 0.2 & 11 & 0.5 & crystalline silica (2), solvents n.o.s. (2) \\
\hline Abnormal CXR (n.o.s.) & 10 & 0.2 & - & - & \\
\hline Symptoms & 8 & 0.2 & 57 & 2.8 & \\
\hline Cough & 2 & 0.1 & 18 & 0.9 & indoor air pollutants (4) \\
\hline Shortness of breath & 5 & 0.1 & 20 & 1.0 & various ( 1 each) \\
\hline Chest pain & - & - & 9 & 0.4 & various ( 1 each) \\
\hline Hoarseness & - & - & 7 & 0.3 & indoor air pollutants (2) \\
\hline Nosebleeds & - & - & 2 & 0.1 & glutaraldehyde (1), chromium n.o.s. (1) \\
\hline Hemoptysis & 1 & 0.1 & 1 & 0.1 & cutting oils (1) \\
\hline Non-specific respiratory disorders & 4 & 0.1 & 21 & 1.0 & indoor air pollutants (2) \\
\hline Chemical poisonings & - & - & 70 & 3.4 & \\
\hline Metal fume fever & - & - & 16 & 0.8 & zinc (5), metal fumes n.o.s. (5) \\
\hline Toxic effects of carbon monoxide or cyanides & - & - & 21 & 1.0 & carbon monoxide (13) \\
\hline $\begin{array}{l}\text { Toxic effects of gas/fumes/vapors/misc. chemicals (excl. } \\
\text { lead, other heavy metals, pesticides, solvents) }\end{array}$ & - & - & 32 & 1.5 & ethylene oxide (6) \\
\hline Hypoxia & - & - & 1 & 0.1 & Freon $®(1)$ \\
\hline Selected miscellaneous conditions & - & - & 87 & 4.2 & \\
\hline Positive PPD skin test & - & - & 83 & 4.0 & tuberculosis (83) \\
\hline Sarcoidosis & - & - & 4 & 0.2 & cleaning materials n.o.s. (2) \\
\hline TOTAL & 4,543 & 100.0 & 2,067 & 100.0 & \\
\hline
\end{tabular}

- indicates no cases

n.o.s. - not otherwise specified

NOTE: In the Symptoms and the Chemical Poisonings categories, all diagnoses in the subcategories shown have been included as "respiratory" in this table, though some may not have been respiratory. In addition to the diagnoses shown in this table, there were 17 non-respiratory diagnoses related to asbestos and 5,413 non-respiratory diagnoses related to other occupational hazards. See appendices for source description.

SOURCE: AOEC Occupational and Environmental Disease Database. 
Table 15-5. Most frequent hazards associated with respiratory diagnoses, patients without asbestos-related diagnoses, selected occupational and environmental medical clinics, 1991-2000

\begin{tabular}{|c|c|c|}
\hline Respiratory hazards & Number & Percent \\
\hline Indoor air pollutants & 320 & 12.8 \\
\hline Crystalline silica & 171 & 6.8 \\
\hline Solvents n.o.s. & 128 & 5.1 \\
\hline Dust n.o.s. & 94 & 3.8 \\
\hline Smoke n.o.s. & 84 & 3.4 \\
\hline Coal & 77 & 3.1 \\
\hline Isocyanates n.o.s. & 72 & 2.9 \\
\hline Welding n.o.s. & 59 & 2.4 \\
\hline Paint & 55 & 2.2 \\
\hline Chemicals n.o.s. & 48 & 1.9 \\
\hline Mold & 47 & 1.9 \\
\hline Cutting oils & 46 & 1.8 \\
\hline Formaldehyde & 36 & 1.4 \\
\hline Latex, natural rubber & 36 & 1.4 \\
\hline Ammonia solution n.o.s. & 29 & 1.2 \\
\hline Glutaraldehyde & 27 & 1.1 \\
\hline Chlorine & 26 & 1.0 \\
\hline Metal fumes n.o.s. & 23 & 0.9 \\
\hline Toluene diisocyanate & 22 & 0.9 \\
\hline Toluene & 22 & 0.9 \\
\hline Epoxy resins & 21 & 0.8 \\
\hline Methyl chloroform (1,1,1-trichloroethane) & 19 & 0.8 \\
\hline Wood dust n.o.s. & 19 & 0.8 \\
\hline Hydrocarbons n.o.s. & 18 & 0.7 \\
\hline Methyl ethyl ketone & 18 & 0.7 \\
\hline Glues n.o.s. & 18 & 0.7 \\
\hline Cleaning materials n.o.s. & 17 & 0.7 \\
\hline Acids, bases, oxidizers n.o.s. & 17 & 0.7 \\
\hline Man-made mineral fibers & 16 & 0.6 \\
\hline Carbon monoxide & 16 & 0.6 \\
\hline Diesel exhaust & 16 & 0.6 \\
\hline Lubricants n.o.s. & 15 & 0.6 \\
\hline Metal dust n.o.s. & 15 & 0.6 \\
\hline Sulfuric acid & 14 & 0.6 \\
\hline Bleach & 12 & 0.5 \\
\hline Xylene & 12 & 0.5 \\
\hline Perchloroethylene & 12 & 0.5 \\
\hline Cigarette smoke & 12 & 0.5 \\
\hline Sodium hydroxide & 11 & 0.4 \\
\hline Pesticides n.o.s. & 11 & 0.4 \\
\hline Hydrochloric acid & 10 & 0.4 \\
\hline Flour n.o.s. & 10 & 0.4 \\
\hline All others & 753 & 30.1 \\
\hline TOTAL & 2,504 & 100.0 \\
\hline
\end{tabular}

n.o.s. - not otherwise specified

NOTE: The hazards in this table relate to the 2,021 patients with respiratory (excluding asbestos-related) diagnoses shown in Table 15-4. Percentages may not total to $100 \%$ due to rounding. See appendices for source description and methods.

SOURCE: AOEC Occupational and Environmental Disease Database. 



\section{Section 16}

Smoking Prevalence by

Industry and

Occupation 

Table 16-1. Smoking status: Estimated prevalence by current industry, U.S. residents age 18 and over, 2000

\begin{tabular}{|c|c|c|c|c|c|c|}
\hline \multirow{3}{*}{$\frac{\text { Industry }}{\text { Repair services }}$} & \multirow{3}{*}{$\begin{array}{c}\begin{array}{c}\text { Number of } \\
\text { Respondents }\end{array} \\
283\end{array}$} & \multicolumn{3}{|c|}{ Current Smokers } & \multicolumn{2}{|c|}{ Former Smokers } \\
\hline & & \multirow{2}{*}{$\begin{array}{c}\begin{array}{c}\text { Prevalence } \\
(\%)\end{array} \\
43.4\end{array}$} & \multicolumn{2}{|c|}{$\begin{array}{c}95 \% \\
\text { Confidence } \\
\text { Interval } \\
\end{array}$} & \multirow{2}{*}{$\begin{array}{c}\begin{array}{c}\text { Prevalence } \\
(\%)\end{array} \\
19.6\end{array}$} & $\begin{array}{c}95 \% \\
\text { Confidence } \\
\text { Interval } \\
\end{array}$ \\
\hline & & & 36.5 & -50.3 & & $14.5-24.7$ \\
\hline Eating and drinking places & 901 & 39.7 & 35.8 & - 43.6 & 12.1 & $9.6-14.6$ \\
\hline Automotive dealers and gasoline stations & 317 & 38.9 & 32.6 & -45.2 & 18.3 & $13.4-23.2$ \\
\hline Construction & 1,226 & 37.4 & 34.1 & - 40.7 & 18.7 & $16.2-21.2$ \\
\hline Food, bakery and dairy stores & 434 & 36.1 & 31.0 & - 41.2 & 13.5 & $10.2-16.8$ \\
\hline Fabricated metal industries, including ordnance & 184 & 34.1 & 26.7 & - 41.5 & 21.4 & $14.0-28.8$ \\
\hline Primary metal industries & 114 & 33.3 & 23.9 & - 42.7 & 26.1 & $16.9-35.3$ \\
\hline Trucking service and warehousing & 367 & 33.2 & 27.7 & -38.7 & 17.4 & $12.9-21.9$ \\
\hline Food and kindred products & 241 & 32.8 & 25.7 & -39.9 & 18.0 & $11.7-24.3$ \\
\hline Mining & 66 & 32.6 & 21.0 & -44.2 & 22.3 & $9.6-35.0$ \\
\hline Machinery, except electrical & 303 & 31.9 & 25.8 & -38.0 & 23.2 & $17.7-28.7$ \\
\hline Furniture, lumber and wood & 207 & 29.9 & 23.6 & -36.2 & 13.8 & $8.5-19.1$ \\
\hline Textile mill and finished textile products & 185 & 29.5 & 21.1 & -37.9 & 14.9 & $8.8-21.0$ \\
\hline Electrical machinery, equipment and supplies & 280 & 29.2 & 23.1 & -35.3 & 14.8 & $9.5-20.1$ \\
\hline Forestry and fisheries & 31 & 28.3 & 8.9 & -47.7 & 16.2 & $4.4-28.0$ \\
\hline Business services & 1,219 & 27.0 & 24.1 & -29.9 & 16.0 & $13.5-18.5$ \\
\hline Other nondurable goods & 229 & 26.7 & 20.0 & -33.4 & 24.0 & $18.1-29.9$ \\
\hline Other personal services & 541 & 26.7 & 22.4 & -31.0 & 18.3 & $14.6-22.0$ \\
\hline Other and not specified durable goods & 245 & 26.4 & 20.5 & -32.3 & 18.7 & $13.4-24.0$ \\
\hline General merchandise stores & 363 & 25.7 & 20.6 & -30.8 & 14.7 & $10.4-19.0$ \\
\hline Transportation equipment & 317 & 25.4 & 19.7 & -31.1 & 22.9 & $18.0-27.8$ \\
\hline Railroads & 44 & 24.8 & 10.5 & -39.1 & 24.0 & $9.5-38.5$ \\
\hline Wholesale trade & 703 & 24.2 & 20.5 & -27.9 & 25.0 & $21.7-28.3$ \\
\hline Entertainment and recreation services & 362 & 24.2 & 19.1 & -29.3 & 16.8 & $12.5-21.1$ \\
\hline Other and not specified retail trade & 922 & 23.9 & 20.8 & - 27.0 & 20.0 & $17.1-22.9$ \\
\hline Other transportation & 453 & 23.5 & 18.6 & -28.4 & 27.0 & $21.7-32.3$ \\
\hline Chemicals and allied products & 167 & 22.9 & 15.5 & -30.3 & 23.4 & $16.7-30.1$ \\
\hline Banking and credit agencies & 421 & 22.4 & 17.9 & -26.9 & 16.8 & $12.7-20.9$ \\
\hline Health services, except hospitals & 1,107 & 21.9 & 19.2 & -24.6 & 18.8 & $16.1-21.5$ \\
\hline Insurance, real estate, and other finance & 852 & 21.0 & 18.1 & -23.9 & 22.0 & $18.7-25.3$ \\
\hline Communications & 334 & 20.6 & 15.7 & -25.5 & 19.7 & $14.8-24.6$ \\
\hline Utilities and sanitary & 178 & 20.6 & 13.7 & -27.5 & 20.0 & $12.9-27.1$ \\
\hline Printing, publishing and allied industries & 243 & 20.3 & 14.2 & -26.4 & 25.5 & $19.4-31.6$ \\
\hline Agriculture & 465 & 20.2 & 16.3 & -24.1 & 17.9 & $14.0-21.8$ \\
\hline Public administrations & 940 & 20.1 & 17.0 & -23.2 & 24.6 & $21.5-27.7$ \\
\hline Private households & 170 & 19.3 & 11.7 & -26.9 & 16.6 & $8.8-24.4$ \\
\hline Hospitals & 823 & 18.8 & 15.9 & -21.7 & 18.0 & $14.9-21.1$ \\
\hline Legal, engineering and other professional services & 720 & 18.7 & 15.2 & -22.2 & 22.2 & $18.9-25.5$ \\
\hline Social services, religious and membership organizations & 793 & 16.9 & 14.2 & -19.6 & 20.7 & $17.6-23.8$ \\
\hline Other educational services & 89 & 12.8 & 3.6 & -22.0 & 30.0 & $19.6-40.4$ \\
\hline Elementary and secondary schools and colleges & 1,710 & 11.8 & 10.0 & -13.6 & 20.9 & $18.7-23.1$ \\
\hline Armed forces & 4 & 0.0 & - & - & 33.3 & $0.0-78.0$ \\
\hline TOTAL & 20,094 & 25.0 & 24.2 & -25.8 & 19.4 & $18.8-20.0$ \\
\hline
\end{tabular}

- No estimates due to no smokers/former smokers among respondents.

NOTE: Industries were classified according to 1995 Standard Industrial Classification System and then regrouped by NCHS. See appendices for source description and methods.

SOURCE: National Center for Health Statistics 2000 National Health Interview Survey. 


\section{Table 16-2. Smoking status: Estimated prevalence by current industry, U.S. male residents age 18 and over, 2000}

\begin{tabular}{|c|c|c|c|c|c|c|c|}
\hline \multirow{3}{*}{$\frac{\text { Industry }}{\text { Repair services }}$} & \multirow{3}{*}{$\begin{array}{c}\begin{array}{c}\text { Number of } \\
\text { Respondents }\end{array} \\
248\end{array}$} & \multicolumn{3}{|c|}{ Current Smokers } & \multicolumn{3}{|c|}{ Former Smokers } \\
\hline & & \multirow{2}{*}{$\begin{array}{c}\begin{array}{c}\text { Prevalence } \\
(\%)\end{array} \\
42.8\end{array}$} & \multicolumn{2}{|c|}{$\begin{array}{c}95 \% \\
\text { Confidence } \\
\text { Interval } \\
\end{array}$} & \multirow{2}{*}{$\begin{array}{c}\begin{array}{c}\text { Prevalence } \\
(\%)\end{array} \\
20.2\end{array}$} & \multicolumn{2}{|c|}{$\begin{array}{c}95 \% \\
\text { Confidence } \\
\text { Interval }\end{array}$} \\
\hline & & & 35.2 & -50.4 & & 14.7 & -25.7 \\
\hline Eating and drinking places & 410 & 40.4 & 34.7 & -46.1 & 10.1 & 7.2 & -13.0 \\
\hline Construction & 1,120 & 38.3 & 34.8 & -41.8 & 18.5 & 15.8 & -21.2 \\
\hline Automotive dealers and gasoline stations & 223 & 37.0 & 29.9 & -44.1 & 20.1 & 14.2 & -26.0 \\
\hline Fabricated metal industries, including ordnance & 142 & 36.0 & 27.6 & -44.4 & 23.4 & 15.2 & -31.6 \\
\hline Food and kindred products & 145 & 35.6 & 26.6 & -44.6 & 20.6 & 12.6 & -28.6 \\
\hline Forestry and fisheries & 21 & 34.8 & 10.3 & -59.3 & 7.9 & 0.0 & - 17.3 \\
\hline General merchandise stores & 108 & 34.2 & 23.2 & -45.2 & 17.9 & 9.5 & -26.3 \\
\hline Other personal services & 162 & 33.5 & 25.9 & -41.1 & 18.7 & 11.4 & -26.0 \\
\hline Other and not specified durable goods & 151 & 33.1 & 25.1 & -41.1 & 17.0 & 10.3 & -23.7 \\
\hline Textile mill and finished textile products & 65 & 32.9 & 18.8 & -47.0 & 15.9 & $6.7-$ & -25.1 \\
\hline Primary metal industries & 86 & 32.6 & 22.4 & -42.8 & 28.7 & 18.3 & - 39.1 \\
\hline Trucking service and warehousing & 283 & 32.6 & 26.7 & -38.5 & 18.5 & 13.2 & -23.8 \\
\hline Machinery, except electrical & 225 & 32.3 & 25.4 & -39.2 & 24.5 & 18.0 & -31.0 \\
\hline Food, bakery and dairy stores & 191 & 30.9 & 23.3 & -38.5 & 16.9 & 11.6 & -22.2 \\
\hline Mining & 57 & 29.3 & 17.9 & -40.7 & 24.3 & 10.0 & - 38.6 \\
\hline Electrical machinery, equipment and supplies & 157 & 28.6 & 20.4 & - 36.8 & 16.4 & 9.7 & -23.1 \\
\hline Furniture, lumber and wood & 147 & 28.5 & 20.7 & -36.3 & 15.8 & 8.9 & -22.7 \\
\hline Other nondurable goods & 165 & 27.1 & 19.1 & -35.1 & 26.3 & 19.0 & -33.6 \\
\hline Printing, publishing and allied industries & 132 & 26.7 & 17.5 & -35.9 & 25.7 & 17.7 & - 33.7 \\
\hline Business services & 642 & 26.3 & 22.2 & - 30.4 & 16.6 & 13.1 & -20.1 \\
\hline Other transportation & 263 & 26.1 & 19.2 & -33.0 & 27.9 & 20.5 & -35.3 \\
\hline Transportation equipment & 220 & 26.0 & 19.7 & -32.3 & 22.3 & 16.8 & -27.8 \\
\hline Private households & 10 & 25.7 & 0.0 & -54.3 & 24.9 & 0.0 & -55.5 \\
\hline Railroads & 41 & 25.5 & 10.8 & -40.2 & 24.6 & 9.7 & -39.5 \\
\hline Wholesale trade & 447 & 25.5 & 20.8 & -30.2 & 26.8 & 22.5 & - 31.1 \\
\hline Other and not specified retail trade & 415 & 24.8 & 19.9 & -29.7 & 23.0 & 18.3 & -27.7 \\
\hline Entertainment and recreation services & 202 & 23.8 & 16.9 & - 30.7 & 14.4 & 9.3 & -19.5 \\
\hline Banking and credit agencies & 106 & 22.6 & 13.8 & -31.4 & 27.8 & 18.6 & -37.0 \\
\hline Agriculture & 360 & 20.9 & 16.4 & -25.4 & 18.6 & 13.9 & -23.3 \\
\hline Chemicals and allied products & 102 & 20.1 & 11.3 & -28.9 & 26.0 & 17.0 & -35.0 \\
\hline Utilities and sanitary & 139 & 19.8 & 12.2 & -27.4 & 20.5 & 12.9 & -28.1 \\
\hline Insurance, real estate, and other finance & 341 & 19.6 & 15.3 & -23.9 & 25.4 & 19.9 & -30.9 \\
\hline Communications & 188 & 18.9 & 12.8 & -25.0 & 22.7 & 16.0 & - 29.4 \\
\hline Public administrations & 468 & 18.0 & 13.9 & -22.1 & 27.0 & 22.1 & - 31.9 \\
\hline Legal, engineering and other professional services & 344 & 16.6 & 12.1 & -21.1 & 20.7 & 16.4 & -25.0 \\
\hline Health services, except hospitals & 181 & 15.1 & 9.6 & -20.6 & 25.3 & 18.2 & - 32.4 \\
\hline Hospitals & 176 & 13.4 & 7.1 & - 19.7 & 20.9 & 14.0 & -27.8 \\
\hline Other educational services & 19 & 11.8 & 0.0 & -26.5 & 55.2 & 31.1 & - 79.3 \\
\hline Elementary and secondary schools and colleges & 468 & 11.2 & 8.1 & -14.3 & 21.2 & 16.9 & -25.5 \\
\hline Social services, religious and membership organizations & 171 & 10.5 & 5.8 & -15.2 & 30.8 & 22.8 & -38.8 \\
\hline Armed forces & 2 & 0.0 & - & - & 28.3 & 0.0 & -84.6 \\
\hline TOTAL & 9,818 & 27.0 & 26.0 & -28.0 & 21.0 & 20.0 & $-\quad 22.0$ \\
\hline
\end{tabular}

- No estimates due to no smokers/former smokers among respondents.

NOTE: Industries were classified according to 1995 Standard Industrial Classification System and then regrouped by NCHS. See appendices for source description and methods.

SOURCE: National Center for Health Statistics 2000 National Health Interview Survey. 
Table 16-3. Smoking status: Estimated prevalence by current industry, U.S. female residents age 18 and over, 2000

\begin{tabular}{|c|c|c|c|c|c|c|c|}
\hline \multirow{3}{*}{$\frac{\text { Industry }}{\text { Mining }}$} & \multirow{3}{*}{$\begin{array}{c}\begin{array}{c}\text { Number of } \\
\text { Respondents }\end{array} \\
9\end{array}$} & \multicolumn{3}{|c|}{ Current Smokers } & \multicolumn{3}{|c|}{ Former Smokers } \\
\hline & & \multirow{2}{*}{$\begin{array}{c}\begin{array}{c}\text { Prevalence } \\
(\%)\end{array} \\
57.4\end{array}$} & \multicolumn{2}{|c|}{$\begin{array}{c}95 \% \\
\text { Confidence } \\
\text { Interval }\end{array}$} & \multirow{2}{*}{$\begin{array}{c}\begin{array}{c}\text { Prevalence } \\
(\%)\end{array} \\
6.6\end{array}$} & \multicolumn{2}{|c|}{$\begin{array}{c}95 \% \\
\text { Confidence } \\
\text { Interval } \\
\end{array}$} \\
\hline & & & 23.7 & - 91.1 & & 0.0 & -19.5 \\
\hline Repair services & 35 & 48.7 & 29.9 & - 67.5 & 14.2 & 1.7 & - 26.7 \\
\hline Automotive dealers and gasoline stations & 94 & 44.4 & 32.2 & -56.6 & 13.1 & 5.5 & -20.7 \\
\hline Food, bakery and dairy stores & 243 & 40.6 & 33.7 & -47.5 & 10.6 & 6.5 & - 14.7 \\
\hline Eating and drinking places & 491 & 39.0 & 33.5 & -44.5 & 14.2 & 10.3 & - $\quad 18.1$ \\
\hline Primary metal industries & 28 & 36.6 & 16.2 & -57.0 & 14.4 & 0.0 & $-\quad 31.1$ \\
\hline Trucking service and warehousing & 84 & 35.9 & 23.2 & -48.6 & 12.8 & 4.0 & -21.6 \\
\hline Furniture, lumber and wood & 60 & 33.3 & 21.7 & -44.9 & 8.8 & 0.8 & -16.8 \\
\hline Machinery, except electrical & 78 & 30.4 & 17.9 & -42.9 & 18.1 & 8.9 & -27.3 \\
\hline Electrical machinery, equipment and supplies & 123 & 30.1 & 20.3 & -39.9 & 12.6 & 5.3 & - $\quad 19.9$ \\
\hline Chemicals and allied products & 65 & 28.3 & 15.8 & -40.8 & 18.1 & 7.7 & -28.5 \\
\hline Business services & 577 & 27.8 & 23.7 & - 31.9 & 15.3 & 12.0 & -18.6 \\
\hline Construction & 106 & 27.5 & 17.1 & - 37.9 & 21.1 & 11.3 & $-\quad 30.9$ \\
\hline Food and kindred products & 96 & 27.3 & 16.7 & - 37.9 & 13.0 & 5.4 & -20.6 \\
\hline Textile mill and finished textile products & 120 & 27.3 & 16.1 & -38.5 & 14.2 & 6.8 & -21.6 \\
\hline Fabricated metal industries, including ordnance & 42 & 26.5 & 12.6 & -40.4 & 13.4 & 0.0 & -26.9 \\
\hline Other nondurable goods & 64 & 25.6 & 13.3 & - 37.9 & 16.1 & 7.7 & -24.5 \\
\hline Utilities and sanitary & 39 & 24.9 & 8.8 & -41.0 & 17.4 & 3.5 & $-\quad 31.3$ \\
\hline Entertainment and recreation services & 160 & 24.7 & 17.3 & -32.1 & 20.5 & 13.6 & $-\quad 27.4$ \\
\hline Communications & 146 & 23.9 & 16.1 & - 31.7 & 14.2 & 7.5 & -20.9 \\
\hline Health services, except hospitals & 926 & 23.5 & 20.4 & - 26.6 & 17.3 & 14.4 & $-\quad 20.2$ \\
\hline Transportation equipment & 97 & 23.4 & 13.8 & -33.0 & 24.4 & 14.2 & -34.6 \\
\hline Other personal services & 379 & 23.2 & 18.1 & -28.3 & 18.0 & 13.7 & $-\quad 22.3$ \\
\hline Other and not specified retail trade & 507 & 23.0 & 18.9 & -27.1 & 17.0 & 13.3 & - $\quad 20.7$ \\
\hline Public administrations & 472 & 22.9 & 18.6 & -27.2 & 21.4 & 16.9 & $-\quad 25.9$ \\
\hline Banking and credit agencies & 315 & 22.3 & 16.8 & -27.8 & 12.1 & 8.0 & -16.2 \\
\hline General merchandise stores & 255 & 22.2 & 16.5 & -27.9 & 13.3 & 8.6 & $-\quad 18.0$ \\
\hline Insurance, real estate, and other finance & 511 & 22.0 & 18.3 & -25.7 & 19.4 & 15.5 & $-\quad 23.3$ \\
\hline Wholesale trade & 256 & 21.4 & 15.9 & -26.9 & 21.0 & 15.5 & -26.5 \\
\hline Legal, engineering and other professional services & 376 & 20.8 & 15.9 & -25.7 & 23.7 & 18.8 & -28.6 \\
\hline Hospitals & 647 & 20.3 & 16.8 & -23.8 & 17.1 & 13.8 & -20.4 \\
\hline Social services, religious and membership organizations & 622 & 19.2 & 15.9 & -22.5 & 17.2 & 13.9 & -20.5 \\
\hline Other transportation & 190 & 19.0 & 12.5 & -25.5 & 25.2 & 18.5 & - $\quad 31.9$ \\
\hline Private households & 160 & 18.9 & 11.1 & -26.7 & 16.1 & 7.9 & -24.3 \\
\hline Agriculture & 105 & 17.6 & 10.0 & -25.2 & 15.6 & 5.8 & -25.4 \\
\hline Forestry and fisheries & 10 & 16.2 & 0.0 & -44.8 & 31.8 & 0.2 & -63.4 \\
\hline Other and not specified durable goods & 94 & 13.2 & 5.8 & -20.6 & 21.9 & 12.7 & $-\quad 31.1$ \\
\hline Other educational services & 70 & 13.1 & 2.1 & -24.1 & 23.0 & 12.2 & $-\quad 33.8$ \\
\hline Elementary and secondary schools and colleges & 1,242 & 12.1 & 9.9 & -14.3 & 20.8 & 18.3 & -23.3 \\
\hline Printing, publishing and allied industries & 111 & 11.4 & 6.1 & - 16.7 & 25.1 & 16.7 & $-\quad 33.5$ \\
\hline Railroads & 3 & 0.0 & - & - & 0.0 & - & - \\
\hline Armed forces & 2 & 0.0 & - & - & 41.5 & 0.0 & -100.0 \\
\hline TOTAL & 10,276 & 22.8 & 21.8 & - 23.8 & 17.6 & 16.8 & $\begin{array}{l}-18.4 \\
\end{array}$ \\
\hline
\end{tabular}

- No estimates due to no smokers/former smokers among respondents.

NOTE: Industries were classified according to 1995 Standard Industrial Classification System and then regrouped by NCHS. See appendices for source description and methods.

SOURCE: National Center for Health Statistics 2000 National Health Interview Survey. 


\section{Table 16-4. Smoking status: Estimated prevalence by current occupation, U.S. residents age 18 and over, 2000}

\begin{tabular}{|c|c|c|c|c|c|c|c|}
\hline \multirow{3}{*}{$\begin{array}{l}\text { Occupation } \\
\text { Machine operators and tenderers, except precision }\end{array}$} & \multirow[b]{2}{*}{$\begin{array}{c}\text { Number of } \\
\text { Respondents }\end{array}$} & \multicolumn{3}{|c|}{ Current Smokers } & \multicolumn{3}{|c|}{ Former Smokers } \\
\hline & & \multirow{2}{*}{$\begin{array}{c}\begin{array}{c}\text { Prevalence } \\
(\%)\end{array} \\
45.9\end{array}$} & \multicolumn{2}{|c|}{$\begin{array}{c}95 \% \\
\text { Confidence } \\
\text { Interval } \\
\end{array}$} & \multirow{2}{*}{$\begin{array}{c}\begin{array}{c}\text { Prevalence } \\
(\%)\end{array} \\
18.2\end{array}$} & \multicolumn{2}{|c|}{$\begin{array}{c}95 \% \\
\text { Confidence } \\
\text { Interval } \\
\end{array}$} \\
\hline & 743 & & 41.9 & -49.8 & & 15.3 & -21.1 \\
\hline Forestry and fishing occupations & 20 & 42.0 & 16.3 & - 67.7 & 2.4 & 0.0 & 7.1 \\
\hline Construction and extractive trades & 806 & 41.3 & 37.4 & -45.2 & 18.6 & 15.3 & -21.9 \\
\hline Material moving equipment operators & 139 & 40.5 & 31.3 & -49.7 & 15.8 & 8.5 & -23.1 \\
\hline Food service & 839 & 39.8 & 35.5 & -44.1 & 11.4 & 9.2 & -13.6 \\
\hline Precision production occupations & 527 & 36.2 & 31.5 & - 40.9 & 19.0 & 15.3 & -22.7 \\
\hline Motor vehicle operators & 597 & 34.8 & 30.5 & - 39.1 & 20.4 & 16.9 & -23.9 \\
\hline Construction laborers & 149 & 34.3 & 26.3 & -42.3 & 13.2 & 6.1 & -20.3 \\
\hline Health service & 475 & 32.8 & 27.7 & - 37.9 & 14.3 & 10.6 & -18.0 \\
\hline Freight, stock and material handlers & 583 & 32.5 & 28.0 & - 37.0 & 13.8 & 10.5 & -17.1 \\
\hline Mechanics and repairers & 623 & 31.5 & 27.2 & -35.8 & 22.0 & 18.5 & -25.5 \\
\hline Cleaning and building service & 534 & 30.8 & 26.3 & -35.3 & 15.0 & 11.7 & -18.3 \\
\hline Other protective service occupations & 158 & 28.6 & 20.6 & -36.6 & 20.0 & 12.9 & -27.1 \\
\hline Farm workers and other agricultural workers & 337 & 28.6 & 22.9 & -34.3 & 14.1 & 9.2 & -19.0 \\
\hline Fabricators, assemblers, inspectors and samplers & 401 & 28.1 & 22.8 & -33.4 & 18.2 & 13.5 & -22.9 \\
\hline Other transportation, except motor vehicles & 36 & 25.3 & 7.9 & -42.7 & 28.8 & 11.6 & -46.0 \\
\hline Managers and administrators, except public administration & 1,857 & 25.2 & 23.0 & -27.4 & 23.0 & 20.8 & -25.2 \\
\hline Health technologists and technicians & 281 & 24.9 & 18.8 & -30.9 & 15.2 & 10.5 & -19.9 \\
\hline Other sales & 905 & 24.4 & 21.3 & -27.5 & 17.2 & 14.3 & -20.1 \\
\hline Supervisors and proprietors & 601 & 23.4 & 19.7 & -27.1 & 24.2 & 19.9 & -28.5 \\
\hline Financial records processing occupations & 343 & 23.1 & 18.0 & -28.2 & 18.1 & 13.4 & -22.8 \\
\hline Personal service & 467 & 20.8 & 16.7 & -24.9 & 17.5 & 13.4 & -21.6 \\
\hline Secretaries, stenographers and typists & 405 & 20.5 & 16.2 & -24.8 & 19.7 & 14.8 & -24.6 \\
\hline Officials and administrators, public health administration & 117 & 20.1 & 11.7 & -28.5 & 31.6 & 22.6 & -40.6 \\
\hline Management related occupation & 793 & 19.9 & 16.6 & -23.2 & 20.2 & 16.9 & -23.5 \\
\hline Mail and message distributing & 133 & 19.3 & 11.9 & -26.7 & 24.0 & 15.2 & -32.8 \\
\hline Other administrative support & 1,941 & 19.3 & 11.8 & -26.7 & 19.0 & 17.0 & -21.0 \\
\hline Sales representatives, commodities and finances & 623 & 18.9 & 15.4 & -22.4 & 26.5 & 22.6 & -30.4 \\
\hline Writers, artists, entertainers and athletes & 343 & 18.6 & 14.1 & -23.1 & 23.3 & 18.4 & -28.2 \\
\hline Technologists, technicians except health & 378 & 18.5 & 14.4 & -22.6 & 20.9 & 16.4 & -25.4 \\
\hline Architects and surveyors & 27 & 17.1 & 1.6 & -32.6 & 16.5 & 0.6 & -32.4 \\
\hline Police and firefighters & 188 & 16.3 & 10.8 & -21.8 & 21.5 & 14.8 & -28.2 \\
\hline Private households occupations & 147 & 16.0 & 8.4 & -23.6 & 18.3 & 9.3 & -27.3 \\
\hline Natural mathematical and computer scientists & 377 & 15.6 & 11.3 & - 19.9 & 19.6 & 15.5 & -23.7 \\
\hline Other professional specialty occupations & 434 & 14.6 & 11.1 & -18.1 & 24.2 & 19.3 & -29.1 \\
\hline Engineers & 292 & 13.4 & 9.3 & -17.5 & 18.6 & 13.9 & -23.3 \\
\hline Farm operators and managers & 127 & 13.2 & 7.9 & -18.5 & 25.9 & 18.1 & -33.7 \\
\hline Health assessment and treating occupations & 510 & 12.5 & 9.8 & -15.2 & 22.5 & 18.6 & -26.4 \\
\hline Computer equipment operators & 42 & 10.3 & 1.9 & -18.7 & 17.4 & 3.3 & -31.5 \\
\hline Teachers, librarians and counselors & 1,115 & 9.6 & 7.6 & -11.6 & 20.9 & 18.0 & -23.8 \\
\hline Health diagnosing occupations & 153 & 3.7 & 0.2 & $-\quad 7.2$ & 18.1 & 11.2 & -25.0 \\
\hline Military & 5 & 0.0 & - & - & 30.9 & 0.0 & -71.9 \\
\hline TOTAL & 20,094 & 25.0 & 24.2 & -25.8 & 19.4 & 18.8 & -20.0 \\
\hline
\end{tabular}

- No estimates due to no smokers/former smokers among respondents.

NOTE: Industries were classified according to 1995 Standard Industrial Classification System and then regrouped by NCHS. See appendices for source description and methods.

SOURCE: National Center for Health Statistics 2000 National Health Interview Survey. 
Table 16-5. Smoking status: Estimated prevalence by current occupation, U.S. male residents age 18 and over, 2000

\begin{tabular}{|c|c|c|c|c|c|c|c|}
\hline \multirow{3}{*}{$\frac{\text { Occupation }}{\text { Food service }}$} & \multirow{3}{*}{$\begin{array}{c}\begin{array}{c}\text { Number of } \\
\text { Respondents }\end{array} \\
339\end{array}$} & \multicolumn{3}{|c|}{ Current Smokers } & \multicolumn{3}{|c|}{ Former Smokers } \\
\hline & & \multirow{2}{*}{$\begin{array}{c}\begin{array}{c}\text { Prevalence } \\
(\%)\end{array} \\
41.9\end{array}$} & \multicolumn{2}{|c|}{$\begin{array}{c}95 \% \\
\text { Confidence } \\
\text { Interval } \\
\end{array}$} & \multirow{2}{*}{$\begin{array}{c}\begin{array}{c}\text { Prevalence } \\
(\%)\end{array} \\
9.3\end{array}$} & \multicolumn{2}{|c|}{$\begin{array}{c}95 \% \\
\text { Confidence } \\
\text { Interval }\end{array}$} \\
\hline & & & 35.2 & -48.6 & & $6.6-$ & 12.0 \\
\hline Construction and extractive trades & 787 & 41.3 & 37.2 & - 45.4 & 18.8 & $15.5-$ & 22.1 \\
\hline Machine operators and tenderers, except precision & 451 & 40.4 & 35.1 & - 45.7 & 20.2 & $16.3-$ & 24.1 \\
\hline Material moving equipment operators & 127 & 40.0 & 30.6 & - 49.4 & 16.5 & $8.9-$ & 24.1 \\
\hline Forestry and fishing occupations & 18 & 39.8 & 13.3 & -66.3 & 2.6 & $0.0-$ & 7.7 \\
\hline Health service & 46 & 38.0 & 20.4 & - 55.6 & 22.0 & $8.3-$ & 35.7 \\
\hline Precision production occupations & 377 & 35.4 & 29.7 & - 41.1 & 20.4 & $15.9-$ & 24.9 \\
\hline Construction laborers & 146 & 34.7 & 26.5 & -42.9 & 13.3 & $6.2-$ & 20.4 \\
\hline Motor vehicle operators & 504 & 34.5 & 30.0 & -39.0 & 20.8 & $16.9-$ & 24.7 \\
\hline Mechanics and repairers & 572 & 32.2 & 27.5 & -36.9 & 22.5 & $18.8-$ & 26.2 \\
\hline Freight, stock and material handlers & 398 & 32.2 & 27.1 & - 37.3 & 14.9 & $10.8-$ & 19.0 \\
\hline Secretaries, stenographers and typists & 8 & 31.6 & 0.0 & -67.9 & 10.4 & $0.0-$ & 30.2 \\
\hline Fabricators, assemblers, inspectors and samplers & 226 & 30.9 & 24.2 & - 37.6 & 21.6 & $15.1-$ & 28.1 \\
\hline Farm workers and other agricultural workers & 275 & 28.7 & 22.6 & -34.8 & 14.7 & $9.4-$ & 20.0 \\
\hline Cleaning and building service & 251 & 28.1 & 22.0 & -34.2 & 18.6 & $13.7-$ & 23.5 \\
\hline Personal service & 63 & 28.1 & 13.6 & -42.6 & 21.4 & $14.1-$ & 28.7 \\
\hline Other protective service occupation & 106 & 26.8 & 17.4 & -36.2 & 24.0 & $15.2-$ & 32.8 \\
\hline Managers and administrators, except public administration & 996 & 25.9 & 23.0 & -28.8 & 23.5 & $20.8-$ & 26.2 \\
\hline Other transportation, except motor vehicles & 35 & 25.8 & 8.0 & -43.6 & 27.5 & $10.3-$ & 44.7 \\
\hline Other administrative support & 456 & 25.6 & 20.9 & -30.3 & 20.4 & $16.1-$ & 24.7 \\
\hline Other sales & 336 & 23.7 & 18.2 & -29.2 & 22.2 & $16.9-$ & 27.5 \\
\hline Supervisors and proprietors & 334 & 22.9 & 17.6 & -28.2 & 26.8 & $20.9-$ & 32.7 \\
\hline Private household occupations & 6 & 22.4 & 0.0 & -60.8 & 30.6 & $0.0-$ & 77.1 \\
\hline Officials and administrators, public health administration & 56 & 21.4 & 9.8 & -33.0 & 36.0 & $21.7-$ & 50.3 \\
\hline Mail and message distributing & 66 & 20.9 & 10.1 & -31.7 & 23.6 & $11.8-$ & 35.4 \\
\hline Architects and surveyors & 22 & 20.1 & 1.9 & -38.3 & 16.4 & $0.0-$ & 34.2 \\
\hline Health technologists and technicians & 49 & 19.6 & 4.7 & -34.5 & 15.9 & $4.1-$ & 27.7 \\
\hline Writers, artists, entertainers and athletes & 176 & 19.3 & 12.4 & -26.2 & 21.0 & $14.5-$ & 27.5 \\
\hline Management related occupation & 292 & 17.9 & 12.2 & -23.6 & 23.9 & $17.8-$ & 30.0 \\
\hline Natural mathematical and computer scientists & 245 & 17.9 & 12.4 & -23.4 & 18.7 & $13.6-$ & 23.8 \\
\hline Sales representatives, commodities and finances & 353 & 17.8 & 13.5 & -22.1 & 30.1 & $24.8-$ & 35.4 \\
\hline Technologists, technicians except health & 234 & 16.9 & 11.8 & -22.0 & 21.0 & $15.7-$ & 26.3 \\
\hline Police and firefighters & 153 & 14.5 & 8.6 & -20.4 & 21.4 & $14.1-$ & 28.7 \\
\hline Financial records processing occupations & 32 & 13.2 & 0.0 & -28.5 & 23.0 & $7.5-$ & 38.5 \\
\hline Farm operators and managers & 104 & 12.7 & 7.2 & -18.2 & 27.0 & $17.6-$ & 36.4 \\
\hline Engineers & 257 & 12.6 & 8.5 & -16.7 & 20.3 & $15.2-$ & 25.4 \\
\hline Health assessment and treating occupations & 62 & 12.5 & 3.9 & -21.1 & 22.9 & $11.5-$ & 34.3 \\
\hline Other professional specialty occupations & 181 & 10.3 & 5.8 & -14.8 & 30.4 & $22.4-$ & 38.4 \\
\hline Computer equipment operators & 23 & 8.6 & 0.0 & -18.8 & 24.0 & $3.4-$ & 44.6 \\
\hline Teachers, librarians and counselors & 292 & 8.3 & 5.0 & -11.6 & 24.0 & $18.7-$ & 29.3 \\
\hline Health diagnosing occupations & 98 & 0.7 & 0.0 & $-\quad 2.1$ & 22.8 & $13.6-$ & 32.0 \\
\hline Military & 3 & 0.0 & - & - & 25.1 & $0.0-$ & 73.7 \\
\hline TOTAL & 9,818 & 27.0 & 26.0 & - 28.0 & 21.0 & $20.0-$ & 22.0 \\
\hline
\end{tabular}

- No estimates due to no smokers/former smokers among respondents.

NOTE: Industries were classified according to 1995 Standard Industrial Classification System and then regrouped by NCHS. See appendices for source description and methods.

SOURCE: National Center for Health Statistics 2000 National Health Interview Survey. 
Table 16-6. Smoking status: Estimated prevalence by current occupation, U.S. female residents age 18 and over, 2000

\begin{tabular}{|c|c|c|c|c|c|c|c|}
\hline \multirow{3}{*}{$\begin{array}{l}\text { Occupation } \\
\text { Forestry and fishing occupations }\end{array}$} & \multirow{3}{*}{$\begin{array}{c}\begin{array}{c}\text { Number of } \\
\text { Respondents }\end{array} \\
2\end{array}$} & \multicolumn{3}{|c|}{ Current Smokers } & \multicolumn{3}{|c|}{ Former Smokers } \\
\hline & & \multirow{2}{*}{$\begin{array}{c}\begin{array}{c}\text { Prevalence } \\
(\%)\end{array} \\
67.8\end{array}$} & \multicolumn{2}{|c|}{$\begin{array}{c}95 \% \\
\text { Confidence } \\
\text { Interval } \\
\end{array}$} & \multirow{2}{*}{$\begin{array}{c}\begin{array}{c}\text { Prevalence } \\
(\%)\end{array} \\
0.0\end{array}$} & \multicolumn{2}{|c|}{$\begin{array}{c}95 \% \\
\text { Confidence } \\
\text { Interval } \\
\end{array}$} \\
\hline & & & 7.2 & -128. & & - & - \\
\hline Material moving equipment operators & 12 & 47.0 & 9.0 & $-85 . \theta$ & 5.2 & $0.0-$ & 15.6 \\
\hline Construction and extractive trades & 19 & 41.1 & 8.6 & -73.6 & 11.3 & $0.0-$ & 25.6 \\
\hline Precision production occupations & 150 & 38.5 & 29.5 & -47.5 & 14.3 & $8.2-$ & 20.4 \\
\hline Food service & 500 & 38.0 & 32.9 & -43.1 & 13.1 & $10.0-$ & 16.2 \\
\hline Motor vehicle operators & 93 & 36.2 & 25.0 & -47.4 & 17.7 & $9.3-$ & 26.1 \\
\hline Cleaning and building service & 283 & 33.9 & 27.4 & -40.4 & 11.0 & $6.5-$ & 15.5 \\
\hline Other protective service occupation & 52 & 33.3 & 18.2 & -48.4 & 9.8 & $0.6-$ & 19.0 \\
\hline Freight, stock and material handlers & 185 & 33.3 & 25.3 & -41.3 & 10.9 & $6.0-$ & 15.8 \\
\hline Health service & 429 & 32.2 & 26.9 & -37.5 & 13.3 & $9.6-$ & 17.0 \\
\hline Police and firefighters & 35 & 28.0 & 9.8 & - 46.2 & 22.4 & $5.2-$ & 39.6 \\
\hline Farm workers and other agricultural workers & 62 & 27.8 & 14.3 & -41.3 & 11.0 & $0.0-$ & 23.9 \\
\hline Machine operators and tenderers, except precision & 292 & 27.7 & 21.4 & -34.0 & 14.6 & $10.7-$ & 18.5 \\
\hline Health technologists and technicians & 232 & 26.3 & 20.0 & -32.6 & 15.0 & $10.1-$ & 19.9 \\
\hline Other sales & 569 & 24.9 & 21.2 & -28.6 & 13.6 & $10.3-$ & 16.9 \\
\hline Supervisors and proprietors & 267 & 24.2 & 18.3 & -30.1 & 20.1 & $14.6-$ & 25.6 \\
\hline Managers and administrators, except public administration & 861 & 24.2 & 20.7 & -27.7 & 22.5 & $19.4-$ & 25.6 \\
\hline Financial records processing occupations & 311 & 24.0 & 18.7 & -29.3 & 17.6 & $12.5-$ & 22.7 \\
\hline Fabricators, assemblers, inspectors and samplers & 175 & 23.4 & 14.8 & -32.0 & 12.7 & $7.4-$ & 18.0 \\
\hline Other administrative support & 1,485 & 23.4 & 21.0 & -25.8 & 18.5 & $16.1-$ & 20.9 \\
\hline Engineers & 35 & 21.7 & 6.4 & - 37.0 & 1.5 & $0.0-$ & 4.4 \\
\hline Technologists, technicians except health & 144 & 21.6 & 14.5 & -28.7 & 20.6 & $13.0-$ & 28.2 \\
\hline Management related occupation & 501 & 21.2 & 17.1 & -25.3 & 17.6 & $13.9-$ & 21.3 \\
\hline Sales representatives, commodities and finance & 270 & 20.7 & 15.6 & -25.8 & 20.5 & $15.2-$ & 25.8 \\
\hline Secretaries, stenographers and typists & 397 & 20.3 & 16.0 & -24.6 & 19.8 & $14.9-$ & 24.7 \\
\hline Mechanics and repairers & 51 & 20.2 & 8.0 & -32.4 & 15.2 & $3.8-$ & 26.6 \\
\hline Personal service & 404 & 19.5 & 15.4 & -23.6 & 17.7 & $13.2-$ & 22.2 \\
\hline Officials and administrators, public health administration & 61 & 18.5 & 8.3 & -28.7 & 26.1 & $13.9-$ & 38.3 \\
\hline Other professional specialty occupations & 253 & 18.3 & 12.8 & -23.8 & 18.8 & $13.5-$ & 24.1 \\
\hline Writers, artists, entertainers and athletes & 167 & 17.7 & 11.2 & -24.2 & 25.8 & $18.7-$ & 32.9 \\
\hline Mail and message distributing & 67 & 17.2 & 8.4 & -26.0 & 24.6 & $11.5-$ & 37.7 \\
\hline Private household occupations & 141 & 15.7 & 7.9 & -23.5 & 17.7 & $8.7-$ & 26.7 \\
\hline Farm operators and managers & 23 & 15.4 & 1.1 & -29.7 & 21.1 & $3.7-$ & 38.5 \\
\hline Computer equipment operators & 19 & 13.4 & 0.0 & -29.1 & 5.3 & $0.0-$ & 12.7 \\
\hline Health assessment and treating occupations & 448 & 12.5 & 9.4 & -15.6 & 22.4 & $18.5-$ & 26.3 \\
\hline Natural mathematical and computer scientists & 132 & 10.6 & 3.7 & -17.5 & 21.7 & $14.1-$ & 29.3 \\
\hline Teachers, librarians and counselors & 823 & 10.2 & 7.8 & -12.6 & 19.7 & $16.6-$ & 22.8 \\
\hline Health diagnosing occupations & 55 & 9.7 & 0.1 & - 19.3 & 8.3 & $1.4-$ & 15.2 \\
\hline Construction laborers & 3 & 0.0 & - & - & 0.0 & - & - \\
\hline Other transportation, except motor vehicles & 1 & 0.0 & - & - & 0.0 & - & - \\
\hline Architects and surveyors & 5 & 0.0 & - & - & 17.3 & 0.0 & 48.9 \\
\hline Military & 2 & 0.0 & - & - & 0.0 & - & - \\
\hline TOTAL & 10,276 & 22.8 & 21.8 & $\begin{array}{l} \\
\end{array}$ & 17.6 & 16.8 & 18.4 \\
\hline
\end{tabular}

- No estimates due to no smokers/former smokers among respondents.

NOTE: Industries were classified according to 1995 Standard Industrial Classification System and then regrouped by NCHS. See appendices for source description and methods.

SOURCE: National Center for Health Statistics 2000 National Health Interview Survey. 


\section{Appendices}




\section{Appendix A \\ Sources of Data}

\section{Annual Survey of Occupational Injuries and Illnesses, BLS}

After passage of the Occupational Safety and Health Act of 1970, the responsibility for collecting statistics on occupational injuries and illnesses was delegated to the Bureau of Labor Statistics (BLS). The BLS Annual Survey of Occupational Injuries and Illnesses, done in cooperation with participating State agencies, involves data collection by mail from a sample of approximately 250,000 establishments each calendar year. Nearly all industries in the private sector (employers covered by the Occupational Safety and Health Act of 1970) are included. Annual BLS reports of these data incorporate corresponding data from mine operators, provided to BLS by the Mine Safety and Health Administration (MSHA), and from railroad transportation employers, provided to BLS by the Federal Railroad Administration. National estimates of injury and illness incidence rates by industry are developed from the survey data. Beginning in 1992, the survey was expanded to provide more information on illnesses resulting in days away from work, allowing for more detailed classification of respiratory system diseases. For this report, annual summary data on respiratory illnesses were abstracted from BLS annual reports on occupational injuries and illnesses.

In contrast with injury data, illness data presented in the BLS annual reports are quite limited because employers typically do not recognize and report illnesses, particularly illnesses with a long latency. Also, the survey does not cover all workers since it excludes the self-employed; farm operators with fewer than 11 employees; private households; employees in federal, state, and local government agencies; and independent mining contractors.

For more information refer to annual reports: Occupational Injuries and Illnesses: Counts, Rates, and Characteristics, Office of Safety, Health and Working Conditions, U.S. Department of Labor, Bureau of Labor Statistics; and www.bls.gov/iif/ home.htm.
Black Lung Benefit Awards, SSA and DOL Title IV of the Coal Mine Health and Safety Act of 1969 authorizes a benefits program, providing medical payments and cash stipends for miners totally disabled because of pneumoconiosis arising out of employment in underground coal mining, as well as for widows of coal miners whose death resulted from the disease or who were entitled to Black Lung benefits at the time of death. The Social Security Administration (SSA) was assigned initial responsibility for operating the benefits program. The Black Lung Benefits Act of 1972 continued SSA responsibility for payments to miners granted claims before July 1973, assigned the Department of Labor responsibility for claims filed after July 1973, and extended eligibility for benefits to surface coal miners and to surviving children of miners. This latter provision allowed children to receive benefits if both parents were deceased, or if a widow ceased to qualify for benefits through remarriage. (In September 1997, in an effort to enhance customer service to Black Lung program beneficiaries, the responsibility for managing all active SSA Black Lung claims was assigned to DOL.)

For more information refer to annual reports: Social Security Bulletin, Annual Statistical Supplements; www.ssa.gov/statistics/Supplement/2000/9d.pdf; annual reports to Congress: Office of Workers' Compensation Programs, U.S. Department of Labor, Employment Standards Administration; and www.dol.gov/esa/regs/compliance/owcp/ bltable.htm.

\section{Coal Mine Employment Data, MSHA}

Initiated in 1970, annual informational reports from the Mine Safety and Health Administration (MSHA) summarize occupational injury and illness experience of United States miners, based on data reported by mine operators. Each operator subject to the Federal Mine Safety and Health Act of 1977 is required to submit annual reports of all injuries and occupational illnesses (see section on Annual 
Survey of Occupational Injuries and Illnesses, above), as well as related data, including average number of employees during the year. The MSHA informational reports on coal mining provide annual estimates for size of the mining workforce, including separate figures for underground mines. Similar estimates are provided based on data reported by contractors performing certain work at mining operations.

For more information refer to annual reports: Injury Experience in Coal Mining, U.S. Department of Labor, Mine Safety and Health Administration; and www.msha.gov/stats/part50/p50y2k/aetable.htm.

\section{Coal Workers' X-ray Surveillance Program, NIOSH}

The Coal Workers' X-ray Surveillance Program (CWXSP) is a NIOSH-administered occupational health program mandated by the Coal Mine Health and Safety Act of 1969. The primary objective of the CWXSP is to screen miners for coal workers' pneumoconiosis (CWP). Since 1970, coal mine operators have been required to offer a chest radiograph to all underground coal miners at the time of hire and again three years later. Subsequently, miners can volunteer for radiographs at approximately five-year intervals. The chest $\mathrm{x}$ rays are taken at no cost to the miners. In addition to the posterior-anterior chest x-ray, other information is collected, including miner identification, age, tenure, and specific job in the mine.

The chest films are read by physicians certified by NIOSH as proficient in use of the International Labour Office (ILO) classification system for radiographs of the pneumoconioses. Each film is read by at least two readers, and a consensus rule is used to reach a final determination for each film. The CWXSP defines CWP as small opacity profusion category of at least $1 / 0$ or large opacities (i.e., larger than one centimeter in diameter). Miners with radiographic evidence of CWP on their chest A-2 radiographs are offered the option to work in an area of the mine with a respirable coal mine dust level of $1 \mathrm{mg} / \mathrm{m}^{3}$ or less and have personal dust exposures monitored at frequent intervals.

The large number of chest $\mathrm{x}$-ray examinations since 1970 provide a means of monitoring the prevalence of CWP among active underground coal miners. However, coal miner participation rates have generally decreased since 1970 to less than $30 \%$ of working underground coal miners. Thus, tenurespecific prevalence estimates may be biased due to selective participation. Also, overall crude prevalence estimates may reflect overrepresentation of newly employed miners. Inferences regarding the entire coal mine work force that are based on CWXSP data should be drawn with caution. Tabulations of CWXSP data presented in this report vary from those presented in some earlier editions of the Work-Related Lung Disease Surveillance Report due to revised criteria for categorizing tenure and round.

For more information: Coal Workers' Health Surveillance Program, Surveillance Branch, Division of Respiratory Disease Studies, NIOSH, 1095 Willowdale Road, Morgantown, WV 26505. Phone (304) 285-5724.

\section{Integrated Management Information System, OSHA}

The Integrated Management Information System (IMIS) includes most of the industrial hygiene sample data from Occupational Safety and Health Administration (OSHA) compliance inspections and consultation surveys conducted since May 1979. The data are reported by OSHA compliance safety and health officers and OSHA state consultants. Each IMIS record includes sample date, substance code, airborne concentration, sample type and exposure type, occupation, OSHA permissible exposure limit (PEL), and standard industrial classification (see Methods, Appendix B). OSHA consultation data were not included in previous 
NIOSH Work-Related Lung Disease Surveillance Reports. Therefore, most numbers of samples reported for a given year, or period of years, are greater than reported previously.

For more information: Directorate of Information Technology, Occupational Safety and Health Administration, 200 Constitution Avenue, NW, Washington, DC 20210. Phone (202) 693-1700.

\section{Metal/Nonmetal Mine Data, MSHA}

The metal/nonmetal mine data (MNMD) are records of industrial hygiene samples collected by Mine Safety and Health Administration (MSHA) inspectors in non-coal surface and underground mines and mills since 1974. This report presents data since 1979, which represent both personal and area samples. Each MNMD record includes sample date, contaminant code, airborne concentration, occupation, MSHA permissible exposure limit (PEL), percent silica and silica concentration where available, standard industrial classification, and the mine and/or mill at which the sample was obtained. In 1982, Congress temporarily removed the surface stone and sand and gravel industries from MSHA's jurisdiction. During this year the number of respirable dust samples collected are fewer than in other years. The quartz reference standard used for MNMD samples changed in 1988. As a result, the reported percent quartz content, quartz concentrations, and the percentage of samples exceeding the PEL increased in 1988 from 1987. MSHA occasionally revises and updates MNMD files, so the number of records reported for a given year, or period of years, may differ from previous reports.

For more information: Metal and Nonmetal Health Division, Mine Safety and Health Administration, Room 2453, 1100 Wilson Blvd., Arlington, VA 22209. Phone (202) 693-9630.

For more information on the quartz reference standard used for the MNMD samples: Dust
Division, Pittsburgh Safety and Health Technology Center, Mine Safety and Health Administration, P.O. Box 18233, Pittsburgh, PA 15236. Phone (412) 3866858 .

\section{Multiple Cause of Death Data, NCHS}

The National Center for Health Statistics (NCHS) has made available annual public-use multiple cause of death data files since 1968. These files contain records of all deaths in the United States (approximately two million annually) that are reported to state vital statistics offices. Each death record includes codes for up to 20 conditions listed on the death certificate, including both underlying and contributing causes of death in two fields: the entity axis, which preserves diagnostic detail for all listed conditions and their placement on the death certificate; and the record axis, which reorders the codes, removes redundancies, and (infrequently) combines some associated conditions (see "Detail Record Layout" at www.cdc.gov/nchs/about/major/ dvs/mcd/1998mcd.htm). Other data include age, race, sex, and state and county of residence at time of death. In addition, usual industry and occupation codes are available for decedents from some states since 1985 . NCHS annually determines that certain quality criteria have been met by usual industry and occupation data from selected states (see Appendix E).

Potential limitations of multiple cause of death data include: under- or over-reporting of conditions on the death certificate by certifying physicians; incomplete or unclassified reporting of usual occupation and industry; and non-specificity of codes.

For more information: Mortality Statistics Branch, Division of Vital Statistics, National Center for Health Statistics, Centers for Disease Control and Prevention, 6525 Belcrest Road, Room 820, Hyattsville, Maryland 20782. Phone (301) 458-4666; and www.cdc.gov/ nchs/products/elec_prods/subject/mortmcd.htm. Also refer to the annual reports: Vital Statistics of the United States, Vol. II Mortality (Parts A and B), Public Health 
Service, National Center for Health Statistics; and www.cdc.gov/nchs/products/pubs/pubd/vsus/ vsus.htm.

For more information on usual industry and occupation codes: see "Technical Appendix for 1995" at www.cdc.gov/nchs/about/major/dvs/mcd/ $1998 \mathrm{mcd} . \mathrm{htm}$.

\section{National Health Interview Survey, NCHS}

The National Center for Health Statistics (NCHS) makes available public-use data from the National Health Interview Survey (NHIS), an annual health survey that has been conducted since 1960. NHIS is a cross-sectional household interview survey on the health of the civilian non-institutionalized population of the United States. The main objective of the NHIS is to monitor the health of the United States population through the collection and analysis of data on a broad range of health topics. NHIS data are collected annually from approximately 40,000 households and include about 100,000 persons. The households selected for interview in the NHIS are a probability sample representative of the target population. The annual response rate of the NHIS is near $90 \%$ of the eligible households in the sample.

For more information: Division of Health Interview Statistics, National Center for Health Statistics, 6525 Belcrest Road, Hyattsville, MD 20782; and www.cdc.gov/nchs/nhis.htm.

\section{National Hospital Discharge Survey, NCHS}

Estimated numbers of hospital discharges presented in this report have been abstracted from National Hospital Discharge Survey (NHDS) reports published by the National Center for Health Statistics (NCHS). The NHDS, conducted yearly by NCHS, collects data on the use of short-stay nonFederal hospitals in the United States. Federal, military, and Department of Veterans Affairs hospitals were excluded in the survey. In recent years, data have been abstracted from approximately 300,000 records from about 500 hospitals. Each A-4 discharge record includes information on patient age, race, sex, ethnicity (since 1985), marital status, length of stay, source of payment (since 1977), diagnoses and surgical procedures, hospital size, ownership, and region of the United States.

Only hospitals with six or more beds for patient use and those in which the average length of stay for all patients is less than 30 days are included in the survey. One limitation of NHDS data is that they represent number of discharges, not number of patients. In addition, information is available only nationally and by region, but not by state. The NHDS relies on the completeness of hospital medical records, and findings can be influenced by diagnostic practices.

For more information: National Hospital Discharge Survey: Annual Summary with Detailed Diagnosis and Procedure Data, Division of Health Care Statistics, National Center for Health Statistics (www.cdc.gov/nchs/about/major/hdasd/ nhdsdes.htm).

\section{Occupational and Environmental Disease Surveillance Database, AOEC}

A database for occupational and environmental diseases and chronic injuries has been developed by the Association of Occupational and Environmental Clinics (AOEC). For inclusion in the database, a case must have at least one diagnosed condition that, in the physician's judgment, is more likely than not to be related to occupational or environmental exposure. Twenty-four AOEC member clinics contributed cases for the period 1991-2000. Six clinics participated over the entire 10-year period and contributed $80 \%$ of the cases. An additional seven clinics contributed over 125 cases each and submitted $14 \%$ of the cases. While not necessarily representative of all patients with work-related conditions, these case reports provide insight into the types of occupational conditions being treated by occupational medicine specialists, as well as into the types of exposures that are causing or exacerbating these diseases. 
For more information: Association of Occupational and Environmental Clinics, 1010 Vermont Ave., NW, \#513, Washington, DC 20005. Phone (202) 347-4976; and www.aoec.org.

\section{Population Data Estimates, BoC and CDC}

National population estimates used in this report are based on national and state level data from the United States Bureau of the Census (BoC). All population estimates used to compute rates in this report have been those obtainable through the CDC computer system. BoC decennial census population data were used for 1970, 1980, and 1990. In all other years prior to 1990 , estimates from intercensal Demo Detail files were used. Estimates from postcensal Demo Detail files were used for 19911995. Since 1996, comparable postcensal population estimates prepared by the $\mathrm{BoC}$ were used. [Note: Comparing population statistics from Demo-Detail and BoC postcensal estimates for each year from 1990 through 1995, we observed a maximum annual difference of less than 0.05 percent, and a difference of 0.01 percent or less in a majority of years. State-specific differences for the same years were less than one percent for all states, with very rare exceptions.]

For more information: 1990 Census of the Population, General Population Characteristics, U.S. Bureau of the Census, Series 1900, CP-1; and www.census.gov/prod/www/abs/ decenial.html. For more information on population estimates: http://eire.census.gov/ popest/estimates.php.

\section{Respirable Coal Mine Dust Data, MSHA}

The data consist of respirable coal mine dust measurements collected by MSHA inspectors and mine operators at surface and underground coal mines and preparation plants since 1974. Each record includes sample date, duration, and airborne concentration, as well as occupation and the mine or preparation plant at which the sample was obtained.
For more information: Information Resource Center, Mine Safety and Health Administration, P.O. Box 25367, Denver, CO 80225. Phone (303) 2315475.

\section{Respirable Coal Mine Quartz Dust Data, MSHA} The data consist of respirable quartz measurements collected by MSHA inspectors and mine operators at surface and underground coal mines and preparation plants since 1982. Each record includes sample date, duration, percent quartz, and airborne concentration, as well as occupation and the mine or preparation plant at which the sample was obtained.

For more information: Dust Division, Pittsburgh Safety and Health Technology Center, Mine Safety and Health Administration, P.O. Box 18233, Pittsburgh, PA 15236. Phone (412) 386-6858.

\section{Sentinel Event Notification Systems for Occupational Risks (SENSOR), NIOSH}

Since 1987, NIOSH has awarded cooperative agreements to various state health departments to develop models for state-based and conditionspecific surveillance and preventive intervention. Two of the conditions for which states have been funded through the SENSOR program are silicosis and work-related asthma. States and years funded for these two conditions are shown in Table A-1.

SENSOR Silicosis. A total of three states (MI, NJ, $\mathrm{OH})$ maintained silicosis surveillance programs during the 10-year period covered by the SENSOR tables included in this report (1989-1998). All three states identified potential cases using a variety of sources: review of state death certificate data, case reports from physicians, review of hospital discharge data or direct hospital reporting to the state health department. In addition, Michigan and Ohio review workers' compensation records.

In all three states, demographic, work history, and medical information used for case confirmation and 
description was obtained through a combination of the initial case ascertainment source, a review of medical records, and follow-up telephone interview with the reported cases or their surviving next of kin. For SENSOR surveillance purposes, silicosis case confirmation requires a history of occupational exposure to airborne silica dust and either: (a) a chest radiograph interpreted as characteristic of silicosis, or (b) lung histopathology characteristic of silicosis (see Appendix G).

For more information: Maxfield R, Alo C, Reilly MJ, et al. Surveillance for silicosis, 1993-Illinois, Michigan, New Jersey, North Carolina, Ohio, Texas, and Wisconsin. MMWR Surveill Summ 1997/46 (SS-1); 13-28 (www.cdc.gov/mmwr/preview/ mmwrhtml/00046046.htm).

SENSOR Work-Related Asthma (WRA). A total of four states (CA, MA, MI, NJ) maintained WRA surveillance programs during the seven-year period covered by the SENSOR tables included in this report (1993-1999). Physician case reports represented the primary ascertainment source in all four states. Massachusetts, Michigan, and New Jersey actively solicited physicians for case reports, whereas California identified potential cases by reviewing data from Doctor's First Reports (DFR) of Occupational Injury or Illness, a longstanding statewide physician reporting system linked to physician reimbursement for medical services. In addition, Michigan and New Jersey actively solicited hospital reports and reviewed hospital discharge records for potential WRA cases. In 1993, Massachusetts also began supplementing case ascertainment with review of state-wide hospital discharge data.

In all four states, surveillance staff collected demographic, work history, and medical information used for case confirmation, classification, and description through a combination of the initial case ascertainment source, a review of medical records, and follow-up telephone interview with reported cases. For SENSOR surveillance purposes, WRA surveillance case confirmation requires a healthcare professional's diagnosis of asthma (or a related diagnosis consistent with asthma) and an association between symptoms of asthma and work. Confirmed WRA cases are classified according to established criteria (see Appendix G). To facilitate consistency in agent coding across states, putative causes of WRA are coded using the Association of Occupational and Environmental Clinics (AOEC) exposure coding scheme (www.aoec.org/aoeccode.htm), which flags "known asthma inducers."

For more information: Jajosky RA, Harrison R, Reinisch F, et al. Surveillance of work-related asthma in selected U.S. states using surveillance guidelines for state health departments-California, Massachusetts, Michigan, and New Jersey, 19931995. MMWR Surveill Summ 1999/48 (SS-3); 120 (www.cdc.gov/mmwr/preview/mmwrhtml/ ss4803a1.htm).

\begin{tabular}{|c|c|c|c|}
\hline State & $\begin{array}{l}\text { Oct. } 1988 \text { - } \\
\text { Sept. } 1992\end{array}$ & $\begin{array}{l}\text { Oct. } 1992 \text { - } \\
\text { Sept. } 1997\end{array}$ & $\begin{array}{l}\text { Oct. } 1997 \text { - } \\
\text { Sept. } 2002\end{array}$ \\
\hline CA & & A & A \\
\hline Co & A & & \\
\hline IL & & $\mathrm{S}$ & \\
\hline MA & A & A & $\mathrm{A}$ \\
\hline MI & $\mathrm{A}, \mathrm{S}$ & $\mathrm{A}, \mathrm{S}$ & $\mathrm{A}, \mathrm{S}^{*}$ \\
\hline NJ & $\mathrm{A}, \mathrm{S}$ & $\mathrm{A}, \mathrm{S}$ & $\mathrm{A}^{*}, \mathrm{~S}$ \\
\hline NY & A & & \\
\hline $\mathrm{NC}$ & & $\mathrm{S}$ & \\
\hline $\mathrm{OH}$ & $\mathrm{S}$ & $\mathrm{S}$ & $S$ \\
\hline TX & & $S$ & \\
\hline WI & $\mathrm{A}, \mathrm{S}$ & $\mathrm{S}$ & \\
\hline
\end{tabular}





\section{Appendix B \\ Methods}

\section{MORTALITY}

\section{Number of Deaths}

In this report, the number of deaths for each occupational respiratory condition is the number of decedents for which the condition was coded as either underlying or contributing cause of death. For the years 1968-1998, these numbers were tabulated from the record axis of the NCHS multiple cause of death data files. Beginning with the 1999 data, these numbers were tabulated from the entity axis and the underlying cause of death of the multiple cause of death data files. (This change was made to permit more complete ascertainment of the diseases of interest.) In the current report, a small number of deaths in 1999 with underlying cause code J65 (pneumoconiosis associated with tuberculosis) were included in the underlying cause of death tabulations of each specific type of pneumoconiosis. Similarly, deaths in 1999 with underlying cause code J92.0 (pleural plaque with asbestos) were included in asbestosis underlying cause of death tabulations. Cause of death codes are defined as shown in Appendix C: International Classification of Diseases (ICD) Codes. The number of deaths by condition are reported both annually and for selected time periods. Reported deaths are restricted to United States residents, 15 years or older, based on state of residence at death. Race was classified as white, black, and all others.

\section{Crude Mortality Rates}

To compute annual cause-specific crude mortality rates, the total number of decedents, 15 years and older, with a specified condition coded as either underlying or contributing cause in a given year was divided by the population, 15 years and older, of the same geopolitical unit in the same year. Raceand sex-specific rates were computed from the appropriate subsets of the data. Crude mortality rates were computed at the national and state level for the multi-year period 1990-1999, and at the county level for the multi-year period 1985-1999. For each time period, the average annual number of decedents, 15 years and older, with a specified condition coded as either underlying or contributing cause was divided by the mid-year population (1995, 1992, respectively), 15 years and older, of the same geopolitical unit.

\section{Age-Adjusted Mortality Rates}

Age-adjusted mortality rates presented in this report were based on deaths with the condition of interest mentioned as either underlying or contributing cause of death. Rates were calculated annually for each specified condition from 1968 through 1999, as well as for selected periods. For a given year, the ageadjusted rates represent the rates that would have been observed if the age-specific rates for specified age groups had occurred in a population with the same age distribution as that of the standard population. To conform with current NCHS guidelines, the U.S. Year 2000 Standard Population was used as the standard. (All earlier editions of the Work-Related Lung Disease Surveillance Report have used the 1940 standard population.) The specific age intervals used were 15-24, 25-34, 35$44,45-54,55-64,65-74,75-84$, and 85 years and older. Rates for the entire United States population and for each sex-race group were age-adjusted separately, using the same standard population.

Age-adjusted rates were computed by the direct method. First, the annual age-specific rates for the population of interest were calculated. The product of the age-specific rates and the number in the comparable age-specific group in the standard population equals the expected number of deaths per million population for each age group. The total expected numbers of deaths were then obtained by summing over all age groups. The total expected number of deaths was divided by the sum of the standard population and the resulting quotient was multiplied by $1,000,000$ to produce the age-adjusted rate (per million).

Age-adjusted rates were computed at the national and state level for the multi-year period 1990-1999. 
Rates also were computed at the county level either for two 15-year periods and one 30-year period (1970-1984, 1985-1999, and 1970-1999), or for a single 20-year period (1980-1999), depending on whether or not the condition of interest was discretely classified during those time periods (see Appendix C). Rates for malignant mesothelioma were computed for 1999 only. For each time period (1970-1984, 1970-1999, 1980-1999, 1985-1999, and 1990-1999), age-specific rates first were computed by dividing the average annual number of deaths in each age group by the corresponding age-grouped, mid-year population (1977, 1985, 1990,1992 , and 1995, respectively) in the comparable geopolitical unit. Age-adjusted rates then were computed as described above.

\section{Years of Potential Life Lost (YPLL)}

YPLL were based on deaths with the condition of interest mentioned as either underlying or contributing cause of death. They were calculated using the method described by the Centers for Disease Control (CDC) (MMWR Surveill Summ 1986/35(2S); www.cdc.gov/mmwr/preview/ mmwrhtml/00001773.htm). YPLL were calculated both to age 65 and to life expectancy. YPLL to age 65 may be considered as a loss of years from a traditional working life, while YPLL to life expectancy may be considered as a loss of years from the overall life span. To compute YPLL to life expectancy, the number of deaths in each race/sex age group (the same age intervals used for computing age-adjusted rates) first was multiplied by the difference between the mid-point of the age group and life-expectancy for that race/sex agegroup. Life tables published annually by NCHS (www.cdc.gov/nchs/products/pubs/pubd/lftbls/life/ 1966.htm) were used to determine race/sex lifeexpectancies for white/male, white/female, black/ male, and black/female. The overall U.S. population life-expectancy was used for other/male and other/ female. To compute YPLL to age 65, the number of deaths in age groups 15-24 through 55-64 was multiplied by the difference between 65 years and B-2 the mid-point of each age group (e.g., 65 minus 20 years for the 15-24 age group). These age-specific YPLLs then were summed over all age groups to obtain total YPLLs (to life expectancy, and to age 65) for each race/sex/year from 1990 to 1999.

State-specific YPLLs (to life expectancy) per death also were calculated for the period 1990-1999. To calculate this index, the total number of all race/sex deaths in each age group was multiplied by the corresponding U.S. population life-expectancy, then summed over all age groups to obtain the total YPLL, and then divided by the total number of deaths for each state during this time period.

\section{Rank Order}

For each state, a rank order is presented for each of several mortality measures. Depending on the specific mortality measures, a rank order of " 1 " indicates the greatest number of deaths, highest mortality rate, or highest YPLL among all states in the U.S.

\section{Most Frequently Recorded Industries/ Occupations}

In this report, the ten most frequently recorded Bureau of Census industries (CIC) and occupations (COC) with at least two decedents have generally been listed for specified causes of death (from selected states and years in Appendix E). Where more than one industry/occupation was tied for tenth place, all those that were tied were listed.

\section{Proportionate Mortality Ratio (PMR)}

The data used for PMR analyses are a subset of the NCHS multiple cause of death files for which usual industry and occupation codes are available and meet quality criteria set by NCHS (see Appendix E for a list of states and years for which data qualified).

The PMR is defined as the observed number of deaths with the condition of interest (mentioned as either underlying or contributing) in a specified industry/occupation (from selected states and years 
in Appendix E), divided by the expected number of deaths with that condition. The expected number of deaths is the total number of deaths in the Bureau of Census industry (CIC) or occupation (COC) of interest multiplied by a proportion defined as the number of cause-specific deaths for the condition of interest in all industries/occupations, divided by the total number of deaths in all industries/ occupations. The PMRs in this report have been internally adjusted by five-year age groups (i.e., 15$19,20-24, \ldots 110-114$, and 115 years and over), sex, and race (i.e., white, black, and all other). (PMRs presented in the 1999 Work-Related Lung Disease Surveillance Report were internally adjusted for age only, using the age groupings 15-34, 35-54, 55-74, and 75 years and over.) Confidence intervals were calculated assuming Poisson distribution of the data.

A PMR greater than 1.0 indicates that there were more deaths associated with the condition in a specified occupation or industry than expected. This report includes only those industries/occupations with five or more decedents with the condition of interest and a lower 95\% confidence limit exceeding 1.0.

\section{MORBIDITY}

\section{Prevalence (Asthma, COPD, and Smoking)}

The prevalence of asthma, chronic obstructive pulmonary disease (COPD), and cigarette smoking was based on the 2000 NHIS data collected from adult (18 years and older) household interview survey. Asthma was defined as a "yes" response to the question, "Have you ever been told by a doctor or other health professional that you had asthma?" COPD was defined as a "yes" response to either of the following questions: (1) "Have you ever been told by a doctor or other health professional that you had chronic bronchitis?" or (2) "Have you ever been told by a doctor or other health professional that you had emphysema?" Cigarette smoking status was classified as three groups: nonsmokers, current smokers, and former smokers. Nonsmokers were defined as those who smoked less than 100 cigarettes during their entire life. Former smokers were defined as those who smoked at least 100 cigarettes in their entire life and do not currently smoke. Information on current occupation and industry was coded according to the revised 1995 Standard Industrial and Occupational Classification. These detailed occupation and industry codes were collapsed in the NHIS public-use data set (available at www.cdc.gov/nchs/about/major/hdasd/ nhdsdes.htm).

Prevalence rates for asthma and COPD were estimated (using sample weights and adjustment for non-responses) by gender, smoking status, industry, and occupation as regrouped by NCHS in the NHIS data files. The prevalence of cigarette smoking was estimated by gender, industry, and occupation. Survey Data Analysis (SUDAAN®) software was used to estimate variances, enabling calculation of 95\% confidence intervals for asthma, COPD, and smoking prevalence rates. Lower $95 \%$ confidence limits less than zero were converted to 0.0 and upper $95 \%$ confidence limits greater than 100 were converted to 100.0 .

\section{Prevalence (CWP)}

Prevalence of CWP, presented by tenure and time period, was based solely on "final determinations" (consensus values) of ILO category $1 / 0$ or higher of chest radiographs taken for the Coal Workers' Xray Surveillance Program (CWXSP). Administrative and regulatory guidelines have varied over the life of the program. From 1970 through 1981, the program was administered in structured rounds. After a change in procedure in 1981, examinations have been arranged on a continual basis. For this report, CWXSP data collected after 1981 are grouped into 5-year periods (referred to as "rounds"), which roughly correspond to cycles during which all working underground coal miners could elect to receive a chest $\mathrm{x}$-ray. In cases where more than one chest $\mathrm{x}$-ray was available for a single participant in the same round-usually due 
to a change in employer-the final determination for the most recent chest $\mathrm{x}$-ray was used. Tenure in underground coal mining was based on summation of years in various mining occupations, as reported by the miner at the time of $\mathrm{x}$-ray.

\section{Incidence Rates (Occupational Respiratory Illnesses)}

Estimated numbers of work-related respiratory illness (with days away from work) and incidence rates of occupational respiratory conditions due to toxic agents were generally abstracted from the BLS annual reports of occupational injuries and illnesses, 1992-2000. Where data were not directly abstracted from BLS reports, incidence rates for occupational respiratory illnesses (with days away from work) were computed by dividing the BLS-estimated annual number of incident cases in the industry by the BLS-estimated industry-specific employment for the corresponding year. The resulting quotients were multiplied by 100,000 to yield rates per 100,000 workers.

\section{Association of Occupational and Environmental Clinics (AOEC) Diagnoses}

In this report, the frequency distributions of workrelated respiratory conditions diagnosed in AOEC clinics and respiratory hazards associated with respiratory diagnoses were tabulated by AOEC from the AOEC database.

\section{EXPOSURE}

\section{Occupational Exposure Limits}

Permissible Exposure Limits. OSHA and MSHA each enforce regulations that establish the legal limits of workplace exposures to pneumoconiotic agents. These legal limits are described in this report as permissible exposure limits (PELs), although the regulations sometimes use the term "standard" or "exposure limit." The current legal limits may be found in the U.S. Code of Federal Regulations (CFR), as follows:

\section{$B-4$}

OSHA

$\begin{array}{ll}\text { general industry } & \text { construction industry } \\ \text { 29 CFR 1910.1000 } & \text { 29 CFR 1926.55 } \\ \text { 29 CFR 1910.1001 } & \text { 29 CFR 1926.1101 }\end{array}$

29 CFR 1910.1043

coal mine industry

30 CFR 70.100

30 CFR 70.101

30 CFR 71.100

30 CFR 71.101

30 CFR 71.700

30 CFR 75.321

30 CFR 90.100

30 CFR 90.101

MSHA

non-coal mining industry

30 CFR 56.5001

30 CFR 57.5001

Although OSHA has PELs for the maritime industry [29 CFR 1915], very few samples have been collected and are not reported here.

The OSHA PELs for several pneumoconiotic agents were changed on March 1, 1989, but a legal challenge to the modified OSHA PELs was upheld, and the modified OSHA PELs reverted to the previous OSHA PELs on March 23, 1993. Therefore, data for respirable quartz, selected pneumoconiotic agents, and all pneumoconiotic agents are reported for the three time periods: 1979 to $1988 ; 1989$ to 1992 ; and 1993 to 1999 . Some pneumoconiotic agents had a substance-specific OSHA PEL only from March 1, 1989 to March 22, 1993, including: aluminum as welding fumes, respirable dust of natural graphite, mica containing less than $1 \%$ crystalline silica, tin oxide, inorganic compounds of tin oxide, fused respirable silica dust, fibrous talc not containing tremolite, talc not containing asbestos, insoluble tungsten \& compounds, and welding fumes (total particulate).

The MSHA metal/nonmetal mining PELs for pneumoconiotic agents were adopted from the 1973 edition of the American Conference of Governmental Industrial Hygienists $\left(\mathrm{ACGIH}^{\circledR}\right)$ 
publication entitled " $T L V^{\circledR} s$ Threshold Limit Values for Chemical Substances in Workroom Air Adopted by ACGIH for 1973." MSHA has not adopted a PEL for the pneumoconiotic agents: tin oxide dust/ fume; inorganic dusts of tin; insoluble tungsten dusts/fumes; and welding fumes (total particulate). In this report a MSHA PEL of 10 milligrams per cubic meter $\left(\mathrm{mg} / \mathrm{m}^{3}\right)$ is used for welding fumes (total particulate) through 1993, but since then it has been MSHA policy not to collect samples for welding fumes.

OSHA and MSHA do not have PELs specific for any form of crystalline silica. The PELs apply to respirable dust containing crystalline silica, and the allowable exposure to respirable dust is reduced as the crystalline silica content increases. The formulas for allowable exposure vary with the agency and the industry. In metal/nonmetal mining, the MSHA PEL is the same as the OSHA PEL for respirable dust containing at least $1 \%$ quartz:

$$
\text { OSHA PEL }=\frac{10 \mathrm{mg} / \mathrm{m}^{3}}{\% \text { Quartz }+2}
$$

However, OSHA adopted a PEL of $0.1 \mathrm{mg} / \mathrm{m}^{3}$ for quartz that was enforced from March 1, 1989 through March 22, 1993.

Since December 1972, the MSHA PEL for respirable coal mine dust has been $2 \mathrm{mg} / \mathrm{m}^{3} \mathrm{MRE}^{1}$ unless the quartz concentration of the respirable coal mine dust at the mine exceeds $5 \%$. When the quartz content of the respirable dust exceeds $5 \%$ in a coal mine sample, the MSHA PEL is reduced based on the following formula:

\footnotetext{
1 The MRE designation refers to the Mining Research Establishment of the National Coal Board, London, England. MSHA's PELs for respirable coal mine dust and respirable coal mine dust containing quartz are based on sampling criteria developed by MRE, but OSHA's are based on different sampling criteria. To clearly indicate the difference, the MSHA PELs and sample results are designated by "MRE" in this report.
}

$$
\text { MSHA PEL }=\frac{10 \mathrm{mg} / \mathrm{m}^{3} M R E}{\% \text { Quartz }}
$$

The OSHA PEL of 2 fibers per cubic centimeter ( $\mathrm{f} / \mathrm{cc})$ for asbestos was reduced to $0.2 \mathrm{f} / \mathrm{cc}$ on July 21,1986 , and to $0.1 \mathrm{f} / \mathrm{cc}$ on October 11, 1994. Therefore, asbestos exposures are reported for three time periods: 1979 to $1986 ; 1987$ to 1994; and 1994 to 1999. The MSHA PEL for asbestos has not changed from $2 \mathrm{f} / \mathrm{cc}$ since it was adopted.

The OSHA PELs for cotton dust (raw) vary by processing operation. They are:

- $1 \mathrm{mg} / \mathrm{m}^{3}$ for the cotton waste processing operations of waste recycling (sorting, blending, cleaning, and willowing) and garneting;

- $0.75 \mathrm{mg} / \mathrm{m}^{3}$ for textile slashing and weaving operations;

- $0.50 \mathrm{mg} / \mathrm{m}^{3}$ for textile mill waste house operations or for dust from "lower grade washed cotton" used during yarn manufacturing; and

- $0.20 \mathrm{mg} / \mathrm{m}^{3}$ for yarn manufacturing and cotton washing operations.

Reporting of cotton dust data began when the process-specific OSHA PELs became effective on March 27, 1980.

Recommended Exposure Limits. NIOSH develops and periodically revises recommended exposure limits (RELs) for hazardous substances or conditions in the workplace. The RELs are then published and transmitted to OSHA and MSHA for use in promulgating legal standards. The RELs for mineral dusts and chemical hazards, including pneumoconiotic agents, are published in the NIOSH Pocket Guide to Chemical Hazards (NIOSH Pub. No. 97-140). The REL for coal mine dust was adopted in September 1995, while RELs for the other pneumoconiotic agents in this report were adopted before 1979, which is the first year OSHA and MSHA data are reported. The REL for beryllium and compounds is based on cancer, rather 
than pneumoconiotic effects. NIOSH has no fullshift RELs for the following pneumoconiotic agents: aluminum oxide; emery; synthetic graphite; rouge; fused respirable silica dust; titanium dioxide; and welding fumes (total particulate).

\section{Data Selection}

MSHA coal mine dust samples included in this report met all of the following criteria:

(1) obtained in the United States or one of its territories;

(2) designated by MSHA as valid;

(3) coded as "designated occupation," "nondesignated occupation," "designated work position," "non-designated work position" with valid occupation codes, or "designated area" other than "intake air."

MSHA Coal Mine Quartz. MSHA coal mine quartz samples included in this report met all of the following criteria:

(1) obtained in the United States or one of its territories;

(2) designated by MSHA as valid;

(3) sample duration greater than zero;

(4) quartz concentration greater than or equal to zero;

(5) coded as "designated occupation," "nondesignated occupation," "designated work position," "non-designated work position" with valid occupation codes, or "designated area" other than "intake air."

MSHA Metal/Nonmetal Mine Data (MNMD). MSHA metal/nonmetal mine data (MNMD) included in this report met the following criteria:

(1) obtained in the United States or one of its territories;

(2) not duplicated by another record, as determined by a comparison of all data fields.

NIOSH staff edited the MNMD provided by MSHA to remove duplicate records and records with B-6 internal inconsistencies, similarly to the methods previously used by the U.S. Bureau of Mines for data presented in earlier Work-Related Lung Disease Surveillance Reports.

OSHA Integrated Management Information System (IMIS). IMIS samples included in this report met all of the following criteria:

(1) the state code was one of the 50 U.S. states, Washington, DC, American Samoa, Guam, Puerto Rico, or the U.S. Virgin Islands;

(2) the sample type was "area" or "personal" (excludes: "bulk," "wipe," "screen," "blood," and "urine" samples);

(3) the exposure type was "time-weighted average," or "not detected" (excludes: "ceiling," "peak," "dose," "sound reading," "not analyzed," and "not valid");

(4) the indicated OSHA PEL and units were applicable to the contaminant indicated by the substance code for the recorded date of sampling.

\section{Data Analysis for MSHA and OSHA Samples}

The reported number of samples for an agent was the total number of samples meeting the above criteria. The percent of samples exceeding the PEL for an agent category was calculated as the number of samples in that category with measured exposures exceeding the PEL enforced at the time the sample was collected, divided by the total number of samples for the agent, and finally multiplying by 100. The percent of samples exceeding the REL for an agent was calculated as the number of samples in that category with measured exposure exceeding the REL, divided by the total number of samples for the agent, and multiplying by 100 .

Exposures are commonly log normally distributed, rather than normally distributed. For this reason we present geometric means of the exposure. To calculate a geometric mean exposure, samples less than the minimum quantifiable concentration $(\mathrm{MQC})$ were assigned a value, either the (MQC/2) 
or $\left(\mathrm{MQC} / 2^{1 / 2}\right)$, depending on the distribution of samples that were quantifiable. ${ }^{2}$ The analytical methods used to calculate the MQC for selected pneumoconiotic agents are presented in Table F-2 of Appendix F. The calculation assumes a sample duration of 6 hours for cotton dust, and 7 hours for other agents.

The OSHA and MSHA asbestos MQCs changed during the 1979 to 1999 period; therefore, appropriate MQCs were used for each time period.

OSHA analyzed cotton dust or welding fumes (total particulate) samples by using their standard operating procedure (SOP) for nuisance dusts. The limit of detection of 10 micrograms was determined by the sensitivity of the balance. Results for cotton dust samples below the MQC, $4.3 \%$ of all cotton dust samples, could not be assigned to a specific cotton dust processing operation and were not included in Figure 4-4 and Table 4-11.

The MSHA respirable coal mine quartz data are based on analyses of respirable coal mine dust samples. However, the quartz content could not be reliably identified for most of these samples. Therefore, in Section 2, the percent of respirable coal mine dust samples exceeding the MSHA PEL were calculated using the MSHA PEL of $2 \mathrm{mg} / \mathrm{m}^{3}$ MRE for respirable coal mine dust containing no more than 5\% quartz.

In Section 3 the geometric means of exposure to quartz are reported for OSHA samples. However, the reported percentage greater than the PEL $(\%>$ PEL) compares only the respirable dust samples containing at least $1 \%$ quartz to the PEL for respirable dust containing at least $1 \%$ quartz. The exception is from March 1, 1989 through March 22, 1993, when OSHA enforced a PEL of $0.1 \mathrm{mg} / \mathrm{m}^{3}$ for

\footnotetext{
${ }^{2}$ Hornung, R., Reed, L. 1990. Estimation of average concentration in the presence of nondetectable values. Applied Occupational and Environmental Hygiene 5:46-51.
}

respirable quartz. During this period the percentage greater than the PEL $(\%>$ PEL) compares the exposure to quartz to $0.1 \mathrm{mg} / \mathrm{m}^{3}$.

\section{Industries with Elevated PMRs and Most} Frequently Recorded on Death Certificates

This report includes number of samples, geometric mean exposures, and percent of samples exceeding the PEL or REL by selected industries for exposure agents related to elevated occupational lung disease mortality. For asbestosis, CWP, silicosis, byssinosis, and all pneumoconiosis, separate tables present data for the ten most frequently recorded industries with five or more decedents and significantly elevated PMRs.

\section{STATE AND COUNTY DESIGNATIONS}

The "number of states" displayed on maps in this report sums to 51 because the District of Columbia is included.

Counties in this report are coded according to the 1990 Federal Information Processing Standards (FIPS) Codes system. A small number of counties or county equivalents have split, merged with, or separated from surrounding or adjacent subdivisions (see Appendix H). Readers should be cautious in assessing geographic patterns and temporal trends for subdivisions that have split or merged.

\section{INDUSTRY/OCCUPATION CODES AND TITLES}

Since 1993, the 1990 Bureau of Census (BoC) Index of Industries and Occupations classification system (see "Technical Appendix for 1995" at www.cdc.gov/nchs/about/major/dvs/mcd/ 1998mcd.htm) has been used for coding death certificate information on the NCHS multiple cause of death data files. Most codes and titles in the 1990 system do not differ from the 1980 system. All tables 
reporting BoC industry (CIC) and occupation (COC) codes and titles that are presented in the mortality and exposure sections of this report, except those listed in Appendix D, follow the 1980 BoC classification system.

Industry/occupation titles ranked by estimated prevalence of asthma, COPD, and smoking, which are presented in the morbidity sections of this report, had been classified according to the 1995 Standard Industrial Classification (SIC) System and then regrouped by NCHS. Incidence rates of the pneumoconioses (including siderosis) and respiratory conditions due to toxic agents follow the 1987 SIC System. Tables summarizing temporal patterns of geometric means in selected exposure sections (i.e., asbestos, silica, and pneumoconiotic agents) of this report also group industries by the 1987 SIC System.

The primary industries associated with silicosis and work-related asthma cases in the SENSOR sections of this report are grouped by the 1987 SIC System; however, the primary occupations (COC) are grouped by the $1990 \mathrm{BoC}$ classification system. 


\section{Appendix C}

\section{International Classification of Diseases (ICD) Codes}

\begin{tabular}{|c|c|c|c|c|c|c|}
\hline \multirow{2}{*}{$\begin{array}{l}\text { Condition } \\
\text { (as defined for this report) }\end{array}$} & \multicolumn{2}{|c|}{ ICD-8 (1968-1978) } & \multicolumn{2}{|c|}{ ICD-9 (1979-1998) } & \multicolumn{2}{|l|}{ ICD-10 (1999) } \\
\hline & Rubrics & Codes & Rubrics & Codes & Rubrics & Codes \\
\hline Asbestosis & Asbestosis & 515.2 & Asbestosis & $\overline{501}$ & \begin{tabular}{|l|}
$\begin{array}{l}\text { Pneumoconiosis due to } \\
\text { asbestos and other } \\
\text { mineral fibers } \\
\text { Asbestosis }\end{array}$ \\
\end{tabular} & J61* \\
\hline $\begin{array}{l}\text { Coal Workers' } \\
\text { Pneumoconiosis }\end{array}$ & $\begin{array}{l}\text { Anthracosilicosis } \\
\text { Anthracosis } \\
\text { Coal miners' lung }\end{array}$ & 515.1 & \begin{tabular}{|l} 
Coal workers' \\
pneumoconiosis \\
Anthracosilicosis \\
Anthracosis \\
Black lung disease \\
Coal workers' lung \\
Miners' asthma \\
\end{tabular} & 500 & $\begin{array}{l}\text { Coal workers' } \\
\text { pneumoconiosis } \\
\text { Anthracosilicosis } \\
\text { Anthracosis } \\
\text { Coal workers' lung }\end{array}$ & J60* \\
\hline \multirow[t]{2}{*}{ Silicosis } & \begin{tabular}{|l} 
Silicosis \\
Calcicosis \\
Chalicosis
\end{tabular} & 515.0 & $\begin{array}{l}\text { Pneumoconiosis due to } \\
\text { other silica or silicates } \\
\text { Pneumoconiosis due to talc } \\
\text { Silicotic fibrosis (massive) of } \\
\text { lung } \\
\text { Silicosis (simple)/ } \\
\text { (complicated) }\end{array}$ & 502 & $\begin{array}{l}\text { Pneumoconiosis due to } \\
\text { dust containing silica } \\
\text { Silicotic fibrosis (massive) } \\
\text { of lung } \\
\text { Pneumoconiosis due to talc } \\
\text { dust } \\
\text { Pneumoconiosis due to other } \\
\text { dust containing silica }\end{array}$ & $\mathrm{J} 62 *$ \\
\hline & $\begin{array}{l}\text { Silicotuberculosis } \\
\text { Colliers' phthisis } \\
\text { Grinders' phthisis } \\
\text { Miners' phthisis } \\
\text { Stonemasons' phthisis }\end{array}$ & 010 & No discrete ICD-9 code & & $\begin{array}{l}\text { No discrete ICD-10 } \\
\text { code }\end{array}$ & \\
\hline Byssinosis & $\begin{array}{l}\text { No discrete ICD-8 } \\
\text { code }\end{array}$ & & \begin{tabular}{|l} 
Pneumonopathy due \\
to inhalation of other \\
dust \\
Byssinosis \\
Cannabinosis \\
Flax-dressers' disease
\end{tabular} & 504 & 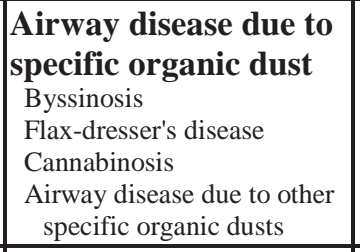 & J66 \\
\hline \multirow[t]{2}{*}{$\begin{array}{l}\text { Unspecified/Other } \\
\text { Pneumoconioses }\end{array}$} & $\begin{array}{l}\text { Pneumoconiosis due } \\
\text { to inhalation of other } \\
\text { inorganic dust } \\
\text { Aluminosis (of lung) } \\
\text { Bauxite fibrosis (of lung) } \\
\text { Berylliosis } \\
\text { Graphite fibrosis (of lung) }\end{array}$ & 516.0 & \begin{tabular}{|l|} 
Pneumoconiosis due to \\
other inorganic dust \\
Aluminosis (of lung) \\
Bauxite fibrosis (of lung) \\
Berylliosis \\
Graphite fibrosis (of lung) \\
Siderosis \\
Stannosis
\end{tabular} & 503 & \begin{tabular}{|l|} 
Pneumoconiosis due to \\
other inorganic dusts \\
Aluminosis (of lung) \\
Bauxite fibrosis (of lung) \\
Berylliosis \\
Graphite fibrosis (of lung) \\
Siderosis \\
Stannosis \\
Pneumoconiosis due to other \\
specified inorganic dusts
\end{tabular} & $\mathrm{J} 63^{*}$ \\
\hline & $\begin{array}{l}\text { Other } \\
\text { pneumoconiosis, } \\
\text { including } \\
\text { unspecified } \\
\text { Pneumoconiosis: n.o.s. } \\
\text { due to: } \\
\quad \text { silicates n.e.c. } \\
\text { talc }\end{array}$ & 515.9 & $\begin{array}{l}\text { Pneumoconiosis, } \\
\text { unspecified }\end{array}$ & 505 & $\begin{array}{l}\text { Unspecified } \\
\text { pneumoconiosis }\end{array}$ & J64* \\
\hline
\end{tabular}

See footnotes at end of table. 


\begin{tabular}{|c|c|c|c|c|}
\hline \multirow{2}{*}{$\begin{array}{l}\text { Condition } \\
\text { (as defined for this report) }\end{array}$} & \multicolumn{2}{|l|}{ ICD-9 (1979-1998) } & \multicolumn{2}{|l|}{ ICD-10 (1999) } \\
\hline & Rubrics & Codes & Rubrics & Codes \\
\hline $\begin{array}{l}\text { Malignant } \\
\text { Mesothelioma }\end{array}$ & No discrete ICD-9 code & & \begin{tabular}{|l|} 
Mesothelioma \\
Mesothelioma of pleura \\
Mesothelioma of peritoneum \\
Mesothelioma of pericardium $* *$ \\
Mesothelioma of other sites \\
Mesothelioma, unspecified \\
\end{tabular} & $\mathrm{C45}$ \\
\hline $\begin{array}{l}\text { Hypersensitivity } \\
\text { Pneumonitis }\end{array}$ & $\begin{array}{l}\text { Extrinsic allergic alveolitis } \\
\text { Farmers' lung } \\
\text { Bagassosis } \\
\text { Bird-Fanciers' lung } \\
\text { Suberosis } \\
\text { Malt workers' lung } \\
\text { Mushroom workers' lung } \\
\text { Maple bark-strippers' 'ung } \\
\text { Ventilation pneumonitis } \\
\text { Other specified allergic alveolitis and } \\
\text { pneumonitis } \\
\text { Unspecified allergic alveolitis and pneumonitis }\end{array}$ & 495 & $\begin{array}{l}\text { Hypersensitivity pneumonitis due to } \\
\text { organic dust } \\
\text { Farmer's lung } \\
\text { Bagassosis } \\
\text { Bird fancier's lung } \\
\text { Suberosis } \\
\text { Maltworker's lung } \\
\text { Mushroom-worker's lung } \\
\text { Maple-bark-stripper's lung } \\
\text { Air-conditioner and humidifier lung } \\
\text { Hypersensitivity pneumonitis due to other organic } \\
\text { dusts } \\
\text { Hypersensitivity pneumonitis due to unspecified } \\
\text { organic dust }\end{array}$ & J67 \\
\hline Asthma & \begin{tabular}{|l|} 
Asthma \\
Extrinsic asthma \\
Intrinsic asthma \\
Asthma, unspecified
\end{tabular} & 493 & $\begin{array}{l}\text { Asthma } \\
\text { Predominantly allergic asthma } \\
\text { Nonallergic asthma } \\
\text { Mixed asthma } \\
\text { Asthma, unspecified } \\
\text { Status asthmaticus } \\
\text { Acute severe asthma }\end{array}$ & J46 \\
\hline $\begin{array}{l}\text { Chronic Obstructive } \\
\text { Pulmonary Disease }\end{array}$ & No data included in this report & & $\begin{array}{l}\text { Bronchitis, not specified as acute or } \\
\text { chronic } \\
\text { Bronchitis: } \\
\text { n.o.s. } \\
\text { catarrhal } \\
\text { with tracheitis n.o.s. } \\
\text { Tracheobronchitis n.o.s. } \\
\text { Simple and mucopurulent chronic } \\
\text { bronchitis } \\
\text { Simple chronic bronchitis } \\
\text { Mucopurulent chronic bronchitis } \\
\text { Mixed simple and mucopurulent chronic bronchitis } \\
\text { Unspecified chronic bronchitis } \\
\text { Emphysema } \\
\text { MacLeod's syndrome } \\
\text { Panlobular emphysema } \\
\text { Centrilobular emphysema } \\
\text { Other emphysema } \\
\text { Emphysema, unspecified } \\
\text { Other chronic obstructive pulmonary } \\
\text { disease } \\
\text { Chronic obstructive pulmonary disease with acute } \\
\text { lower respiratory infection } \\
\text { Chronic obstructive pulmonary disease with acute } \\
\text { exacerbation, unspecified } \\
\text { Other specified chronic obstructive pulmonary } \\
\text { disease } \\
\text { Chronic obstructive pulmonary disease, unspecified } \\
\text { Bronchiectasis }\end{array}$ & $\begin{array}{l}\mathrm{J} 42 \\
\mathrm{~J} 43\end{array}$ \\
\hline
\end{tabular}

See footnotes at end of table.

$C-2$ 


\begin{tabular}{|c|c|c|c|c|}
\hline \multirow{2}{*}{$\begin{array}{l}\text { Condition } \\
\text { (as defined for this report) }\end{array}$} & \multicolumn{2}{|l|}{ ICD-9 (1979-1998) } & \multicolumn{2}{|l|}{ ICD-10 (1999) } \\
\hline & Rubrics & Codes & Rubrics & Codes \\
\hline $\begin{array}{l}\text { Respiratory } \\
\text { Tuberculosis }\end{array}$ & $\begin{array}{l}\text { Primary tuberculous infection } \\
\text { Primary tuberculous infection } \\
\text { Tuberculous pleurisy in primary progressive } \\
\text { Other primary progressive tuberculosis } \\
\text { Primary tuberculous infection, unspecified } \\
\text { Pulmonary tuberculosis } \\
\text { Tuberculosis of lung, infiltrative } \\
\text { Tuberculosis of lung, nodular } \\
\text { Tuberculosis of lung with cavitation } \\
\text { Tuberculosis of bronchus } \\
\text { Tuberculous fibrosis of lung } \\
\text { Tuberculous bronchiectasis } \\
\text { Tuberculous pneumonia [any form] } \\
\text { Tuberculous pneumothorax } \\
\text { Other specified pulmonary tuberculosis } \\
\text { Pulmonary tuberculosis, unspecified } \\
\text { Other respiratory tuberculosis } \\
\text { Tuberculous pleurisy } \\
\text { Tuberculosis of intrathoracic lymph nodes } \\
\text { Isolated tracheal or bronchial tuberculosis } \\
\text { Tuberculous laryngitis } \\
\text { Other specified respiratory tuberculosis } \\
\text { Miliary tuberculosis } \\
\text { Acute miliary tuberculosis } \\
\text { Other specified miliary tuberculosis } \\
\text { Miliary tuberculosis, unspecified } \\
\text { Late effects of tuberculosis } \\
\text { Late effects of respiratory or unspecified } \\
\text { tuberculosis }\end{array}$ & 018 & $\begin{array}{l}\text { Respiratory tuberculosis, } \\
\text { bacteriologically and histologically } \\
\text { confirmed } \\
\text { Tuberculosis of lung, confirmed by sputum } \\
\text { microscopy with or without culture } \\
\text { Tuberculosis of lung, confirmed by culture only } \\
\text { Tuberculosis of lung, confirmed histologically } \\
\text { Tuberculosis of lung, confirmed by unspecified } \\
\text { means } \\
\text { Tuberculosis of intrathoracic lymph nodes, confirmed } \\
\text { bacteriologically and histologically } \\
\text { Tuberculosis of larynx, traches and bronchus, } \\
\text { confirmed bacteriologically and histologically } \\
\text { Tuberculous pleurisy, confirmed bacteriologically } \\
\text { and histologically } \\
\text { Primary respiratory tuberculosis, confirmed } \\
\text { bacteriologically and histologically } \\
\text { Other respiratory tuberculosis, confirmed } \\
\text { bacteriologically and histologically } \\
\text { Respiratory tuberculosis unspecified, confirmed } \\
\text { bacteriologically and histologically } \\
\text { Sequelae of respiratory and unspecified tuberculosis } \\
\text { Respiratory tuberculosis, not confirmed } \\
\text { bacteriologically or histologically } \\
\text { Tuberculosis of lung, bacteriologically and } \\
\text { histologically negative } \\
\text { Tuberculosis of lung, bacteriological and histological } \\
\text { examination not done } \\
\text { Tuberculosis of lung, without mention of } \\
\text { bacteriological or histological confirmation } \\
\text { Acute miliary tuberculosis of multiple sites } \\
\text { Acute miliary tuberculosis, unspecified } \\
\text { Tuberculosis of intrathoracic lymph nodes, without } \\
\text { mention of bacteriological or histological } \\
\text { confirmation } \\
\text { Tuberculosis of larynx, trachea, and bronchus, } \\
\text { without mention of bacteriological or } \\
\text { histological confirmation } \\
\text { Tuberculous pleurisy, without mention of } \\
\text { bacteriological or histological confirmation } \\
\text { Primary respiratory tuberculosis without mention of } \\
\text { bacteriological or histological confirmation } \\
\text { Other respiratory tuberculosis, without mention of } \\
\text { bacteriological or histological confirmation } \\
\text { Respiratory tuberculosis unspecified, without } \\
\text { mention of bacteriological or histological } \\
\text { confirmation }\end{array}$ & B90.9 \\
\hline
\end{tabular}

See footnotes at end of table. 


\begin{tabular}{|c|c|c|c|c|}
\hline \multirow{2}{*}{$\begin{array}{l}\text { Condition } \\
\text { (as defined for this report) }\end{array}$} & \multicolumn{2}{|l|}{ ICD-9 (1979-1998) } & \multicolumn{2}{|l|}{ ICD-10 (1999) } \\
\hline & Rubrics & Codes & Rubrics & Codes \\
\hline $\begin{array}{l}\text { Respiratory } \\
\text { Conditions due to } \\
\text { Chemical Fumes and } \\
\text { Vapors }\end{array}$ & $\begin{array}{l}\text { Respiratory conditions due to } \\
\text { chemical fumes and vapors } \\
\text { Bronchitis and pneumonitis due to fumes and } \\
\text { vapors } \\
\text { Acute pulmonary edema due to fumes and } \\
\text { vapors } \\
\text { Upper respiratory inflammation due to fumes } \\
\text { and vapors } \\
\text { Other acute and subacute respiratory } \\
\text { conditions due to fumes and vapors } \\
\text { Chronic respiratory conditions due to fumes } \\
\text { and vapors } \\
\text { Unspecified respiratory conditions due to } \\
\text { fumes and vapors }\end{array}$ & 506 & $\begin{array}{l}\text { Respiratory conditions due to inhalation } \\
\text { of chemicals, gases, fumes, and vapors } \\
\text { Bronchitis and pneumonitis due to chemicals, gases, } \\
\text { fumes, and vapors } \\
\text { Acute pulmonary edema due to chemicals, gases, } \\
\text { fumes, and vapors } \\
\text { Upper respiratory inflammation due to chemicals, } \\
\text { gases, fumes, and vapors, n.e.c. } \\
\text { Other acute and subacute respiratory conditions due } \\
\text { to chemicals, gases, fumes, and vapors } \\
\text { Chronic respiratory conditions due to chemicals, } \\
\text { gases, fumes, and vapors } \\
\text { Other respiratory conditions due to chemicals, gases, } \\
\text { fumes, and vapors } \\
\text { Unspecified respiratory condition due to chemicals, } \\
\text { gases, fumes, and vapors }\end{array}$ & J68 \\
\hline Lung Cancer & No data included in this report & & $\begin{array}{l}\text { Malignant neoplasm of trachea } \\
\text { Malignant neoplasm of bronchus and } \\
\text { lung } \\
\text { Main bronchus } \\
\text { Upper lobe, bronchus or lung } \\
\text { Middle lobe, bronchus or lung } \\
\text { Lower lobe, bronchus or lung } \\
\text { Overlapping lesion of bronchus and lung } \\
\text { Bronchus or lung, unspecified }\end{array}$ & $\begin{array}{l}\text { C33 } \\
\text { C34 }\end{array}$ \\
\hline $\begin{array}{l}\text { Other Interstitial } \\
\text { Pulmonary Diseases }\end{array}$ & No data included in this report & & $\begin{array}{l}\text { Other Interstitial Pulmonary Diseases } \\
\text { Alveolar and parietoalveolar conditions } \\
\text { Other interstitial pulmonary diseases with fibrosis } \\
\text { Other specified interstitial pulmonary diseases } \\
\text { Interstitial pulmonary diseases, unspecified }\end{array}$ & J84 \\
\hline
\end{tabular}

n.o.s. - not otherwise specified

n.e.c. - not elsewhere classified

* A small number of deaths with underlying cause equal to ICD-10 code J65 or J92.0 are included in underlying cause of death tabulations. See methods for more detailed explanation.

** In this report, mesothelioma of the pericardium is grouped into mesothelioma of other sites.

SOURCES: U.S. Department of Health, Education, and Welfare: Eighth Revision International Classification of Diseases, Volume 1.

U.S. Department of Health and Human Services: International Classification of Diseases 9th Revision, Volume 1.

World Health Organization: International Statistical Classification of Diseases and Related Health Problems 10th Revision, Volume 1. 


\section{Appendix D}

\section{Changes in Bureau of Census Industry and Occupation Codes and Titles}

\begin{tabular}{|c|c|c|c|}
\hline \multicolumn{2}{|r|}{1980} & \multicolumn{2}{|r|}{1990} \\
\hline Code & Title & Code & Title \\
\hline \multicolumn{4}{|c|}{ Industry: 1980 codes merged into 1990 codes } \\
\hline 382 & Not specified professional equipment (manufacturing) & \multirow{2}{*}{392} & \multirow{2}{*}{ Not specified manufacturing industries } \\
\hline 392 & Not specified manufacturing industries & & \\
\hline 510 & Sporting goods, toys and hobby goods & \multirow{3}{*}{532} & \multirow{3}{*}{ Miscellaneous wholesale, durable goods } \\
\hline 522 & Not specified electrical and hardware (wholesale trade) & & \\
\hline 532 & Miscellaneous wholesale, durable goods & & \\
\hline 730 & Commercial research, development, and testing labs & \multirow{2}{*}{891} & \multirow{2}{*}{ Research, development and testing services } \\
\hline 891 & Noncommercial educational and scientific research & & \\
\hline \multicolumn{4}{|c|}{ Industry: 1980 title changed to 1990 title } \\
\hline 042 & Crude petroleum and natural gas extraction & 042 & Oil and gas extraction \\
\hline 102 & Canned and preserved fruits and vegetables & 102 & Canned, frozen and preserved fruits and vegetables \\
\hline 141 & Floor coverings, except hard surface & 141 & Carpets and rugs \\
\hline 281 & Cutlery, handtools, and other hardware & 281 & Cutlery, handtools, and general hardware \\
\hline 322 & Electronic computing equipment & 322 & Computers and related equipment \\
\hline 372 & Optical and health services supplies & 372 & Medical, dental, optical instruments and supplies \\
\hline 440 & Radio and television broadcasting & 440 & Radio and television broadcasting and cable \\
\hline 441 & Telephone (wire and radio) & 441 & Telephone communications \\
\hline 672 & Fuel and ice dealers & 672 & Fuel dealers \\
\hline 701 & Savings and loan associations & 701 & Savings institutions, including credit unions \\
\hline 712 & Real estate, including real estate-insurance-law offices & 712 & Real estate, including real estate-insurance offices \\
\hline 751 & Automotive repair shops & 751 & Automotive repair and related services \\
\hline 812 & Offices of physicians & 812 & Offices and clinics of physicians \\
\hline 820 & Offices of dentists & 820 & Offices and clinics of dentists \\
\hline 821 & Offices of chiropractors & 821 & Offices and clinics of chiropractors \\
\hline 822 & Offices of optometrists & 822 & Offices and clinics of optometrists \\
\hline 830 & Offices of health practitioners, n.e.c. & 830 & Offices and clinics of health practitioners, n.e.c. \\
\hline 851 & Business, trade, and vocational schools & 851 & Vocational schools \\
\hline 961 & Homemaker, student, unemployed, volunteer & 961 & Non-paid worker or non-worker or own home/at home \\
\hline \multicolumn{4}{|c|}{ Occupation: 1980 codes merged into 1990 codes } \\
\hline 349 & Telegraphers & \multirow{2}{*}{353} & \multirow{2}{*}{ Communications equipment operators, n.e.c. } \\
\hline 353 & Communications equipment operators, n.e.c. & & \\
\hline 368 & Weighers, measurers, and checkers & \multirow{2}{*}{368} & \multirow{2}{*}{ Weighers, measurers, checkers, and samplers } \\
\hline 369 & Samplers & & \\
\hline 436 & Cooks, except short-order & \multirow{2}{*}{436} & \multirow{2}{*}{ Cooks } \\
\hline 437 & Short-order cooks & & \\
\hline 673 & Apparel and fabric patternmakers & \multirow{2}{*}{674} & \multirow{2}{*}{ Miscellaneous precision apparel and fabric workers } \\
\hline 674 & Miscellaneous precision apparel fabric workers & & \\
\hline 794 & Hand grinding and polishing occupations & \multirow{2}{*}{795} & \multirow{2}{*}{ Miscellaneous hand working occupations } \\
\hline 795 & Miscellaneous hand working occupations & & \\
\hline 804 & Truck drivers, heavy & \multirow{2}{*}{804} & \multirow{2}{*}{ Truck drivers } \\
\hline 805 & Truck drivers, light & & \\
\hline \multicolumn{4}{|c|}{ Occupation: 1980 title changed to 1990 title } \\
\hline 098 & Inhalation therapists & 098 & Respiratory therapists \\
\hline 558 & Supervisors, n.e.c. & 558 & Supervisors, construction, n.e.c. \\
\hline 734 & Printing machine operators & 734 & Printing press operators \\
\hline
\end{tabular}

n.e.c. - not elsewhere classified 



\section{Appendix E}

States (and Years) for which Industry and Occupation Codes from Death Certificates Met NCHS Quality Criteria, 1985-1999

\begin{tabular}{|c|c|c|c|c|c|c|c|c|c|c|c|c|c|c|c|}
\hline State & 1985 & 1986 & 1987 & 1988 & 1989 & 1990 & 1991 & 1992 & 1993 & 1994 & 1995 & 1996 & 1997 & 1998 & 1999 \\
\hline Alaska & & & $X$ & $X$ & & & & & & & & & & & \\
\hline Colorado & $X$ & $X$ & $X$ & $X$ & $X$ & $X$ & $X$ & $X$ & $X$ & $X$ & $X$ & $X$ & $X$ & $X$ & $X$ \\
\hline Georgia & $X$ & $X$ & $X$ & $X$ & $X$ & $X$ & $X$ & $X$ & $X$ & $X$ & $X$ & $X$ & $X$ & $X$ & $X$ \\
\hline Hawaii & & & & & & & & & $X$ & $X$ & & $X$ & & $X$ & $X$ \\
\hline Idaho & & & & $X$ & $\mathrm{X}$ & $X$ & $X$ & $\mathrm{X}$ & $\mathrm{X}$ & $\mathrm{X}$ & $X$ & $X$ & $X$ & $X$ & $X$ \\
\hline Indiana & & $X$ & $X$ & $X$ & $X$ & $X$ & $X$ & $X$ & $X$ & & $X$ & & & $X$ & $X$ \\
\hline Kansas & $X$ & $X$ & $X$ & $X$ & $X$ & X & $X$ & $X$ & X & $X$ & $X$ & $X$ & X & $X$ & $X$ \\
\hline Kentucky & $X$ & $\mathrm{X}$ & $X$ & $X$ & $X$ & $X$ & $X$ & $\mathrm{X}$ & $X$ & $X$ & $X$ & $X$ & $X$ & $X$ & $X$ \\
\hline Maine & $X$ & $X$ & $X$ & $\mathrm{X}$ & $\mathrm{X}$ & $X$ & $X$ & $X$ & $X$ & $\mathrm{X}$ & $X$ & $X$ & & $X$ & \\
\hline Missouri & $X$ & $X$ & & & & & & & & & & & & & \\
\hline Nebraska & $X$ & & & & & & & & & & & & & & $\mathrm{X}$ \\
\hline Nevada & $X$ & $X$ & $X$ & $X$ & $X$ & $X$ & $X$ & $X$ & $X$ & $\mathrm{X}$ & $X$ & $X$ & $X$ & $X$ & $X$ \\
\hline New Hampshire & $\mathrm{X}$ & $\mathrm{X}$ & $\mathrm{X}$ & $\mathrm{X}$ & $X$ & $\mathrm{X}$ & $X$ & $\mathrm{X}$ & $X$ & $\mathrm{X}$ & $\mathrm{X}$ & $X$ & & $X$ & $X$ \\
\hline New Jersey & & & & $\mathrm{X}$ & $\mathrm{X}$ & $\mathrm{X}$ & $X$ & $\mathrm{X}$ & $\mathrm{X}$ & $\mathrm{X}$ & $X$ & $\mathrm{X}$ & $\mathrm{X}$ & $X$ & $X$ \\
\hline New Mexico & & $X$ & $X$ & $\mathrm{X}$ & $X$ & $X$ & $X$ & $X$ & $X$ & $X$ & $X$ & $X$ & $X$ & $X$ & $X$ \\
\hline North Carolina & & & $\mathrm{X}$ & $\mathrm{X}$ & $\mathrm{X}$ & $\mathrm{X}$ & $X$ & $X$ & $X$ & $\mathrm{X}$ & $X$ & $\mathrm{X}$ & $\mathrm{X}$ & $X$ & $\mathrm{X}$ \\
\hline Ohio & $X$ & $\mathrm{X}$ & $\mathrm{X}$ & $X$ & $\mathrm{X}$ & $\mathrm{X}$ & $X$ & $\mathrm{X}$ & $X$ & & $\mathrm{X}$ & $\mathrm{X}$ & $\mathrm{X}$ & $X$ & \\
\hline Oklahoma & $X$ & $X$ & $X$ & $X$ & $X$ & $\mathrm{X}$ & $X$ & $X$ & $X$ & & & & & & \\
\hline Rhode Island & $X$ & $X$ & $X$ & $X$ & $\mathrm{X}$ & $X$ & $X$ & $X$ & $X$ & $X$ & $X$ & $\mathrm{X}$ & $\mathrm{X}$ & $X$ & $X$ \\
\hline South Carolina & $X$ & $\mathrm{X}$ & $X$ & $\mathrm{X}$ & $X$ & $\mathrm{X}$ & $\mathrm{X}$ & $X$ & $X$ & $\mathrm{X}$ & $X$ & $\mathrm{X}$ & $\mathrm{X}$ & $X$ & $X$ \\
\hline Tennessee & $\mathrm{X}$ & $\mathrm{X}$ & $\mathrm{X}$ & $\mathrm{X}$ & & & & & & & & & & & \\
\hline Utah & $\mathrm{X}$ & $\mathrm{X}$ & $\mathrm{X}$ & $\mathrm{X}$ & $\mathrm{X}$ & $X$ & $X$ & $\mathrm{X}$ & $X$ & $\mathrm{X}$ & $X$ & $X$ & $X$ & $X$ & $X$ \\
\hline Vermont & & $\mathrm{X}$ & $\mathrm{X}$ & $X$ & $\mathrm{X}$ & $\mathrm{X}$ & $X$ & $\mathrm{X}$ & $\mathrm{X}$ & $X$ & $X$ & $X$ & $\mathrm{X}$ & $X$ & $X$ \\
\hline Washington & & & & & $\mathrm{X}$ & $\mathrm{X}$ & $X$ & $\mathrm{X}$ & & & & & & & \\
\hline West Virginia & & & & $\mathrm{X}$ & $\mathrm{X}$ & $X$ & $\mathrm{X}$ & $\mathrm{X}$ & $X$ & $X$ & $X$ & $\mathrm{X}$ & $\mathrm{X}$ & $\mathrm{X}$ & $\mathrm{X}$ \\
\hline Wisconsin & $X$ & $X$ & $X$ & $X$ & $X$ & $X$ & $X$ & $X$ & $X$ & $\mathrm{X}$ & $X$ & $X$ & $X$ & X & $X$ \\
\hline
\end{tabular}





\section{Appendix F \\ Exposure Categories}

\section{Table F-1. Pneumoconiotic agent categories for MSHA and OSHA data}

\begin{tabular}{|c|c|c|}
\hline $\begin{array}{l}\text { Pneumoconiotic } \\
\text { Agent Category } \\
\text { (as defined for } \\
\text { this report) }\end{array}$ & MSHA Agents in Category & OSHA Agents in Category \\
\hline Cotton Dust & & $\begin{array}{l}\text { Cotton dust (raw) } \\
\text { Flax dust* }\end{array}$ \\
\hline Quartz & $\begin{array}{l}\text { Respirable coal mine dust, > 5\% quartz } \\
\text { Respirable dust, }>1 \% \text { quartz } \\
\text { Nuisance dust (respirable fraction), }<1 \% \text { quartz** } \\
\text { Unlisted particulate (respirable fraction), }<1 \% \text { quartz } * * \\
\text { Respirable dust (not analyzed or below detection } \\
\text { limit)** }\end{array}$ & $\begin{array}{l}\text { Respirable crystalline silica (as quartz) } \\
\text { Respirable crystalline silica/tripoli (as quartz) } \\
\text { Respirable coal dust, }>5 \% \text { quartz }\end{array}$ \\
\hline Other & 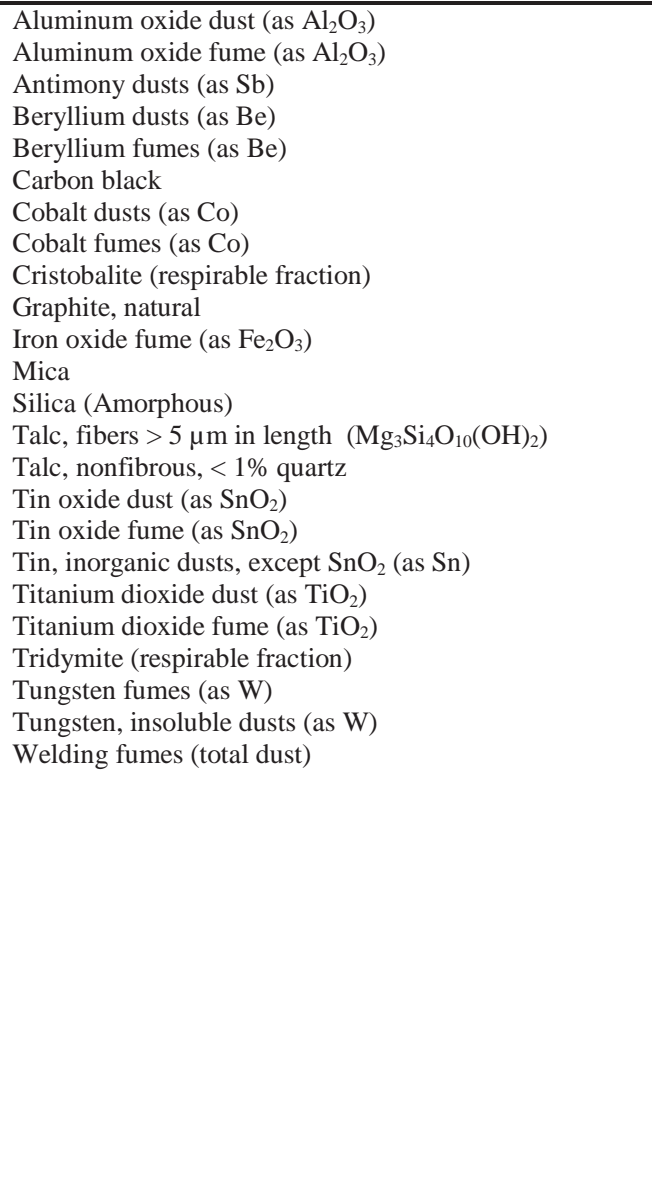 & $\begin{array}{l}\text { Alpha-alumina (total dust) } \\
\text { Alpha-alumina (respirable fraction) } \\
\text { Aluminum oxide } \\
\text { Aluminum (as Al), metal (total dust) } \\
\text { Aluminum (as Al), metal (respirable fraction) } \\
\text { Aluminum (as Al), welding fumes } \\
\text { Antimony and compounds (as Sb) } \\
\text { Barium (insoluble compounds) } \\
\text { Barium sulfate (total dust) } \\
\text { Barium sulfate (respirable fraction) } \\
\text { Beryllium and compounds (as Be) } \\
\text { Carbon black } \\
\text { Cobalt, metal, fume and dust (as Co) } \\
\text { Emery (total dust) } \\
\text { Emery (respirable fraction) } \\
\text { Graphite, natural (respirable fraction) } \\
\text { Graphite, synthetic (total dust) } \\
\text { Graphite, synthetic (respirable fraction) } \\
\text { Iron oxide fume (as Fe } \mathrm{O}_{3} \text { ) } \\
\text { Kaolin (total dust) } \\
\text { Kaolin (respirable fraction) } \\
\text { Magnesite (total dust) } \\
\text { Magnesite (respirable fraction)* } \\
\text { Mica (< } 1 \% \text { crystalline silica) } \\
\text { Rouge (total dust) } \\
\text { Rouge (respirable fraction) } \\
\text { Silica, amorphous, diatomaceous earth ( }<1 \% \text { crystalline silica) } \\
\text { Silica, respirable cristobalite } \\
\text { Silica, respirable tridymite } \\
\text { Silica, fused (respirable fraction) } \\
\text { Talc (containing no asbestos) } \\
\text { Talc, fibrous non-tremolite } \\
\text { Tin, inorganic compounds, except oxide (as Sn) } \\
\text { Tin oxide (as Sn) } \\
\text { Titanium dioxide (total dust) } \\
\text { Tungsten and compounds (insoluble asW) } \\
\text { Welding fumes (total dust) }\end{array}$ \\
\hline
\end{tabular}

* No data reported for these agents in most recent provisional data.

** See Selected Limitations section.

The following documents were reviewed to identify pneumoconiotic agents: ACGIH ${ }^{\circledR}$ Documentation of TLV®s, $6^{\text {th }}$ edition; Occupational Respiratory Diseases Report, NIOSH Pub. No. 86-102; The NIOSH Pocket Guide to Chemical Hazards, NIOSH Pub No. 97-140; and NIOSH Criteria Documents. 


\section{Table F-2. MSHA analytical methods for selected pneumoconiotic agents}

\begin{tabular}{|c|c|c|}
\hline $\begin{array}{l}\text { Pneumoconiotic } \\
\text { Agent Category } \\
\text { (as defined for } \\
\text { this report) }\end{array}$ & MSHA Agents in Category & MSHA Analytical Method \\
\hline Asbestos & Asbestos, fibers $>5 \mu \mathrm{m}$ in length $\left(3 \mathrm{MgO} 2 \mathrm{SiO}_{2} \cdot 2 \mathrm{H}_{2} \mathrm{O}\right)$ & NIOSH 7400 \\
\hline Coal Mine Dust & Respirable coal mine dust, $<=5 \%$ quartz & NIOSH 7603/MSHA P7 \\
\hline Quartz & $\begin{array}{l}\text { Respirable coal mine dust, }>5 \% \text { quartz } \\
\text { Respirable dust, }>1 \% \text { quartz } \\
\text { Nuisance dust (respirable fraction), }<1 \% \text { quartz } \\
\text { Unlisted particulate (respirable fraction), <1\% quartz } \\
\text { Respirable dust (not analyzed or below detection limit) }\end{array}$ & $\begin{array}{l}\text { MSHA coal: MSHA P7/NIOSH 7300 } \\
\text { MSHA metal/nonmetal: MSHA P2/NIOSH } 7500\end{array}$ \\
\hline \multirow[t]{4}{*}{$\begin{array}{c}\text { Selected } \\
\text { Pneumoconiotic } \\
\text { Agents }\end{array}$} & $\begin{array}{l}\text { Aluminum oxide dust }\left(\text { as } \mathrm{Al}_{2} \mathrm{O}_{3}\right) \\
\text { Aluminum oxide fume }\left(\text { as } \mathrm{Al}_{2} \mathrm{O}_{3}\right) \\
\text { Beryllium dusts (as } \mathrm{Be}) \\
\text { Beryllium fumes (as Be) } \\
\text { Cobalt dusts (as Co) } \\
\text { Cobalt fumes (as Co) } \\
\left.\text { Iron oxide fume (as } \mathrm{Fe}_{2} \mathrm{O}_{3}\right) \\
\text { Titanium dioxide dust }\left(\text { as } \mathrm{TiO}_{2}\right) \\
\text { Titanium dioxide fume }\left(\text { as } \mathrm{TiO}_{2}\right)\end{array}$ & OSHA $121 / 125$ \\
\hline & Cristobalite (respirable fraction) & MSHA P2/NIOSH 7500 \\
\hline & Welding fumes (total dust) & OSHA $121 / 125$ \\
\hline & Talc, nonfibrous, $<1 \%$ quartz & NIOSH 0600 (gravimetric)/MSHA P8 (impinger) \\
\hline
\end{tabular}




\section{Table F-3. Most commonly used OSHA analytical methods for selected pneumoconiotic agents}

\begin{tabular}{|c|c|c|c|}
\hline $\begin{array}{l}\text { Pneumoconiotic } \\
\text { Agent Category } \\
\text { (as defined for } \\
\text { this report) }\end{array}$ & OSHA Agents in Category & $\begin{array}{l}\text { OSHA Compliance } \\
\text { Analytical Method } \\
\text { (SLCTC) }\end{array}$ & $\begin{array}{l}\text { OSHA Consultation } \\
\text { Analytical Method } \\
\text { (WOHL) }\end{array}$ \\
\hline Asbestos & $\begin{array}{l}\text { Asbestos [actinolite, anthophyllite, chrysotile, } \\
\text { crocidolite, tremolite] } \\
\text { Talc containing fibrous tremolite }\end{array}$ & ID-160 & $\begin{array}{l}\text { WOHL method (based on NIOSH } 7400 \text { and } \\
\text { OSHA ID-160) }\end{array}$ \\
\hline Cotton Dust & Cotton dust (raw) & $\begin{array}{l}\text { 1910.1043 - Appendix A; } \\
\text { SOP for nuisance dust }\end{array}$ & WW001.6.0 (5um PVC filter) \\
\hline Quartz & $\begin{array}{l}\text { Respirable crystalline silica (as quartz) } \\
\text { Respirable crystalline silica/tripoli (as quartz) } \\
\text { Respirable coal dust, > 5\% quartz }\end{array}$ & ID-142 & $\begin{array}{l}\text { WOHL method (based on NIOSH } 7500 \\
\text { and OSHA ID-142) }\end{array}$ \\
\hline \multirow[t]{4}{*}{$\begin{array}{c}\text { Selected } \\
\text { Pneumoconiotic } \\
\text { Agents }\end{array}$} & $\begin{array}{l}\text { Alpha-alumina (total dust) } \\
\text { Aluminum oxide } \\
\text { Antimony and compounds (as } \mathrm{Sb} \text { ) } \\
\text { Beryllium and compounds (as Be) } \\
\text { Cobalt, metal, fume and dust (as Co) } \\
\text { Iron oxide fume (as Fe) } \\
\text { Tin (Inorganic Compounds, Except oxide as } \\
\quad \text { Sn) }\end{array}$ & ID-125G & WW001.3.1 \\
\hline & Silica, respirable crystalline cristobalite & ID-142 & $\begin{array}{l}\text { WOHL method (based on NIOSH } 7500 \text { and } \\
\text { OSHA ID-142) }\end{array}$ \\
\hline & Carbon black & ID-196 & WC019cb.4.0 \\
\hline & Welding fumes (total particulate) & SOP for nuisance dust & WW001.6.0 (5um PVC filter) \\
\hline
\end{tabular}





\section{Appendix G \\ Surveillance Guidelines for State Health Departments}

\section{Silicosis}

\section{Reporting Guidelines}

State health departments should encourage physicians, including radiologists and pathologists, as well as other health-care professionals, to report all diagnosed or suspected cases of silicosis. These reports should include persons with:

A. A physician's provisional or working diagnosis of silicosis.

\section{OR}

B. A chest radiograph interpreted as consistent with silicosis. OR

C. Pathologic findings consistent with silicosis.

State health departments should collect appropriate clinical, epidemiologic, and workplace information on reported persons with silicosis as needed to set priorities for workplace investigations.

\section{Surveillance Case Definition}

A. History of occupational exposure to airborne silica dust.*

\section{AND EITHER OR BOTH OF THE FOLLOWING:}

B1. Chest radiograph or other imaging technique interpreted as consistent with silicosis. ${ }^{+}$ B2. Pathologic findings characteristic of silicosis. ${ }^{\S}$

* Exposure settings associated with silicosis are well characterized and have been summarized in several reviews. The induction period between initial silica exposure and development of radiographically detectable nodular silicosis is usually $>10$ years. Shorter induction periods are associated with heavy exposures, and acute silicosis may develop within months following massive silica exposure.

+ Cases can be classified as nodular or acute. Common radiographic findings of nodular silicosis include multiple, bilateral, and rounded opacities in the upper lung zones; other patterns have been described. Since patients may have mixed dust exposure, irregular opacities may be present or even predominant. To be considered consistent with silicosis, radiographs of nodular silicosis classified by NIOSH-certified "B" readers should have small opacity profusion categories of $1 / 0$ or greater by the International Labour Organization classification system. If the largest opacity is $>1 \mathrm{~cm}$ in diameter, progressive massive fibrosis [PMF] (also known as 'complicated' silicosis) is present. A bilateral alveolar filling pattern is characteristic of acute silicosis and may be followed by rapid development of bilateral small or large opacities.

$\S \quad$ Characteristic lung tissue pathology in nodular silicosis consists of fibrotic nodules with concentric "onion-skinned" arrangement of collagen fibers, central hyalinization, and a cellular peripheral zone, with lightly birefringent particles seen under polarized light. In acute silicosis, microscopic pathology shows a periodic acid-Schiff positive alveolar exudate (alveolar lipoproteinosis) and a cellular infiltrate in the alveolar walls. 


\section{Work-Related Asthma}

\section{Reporting Guidelines}

State health departments should encourage health-care professionals to report all diagnosed or suspected cases of asthma that are caused by or exacerbated by workplace exposures or conditions. Reported cases should include asthma caused by sensitizers or irritants and should include cases of reactive airways dysfunction syndrome (RADS).

\section{Surveillance Case Definition}

A. Healthcare professional's diagnosis consistent with asthma.* AND

B. An association between symptoms of asthma and work. ${ }^{+}$

\section{Surveillance Case Classification Criteria (see next page)}

* Asthma is a chronic condition characterized by inflammation of the tracheobronchial tree associated with increased airways responsiveness to a variety of stimuli. Symptoms of asthma include episodic wheezing, chest tightness, cough, and dyspnea, or recurrent attacks of bronchitis with cough and sputum production. The primary physiologic manifestation of airways hyperresponsiveness is variable or reversible airflow obstruction. It is commonly demonstrated by significant changes in the forced expiratory volume in 1 second ( $\mathrm{FEV}_{1}$ ) or peak expiratory flow rate (PEFR). Airflow changes can occur spontaneously, with treatment, with a precipitating exposure, or with diagnostic maneuvers such as nonspecific inhalation challenge.

+ Patterns of association can vary and include: (1) symptoms of asthma that develop or worsen after a worker starts a new job or after new materials are introduced on a job (a substantial period can elapse between initial exposure and development of symptoms); (2) symptoms that develop within minutes of specific activities or exposures at work; (3) delayed symptoms that occur several hours after exposure (e.g., during the evenings of workdays); (4) symptoms that occur less frequently or not at all on days away from work and on vacations; (5) symptoms that occur more frequently when the affected worker returns to work; and (6) symptoms that are temporally associated with workplace exposure to an agent with irritant properties. Work-related changes in medication requirements can accompany these symptom patterns. 


\section{Work-Related Asthma (continued)}

\section{Surveillance Case Classification Criteria (see decision logic on next page)}

C1) Increased asthma symptoms or increased use of asthma medication (upon entering an occupational exposure setting) experienced by a person with preexisting asthma who was symptomatic or treated with asthma medication within the two years prior to entering that occupational setting.

C2) New asthma symptoms that develop within 24 hours after a one-time high-level inhalation exposure (at work) to an irritant gas, fume, smoke, or vapor and that persist for at least three months.

C3) Workplace exposure to an agent previously associated with occupational asthma.*

$\mathrm{C4})$ Work-related changes in serially measured forced expiratory volume in one second $\left(\mathrm{FEV}_{1}\right)$ or peak expiratory flow rate (PEFR). ${ }^{+}$

C5) Work-related changes in bronchial responsiveness as measured by serial nonspecific inhalation challenge testing. ${ }^{\S}$

C6) Positive response to specific inhalation challenge testing with an agent to which the patient has been exposed at work.

* Many agents can induce occupational asthma via a specific hypersensitivity mechanism. A comprehensive list of these asthma inducers is used for this criterion. Known asthma inducers have been designated with the letter "A" in the Association of Occupational and Environmental Clinics (AOEC) coding scheme (www.aoec.org/aoeccode.htm).

+ Spirometric measurements (e.g., $\mathrm{FEV}_{1}$ ) can be obtained before and after a person's work shift (i.e., cross-shift spirometry). However, many cases of occupational asthma can fail to demonstrate a significant cross-shift reduction in $\mathrm{FEV}_{1}$, either because of a delayed bronchoconstrictor response or because of intermittent exposure patterns. Cross-shift spirometry testing on multiple days might help confirm the association with work.

Alternatively, PEFR can be measured serially throughout the day on multiple days at and away from work using a portable peak flow meter.

$\S$ Changes in bronchial responsiveness can be measured by serial inhalation challenge testing with nonspecific agents (e.g., using methacholine or histamine). Evidence of work-relatedness is manifested by increased bronchial responsiveness (i.e., bronchoconstriction at lower inhaled doses of methacholine or histamine) following work exposures and decreased or normal bronchial responsiveness after a period away from work.

I Specific inhalation challenge testing has distinct objectives, including the following: (1) identifying previously unrecognized causes of occupational asthma; (2) confirming a diagnosis of occupational asthma; and (3) identifying the causative agent when more than one allergen is present in the occupational environment and identification of the causative agent is essential for management. Specific inhalation challenge testing is potentially dangerous and should be performed by experienced personnel in a hospital setting where resuscitation facilities are available and frequent observations can be made over sufficient time to monitor for delayed reactions. Specific inhalation challenge testing is usually not necessary for clinical diagnosis of occupational asthma. 


\section{Decision Logic for Work-Related Asthma}

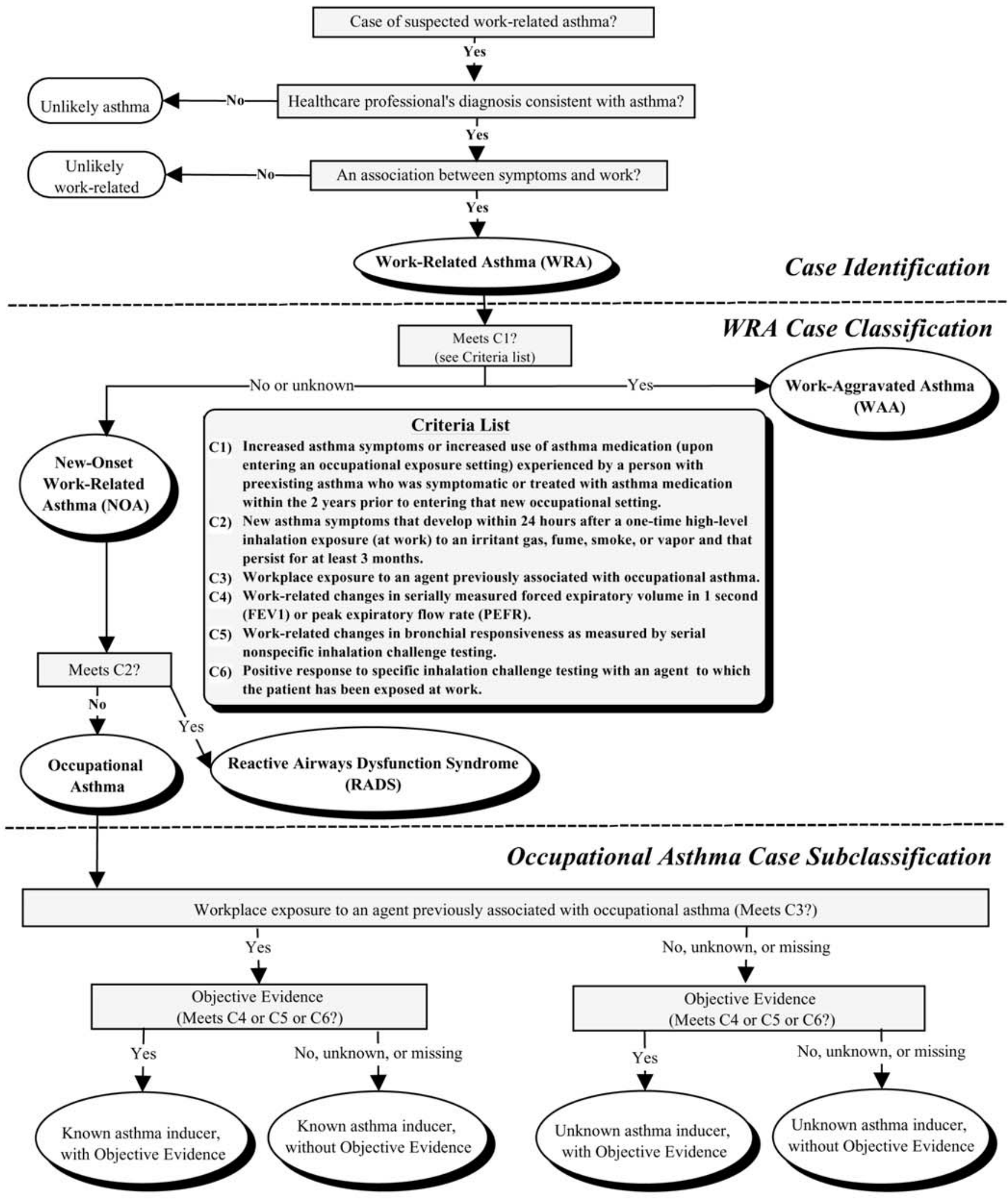




\section{Appendix $\mathbf{H}$ \\ Split, Merged, or Renamed Counties and County Equivalents}

\begin{tabular}{|c|c|c|c|c|}
\hline \multirow[b]{2}{*}{ State } & \multirow[b]{2}{*}{ Subdivision } & \multicolumn{3}{|c|}{ County Representation in this Report } \\
\hline & & Same* & Other & \\
\hline \multirow[t]{22}{*}{ Alaska } & Aleutian Islands & - & Aleutians East Borough & $1968-1993$ \\
\hline & Aleutians West Census Area & 1994-1999 & *** & $* *$ \\
\hline & Anchorage District & - & Anchorage Borough & $1968-1981$ \\
\hline & $\begin{array}{l}\text { Bethel District } \\
\text { Kuskokwim District }\end{array}$ & $\begin{array}{ll}- \\
-\end{array}$ & Bethel Census Area & $\begin{array}{l}1968-1981 \\
1968-1981\end{array}$ \\
\hline & $\begin{array}{l}\text { Bristol Bay Division } \\
\text { Dillingham Census Area }\end{array}$ & $\begin{array}{c}- \\
1982-1999\end{array}$ & Bristol Bay Borough & $\begin{array}{l}1968-1981 \\
1968-1981\end{array}$ \\
\hline & Lake and Peninsula Borough & 1994-1999 & Dillingham Census Area & $1982-1993$ \\
\hline & $\begin{array}{l}\text { Southeast Fairbanks Census Area } \\
\text { Fairbanks District }\end{array}$ & $\begin{array}{c}1982-1999 \\
-\end{array}$ & Fairbanks North Star Borough & $\begin{array}{l}1968-1981 \\
1968-1981\end{array}$ \\
\hline & Juneau District & - & Juneau Borough & 1968-1981 \\
\hline & $\begin{array}{l}\text { Kenai-Cook Inlet District } \\
\text { Seward District }\end{array}$ & $\begin{array}{ll}- \\
-\end{array}$ & Kenai Peninsula Borough & $\begin{array}{l}1968-1981 \\
1968-1981 \\
\end{array}$ \\
\hline & Ketchikan District & - & Ketchikan Gateway Borough & $1968-1981$ \\
\hline & Kodiak District & - & Kodiak Island Borough & 1968-1981 \\
\hline & Palmer-Wasilla District & - & Matanuska-Susitna Borough & 1968-1981 \\
\hline & Nome District & - & Nome Census Area & $1968-1981$ \\
\hline & Barrow District & - & North Slope Borough & $1968-1981$ \\
\hline & $\begin{array}{l}\text { Kobuk District } \\
\text { Kobuk Borough }\end{array}$ & $\begin{array}{l}- \\
-\end{array}$ & Northwest Arctic Borough & $\begin{array}{l}1968-1981 \\
1982-1993\end{array}$ \\
\hline & $\begin{array}{l}\text { Outer Ketchikan District } \\
\text { Prince of Wales District }\end{array}$ & $\begin{array}{l}- \\
-\end{array}$ & Prince of Wales-Outer Ketchikan Census Area & $\begin{array}{l}1968-1981 \\
1968-1981 \\
\end{array}$ \\
\hline & Sitka District & - & Sitka Borough & 1968-1981 \\
\hline & $\begin{array}{l}\text { Haines Borough } \\
\text { Lynn Canal-Icy District } \\
\text { Skagway-Yakutat District } \\
\text { Skagway-Hoonah-Angoon Census Area } \\
\text { Yakutat Census Area }\end{array}$ & $\begin{array}{c}1982-1999 \\
- \\
- \\
- \\
- \\
\end{array}$ & Skagway-Yakutat-Angoon Census Area & $\begin{array}{l}1968-1981 \\
1968-1981 \\
1968-1981 \\
1994-1999 \\
1994-1999 \\
\end{array}$ \\
\hline & $\begin{array}{l}\text { Cordova-McCarthy District } \\
\text { Valdez-Chitina-Whittier District }\end{array}$ & - & Valdez-Cordova Census Area & $\begin{array}{l}1968-1981 \\
1968-1981 \\
\end{array}$ \\
\hline & Wade Hampton District & - & Wade Hampton Census Area & 1968-1981 \\
\hline & Wrangell District & - & Wrangell-Petersburg Census Area & 1968-1981 \\
\hline & $\begin{array}{l}\text { Upper Yukon District } \\
\text { Yukon-Koyukuk District } \\
\text { Denali Borough }\end{array}$ & $\begin{array}{ll}- \\
- \\
-\end{array}$ & Yukon-Koyukuk Census Area & $\begin{array}{l}1968-1981 \\
1968-1981 \\
1994-1999\end{array}$ \\
\hline$\overline{\text { Arizona }}$ & La Paz County & 1994-1999 & Yuma County & $1968-1993$ \\
\hline Hawaii & Kalawao County & $1982-1999$ & Maui County & 1968-1981 \\
\hline New Mexico & Cibola County & $1982-1999$ & Valencia County & 1968-1981 \\
\hline \multirow[t]{5}{*}{ New York } & Bronx Borough & - & Bronx County & 1968-1981 \\
\hline & Brooklyn Borough & - & Kings County & $1968-1981$ \\
\hline & Manhattan Borough & - & New York County & 1968-1981 \\
\hline & Queens Borough & - & Queens County & 1968-1981 \\
\hline & Staten Island Borough & - & Richmond County & 1968-1981 \\
\hline South Dakota & Washabaugh County & - & Jackson County & 1968-1979 \\
\hline \multirow[t]{8}{*}{ Virginia } & Waynesboro City & 1970-1999 & Augusta County & 1968-1969 \\
\hline & $\begin{array}{l}\text { Portsmouth City } \\
\text { Virginia Beach City }\end{array}$ & $\begin{array}{l}1970-1999 \\
1970-1999 \\
\end{array}$ & Chesapeake City & $\begin{array}{l}1968-1969 \\
1968-1969\end{array}$ \\
\hline & Winchester City & 1970-1999 & Frederick County & 1968-1969 \\
\hline & South Boston City & 1968-1995 & Halifax County & 1996-1999 \\
\hline & Williamsburg City & 1970-1999 & James City County & 1968-1969 \\
\hline & $\begin{array}{l}\text { Manassas City } \\
\text { Manassas Park City }\end{array}$ & $\begin{array}{l}1982-1999 \\
1982-1999\end{array}$ & Prince William County & $\begin{array}{l}1968-1981 \\
1968-1981 \\
\end{array}$ \\
\hline & $\begin{array}{l}\text { Nansemond County } \\
\text { Nansemond City }\end{array}$ & $\begin{array}{ll}- \\
-\end{array}$ & Suffolk City & $\begin{array}{l}1968-1974 \\
1970-1981\end{array}$ \\
\hline & Poquoson City & 1982-1999 & York County & 1968-1981 \\
\hline
\end{tabular}

- indicates subdivisions that no longer existed as of the 1990 Census, or were renamed or split after the 1990 Census.

* During the indicated years, population and mortality data are assigned to the subdivision itself.

** All data from Aleutian Islands (1968-1993) were assigned to Aleutians East Borough.

NOTE: The primary political divisions of most states are termed counties. Prior to 1982, Alaskan county equivalents were called districts, divisions, or islands. Since 1982,

Alaskan county equivalents were reclassified as boroughs or census areas. Virginia has many county-equivalent cities that are or were independent of any county organization. 
